\title{
Multi-parameter assessment of mechano-sensitivity driven differentiation of human mesenchymal stem cells
}

\author{
Dissertation for the award of the degree
}

\section{"Doctor rerum naturalium"}

of the Georg-August University Göttingen within the doctoral International Max Planck Research School programm:

"Physics of Biological and Complex Systems"

of the Göttingen Graduate School for Neurosiences, Biophysics, and Molecular Biosciences (GGNB)

\section{Lara Hauke}

from

Celle, Germany

Göttingen, 22.10.2020 



\section{Thesis Committee}

First Member

Dr. Florian Rehfeldt

Experimental Physics I, University of Bayreuth

Second Member

Prof. Dr. Sarah Köster

Institute for X-ray Physics, University of Göttingen

Third Member

Prof. Dr. Stefan Klumpp

Institute for the Dynamics of Complex Systems, University of Göttingen

Members of the examination board:

First Referee

Dr. Florian Rehfeldt

Experimental Physics I, University of Bayreuth

Second Referee

Prof. Dr. Sarah Köster

Institute for X-ray Physics, University of Göttingen

Further Members of the examination board:

Prof. Dr. Stefan Klumpp

Institute for the Dynamics of Complex Systems, University of Göttingen

Prof. Dr. Jörg Enderlein

Third Institute of Physics - Biophysics, University of Göttigen

Prof. Dr. med. Wolfram-Hubertus Zimmermann

Institute of Pharmacology and Toxicology, University Medical Center Göttingen

Prof. Dr. Fred Wouters

Institut of Neuropathology, University Medical Center Göttingen

Duration of thesis: 01.09.2017 - 22.10.2020, Göttingen

Date of Examination: 26.11.2020 



\section{A davit}

I hereby confirm that this thesis has been written independently, with no other sources and aids than quoted. It is based on my own work and has not been submitted for any other degree.

Göttingen, den 22.10.2020, 


\section{Inge}

$1956-2020$

\section{Herbert}

$1930-2020$ 


\section{Abstract}

What factors and conditions control stem cell differentiation is currently one of the major questions in biomedical research. What factors ultimately influence the cells' decision is unclear but it has been shown, that stem cells differentiate reversibly through mechanical clues alone, expressing specific tissue markers as early as several days after plating on a substrate with similar stiffness $[67,86]$.

Stem cells cultured on elastic substrates with different Young's moduli $E$ exhibit distinct structure formation of their cytoskeletal organization already within the first 24 hours. Whether this is a solely mechanical response or is also accompanied and impacted by early transcriptome changes, is an open question. To assess this, I investigate and quantify both structural changes of the cell's cytoskeleton and focal adhesions as well as transcriptomic alterations in cells seeded on polyacrylamide (PAA) gels of different stiffness.

For a quantitative analysis of the cytoskeleton, I further developed the FilamentSensor software for near real-time stress fiber detection. Besides runtime improvement, I added features for the assessment of the mechanical active area and multi-cell separation. I quantified the experimental effects of the lot-to-lot variation, various culture conditions, actin vs. NMM2a staining, and intrinsic variations of mesenchymal stem cells (hMSCs) as they are primary cells. For a data set of 690 time-lapse movies of live hMSCs on different stiffnesses, I showed that cell area, aspect ratio, filament number per cell, and filament width are reliable parameters to distinguish differentiating hMSC on substrates of different stiffness. To enhance the understanding of the mechanical interplay between cell and matrix, I used Metal-Induced and Förster Resonance Energy Transfer (MIET) to understand stress fiber arrangement in $Z$ and the Focal Adhesion Filament Cross-correlation Kit (FAFCK), a novel functionality of the FilamentSensor, to investigate the correlation of stress fibers and focal adhesions.

To investigate the effect of substrate stiffness on the transcriptomic level, I first enucleated cells to rule out de novo transcription and second did full transcriptome RNA-Sequencing of hMSCs after 24 hours on different substrates as well as qRT-PCR of selected targets for multiple time points. Cells seeded on PAA gels show up-regulation in ECM constituent secretion and stress response, but down-regulation in proliferation pathways. The down-regulation of proliferation is more pronounced in higher passage numbers. 



\section{Contents}

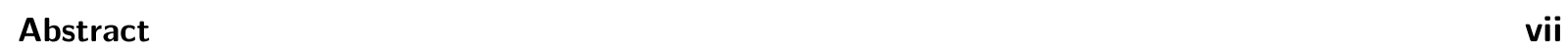

$\begin{array}{ll}\text { 1. Introduction } & 3\end{array}$

2. Mechanosensing of stem cells $\quad \mathbf{5}$

2.1. Human Mesenchymal Stem Cells (hMSCs) . . . . . . . . . . . . . . . . . . . . . 5

2.1.1. Mesenchymal stem cells in science and medicine . . . . . . . . . . . . . 5

2.1.2. Classical hMSC differentiation . . . . . . . . . . . . . . . . . . . . 6

2.1.2.1. Neurogenic differentiation . . . . . . . . . . . . . . . 6

2.1.2.2. Myogenic differentiation ................ . . 7

2.1.2.3. Osteogenic differentiation . . . . . . . . . . . . . . 7

2.1.3. Differentiation of hMSC by mechanical cues . . . . . . . . . . . . . . . 8

2.2. How do cells interact with their environment? . . . . . . . . . . . . . . . . . . 9 9

2.2.1. Cytoskeleton . . . . . . . . . . . . . . . . . . . . . . . 9

2.2.2. Stress fibers . . . . . . . . . . . . . . . . . . 10

2.2.2.1. Stress fibers and related proteins . . . . . . . . . . . . 11

2.2.2.2. Subtypes of stress fibers . . . . . . . . . . . . . . . 11

2.2.3. Anchor points - Focal adhesions . . . . . . . . . . . . . . . . . . . . . 12

2.2.3.1. Focal adhesions as force sensors . . . . . . . . . . . . . . . 15

2.2.4. Reactions to artificial substrates . . . . . . . . . . . . . . . . . 15

3. FilamentSensor software for fast and reliable fiber recognition 17

3.1. Why is a software needed to quantify stress fibers? . . . . . . . . . . . . . . . . . . 18

3.1.1. Parameterization of stress fiber features . . . . . . . . . . . . . . 19

3.1.1.1. Bias in human quantification . . . . . . . . . . . . . . 20

3.2. The FilamentSensor software . . . . . . . . . . . . . . . . . . . . . . . . . . . . . . 20

3.2.1. Fast and accurate recognition of curved filaments . . . . . . . . . . . . . . 22

3.2.2. Cell area . . . . . . . . . . . . . . . . . . . . 22

3.2.3. Aspect ratio . . . . . . . . . . . . . . . . . 23

3.2.4. Order parameter . . . . . . . . . . . . . . . . . 23

3.2.5. Migration statistics ........................ 24

3.3. Novel features in Alpha version . . . . . . . . . . . . . . . . . . . . . . 26

3.3.1. Refactoring ....................... 26

3.3.2. Stack processing . . . . . . . . . . . . . . . . . . . . 27

3.3.3. Bounding boxes for multiple cells in field of view . . . . . . . . . . . . . . . . . 27

3.3.4. Recognition of mechanically active area . . . . . . . . . . . . . . . . . . . . 28

3.3.5. Better runtime: more possibilities . . . . . . . . . . . . . . . . . 29

3.4. The FAFCK: A cross-correlation subroutine . . . . . . . . . . . . . . . . . . . . . . . 31

3.5. MS-TIRF used for imaging of focal adhesion localized paxillin . . . . . . . . . . . . . . 46 
4. Substrates for stem cell differentiation $\mathbf{5 7}$

4.1. Polyacrylamide gel substrates . . . . . . . . . . . . . . . . . . . . . . . . . 57

4.1.1. Glass preparation .................... 57

4.1.1.1. Cover glass preparation . . . . . . . . . . . . . . 58

4.1.1.2. Bottom glass preparation . . . . . . . . . . . . . . 59

4.1.2. Manufacturing of polyacrylamide gels . . . . . . . . . . . . . . . 60

4.1.3. Collagen coating . . . . . . . . . . . . . . . . . . . . . . 62

4.2. Stiffness . . . . . . . . . . . . . . . . . . . . . . . 63

4.3. Possible modulations of PAA gels . . . . . . . . . . . . . . . . . . . . . . 65

4.4. Rheology . . . . . . . . . . . . . . . . . . . . . . . . . 66

4.4.1. Viscoelasticity . . . . . . . . . . . . . . . . . . . . 68

4.5. Rheological measurements of gels used in this thesis . . . . . . . . . . . . . . . . 69

$\begin{array}{ll}\text { 5. Live cell experiments with hMSC } & 71\end{array}$

5.1. Preparation of cells . . . . . . . . . . . . . . . . . . . . . . . . 71

5.1.1. Transfection ..................... . . . 72

5.1.1.1. LifeAct controversy . . . . . . . . . . . . . . . . . . 74

5.2. Live-cell imaging . . . . . . . . . . . . . . . . . . . . . . . . . . . 75

5.2.1. Sample preparation .................... 75

5.2.2. Microscopy ......................... 76

5.2.2.1. The Cell Observer setup . . . . . . . . . . . . . . . . 76

5.2.2.2. Multi-dimensional acquisition with Micro-Manager . . . . . . . . . 77

5.3. Immunostaining . . . . . . . . . . . . . . . . . . . . . . . . . . . 78

5.4. Image analysis . . . . . . . . . . . . . . . . . . . . . . . . . . . . 78

5.4.1. ImageJ . . . . . . . . . . . . . . . . . . . . 78

5.4.2. FilamentSensor ... . . . . . . . . . . . . . . . . . . . . 79

5.5. Results .............................. . . . 79

5.5.1. Different lots of hMSCs . . . . . . . . . . . . . . . . . . . . . 79

5.5.2. Sources of statistical variation in hMSC data . . . . . . . . . . . . . . . 81

5.5.3. Effect of stiffness on untransfected hMSC . . . . . . . . . . . . . . . . . 86

5.5.4. Comparing live cell movies to existing results of different experimental origin . . . 88

5.5.5. Comparison of cells transfected (LifeAct) and immunostained (phalloidin) . . . . 93

5.5.6. Comparison of phalloidin and NMM2a staining for stress fibers . . . . . . . . . . 94

5.6. Evolution of stem cells in the first 24 hours after seeding . . . . . . . . . . . . . . . . 96

5.6.1. Whole cell behavior . . . . . . . . . . . . . . . . . . . . 96

5.6.2. Filament network behavior ....................... 98

5.6.3. Migration ......................... 104

$\begin{array}{lr}\text { 6. MIET } & 107\end{array}$

6.1. MIET ............................ . . . . . . . . . . . . . . . . . . .

6.2. liveMIET ............................. 117

$\begin{array}{lr}\text { 7. Enucleation } & 123\end{array}$

7.1. The contribution of the nucleus to force sensing . . . . . . . . . . . . . . . . 123

7.2. Enucleation . . . . . . . . . . . . . . . . . . . 125

7.2.1. Difference in the enucleation of multiple cell lines . . . . . . . . . . . . . . . . 126

7.2.2. Stress fiber networks of different cell lines . . . . . . . . . . . . . . . . . . . 128

7.2.2.1. Spreading on glass . . . . . . . . . . . . . . . . . . . . . 128

7.2.2.2. Ambivalent behavior . . . . . . . . . . . . . . . 130

7.2.2.3. Spreading on $11 \mathrm{kPa}$ PAA gel . . . . . . . . . . . . . . 131 
7.2.3. Results of enucleation . . . . . . . . . . . . . . . . . . 133

7.2.3.1. Cos-7 cells . . . . . . . . . . . . . . . . . . . 134

7.2.3.2. MCF-7 cells . . . . . . . . . . . . . . . . . . 135

7.2.3.3. NIH-3T3 cells . . . . . . . . . . . . . . . . . . . 137

7.2.3.4. REF-52 (YFP-Pax) cells . . . . . . . . . . . . . . 139

7.2.4. Statistics of cytoplasts fixed after 24 hours . . . . . . . . . . . . . . 141

$\begin{array}{ll}\text { 8. Investigating the transcriptome of differentiating hMSCs } & 147\end{array}$

8.1. RNA extraction of hMSCs on substrates of different stiffnesses . . . . . . . . . . . . 147

8.2. Full RNA Sequencing after 24 hours . . . . . . . . . . . . . . . . 147

8.2.1. KEGG pathway analysis . . . . . . . . . . . . . . . . 149

8.3. Comparison of my results and Wollnik (2015) results regarding sample quality . . . . . 149

8.3.1. Gene and pathway overlap . . . . . . . . . . . . . . . . 152

8.4. Influence of stiffness on early hMSC transcriptome . . . . . . . . . . . . . . 154

8.4.1. Wollnik data $(2015) \ldots \ldots \ldots \ldots \ldots$

8.4.1.1. Transcriptomal differences between gel stiffnesses with controls omitted . 156

8.4.2. Hauke data . . . . . . . . . . . . . . . . . . . . . 158

8.4.2.1. Reduced data set according to Wollnik samples compared to zero-hour

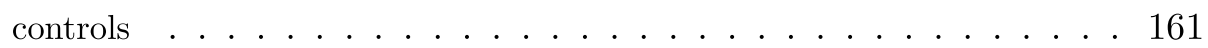

8.4.2.2. Full data set compared to glass and cell culture flasks as controls . . . . 162

8.4.2.3. Full data set analyzed for passage effects . . . . . . . . . . . 163

8.4.2.4. Transcriptomal differences between gel stiffnesses with control groups omitted 165

8.5. qRT-PCR of selected genes for multiple time points . . . . . . . . . . . 167

8.5.1. Why these targets? . . . . . . . . . . . . . . . . 168

8.5.2. Results . . . . . . . . . . . . . . . . . . . . 168

$\begin{array}{ll}\text { 9. Summary } & 173\end{array}$

$\begin{array}{ll}\text { 10. Outlook } & 179\end{array}$

$\begin{array}{ll}\text { A. Appendix } & 187\end{array}$

A. Cell culture . . . . . . . . . . . . . . . . . . . . . . . . . . 188

A.1. Thawing . . . . . . . . . . . . . . . . . . . . . . . . 189

A.2. Freezing . . . . . . . . . . . . . . . . . . . . . . 191

A.3. Splitting . . . . . . . . . . . . . . . . . . . . . . . . 192

A.4. Transfection . . . . . . . . . . . . . . . . . . 193

A.5. Enucleation . . . . . . . . . . . . . . . . . 195

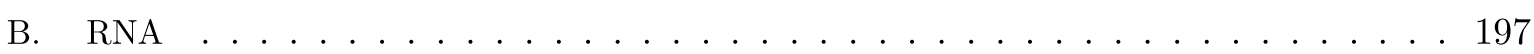

B.1. RNA extraction . . . . . . . . . . . . . . . . . . 197

B.2. Writing RNA in cDNA . . . . . . . . . . . . . . . 199

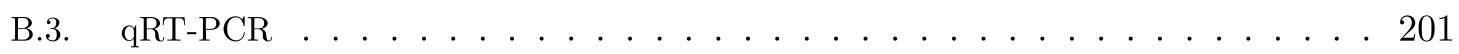

B.3.1. Sample and Plate Preparation . . . . . . . . . . . . . . 201

B.3.2. Software settings and Run . . . . . . . . . . . . . . . 202

C. Polyacrylamide gels . . . . . . . . . . . . . . . . . . . 203

C.1. Cover glass preparation . . . . . . . . . . . . . . . . . . . . 204

C.2. Bottom glass preparation . . . . . . . . . . . . . . . . . 205

C.3. Gels . . . . . . . . . . . . . . . . . . . . 206

C.4. Coating . . . . . . . . . . . . . . . . . 208

C.5. Preparing dishes for live cell imaging . . . . . . . . . . . . . . . . . . . . . . . . . 209

D. Imunostaining . . . . . . . . . . . . . . . . . . . . . . . 210 
E. Live-cell movies . . . . . . . . . . . . . . . . . . . . . . . . . . . . . . . 213

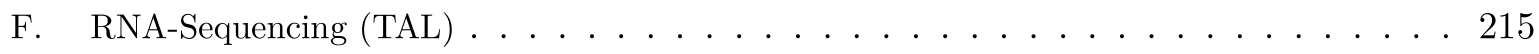

Bibliography 


\section{List of Figures}

2.1. Mechanical cell response to substrate. . . . . . . . . . . . . . . . . . . 8

2.2. Schematic drawing of the cytoskeleton. . . . . . . . . . . . . . . . . . . 9

2.3. Different arrangement of actin fibers in adherent cells. . . . . . . . . . . . . . . . . . . 10

2.4. Composition of stress fiber subtypes. . . . . . . . . . . . . . . . . . . . . . . . 12

2.5. Junction types in adhesive cells. . . . . . . . . . . . . . . . . . . . . . . . . 13

2.6. Proteins associated with focal adhesions. . . . . . . . . . . . . . . . . . . 14

3.1. Comparison of different methods for fiber tracing. . . . . . . . . . . . . . . . . . . . . . . 17

3.2. Differentiation markers in hMSCs. . . . . . . . . . . . . . . . . . . . . . . 19

3.3. FilamentSensor workflow. . . . . . . . . . . . . . . . . . . . . . . . 21

3.4. Migration distance. . . . . . . . . . . . . . . . . . . . . . . . 24

3.5. Migration distance calculations comparison. . . . . . . . . . . . . . . . . . . 25

3.6. MSD curve overview. . . . . . . . . . . . . . . . . . . . . . . . 25

3.7. Interplay between mechanical environment, signalling, cytoskeleton and motility. . . . . . 26

3.8. FilamentSensor Bounding box feature. . . . . . . . . . . . . . . . . . . . . 28

3.9. Comparison of area detection algorithms in FilamentSensor. . . . . . . . . . . . . . . . 29

4.1. Gel polymerization in glass-sandwich. . . . . . . . . . . . . . . . . . . . 58

4.2. Silanization reaction. . . . . . . . . . . . . . . . . . . . . 58

4.3. Glass functionalization reaction with APTES. . . . . . . . . . . . . . . . . . 59

4.4. PAA gel polymerization reaction with TEMED and APS. . . . . . . . . . . . . . . . 60

4.5. Linkage reaction between PAA gel and glass. . . . . . . . . . . . . . . . . . . . 61

4.6. Sulfo-SANPAH activation. . . . . . . . . . . . . . . . . . . . . . 63

4.7. Stiffness in different biological environments. . . . . . . . . . . . . . . . . . . . . 64

4.8. AFM measurement of PAA gels. . . . . . . . . . . . . . . . . . . . . . 65

4.9. Measurement of material deformation. . . . . . . . . . . . . . . . . . . . . 66

4.10. Rheological measurement. . . . . . . . . . . . . . . . . . . . . . . 67

4.11. Storage and loss modulus in PAA gel. . . . . . . . . . . . . . . . . . . . . . . 67

4.12. Cell behavior on PAA gel of different stiffness. . . . . . . . . . . . . . . . . 69

5.1. Nucleofection process. . . . . . . . . . . . . . . . . . . . . 73

5.2. pCMV-LifeAct-TagRFP plasmide. . . . . . . . . . . . . . . . . . . . . . 74

5.3. Bottom glass glueing process. . . . . . . . . . . . . . . . . . . . . . 76

5.4. Live-cell imaging setup. . . . . . . . . . . . . . . . . . . . . . . . . 77

5.5. Area and AR of hMSCs on $11 \mathrm{kPa}$ PAA gel and glass in 24 hours. . . . . . . . . . . . . 80

5.6. Effect of different FBS batches on fixed hMSCs. . . . . . . . . . . . . . . . . . . . . . 81

5.7. Effect of different incubating conditions on fixed hMSCs. . . . . . . . . . . . . . . . 83

5.8. Difference in fit curves. . . . . . . . . . . . . . . . . . . . . . . . . . . . . . . . . . . . 84

5.9. Effect of different fits. . . . . . . . . . . . . . . . . . . . . . . . 85

5.10. Area and aspect ratio differences in untransfected, fixed hMSCs. . . . . . . . . . . 86 
5.11. Area, aspect ratio, and filament number differences in untransfected, fixed hMSCs for whole cell and nucleus. . . . . . . . . . . . . . . . . . . . . . 87

5.12. Difference between two large data sets of untransfected, fixed hMSCs. . . . . . . . . . . 89

5.13. Differeences in five fixed data sets of hMSCs. . . . . . . . . . . . . . . . . . 91

5.14. Area, aspect ratio and filament number in 24 hours hMSC live cell movies. . . . . . . . . 93

5.15. Difference between lifeact and NMM2a staining in hMSCs. . . . . . . . . . . . . . . . . . 94

5.16. Area, aspect ratio, and long half axis in 24-hour live cell movies of hMSCs. . . . . . . . . 97

5.17. Order parameter in 24-hour live cell movies of hMSCs. . . . . . . . . . . . . . . . . 98

5.18. Order parameter convergence in live cell movies. . . . . . . . . . . . . . . . . . 99

5.19. Order parameter difference in fixed cells. . . . . . . . . . . . . . . . . . . 100

5.20. Filament number in 24-hour live cell movies of hMSCs. . . . . . . . . . . . . . . . . 100

5.21. Filament length in 24-hour live cell movies of hMSCs. . . . . . . . . . . . . . . . . . . 101

5.22. Filament angle in 24-hour live cell movies of hMSCs. . . . . . . . . . . . . . . . . 102

5.23. Filament width in 24-hour live cell movies of hMSCs. . . . . . . . . . . . . . . . . . . 103

5.24. Filament curvature in 24-hour live cell movies of hMSCs. . . . . . . . . . . . . . . . . 103

5.25. MSD example curves. . . . . . . . . . . . . . . . . . . . . . . . . . . . . . 104

5.26. MSD in 24-hour live cell movies of hMSCs. . . . . . . . . . . . . . . . . . . . 105

5.27. Trajectories in 24-hour live cell movies of hMSCs. . . . . . . . . . . . . . . . 106

7.1. Nuclear envelope stretching. . . . . . . . . . . . . . . . . . . . . . . 123

7.2. Proteins connecting cytoskeleton with nuclear envelope. . . . . . . . . . . . . . . . 124

7.3. Ultracentrifugation gradient. . . . . . . . . . . . . . . . . . . . . 126

7.4. Nuclear stiffness and lamin composition of different tissues. . . . . . . . . . . . . . . . 127

7.5. Area development in 24 hours of Cos-7, hMSC, MCF-7, and SAOS-2 . . . . . . . . . . 128

7.6. Aspect ratio in 24 hours of Cos-7, hMSC, MCF-7, and SAOS-2 . . . . . . . . . . . . 129

7.7. Filament number in 24 hours of Cos-7, hMSC, MCF-7, and SAOS-2. . . . . . . . . . . 129

7.8. Area development in 24 hours of 3T3, Vero, and NIH-3T3. . . . . . . . . . . . . . . . . 130

7.9. Aspect ratio development in 24 hours of 3T3, Vero, and NIH-3T3. . . . . . . . . . . . . 131

7.10. Filament number development in 24 hours of 3T3, Vero, and NIH-3T3. . . . . . . . . . . . 131

7.11. Area development in 24 hours of 293, HeLa, and REF-52 (YFP-Pax) cells. . . . . . . . . . 132

7.12. Aspect ratio development in 24 hours of 293, HeLa, and REF-52 (YFP-Pax). . . . . . . . 132

7.13. Filament number development in 24 hours of 293, HeLa, and REF-52 (YFP-Pax). . . . . 133

7.14. Area of enucleated Cos-7 cells. . . . . . . . . . . . . . . . . . . . . . . . . . . 134

7.15. Aspect ratio of enucleated Cos-7 cells. . . . . . . . . . . . . . . . . . . . . . 135

7.16. Filament number of enucleated Cos-7 cells. . . . . . . . . . . . . . . . . . . 135

7.17. Area of enucleated MCF-7 cells. . . . . . . . . . . . . . . . . . . . . . . 136

7.18. Aspect ratio of enucleated MCF-7 cells. . . . . . . . . . . . . . . . . . . . . 136

7.19. Filament number of enucleated MCF-7 cells. . . . . . . . . . . . . . . . . . . 137

7.20. Area of enucleated NIH-3T3 cells. . . . . . . . . . . . . . . . . . . . 138

7.21. Aspect ratio of enucleated NIH-3T3 cells. . . . . . . . . . . . . . . . . . 138

7.22. Filament number of enucleated NIH-3T3 cells. . . . . . . . . . . . . . . . . . . . 138

7.23. Area of enucleated REF-52 (YFP-Pax) cells. . . . . . . . . . . . . . . . . . 139

7.24. Aspect ratio of enucleated REF-52 (YFP-Pax) cells. . . . . . . . . . . . . . . . . . 140

7.25. Filament number of enucleated REF-52 (YFP-Pax) cells. . . . . . . . . . . . . . . 140

7.26. Area, aspect ratio and filament number of enucleated C2-C12 cells. . . . . . . . . . . . . 141

7.27. Area, aspect ratio and filament number of enucleated 293 and Vero cells. . . . . . . . . . . 142

7.28. Area, aspect ratio and filament number of enucleated HeLa cells. . . . . . . . . . . . . . 143

7.29. Area, aspect ratio and filament number of enucleated SAOS-2 cells. . . . . . . . . . . . . 144

7.30. Area, aspect ratio and filament number of enucleated 3 T3 cells. . . . . . . . . . . . . 145 
7.31. Area, aspect ratio and filament number of enucleated hMSC cells. . . . . . . . . . . 146

8.1. Percentages for aligned and assigned reads, duplicates and GC content. . . . . . . . . . 149

8.2. PCA plots for Hauke (top) and Wollnik (bottom) data. . . . . . . . . . . . . . 150

8.3. Comparison of sample distance heatmaps. . . . . . . . . . . . . . . . . . . . 151

8.4. Top 20 up- and downregulated pathways in Hauke and Wollnik data sets. . . . . . . . 153

8.5. Heatmap and clustering of the top 50 genes in Wollnik data set. . . . . . . . . . . 155

8.6. Heatmap and clustering of the top 50 genes in Hauke data set. . . . . . . . . . . . 158

8.7. Heatmap and clustering of the top 50 genes in Hauke data set with groupings. . . . . . 159

8.8. Expression difference of vimentin. . . . . . . . . . . . . . . . . . . . . . 169

8.9. Expression difference of Actin Alpha 2. . . . . . . . . . . . . . . . . . . . . 169

8.10. Expression difference of Actin Beta. . . . . . . . . . . . . . . . . 170

8.11. Expression difference of Aniline. . . . . . . . . . . . . . . . . . 170

8.12. Expression difference of myosin heavy chain $9 . \ldots \ldots \ldots \ldots \ldots$

10.1. Preliminary visualization of single filament tracking. . . . . . . . . . . . . . . . . 181 



\section{List of Tables}

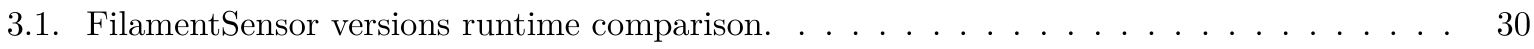

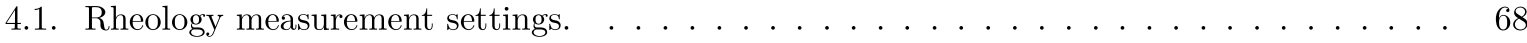

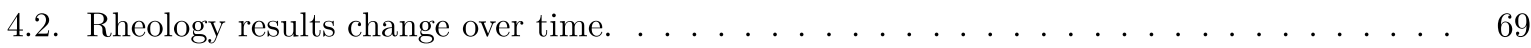

4.3. Rheology results of $11 \mathrm{kPa}$ gels for live cell experiments. . . . . . . . . . . . . . 70

4.4. [Rheology results for RNA experiments.]Results of rheological measurements for RNA experiments. Dashed lines indicate stock solution was not mixed. . . . . . . . . . . . . 70

5.1. hMSC Lots. . . . . . . . . . . . . . . . . . . . . . . . 72

5.2. pCMV LifeAct-TagRFP Plasmid details. . . . . . . . . . . . . . . . . 73

5.3. Effect of different FBS batches on fixed hMSCs. . . . . . . . . . . . . . . . . . 82

5.4. Effect of different incubating conditions on fixed hMSCs. . . . . . . . . . . . . . 84

5.5. Area, aspect ratio, and filament number differences in untransfected, fixed hMSCs for whole

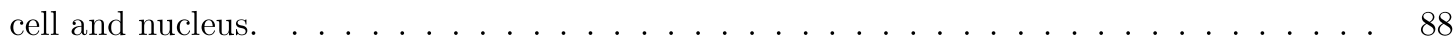

5.6. Difference between two large data sets of untransfected, fixed hMSCs. . . . . . . . . . 90

5.7. Difference in five data sets of fixed hMSCs. . . . . . . . . . . . . . . . . . . 92

5.8. Area, aspect ratio, filament number, and order parameter in hMSC live cell movies and fixed samples. . . . . . . . . . . . . . . . . . . . . . 93

5.9. Difference between LifeAct and NMM2a staining in hMSCs. . . . . . . . . . . . . . 95

7.1. Used cell lines. . . . . . . . . . . . . . . . . . . . . . . . . . . . . . . . 125

7.2. Cell lines surviving enucleation. . . . . . . . . . . . . . . . . . . . . . . 134

8.1. Top 10 upregulated KEGG pathways in Hauke and Wollnik data sets. . . . . . . . . . . 154

8.2. Top 10 downregulated KEGG pathways in Hauke and Wollnik data sets. . . . . . . . . . . 154

8.3. Top 10 upregulated KEGG pathways in the Wollnik data set. . . . . . . . . . . . . . . 156

8.4. Top 10 downregulated KEGG pathways in the Wollnik data set. . . . . . . . . . . . . 156

8.5. Top 50 genes from Wollnik data set with control group omitted. . . . . . . . . . . . . . 157

8.6. The top 50 genes in Hauke data set. . . . . . . . . . . . . . . . . . . . . . . . 160

8.7. Top 10 upregulated KEGG pathways in Hauke data set. . . . . . . . . . . . . . . . . . 161

8.8. Top 10 downregulated KEGG pathways in Hauke data set. . . . . . . . . . . . . . . . 161

8.9. Top 10 upregulated KEGG pathways in Hauke data set vs. glass/plastic. . . . . . . . . . 162

8.10. Top 10 downregulated KEGG pathways in Hauke data set vs. glass/plastic. . . . . . . . . 162

8.11. Top 10 upregulated KEGG pathways in Hauke data set with glass/plastic excluded. . . . 164

8.12. Top 10 downregulated KEGG pathways in Hauke data set with glass/plastic excluded. . . 164

8.13. Top 10 upregulated KEGG pathways in Hauke data set between passages. . . . . . . . . . 165

8.14. Top 10 downregulated KEGG pathways in Hauke data set between passages. . . . . . . . 165

8.15. Top 50 genes in Hauke data set. . . . . . . . . . . . . . . . . . . . . . . 166

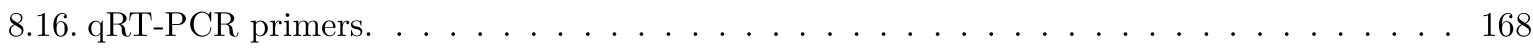

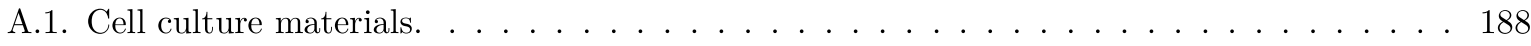


A.2. Used medium and flasks. . . . . . . . . . . . . . . . . . . . . . . . . . 189

A.3. Transfection materials. . . . . . . . . . . . . . . . . . . . . . . . 193

A.4. Transfection solutions. . . . . . . . . . . . . . . . . . . . . . . . . . 194

A.5. Enucleation materials. . . . . . . . . . . . . . . . . . . . . . . 195

B.6. RNA extraction reagents. . . . . . . . . . . . . . . . . . . . 197

B.7. qRT-PCR reagents. . . . . . . . . . . . . . . . . . . . . . . 199

C.8. PAA gel manufacturing materials. . . . . . . . . . . . . . . . . . . . . 203

C.9. Rheometer equipment. . . . . . . . . . . . . . . . . . 206

C.10.Polyacrylamide gel mixtures. . . . . . . . . . . . . . . . . . . . . 206

C.11.Materials for dish preparation. . . . . . . . . . . . . . . . . . . . 209

D.12.Imunostaining materials. . . . . . . . . . . . . . . . . . . . . . 210

D.13.Antibodies used in immunostaining. . . . . . . . . . . . . . . . . . . . 211

E.14.Live cell imaging microscope parts. . . . . . . . . . . . . . . . . . . . . . 213 


\section{Terms and Abbreviations}

\begin{tabular}{l|l}
\hline AA & Acetic acid \\
AA & Amino acids \\
ACAFAs & Actin cap associated focal adhesions \\
Acrylamide & $40 \%$ Acrylamide solution, acrylic amide \\
APS & Ammonium persulfate, oxidizing agent \\
APTES & 3-Aminopropyltriethoxysilan \\
ATP & Adenosintriphosphat \\
Aza & 5-Azacytidine \\
BDNF & Brain-derived neurotrophic factor \\
bFGF & Basic fibroblast growth factor \\
BHA & Butylhydroxyanisol \\
bHLH & basic helix loop \\
Bis-Acrylamide & 2\% Bis-Acrylamide solution, N,N'-Methylenbis(acrylamide), \\
BM-hMSC & cross-linking agent \\
BME & Bone marrow derived human mesenchymal stem cells \\
BMP-2 & Beta-mercaptoethanol \\
BSA & Morphogenetic protein-2 \\
CAS & Bovine serum albumin \\
CNS & Crk-associated substrate \\
Col I & Central nervous system \\
Dex & Rat tail Collagen I \\
DMCS & Dexamethasone \\
DMEM & Dimethyldichlorosilane \\
DMSO & Dulbecco's Modified Eagle Serum \\
DNA & Dimethyl sulfoxide \\
ECM & Deoxyribonucleic acid \\
EGF & extracellular matrix \\
FA & Epidermal-growth factor \\
FDR & Focal adhesions \\
FRET & False discovery rate \\
GAPDH & Förster resonance energy transfer \\
GFAB & Glyceraldehyde 3-phosphate dehydrogenase \\
GLY & (used as housekeeping gene in qRT-PCR) \\
GUI & Glial fibrillary acidic protein \\
FBS & Glycine \\
&
\end{tabular}




\begin{tabular}{|c|c|}
\hline HEPES & 2-(4-(2-Hydroxyethyl)-1-piperazinyl) ethansulfon acid, buffer \\
\hline hMSCs & Human mesenchymal stem cells \\
\hline HYP & Hydroxyproline \\
\hline IBMX & 3-isobutyl-1-methylxanthine \\
\hline KEGG & Kyoto Encyclopedia of Genes and Genomes \\
\hline Lifeact-TagRFP & pCMV Lifeact-TagRFP, actin labelin plasmid \\
\hline LINC & Linker of nucleoskeleton and cytoskeleton \\
\hline McCoy's 5A & McCoy's 5A Medium \\
\hline MIET & Metal-induced and Förster resonance energy transfer \\
\hline MRF-4 & Myogenic regulation factor 4 \\
\hline MS-TIRF & metasurface-based total internal reflection microscopy \\
\hline MSCs & Mesenchymal stem cells \\
\hline MSD & Mean square displacement \\
\hline Myf-5 & Myogenic factor 5 \\
\hline MyoD & Myoblast determination protein 1 \\
\hline N.A. & numerical aperture \\
\hline NHS & N-Hydroxysuccinimid \\
\hline NMM & Non-muscle myosin \\
\hline Nucleofector & Electroporation machine by Lonza \\
\hline P1 (buffers) & Buffer solutions used for electroporation by Lonza \\
\hline PAA & Polyacrylamide \\
\hline PBS & Dulbecco's Phosphate Bufferes Saline, buffer \\
\hline PCR & Polymerase chain reaction \\
\hline PDMS & Polydimethylsiloxan \\
\hline $\mathrm{P} / \mathrm{S}$ & Penicillin/streptomycin, antibiotics \\
\hline PRO & Proline \\
\hline qRT-PCR & Real-time quantitative reverse transcription PCR \\
\hline RA & Retinoic acid \\
\hline RAM & Random-access memory \\
\hline RFP & Red fluorescent protein \\
\hline RNA & Ribonucleic acid \\
\hline RNASeq & RNA sequencing \\
\hline ROCK & Rho-associated protein kinase \\
\hline SEM / sem & Standard error of the mean \\
\hline SE (buffers) & Buffer solutions used for electroporation by Lonza \\
\hline SF (buffers) & Buffer solutions used for electroporation by Lonza \\
\hline SFs & Stress fibers \\
\hline SNP & Single nucleotide polymorphism \\
\hline Std / std & Standard deviation \\
\hline Sulfo-SANPAH & $\begin{array}{l}\text { Sulfosuccinimidyl 6-(4'-azido-2'-nitrophenylamino) hexanoate, } \\
\text { cross-linker }\end{array}$ \\
\hline Syncytium & Cell with multiple nuclei as a result of uninuclear cell fusion \\
\hline TAZ & Transcriptional coactivator with PDZ-binding motif \\
\hline TEMED & Tetramethylethylenediamine \\
\hline TGF & Transforming growth factor \\
\hline TFM & Traction force microscopy \\
\hline
\end{tabular}




\begin{tabular}{l|l} 
TIRF & Total internal reflection microscopy \\
Triton X-100 & Polyethylene glycol alkylphenyl ether \\
Trypsin & $0.05 \%$ Trypsin-EDTA (with Phenol red) \\
UV & Ultra violett \\
WT & Wild-type \\
YAP & Yes-associated protein \\
\hline
\end{tabular}






\section{Introduction}

Wherever matter interacts, forces can be measured. In biophysics, mechanical forces on the cell, cell-tocell, and tissue levels have attracted interest not only as driving forces in healthy processes but also as contributors in various pathological processes. Thus, the biophysical study of these mechanical forces closely relates to the field of medicine and can provide fundamental research.

This not only affects processes in which the mechanical contribution is obvious, such as cartilage loss, scarification, and inflammation, but also metastasis, myocardial infarction, or organ donation. In these cases, changes in tissue stiffness or composition contribute to the medical condition or prevent healing. Especially for myocardial infarction, in which scarification and an increase in tissue stiffness alters electrical wave propagation and leads to heartbeat rate and chronic arrhythmia, mechanical properties are a major topic in recent medical research. One approach there is not to alter the scarification caused by infarction but to bridge these regions with a tissue patch to restore electrical wave propagation. In this case, the tissue patch consists of an ECM-mimicker and either a mixture of endothelial cells, smooth muscle cells, and cardiomyocytes, or human-induced pluripotent stem cells, which can differentiate into any of these cells. Similar tissue patches, composed for specific uses, could offer similar benefits for various conditions, and organoid production for in vivo studies follow a similar pattern of production.

For such applications, mechanical surroundings and cell response have to be understood and monitored to obtain results. In this thesis, I aim to show the differentiation of human mesenchymal stem cells (hMSC) influenced by different substrate stiffness in the first 24 hours, and to quantify these via fluorescence microscopy of the cytoskeletal structures and transcriptome analysis.

It has already been shown that hMSCs can be differentiated by mechanical clues alone. Additionally, it has been demonstrated that this method of differentiation is reversible, in contrast to a chemically induced differentiation. In Chapter 2, I introduce hMSC, and give an overview of their use in science and medicine as well as an overview of differentiation strategies. Furthermore, in this chapter I give a basic introduction of the components of the cytoskeleton and of related structures involved in the mechanical response in a cell. For the microscopy portion of this thesis, stress fibers, which are a main component of the cytoskeleton, are labelled, as well as, in some experiments, focal adhesions, which anchor the cytoskeleton to the surrounding extracellular matrix to exert forces.

In Chapter 3, our software, developed for fast, high-throughput stress fiber analysis, is introduced and described. Additionally, two methods for parallel analysis and correlation of stress fibers and focal adhesions are shown, information that is essential for a full understanding of force generation and transmission between cells and matrix that could also help to improve traction force microscopy analysis. The first method, the MS-TIRF, introduces TIRF of paxillin stained focal adhesions with a large field of view using microstructures. The second, a cross-correlation subroutine of FilamentSensor for stress fiber analysis, provides possibilities to relate stress fiber and focal adhesion data.

Polyacrylamide (PAA) gels covalently coated with collagen are used as substrates for differentiation throughout the thesis. Chapter 4 introduces the fabrication of these gels as well as the concept of stiffness and rheology as a methodology to measure this. Possible modulations of PAA gels are also briefly discussed. These first three chapters provide the background information for the following ones. In Chapter 5, all 
microscopy data concerning the stem cell differentiation and generation thereof is shown. This includes not only live cell movies of differentiating hMSCs and comparison to past experiments, but also quantification of other effects such as staining targets, staining protocol, and intrinsic variation in hMSCs. In this chapter, epifluorescence microscopy is used, which is limited in $z$-resolution but can provide enough photons for long-term live cell measurements of whole cells. In contrast to this, in Sections 3.5 and 3.4, only structures in the plane closest to the surface were imaged. In Chapter 6, a combined metal-induced and Förster resonance energy transfer (dc-MIET) is described as a method to measure distance between stress fibers and the surface, as well as distance from other structures (in this case, vinculin-labelled focal adhesions). The resulting measurements of inclination angles of stress fibers are shown both in fixed and live cells, introducing liveMIET as a method to obtain more detailed information about the stress fiber architecture in 3D in living cells over time.

To elucidate further the role of immediate transcriptional changes and de novo protein production in the early regime of mechanical cell-matrix interaction, enucleation of hMSCs was attempted to determine whether differentiation-related stress fiber patterns occur in the absence of the nucleus was a reasonable approach. Chapter 7 gives an introduction to the role of the nucleus in the mechanical response of the cell, and decribes the enucleation protocol used. It shows the enucleation attempt not only of hMSCs, but also of various other cell lines, for which it also gives short descriptions of cell and stress fiber networks in a non-enucleated state.

In Chapter 8, a transcriptome analysis is presented. This is done first as an RNA-Seq. The analysis is also compared to previous results. Samples described in this chapter are seeded on six different substrates, which consist of three PAA gels and three different controls. The analysis not only gives top genes and KEGG pathways for the different substrates' stiffnesses, but also compares controls and evaluates differences between them. Second, for specific targets, qRT-PCR of samples of various stiffnesses and for multiple time points is shown.

In the discussion, (Chapter 9), all results are compiled. The Outlook chapter, (Chapter 10), hints at possible future developments that could result from applying and expanding upon this thesis. Lastly, in the Appendix, (Chapter A), all protocols are reproduced in detail, including materials used. The Appendix also contains the list of references and the author's curriculum vitae (CV). 


\section{Mechanosensing of stem cells}

\subsection{Human Mesenchymal Stem Cells (hMSCs)}

Throughout this thesis, hMSCs are primarily used for early onset differentiation experiments. These cells are easy to isolate in huge numbers from the bone marrow of donors, and research suggests they can be expanded for up to 20 passages without losing their characteristics [37, 213].

While all cells that can differentiate into other cell types are grouped under the label of "stem cells", these cells differ in the number of cell types into which they can differentiate. Totipotent stem cells are found in zygotes and can form a whole organism. In later stages of development, cells lose totipotency and become pluripotent, meaning they can differentiate into any of an organism's cell types. In adults, only unipotent and multiipotent stem cells are found in bone marrow, fat tissue, or molars. These uni- or multipotent stem cells, in contrast to embryonic stem cells, lose their differentiation potential over time [76, 116]. They can become a limited number of cell types from either one or two cell layers, respectively. These layers are the endoderm, mesoderm, and ectoderm. The ectoderm consists of skin and neural cells. The mesoderm comprises muscles, kidneys, blood cells, bones, cartilage, and fat. The endoderm's main parts are the pancreas, liver, lung, and the thyroid. Mesenchymal stem cells are multipotent, and can transdifferentiate into ectodermal and endodermal lineages.

In this thesis, I use human mesenchymal stem cells extracted from bone marrow (BM-hMSCs) of donors (Lonza, PT-2501). BM-hMSCs have been shown to differentiate not only into osteoblasts, chondrocytes, and adipocytes (mesodermal cell types), but also into fibroblasts, and neural cells (ectodermal) [129, 150, 161].

They fulfill the International Society for Cellular Therapy's general definition of human MSCs (hMSCs), which requires MSCs to express CD73, CD90, and CD105, but be free of expression of CD11b, CD14, CD19, CD34, CD45, CD79a, and HLA-DR [56]. Also, like all stem cells, they can follow two patterns for cellular division. The symmetric pattern will lead to two stem cells, whereas the asymmetric pattern will yield one stem and one committed cell [181]. These division patterns yield mutations at a different rate, with the asymmetrical division leading to more carcinogenic mutants [181]. For clinical studies, another property of hMSCs is usually of interest: they can secrete immunological active cytokines and present immune relevant receptors that interact with, and alter the immune environment of, the host [213].

\subsubsection{Mesenchymal stem cells in science and medicine}

The potential of hMSCs to influence the immune system is of great interest in medicine, especially in replacement therapy. Reviews find that most applications are tested in the fields of autoimmune, cardiovascular, and neurodegenerative diseases [9, 20, 1, 213, 238].

Although theses uses of hMSCs are promising in theory, there are several reported incidents of neoplastic tissue and lesions at stem cell injection sites both in animals and humans [6, 77, 141, 206]. Because most experiments with hMSCs in a medical context use chemically guided differentiation, it also has to be explored whether tumorigenesis occurs in cases of mechanically guided differentiation. A common 
application of hMSCs is the restoration of cartilage, either by direct injection of stem cells or by insertion of a matrix seeded with stem cells [186]. For type 1 diabetes, clinical trials injecting MSCs to stabilize pancreatic islets are on-going; this would allow for treatment without requiring donated pancreases - which are rare $[87,182,194]$. MSC injections also show a slightly positive effect in patients with type 2 diabetes, leading to higher insulin production, although the mechanism is unclear [212]. Additionally, seeding damaged neuronal tissue with neuronal progenitor cells has been shown to induce central nervous system repair [209].

Stem cell grafts could also be used for the treatment of more complex medical issues such as cardiac diseases. After a cardiac event, the scarred muscle often hinders proper contraction [16], resulting in prolonged side effects and subsequent cardiac events [49]. While some groups have experimented with a wire sleeve around the heart [229], forcing it to beat, another idea is to replace damaged tissue using stem cells, with or without a suitable matrix [172]. However, all of this approaches come with a different set of risks that have to be assessed.

Another issue is the quality control of the cells that are used. For initial in vitro experiments, this might not be the focus of researchers; but for injection into humans, the highest standards have to be met. Cell viability, endotoxin assays, and oncogenic tests have to be performed. For each treatment, optimal dose and administration are crucial [150]. Additionally, cells meeting these standards have to be available in large numbers and low passage to be considered an option for routine treatment, as they lose their stemness with increasing passage number [150].

\subsubsection{Classical hMSC differentiation}

Since it is crucial to understand the mechanism of differentiation in order to advance clinical applications of hMSCs, I give a short overview here of distinct differentiation techniques in vitro. Research has found several ways to differentiate them into desired lineages. Multiple chemicals and hormones can be used with varying outcomes and side effects, as is explained in detail in each subsection. However, these side effects often prevent the critical development from basic cell culture experiments to clinical trials in patients. Thus, they indicate the importance of exploring non-chemical alternatives.

\subsubsection{Neurogenic differentiation}

Chemical agents for neurogenic differentiation applicable to hMSCs are remarkable, as mesenchymal stem cells were originally defined as unipotent stem cells of the mesenchymal lineage. Neural cells, in contrast, belong to the ectodermal lineage. After successful attempts to get functional neurons from hMSCs, their classification has been revised from unipotent to pluripotent stem cells. Nevertheless, we know of many pluriponent stem cells, like beta-mercaptoethanol (BME) [148], 3-isobutyl-1-methylxanthine (IBMX) [20, 209], butylhydroxytoluene, butylhydroxyanisol (BHA) [209], epidermal-growth factor (EGF) $[113,170,176]$, and brain-derived neurotrophic factor (BDNF) $[20,30,176]$. Dimethyl sulfoxide (DMSO) [38] and 5-Azacytidine (Aza) [11, 119, 240] have to be named here as well, as myogenic differentiation agents.

In humans, it has been shown that bone marrow-derived hMSCs (BM-hMSCs) are able to differentiate into functional astrocyte-like cells [10, 40], as well as towards neuroglia [22] and neuron-like cells [70, 223, 237, 238]. Additionally, BM-hMSCs can fuse with Purkinje neurons and develop into functional central nervous system (CNS) neurons [113], and can also produce action potentials [70].

In rats, there has been successful differentiation of MSCs into excitable neuron-like cells $[100,166]$. Differentiation towards nestin-positive neurospheres can be archived in the presence of EGF and basic fibroblast growth factor (bFGF) $[113,166]$. After discarding mitogens, this combination can also yield neurofilament-positive neurons or glial fibrillary acidic protein (GFAP)-positive glia [113]. MSC-derived 
rat neuron-like cells can be transferred into injured rat brains and integrated into the transected spinal cord [238]. Also, after injecting MSCs into the postnatal murine brain, a phenotype change towards astrocytes and neurons can be observed [120].

\subsection{Electrical stimulation}

In addition to chemical agents, electrical currents are used to enhance neurogenic differentiation. Here, the applied frequency is important. While $50 \mathrm{~Hz}$ leads to neuronal differentiation-over 6 days in neuronal induction medium [42] or 12 days in non-hematopoietic cell medium [40] — a frequency of $10 \mathrm{~Hz}$ will lead to osteogenic differentiation instead [47].

\subsubsection{Myogenic differentiation}

There are some biological factors known to contribute to skeletal muscle differentiation, of which myoblast determination protein 1 (MyoD), Myogenic factor 5 (Myf-5), myogenic regulation factor 4 (MRF-4), and Myogenin are best understood [104, 139]. These factors can be used to differentiate cells towards a myogenic lineage, but other methods have been proven to work as well [11, 18, 72]. I describe each of these common myogenic differentiation agents here.

MyoD is a protein of the bHLH (basic helix loop) family, which binds to DNA and acts as the regulatory factor in myogenesis. MyoD can convert cell types like fibroblasts, chondroblasts, smooth muscle cells, and retinal pigmented epithelial cells into striated myoblasts and myotubes [41, 74, 203, 220]. In contrast, Myf-5 is a reporter gene specific to muscles. It is inactive in non-muscle cells but leads eventually to the formation of a myogenic phenotype [41, 50]. MFR-4 can determine whether MyoD and Myf- 5 are absent, acting as a determination gene upstream of $\mathrm{MyoD}$ and Myf-5 to direct multipotent cells into the myogenic lineage [104].

Finally, Myogenin is an essential muscle differentiation factor causing the fusion of myoblasts to multinucleated myofibers. Interestingly, a downregulation of myogenin in mouse myotubes reverses their differentiation and causes cell cycle re-entry via downregulation of MyoD [83, 139, 180].

Chemicals usable for muscle differentiation are mostly 5-Azacytidine (Aza) and, to some extent, L-ethionine. The toxicity of Aza is proven, but whether the toxic effects exceed the differentiation effects is disputed $[11,119,240]$. Despite these disputes about its toxicity, Aza has been used to differentiate cells in myogenic or cardiomyogenic lineages. Lower concentrations $(<15 \mu \mathrm{l})$ seemingly favor the expression of myogenin in hMSCs, while higher concentrations $(>15 \mu \mathrm{l})$ favor expression of Troponin T, a cardiac-specific marker [200]. Using Aza for muscle differentiation in this way, a decrease in proliferation speed was observed but viability was not affected [239]. Applying Aza to human umbilical cord-derived mesenchymal stem cells (hucMSCs) leads not only to the expression of myogenic and cardiac-specific markers, but also to a decrease of stem cell-specific markers such as Sox2 and Nanog [165].

L-ethionine is used for erythroid and myeloid maturation. It arrests cells in the G1 phase of the cell cycle by inhibition of DNA and tRNA maturation [4].

\subsubsection{Osteogenic differentiation}

There are few chemicals able to induce osteogenic differentiation in mammalian cells, and they are often used in combination. These are Dexamethasone (Dex) [92, 114, 171, 173], the morphogenetic protein-2 (BMP-2) [99, 127, 171], beta-glycerophosphate (beta-GP) [92], and ascorbic acid (AA) [92, 99, 127].

Dex is a steroid commonly used in osteogenesis and osteoblast maturation, inducing an osteoblast-like phenotype and increasing mineralization [99, 114]. However, high concentrations reduce viability and enhance phosphatase activity [114]. 
To also stimulate collagen production, BMP-2 - from the transforming growth factor (TGF) superfamily - can be used [127]. It induces type I collagen production and is involved in chondro-osteogenic differentiation $[5,30]$.

Beta-GP becomes hydrolyzed by alkaline phosphatases bound to the membrane extracellular of bone cells. This leads to a rapid (nonapatitic) mineral deposition [92].

One very useful chemical, because it is less toxic, is AA. $0.25 \mathrm{mM}$ of AA stimulates cell proliferation $[32,127]$. Exposure to AA for 10 hours activates post-transcriptional mechanisms that enhance collagen synthesis levels, but not collagen mRNA [32]. In human fibroblasts, exposure to AA and $\mathrm{Fe}^{2+}$ increase collagen gene transcription, mRNA, and production [43].

\subsubsection{Differentiation of hMSC by mechanical cues}

In recent years there has been the realization that stem cells can be differentiated using mechanical clues, foremost stiffness of the substrate, thus avoiding the side effects of chemical differentiation $[67,86]$. Figure 2.1 gives a short overview of the coupling of substrate and cell reaction.

Because cells in tissues are mechanically coupled to their neighbors and the extracellular matrix (ECM) through cell-to-cell and cell-matrix junctions (see Figure 2.3), it is straightforward to assume that cells must have means to sense their surroundings in order to adapt to changes or communicate [95, 155]. Further, motile cells need a way to exert forces in order to move through the bloodstream or tissues, close wounds, or engulf particles [134]. These are performed with the help of the cytoskeleton, which consists of actin stress fibers, microtubules, and intermediate filaments, and which forms a backbone of filamentous structures inside the cell (see Figure 2.2).

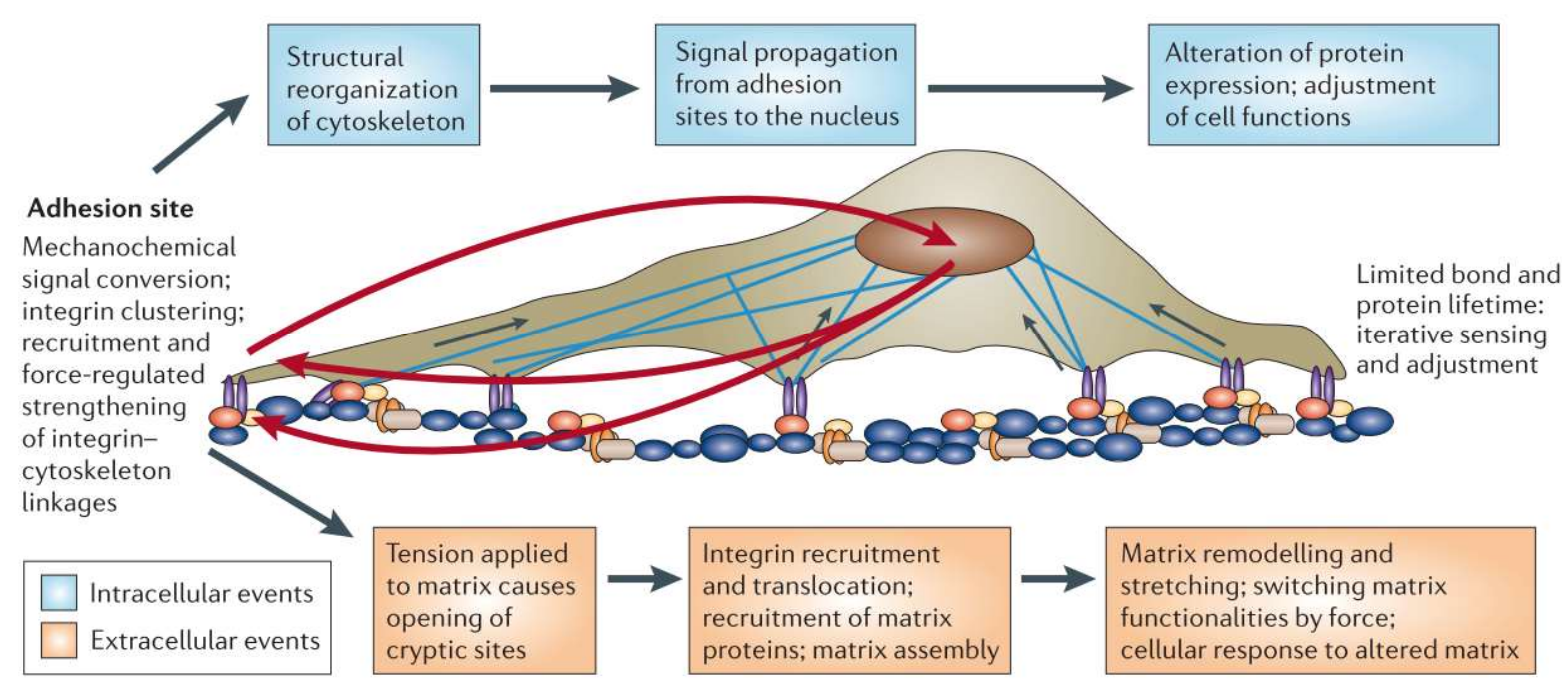

Figure 2.1.: Schematic of basic mechanical cell response to substrate [216].

Of these components, the most important for the mechanical feedback are stress fibers. These fibers connect focal adhesions, the anchor points for force transmission to neighboring cells or substrates [54]. Forces can be generated by myosin motor proteins, which move along the stress fibers while remaining attached to other stress fibers, thus shortening or lengthening stress fiber bundles [35, 34]. This stress fiber network is connected not only to the cell membrane but also to the nuclear membrane, enabling the cell to use the nucleus as a counterweight to probe the stiffness of the surrounding [216]. This stress on the nucleus, however, influences the morphology of the nucleus and, presumably, the availability of certain chromosomes for transcription. This is because chromosomes are connected to the nuclear envelop 
and located in specific domains. With alteration of the availability for transcription, the transcriptome, proteasome, cell behavior, and finally differentiation are each affected. This is how substrate stiffness directly influences differentiation $[28,201,205]$. Here, we see that a certain stiffness range of the substrate is necessary. For optimal results in already differentiated cells, the stiffness should match the tissue [66]. For stem cells, differentiation will depend on the substrate stiffness. The cells will differentiate into those cells for which the surrounding substrate has optimally defined stiffness. [65, 155, 210].

Although it is clear that stem cells differentiate based on the mechanical forces imposed on them by the surrounding substrate, we must still figure out which component of the mechanical surrounding is the main contributor to the differentiation [137]. Is the stiffness alone sufficient, or do we also have to take into account surface coating, surface topology, and other factors like material? There have been recent attempts not only to mimic a tissue as closely as possible, but also to find sufficient materials that are easy to use in cell culture $[115,131,133,188]$. For example, one can grow cells on titanium oxide tubes manufactured by galvanization. These are of course much stiffer than any tissue, but mimic nanoscopic topology and seem to provide sufficient support for cells [132, 138, 149, 175].

An interesting fact is that, in contrast to those produced by chemical differentiation, mechanically differentiated cells are not fixed in their path. Cells grown for up to 2 weeks on $11 \mathrm{kPa}$ gels and that show signs of myogenic differentiation can be seeded to substrates with different stiffness and readjust within 24 hours [121].

\subsection{How do cells interact with their environment?}

Before we can try to understand the cells' interactions with the environment, we have to get an idea about the complex mechanisms inside the cell. There are three basic structures involved in force generation within the cell: focal adhesions, as contact points to the surrounding; stress fibers, as part of the cytoskeleton interconnecting the focal adhesions; and, finally, myosin motor proteins lengthening and shortening the fibers, thus exerting forces $[31,35,155]$.

\subsubsection{Cytoskeleton}

A cell is often imagined as a spherical object when unattached, and half-spherical when attached to a 2D substrate. However, the shape of a fried egg is closer to the truth. The nucleus is slightly compressed, but it is still the highest point of the cell by far. All other structures are close to the surface of the cell, with almost no height difference between them $[28,39]$.

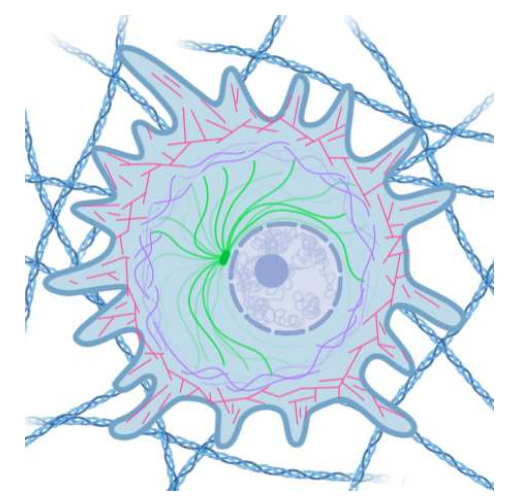

Figure 2.2.: Schematic drawing of the cytoskeleton. Microtubules in green, intermediate filaments in violet, and stress fibers in red, shown from atop. 
In the cell, the cytoskeleton is used to transport cell organelles, proteins, and vesicles, or to aid in migration. The cytoskeleton consists of stress fibers, microtubules, and intermediate filaments; the ratio and composition vary with cell type. All these filaments work together, along with the help of multiple proteins, including motors and linkers, during cell adhesion and migration [187, 214].

Microtubules are anchored to the nucleus and are mainly located in the cell body [241]. They are used by kinesin and dynein motors to transport loads [167]. Because microtubules are anchored at the nuclear membrane, they are important for cell division [226] and interact with the stress fiber network [55, 219, 215]. Intermediate filaments are not used by motors to transport loads but seem to be involved in cell signaling [68]. The main intermediate filament in hMSCs is vimentin, which is believed to be important for many cellular functions and the morphology of the cell [45].

Focal adhesions, which connect the cell to a substrate, have polar structures stretching throughout the cell between them. These structures are actin stress fibers. Myosin motor proteins use the network of stress fibers to transport vesicles. The stress fibers can exert forces towards the surrounding substrate, and thus the stress fiber network and the dynamics of the force it exerts will be the focus of this thesis [19]. Microtubules and intermediate filaments will not be discussed further.

\subsubsection{Stress fibers}

Stress fibers are composed of actin and non-muscle myosin. Actin is a well-studied filament of monomers (42 kDa, 375 amino acids, called G-actin) interacting with many proteins and protein complexes. A stress fiber usually consists of 10-30 actin filaments. On the opposing sites of the monomer, a hydrolytic site and an adenosintriphosphat (ATP) binding site are located. Binding of the monomers occurs when dephosphorylation of ATP causes a conformational change [208]. In the growing filament, the ATP-containing end is called the barbed end, while the hydrolytic end is called the pointed end. The ATP end is the faster-growing end; because of this, in some descriptions it is also called the plus end. This polar-structural arrangement is used by motor proteins, most prominently non-muscle myosin (NMM), which belong to the myosin motor protein subfamily of motor proteins. These create motion and stresses by "walking" along the actin fibers.

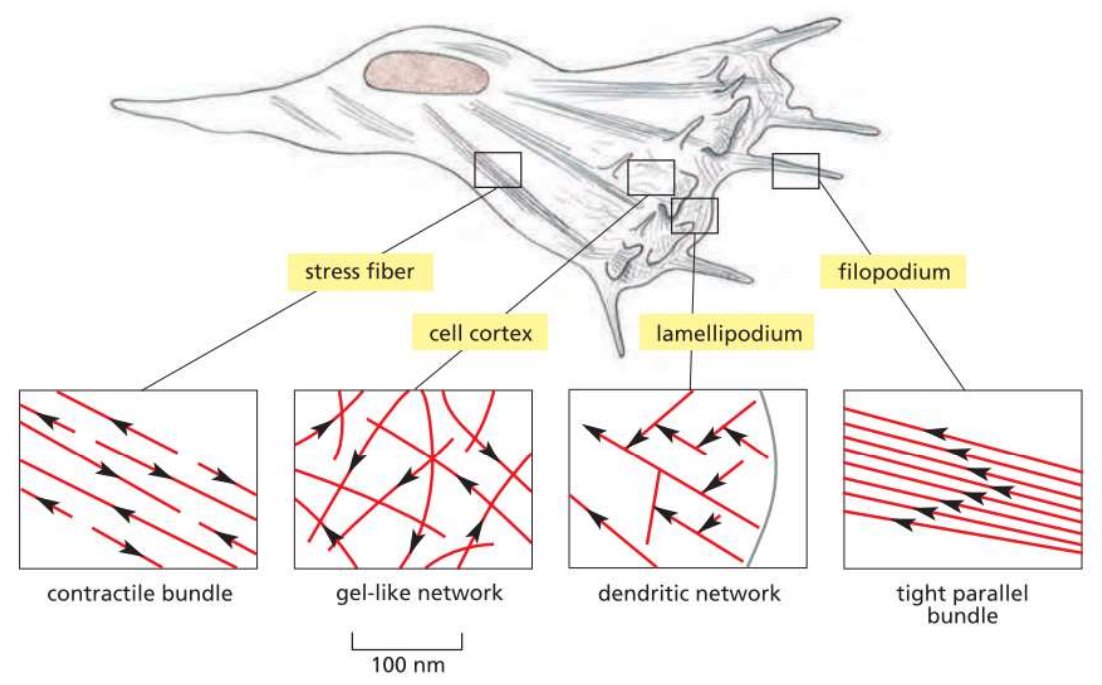

Figure 2.3.: Overview of different arrangements of actin fibers in adherent cells. Actin fibers are shown in red, arrowheads pointing to the plus end. Contractile stress fibers exert tension, cell cortex underlies plasma membrane, filopodia are projections of the plasma membrane to explore the environment. From [2]. 


\subsubsection{Stress fibers and related proteins}

Besides NMMs, several other proteins interact with the actin stress fibers. Where NMMs use the actin network for transport of vesicles and organelles, and move the fibers to accomplish this, other proteins are involved in bundling, nucleation, depolymerization, or interlinkage to other structures such as focal adhesions or the nucleus. In muscle cells for example, bundles of myosin motor proteins - in structures called sarcomeres - contract actin fibers by moving the fibers in opposite directions [106].

The formation of stress fibers is mainly regulated by Rho GTPases. These activate ROCK (Rho-associated protein kinase), and subsequently the nucleation and polymerization of actin filaments, through formins. ROCK also mediates activation of myosin, which accumulates at the actin filaments and leads to the assembly of stress fibers, and inactivates cofilin, the protein responsible for disintegrating stress fibers [19]. In fully developed stress fibers, $\alpha$-actinin bundles the actin filaments by alternating polarity [190]. $\alpha$-actinin can be found periodically along the stress fibers accompanied by tropomyosin, zyxin, and caldesmon [27]. Other associated proteins are filamin and fascin [106].

The contraction of stress fibers is facilitated by myosins and cross-linkers. NMM II motor proteins walk on parallel filaments or bundles in opposite directions using focal adhesions as handles, causing stress throughout the cell [35]. NMM II-mediated contractility - in close cooperation with associated focal adhesions - also regulates other spatial and temporal cellular activities, like directional cell migration, establishing the front-to-back polarity, ECM remodeling, and retraction of the trailing edge [117, 214]. Also, NMM II-derived force plays an important role in actin assembly and disassembly, as well as in alignment in migrating cells by inhibiting VASP phosphorylation, fiber elongation, and ADF/cofilin-mediated disassembly of non-contractile fibers [207].

Most of the stress fiber subtypes are connected to focal adhesions at one end at least [26] via actin cross-linking proteins like Arp2/3 and vinculin [34]. When forces enacted on the focal adhesions are too high, these complexes disengage. At this time, tension-sensitive proteins are released from the adhesion complex [73]. The integrin clusters are left behind, while the stress fibers are repaired by zyxin, $\alpha$-actinin, and VASP [192].

Historically, one distinguishes between different types of stress fibers according to the position in the cell—but there are differences in composition, too (see Figure 2.4).

\subsubsection{Subtypes of stress fibers}

Recently, various experimental techniques have been developed to elucidate both the mechanical properties and protein composition of single filaments. Subcellular laser ablation gives insight into force load on single stress fibers, as well as their connections to other structures $[105,122]$. Protein knockouts, or fluorescently labeled proteins, give insights into both localization and function of specific proteins. Nowadays, we know that stress fibers can be distinguished not only visually through their location inside the cell but also via their biochemical composition - especially NMM-IIA and NMM-IIB content-and thus the fibers must fulfill different roles inside the cell $[25,27,106,123,128,202]$. Subtypes of stress fibers are:

- Dorsal stress fibers connect the leading edge of the cell to the nucleus, and thus play an important role in adhesion regulation in the leading edge. They lack myosin II and develop through formindriven actin polymerization at the focal adhesion site[19].

- Transverse arcs are a type of stress fiber consisting of Arp2/3-nucleated actin bundles connected to myosin bundles. They are usually not connected to focal adhesions but rather to other stress fibers. These other fibers, for example dorsal stress fibers, use transverse arcs to mediate the tension of the leading edge adhesion $[3,85]$.

- Ventral stress fibers maintain adhesion at, and retraction of, the trailing edge. They are connected to focal adhesions at both ends and are also connected to both other types of stress fibers. They are 
important for cell migration and regulate the polarity of the cell [160]. Ventral stress fibers are less understood than transverse arcs. It has been proposed that transverse arcs and dorsal stress fibers fuse to generate ventral stress fibers in the central region of the cell [85]. However, simultaneous formation of transverse arcs and ventral stress fibers has been documented, contradicting this proposal $[13,123]$.

While in 2D these definitions are reasonable and even visible in the cell, in 3D they are harder to follow. Polarization, directed movement, and stress fiber compositions remain present in 3D, although they are harder to observe and probe. Further complicating potential research, both mechanical probing and protein alterations disturb the biological behavior of the cell we want to study. For prolonged studies on living cells or tissues without alteration of their morphology or behavior, there must be other approaches. Here, localization can be the key. When we have evidence for a strong correlation of protein content and localization, thus stress fiber type and localization, we can try to classify stress fiber dynamics with longterm fluorescent microscopy. In the following, we attempt to group stress fibers by localization, nucleation speed, length, and persistence over time, and gather information about cytoskeletal processes inside the cells.

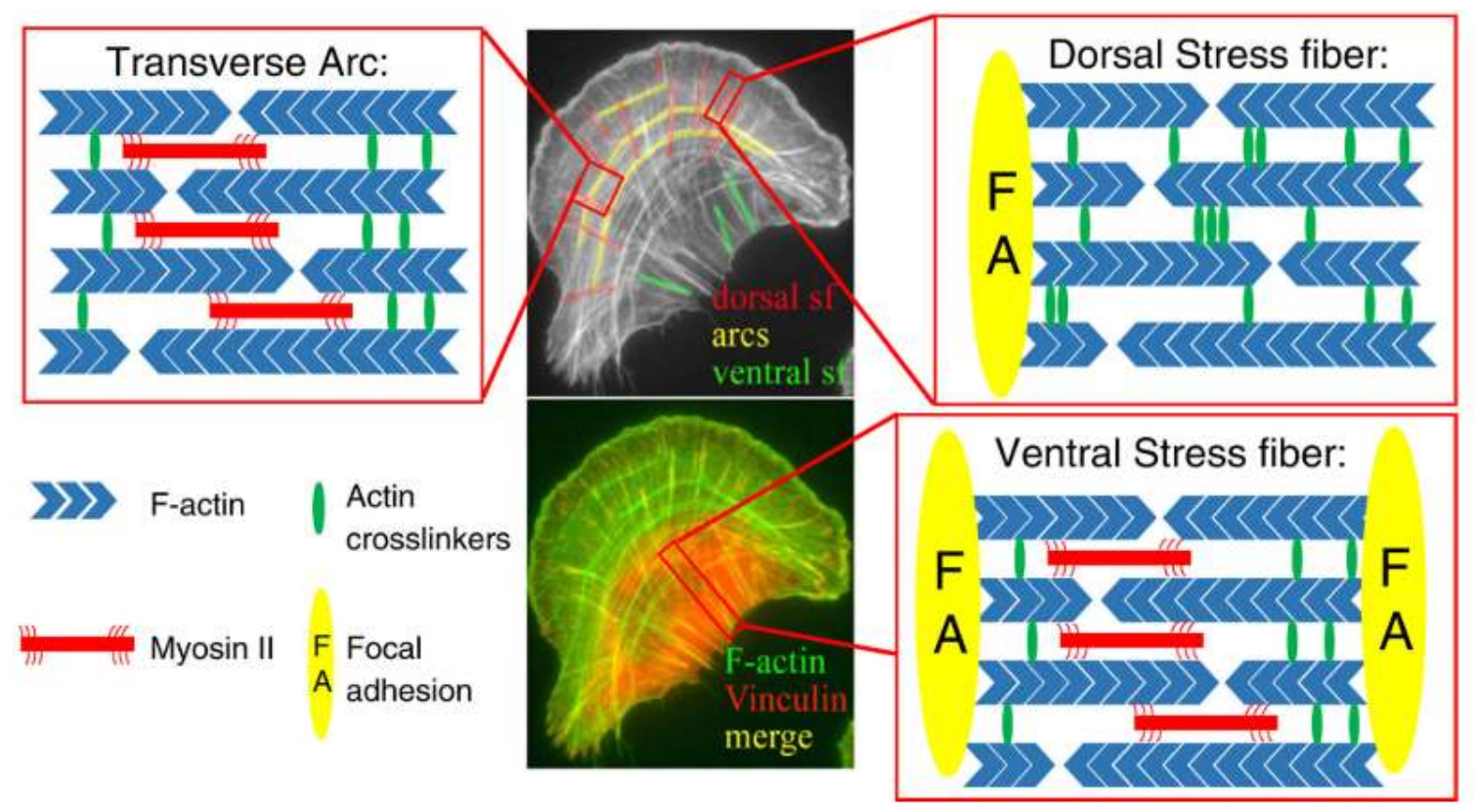

Figure 2.4.: Schematic of the occurrence of focal adhesions, motor proteins, and cross-linkers in different stress fiber subtypes, taken from [106].

\subsubsection{Anchor points - Focal adhesions}

To exert forces, the stress fibers contracted by myosin motors must have stable anchor points and sense forces (see Subsection 2.2.3.1). When considering single cells on artificial substrates, as in this thesis, actin-linked cell-matrix junctions, or focal adhesions, are of great importance.

Focal adhesions are point-like adhesions scattered over the cell's contact surface with the substrate. Cells on stiffer substrates have more focal adhesions [118]. The descriptions here are written from a 2D view. The ways adhesions are distributed and maintained in 3D differs [154].

Focal adhesions usually develop under induction by small GTPase Rho-A (which also activates myosins that bundle actin filaments to stress fibers). In the cell periphery, focal adhesions are connected to stress fibers via integrins and vinculin. Vinculin also recruits talin, which connects integrins to filamentous actin (f-actin). Talin reacts to stress and facilitates the growth of focal adhesions by exposing new vinculin 
binding sites after stretching. The growth of focal adhesions is called focal adhesion maturation. While they mature and recruit integrins linking the cytoskeleton to the substrate, the focal adhesions change shape from a point-like to an oval, elongated shape. This mature focal adhesion can contain over 60 different proteins including vinculin, paxillin, talin, zyxin, tyrosine-phosphorylated proteins, and focal adhesion kinase [154, 236]. A large, mature focal adhesion found at the edge of he lamellipodium is called a focal complex [19].

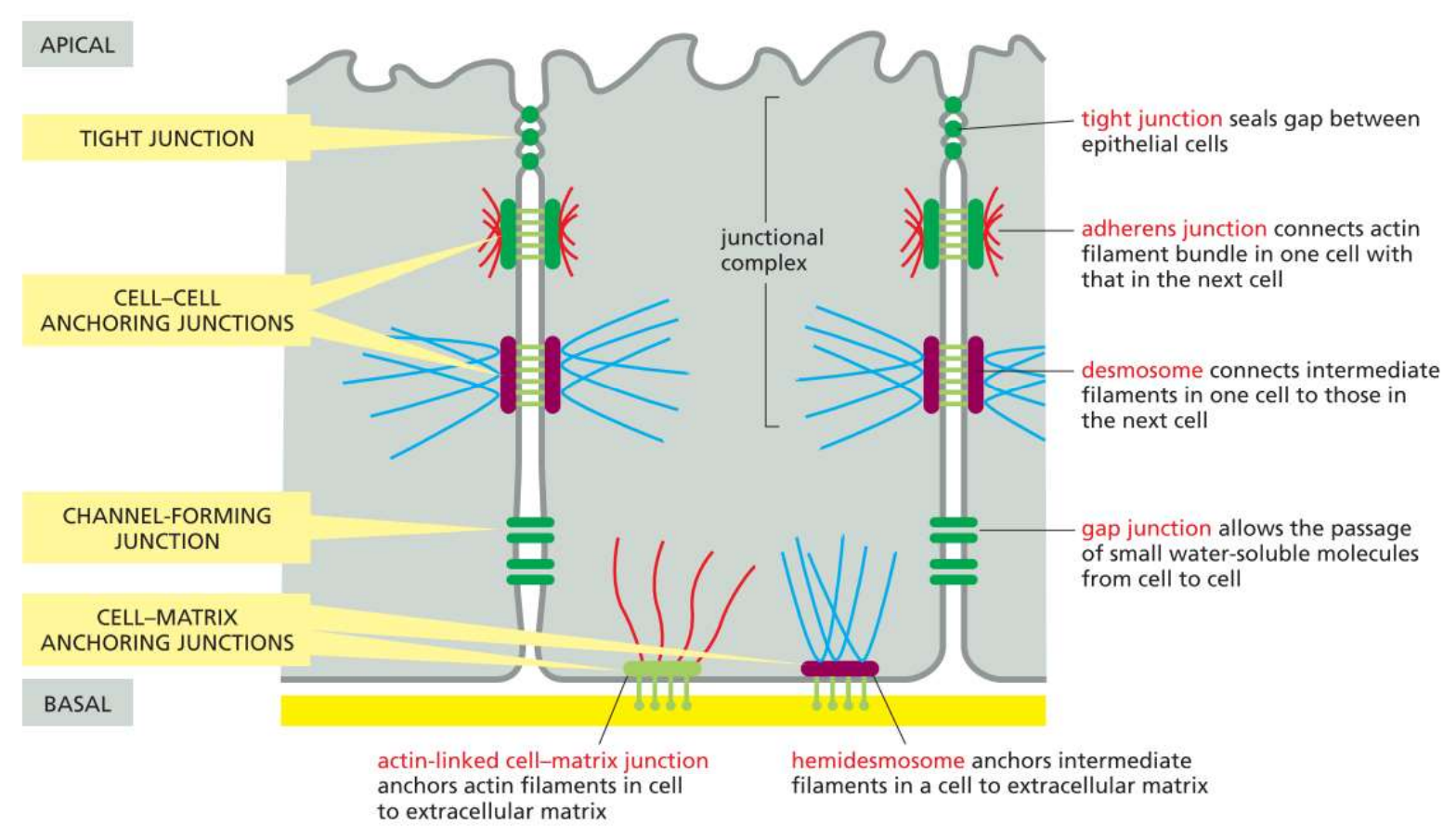

Figure 2.5.: Overview of junction types in adhesive cells, from [2].

The focal adhesions are interconnected by ventral stress fibers. The vinculin bound to the focal adhesion provides binding sites for the actin-cross-linking proteins like Arp2/3. Arp2/3 is also necessary to form lamellipodia. Cells lacking Arp2/3 show no haptotaxis and also fail to globally align focal adhesions [228]. Forces exerted on the stress fibers by NMM II activity contribute to the maturation of focal adhesions by stress-responsive proteins [26]. However, it has been shown that maturation also occurs when NMM II activity is reduced by $80 \%$, suggesting that forces alone do not propagate maturation. Rather, it seems that stress fiber assembly at adhesions serves as the template that facilitates adhesion maturation [147]. Stages of the focal adhesion maturation during cell migration are:

- Focal complexes form at the edge of the lamellipodium, and are phenotypically induced by Rac. They are dot-like contact sites and are typically constituted of paxillin, vinculin, and tyrosinephosphorylated proteins [19]. If the lamellipodium is not withdrawn but the cell moves forward, the focal complex will not dissolve but mature.

- Focal adhesions are found at the cell periphery and maturation is induced by Rho. They are oval-shaped, elongated, and typically constituted of integrin, paxillin, vinculin, $\alpha$-actinin, talin, focal adhesion kinase, and tyrosine-phosphorylated proteins. They serve as anchor points for migration until reaching the trailing edge [236].

- Fibrillar adhesions are a subtype of mature focal adhesions typically located at the cell center. With growing tension and translocation the amount of bound tensin increases, which is one of the characteristic factors of fibrillar adhesions. The shift is facilitated by Rho and is typically maintained 
by integrins, and by tension from outside the cell as well as from the cytoskeleton [17]. The adhesion itself benefits from the intertwined binding of the proteins, which stabilizes the entire adhesion site $[26]$.

- Actin cap associated focal adhesions (ACAFAs) are a small subset of focal adhesions that terminate fibers in the actin cap. The actin cap is anchored to the top of the nucleus by the linkage of nucleoskeleton and cytoskeleton (LINC) complexes. These ACAFAs exhibit different morphology and turnover, and respond to stimuli differently. While regular focal adhesions are involved in mechanosensing only on very soft substrates, ACAFAs span a wide range of stiffness [112].

In the vertical direction, focal adhesions form three distinct layers: the integrin layer close to the membrane; the force transduction layer, containing force-sensitive proteins; and the actin regulatory layer [103].

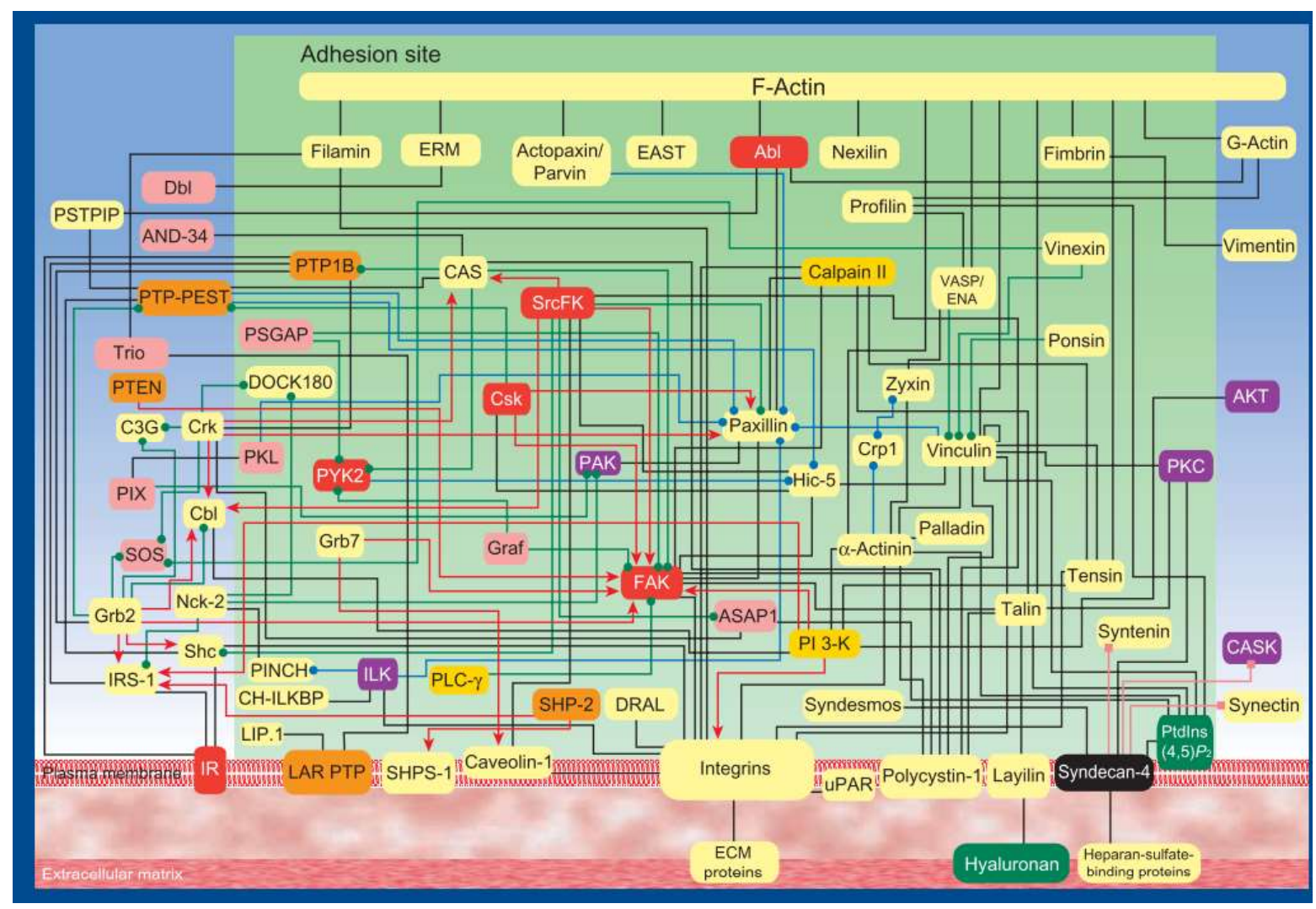

Figure 2.6.: Attempt of a simple illustration of known proteins associated to focal adhesions and main domain interactions. Figure from [236], legend omitted.

Since so many proteins are associated with the focal adhesion structure itself or with its function in the cell, all descriptions and rigid categorizations fall short. Nevertheless, it will be useful to gain a basic understanding. Figure 2.6 tries to provide an overview of focal adhesion-related proteins and their interactions. In this figure, proteins are color-coded according to their family and arrows are color-coded based on their domain interactions. Additionally, all interactions inside the green box are stable while unstable interactions are placed outside. With this depiction, one can begin to grasp the complexity of these cell anchors. 


\subsubsection{Focal adhesions as force sensors}

There are many force-sensitive proteins associated with focal adhesions that not only are involved with maturation but also are able to sense and relay forces, inducing changes in protein expression. Because focal adhesions are submitted to the strongest forces compared to other cellular structures, force-sensitive proteins coupled to them also react comparatively faster.

In contrast to focal adhesion maturation, which occurs by means of additional binding of molecules with increasing force, sensed stress is mostly communicated to the cell by the unbinding of overstressed proteins. The force exerted on a single focal adhesion is not dependant on size, but on function and location [106], with leading-edge adhesions bearing more stress (in migrating cells) than those in the middle and back regions. Transmitted stresses also correlate with the stiffness of the substrate. Although constant low tension facilitates focal adhesion maturation and growing stress fiber network stability through reduced cofilin binding and stress fiber cleavage, above a certain threshold force disruption occurs [124].

There are two types of disruptive mechanisms involved in force disruption. First, some proteins unbind. For example, p130cas activated through vinculin binding and a threshold amount of stress will subsequently facilitate focal adhesion assembly. In the same fashion, stretched Crk-associated substrate (CAS) bound to vinculin provides phosphorylation sites when stretched. As a side note, the prevention of CAS-vinculin binding can reduce traction forces inside the cell [94]. A second mechanism is the variable localization of proteins inside the cell. In hMSC for example, YAP/TAZ (Yes-associated protein/transcriptional coactivator with PDZ-binding motif) changes localization and can be found in the cytoplasm on soft substrates and in the nucleus on stiff substrates. YAP/TAZ switches in and out in response to substrate stiffness while the total amount in the cell remains constant [58].

The forces inside the cell also depend on the cell itself. A stem cell following osteogenic differentiation, and thus exhibiting a very detailed stress fiber network, will show higher traction forces compared to a stem cell undergoing adipogenic differentiation or to an undifferentiated stem cell [221]. Interestingly, non-differentiating stem cells show higher traction forces in their early stages, which decrease later. What stays constant in non-differentiating stem cells is their higher contractility compared to differentiating ones [58].

\subsubsection{Reactions to artificial substrates}

Knowing that cells exert forces on their environment and probe their surroundings, combined with knowing that stem cells can be differentiated using the stiffness of the substrate, we can conclude that the cell's reactions can be measured with as much detail as possible using artificial substrates with defined physical properties. This can be done either indirectly, by measuring the forces exerted from the cell onto the substrate, or directly, by measuring inside the cell. Very early indirect measurements have been done on silicone films, quantifying the wrinkling of these films [82]. A more modern approach toward indirect measurement is traction force microscopy (TFM), in which the forces exerted by the cell can be extrapolated from the movement of fluorescent beads in a gel substrate. Although their use for measuring cell forces is declining, silicone films are still used for stretching and wound healing experiments [84].

Direct measurements from outside of the cell can use optical or magnetic traps, or magnetic tweezers. With each of these tools, a bead can be trapped. If this bead is coated with binding proteins and attached to a cell, one can pull directly on the cell and study forces in the $\mathrm{pN}$ regimen with low noise. Also, shear stress can be applied and the stiffening of the cell recorded. This can be done either with one bead on an adherent cell or with multiple beads adhered to a cell in solution [146, 178].

A direct intracellular measurement of stress fibers can also be done with DNA force sensors. There, ssDNA strands with a FRET (Förster resonance energy transfer) pair (fluorophore and quencher) are bound to a backbone and to the cytoskeleton, then the FRET is measured. From known FRET efficiency of the sensor and known force needed to open the sensor, forces inside the cell can be estimated. Also, the 
force range of these sensors can be adjusted [164]. A detailed explenation of FRET and ssDNA sensors is beyond the scope of this thesis but can be found in the theses of Jayachandran [98] and Prabhune [163]. However, for all these experiments we need adjustable substrates. Besides needing to be nontoxic, these substrates must also be adjustable in stiffness to match various tissues types, from very soft tissue like the brain to stiff tissues like bone. Furthermore, modulation of topology, surface coating, or surface shape must be possible, and cells should naturally adhere to them. Additionally, the substrates have to offer the possibility of simulating conditions like chemo- or durotaxis occurring in the tissue.

The next section will broach the issue of polyacrylamide gel as a substrate for live cell experiments. Polyacrylamide gels offer great possibilities, as they are adjustable over a wide range of stiffness and easy to modify. 


\section{FilamentSensor software for fast and reliable fiber recognition}

The improvement of the FilamentSensor software, starting from the version mentioned in [62], was done with the help of working student Andreas Primeßnig.

To quantify a structure in the living cell, one has to keep in mind several issues. From the experimental point of view, we have to reliably label the structure of interest. This means that it should be well about background noise for the duration of the experiment, and in compliance with experiment goals (no change of morphology). Additionally, one should not mislabel anything by staining other structures or missing parts of the structure of interest. The signal-to-noise ratio is also important from the point of view of the downstream analysis. For this step, we use the LifeAct protein to reversibly label stress fibers without disturbing natural actin expression (for the detailed description, see Subsection 5.1.1.1).

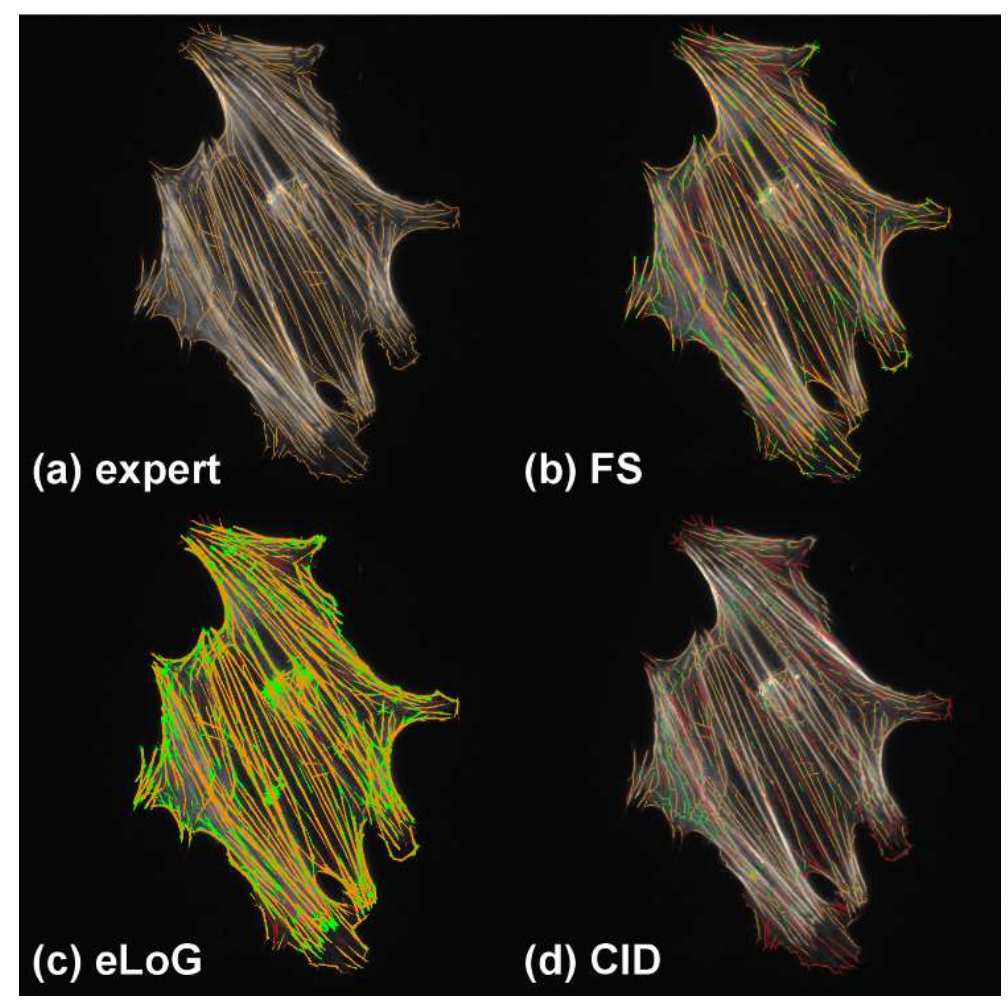

Figure 3.1.: Comparison of different methods for fiber tracing. a) Human expert marking fibers by hand. b) FilamentSensor. c) eLoG. d) CID. Fibers also matching human expert are marked in orange, additional fibers or parts in green, missing fibers or parts in red. From [62]. 
To analyze structures of interest one has to think about all steps from detection to data storage. How reliable should the detection be? What are my error margins? What artifacts do I want to avoid? What information do I want to save, and how? Do I need unique identifiers or not? What data formats are easiest to use in further analysis, or are most commonly used? For the FilamentSensor software, most of these questions have already been answered by Benjamin Eltzner and Carina Wollnik, the developers of the program [62]. FilamentSensor uses an algorithm developed for fingerprint tracking and many functionalities of the ImageJ freeware (for a detailed description of the software see Section 3.2). Besides FilamentSensor, there are other approaches to the same problem that should also be investigated.

In the Eltzner paper, FilamentSensor was compared to the CID and eLog methods. While CID collects data just on line pixels and has a high runtime, it misses most parallel lines and finds an additional 'mesh' of lines when lines are too close together. The eLoG method collects data on line pixels, angular histograms, and pixel-wise orientations. It performs significantly better than the CID method, but still misses close parallel lines and adds additional lines to the fiber ends.Also, while CID and eLoG find most crossing fibers in images with excellent signal-to-noise ratios, they are both severely impaired when there is blur or noise, and misidentify more fibers than they identify (see Figure 3.1) [62]. FilamentSensor outperforms both the CID and eLoG methods, while additionally collecting individual and overall fiber length and width. Common problems of CID and eLoG algorithms - overestimation of ends and misinterpretation of parallel or crossed lines-do not occur with FilamentSensor.

Besides single scripts of various languages (including FilamentSensor, CID, and eLog) for fiber identification, there are also other softwares available. A prominent example is the SOAX software, which uses "Stretching Open Active Contours" to identify filaments and network junctions. The SOAX application was built for 3D networks imaged with confocal or super-resolution microscopy [231]. However, the software has recently been updated with a global and local correspondence algorithm, called TSOAX, which has been shown to be capable of loop detection [230]. Jasnin et al. has also described a workflow to use the 3D modeling software IMOD to obtain filament data using a generic filament as a template [96].

\subsection{Why is a software needed to quantify stress fibers?}

Trustworthy automated analysis of filaments offers huge benefits on multiple levels. First, it reduces bias introduced by manual labeling. Second, data can be reanalyzed, or analysis can be crosschecked easily, when open source software is used and the version is mentioned, in contrast to self-written pipelines. Finally, it saves time. This saved time can be invested to generate larger data sets with stable means to detect subtle differences and variations.

As mentioned in Chapter 5, the behavior and morphology of stem cells is more diverse than in immortalized cell lines. Hence, we need bigger sample sizes to make reliable claims about general behavior. In Subchapter 5.5.2, this is discussed further. In this thesis, I want to show that for the distinction of early differentiation of hMSCs, the stress fiber patterns alone are sufficient. To show this, I must discriminate between very similar treated cells in an early stage, using morphological descriptors of a highly variable cell line. Adherent hMSCs sense substrate stiffness. In Figure 3.2 the expression of differentiation markers in hMSCs is shown, as well as the cancellation of these by blebbistatin. Blebbistatin is an NMM inhibitor and thus hinders force transduction; in hMSCs, this accordingly hinders mechanically induced differentiation [121].

While the differentiation marker onset takes a couple of days, the stress fiber pattern adjusts within 24 hours. This is faster than the test of markers and, since testing stress fiber patterns is noninvasive, it can be done at multiple time points for the same sample without altering behavior.

On substrates of different stiffnesses, we see varying numbers and arrangements of stress fibers per cell. However, the number of movies necessary to show this behavior in stem cells, which do not behave as uniformly as a cancer cell line, would be impossible to annotate by hand and analyze. Because of the intrinsic variability of the hMSC in contrast to cancer cell lines, the variability is already very high even 
within one condition. Furthermore, distributions are very similar. Hence, we need to have huge numbers to distinguish the differences. Interestingly, with around 150 movies we can discriminate between substrates of sufficiently different stiffness with $95 \%$ significance (11 kPa vs. glass; 1 vs. $30 \mathrm{kPa})$. Analyzing this amount of movies by hand is not feasible, as it would require one to mark every stress fiber by hand for all time points taken, 144 in this case. For similar substrates like 20 vs. $30 \mathrm{kPa}$ PAA gels, this is not yet possible. However, it is unlikely the stress fiber pattern would differ anyway, as both would lead to a myogenic differentiation.

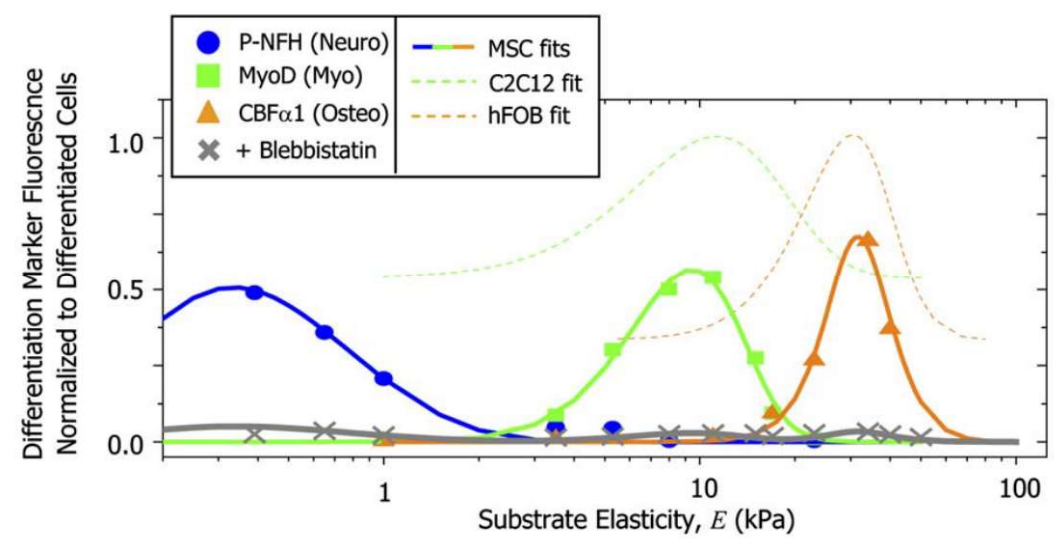

Figure 3.2.: Differentiation marker intensity for mesenchymal stem cells grown on gels with different stiffness for 1 week with or without blebbistatin treatment. Taken from [65].

\subsubsection{Parameterization of stress fiber features}

For quantification, we can obtain descriptors of individual fibers, fiber networks in an individual cell, and/or whole group of cells. For a single fiber, the set of descriptors is comprised of start- and endpoint, length, thickness, position, and curvature. For a fiber network we further quantify mean angle, order parameter, mean length, and distribution of fibers. Finally, with the metadata of the sample saved by the microscope (i.e., imaging position, duration), we can put an imaged cell into perspective by comparing it with the whole sample to determine effects that may occur on the edge of the sample.

The more descriptors we measure, the more possibilities we have in later analysis, and the more detailed are the answers we can provide for our questions. Additionally, some of these descriptors can help monitor our experimental approach. Mean brightness over time might give insight into maximal experiment length. Differences in cell shape (aspect ratio) and cell area between the middle of sample and its boundary area might make it necessary to define not only minimal gel thickness, but also minimal distance to gel edge. This is because cells can sense underlying glass and react as if directly seeded on glass where gel is too thin. Similarly, on the gel edge cells pulling on the substrate get aberrant sensations. The larger sample sets made possible with automated analysis allow the statistical significance of trends to be evaluated; thus, they can help improve experimental approaches.

The ability to quantify stress fibers also depends on the image acquisition technique used. On an epifluorescence microscope, for example, a fiber leaving the focal plane will appear wider, skewing all other data for that descriptor. On a confocal microscope, fibers with an inclination above $50^{\circ}$ will not be detected, similarly skewing the data [97]. The software might cut outliers, like cells expressing very curved filaments, into pieces. In other cells, stress fibers might be overly frequent but very short. In such cases, one has to balance between detection of fibers and noise.

In conclusion, when using automated analysis one has to know the pitfalls, limitations, and corner cases of the experiment. While with human annotation of a small data set this will be done while annotating, in 
large data sets detection (hyper-) parameters have to be adjusted, and trends in data interpreted correctly, to get proper results.

\subsubsection{Bias in human quantification}

A serious problem in microscopy is human bias. This can be an issue both in the search for cells worthy of imaging and in the selection of structures during expert labeling. During the selection of cells, when the same person selects images and does subsequent analysis, one tends to exclude everything that does not fit the expected morphology. Thus, the experimenter is preselecting the data, which will be more likely to later yield the expected outcome. A common approach to reduce this is to have a set of identifiers for a desirable cell and split channels, so that bias by seeing the desired structure is completely excluded. Here, one identifier in the phase channel could be to select a single cell, with no contact with any other cell. For another identifier, a nucleus stain could be used to ensure the cell is healthy and not undergoing division . When it is not possible to have the structure out of view, it is possible to give the set of identifiers to a nonexpert person and have them do the imaging.

Human bias is an even bigger problem during the analysis of the images. The human eye is a great detector of patterns but also gets trained to recognize the patterns it searches for. And since perception is subjective, different people will interpret data differently. This occurs even when the same expert is asked to relabel data, since subjective assessment changes with knowledge gain. An algorithm, on the other hand, is truly objective, and its parameters are comprehensible and reproducible.

\subsection{The FilamentSensor software}

FilamentSensor is an open-source filament-tracking program developed in a collaboration between the teams of Dr. Florian Rehfeldt and Prof. Stefan Huckemann in the SFB 755, project B8. Large parts of the program are referencing ImageJ, used as an internal library. This enables one to use all the ImageJ functionalities, including the magic wand tool, thresholding algorithms for area estimations, and filter sets. These are useful tools to accompany the main part of the software, which is designed to detect filamentous structures in fluorescence images, based on an algorithm for fingerprint analysis. This theoretical groundwork was done by Dr. Benjamin Eltzner [62], while he was a Postdoc in Prof. Huckemann's group in the Institute for Mathematical Stochastics at the Georg August University of Göttingen. Figure 3.3 shows the workflow of fiber tracking with FilamentSensor. 


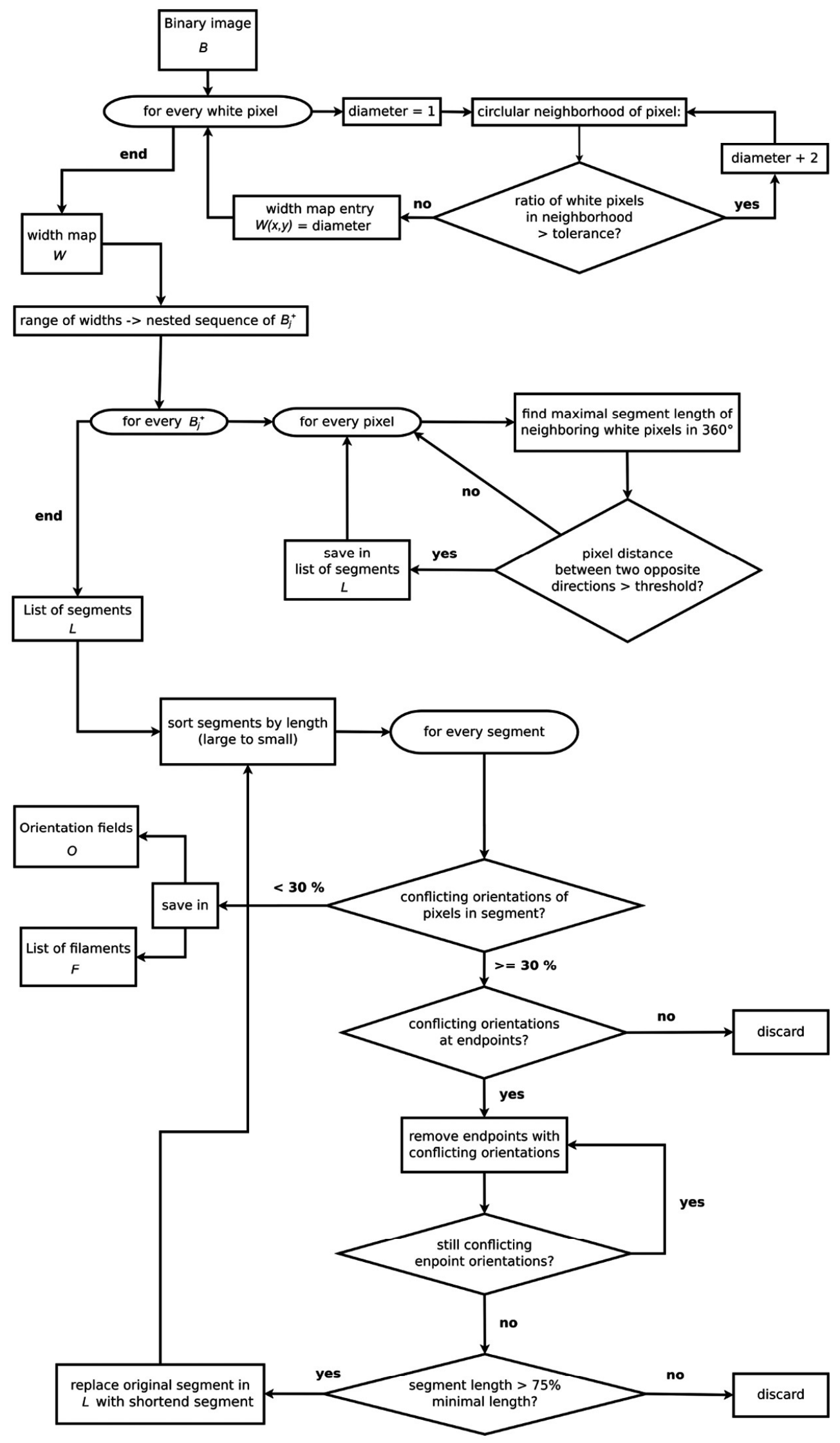

Figure 3.3.: FilamentSensor filament tracking workflow. Image from [62]. 


\subsubsection{Fast and accurate recognition of curved filaments}

While the original paper introducing FilamentSensor [62] presented a tool only applicable for straight fibers, fibers within the cell are curved most of the time due to forces exerted or transferred onto the fibers. Hence, a search for straight fibers will find these curved ones but break them down into small pieces. The functionality of the FilamentSensor was enhanced to curved fibres.

Within the software, the CurveTracer class appends the LineTracer class. There, a WidthMap is initialized. The WidthMap is a matrix containing, for each white pixel, the largest diameter of a circular mask in which the ratio of black pixels to total pixels is above the threshold. For every circle diameter, starting with the widest, the LineTracer follows the following steps:

- It generates a Boolean matrix, and sets "true" for every diameter smaller than the present one.

- For each cell containing "'false," a LineInfo object is generated.

- The LineInfo object tests lines in all directions from the current point and saves points with maximal distance.

- A map is generated from all the points located on lines with length above minimal length, ordered by length.

- This map is iterated starting with the longest line.

- While iterating, conflicts for points located on multiple lines are solved and FilamentObjects are generated and saved to a list.

The CurveTracer first initializes the WidthMap but groups all points with the same diameter. For each point, starting with the largest:

- Scores and gatherers are initialized. The score is the sum of all diameters from the WidthMap per point.

- Gatherers collect points on curves and save them to a map of scored lines.

- After collecting 10,000 lines, cleanup is started. Longest lines are marked, shorter ones discarded. This threshold is set because, with the combination of background noise and the fingerprint algorithm, the software could find a high number of very short filaments in the background. With the new possibility to restrict filament detection to cell area (see Subsection3.2.2) this problem can be solved, too. However, as some users might not restrict detection, we kept this threshold in the code.

- Longest lines are iterated and any conflicts for points found on multiple lines are solved.

- Lines are saved as CurvedFilament and saved to a list.

With the CurveTracer, parameters for curvature are available up to a set maximal angle. Furthermore, filaments can still be filtered for minimal and maximal length.

\subsubsection{Cell area}

To correctly determine the cell area, there is a line of processing in action that mainly consists of three steps: a filter queue, mainly consisting of Gaussian filters; thresholding; and enhanced binarization. In contrast to the FilamentSensor version described in [62], many parts of the analysis pipeline are now freely adjustable.

The filter queue consists of several steps of Gaussian, Laplace, line-Gaussian, and cross-correlation filters, alternating with contrast enhancement to blur out noise without disturbing the general cell features. The order and number of filters can be adjusted by the user, who can also import self-written filters. The most 
important filter is the Gaussian filter, which applies a Gaussian distribution to each pixel depending on its distance from the central pixel. This is also an important preprocessing step used for the filament detection.

Thresholding is used to reduce background noise by setting all pixels with a value below the threshold to zero. FilamentSensor offers the same algorithms as ImageJ with the option to write one's own. This allows the user to adjust the algorithm's filters and thresholds to suit various data sets. Furthermore, both automatic and manual setting of thresholds, filters, and additional parameters are available. Both the filter queue used and the thresholding algorithm are saved in a project file that can be read or reimported to keep track of the processing steps done for a certain data set.

Finally, the image is binarized using the thresholded image from the previous step. On this mask of zero and 1 values, a closing-holes operation from the ImageJ library is applied and all but the largest area (patch of 1 values) are discarded. Fiber detection can be applied to either the whole picture or to this restricted area; the latter is recommended. This area will also be exported as one of the whole cell descriptors in the serialized .csv files. However, parts of the cell that are too dim to pass the thresholding value will be omitted. This poses a problem with filopodia or similar structures. Thus, we established another method for area recognition, namely the "mechanically active area." While the regular area calculation starts from the original image, the mechanically active area is an envelope of all found fibers. The fibers are thickened, spaces are filled by an ImageJ "close holes" operation, and, via a series of thickening, closing, filling, and shrinking operations, convex spaces are corrected (see Subchapter 3.3.4).

\subsubsection{Aspect ratio}

The aspect ratio is a valuable piece of information about single cells, and different cell types have different characteristic mean aspect ratios. In addition, differentiation of hMSCs shows different aspect ratios for hMSCs following their neurogenic, myogenic, or osteogenic differentiation.

The aspect ratio is calculated using the binarized image from the area calculation described in the previous subsection. To calculate the aspect ratio, we define the moments of this binarized image using the number of white pixels and the covariance. From the covariance we get the larger and smaller eigenvalue. The aspect ratio is defined as the square root of the larger divided by the smaller eigenvalue.

When approximating the area of a cell with an elliptical shape, with a long half axis $\alpha$ and short half axis $\beta, \beta=\alpha / \rho$. For area, $\mathrm{A}, \mathrm{A}=\alpha^{2} / \rho$. The length of the long half axis is thus $\alpha=\sqrt{A \cdot \rho}$ pixels.

The orientation of the cell can then be calculated using -arctan 2 of the larger eigenvalue, minus the covariance of the larger eigenvalue combined with the covariance of the larger with the smaller eigenvalue. This orientation can show the cell's behavior but does not follow global trends like the aspect ratio, which converges against specific metrics for certain cell types or environments. A sudden change in orientation should be evaluated more closely than a slow change; it might be due to dirt, division onset, or other irregular events. Taking into account all orientations of all cells in one sample, one might encounter a nonrandom distribution of cell orientations and investigate this effect closer. Thus, the orientation serves mostly as a parameter of sample quality in this thesis.

Beside this, FilamentSensor offers a possibility of grouping fibers in a single cell by orientation, as well as a proximity parameter to "orientation fields." Number of filaments in single-orientation fields and number of fields give further insight into differentiation and behavior of the cells.

\subsubsection{Order parameter}

The order parameter describes how well the fibers found in one cell are aligned to the long axis of the ellipse fitted to the cell. As for elongated cells, it is probable that the main fraction of fibers is aligned to the long axis. 
The order parameter $S$ for the cell is defined as a set of lines $n$ with distinct length $l_{k}$, width $w_{k}$, and orientation $\phi_{k}$ ( $\phi_{c}$ being the orientation of the whole cell). For the whole set we can summarize:

$$
S=\sum_{j=1}^{n} l_{k} \cdot w_{k} \cdot \cos \left(2\left(\phi_{k}-\phi_{c} e l l\right)\right) / \sum_{j=1}^{n} l_{k} \cdot w_{k}
$$

$S$ here is a weighted mean of terms $\cos \left(2\left(\phi_{\mathrm{k}}-\phi_{\text {cell }}\right)\right)$. The subtraction of the cell's orientation makes the order parameter nondependent on the orientation of the cell, so that we can compare cells regardless of their location. An order parameter of 1 means all filaments are parallel to the long axis of the cell, -1 means all are parallel to the short axis.

\subsubsection{Migration statistics}

All cells can move in some way, with some movements almost imperceptible while other movements are very fast or take place over great distances. There can be both random and directed movement according to the cell's function. All motion follows some basic steps, however: polarization, membrane extension, formation and stabilization of attachments, contractile forces and traction, rear release, and, finally, a phase of overall coordination $[179,242]$. In vivo, these steps can often not be separated; but, to initiate movement, there must be a polarization of the cytoskeleton to enable exertion of forces in different parts of the cell [155].

Lamella protrusions probe the substrate's adhesive properties and get pushed outward by actin filaments extending from the cell membrane towards the cytosol [156]. After probing the substrate, focal adhesions are formed to connect cell to substrate [19]. These adhesions can mature over time and with more forces applied form stable adhesions. The stress fiber network, which is connected to adhesions in both the front and back, contracts and pulls the cell in the direction of the protrusion while increasing tensions on the rear focal adhesions [155]. The adhesions will be disassembled after detection of stress-sensible proteins on a certain strain. The proteins of disassembled focal adhesions will be used for building new focal adhesions or maturing existing ones $[24,26]$.

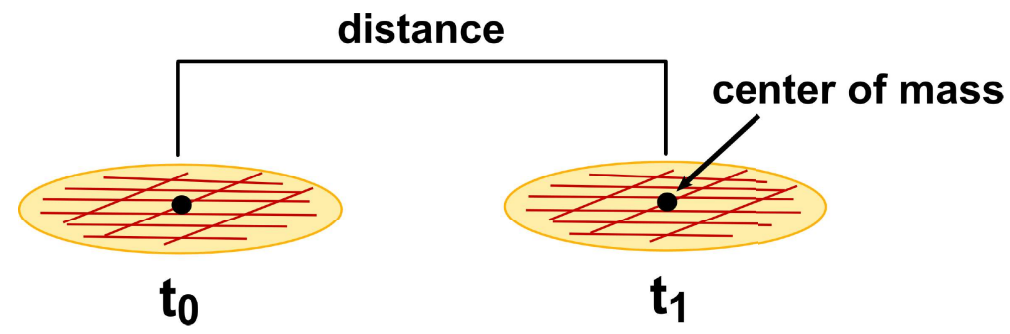

Figure 3.4.: Migration distance can be calculated by either comparing the position of the center of mass in the first and last frame, or by following the whole path of migration over time. Image from [225].

Because FilamentSensor fits an ellipse to the cell to extract multiple parameters, we know the centroid of the cell's contour (see Figure 3.4). Ignoring possible discussions about whether we should use this point, obtain the center of mass for the cell, or use any other possible point, we can use this approximation as a proxy for the cell's real center in order to do basic migration statistics.

For migration of single cells, two main parameters are of interest. These are the velocity of the migrating cell and the distance. The distance can be defined as the cell's migrated path or as the distance between start and end of the path (see Figure 3.5). However, taking into account just start and end points neglects to allow for the possibility of the cell returning to its original position. 


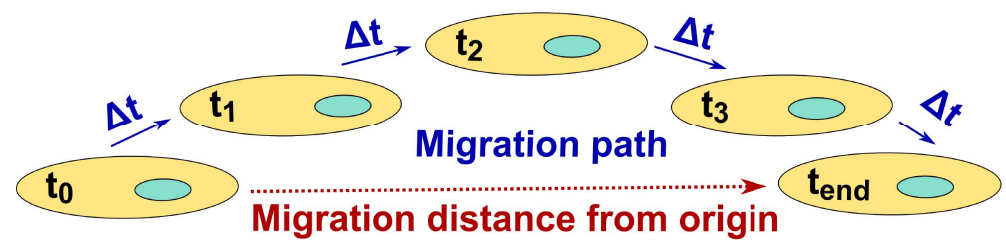

Figure 3.5.: Comparing the migration path over time to the distance between the center of mass in the first and last frame, the path might be longer. To get real migration speed and distance, taking into account all frames gives more accurate numbers. Image from [225].

For migration analysis as done in Chapter 5 the experimental setup itself poses a problem. Because in this thesis we aimed to examine cells staying in the field of view for 24 hours, we could have subsampled. This would have meant focusing on a percentage of the whole sample, which might not have reflected the sample as a whole. To correct for this, I compared the cells used for 24 hour differentiation statistics with cells that were discarded because they left the field of view within 18 or 12 hours.

The common method of migration analysis is the mean square displacement (MSD). This descriptor is generally used in single-particle trajectory analysis and provides information about the type of movement, generally dividing between directed and random movement (see Figure 3.6).

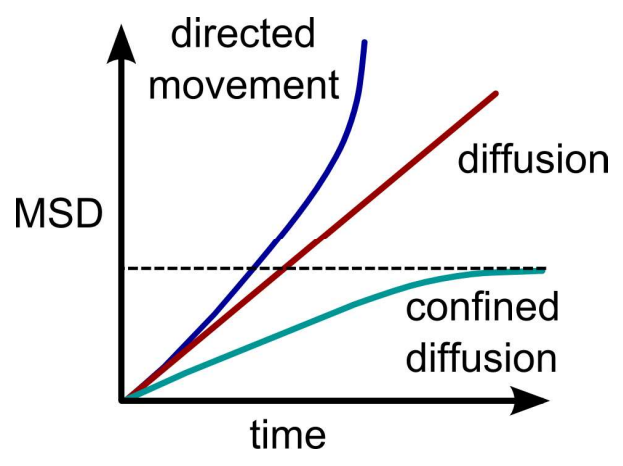

Figure 3.6.: Schematic of mean square displacement (MSD) curves. Diffusion is used as reference to distinguish between confined diffusion (slower) and directed movement (faster). Image from [225].

When subsampling is excluded from the experiment, the MSD can also be used to describe the general behavior of one cell line in the experiment or for comparison of cell lines. However, one should keep in mind that migration not only depends on the internal signalling of the cell (see Figure 3.7) but can also be influenced by the experimental environment. Differences in the "stickiness" of protein coatings facilitating adhesion, flexibility of substrate [156], or patterns can be especially problematic, as they significantly influence adhesion, polarization [134], and migration [124]. 


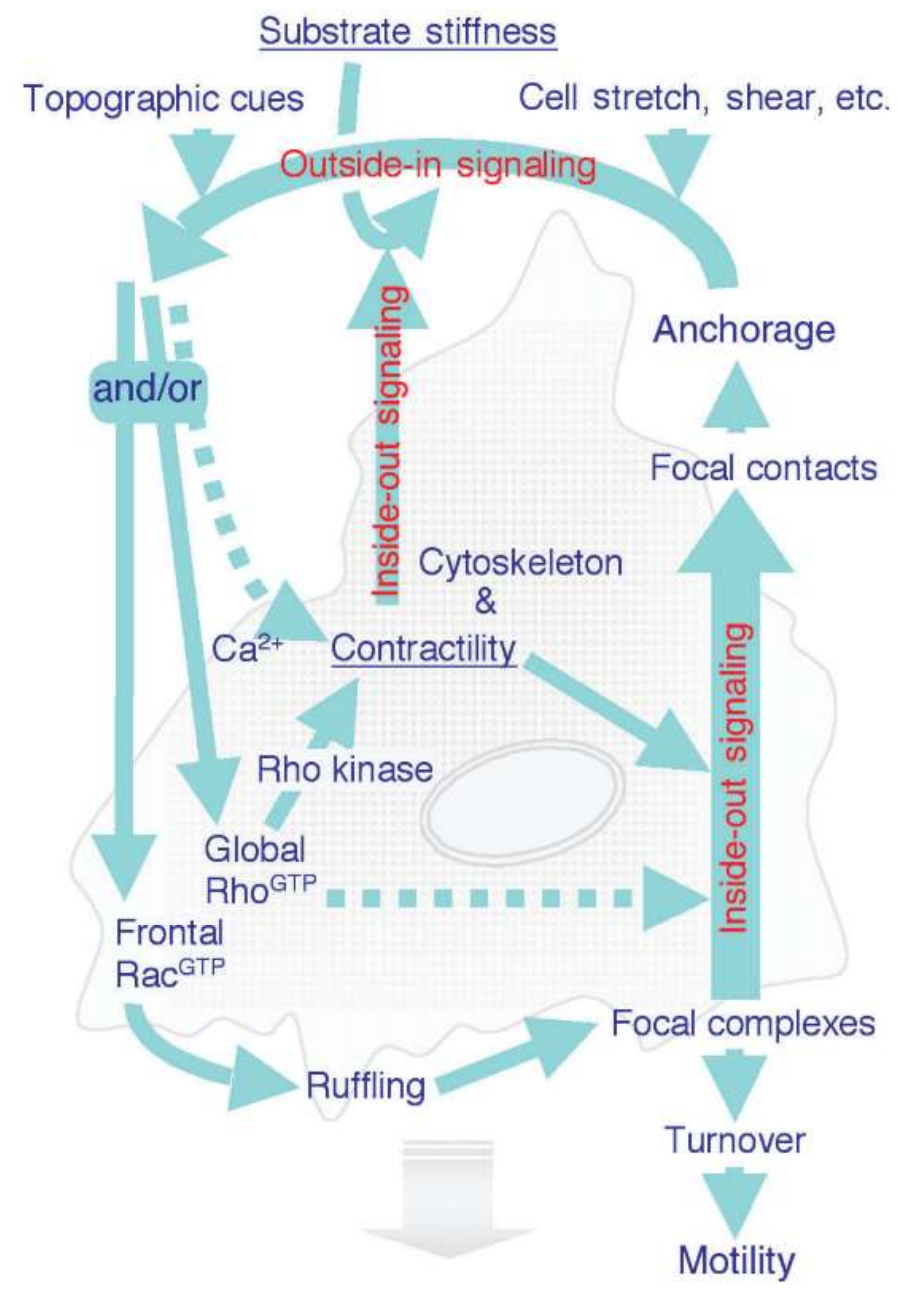

Figure 3.7.: Interplay between mechanical environment, signalling, cytoskeleton and motility, taken from [54].

\subsection{Novel features in Alpha version}

While the FilamentSensor version published in [62] was a great start and provided algorithms unchanged in later versions, there were some areas to be improved. Mainly, usability and runtime had to be improved as well as the documentation and structure of the program itself to make it usable for other projects.

In this section some of the biggest improvements are described.

\subsubsection{Refactoring}

The term refactoring describes the improvement of readability, clarity, comprehensibility, expandability, and testability, as well as avoidance of redundancy. While it takes a lot of work, its use is often underestimated. This is because the results are not visible to the user in a GUI-based program. However, when writing code, we should always have in mind that it should be made available to colleagues or even published. A clean and well-documented code will outlive the original coder and contribute to the whole community. Basic work within the refactoring consists of simple tasks. First, one should split code into classes according to the tasks performed. This way, blocks of code can be reused (also with modifications) for different tasks, slimming the whole code. Second, variables and methods should be well-named. This means names should conform to standards, where they exist; should be unique, where they do not; and should be 
self-explanatory, where possible. This second task already suggests the third task, documentation, in which every variable, method, and possible variation is commented on in the code.

Additionally, the code should include test-routines and benchmark data for intern testing and testing by other developers. This testing should be done after every major change. A well-structured, documented, and tested code provides the possibility for easy expansion of the main program with additional features if needed.

Another part of refactoring is the adaption of multi-threading. Where multiple processor cores are available, and code is structured accordingly, methods can be run in parallel, which significantly improves overall runtime.

In the case of FilamentSensor, the program was transformed from a single-class piece of code using just one thread into a well-documented, multi-package, and multi-class project available on multiple sources, predominantly www.filament-sensor.de. The most prominent result for users, however, is the decrease in runtime for a specific complicated test set (3 intertwined cells with about 3,000 fibers) from 48 to 8 minutes.

\subsubsection{Stack processing}

Most of the time we do not collect single images of a cell but a stack of images. This stack could be over time or in the z-direction. Also, most 3D-images are saved in a hyperstack format which can be treated as a stack. To show and process pictures in the FilamentSensor software as stacks is thus a reasonable next step.

Like the other functionalities inherited from ImageJ (filters, thresholding, image formats), the stack methods are called from the internal ImageJ library. In addition to the stack view, FilamentSensor offers various functionalities. Because for live-cell movies taken over the course of several days bleaching occurs, contrast and brightness adjustment can be done for single pictures or for the whole stack. For another enhancement of the FilamentSensor, which allows it to follow single fibers over time, we implement a Wasserstein transport plan to compare fibers frame-to-frame and match them. For 3D stacks this application can be used to stitch fiber fragments together through the whole 3D model (see Subchapter 10).

Calling the stack methods has downsides as well, as the RAM usage increases significantly in comparison to the single-picture view. While most modern computers (minimum 4 GB RAM) can handle basic stack view, when z-slides are added needed RAM increases to 64 GB or more.

We use stack view not only for data of stacks in which picture size is continuous, but also for data of variable dimensions, by using flexible parameters. Data of this latter type could originate from cropped pictures of fixed cells, for example.

\subsubsection{Bounding boxes for multiple cells in field of view}

A recurring problem with detection of particles is the identification of the particle of interest, especially when there are other fluorescent cells, dirt, or floating dead cells in the peripheries. A brute force method is the cropping of the field of view to the desired object. An improved method is to build a tool to select the desired object.

We used two solutions for the particle selection problem. In its earliest versions, the FilamentSensor program would always pick the largest particle for analysis. This posed a problem in rare cases, such as when cells in the field of view switch sizes. The newer versions use a bounding box around each particle of reasonable size. One bounding box can be selected for analysis by clicking on it; the program then uses the overlap of bounding boxes from frame to frame to follow a particle. Each detected particle is assigned a unique identifier. This identifier is kept even when cells merge; for dividing cells, new identifiers are created. 


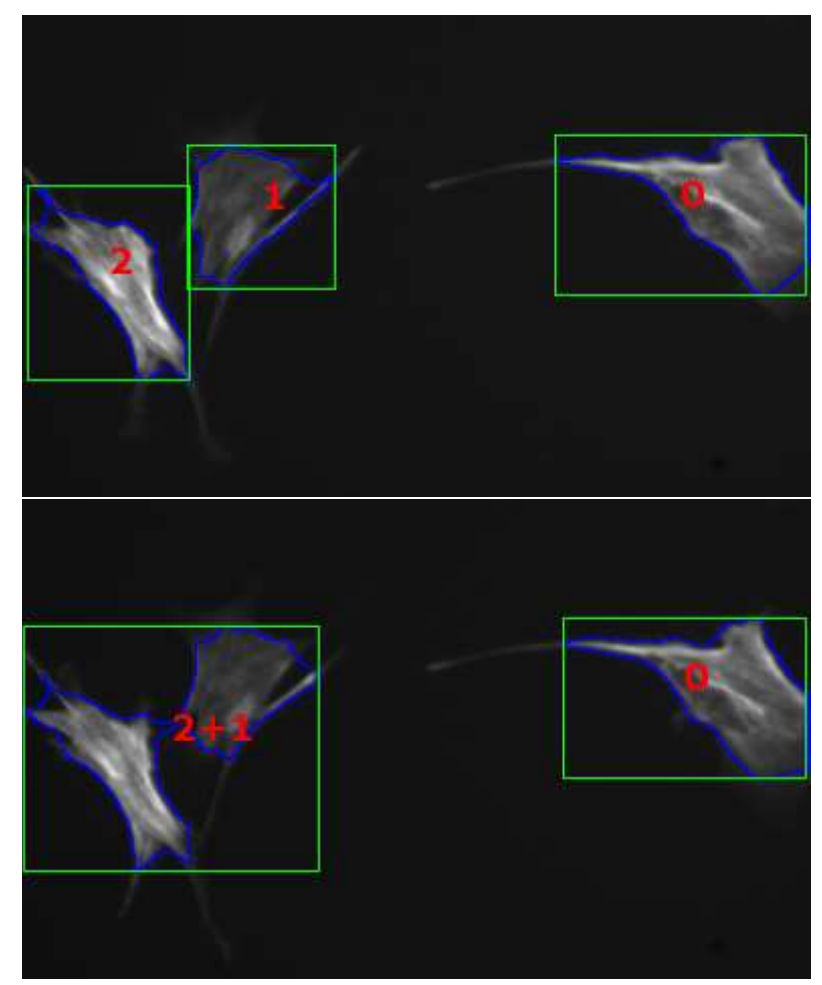

Figure 3.8.: Bounding box feature of the FilamentSensor. Each detected separated cell area is assigned an unique identifier. This identifier is kept even when cells merge and for dividing cells new identifiers are created. left: Three separate cells detected. right: Two of the cells touch and are detected as fused cells.

This bounding box application will, in later versions, also be used to follow dividing cells or -in migration statistics, colliding cells - with the addition of a filter to distinguish both cells upon collision. In early tests, brightness was used.

\subsubsection{Recognition of mechanically active area}

Area recognition was an important tool from the first FilamentSensor versions onward. There are, however, some issues with what to assume as an "area worth detection" and how to detect this area.

As already described in Subchapter 3.2.2, when a binarized version of the thresholded original image is used to determine the cell area some structures are omitted. For movies used in this thesis, actin stress fibers are labelled most of the time. The LifeAct protein used (see Subsection 5.1.1.1) also stains peripheral actin. However, in comparison to the stress fibers especially, filopodia do not pass the threshold.

In practice, this leads to different percentages of the total cell area being neglected for different cells. Thus, we cut the area down to the mechanically active area for a straightforward comparison of cells.

To accomplish this, the detected fibers are used instead of the original image. The binary mask of these fibers, which is a intermediate step in fiber detection, is subjected to a series of operations derived from ImageJ. The binarized lines of the stress fibers are treated with a closing operation with a circle mask of 30 pixel diameters. The closing operation is composed of a dilate operation followed by an erosion. This is followed with a clear-edges operation and another closing of holes. The challenge here is to close holes between the fibers but leave inbound areas in the cell outline. 


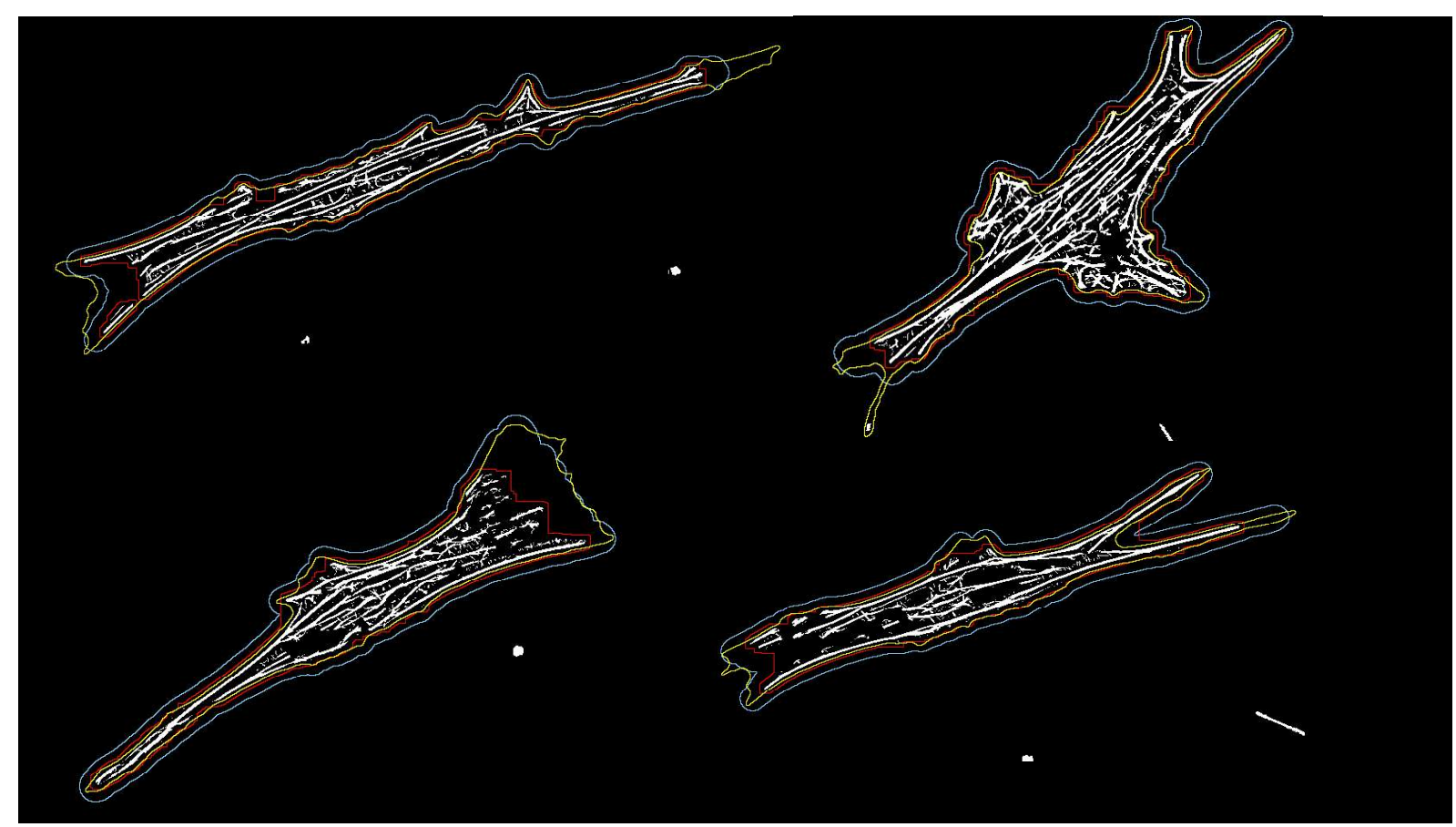

Figure 3.9.: Examples of different area detection results in FilamentSensor. Yellow: Best possible fit to the cell outline detected. Blue: Smoothed and slightly enlarged cell outline. Red: Mechanically active area fitted as an envelope around the detected fibers.

\subsubsection{Better runtime: more possibilities}

A side effect of the refactoring process is an improved runtime. We tested different versions of FilamentSensor to measure the improvement.

The test system used had the following specifications:

- OS: Windows 10 64bit

- Java: java version "1.8.0_172" 64bit

- Processor: Ryzen 2700X (8Core $16 \mathrm{Threads}, 3.7 \mathrm{GHz}-4.3 \mathrm{GHz}$ )

- Hard Disk-data was written to: USB 3.0 external hard disk

- RAM: 32GB @2933Hz (dual-channel)

- NVME SSD: Read/Write $>2000 \mathrm{MB} / \mathrm{s}$

Test methodology used:

Time is measured from Batch Processing from start of Java main until end of main method. Timestamps are taken via System.currentTimeMillis(). Each time a cold start is done (one run of batch processing, then end).

Test data is the same for every version of FilamentSensor. For comparison, three runs of the same version are done and the average time is taken. The FilamentSensor versions are modified by adding a method-call to start batch processing from an external program. To see the impact of the file write and read cycle, a fourth run is added for some versions. The fourth run is executed on a NVME SSD. The test data set consists of 144 images, each with dimensions of $1560 \times 1866$, gray scale. We tested three versions of FilamentSensor before refactoring, as well as the developer version from April 2019. All versions are available online at www.filament-sensor.de. 


\begin{tabular}{l|l|l|l} 
FS Vers. 0.2.2j & FS Vers. 0.2.2l & FS Vers. 0.2.3 & DevVers. Apr2019 \\
\hline $1323.973 \mathrm{~s}$ & $1310.342 \mathrm{~s}$ & $1593.324 \mathrm{~s}$ & $218.803 \mathrm{~s}$ \\
$1322.253 \mathrm{~s}$ & $1324.484 \mathrm{~s}$ & $1561.271 \mathrm{~s}$ & $214.971 \mathrm{~s}$ \\
$1321.254 \mathrm{~s}$ & $1319.306 \mathrm{~s}$ & $1574.934 \mathrm{~s}$ & $210.576 \mathrm{~s}$ \\
$1303.887 \mathrm{~s}$ & not done & not done & $216.894 \mathrm{~s}$
\end{tabular}

Table 3.1.: Comparison of runtimes of different FilamentSensor (FS) versions before and after refactoring. Versions 0.2.2 $\mathrm{j}$ and 0.2.21 were developed by Benjamin Eltzner, version 0.2.3 is the first version after start of refactoring process. The version Apr2019 is an unpublished developer version (DevVers.) with refactoring process completed.

- FS Vers. 0.2.2j: CPU usage 0-10\%, memory usage up to $3 \mathrm{~GB}$, most of the time below 2GB.

- FS Vers. 0.2.21: CPU usage 0-11\%, memory usage up to $3 \mathrm{~GB}$, most of the time below $2 \mathrm{~GB}$. A fourth run was not done as there was no difference in performance compared to Version 0.2.2j.

- FS Vers. 0.2.3: CPU usage 0-11\%, memory usage up to 3 GB. Again, no performance difference when compared to Version $0.2 .2 \mathrm{j}$.

- DevVers. Apr2019: CPU usage 0-100\%, memory usage up to 6 GB. Note that process exited about 59 seconds after batch processing finished, this is because the Java threads from Stream API and CompletableFuture.runAsync are shutting down. This has no practical impact on performance.

On average, the current, refactored developer version (DevVers. Apr2019) needs only $16 \%$ of runtime in comparison to previous versions, while also using available CPU and RAM more efficiently. This is the first step to expand FilamentSensor. New tasks that are now more feasible with multithreading established include stack processing, cross-correlation (see 3.4), single filament tracking over time (see Subchapter 10), and processing of $3 \mathrm{D}$ data.

Furthermore, we plan a MicroManager integration with FilamentSensor, which will allow for full real-time analysis of fluorescent-labelled fibers during microscopy. 


\title{
3.4. The FAFCK: A cross-correlation subroutine
}

To enhance the understanding of the cells' response to mechanical stimuli, a simultaneous examination of stress fibers and related fibers is useful. Here, we describe a spin-off of the FilamentSensor that can correlate fiber and focal adhesion data.

The Focal Adhesion Filament Cross-correlation Kit (FAFCK) combines all functionalities of the newest FilamentSensor version with several options of either automatized and unsupervised or manual and userassisted focal adhesion detection. All object data can be imported for either fibers or focal adhesions alone or in a correlated manner. The correlation separates data according to overlaps with other structures (not used, single used, multiple used) and thus can help to categorize stress fiber and focal adhesion subtypes. Additionally, the software offers a validation tool to compare software-generated object masks to user-drawn ones.

In the future, this software will be improved to offer stress fiber and focal adhesion tracking in time to further amplify the understanding of the generation and interplay of different stress fiber subtypes during migration.

This manuscript is on the day of submission still unpublished.

My own contribution to this paper consists of the development of the software. There, I am responsible for the idea, management, and testing, while Andreas Primeßnig does the coding itself. Furthermore, I did the initial data analysis and part of the analysis that is used in the manuscript. Lastly, I wrote the manuscript that was then revised by the other authors.

\section{A Focal Adhesion Filament Cross-correlation Kit for fast, automated segmen- tation and correlation of focal adhesions and associated stress fibers in cells.}

Lara Hauke ${ }^{1, \uparrow}$, Shwetha Narasimhan $^{2,}$, Andreas Primeßnig ${ }^{1}$, Irina Kaverina $^{2, \ddagger^{*}}$, Florian Rehfeldt ${ }^{1,3, \sharp^{* *}}$

1 Third Institute of Physics - Biophysics, Georg-August-University Göttingen, D-37077 Göttingen, Germany

2 Department of Cell and Developmental Biology, Vanderbilt University, Nashville, TN, USA

3 Experimental Physics I, University of Bayreuth, D-95440 Bayreuth, Germany

A These authors contributed equally to this work.

$\ddagger$ These authors also contributed equally to this work.

* irina.kaverina@vanderbilt.edu

** florian.rehfeldt@uni-bayreuth.de

\begin{abstract}
Focal adhesions (FAs) and associated actin stress fibers (SFs) form a complex mechanical system that mediates bidirectional interactions between cells and their environment. This linked network facilitates mechanosensing, force production and transduction, thus directly governing cellular processes like polarization, migration and extracellular matrix remodeling. We introduce a tool for fast and robust coupled analysis of both FAs and SFs, named the Focal Adhesion Filament Cross-correlation Kit (FAFCK). Our software can detect and record location, length, area, orientation and aspect ratio for ellipsoid adhesive structures as well as the location, length, width and orientation for filamentous structures. This enables users to automate analysis of linked adhesions and filaments and study the SF system in a higher degree, which is pivotal to accurately evaluate the SF organization and functions such as transmission of forces
\end{abstract}


between the cell and the ECM. For validation, we provide comparison datasets of real cells of varying quality that are labelled by a human expert. Both datasets and FAFCK are freely available as open source under the GNU General Public License.

\section{Author summary}

Our novel Focal Adhesion Filament Cross-correlation Kit (FAFCK) allows for fast, reliable and unbiased detection of focal adhesions and acto-myosin stress fibers in cells and their mutual correlation. Detailed information of those both types of key elements in cellular mechano-transduction will be of essential help for quantitative analysis of cell-matrix-mechanics experiments, including sophisticated traction force microscopy analysis based on the correlation of stress fibers and focal adhesions.

\section{Introduction}

The shape and mechanics of motile vertebrate cells relies on a dynamic system that produces both the cellular endoskeleton and the tensile forces exerted to their environment, such as the extracellular matrix (ECM) or neighboring cells. A predominant and essential part of this system is the actin cytoskeleton. Actin filament bundles act as structural elements and, importantly, are capable of producing force when co-assembled with myosin II into contractile stress fibers.[8]

Geometry and rearrangement of stress fibers (Figure 1) is a critical factor during cell migration and have to be quantitatively assessed when studying mechanisms of motility. Various approaches exist to address this task, among which our recently developed Filament Sensor analysis tool allows for automated detection and quantification of stress fibers[5]. However, analytical functional analysis of SFs requires more than detection of filamentous structures in a cell. The ability of actin bundles to exert force to the environment is critical for their function in migration and has to be taken into account. To achieve this goal, actin organization has to be evaluated in conjunction with the organization of cellular adhesion sites.

Cells adhere to the extracellular matrix (ECM) or surrounding cells via cell-matrix and cell-cell contacts, respectively. These structures function as biochemical anchors and are key to the signaling and mechanical interactions of cells with their surroundings. Focal Adhesions (FAs) are cell-matrix anchors based on trans-membrane proteins integrins, with a multitude of associated proteins on the cytosolic side. They have several functions, such as providing cellular attachment to the substrate, transducing cytoskeletal forces to the ECM and facilitating bi-directional transmembrane signaling.[7]

At the cytosolic side, FAs are structurally and dynamically linked to the ends of SFs (Figure 1). The formation and maturation of FAs is dependent on actomyosin-driven tensile forces applied on them through the associated SFs.[7] In turn, signaling pathways that are mechanically triggered at adhesions lead to actin polymerization and elongation of the fibers at their FA-associated termini.[4] Thus, FAs and SFs exist in a constant dynamic feedback.

Because FAs serve as an interface between the SFs and the ECM, they are responsible for the transmission of intracellularly generated contractile forces to the environment. This function is pivotal for the mechanical regulation of cell-matrix interactions. Cellular SFs are broadly classified as transverse arcs, dorsal SFs and ventral SFs based on their FA association, which underlies their varied functionality. Actin transverse arcs which are not associated with FA (Figure 1) but rather embedded into the cortical actin meshwork at their termini, are contractile structures that contribute to cell shape but not do not directly exert force into the environment. Dorsal SFs are associated with FAs at one end and with transverse arcs on the other end (Figure 1).Though they are non-contractile due to their negligible myosin II content, they can exert forces on their terminal adhesion through their association with transverse arcs. Ventral SFs, which are connected to FAs at both ends (Figure 1),are contractile structures that generate majority of cellular traction forces on the substrate.[11,12]. Dynamic interplay and transitions between these actin structure types underlie complex cell motility phenotypes in health and disease. Therefore, detection of SF-FA coupling for the recognition of actin bundle types is critical for understanding of cell mechanics 
and motility.

Manual evaluation of FAs and SFs in cells to analyze them is a laborious, time-intensive process. Recently, this process has been aided by several automated analysis tools and algorithms that are optimized either for focal adhesion analysis (such as the Focal Adhesion Analysis Server [1] and others [2,3], or stress fiber analysis (such as previous version of Filament Sensor [5], CytoSeg [6] and others [9,13].

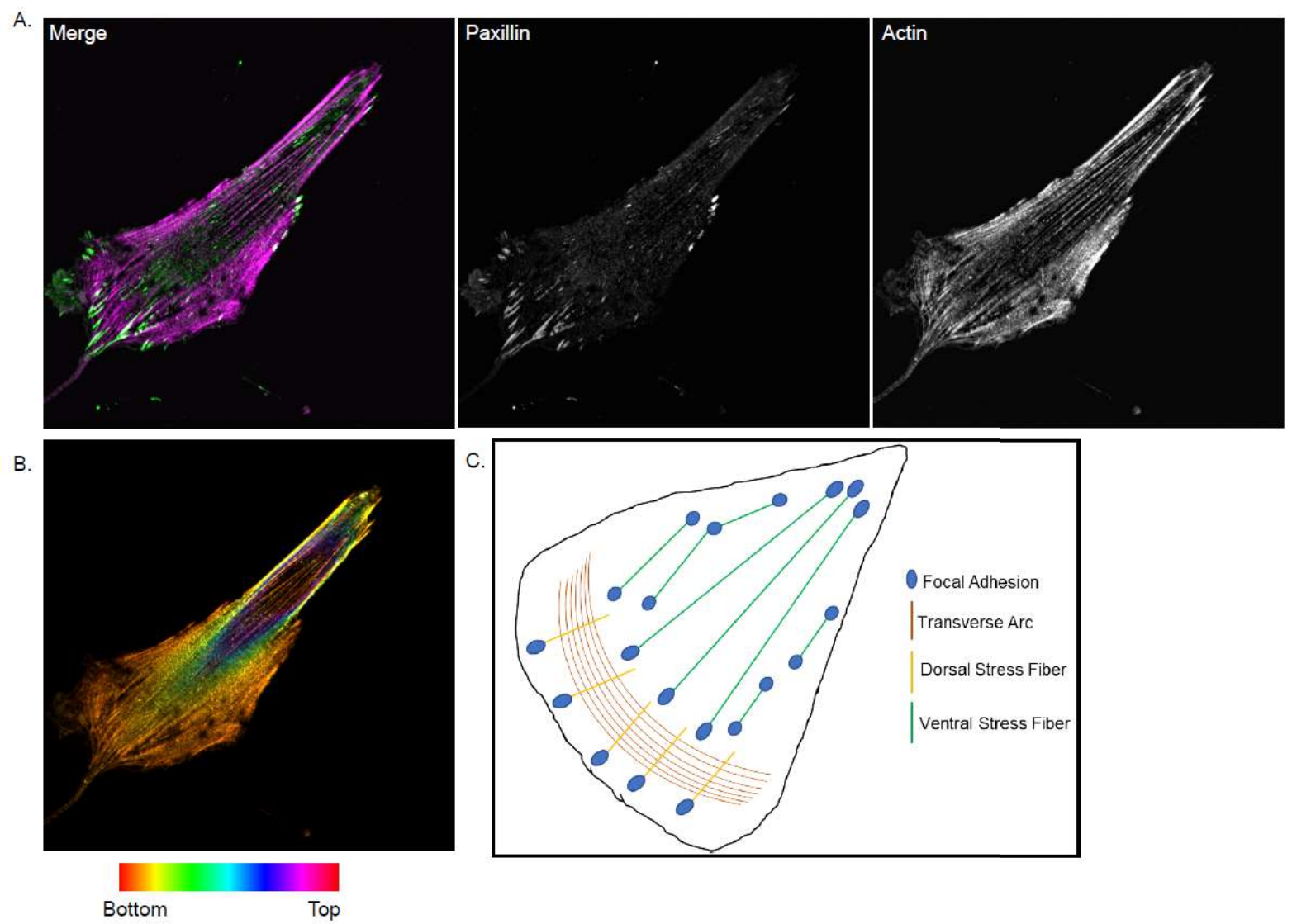

Fig.1: Actin filaments are associated with adhesions in stress fibers of the cell. Representative images of MRC5 cell immuno-stained for actin (phalloidin) and adhesions (paxillin) and imaged with confocal microscope is shown. A) (Left) Merged image of the cell with actin filaments in magenta and adhesions in green, ventral plane. (Center) Greyscale image of the adhesions, ventral plane. (Right) Greyscale image of actin, ventral plane. Scale bar- 10 m. B) Falsely colored z-stack of the actin filaments in the cell using temporal color-coding with Rainbow LUT. Color scale as below image. C) Schematic of the stress fiber subtypes in a cell.

However, a tool for unbiased quantification of SF-FA coupling is yet missing. Here, we present an integrated FA-SF analysis module called the Focal Adhesion Filament Cross-correlation Kit (FAFCK). This tool is based on our previously published Filament Sensor analysis tool, with added capacities for adhesion detection and characterization, filament analysis and coupled FA-SF correlation along with batch processing capabilities to streamline analysis of huge datasets. FAFCK detects and quantifies FAs and SFs by means of location, area, length, width, aspect ratio and orientation, with capacity for exporting this information enumerated for each frame, allowing for a multitude of further data analysis. Our software package will be particularly helpful for sophisticated methods such as model based traction force microscopy (TFM) experiments that are based on knowing the positions of focal adhesions and stress fiber types in addition to the displacement field in the substrate. [11] 


\section{Results:}

\section{Detection of focal adhesions with FAFCK}

The Focal Adhesion Filament Cross-correlation Kit (FAFCK) is a comprehensive software consisting of two modules: the FAFCK, for adhesion detection and FilamentSensor, for filament detection. This setup couples the analysis of FAs and filaments in a cell. As stand- alone programs with a shared GUI, both routines can be used independently. To correlate adhesion with associated stress fibers in the cell, the software has a function that relates each adhesion object detected by FAFCK with corresponding filaments that are detected by the FilamentSensor module.

The FAFCK is the adhesion detection module in the software. It is a robust tool for detection of point-like structures partly based on the FilamentSensor [5]. Based on adapted ImageJ routines (Figure 2), it analyzes the adhesions as objects which can be exported with characteristics and IDs, with myriad customization options to improve accuracy as desired by user.

User inputs IF image of the cellular adhesions in recommended formats based on image type. The module is split into main, pre-processing and FA output sections. All images are shown in panels on the rightincluding original image of adhesions, pre-processed image, thresholded image and image with overlay of filaments detected (Stress Fiber Overlay). The windows can be split from the interface and zoomed in for user ease. Pre-processing tab allows user to add optional filters to image to improve signal to noise ratio and normalize the image. Filters included are the Gauss filter, Laplace filter, Line gauss filter, Cross-correlation filter and Enhance contrast filter. Filter queues can be saved for reuse. The main tab has thresholding controls with automated protocols. The levels can be altered manually to produce the desired binarized image. Additional filters are provided for deciding how many minimum or maximum pixels to include for an adhesion and the maximum amount of clusters allowed in one image.

For each adhesion detected, the outline is derived, and a convex hull is calculated. The main axis is set for the points farthest away on the convex hull and for the points farthest away from the main axis, the minor axis is set. The aspect ratio, orientation, and center for each focal adhesion is also calculated. The module also allows for further close customization of the detected objects by user to obtain the most accurate result. In cases where nearby adhesions have been detected as one due to poor signal separation, overlap, artifacts and more, the user has the option to draw a line on the thresholded image and separate them at their discretion.

Once the lines have been drawn to separate all adhesions as desired, the adhesions can be re-processed to get the split objects in a new map. Adhesions detected are displayed in the table in the Focal adhesions tab. The ID, XY center position, Length of main axis, Length of side axis, Angle, Area and Area ellipse of each adhesion is characterized. User can choose to discard detected adhesion objects by selecting the objects in the Focal Adhesion original window, on which the boundary turns red, and clicking the remove button under the table. This allows user to closely edit the adhesion map obtained from the software to remove any inconsistencies based on their expertise.

The output focal adhesion map image can be exported as a mask with optional numbering with IDs. The table can be exported as a 'CSV' file and the adhesion detection can be exported as a project 'XML' file. We illustrate the usage of the adhesion detection module with input of IF image of ventral plane of adhesions in a MRC5 cell that has been labelled with paxillin and imaged in-focus on a confocal microscope Figure 3A). The image was preprocessed using Laplace and Gauss filters and thesholded using Intermodes algorithm in manual mode. With a minimum limit of 10 pixels per adhesion, objects were detected and optimized by the user, giving an output of numbered adhesion map (Figure 3B). User customizations include splitting adhesions according to user discretion (Figure 3C) and deleting inaccurate objects (not shown).

In the Post thresholding section, there is the option to add or opt-out of the closing algorithm and filling 
holes algorithm, which allow for seemingly disparate objects to be detected as one, especially in cases of large, single adhesion plaques whose signal is not uniform. (Figure 3D) A large, boundary adhesion plaque that is detected as split pieces without the closing and filling holes algorithm, can be re-processed with the algorithm allows for its detection as a single object, which is accurate with user's expert perception of the IF image.

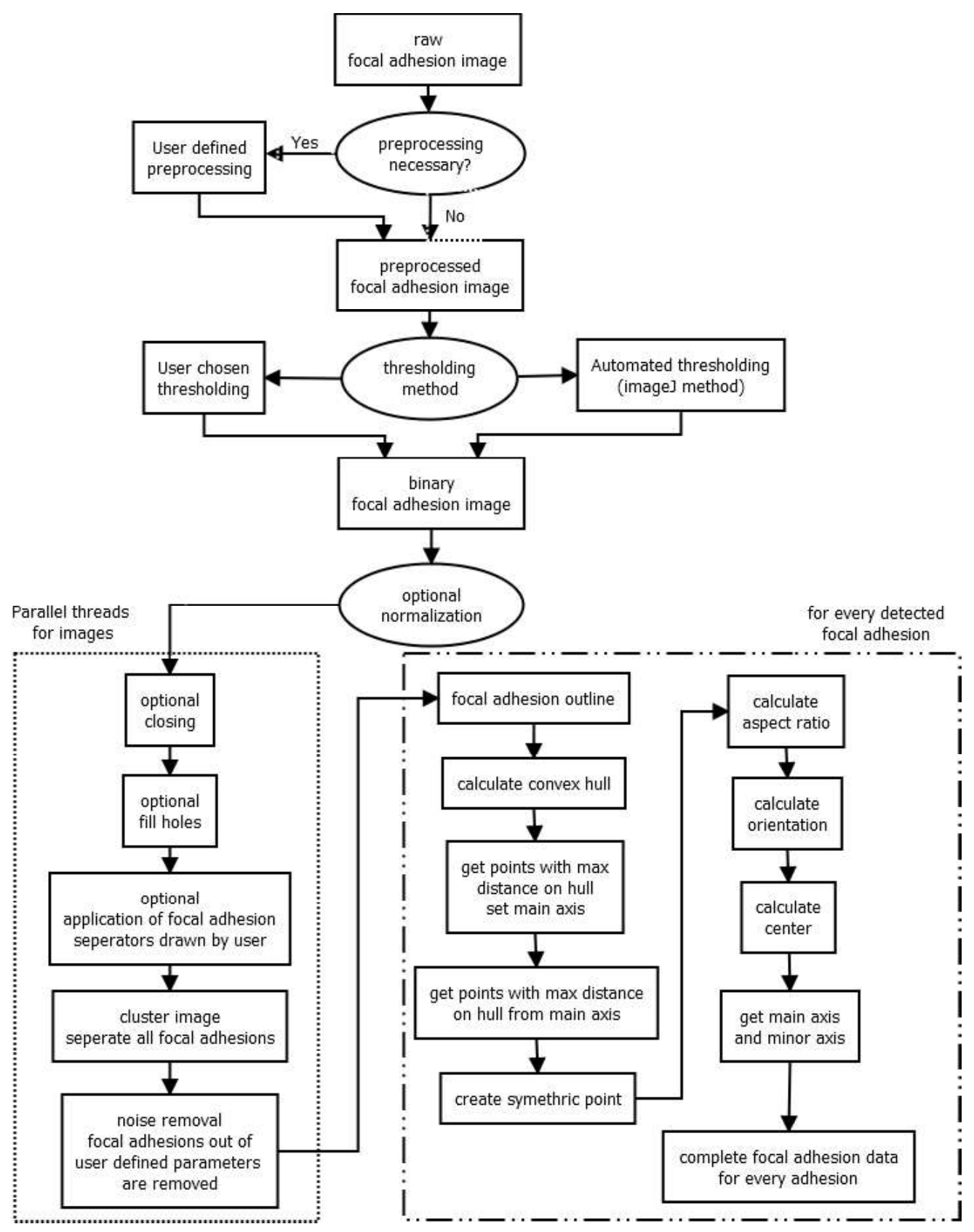

Fig.2: Workflow for adhesion detection. 
A.

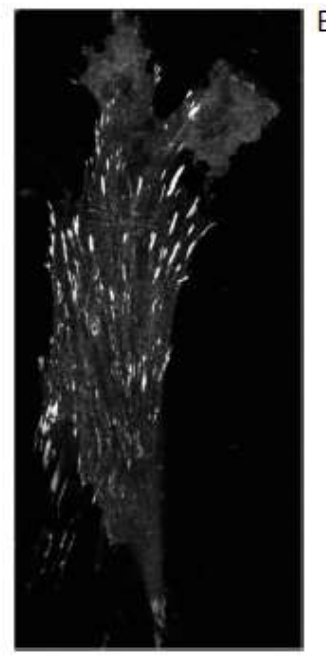

B.

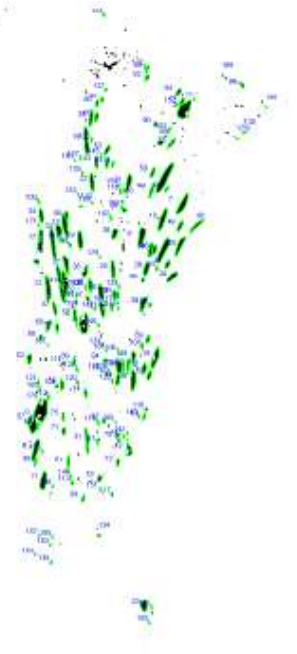

C.
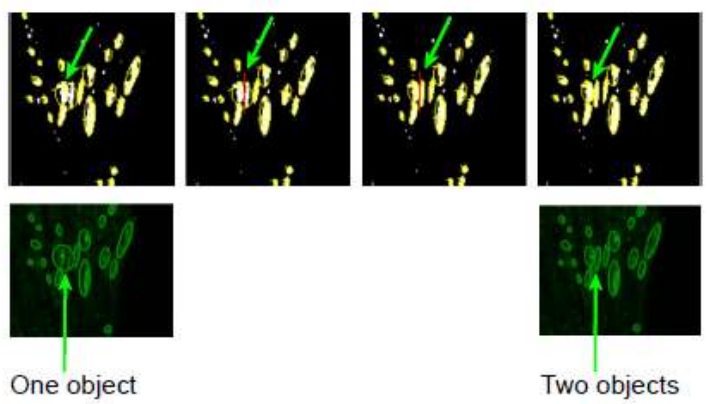

D.
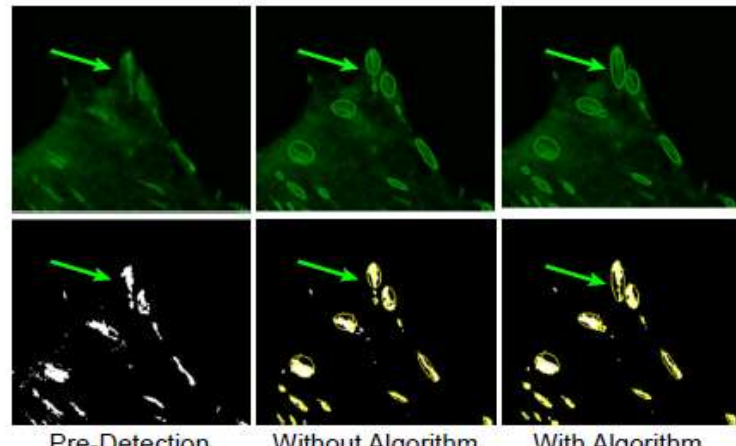

Pre-Detection

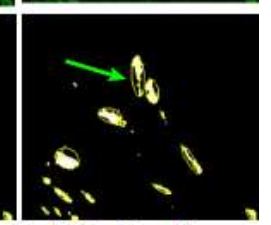

With Algorithm

Fig.3: Introduction of adhesion detection module in FAFCK. Ventral plane of MRC5 cell stained for paxillin and imaged with confocal microscope is input for adhesion detection. Adhesions are in-focus. A) Greyscale image of adhesions, ventral plane. Scale bar- $10 \mathrm{~m}$. B) Map of detected adhesion objects circled by green, numbered C) Montage of adhesion splitting capacity of the module, cell input is same as A (Top) Thresholded objects detected by module are circled by yellow. Red line is drawn by user to split where desired. After processing adhesions again, the objects are split. Green arrow for indication of splitting objects. (Bottom) Before and after images of objects detected on IF signal that are split. D) Montage of closing/filling holes algorithms of the module. IF adhesion image input of a different MRC5 cell than A, imaged with confocal microscope and adhesions are in focus. (Top) Objects detected by software on IF image of the cell (Bottom) Objects circled by yellow on thresholded image of the cell. (Left) Pre-detection by module (Center) Objects detected by module when closing/filling holes algorithms are not applied. (Right) Objects detected by module when closing/filling holes algorithms are applied. Green arrow indicates the adhesion object which is multiple without the algorithm and detected as a single object with the algorithm.

\section{Evaluation of the FAFCK output with user generated mask}

For those considering this software to analyse structures in cells, it is important to understand how the results compares to their expert opinion and any pre-established routines they already use. To accurately assess the differences between user and software in adhesion detection, our module offers an evaluation option. The ground truth image of adhesions marked and provided by the user (Figure 4A) is compared with the image generated by software from IF image input after processing (Figure 4B). The comparison is done in a pixelwise (Figure 4C) and object-wise (Figure 4D) fashion to provide evaluation of the similarity in pixels and objects detected by user vs software and the results can be exported in a csv file. The ground truth image should be a binary mask provided by user. In the example, the mask has been generated by an expert manually drawing the adhesions using Fiji, but it can also be from a Fiji routine or other sources as applicable. 
A.
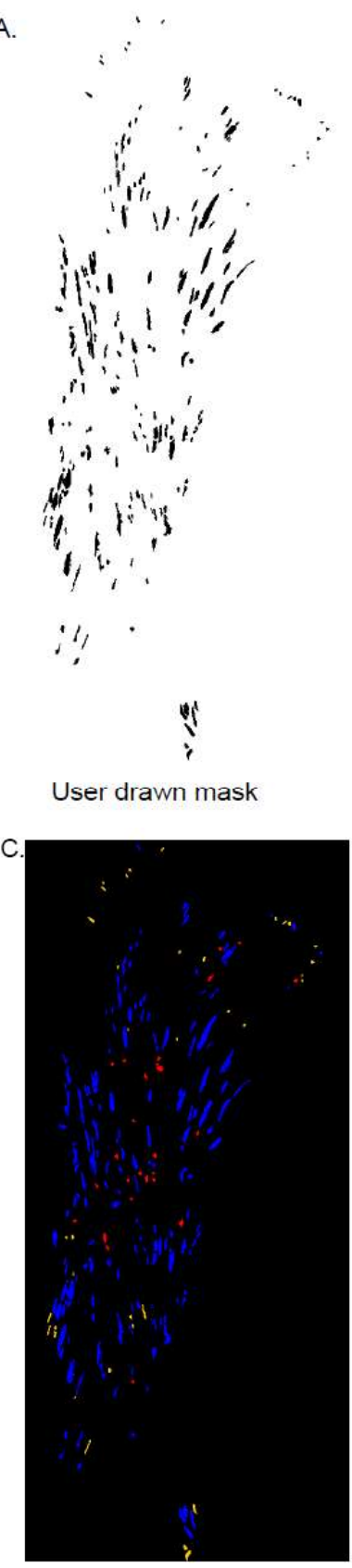

Object-wise comparison
B

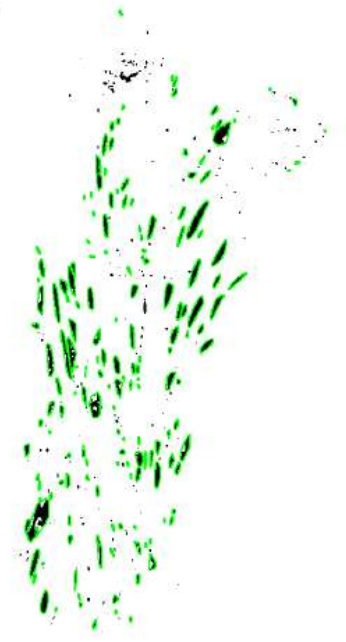

Software generated mask

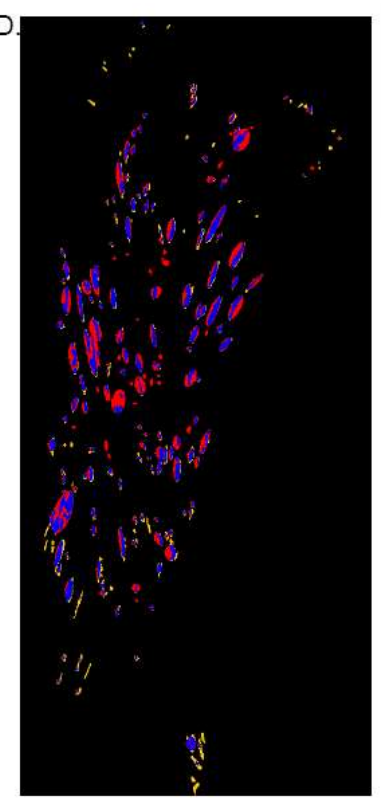

Pixel-wise comparison

Fig.4: Comparison of adhesion detected by user expert in Fiji vs detected by the software module. Input adhesion image same as panel A in Fig 4. A) Binary mask of adhesion ROIs drawn by user with the Freehand tool in Fiji B) Map of adhesion objects detected by software, same as Fig 4 B, not numbered for clarity C) Objectwise comparison of user mask vs software output. D) Pixelwise comparison of user mask vs software output. Blue- detected by user and adhesion module, Yellow- not detected by adhesion module, Red- excluded by optional filters in the module. With pixelwise comparison, hitrate is 0.7471 , missrate is 0.2529 . With objectwise comparison, 168 objects were found, 37 were missed, 5 cases where sensor finds multiple objects for one object marked by expert (multiMatchesOneToN) and 22 cases where sensor finds one object for multiple objects marked by expert (multiMatchesNToOne). 


\section{Detection of Stress Fibers with FilamentSensor}

The Filament Sensor, as integrated here in the FAFCK, is based on the version published by Eltzner et al [5], in 2015, adjusted to feature stack handling of image sequences and drastically reduced runtime as well as some additions for area calculation. The plugin featured in the FAFCK includes all components included in the stand-alone software, being a preprocessing, line sensor, and filament submenu. The workflow of the software as published before is included in Suppl 1.

During preprocessing, contrast and brightness can be adjusted for either individual pictures or a whole stack, if needed. These routines are based on ImageJ [10], which is included as an internal library and used wherever possible, as the ImageJ routines are fast and well tested. The main preprocessing step consists of a filter queue which the user can customize to their needs. This serves as the 'artificial retina' to prepare the original fiber image for binarization, tackling the issue of crossing fibers that have to be recognized as individual structures. On this image, the binarization is applied and fiber objects extracted according to the flowchart shown in Suppl Fig 2. This is done in parallel threads to improve runtime and subjected to several boundaries the user can determine including minimal and maximal length, maximal curvature, width, restriction to cell area mask, and more. This flexibility allows for the program to be utilized for a wide variety of fiber types. Lastly, the filament subsection allows to filter filament data for export. The Filament Sensor module offers a set of descriptors of the whole cell such as IDs, area, aspect ratio, center of mass, length of axes, number of filaments, orientation, brightness, and for each individual fiber such as endpoints, length, curvature, width, brightness and orientation. For each image file, the fiber objects are assigned an individual identifier as done for the focal adhesion objects and a variety of export types are available with the option of superimposing filaments as required.

\section{Correlation of focal adhesions and stress fibers in FAFCK}

As focal adhesions and actin filaments are innately linked cellular structures, the module offers correlation of detected focal adhesions from FAFCK and filaments from FilamentSensor. The workflow is illustrated in Figure 5.

Using the file name of the original images or image stacks loaded, it is first checked whether data for both conditions exist and single sets are ignored. The filaments are derived from the IF image of the cell and paired with focal adhesion objects detected by the software. (Figure 6A) For every fiber in an image, every detected focal adhesion is tested with the requirement that focal adhesion main axis has to be longer than the fiber length. For the focal adhesion objects, the user decides whether the convex hull or a fitted ellipse is used for verification purpose. This ellipse is calculated by setting the line between the two points with the greatest distance on the convex hull as long axis and the axis orthogonal to that and with the greatest length as short axis. Furthermore, the area can be artificially increased by increasing the neighborhood in which verification is done. Now, starting from the ends, for each point on the fiber, a neighborhood rectangle is created and in the list of focal adhesions with main axis length below fiber length, intersecting objects are searched.

The correlation can be done with condition of validating all filaments that are attached to atleast a single adhesion (Figure 6B), or multiple structures along the filament can be validated (Figure 6C), providing a breakdown of the percentage of filaments by adhesion association in the cell (Figure 6D).

The output of the correlation routine consists of the identifier numbers of the respective objects and can consecutively be matched to the data output of the previous routines. Also, verified filaments will be highlighted in the fiber overlay and after verification the fiber data export will be expanded by a 'verification' column with booleans. Overlay images can be exported as well with structures painted in different, fixed colors to ease reuse. In contrast to the FilamentSensor, which offers a 'Batch analysis' option to automate fiber detection in large datasets consisting of multiple stacks, for the FAFCK this was not an option, as the goal is the correlation of two separate analysis steps. 
The output is displayed in the Stress Fiber Overlay window in the main tab in FAFCK. The resulting paired filament and focal adhesion IDs are displayed in a table in the Focal Adhesion tab and can be exported as a 'csv' file. Thus, this correlation provides an overview of adhesion and filament patterning and pairing in cells that can be used to streamline quantitative evaluation of alterations in stress fibers organization.

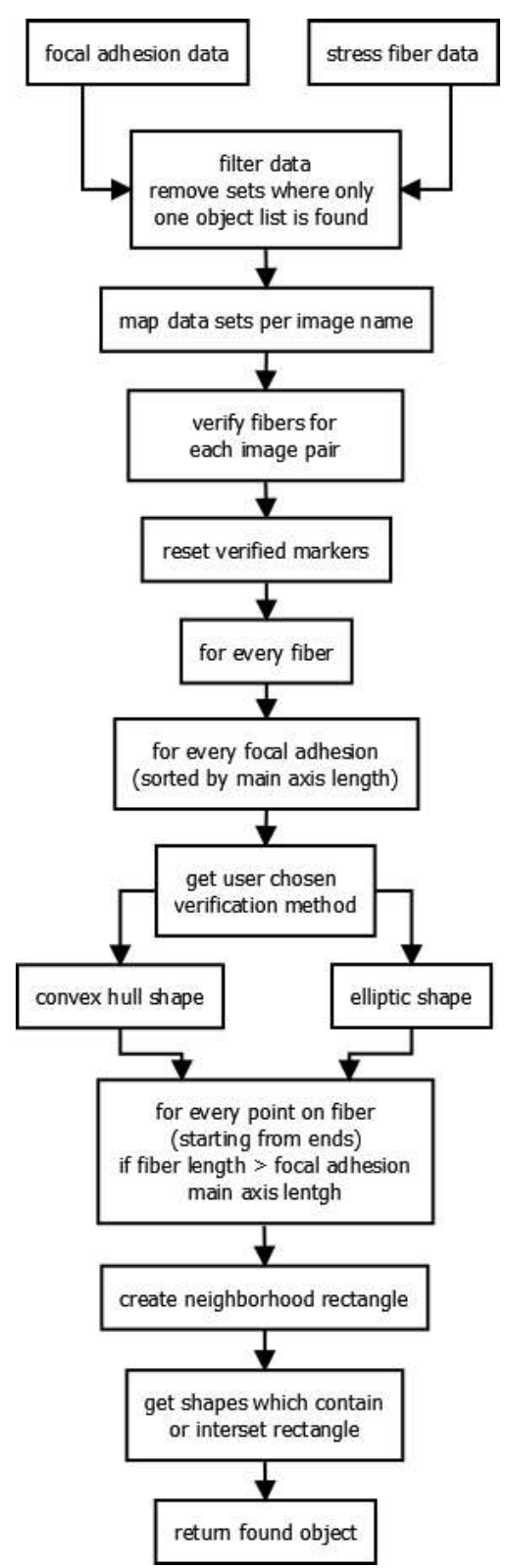

Fig.5: Workflow for correlation between detected adhesion objects and filaments. 


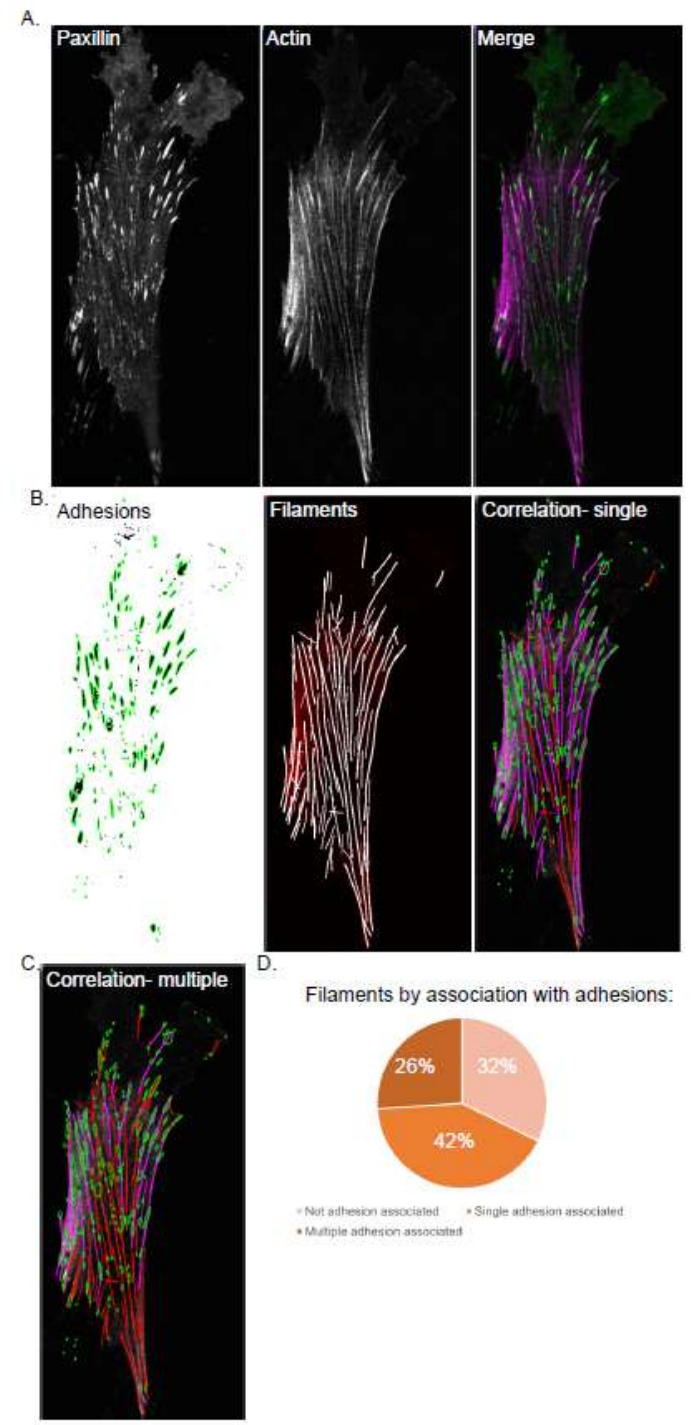

Fig.6: Correlation of detected adhesion objects with detected filaments by FAFCK. Ventral plane of MRC5 cell stained for paxillin and phalloidin and imaged with confocal microscope is input for detection. Adhesions and filaments are in-focus, all images are of ventral plane of the cell. Cell corresponds to Fig 4 and 5. A) (Left) Greyscale image of adhesions. (Center) Greyscale image of actin filaments. (Right) Merged image of the cell with actin filaments in magenta and adhesions in green. Scale bar- $10 \mathrm{~m}$. B) (Left) Map of adhesion objects detected by software, same as Fig 4 B, not numbered for clarity (Center) Map of filaments detected by software, superimposed on IF image of filaments (Right) Map after running correlation of filaments with adhesion objects, with condition that a validated filament has atleast a single adhesion object associated. Superimposed on IF image of filaments. Green- adhesion objects, Magenta- validated filaments, Red- Filaments not validated C) Map after running correlation of filaments with adhesion objects, with condition that a validated filament has multiple adhesion objects associated. Superimposed on IF image of filaments. Green- adhesion objects, Magentavalidated filaments, Red- Filaments not validated D) Pie chart of percentages of detected filaments by association with adhesion objects. Total filaments detected $=96$, corresponding to $100 \%$. Not adhesion associated $=31$ filaments, Single adhesion associated $=40$ filaments, Multiple adhesion associated $=25$ filaments. 


\section{Comparison of manual annotation with software output in varied imaging conditions}

As the FAFCK should offer robust detection of focal adhesions, fibers, and verification of both on a wide variety of image qualities, we compiled comparison datasets of different imaging conditions in which structures were manually labeled by a human expert for comparison.

The dataset consists of 50 images with 10 images in each condition, showcased by representative images in Suppl Fig 1. MRC5 cells were grown on coverslips overnight, fixed with PFA and immuno-stained for adhesions (paxillin) and actin filaments (phalloidin). Z-stacks were taken with varying imaging conditions as follows- 3 sets taken with confocal line-scanning microscope as Confocal in-focus dataset (S1A), Confocal mild-blur dataset (S1B) and Confocal severe blur dataset (S1C). Blur was introduced in images by altering size of pinhole to include out-of-focus light. 2 sets were taken with epifluorescence microscope in in-focus (S1D) and out of focus conditions (S1E), to introduce blur far beyond the range of the confocal microscope. For manual annotation, all the images were marked for adhesions and actin filaments. With the aim of being natively user- detected, adhesions were traced using the freehand selection tool in Fiji and actin filaments were traced using the segmented line tool. Images were traced with minimal signal manipulation to compare the base-level manual annotation by eye with the objects traced by FAFCK after processing by software in the respective modules for adhesions and filaments.

\section{Discussion:}

We have developed the FAFCK that allows for fast and reliable extraction of fiber and point-like structure data as well as cross-correlation of both. While analysis of both types of structures alone is useful, cross-correlation in the module can also answer many underlying questions of the coupled function of these cytoskeletal features.

There are several notable advantages to out new tool. Importantly, our tool allows to identify groups of SF associated with 0,1 , or more than one FA. This allows for an approximated quantification of the relative number and characteristics of transverse arcs (0 FA per filament), dorsal SFs (1 FA per filament), and ventral SFs (2 FA per filament) in large data sets. This type of analysis allows to recognize different types of actin bundles in 2-dimentional images, such as maximum intensity projections of confocal stacks, which significantly quickens the quantification.

Furthermore, application of all the filters and optimizations allows us to analyze wide-field epifluorescence images and images with high blur and/or background.

Of course, there is a degree of error in the computational recognition as well as in the user detection of cellular features. The main idea is that this software allows for the comparison of large data sets where small errors can be neglected. In addition, we provide an option to customize output in each image, which also allows for more precise detection of SF types in smaller data subsets.

Also, it is important that we provide an option to import detection data of filaments and FAs from other software or manual detection. On one hand, it allows for comparison of the computational recognition with individual user perception of the biological reality. On the other hand, it accounts for the continuous improvement of image recognition software in the field and provides a possibility of importing data from a potentially more precise recognition platforms to be developed in the future. Thus, this tool is designed for a long life in the rapidly developing world of computational image analysis.

\section{Materials and methods:}

\section{Cell culture:}

MRC5 cells (ATCC ${ }^{\circledR}$ CCL-171) were maintained in MEM media (Thermo Fisher Scientific) supplemented with $10 \%$ FBS (fetal bovine serum), $100 \mathrm{U} / \mathrm{ml}$ penicillin and $0.1 \mathrm{mg} / \mathrm{ml}$ streptomycin in $5 \% \mathrm{CO}_{2}$ at $37^{\circ} \mathrm{C}$. 
Media was supplemented with $5 \mathrm{~g} / \mathrm{ml}$ Plasmocin (InvivoGen) as a prophylactic against mycoplasma contamination.

\section{Sample preparation:}

MRC5 cells (human lung fibroblasts, ATCC ${ }^{\circledR}$ CCL-171) are seeded on glass coverslips (No. 1.5, $12 \mathrm{~mm}$, circular, Fisher Scientific, Hampton, NH) that have been coated with $10 \mathrm{~g} / \mathrm{ml}$ fibronectin (Biological Industries, Beit HaEmek, Israel) for 1 hour. After 24 hours cells are fixed. 4\% PFA (Paraformaldehyde) was prepared in CB (Cytoskeletal Buffer- $150 \mathrm{mM} \mathrm{NaCl}, 5 \mathrm{mM} \mathrm{MgCl}_{2}, 5 \mathrm{mM}$ EGTA, $5 \mathrm{mM}$ glucose, $10 \mathrm{mM}$ MES). Coverslips with cells were fixed with $4 \%$ PFA in CB for $10 \mathrm{~min}$ at room temperature and washed with CB thrice after fixation. They were permeabilized with $0.25 \%$ Triton X-100 in CB and then blocked with 1\% BSA (bovine serum albumin, Fisher) and 5\% DHS (donor horse serum, Atlanta Biologicals) in PBS for 1 hour.

\section{Fixation and Immunofluorescence staining:}

Coverslips were incubated with a primary anti-paxillin mouse antibody (1:200, BD Transduction Laboratories, 610051) overnight at $4^{\circ} \mathrm{C}$, washed thrice with PBS and incubated with Alexa Fluor 568 Phalloidin (1:300, Invitrogen, A12380) to visualize F-actin and a secondary anti-mouse antibody conjugated with Alexa Fluor 568 (1:300, Invitrogen, A12380) for 1 hour at room temperature. They were washed thrice with PBS, fixed again for $10 \mathrm{~min} 4 \% \mathrm{PFA}$ in $\mathrm{CB}$ at room temperature and mounted with Vectashield Mounting Medium (Vector Labs).

\section{Confocal Imaging:}

Fixed samples were imaged using a laser scanning confocal microscope- Nikon A1r based on a TiE Motorized Inverted Microscope using 100 lens, NA 1.49, run by NIS Elements C software. Three different pinhole settings were used to adjust the amount of out-of-focus light in the images- $1.2 \mathrm{AU}$ (small, in focus), $3 \mathrm{AU}$ (intermediate, light blurr) and $4.7 \mathrm{AU}$ (large, severe blurr). For FITC: Emission wavelength: $525.0 \mathrm{~nm}$, Excitation wavelength: $488.0 \mathrm{~nm}$. For TRITC: Emission wavelength: $595.0 \mathrm{~nm}$, Excitation wavelength: $561.0 \mathrm{~nm}$.

\section{Epifluorescence Imaging:}

Fixed samples were imaged using a Nikon Eclipse 80i fluorescence microscope, using 100x lens, NA 1.40. Micro-Manager 1.4.22 software was used to acquire images.

\section{Manual Annotation by Human Expert:}

Images from all the conditions were completely manually marked for FAs and SFs by a human expert (10 images each for confocal in-focus set, confocal mild blur set, confocal severe blur set, epifluorescence in focus set and epifluorescence blur set). FAs and SFs were marked using the freehand selection tool and segmented line tool respectively in Fiji. Some limitations of manual tracing of fibers are the inability to resolve and separately trace fibers that are close together, such as in fine arcs and the cell edges. To investigate the coupled FAs and SFs, sets of stress fibers that were associated with adhesions in the cell were also marked by the human expert. In cases of images with areas of increased background signal, artifacts and hard-to distinguish areas, user annotated structures by following the fluorescence pixels instead of arbitrarily marking the area. 


\section{Supporting information}

\section{S1 Fig.}

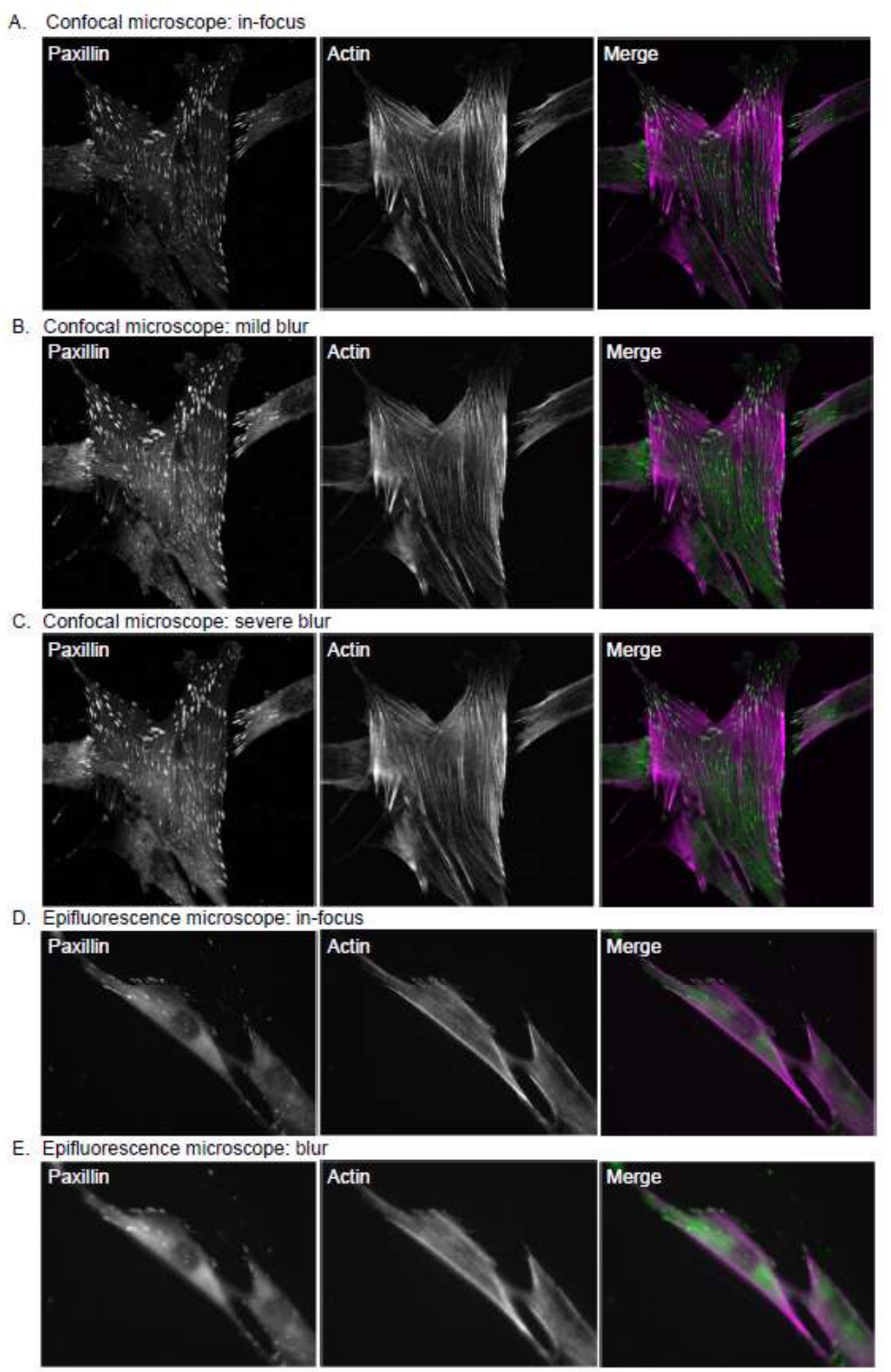

Imaging conditions for the bulk datasets used for comparison between base-level manual annotation and software detection. Representative images of MRC5 cells immuno-stained for actin (phalloidin) and adhesions (paxillin) is shown. Scale bar- $10 \mathrm{~m}$. All images are of the ventral plane of the cell. (Left) Greyscale image of the adhesions (Center) Greyscale image of actin (Right) Merged image of the cell with actin filaments in magenta and adhesions in green A) In-focus images taken with confocal microscope, pinhole at 1.2 AU B) Mild blur images taken with confocal microscope, pinhole at 3 AU C) Severe blur images taken with confocal microscope, pinhole at 4.7 AU D) In-focus images taken with epifluorescence microscope $\mathbf{E}$ ) Blurred images taken from out of focus plane with epifluorescence microscope. 


\section{S2 Fig.}

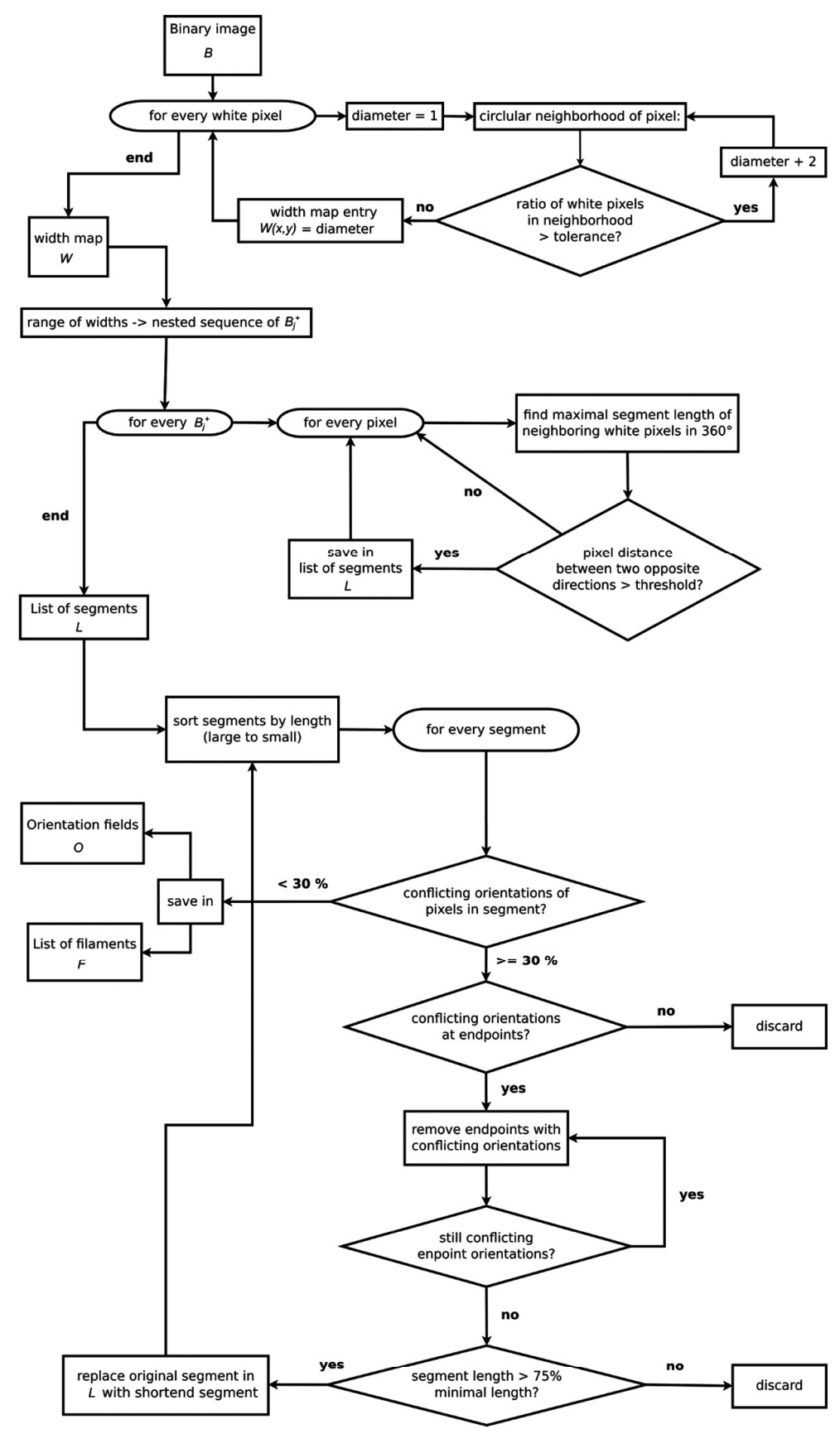

Workflow for fiber detection using Filament Sensor. 


\section{References}

1. Berginski, M.E.; Gomez, S.M., "The Focal Adhesion Analysis Server: a web tool for analyzing focal adhesion dynamics", F1000Research 2013, 10.12688/f1000research.2-68.v1.

2. Broussard, J.A.; Diggins, N.L.; Hummel, S.; Georgescu, W.; Quaranta, V.; Webb, D.J., "Automated Analysis of Cell-Matrix Adhesions in 2D and 3D Environments", Scientific Reports 2015, $10.1038 /$ srep08124.

3. Buskermolen, A.B.C.; Kurniawan, N.A.; Bouten, C.V.C., "An automated quantitative analysis of cell, nucleus and focal adhesion morphology", PLOS ONE 2018, 10.1371/journal.pone.0195201.

4. Ciobanasu, C., Faivre, B., Le Clainche, C., "Actin dynamics associated with focal adhesions", International journal of cell biology 2010 .

5. Eltzner B, Wollnik C, Gottschlich C, Huckemann S, Rehfeldt F., "The Filament Sensor for Near Real-Time Detection of Cytoskeletal Fiber Structures.", PLOS ONE 10 2015; e0126346.

6. Nowak, J.; Gennermann, K.; Persson, S.; Nikoloski, Z., "CytoSeg 2.0: automated extraction of actin filaments", Bioinformatics 2020, 36(9); 10.1093/bioinformatics/btaa035.

7. Parsons, J.T., Horwitz, A.,R., Schwartz, M.A."Cell adhesion: integrating cytoskeletal dynamics and cellular tension", Nature reviews Molecular cell biology 2010, 11(9).

8. Pellegrin, S., Mellor, H., "Actin stress fibres", Journal of cell science 2007, 120(20).

9. Rogge, H.; Artelt, N.; Endlich, N.; Endlich, K., "Automated segmentation and quantification of actin stress fibres undergoing experimentally induced changes", Journal of Microscopy 2017, 268(2); 10.1111/jmi.12593.

10. Schneider, C. A.; Rasband, W. S. Eliceiri, K. W., "NIH Image to ImageJ: 25 years of image analysis", Nature methods 2012; 9(7): 671-675, PMID 22930834.

11. Soine, J.R.D.; Brand, C.A.; Stricker, J.; Oakes, P.W.; Gardel, M.L.; Schwarz, U.S., "Modelbased Traction Force Microscopy Reveals Differential Tension in Cellular Actin Bundles", PLOS Computational Biology 2015; 10.1371/journal.pcbi.1004076.

12. Vallenius, T., "Actin stress fibre subtypes in mesenchymal-migrating cells", Open biology 2013, 3(6).

13. Zhang, Z.; Xia, S.; Kanchanawong, P., "An integrated enhancement and reconstruction strategy for the quantitative extraction of actin stress fibers from fluorescence micrographs", BMC Bioinformatics 2017, 18(1); 10.1186/s12859-017-1684-y. 


\subsection{MS-TIRF used for imaging of focal adhesion localized paxillin}

As described in Section 3.4, a simultaneous analysis of stress fibers and focal adhesions can yield additional information. Proteins like paxillin are, however, present not only in the focal adhesions but also in other structures. This presents a major difficulty for proper analysis.

Total Internal Reflection Microscopy (TIRF) is a technique to limit observation to the cells' membrane bordering the substrate. However, usually the field of view is limited. In this paper, we describe a method for TIRF using a metasurface. This allows for a significantly larger field of view, and thus has the potential to be used for live cells and in combination with time-lapse imaging. The paper is integrated here as submitted to Biomedical Optics Express [78].

My contribution to this paper is the fabrication of biological samples, in detail culture of BM-hMSCs, staining, and preserving. Additionally I wrote the respective section in the paper and gave advise on the biological implications to Antu Gortari.

\section{Metasurface-based Total Internal Reflection Microscopy}

Antu Nehuen Gortari ${ }^{\mathrm{a}, *}$, Sophie Bouchoule ${ }^{\mathrm{a}}$, Edmond Cambril ${ }^{\mathrm{a}}$, Andrea Cattoni ${ }^{\mathrm{a}}$, Lara Hauke ${ }^{\mathrm{b}}$, Jörg Enderlein $^{\mathrm{b}}$, and Florian Rehfeldt ${ }^{\mathrm{b}}$, Alejandro Yacomotti ${ }^{\mathrm{a}, *}$

${ }^{a}$ Ctr. de Nanosciences et de Nanotechnologies, Univ. Paris-Sud, Univ. Paris-Saclay, France;

${ }^{\mathrm{b}}$ Third Institute of Physics-Biophysics, Georg August University, Göttingen, Germany

*antu-nehuen.gortari@c2n.upsaclay.fr

\section{ABSTRACT}

Recent years have seen a tremendous progress in the development of dielectric metasurfaces for visible light applications. Such metasurfaces are ultra-thin optical devices that can manipulate optical wavefronts in an arbitrary manner. Here, we present a newly developed metasurface which allows for coupling light into a microscopy coverslip to achieve total internal reflection (TIR) excitation. TIR fluorescence microscopy (TIRFM) is an important bioimaging technique used specifically to image cellular membranes or surface-localized molecules with high contrast and low background. Its most commonly used modality is objective-type TIRFM where one couples a focused excitation laser beam at the edge of the back focal aperture of an oil-immersion objective with high numerical aperture (N.A.) to realize a high incident-angle plane wave excitation above the critical TIR angle in sample space. However, this requires bulky and expensive objectives with a limited field-of-view (FOV). The metasurface which we describe here represents a low cost and easy-to-use alternative for TIRFM. It consists of periodic 2D arrays of asymmetric structures fabricated in $\mathrm{TiO}_{2}$ on borosilicate glass. It couples up to $70 \%$ of the incident non-reflected light into the first diffraction order at an angle of $65^{\circ}$ in glass, which is above the critical TIR angle for a glass-water interface. Only $\sim 7 \%$ of the light leaks into propagating modes traversing the glass surface, thus minimizing any spurious background fluorescence originating far outside the glass substrate. We describe in detail design and fabrication of the metasurface, and validate is applicability for TIRFM by imaging immunostained human mesenchymal stem cells over a FOV of $200 \mu \mathrm{m}$ x $200 \mu \mathrm{m}$. We envision that these kinds of metasurfaces can become a valuable tool for low-cost and TIRFM, offering high contrast, low photodamage, and high surface selectivity in fluorescence excitation and detection.

\section{Introduction}

During the last three decades, TIRFM has become an important microscopy technique for surface-restricted high-contrast and low-background fluorescence microscopy. [1-3] It is ideally suited to study cell membranes or surface-bound molecules. Most TIRFM applications use so-called objective-type TIR excitation (see 
e.g. $[4,5])$, where a focused laser beam is coupled into a high-N.A. objective at the edge of the back focal aperture to generate a plane-wave illumination in sample space, with an incidence angle above the critical TIR angle of the glass-sample interface. This then generates an evanescent excitation above the glass interface with a field intensity that exponentially decays with increasing distance from the interface. Such an approach requires oil-immersion objectives with a N.A. significantly larger than the refractive index of the cytoplasmic of a cell that can be as high as 1.4, i.e. an objective with N.A. $>1.4$. Besides being bulky and expensive, such objectives typically do not provide a large FOV.

In recent years, one has seen the rapid and broad development of a new generation of optical components, so-called optical metasurfaces (MSs), that can be seen as the two-dimensional variant of optical metamaterials and are expected to have a major impact on a wide range of next-generation of optical systems. MSs typically consist of periodic or quasi-periodic arrays of nanoresonators which modulate the phase of an incident wavefront in an arbitrary manner. This allows tailoring the phase properties of a wavefront in any desirable way, both in reflection and transmission mode. In contrast to traditional refractive optical elements, where phase changes of a propagating wavefront are accumulated rather gradually, a MS imparts an abrupt phase change on a propagating wavefront, usually on a length scale below one wavelength. This unique characteristic, along with the possibility to achieve arbitrary spatial phase modulation that is rather impossible to realize with conventional optical elements, has proven to be useful for a wide range of applications such as spectroscopy [6], integrated optics [7], optical filtering [8], multi-color imaging [9], holography [10,11], or biosensing [12].

State-of-the-art metasurfaces that are adapted to the visible spectrum can be especially important for optical microscopy, despite the relative scarcity of current efforts in this direction.Besides some impressive ongoing progress towards the development of achromatic metalenses (whose scope of applications goes well beyond microscopy), only few publications with relation to microscopy can be mentioned. Among them are the use of MSs to improve the axial resolution in laser scanning microscopy [13], the employment of metalenses for two-photon microscopy [14], the improvement of fluorescence-microscopy image contrast by using resonant structures [15], or the removal of orientation-induced localization errors in single-molecule localization microscopy $[16,17]$.

Beam steering has been among the first pursued objectives in all MS developments, usually as first step towards more complex goals such as beam focusing. One of the first experimental realizations of this concept dates back by more than 20 years [18], with a binary grating more efficient than conventional "échelette" blazed gratings at high angles, where they are limited by their shadowing effect [19]. While the efficiency and complexity of these beam-steering MSs has increased significantly [20-23] with the advent of more advanced design tools (e.g. inverse design algorithms [21-23]) and nanofabrication technologies, to the best of our knowledge theywere never used for MS-based TIRF microscopy.

The MS-based TIRF approach presented in this paper stems from a custom-designed MS and does not require any TIR-capable high-N.A. objective. Instead, a dedicated MS grating on the bottom of a glass coverslip redirects most of the incoming laser light (plane wave with perpendicular incidence angle with respect to the glass surface) into one of the first diffraction orders, thus acting as a blazed grating light-coupler. This generates a high-incidence angle widefield TIR illumination on the top side of the coverslip, with negligible propagation losses due to the extremely low extinction coefficient of the borosilicate glass in the visible range. We experimentally demonstrate that our MSs can couple up to $70 \%$ of the incident non-reflected light into the first diffraction order with a propagation angle of $65^{\circ}$ with respect to the normal, which is sufficient for TIR excitation at the coverslip's top surface. We demonstrate the applicability of these MS-substrates for TIRFM by imaging immunostained human mesenchymal stem cells (hMSCs) derived from whole bone marrow, which are often too large to be fully imaged by an objective-type TIRF system. In contrast to other recent experimental large-FOV TIRF techniques [24,25], this approach requires a single objective and the evanescent field's penetration depth can be modified by adjusting the MS's period or the angle of the incident beam.This paper is organized as follows: in Section 2 we discuss the design, fabrication, and characterization of the TIRF-MS-Substrates. In Section 3, we 
apply them to fluorescence microscopy and bioimaging. Section 4 provides the final conclusions.

\section{Metasurface design, fabrication, and characterization}

When designing our MS we wanted to use only biocompatible materials that are transparent to light in the visible spectrum while maximizing the refractive index contrast between the MS nanostructure material and the substrate. Thus, we have chosen $\mathrm{TiO}_{2}$ for the MS nanostructure material, and borosilicate glass for the substrate. The fabrication process of our MSs is as follows: A $230 \mathrm{~nm}$-thick layer of amorphous $\mathrm{TiO}_{2}$ is deposited (Plassys MEB $800 \mathrm{IAD}$ e-beamgun system) on a $25 \times 50 \mathrm{~mm}$ borosilicate glass. Two positive electron beam resists (A2 PMMA and EL10 MMA) are sequentially spin-coated to create a bilayer stack with a higher-sensitivity lower section, metalized with $20 \mathrm{~nm}$ of aluminum, and patterned using an electron beam writer(Raith EBPG-5000+). After a MIBK/IPA (1:3) resist development, the samples are rinsed in IP alcohol and a $20 \mathrm{~nm}$ nickel layer is deposited (Plassys MEB550SL e-beam evaporator). A lift-off process is performed in a trichloroethylene bath $\left(85^{\circ} \mathrm{C}\right)$ followed by a $\mathrm{HBr} / \mathrm{BCl}_{3}$ TPSA-ICP $\mathrm{TiO}_{2}$ reactive-ion etching. The remaining $\mathrm{Ni}$ is finally removed with a 1-min immersion in nitric acid $26 \%$.

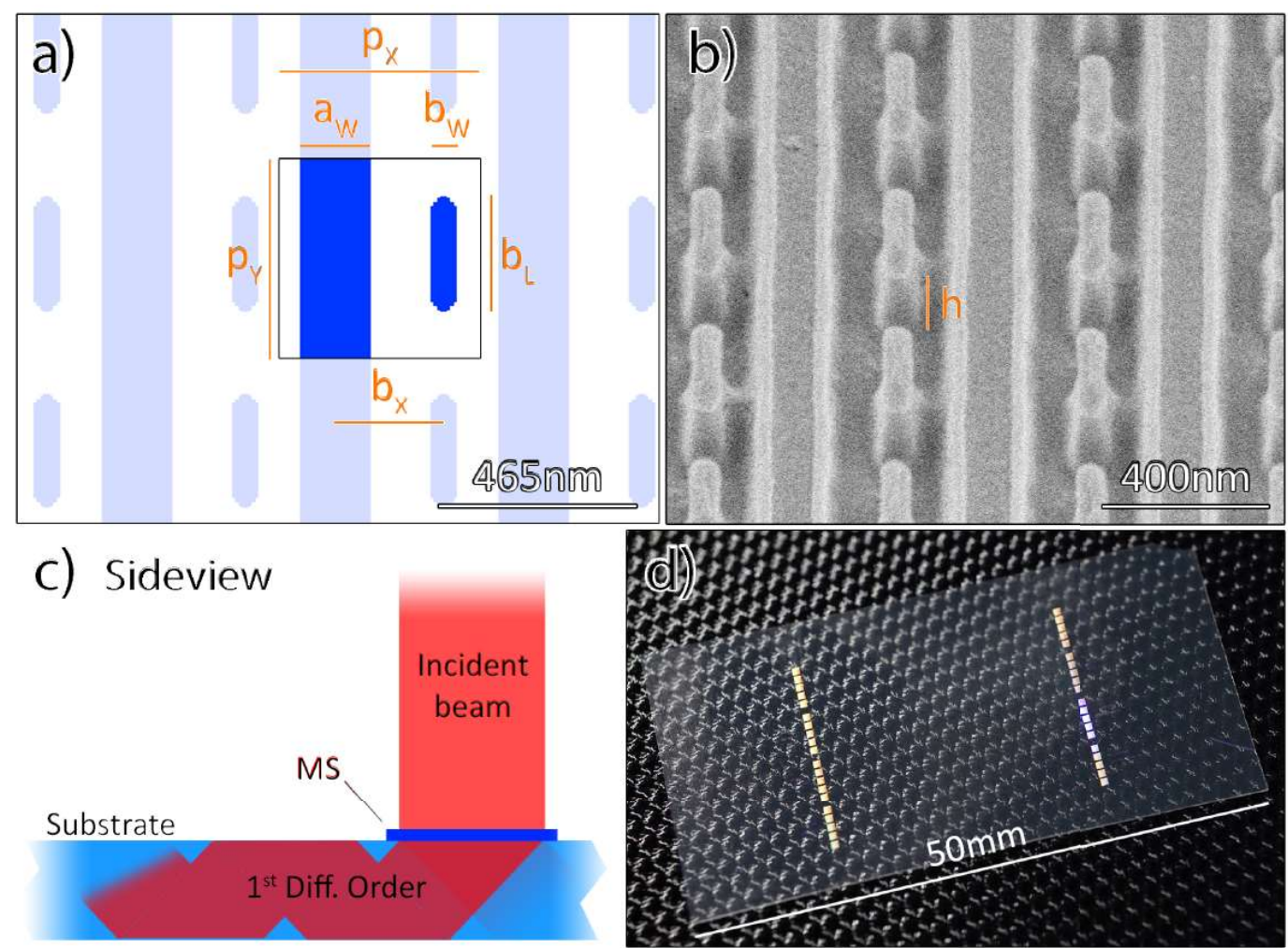

Fig.1: Metasurface design for MS-TIRF. (a) The horizontal geometry of the chosen 2D metasurface design for $\lambda 0=640 \mathrm{~nm}$, with $\mathrm{pX}=\mathrm{pY}=465 \mathrm{~nm}=0.73 \lambda 0, \mathrm{aW}=160 \mathrm{~nm}$, bW $=61 \mathrm{~nm}, \mathrm{bX}=186 \mathrm{~nm}, \mathrm{bL}=322 \mathrm{~nm}$, and a height $\mathrm{h}=230 \mathrm{~nm}$ a long with (b) a scanning electron microscope (SEM) image ( $45^{\circ}$ tilt) of the fabricated sample. (c) When TE-polarized light hits the MS, up to $70 \%$ of the incident light is coupled into one of the first diffraction orders towards the center of the substrate, $21 \%$ is coupled into the opposite first order, and only $9 \%$ into the traversing zeroth order. (d) The entire $25 \times 50 \mathrm{~mm}$ substrate can accommodate multiple MSs, with 40 shown in this sample.

For the MS's design, we utilized the Rigorous Coupled-Wave Analysis (RCWA) software RETICOLO [26] to calculate the diffraction efficiencies and amplitudes of 2D stacked gratings. Starting from a 1D double 
groove structure (similar to those of [27] and [28]) and using a modified gradient ascent algorithm over hundreds of sets of 9 random initial dimensional parameters that define a generic structure layout, we converged to the design shown in Figure 1 (a) and (b). For this purpose, we defined and used a figure of merit (FOM) defined as the ratio between the diffraction efficiency of one of the first orders (-1) and the sum of the other two orders (i.e. 0th and 1st),resulting in an asymmetric composite layout of alternating continuous and segmented lines. This line segmentation was the optimal $\mathrm{TiO}_{2}$ distribution when our current fabrication constrains we reconsidered and allowed us to obtain the highest possible efficiency for a given maximum aspect ratio of the $\mathrm{TiO}_{2}$ structures. The final sample is shown in Figure 1 (d).

The MSs were characterized by using an Olympus UApo N 100X 1.49 oil objective to collect all transmitted diffraction orders from the opposite side of the $170 \mu \mathrm{m}$ glass substrate (the pair of first orders at a $65^{\circ}$ angle would be internally reflected without the index-matching oil). The three transmitted beams are imaged by the objective into its back focal plane (BFP), which is then re-imaged on a CCD camera, thus allowing for the measurement of the three beam intensities and angles.

The first order diffraction angle, being so relevant for our MS-TIRF device, was measured a second time with the help of a $100 \mathrm{~mm}$ semi cylindrical prism oil-adhered to the MS coverslip, in which the beams can propagate long enough for a direct measurement using a photograph. Both measurements were in agreement with the theoretical value of $64.89^{\circ}$. When the MS is illuminated at $90^{\circ}$ incidence with TE-polarized light of $\lambda 0=640 \mathrm{~nm}$ wavelength, we observe that $70 \%$ of the light entering the substrate is diffracted into the first diffraction order of interest (by design the one directed towards the center of the substrate), $21 \%$ is coupled in the opposite first order, and only $9 \%$ into the traversing zeroth order, as seen in Figure 2. In terms of the total incidence intensity, these numbers are $43 \%$ and $13 \%$ for the first diffraction orders and $6 \%$ for the zeroth diffraction order, which means that $38 \%$ of the incident light is back-reflected. These values are in good agreement with the theoretically values of our RCWA calculations (55\% and $16 \%$ for the first diffraction orders, $4 \%$ for the zeroth diffraction order, and $25 \%$ back-reflection). The small direct transmission of $4 \%$ is of key importance for the application of the MS to TIRFM, because it defines how much undesired fluorescence is excited deep in a sample far from the surface. The fact that most light sources in fluorescence microscopy are already polarized lasers greatly eliminates hurdles associated to using a TE-polarized only MS design.

a)

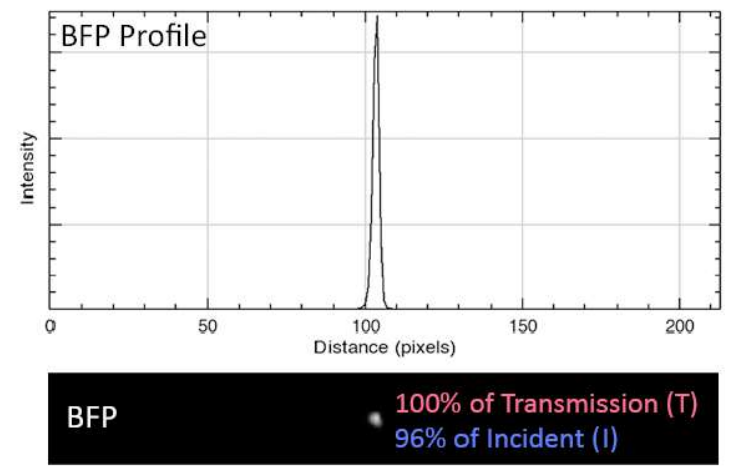

b)

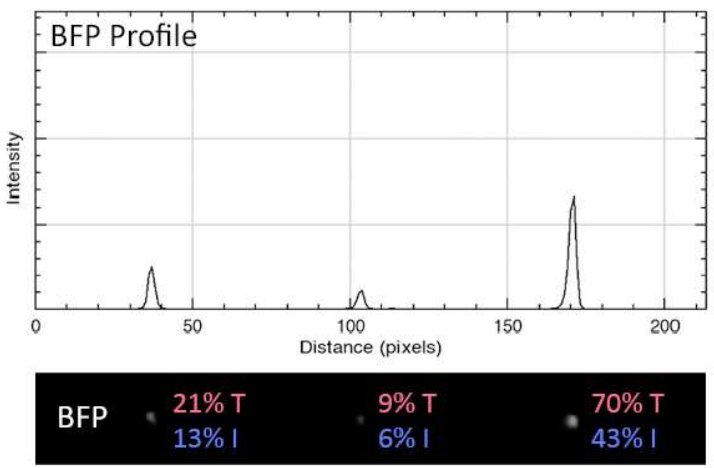

Fig.2: Diffraction during MS-TIRF. When a TE incident beam hits the (a) plain glass substrate (reference) or (b) the MS,the images of the BFP allow for measuring the relative intensities of the different diffraction orders. Our MSs couples up to $70 \%$ of the light that enters the substrate into a diffraction order which propagates at a $65^{\circ}$ angle with respect to the vertical, which being superior to the critical TIR angle, generates the desired evanescent field in sample space. 


\section{Fluorescence microscopy experimental setup and results}

For MS-TIRFM we used the setup shown in Figure 3. A motorized xy-stage with a dedicated metasurface holder is used for holding and moving the MS-substrate. Excitation and imaging is done with an Olympus UPlanSApo 40x NA 0.95 air objective mounted on both a motorized and a piezo z-stage (PIFOC, Physik Instrumente $\mathrm{GmbH}$ ) allowing for fine and course vertical motion. Two continuous-wave diode lasers at $637 \mathrm{~nm}$ wavelength are used for simultaneous widefield (WF) and TIR illumination (Coherent OBIS $140 \mathrm{~mW}$ with a ZET 640/10 Laser Clean-up Filter). Imaging is done with an ANDOR iXon camera (Ultra DU-897U-CS0-\#BV) which has a 512x512 pixel sensor (16 $\mu \mathrm{m}$ pixel size). The excitation light used for illuminating the MS was TE polarized using a Thorlabs CM1-PBS251 polarizing beamsplitter cube, and it was focused on to the MS by a Thorlabs AC254-300-A-ML $300 \mathrm{~mm}$ achromatic doublet.

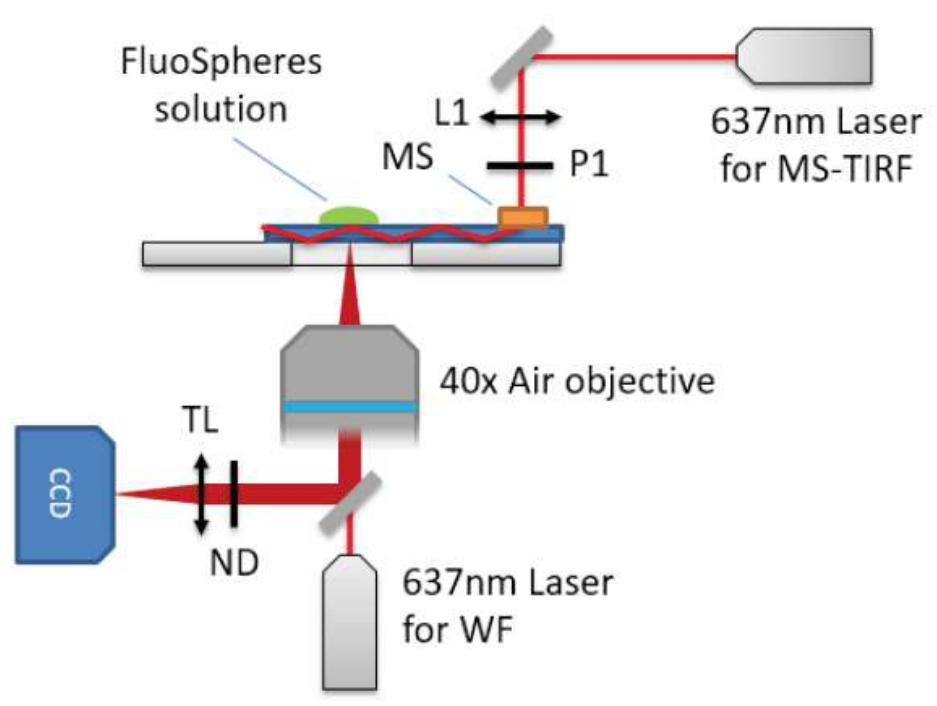

Fig.3: MS-TIRF microscopy setup. The used microscopy setup allows to illuminate the sample both in TIR and WF illumination mode, using two $637 \mathrm{~nm}$ continuous-wave lasers. An Olympus UPlanSApo 40X NA 0.95 air objective and an ANDOR iXon Ultra camera are used to image the fluorescent sample which is mounted on a custom motorized stage. The lens L1 weakly focuses the incoming beam, which is linearly polarized by P1. The tube lens (TL) focuses the fluorescence light that is collected by the objective onto the CCD.

As a first test to verify we could selectively excite fluorophores in close proximity to the substrate's surface, while not exciting the bulk of a specimen, we placed and dried a highly diluted solution of fluorescent particles (Invitrogen FluoSpheres Carboxylate-Modified Microspheres, $0.2 \mu \mathrm{m}$, dark red fluorescent 660/680 $0.0001 \%$ in volume) onto the substrate. This was followed by a second, larger (5-10 $\mu \mathrm{l})$ droplet of solution, thus generating a sample with few particles attached to the surface and other particles diffusing freely in solution. After placing the sample on the microscope, we recorded continuous movies of fluorescence images while alternating between WF and MS-TIRF illumination, or both.

Figure 4 (c) and (d) shows two frames of a series of 200 images, each with $100 \mathrm{~ms}$ integration time, resulting in a 20 seconds video. With 10 seconds of WF illumination, followed by 10seconds of MS-TIRF illumination, we observe a clear difference between bright and dim particle images, corresponding to fluorescent beads attached to the surface, and to beads diffusing freely in solution but coming, for a short time, close to the surface into the evanescent field. With a frame time of $100 \mathrm{~ms}$ which is much longer than the average time needed by a diffusing particle to diffuse into and out of the evanescent field, no dynamics of this diffusion can be observed. However, when reducing the frame time, we more and more 
observe dynamic "blinking" events in a movie which capture a particle's diffusion into and out of the evanescent field in a time-dependent manner.

Finally, by analyzing images recorded with MS-TIR excitation, see Figure 4 (d), we determined the maximum and mean brightness per pixel for the observed stationary fluorescent particles, and the standard deviation of the background (pixels not corresponding to particles). This allowed us to estimate a MS-TIRFM peak-signal-to-noise ratio (PSNR) superior to 700 .
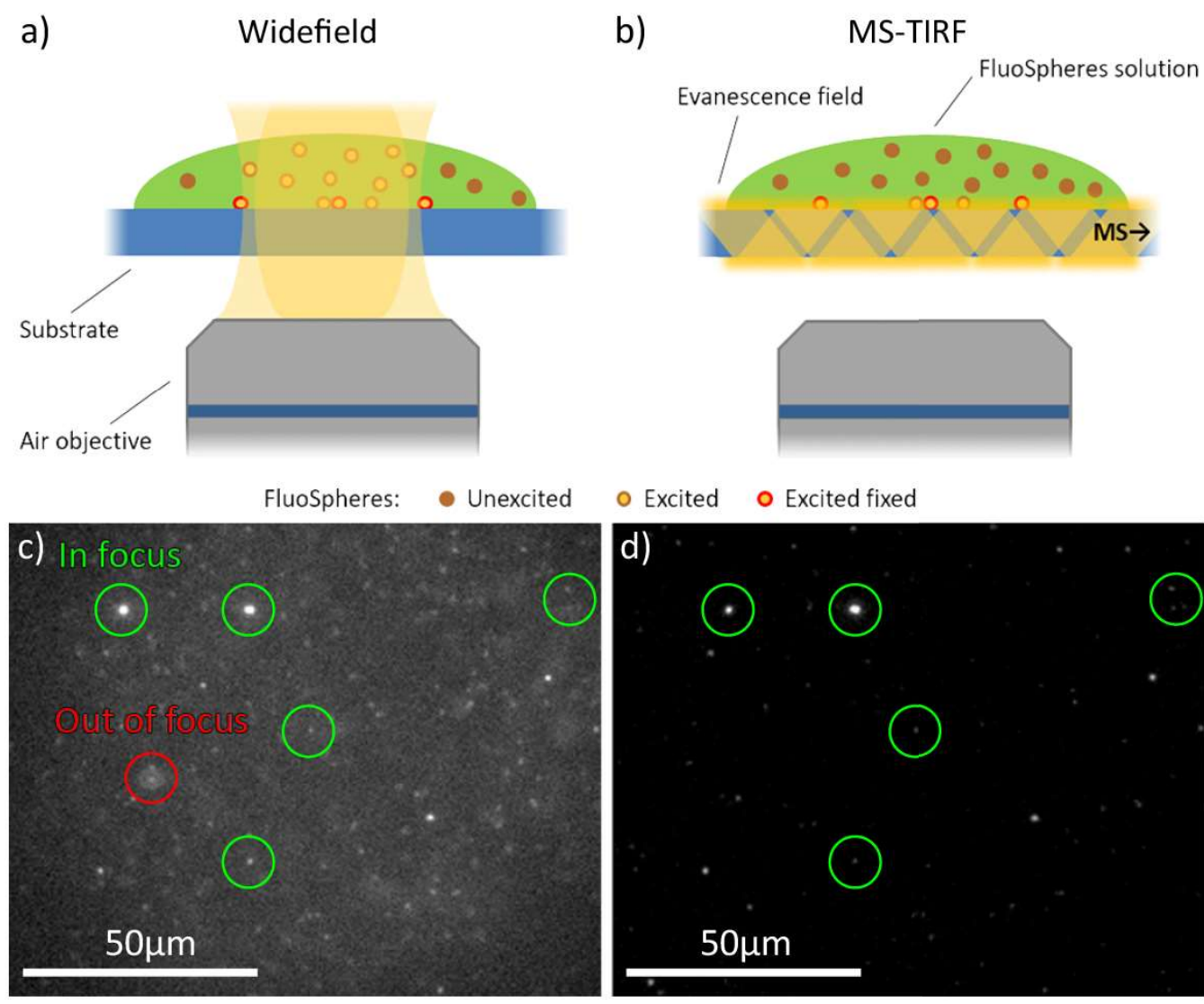

Fig.4: Difference between widefield and MS-TIRF microscopy. The experimental setup visible in Figure 3 was used to achieve widefield illumination(a), exciting all the fluorescent particles on the beam's vertical path, or TIRF illumination (b), exciting only those particles in close proximity to the substrate. Two frames of an image series of $0.2 \mu \mathrm{m}$ Invitrogen FluoSpheres before (c) and after (d) switching from solely WF to solely MS-TIRF illumination. Integration time: $100 \mathrm{~ms} /$ frame. While WF illumination allows us to see the rapid motion of the diffusing particles in the bulk of the solution, it partially hides the fixed particles on the glass surface. MS-TIRF illumination selectively excites only those microspheres on the surface, eliminating the intense background generated by the diffusing particles in WF illumination (see Visualization 1 for the full series). For shorter integration times, one observes occasionally particles diffusing from the bulk close to the surface generating short-time fluorescence intensity peaks when they pass through the evanescent excitation light.

In order to exploit the exceptionally large FOV offered by MS-TIRF, we decided to image fixed hMSCs that were immunostained with anti-paxillin antibody $(\mathrm{AB})$ and corresponding anti-rabbit IgG Atto 647N AB. Paxillin is an integral protein of the cell's focal adhesions, and it plays essential roles in important biological processes including motility, proliferation, differentiation,regulation of gene expression, and cell 
survival. The used primary antibody against paxillin does often also bind to targets in the cytosol making TIRF imaging particularly useful (see Figure 5) for imaging paxilin in focal adhesions. In cell cultures, hMSCs can reach several hundred microns in diameter, often well beyond the FOV of conventional TIRF objectives. The detailed process for the sample preparation is found in the Appendix.

Using our MS-TIRF setup, we recorded a series of high-contrast images with the immunostained cells exclusively excited by the MS-generated evanescent field. Similarly to the FluoSpheres samples, one observes only a tiny slice of the cells close to the surface, thus greatly reducing background from the cells' interior. A representative image is shown in Figure 5 (b), where a clear distinction between the focal adhesions and the cell interior can be seen. Besides specific regions with high fluorophore concentration, Figure 5 (a) shows the existence of an even higher concentration in the endoplasmatic reticulum around the cell's nucleus, which is virtually invisible in the MS-TIRF image.

a)

Widefield

b)

MS-TIRF

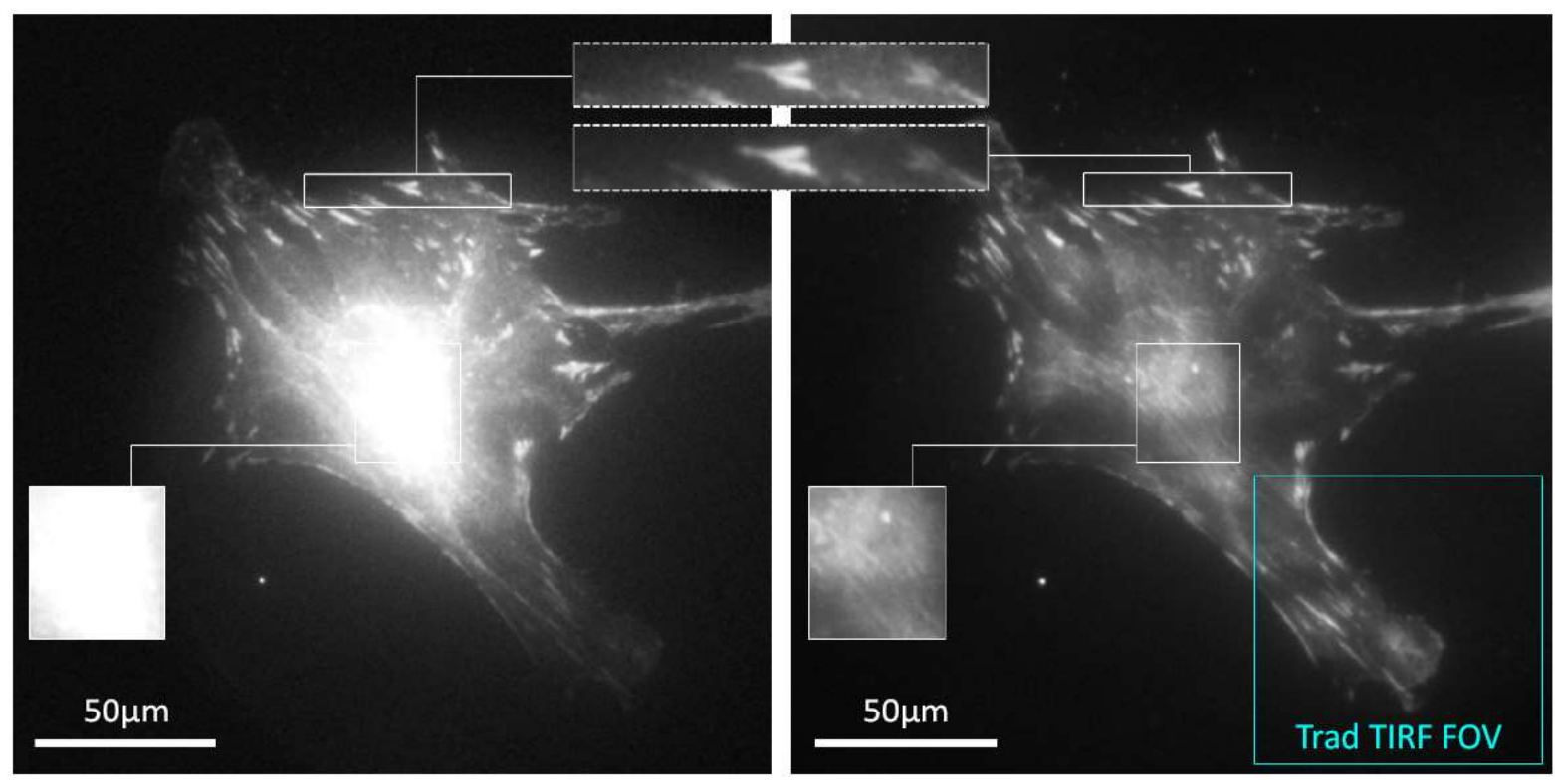

Fig.5: MS-TIRF and widefield microscopy of hMSC. Bone marrow hMSCs immunostained for focal adhesions with anti-paxillin $\mathrm{AB}$ and corresponding anti-rabbit IgG Atto $647 \mathrm{~N} \mathrm{AB}$ and imaged with (a) a standard WF microscope and (b) a MS-TIRF substrate. When equalizing the images' intensity with the brightness of the zones of interest (top dotted frames), background fluorescence originating from the cell's nucleus hides any information from the membrane below it (center (a) and (b)). For reference, the blue frame indicates the FOV of 80-100 $\mu \mathrm{m}$ obtainable with a typical 100x TIRF objectiv.

To better quantify the combined spatial resolution and FOV of our imaging modalities, we use the SpaceBandwidth Product (SBP), which can also be understood as the amount of information that an imaging system can transmit. The SBP can be expressed as the minimum amount of pixels on a sensor needed to capture all the information provided by the objective's resolution FOV. It can be easily calculated as the FOV surface divided by the pixel area required to achieve Nyquist sampling at the resolution given by the objective's N.A. and illumination wavelength. For the laser source and objectives as shown in Fig. 3, we calculated a SBP of 2.2 megapixels $(100 x$, N.A. $1.49, \mathrm{FN}=22, \mathrm{FOV}=220 \mu \mathrm{m})$ and 8.2 megapixels $(40 \mathrm{x}$, N.A. $0.95, \mathrm{FN}=26.5, \mathrm{FOV}=660 \mu \mathrm{m})$. Thus, MS-based TIRFM delivers almost 4 times as much information than objective-type TIRFM. This number could climb up to almost 6 times by using a water 40x N.A. 1.2 water immersion objective, further reducing the resolution difference between the lower-N.A. 
objective used for MS-based TIRFM and the higher-N.A. oil immersion objective used for conventional objective-type TIRFM. Such an enlargement of the FOV and information bandwidth can be a valuable asset to automate imaging of slow-moving or large samples (e.g. the cells shown in this work), as well for biopsies of large specimens.

\section{Conclusion}

In this work, we introduced the concept of MS-TIRF microscopy. We designed, fabricated and experimentally tested various $\mathrm{TiO}_{2}$ metasurface gratings capable of coupling as much as $70 \%$ of the incident non-reflected light into one of the two first diffraction orders, while greatly suppressing the direct transmission. Both are important conditions for a powerful TIRF coupling device. We used fluorescent micro-spheres to verify the selective illumination of MS-TIRF, and we measured the resulting signal-tonoise ratio. Moreover, we expect the efficiency of our MSs to increase significantly as recent improvements on the fabrication process allow for a $13 \%$ increase on the $\mathrm{TiO}_{2}$ layer thickness, which translates in a full $2 \pi$ phase coverage at $\lambda 0=640 \mathrm{~nm}$. Finally,we imaged immunostained hMSCs using our MS-TIRF device and a 40x air objective, and we were able to obtain very high-contrast images over a FOV that was 9 times larger than that of traditional TIRF system. Furthermore, we calculated that the maximum SBP can be four times larger for the MS-TIRF system than for an objective-type TIRFM. While we have only used fixed cells in our study, no performance difference is to be expected for similar live samples.

In conclusion, we have shown that $\mathrm{TiO}_{2}$ metasurface substrates provide a powerful alternative to high-N.A. objectives, enabling TIRFM with low-N.A. objectives. The wide flexibility of the MS design could also allow for implementing more advanced design features to tackle common weaknesses of multiple TIRFM techniques such as excitation inhomogeneity, that currently require expensive optomechanical systems (TIRF illuminators) to generate a spinning azimuthal incidence angle on the sample [29,30]. Future MSs could also allow for even higher axial selectivity by reducing the structure's period and thus increasing the TIR angle even beyond what is currently possible with TIRF objectives.

\section{Appendix}

The sample preparation consisted on the hMSC-bm cells (Lonza Ref. \#PT-2501) grown in T75 cell culture flasks (Corning Inc., New York, NJ, USA, 43061) in Dulbecco's Modified Eagle Medium (DMEM Gibco, Thermo Fisher Scientific Inc., Waltham, MA, USA, A18967-01) supplemented with $10 \%$ fetal bovine serum (Sigma-Aldrich Co., St. Louis, MO, USA, F2442-500ML) and $1 \%$ antibiotics (penicillin/streptomycin, Life Technologies, Thermo Fisher Scientific Inc., Waltham, MA, USA, 15140- 122) at $37^{\circ} \mathrm{C}$ and $5 \% \mathrm{CO} 2$ passaged every 2-3 days, with passage \#5 was used for this experiment. Cells were seeded on non-coated $25 \mathrm{~mm}$ glass coverslips at a density of $10.000 \mathrm{hMSCs}$ per glass in 6- well plates (Sarstedt AG \& Co., Nuembrecht, Germany, 83.3920) with $2 \mathrm{~mL}$ growth medium per well and grown at $37^{\circ} \mathrm{C}$ and $5 \% \mathrm{CO} 2$. Cells were chemically fixed $24 \mathrm{~h}$ after seeding in a $10 \%$ formaldehyde (Sigma- Aldrich Co., St. Louis, MO, USA, 47608-250ML-F) in PBS for $10 \mathrm{~min}$. Subsequently, cells were permeabilized using $0.5 \%$ Triton X 100 (Carl Roth GmbH \& Co. KG, Karlsruhe, Germany, 6683.1) in PBS for 10 min and blocked with $3 \%$ BSA (Sigma-Aldrich Co., St. Louis, MO, USA, A9418-100G) in PBS for 30 min, incubated again with Triton X for 5 min and thoroughly washed with PBS. All antibodies were kept in a $3 \%$ BSA PBS solution. Immunostaining was performed with anti-Paxillin (Abcam, ab32084) [1:400] for $10 \mathrm{~h}$, then anti-rabbit IgG Atto 647N (Sigma-Aldrich Co., St. Louis, MO, USA, 40839) [1:400] for $2 \mathrm{~h}$. Finally, the samples were covered with $24 \mathrm{~mm}$ glass coverslips using Fluoroshield mounting medium (Sigma- Aldrich Co., St. Louis, MO, USA, F6182-20ML).

\section{Funding}

H2020 Marie Skłodowska-Curie Actions (675512). 


\section{Acknowledgments}

We thank Ingo Gregor, Soheil Mojiri, and Oleksii Nevskyi (Third Institute of Physics - Biophysics, Georg August University, Göttingen, Germany) for their help with the TIRFM measurements.

\section{Disclosures}

The authors declare no conflicts of interest.

\section{References}

1. D. Axelrod, N. L. Thompson, and T. P. Burghardt, "Total internal reflection fluorescent microscopy," J. microscopy 129, 19-28 (1983).

2. D. Axelrod, T. P. Burghardt, and N. L. Thompson, "Total internal reflection fluorescence," Annu. review biophysics bioengineering 13, 247-268 (1984).

3. D. Axelrod, E. H. Hellen, and R. M. Fulbright, "Total internal reflection fluorescence," in Topics in fluorescence spectroscopy, (Springer, 2002), pp. 289-343.

4. M. Tokunaga, K. Kitamura, K. Saito, A. H. Iwane, and T. Yanagida, "Single molecule imaging of fluorophores and enzymatic reactions achieved by objective-type total internal reflection fluorescence microscopy," Biochem. biophysical research communications 235, 47-53 (1997).

5. M. Paige, E. Bjerneld, and W. Moerner, "A comparison of through-the-objective total internal reflection microscopy and epifluorescence microscopy for single-molecule fluorescence imaging," Single Mol. 2, 191-201 (2001).

6. A. PORS, M. G. NIELSEN, and S. I. BOZHEVOLNYI, "Plasmonic metagratings for simultaneous determination of stokes parameters," Optica 2, 716-723 (2015).

7. D. T. et al., "Grating couplers for coupling between optical fibers and nanophotonic waveguides," Jpn. J. Appl. Phys. 45, 6071-6077 (2006).

8. C. J. Chang-Hasnain and W. Yang, "High-contrast gratings for integrated optoelectronics," Adv. Opt. Photonics 4, 379-440 (2012).

9. B. H. Chen, P. C. Wu, V.-C. Su, Y.-C. Lai, C. H. Chu, I. C. Lee, J.-W. Chen, Y. H. Chen, Y.-C. Lan, C.-H. Kuan, and D. P. Tsai, "Gan metalens for pixel-level full-color routing at visible light," Nano Lett. 17, 6345-6352 (2017).

10. P. Genevet and F. Capasso, "Holographic optical metasurfaces: a review of current progress," Reports on Prog. Phys. 78 (2015).

11. H. Ren, G. Briere, X. Fang, P. Ni, R. Sawant, S. HÃlron3, S. Chenot, S. VÃlzian, B. Damilano, V. BrÃdndli, S. A. Maier, and P. Genevet, "Metasurface orbital angular momentum holography," Nat. Commun. 10 (2019).

12. G. J. Triggs, Y. Wang, C. P. Reardon, M. Fischer, G. J. O. Evans, and T. F. Krauss, "Chirped guided-mode resonance biosensor," Optica 4, 229-234 (2017).

13. D. Lee, M. Kim, J. Kim, H. Hong, T. Badloe, D. S. Kim, and J. Rho, "All-dielectric metasurface imaging platform applicable to laser scanning microscopy with enhanced axial resolution and wavelength selection," Opt. Mater. Express 9, 3248-3259 (2019).

14. E. Arbabi, J. Li, R. J. Hutchins, S. M. Kamali, A. Arbabi, Y. Horie, P. Van Dorpe, V. Gradinaru, D. A. Wagenaar, and A. Faraon, "Two-photon microscopy with a double-wavelength metasurface objective lens," Nano Lett. 18, 4943-4948 (2018). PMID: 30016110. 
15. M. Brunstein, A. Cattoni, L. Estrada, and A. M. Yacomotti, "Improving image contrast in fluorescence microscopy with nanostructured substrates," Opt. Express 23, 29772-29778 (2015).

16. M. D. Lew and W. Moerner, "Azimuthal polarization filtering for accurate, precise, and robust single-molecule localization microscopy," Nano letters 14, 6407-6413 (2014).

17. M. P. Backlund, A. Arbabi, P. N. Petrov, E. Arbabi, S. Saurabh, A. Faraon, and W. E. Moerner, "Removing orientation-induced localization biases in single-molecule microscopy using a broadband metasurface mask," Nat. Photonics 10, 459 EP-462 (2016).

18. P. Lalanne, S. Astilean, P. Chavel, E. Cambril, and H. Launois, "Blazed binary subwavelength gratings with efficiencies larger than those of conventional échelette gratings," Opt. Lett. 23, 1081-1083 (1998).

19. P. Lalanne, S. Astilean, P. Chavel, E. Cambril, and H. Launois, "Blazed binary subwavelength gratings with efficiencies larger than those of conventional échelette gratings," Opt. Lett. 23, 1081-1083 (1998).

20. N. Yu, P. Genevet, M. A. Kats, F. Aieta, J.-P. Tetienne, F. Capasso, and Z. Gaburro, "Light propagation with phase discontinuities: Generalized laws of reflection and refraction," Science 334, 333-337 (2011).

21. D. Sell, J. Yang, S. Doshay, R. Yang, and J. A. Fan, "Large-angle, multifunctional metagratings based on freeform multimode geometries," Nano Lett. 17, 3752-3757 (2017).

22. D. Sell, J. Yang, E. W. Wang, T. Phan, S. Doshay, and J. A. Fan, "Ultra-high-efficiency anomalous refraction with dielectric metasurfaces," ACS Photonics 5, 2402-2407 (2018).

23. M. M. R. Elsawy, S. Lanteri, R. Duvigneau, M. S. Mohamed, G. Brière, and P. Genevet, "Global optimization of metasurface designs using statistical learning methods," (2019). Working paper or preprint.

24. S. Ramachandran, D. A. Cohen, A. P. Quist, and R. Lal, "High performance, led powered, waveguide based total internal reflection microscopy," Sci. Rep.3(1), 2133 (2013).

25. I. Helle, D. A. Coucheron, J.-C. Tinguely, C. I. Øie, and B. S. Ahluwalia, "Nanoscopy on-a-chip: super-resolution imaging on the millimeter scale," Opt. Express27(5), 6700-6710 (2019).

26. J. Hugonin and P. Lalanne, "Reticolo code 2d for analyzing 2d crossed grating," (2013).

27. T. Matsui, A. Miura, N. Ikeda, H. Fujikawa, Y. Sugimoto, N. Engheta, and H. Iizuka, "Experimental investigation of double-groove grating satisfying total internal reflection condition," Opt. Express 22, 25362-25370 (2014).

28. D. Lin, M. Melli, E. Poliakov, P. S. Hilaire, S. Dhuey, C. Peroz, S. Cabrini, M. Brongersma, and M. Klug, "Optical metasurfaces for high angle steering at visible wavelengths," Sci. Reports 7 (2017).

29. A. L. Mattheyses, K. Shaw, and D. Axelrod, "Effective elimination of laser interference fringing in fluorescencemicroscopy by spinning azimuthal incidence angle," Microsc. Res. Tech.69(8), 642-647 (2006).

30. K. L. Ellefsen, J. L. Dynes, and I. Parker, "Spinning-spot shadowless tirf microscopy," PLoS One10(8), e0136055 (2015). 



\section{Substrates for stem cell differentiation}

There are many possibilities to culture cells nowadays. Unlike suspended cells, for which the material of their container has no big influence, for adherent cells it makes quite a difference. Usually, one uses cell culture plastic, which is physically modified to provide adhesion for cells. There are also cell culture flasks coated with proteins, but since these are not easily storable they are not widely used. Other substrates are, for example, chambers custom-made from polydimethylsiloxane (PDMS) or similar materials. Additionally, cells adhere to metals like gold or titanium [132, 138, 149, 175], which can also be used to coat other materials. In general, all materials without ECM components have the benefit of being storable and preparable in advance.

The mechanism to tailor those aforementioned materials is topology modulation. This poses a problem, however. Although topology can be a tool to influence cell mechanics, one always has to be able to distinguish the influence of all factors in an experiment [210]. Here, one would have to distinguish between stiffness and topology. Directly adjusting the substrate is much more straight forward. Hydrogels are polymers of variable interlinkage and thus varying stiffness. They are a viable tool in medicine, for example in drug delivery [115], and for the pharmaceutical and functional food industries [133]. There are a variety of usable gels, but none entirely spans the range from fat tissue (17 Pa) to tendons (900 MPa) [131]. In this thesis, polyacrylamide (PAA) gels will be used. These gels can easily be adjusted in a range of 1-150 $\mathrm{kPa}$ (see Table C.10), are easy to manufacture and chemically inert, and are sterilizable and coatable with various ECM mimickers [188].

Furthermore, polyacrylamide gels can be stamped with proteins, be made into gradient gels for durotaxis experiment, or be loaded with water-soluble drugs for chemotaxis [54]. For durotaxis, however, the gradient strength is an influential factor and needs to be taken into account [89].

\subsection{Polyacrylamide gel substrates}

Protocols in this section have been modified in the Rehfeldt lab over a decade. Detailed protocols for glass and gel preparation, as well as coating, can be found in the appendix (Section C, Subsection C.1, Subsection C.2, and Subsection C.4). In general, there are two sizes of gels prepared: small gels with 25 $\mathrm{mm}$ glasses to be used in bottomless dishes or 6-well plates (with 6,000-10,000 cells), and big gels to be used pairwise in 1-well plates for large numbers of cells (150,000-300,000 cells).

Regardless of the size, the preparation of the polyacrylamide hydrogels is the same. Firstly, one needs a support structure to polymerize and handle the gels. Here, we use glutaraldehyde-treated glasses for bottom support and silanized glasses to accomplish a smooth, flat surface during polymerization.

\subsubsection{Glass preparation}

To manufacture polyacrylamide gels, one needs a carrier glass that the gel can be bound to in order to handle it, as well as a top glass to get a smooth surface during polymerization. The bottom glass has to 


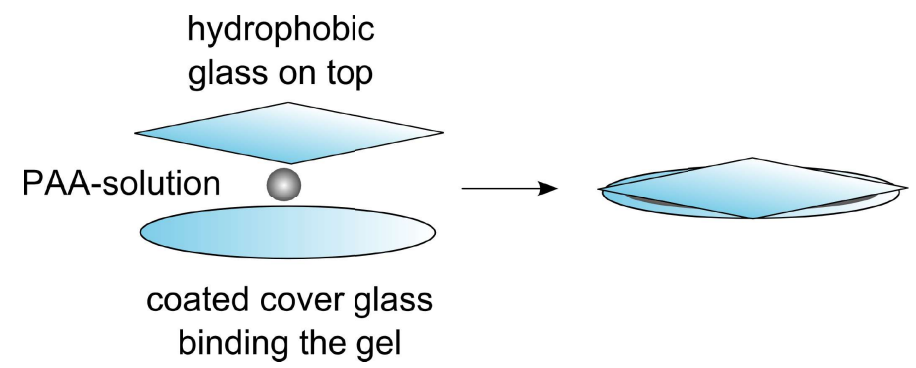

Figure 4.1.: Schematic drawing of gel polymerization in glass-sandwich. Figure from [225].

be chemically treated to provide a cross-linker, while the top glass has to be hydrophobic so it can be removed from the hydrogel after polymerization.

It might not seem like an important step, but any contamination or sloppy treatment will show later in the gels, thus significantly affecting the experiment.

\subsubsection{Cover glass preparation}

Since polyacrylamide gels are hydrogels, they consist mainly of water; water also covers the surface of the gel. Silanization of the cover glasses will prevent sticking to the gels, while the pressure ensures a flat surface of the gel. A detailed protocol can be found in Subsection C.1.

Silanization is done with dimethyldichlorosilane (DMCS), which binds to silanol groups on the glass surface under the elimination of hydrochloric acid. After treatment, the glasses can be stored dry and used multiple times. Rinsing before application to a hydrogel will wash away dirt and ensure the hydrophobic surface is still intact. This is important, as all dirt on the cover glass surface will transfer to the hydrogel. This feature can be used to transfer protein shapes to the top of the gel.

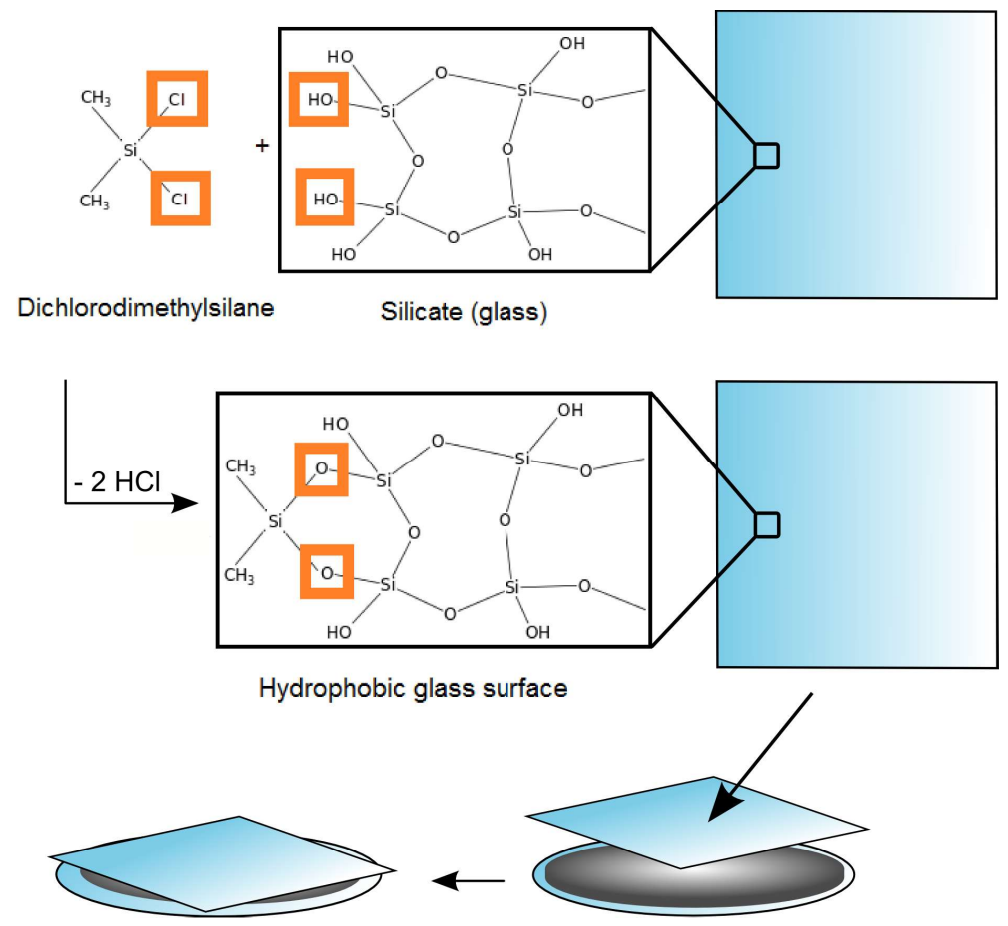

Figure 4.2.: Silanization of cover glass. Figure from [225]. 


\subsubsection{Bottom glass preparation}

Glasses used to support polyacrylamide gels have to be thoroughly cleaned and treated in order to offer binding sites to the polyacrylamide gels. A detailed protocol can be found in Subsection C.2.
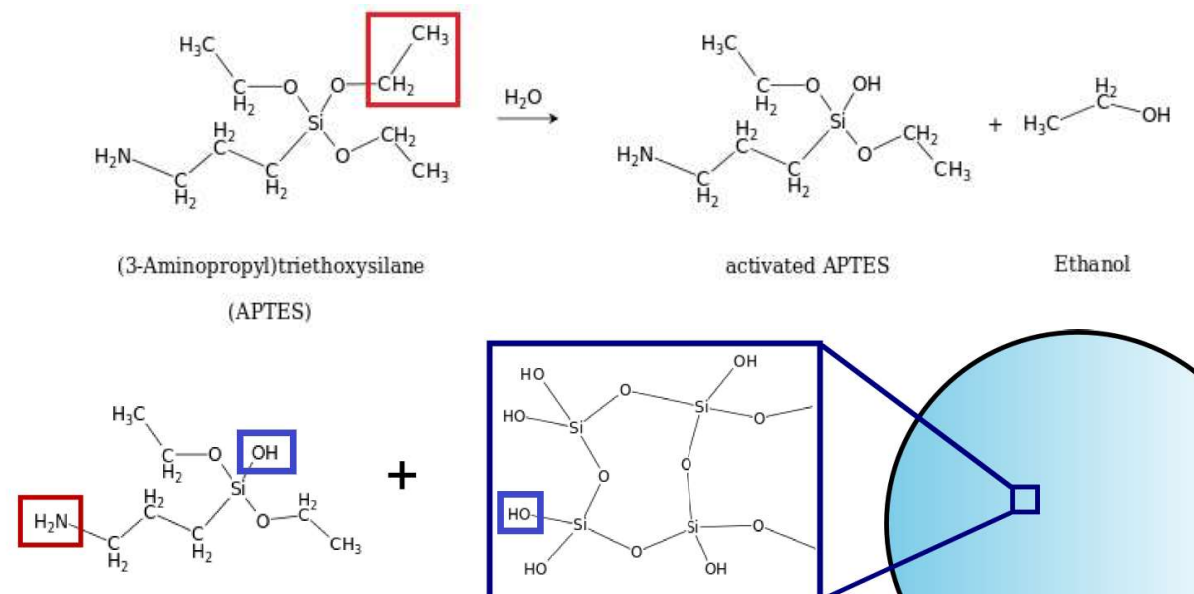

activated APTES
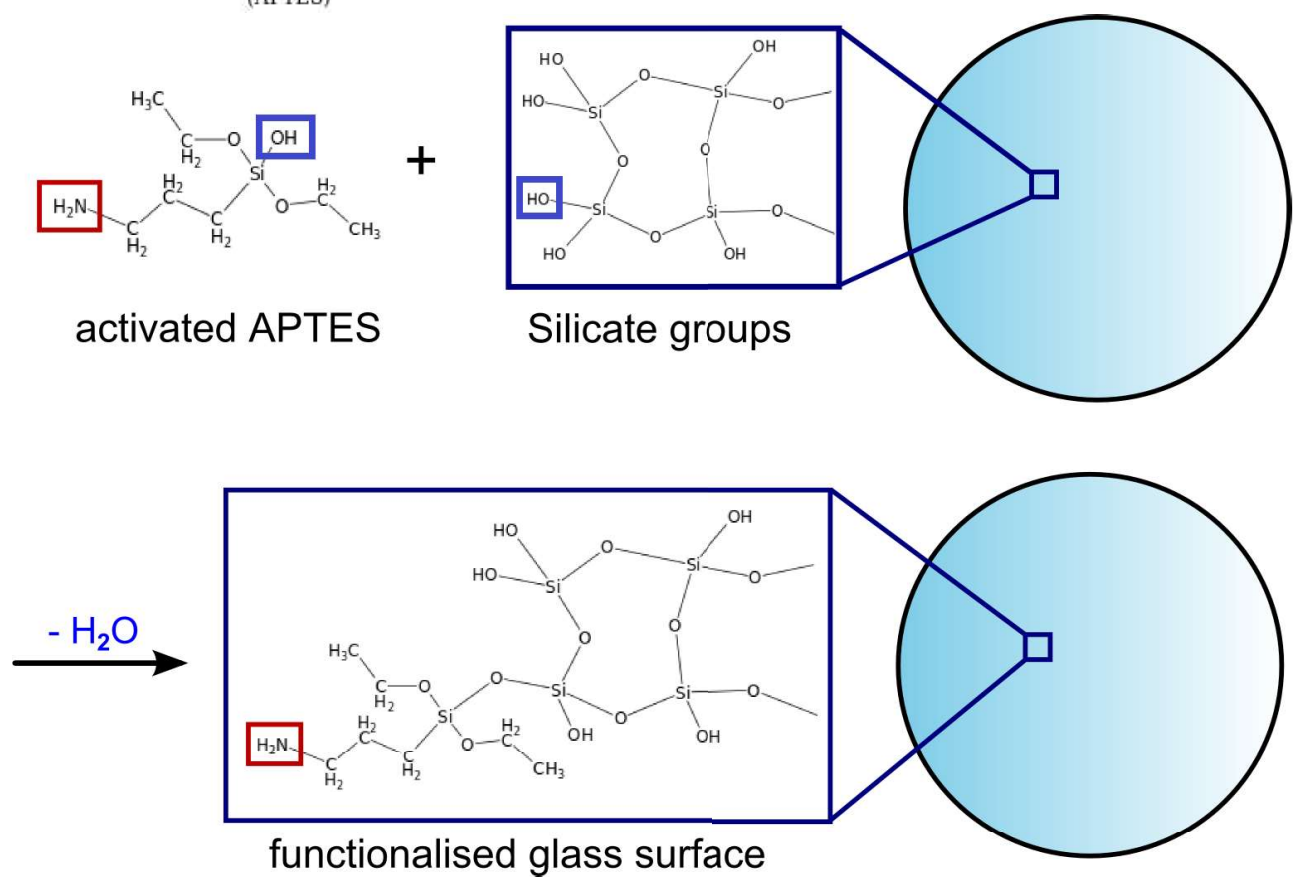

Figure 4.3.: Schematic of glass functionalization with APTES. Figure from [225].

Because purchased glasses are contaminated by particles, lipids, and other substances, we need to clean them in multiple steps. First, the glasses are treated in a plasma cleaner to remove non-sticking particles from the surface. The low-pressure environment together with the oscillating electrical field causes radical formation, as well as movement of remnant molecules and ions. Purple light visible inside the cleaner is due to parts of the energy transformed locally to heat or light. In the second step, glasses are washed in $99 \%$ ethanol to kill bacteria and solve soluble residues. Ultrasound is used to remove more persistent contamination via cavitation bubbles formed by the sound waves. Third, a second washing step is also done in ultrasound, but the 3-aminopropyltriethoxysilan (APTES) added in this step also already provide amino groups on the glass for the later coating. The APTES will react with the fraction of water in the surrounding ethanol to become its activated form, losing its ethane group and forming one ethanol molecule. Activated APTES can covalently bind to the glass surface, reacting with the silicate's hydrogen and forming an oxygen-bridge bond. The amino group of the now bound APTES provides an amino-activated surface to bind the cross-linker glutaraldehyde, which in turn can be be used to bind the polyacrylamide gel.

Glutaraldehyde is a cross-linking agent also frequently used to fix cells. Binding to the free amino group of APTES, it provides a binding partner for the polyacrylamide gel. The use of APTES is necessary because 
the bond of glutaraldehyde to silicate would have been too weak. It is added as a solution in water and left at room temperature to incubate.

When adding polymerizing hydrogel, the free aldehyde group of glutaraldehyde reacts with free amino groups in the polyacrylamide network, forming a carbon-nitrogen bond under the elimination of water. This reaction provides a bond strong enough to fix the gels to the glasses even during rough washing steps.

\subsubsection{Manufacturing of polyacrylamide gels}

The stiffness of polyacrylamide (PAA) hydrogels can be adjusted over a wide range (see Table C.10) and stored for a prolonged time (see Table 4.2 for rheological measurements of PAA gels of up to 10 months). After the first experiments by Pelham and Wang in 1997 [159], polyacrylamide gels have been used for a wide range of experiments, often coated with collagen I. However, the downside of polyacrylamide gels is that acrylamide is toxic in its unpolymerized state. Washing removes all toxic unbound molecules, allowing cells to grow on 2D gels, but a 3D culture with cells engulfed in polyacrylamide hydrogel is still not feasible [169]. Thus, other hydrogels - for example, those made from collagen or hyaluronic acid - are a valuable tool, although their stiffness is not as easily adjustable, or adjustable in a wide range, as in polyacrylamide gels $[52,177]$.

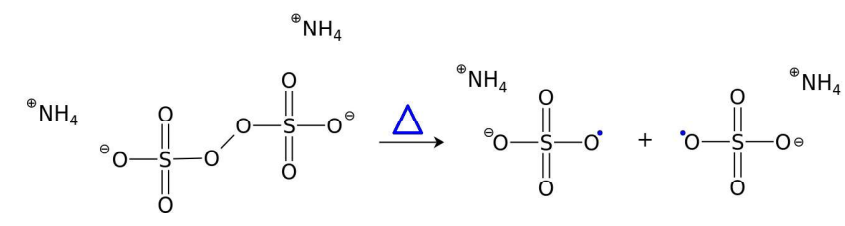

Reaction start by thermal radicals from ammonium persulfate (APS)

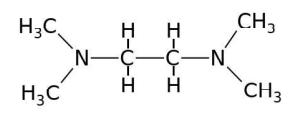

Radical transfer to TEMED

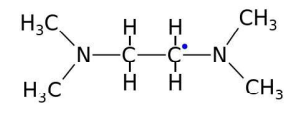
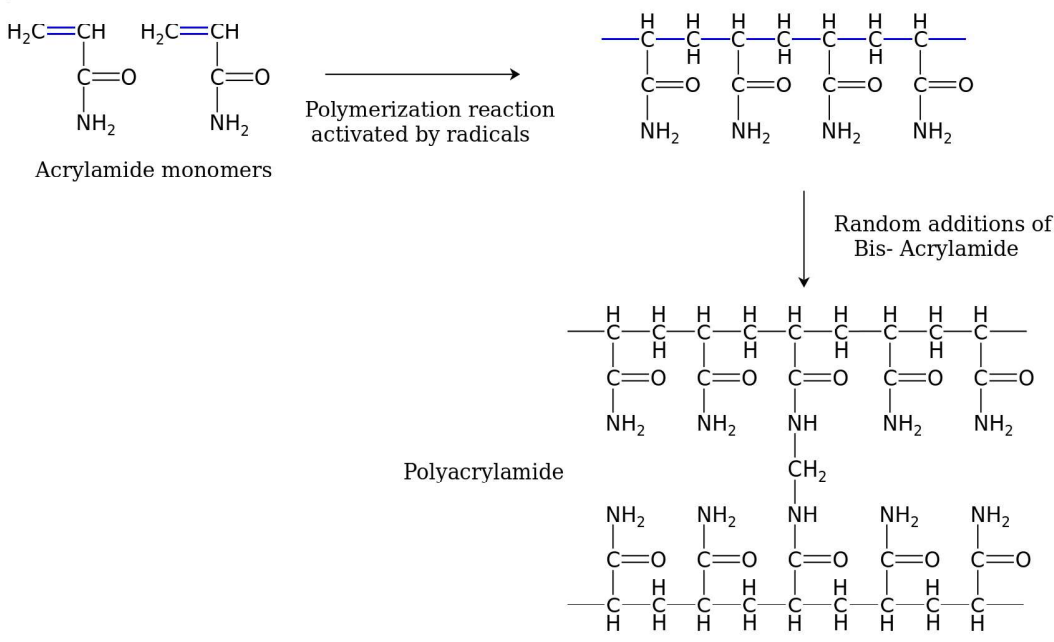

Figure 4.4.: Reactions of polyacrylamide gel polymerization in presence of TEMED and APS.

Figure from [225]. 
The protocol for polyacrylamide gel preparation was used and modified in the Rehfeldt lab for several years. A detailed protocol can be found in the appendix (Subsection C.3), along with the instructions to manufacture polyacrylamide gels of stiffnesses from 1 to $128 \mathrm{kPa}$ (Table C.10). Gels are characterized by the percentages of monomer (acrylamide) and cross-linker (bis-acrylamide) they contain. Pore size and stiffness is varied by adjusting the percentages of those two. It should be noted that premixed polyacrylamide solutions are not stable for more than 6-8 weeks. Acrylamide and bis-acrylamide stock solutions can be used in multiple experiments, but must be discarded after 6 months. During this time, the resulting gels get gradually softer. Initially, the stiffness has to be measured, which is typically done with a rheometer (see section 4.4).

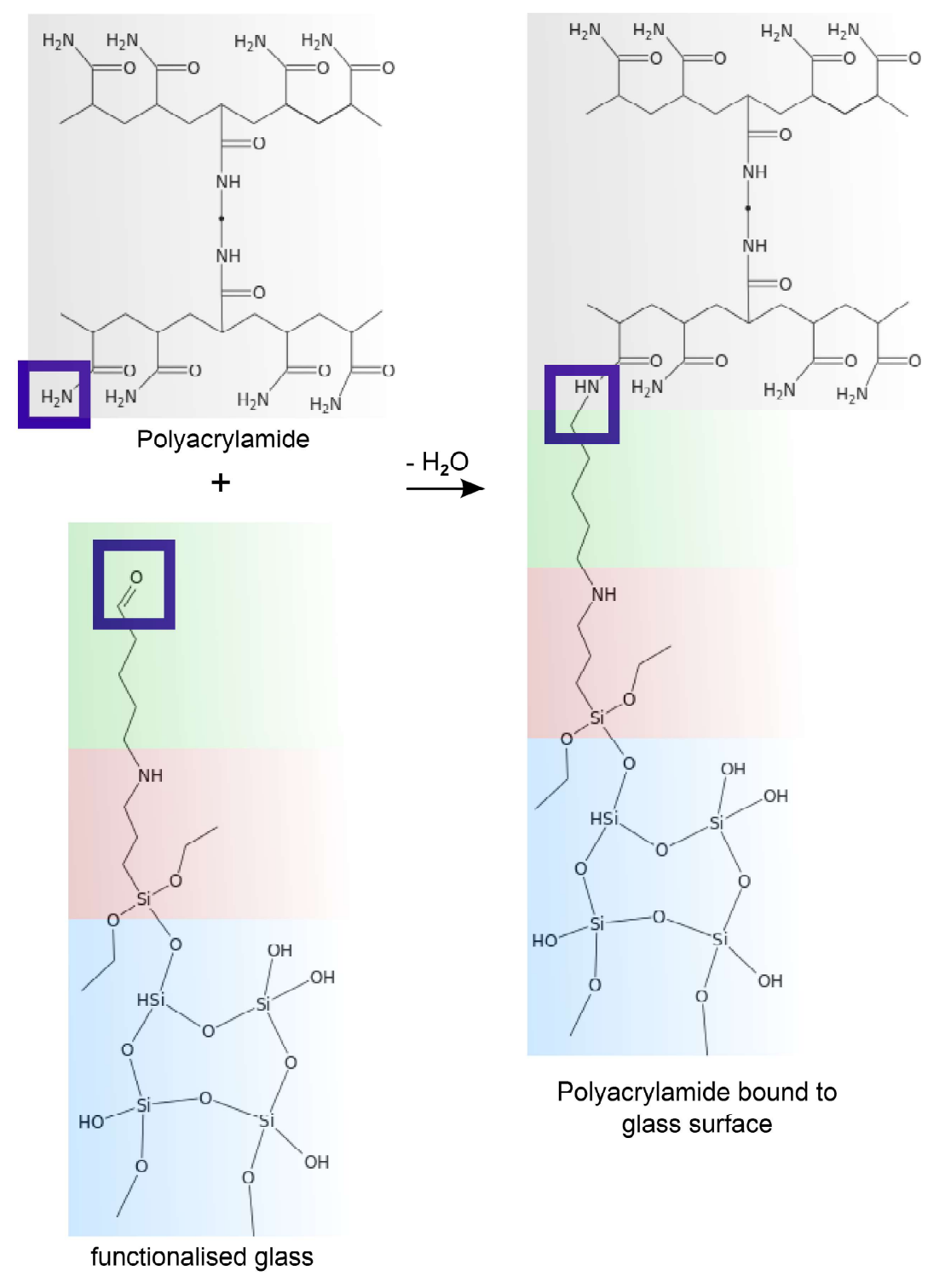

Figure 4.5.: Mechanism of linkage between polyacrylamide gel and bottom glass. Figure from [225].

Polyacrylamide gels are made by mixing four chemicals: acrylamide (acr) and the cross-linker N,N'methylene-bisacrylamide (bis), the catalysators tetramethylethylenediamine (TEMED), and ammonium persulfate (APS) (See Figure 4.5). Usually, 1/1000 volume TEMED and 1/100 volume APS are added. 
APS directly catalyzes the polymerization reaction, while TEMED can exist in a free radical state and accelerates the free radical formation of APS. There are other chemicals with the same properties. For example, riboflavin can supplant APS/TEMED or be added as an enhancer. The reaction speed depends on various factors, such as external temperature, as well as the purity of reagents and monomer and catalyst concentration. The reaction is exothermic and rapid polymerization will generate nonuniform pore structure.

$35 \mu \mathrm{l}$ gel solution is then quickly added to a $25 \mathrm{~mm}$ glutaraldehyde-treated bottom glass. The hydrophobic top glass is placed upon the drop of gel in a way that the gel distributes evenly between the whole surfaces of the glasses. This should yield a gel thick enough to prevent the cells from sensing the underlying glass slide.

The resulting hydrogel is chemically inert and can be functionalized with Sulfo-SANPAH to provide binding sites for a protein coating, facilitating the adhesion of cells to the gel (see 4.1.3). The coating usually consists of proteins from the extracellular matrix (ECM), in order to mimic biological conditions and enable cells to bind to the hydrogel [243]. A way to coat without the addition of Sulfo-SANPAH is to coat flat or patterned PDMS stamps with Synthamax or ECM molecules and print this on glass coverslips. In a humid chamber, these can be directly transferred to the PAA gels during polymerization.

\subsubsection{Collagen coating}

A detailed protocol for collagen coating can be found in (Subsection C.4). To provide adhesion sites for the cells, the polyacrylamide gels have to be coated. This is because the cells can not adhere to the polyacrylamide gels. The most commonly used ECM mimicker is type I collagen. To link the inert hydrogels with collagen I, the gels must be cross-linked. Sulfo-SANPAH is a water-soluble cross-linker providing an NHS ester (N-hydroxysuccinimid) binding and a UV-activatable nitrophenyl azide binding site. Upon applying a wavelength of 320-350 $\mathrm{nm}$ for $10 \mathrm{~min}$, the nitrophenyl azide binds covalently but nonspecifically to the hydrogel, leaving the NHS ester group available for bonding. After thoroughly washing with a $50 \mathrm{mM}$ HEPES buffer to rinse remaining Sulfo-SANPAH, the remaining sulfosuccinimidyl group binds with the collagen's amino groups during 10 hours of incubation, requiring no extra steps. Collagen is a protein consisting of amino acids with at least two functional groups: a carboxyl group and an amino group. This amino group binds to the Sulfo-SANPAH's sulfosuccinimidyl groups. The collagen does not bind to the gel as a film of single amino acids but rather as fibers, which it forms under physiological conditions. A collagen fiber consists of bundles of collagen fibrils that consist of at least two collagen I $\alpha 1$ and one collagen I $\alpha 2$ helical subunits. These are built of linked amino acids like glycine (GLY), proline (PRO), and hydroxyproline (HYP). One has to make sure the collagen forms a thin network and not thick fibers, as it would do when the protocol is done incorrectly. The fibril diameter has an influence on the fiber's mechanical properties [44].

The pore size of the PAA gel and the amount of Sulfo-SANPAH used also have an influence on the mechanosensing of the cell, however. With softer gels and a higher pore size, even when adding maximal amount of Sulfu-SANPAH, as was done in this study, the mechanical properties will therefore vary as the number of possible binding points decreases. 


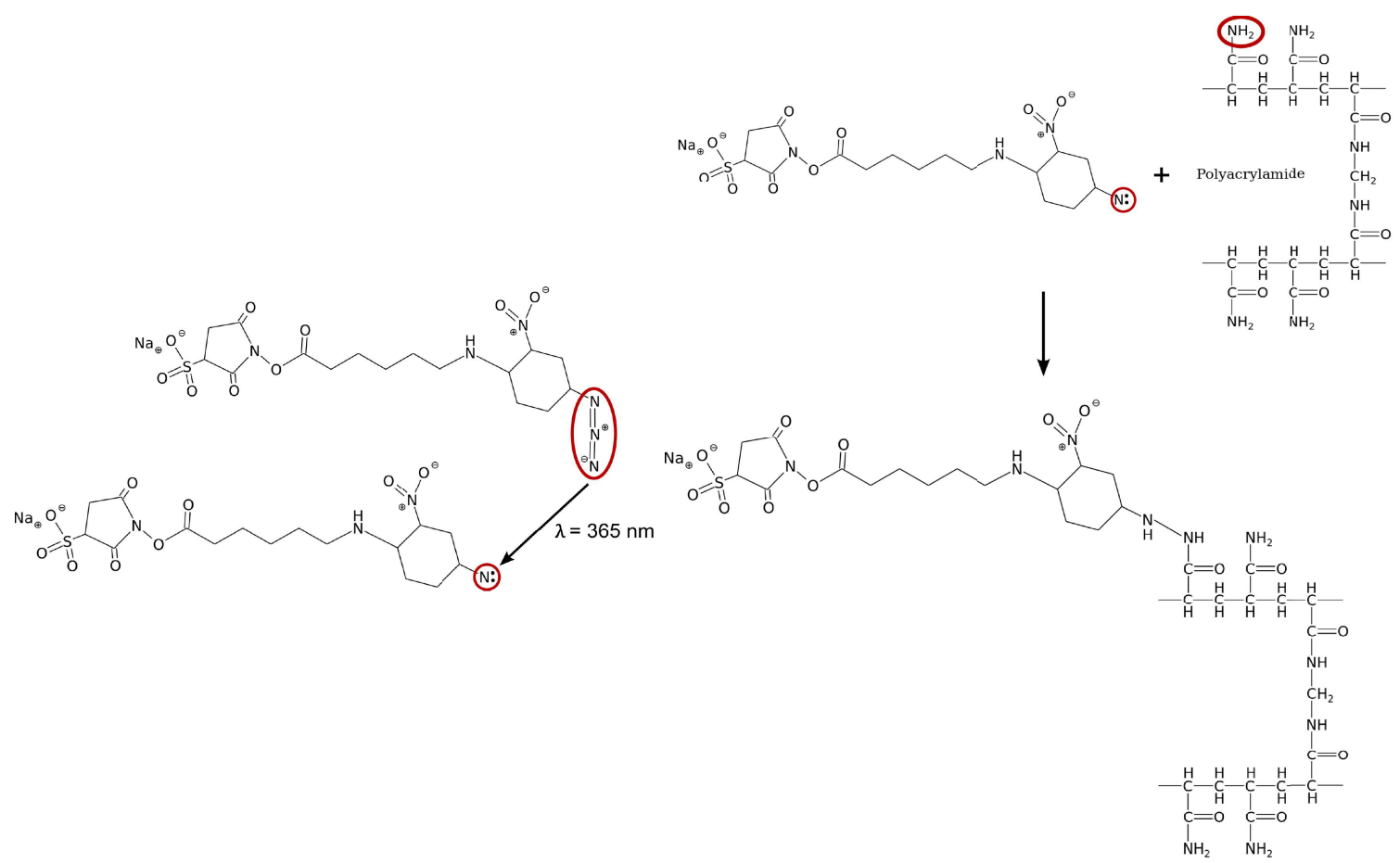

Figure 4.6.: Left: Activation of Sulfo-SANPAH with UV light and Right: reaction with PAA gel. Figure from [225].

\subsection{Stiffness}

While polyacrylamide gels can be tuned to a wide range of stiffnesses, tailoring them to the needs of the cells and the experiment is crucial. Because cells can probe the substrate and react to it, the individual preference of a cell line is important [12]. As depicted in Figure 4.7, cells in tissues operate in a range of stiffnesses [131]. One can find the preferred stiffness of a cell type or line with durotaxis experiments, in which cells show different morphology on a stiffness gradient $[199,234]$ and migrate to their optimal stiffness [134, 210, 211, 227].

Mature cells prefer substrates that resemble their natural environment. For example, while brain tissue is very soft and is ideal for neurons, cells like osteoclasts prefer stiffer substrates, as they naturally operate on the precalcified bone. However, for hMSC, the stiffness acts as a guideline for differentiation. hMSCs will probe substrate stiffness, as would any other cell, but differentiate into the cell types indicated by the stiffness. As shown in Figure 4.7, given enough time, at $1 \mathrm{kPa}$ we will get neurons, at $10 \mathrm{kPa}$ myocytes, and at $>30 \mathrm{kPa}$ osteoblasts from our hMSC culture. However, intermediate stiffnesses will also yield adipocytes and chondrocytes. Hence, we need to make sure substrates have the desired stiffness.

To measure the exact stiffness of the substrates, there are bulk methods and single-point ones. For single-point measurements, AFM, or nanoindenting, is a common technique. With a nanoindenter, the substrate is probed locally. This can be done in air or solution, with substrates or biological material, and point-, line-, or grid-wise. In Figure 4.8 (A), the manufacturing of a PAA gel with defined thickness using beads is shown. These gels are probed with a bead tip nanoindenter (B). In (C), one can see higher stiffness for thin gels. However, Kudryasheva shows that even rheometer and AFM measurements of the same thick gels vary significantly, with gels below $5 \mathrm{kPa}$ being measured stiffer in AFM and over $5 \mathrm{kPa}$ softer [121]. 


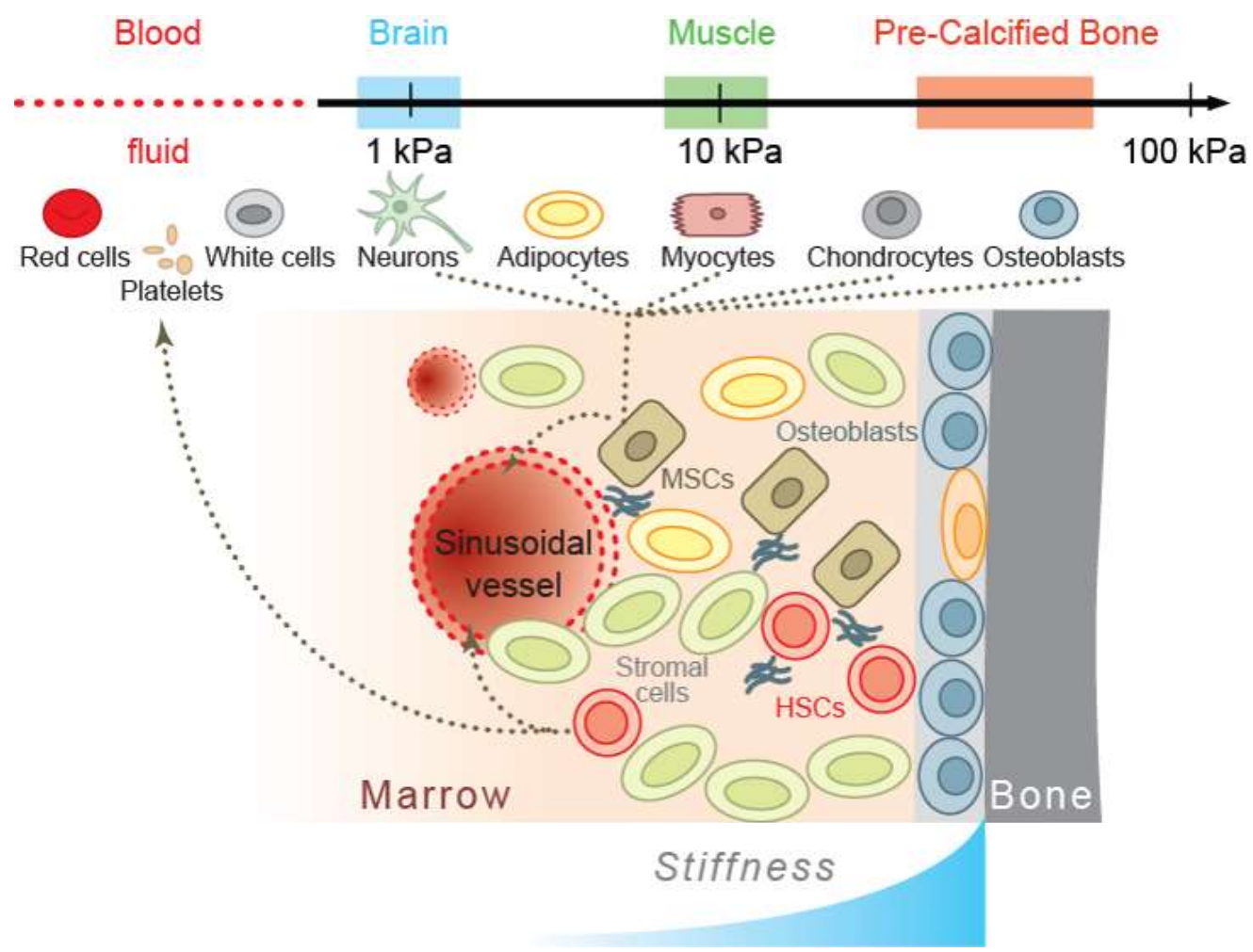

Figure 4.7.: Stiffness in different biological environments. Figure from [109].

This is an observation only possible with indenting techniques. As the stiffness seems to get higher with thinner gels, one can assume that cells would also sense higher stiffness. Several experiments show that one needs to have defined minimal stiffness of gel to prevent cells from sensing the support material [29, 28, 65]. Throughout this thesis, PAA gels with a thickness of 70-100 $\mu \mathrm{l}$ are used.

To do bulk measurements of gel stiffness, the gel is polymerized in a rheometer. This gives values for a stock solution of gel later used in experiments, but not for the exact gel used. This might be important when insufficient mixing or longer times between preparation of stock solution and experiment come into play. This can be used as a stand-alone technique to characterize gel stiffness; however, interactions with the supporting structure or inhomogeneities in substrate stiffness will only be detected using indentation on large surface areas of prepared gels. 
A

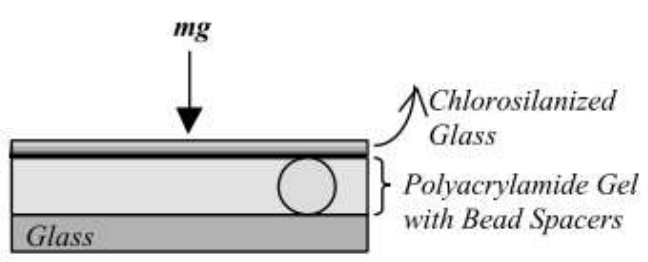

B
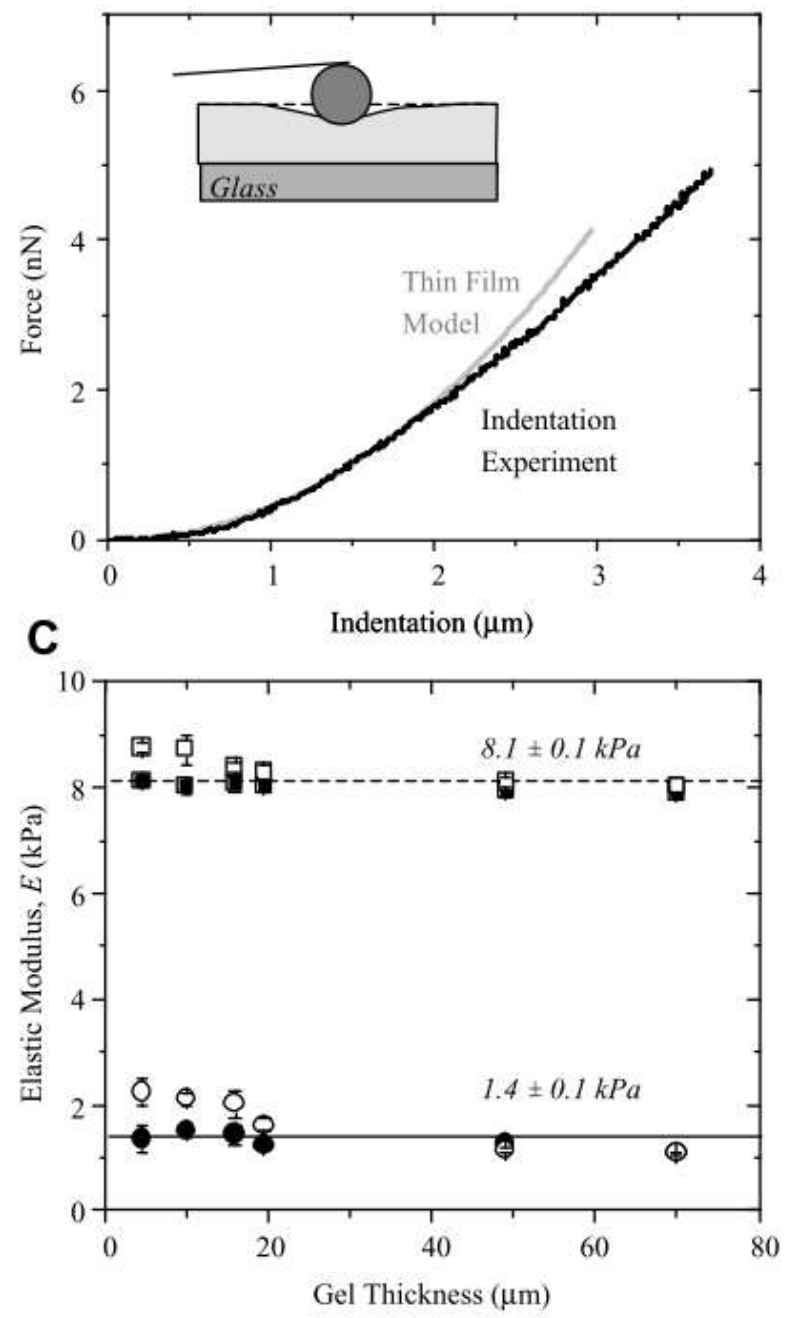

Figure 4.8.: AFM measurement of PAA gels of different thickness and stiffness. Figure from [65].

\subsection{Possible modulations of PAA gels}

PAA gels can be modified in various ways for multiple experimental reasons. We can distinguish modulations of the gel itself, insertions into the gel, or modulations of the surface and coating.

The stiffness of the hydrogel can be adjusted, in general, by varying the content of acrylamide and bis-acrylamide. While polymerizing, PAA gels can be mixed in various manners, the easiest being to polymerize different stiffnesses next to each other or within each other, using the placement of the PAA gel drops on glass slides before adding the cover glass [134]. However, the stiffness for these gradients has to be measured throughout the whole gel. Gradients can also be generated in a generalized fashion by microfluidics. Here, bis-acrylamide is added through multiple inlets in different concentrations to 
acrylamide. The polymerization is initiated by UV light and the gradients measured [234]. This can also be achieved with a uniform gel mixture and varied UV illumination time, by sliding an opaque mask at defined speeds across a substrate [199]. The latter method also yields better-defined gradients than the microfluidic method.

Beads of a defined diameter can be inserted into the PAA gel to ensure correct height [65], or can be used for traction force microscopy in 2D [7, 143,217] and 3D [195].

As already mentioned, one can transfer micropatterns onto PAA gels by stamping them onto a silanized glass slide and using it as cover glass during polymerization [204]. This will transfer the patterns to the gel. Additional steps like blocking with Pluronic-127 could be necessary, but this is a very straightforward technique. Also, one can use micropatterned glass stamps, in which the desired protein fills the patterns $[66,80]$. Here, a cross-linker is needed to bond the protein to the gel and residues may remain in the stamp. However, both techniques have problems with very thin structures and pointed angles. Here, micropatterning using machines has become a more widely used technique. Using software, every imaginable pattern can be rapidly designed and printed [211].

Topography can also be modulated by changing the cover glass to a 3D structure. PDMS molds can be used to microfabricate cell culture chambers from PAA gels [145]. Also, sinusoidal topographies for curvotaxis experiments made from PDMS [162] can be used as well as laser-ablated surfaces [14].

\subsection{Rheology}

Rheology, as a method for bulk measurement of deformation, can be applied to almost any material. In theory, for fluids, shear force can be applied and described using Newton's law. For solids, stress can be applied and described by Hooke's law. Hooke's law states that a solid material will deform when stress is applied; however, the energy needed for deformation is stored in the material and the material will regain its original shape once the force is removed. An ideal liquid, in contrast, will deform under the force applied and keep the conformation even after the force is removed. An ideal solid stores the applied force and returns to its initial conformation after the force is removed. This is called elastic deformation.
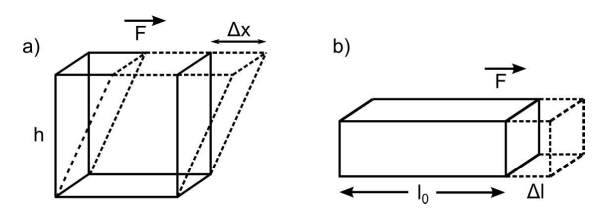

Figure 4.9.: Deformation of material. a) shear, b) strain. Figure from [225].

However, no material is purely solid or purely liquid. For these anisotropically deforming, nonideal materials, we are in the viscoelastic regimen, unifying components of both. With rheology, we try to separate the influences of stress and strain. In this process, stress is defined as the force per area and strain as the deformation of the sample height relative to length with constant volume. Stress and strain are related by the elastic shear modulus $G$, which gives the stiffness of the system. The Young's modulus - often used to describe gels for biophysical uses - describes the ability of a material to resist deformation and is, therefore, a constant related to the elasticity of the material.

$$
G=\frac{\sigma}{\gamma}
$$

Rheology can be divided into macro- and microrheology. Here, microrheology is often done via beads and optical or magnetic tweezers, as already mentioned, for direct probing of cells from the outside; but single molecules or polymers can be probed, too. The required sample size is low, which benefits biological 
experiments with limited sampling options. Additionally, the usable frequency range ranges from $0.1 \mathrm{~Hz}$ to $100 \mathrm{kHz}[8]$.

In contrast, macrorheology is done on larger sample volumes to study bulk behavior, using a rheometer featuring a fixed bottom plate and a rotating top probe. The geometry of the probe used is quite important, as parallel and cone probes result in different geometries. Parallel plates are used where uniform gap conditions are required or when the sample shows a gap-dependent behavior. However, the shearing is not uniform and a large sample volume is needed. In contrast, cone plates show uniform shearing and require less sample volume.

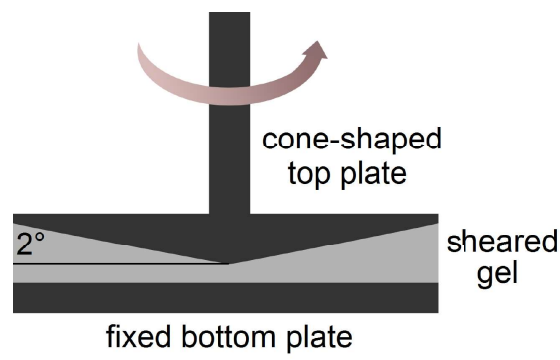

Figure 4.10.: Rheology measurement with the 25-2 cone plate. Figure from [225].

In general, rheometers will be operated as either stress or strain-controlled. We operate ours as straincontrolled. During rheometer measurements, oscillatory strains and stresses are applied to the sample, with amplitude and frequency being varied.

With PAA gels, we use the Young's modulus to describe the stiffness of our samples. This is related to the elasticity of the material and describes the resistance to deformation. In polyacrylamide gels, the loss modulus, $G$, is mainly elastic, as the loss modulus is two orders of magnitude smaller than the storage modulus (compare Figure 4.11). For other materials, like artificial actin networks, there are other characteristic curves [136].

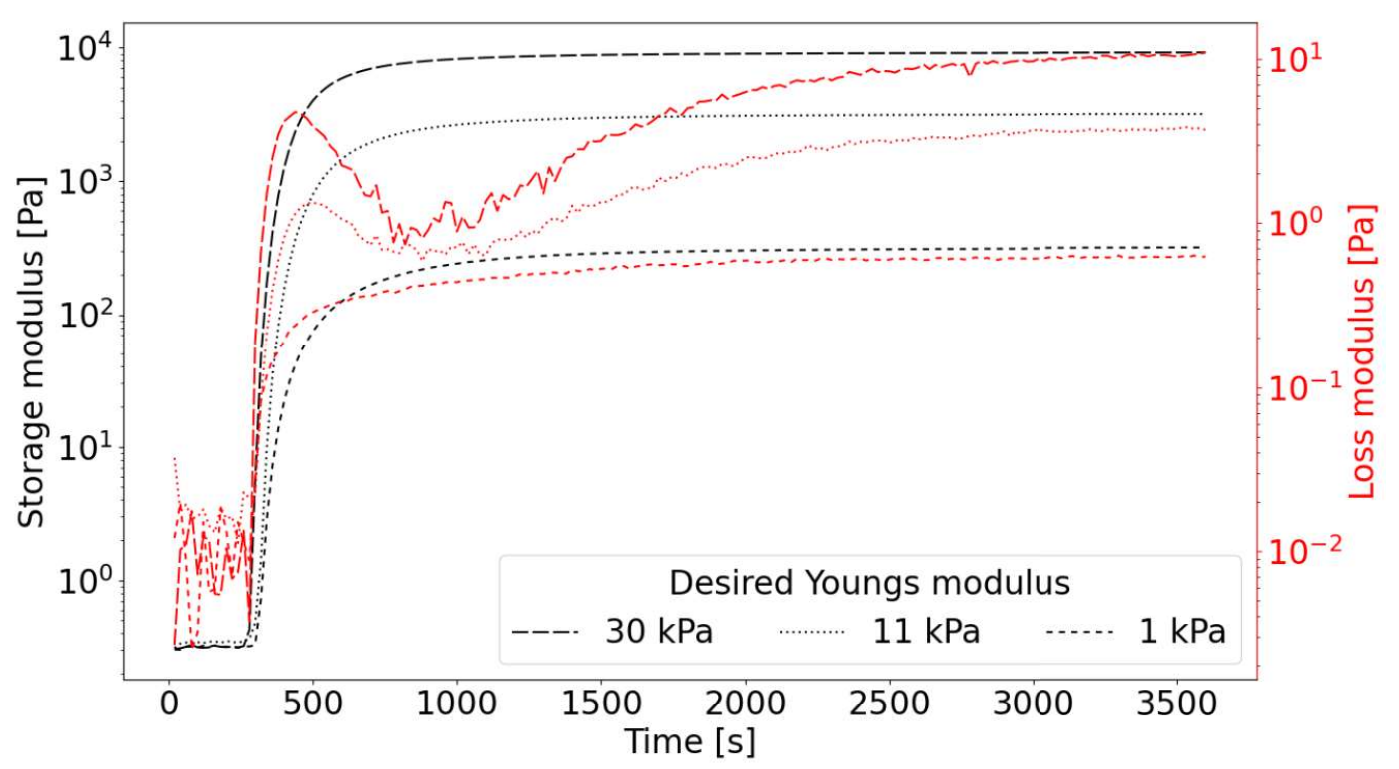

Figure 4.11.: Example plots of storage and loss modulus during a time sweep measurement for gels of different desired Young's moduli. Measurements were done with a rheometer and a 25-2 cone plate. 
Premixed polyacrylamide gel solutions used for experiments were only stored for 6-8 weeks. Control experiments after this amount of time have been done on three occasions for this study (see Table 4.2), but have been done extensively by master's student Martin Schilling for polyacrylamide and other gels in his thesis [177].

Rheometer measurements often apply small, varying oscillatory strains or stresses. The material is added between a fixed steel bottom plate and a rotatable top plate of varying diameter and angle. We used a 25 $\mathrm{mm}$ diameter cone-shaped plate with a $2^{\circ}$ angle and with a sample volume of $140 \mu \mathrm{l}$. To characterize the gel, a series of three experiments were done at room temperature. To prevent the sample from drying out, wet paper towels were placed around the probe. A time, frequency and amplitude sweep were done consecutively, because the amplitude sweep destroys the polymerized gel.

\begin{tabular}{l|c|l|l} 
Sweep & Measurement & Strain & Frequency \\
\hline Time & every $30 \mathrm{~s}(1 \mathrm{~h}$ total $)$ & $1 \%$ & $1 \mathrm{~Hz}$ \\
Frequency & 5 per decade & $1 \%$ & $0.01-100 \mathrm{~Hz}$ \\
Amplitude & 5 per decade & $0.01-100 \%$ & $1 \mathrm{~Hz}$
\end{tabular}

Table 4.1.: Rheology measurement settings.

Polyacrylamide polymerization takes longer than 1 hour. Nevertheless, as can be seen in Figure 4.11, after 1 hour the polymerization is close to plateauing. During the experimental preparation of the hydrogels, 1 hour polymerization time is deemed sufficient. This is the reason time sweep measurements of our PAA gels were also restricted to 1 hour (See Table 4.1).

\subsubsection{Viscoelasticity}

Since we work with materials with mainly viscoelastic properties, measuring those is of the greatest importance to us. In a viscous material, the stress is not proportional to the strain, but rather to the rate of strain $(\mathrm{d} \gamma / \mathrm{d} t)$ with unit $\mathrm{sec}^{-1}$. While in the rheometer the stress is measured through the applied shear force and the strain via the rotation speed of the probe, viscosity describes the resistance to the flow. Viscoelastic measurements are done over large time scales and described by $G(t)$, a time-dependent modulus. To measure the linear response, we applied sinusoidal oscillations with small amplitudes. At frequency $\omega$, the stress will oscillate with time $t$, but will be phase shifted by $\delta$ with respect to strain as

$$
\begin{gathered}
\gamma(t)=\gamma_{0} \sin \omega t \\
\sigma(t)=\sigma_{0} \sin (\omega t+\delta)
\end{gathered}
$$

where $\sigma=$ stress, $\gamma=$ strain, $\sigma_{0}=$ amplitude of stress, $\gamma_{0}=$ amplitude of strain, $\omega=2 \pi \nu=$ angular frequency $(\mathrm{rad} / \mathrm{s})$, where $\nu=$ frequency $(\mathrm{Hz})$ and $\delta=$ phase shift. This phase shift is between $0^{\circ}$ and $90^{\circ}$ for viscoelastic materials [93]. Stress and strain for viscoelastic materials relate as

$$
\sigma(t)=\gamma_{0}\left(G^{\prime} \sin \omega t+G^{\prime \prime} \cos \omega t\right)
$$

where $G^{\prime}$ is the elastic storage modulus and $G^{\prime \prime}$ is the viscous loss modulus, which is out of phase by $90^{\circ}$. In an ideal elastic system $G^{\prime}=G$ and $G^{\prime \prime}=0$. For small strains, the ratio of stress and strain are not dependent on the magnitude of strain, thus giving a linear viscoelastic regime. For larger strains, the ratio will not be proportional and the material will exhibit a strain-stiffening or -softening behavior [197]. 


\subsection{Rheological measurements of gels used in this thesis}

As premixed PAA gel solutions are used by all lab members, rheological measurements of these gels have been done by various people and made public. I show here all measurements of gels used for experiments in Chapters 5, 7, and 8, divided by type of experiment.

Something important to keep in mind is that polyacrylamide solutions and polymerized gels age. This is why pure solutions are discarded after 6 months and premixed gel solutions after 8 weeks. Usually, we measure gel stiffness of new solutions before the first use and never again till they get discarded. However, if we do this, we already see a significant drop in stiffness after 8 weeks (see Table 4.2). Whenever using PAA gels, one has to keep this gradual softening in mind, effects of which become more obvious the stiffer the desired gel would be.

\begin{tabular}{l|l|l|l} 
Desired & $\mathbf{3 0 . 1 1 . 1 8}$ & $\mathbf{2}$ months later & 10 months later \\
\hline $1 \mathrm{kPa}$ & 0.98 & 0.72 & 1.05 \\
$11 \mathrm{kPa}$ & 10.15 & 8.8 & 6.7 \\
$30 \mathrm{kPa}$ & 30.45 & 27.55 & 31.03
\end{tabular}

Table 4.2.: Results of rheological measurements on multiple time points.

For experiments studying pattern changes in the stress fiber networks of stem cells on different substrates, $11 \mathrm{kPa}$ PAA gels and glass slides have been used. Both were collagen-coated. We chose these two regimes because hMSCs show a maximum in spindle factor at $11 \mathrm{kPa}$ (see Figure 4.12). Thus, differences in morphology should be strongest in these two conditions.
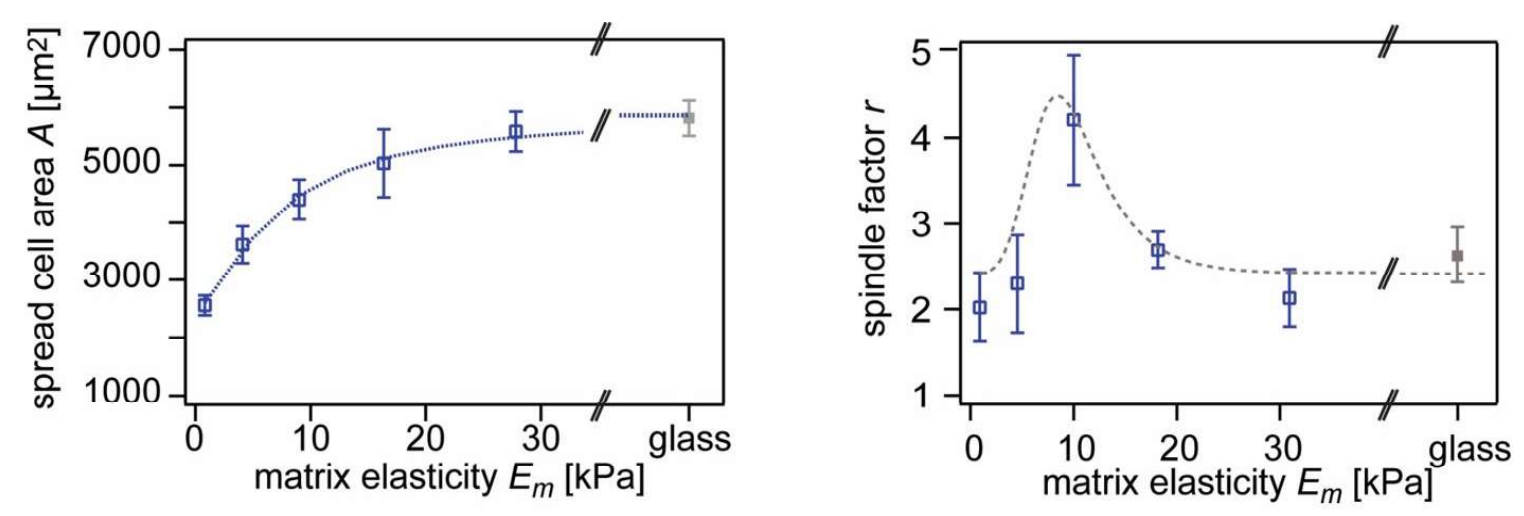

Figure 4.12.: Behavior of stem cells on PAA gels of different stiffness. Left: Spreading area increases with stiffness. Right: Spindle factor has a maximum at $11 \mathrm{kPa}$. Figure adapted from [169].

In Table C.10 one can see two recipes: one for $10 \mathrm{kPa}$, one for $11 \mathrm{kPa}$ PAA gels. Initial measurements with the $10 \mathrm{kPa}$ recipe showed stiffness of around $9 \mathrm{kPa}$. This was determined to be too low, keeping in mind that PAA gel stock solutions age with time and resulting gels keep getting softer (see Table 4.2). With the changed recipe, the resulting gels were measured more closely to the desired $11 \mathrm{kPa}$.

For RNA experiments (see Chapter 8), we needed large numbers of gels on large microscope slides. Here, we used various gel stiffnesses, in addition to glass and cell culture plastic, to capture the whole range of reactions. For qPCR (8.5), all stiffnesses were used. For RNASeq (8.2), only 1, 11, and $30 \mathrm{kPa}$ gels were manufactured. In Table 4.4, gels depicted in the first column were used for initial experiments, the second column for the first two experiments of qRT-PCR, the third column for RNASeq, and a combination of 
columns three and four for the third experiment of qRT-PCR.

\begin{tabular}{l|l|l|l|l|l} 
Desired & $\mathbf{2 9 . 1 . 1 8}$ & $\mathbf{7 . 5 . 1 8}$ & $\mathbf{1 3 . 8 . 1 8}$ & $\mathbf{3 . 1 . 1 9}$ & $\mathbf{2 6 . 5 . 2 0}$ \\
\hline $11 \mathrm{kPa}$ & 10.08 & 10.06 & 11.34 & 10.15 & 12.1
\end{tabular}

Table 4.3.: Results of rheological measurements of gels used for live cell experiments for comparison of behavior on $11 \mathrm{kPa}$ PAA gels and glasses. They were used for stem cell as well as enucleation experiments.

\begin{tabular}{l|l|l|l|l} 
Desired & $\mathbf{1 7 . / 2 2 . 5 . 1 8}$ & $\mathbf{3 0 . 1 1 . 1 8}$ & $\mathbf{3 . 1 . 1 9}$ & $\mathbf{1 2 . 2 . 1 9}$ \\
\hline $1 \mathrm{kPa}$ & 1.07 & 0.977 & 1.044 & - \\
$2 \mathrm{kPa}$ & 1.54 & 2.073 & - & 3.39 \\
$5 \mathrm{kPa}$ & 4.7 & 5.3 & - & 5.13 \\
$10 \mathrm{kPa}$ & $9.01(7.77)$ & - & - & - \\
$11 \mathrm{kPa}$ & - & 10.4 & 10.15 & $(8.87)$ \\
$20 \mathrm{kPa}$ & $(12.62) 20.9$ & 15.42 & - & 14.41 \\
$30 \mathrm{kPa}$ & 28.33 & 30.16 & 30.45 & -
\end{tabular}

Table 4.4.: [Rheology results for RNA experiments.]Results of rheological measurements for RNA experiments. Dashed lines indicate stock solution was not mixed. 


\section{Live cell experiments with hMSC}

In this thesis, the main focus is on the differentiation of hMSCs and the correlated changes in the stress fiber network. There are some aspects that one has to keep in mind when working with live cells. These aspects are explored in this section.

For the main results, I use hMSCs, transfected with pCMV-LifeAct-TagRFP, on different substrates. These are imaged on an inverted microscope with a heating chamber and gas mixer for 24 hours. Starting from the cell line, I have to make sure all cells behave similarly, which is not true for hMSCs from different donors (see 5.5.1). Also, the transfection should not affect the morphology and behavior of the cells. I use the reversibly binding LifeAct protein from yeast, which, in comparison to other techniques, has the least effect on the behavior [69]. However, even the process of transfection, without expression, could influence the cells. Thus, I compare untransfected cells with transfected cells (see 5.5.4). Additionally, because some people report localization differences between LifeAct labeling of stress fibers and Phalloidin labeling, I compare these (see 5.5.5). To ensure the microscope provides good conditions for the cells during the experiment, I fix and stain imaged samples and control samples kept in the incubator and compare the results (see 5.5.5). Comparing the results of these fixed samples to the previously acquired images also provides a way to estimate the subsampling effect. Finally, I look into the differences between actin and NMM IIa localization with a phalloidin and Anti-NMM IIa costaining (see 5.5.6).

\subsection{Preparation of cells}

Stem cells were maintained in a subconfluent culture and reseeded every 2 to 4 days using $0.05 \%$ Trypsin. Medium was used according to Table A.2. Cells were kept at $37^{\circ} \mathrm{C}$ and $5 \% \mathrm{CO}_{2}$. Protocols for thawing (Subsection A.1), freezing (Subsection A.2), and splitting (Subsection A.3), as well as other cell culturerelated protocols, can be found in the Appendix (Chapter A). General materials are used according to A.1. Where special reagents are used, as for transfection (Subsection A.4) and RNA extraction (Subsection B.1), additional reagents can be found in the respective special sections.

For cell lines, especially non-cancer ones, one prefers to know their origin and performance, as well as general behavior as observed by other scientists. When obtained from a vendor, we usually know doubling times, size, and other factors. During expansion, we first check these and compare to the vendors information as well as looking for contamination with morphologically different cells. The doubling time, for example, is also dependent on the media used, and will sometimes vary significantly from the information given.

For hMSCs, purchased from Lonza, additional information is given in Table 5.1. Due to the high variability in stem cells derived from donors in contrast to immortalized cell lines, it is important to know what you are working with. In general, we try to use male donors under the age of 30 for consistent results but have to make work with what is offered sometimes. Also, he most offered race is B, which denotes "African American", and we usually stick to that $(\mathrm{C}=$ White, $\mathrm{O}=$ Other $)$. Since there is an observable difference between the lots, you can find results comparing them in Subsection 5.5.1. 


\begin{tabular}{l|l|l|l|l} 
Experimenter & Lot Number & Age & Sex & Race \\
\hline Wollnik & 0F 4266 & 33 & M & B \\
& 2 F 3478 & 34 & M & B \\
& 351482 & 20 & M & C \\
& 305526 & 22 & F & B \\
\hline Hauke & 423370 & 23 & F & B \\
& 451491 & 20 & M & O \\
& 603525 & 37 & M & B
\end{tabular}

Table 5.1.: Lots of Bone Marrow-Derived Mesenchymal Stem Cells (BM-hMSC) used in our lab.

After examination of general cell behavior, calls have to be additionally treated for experiments. As in phase contrast imaging single structures are hard to visualize, introduction of fluorescent labels is common. Where this is done in living cells, one has to carefully choose the method in order to avoid altering their behavior. In this thesis, LifeAct linked to a fluorophore is used to label actin stress fibers. A discussion of the possible effects of LifeAct on the cell morphology can be found in 5.1.1.1. Furthermore, as I am interested in the dynamics of the stress fiber network, which is dependent on the nucleus, it is useful to remove it. This is done via an ultracentrifugation protocol. A detailed explanation of the technique can be fount in Subsection A.5. Theoretical background and experimental results of the generation of cytoplasts can be found in Chapter 7 .

\subsubsection{Transfection}

Transfection is a common technique to label specific proteins inside a living cell for microscopy which includes different techniques to insert genomic information into a cell. Electroporation, more precisely nucleofection, as done here with the Lonza $4 \mathrm{D}-\mathrm{Nucleofector}$, is a very fast technique in comparison to lipofection. It uses an electric pulse to create pores in the negatively charged membrane, through which the plasmid can enter the cell and the nucleus. An overview of techniques for transfection, as well as suggestions for deciding the right technique, can be found in [101, 126].

After insertion of the plasmid, the cell will start manufacturing the encoded protein - just as it does with its own DNA. However, after some time the cell recognizes the alien DNA and stops translation. Thus, nucleofection can have two outcomes in the cell. The DNA can be either integrated into the cell's DNA, or not; these outcomes are called stable and transient transfection, respectively. In case of integration, the fluorescence signal will be stable, whereas in the case of a transient transfection the signal will become weaker within a few days.

The protocol used is adapted from the protocol given by Lonza. Detailed steps are given in the Appendix (Subsection A.4). To maximize the effect of the nucleofection, Lonza sells buffer solutions to use with their Nucleofector. Transfection kits used, organized according to cell line and amount of plasmid, can be found in Table A.4. Besides choosing the correct solutions, one must also choose the right pulse code and amount of plasmid. Pulse codes are either provided by Lonza upon request or can be determined by trial and error for uncommon cell lines. Amount of plasmid has to be determined experimentally as well, as an insufficient amount of plasmid leads to too few transfected cells, whereas excessive amounts will interfere with cell viability.

The amount of time a cell requires to produce the desired protein-fluorophore construct varies between cell lines. The expression maximum is usually reached after 24 hours. For this research, since for most experiments cells had to be reseeded to substrates and reacted negatively to trypsinization after 24 hours, the cells were rested for 48 hours before the start of experiments. 


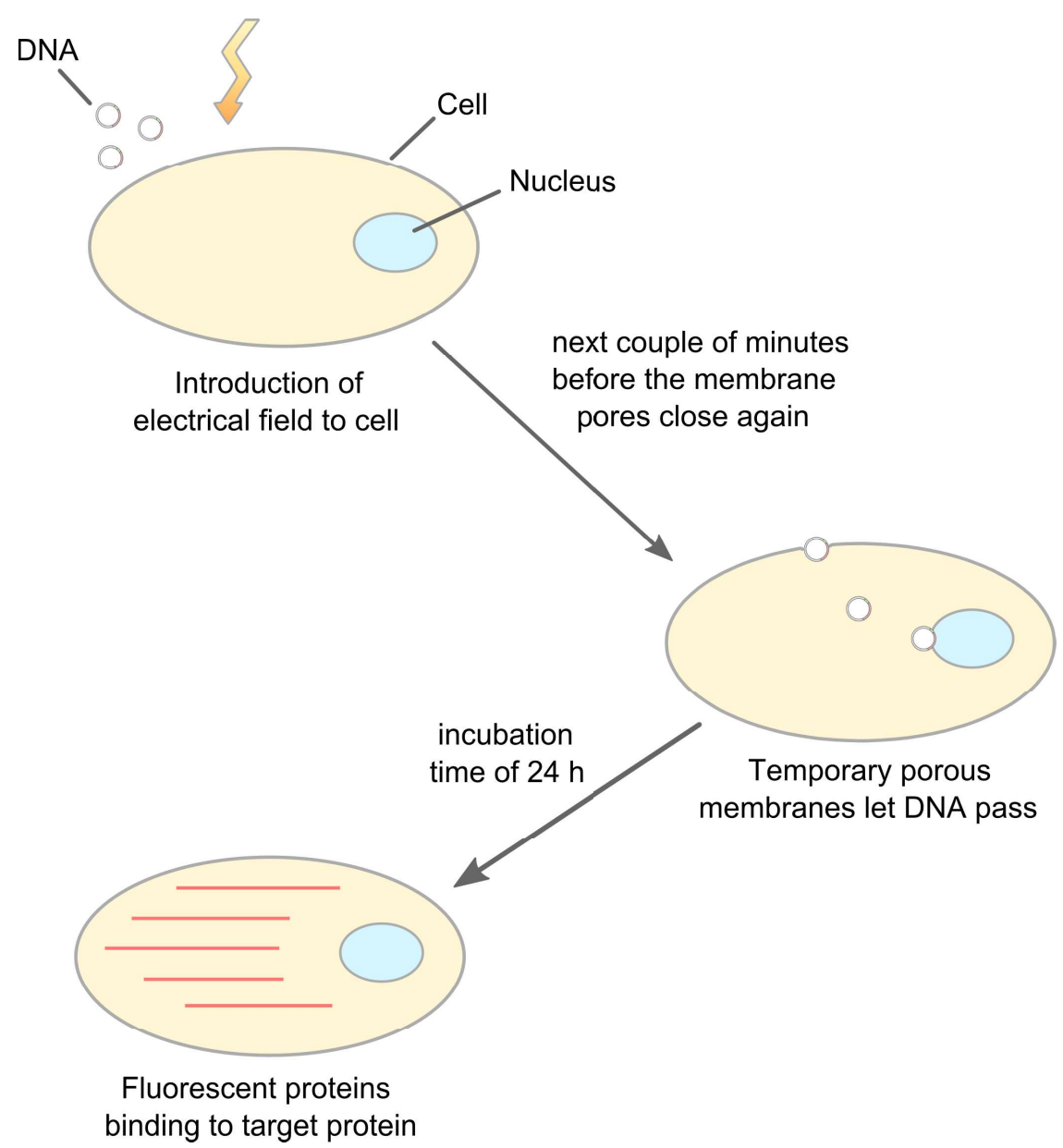

Figure 5.1.: Schematic drawing of nucleofection. Figure from [225].

Using LifeAct is common for stress fiber visualization and anylsis, as LifeAct is a small, transiently binding, protein (17 AA) from Saccharomyces cerevisiae with a halflife (t1/2) of $17.8 \pm 3.9$ seconds [189]. Using a plasmid to introduce LifeAct avoids problems that would arise from additional expression of fluorescently labeled actin as well as the "locking" of the fibers that would occur with intercalating dyes like phalloidin. We tested LifeAct coupled to RFP and GFP, but choose to use RFP as this would work best with the microscope used. For all experiments described here, the same plasmid (pCMV-LifeAct-TagRFP, see Figure 5.2) was used.

\begin{tabular}{l|l|l|l|l|l} 
Plasmid & Strain number & Strain & Vector & Resistance & Supplier \\
\hline pCMV LifeAct-TagRFP & BH43 & DH5 $\alpha$ & pCMV & Neomycin & Ibidi
\end{tabular}

Table 5.2.: pCMV LifeAct-TagRFP plasmid manufacturer information.

The CMV promotor used was proven to be the least troublesome for use with LifeAct, compared with other promotors $[59,158]$. On plasmids, the promotor should not only be compatible with the expressing organism, but also be chosen based on the amount of product. Here, we want the fluorescence to be bright enough to be imaged over a long timescale with minimal bleaching.

However, there are new reports on altered morphology and migration behavior when using LifeAct as actin stain [107], as described in Subsection 5.1.1.1. We observed altered behavior in our research as well, 
and show data on this in 5.5.

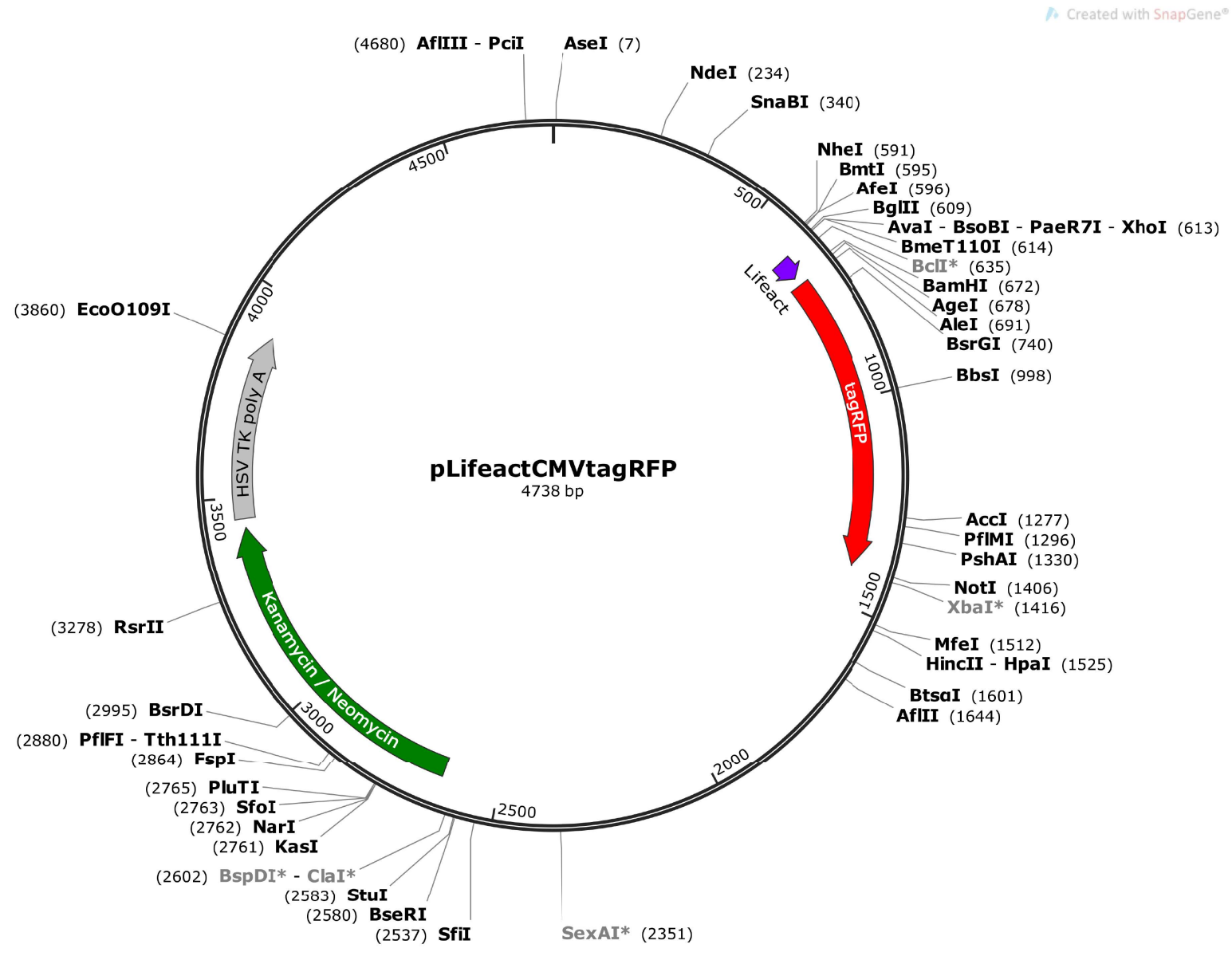

Figure 5.2.: Schematic drawing of pCMV-LifeAct-TagRFP plasmide.

\subsubsection{LifeAct controversy}

LifeAct, as a small protein binding reversible to actin, should provide some substantial advantages compared to other stains. First, it should not alter the amount of actin, as the introduction of a fluorescently labeled actin would. Second, because of its reversible binding it should not influence the actin dynamics as, for example, phalloidin does. However, recent studies have uncovered disadvantages in the usage of the LifeAct protein.

First, there is a problem with the localization of LifeAct. Not only are there general discrepancies reported for the LifeAct localization in comparison to actin or other actin-probes [15], there are also some types of actin, like twisted f-actin, that LifeAct does not stain at all [144].

For cell morphology, some people claim that LifeAct does not alter the cellular behavior [191], while others disagree. A recent paper by Flores et al. [69] investigated in detail the influence of LifeAct expression on hMSCs, as well as on COS-7 and NIH-3T3. The source of hMSC and treatment used by Flores et al., as well as the transfection method, differ from those in my experiments. However, like in my experiments, they use a CMV-promotor, which was determined by [158] to be the least morphogenetic change-inducing promotor. They could link LifeAct-GFP2 expression-but not GFP alone-to the altered cell area, nucleus size, migration speed, and direction. Additionally, they identified the localization of cofilin onto LifeAct-GFP-expressing cells, but not GFP-expressing or control cells. This led them to investigate more deeply into the YAP/TAZ-pathway, where they found alterations in the stress fiber turnover and thus 
effects on both stress fiber network reorganization speed and the total number of filaments. As YAP/TAZ has already been found to play a significant role in mechanotransduction, ECM probing, and migratiory behavior, it seems reasonable to conclude that it is affected by alterations caused by blocking of stress fiber movement [58].

Furthermore, Flores et al. estimate only $20 \%$ of transfected cells to be in a regime unaffected by LifeActexpression effects. A set of cells selected for analysis will thus always contain cells with altered morphology not visible to the naked eye, since only strong effects on morphology will be excluded by the experimenter. A big enough sample size and estimation of the starting point of dose-dependent behavior can aid in excluding this skewed data.

Despite these disadvantages and the surrounding controversy, LifeAct remains the easiest and bestunderstood tool for live-cell stress fiber studies;. For this reason, I decided to use it despite its weaknesses.

\subsection{Live-cell imaging}

Live-cell imaging is widely used to gather insights about cell behavior over large timescales. One can image single cells, clusters, layers, single-cell types, or mixtures for different purposes. These purposes could be to study motility, taxis of any kind, wound healing assays, signaling between different cell types, and many more behaviors.

Here, I use live-cell imaging of single cells to look into the early formation of stress fiber networks after plating and during differentiation. For this, I seed hMSC (or other cell lines for comparison, see 7.1) on substrates of different stiffnesses (see 4.1) and start 24 hours of $10 \mathrm{~min}$ interval imaging. With this imaging, I am able to obtain stop motion pictures of the cells in a reasonable time period without bleaching the cells. The imaging is started 1 hour after plating of the cells. This gives the cells enough time to adhere, but some cells will not be fully spread by then. So, one has to differentiate between reorganization of the stress fiber network during spreading and reorganization during differentiation.

To image the stress fibers, I transfect cells with LifeAct-TagRFP (see Subsection 5.1.1 and Subsection A.4). Besides effects of the plasmid itself ([107], and see 5.1.1.1), stress during trypsinization has an observable effect on the cells. Because I want to image single cells, and first cell division would occur within 24 hours of transfection, I have to reseed the cells to the substrates. To avoid stress effects from trypsinization and transfection, I reseed 48 hours later but miss the peak fluorescence at 36 hours. Furthermore, viability after transfection can vary based on a multitude of influences, and as a result plated cell density would vary from experiment to experiment. Thus, reseeding at a certain density has the additional benefit of unitizing experiments.

Besides factors necessary for later analysis such as brightness, cell count, and transfection efficiency, one must also bear in mind the influence of measurement environment on the cells. Even with $37^{\circ} \mathrm{C}$ and $5 \%$ $\mathrm{CO}_{2}$, the same environment as in an incubator, after 24 hours of measurement cells will behave slightly differently, having altered morphology; additionally, the $\mathrm{PH}$ of the medium will be observably different. For every live cell measurement shown here, there is a control group kept in the incubator. Both populations are fixed after 24 hours and stained for nucleus and actin fibers. Differences between these are shown in 5.5.4.

\subsubsection{Sample preparation}

Substrates most suitable for live-cell movies depend on the setup used and the requirements of the substrate preparation. During this thesis ibidi $\mu$-Dishes with $35 \mathrm{~mm}$ diameter have been used, as they fit the 2-dish ibidi heating chamber of the setup. These are obtainable in different types: high or low, and with or without grid or special coating. For live-cell movies, I have used raw dishes, which means lacking a bottom. For these experiments, the $\mu$-Dishes are precleaned with isopropanol. Then, prepared bottom glasses with PA-gels and collagen I coating (preparation see Section 4.1 and the respective subchapters or Appendix 
Section C) are glued into them. A ring of glue is applied as thinly as possible to the edge of each bottomless $\mu$-Dish. The glasses are placed onto the glue and gently pushed down to be as level as possible. There should be neither air bubbles nor regions not covered in the glue. The dishes are placed under a UV lamp for 90 seconds twice, first right-side up then upside down. After the total 180 seconds, the glue should be firm. To check for leakage, dishes can be placed onto paper while filling them with $2 \mathrm{ml}$ of PBS to avoid drying of the hydrogel. Leaky dishes should be trashed, as filling holes with glue afterward does not prevent leaking entirely.

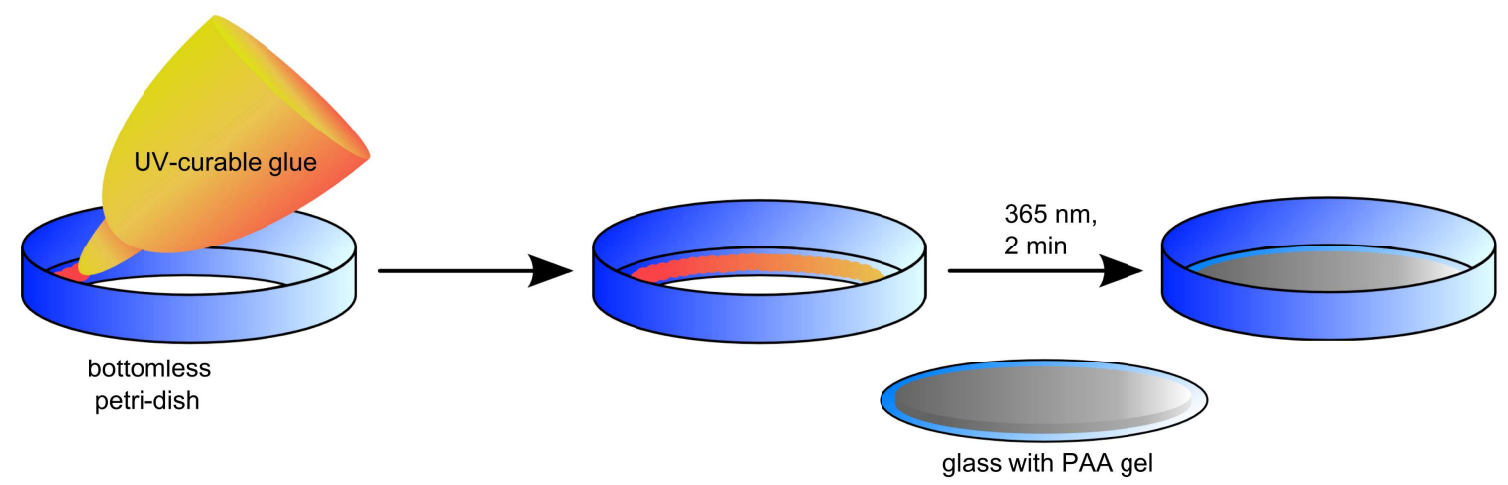

Figure 5.3.: Applying UV curable glue to bottomless petri dishes. Figure from [225].

Dishes with PBS are placed, open, in the cell culture bench with the UV lamp turned on for 2 hours to sterilize them. Afterward, they are washed three more times with sterile PBS to remove possible traces of glue. These sterile dishes can now be used for cell culture.

While collagen I coating tolerates UV radiation for a prolonged time, this is not the case for other coatings. For synthamax stamping on PAA gels, which is also done in our lab, we cannot sterilize using UV. For these experiments, PAA gel preparation and stamping is done under sterile conditions. Glasses bearing these gels are not glued in, but placed in common glass-bottom dishes and fixed using spacers, so they do not move during stage movements in the automated 24-hour measurement.

\subsubsection{Microscopy}

Choosing the right microscope is as important as every other step in the experiment. For these experiments, I prefer the widefield microscope over the confocal microscope for three main reasons. First, I want to image the whole cell for 24 hours. With moving cells, the field of view has to be quite big; thus, I use a 20x air objective. Second, I have to see as many fibers as possible for statistical analysis. With a confocal microscope, I would miss most fibers in different z-planes. Furthermore, fibers with above $50^{\circ}$ inclination would not be detected [97]. Third, in prolonged measurements, Z-drift is a major problem. Caused by thermal fluctuations in the environment mechanical parts of the microscope will expand and shift the sample out of focus. The widefield setup has been proven to be more temperature stable.

Imaging is done in $10 \mathrm{~min}$ intervals (see 5.2.2.2). This has been proven to yield best results for bleaching. Additionally, highly dynamic regions show visible changes in a timeframe of 5-10 minutes, whereas slower shifts are visible in 20 minutes or longer [174].

\subsubsection{The Cell Observer setup}

Live-cell microscopy was done with a Zeiss inverted microscope (Observer A.1) with an added heating chamber, humidifier column, and gas mixing system (see Figure 5.4). The microscope itself has a 
motor-controlled stage to automate long-term measurements of multiple positions. For a detailed list of microscopy parts, as well as a detailed description of live-cell microscopy, please see Section E.

The main problem with live-cell imaging is Z-drift. This is mainly due to the expansion of metal parts with rising temperatures, both in the microscope as well as in the camera. To prevent this issue, the system must be preheated at least 2 to 3 hours, ideally over night, before the start of the measurements with the same settings as will be used later. An additional problem can arise from room temperature fluctuations and air conditioning. An air outlet directly above the setup, for example, may ruin all measurement attempts. Strong fluctuations with the outside temperature will also pose a problem.

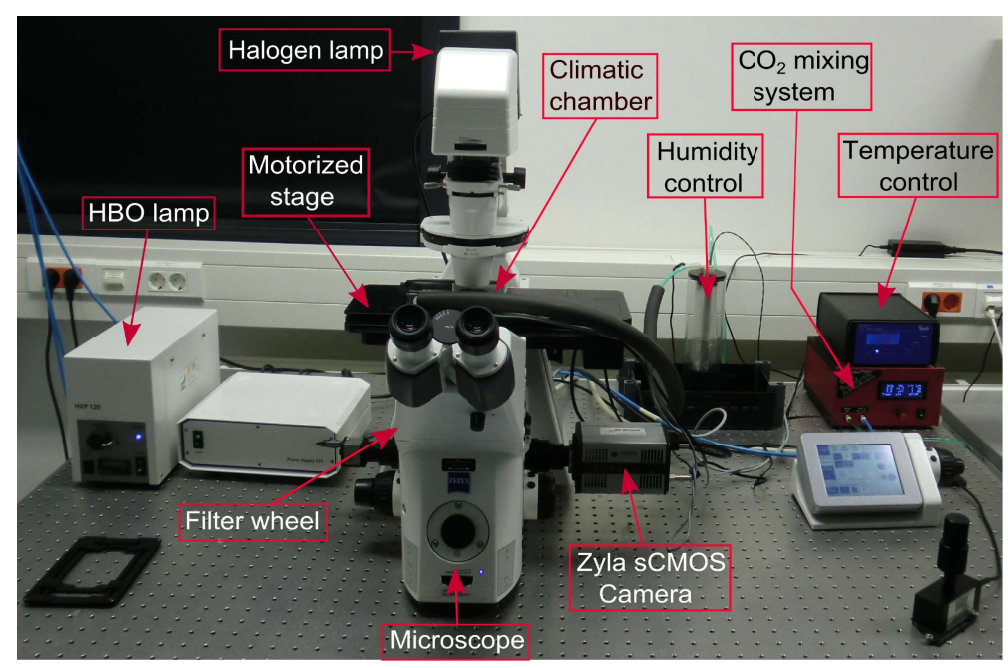

Figure 5.4.: Live-cell imaging setup. Image from [225].

The Cell Observer is used for all live-cell movies as well as imaging immunostained fixed samples, as with its mercury lamp it is best equipped for a wide range of stainings and can also image near-infrared staining.

\subsubsection{Multi-dimensional acquisition with Micro-Manager}

For automated measurements over the course of one or multiple days, the Micro-Manager ( $\mu$ Manager) software can be used. It is a free software compatible with most available microscopes, stages, and cameras [60, 61, 198]. With its "Multi-dimensional acquisition" tool, one can automate loops through multiple positions, channels, and even z-stacks for multiple time points, including adjusting the order of these filters.

Here, I mostly use it for 144 10-min intervals - which adds up to 24 hours - and two channels, the phase contrast and RFP channel. The phase contrast channel is always imaged in addition to any fluorescence channel to discover untransfected cells interfering with the desired transfected ones, as well as dirt, dead cells, or inhomogeneities in the underlying PA gel substrate. A detailed description of the Multi-dimensional acquisition tool can be found in Section E.

This tool can also come in handy for large amounts of fixed cells that need to be imaged in multiple channels. Whereas normally one would have to switch channels manually and save the images, with the Multi-dimensional acquisition tool one can mark the positions and use the tool to loop automatically through all selected channels. 


\subsection{Immunostaining}

In contrast to transfection, in which only a fraction of the cells (depending on transfection efficiency) are labeled for the desired target, immunostaining labels every cell accurately. The latter method uses different agents in PBS, and thus can be done in any container regardless of substrate. A detailed protocol can be found in Appendix Section D. Whereas some immunostaining agents, like antibodies for intracellular structures, require the permeabilization of the membrane, which is only possible for a dead cell, other stainings like phalloidin or Hoechst can be used on living cells. However, such intercalating agents change the morphology and behavior of the cell, and, in the case of phalloidin, will immobilize and kill the cell. So, for live-cell imaging, transfection is preferable.

For immunostaining protocols, we have to make sure all liquids are distributed equally. Thus, all stainings are done on a rocker either at room temperature or, when staining is done overnight, in the cold room. After fixation with formaldehyde solution (or glutaraldehyde for enucleated cells), cells are washed with PBS and incubated with Triton X-100 for 10 minutes to permeabilize the membrane. To block molecules and enhance specific binding, cells are treated 30 minutes with 3\% BSA (bovine serum albumin) in PBS. After another washing step with Triton X-100 for 5 minutes, the primary antibody is introduced. After treatment, the antibody is either stored at $4^{\circ} \mathrm{C}$ or discarded. When other antibodies need to be applied, the washing steps are repeated. These can be antibodies directly linked to fluorescent probes or combinations of unlabeled specific antibodies and unspecific secondary antibodies with an added fluorophore. Furthermore, chemicals like Hoechst or intercalating agents like phalloidin can be used.

The cells can be mounted or stored in PBS in containers sealed with parafilm for several months. For mounting cells, samples are first rinsed three times with deionized water. Microscopy slides are then wiped with isopropanol and a drop of fluoroshield is added. Finally, the samples are dried gently with lint-free wipes and placed on the drop of fluoroshield with the cell side down.

All used antibodys can be found in Table D.13.

\subsection{Image analysis}

In this thesis, I use two software programs for image analysis. ImageJ is used for ground truth validation and nuclei analysis. FilamentSensor, a software mainly written by Benjamin Eltzner and Carina Wollnik and first published in 2015 ([62]), will be used for stress fiber analysis and estimation of basic cell descriptors like area, aspect ratio, and position.

During my thesis, I made major changes to this software with the help of working student Andreas Primeßnig. Here, only the use of the software will be described. A detailed explanation of FilamentSensor can be found in the eponymous Chapter 3 .

\subsubsection{ImageJ}

The FilamentSensor not only gives data on the fluorescently labeled stress fibers but also provides basic descriptors of the cell like area, aspect ratio, length of axes and position. To test the accuracy of the FilamentSensor, randomly selected pictures of different quality were analyzed using the ImageJ magic wand tool and FilamentSensor. For this, fluorescently labeled cells are first thresholded and the resulting binarized area then analyzed with the magic wand tool. This counts the connected pixels within, resulting in a calculation of the cell area, which the program then fits to an ellipse. The software can be used to obtain the minor and major axis of this imaginary ellipse fitted to the thresholded region by calculating the second order moment. The first eigenvalue corresponds to the long axis, while the second eigenvalue reflects the short axis of the chosen region. From this, the aspect ratio can be calculated as well.

For the analysis of nuclei, a thresholding step and the magic wand tool are used as well. Here, the circularity is also taken into account. 


\subsubsection{FilamentSensor}

For a detailed description of the FilamentSensor software and its further development, please see Chapter 3 .

The new version of the FilamentSensor software offers some possibilities for automating the analysis of large data sets. Most important for live-cell movies is the stack view, with which one can scan through a whole movie quickly. This is also important for testing to see the resulting traced fibers, which can help to change preprocessing steps quickly. The second important tool is batch-processing. With the filter queue saving successful filter sets, the sets can be stored for other data sets. Because for one experiment the movies for all positions have similar properties regarding brightness and bleaching, one filter set deemed applicable for one movie should be applicable for all movies of the same experiment. The batch processing tool will, with one given filter set, analyze all movies in a given root folder.

To find errors in the automated routines, a simple visualisation of the results is enough. Wherever the number of fibers or recognized area drops significantly, there are probably difficulties with illumination or fluorescent dead cells floating through the field of view. This movie can then be reanalyzed and background-thresholding and bleach correction applied. The FilamentSensor software offers the possibility to correct the full stack for bleaching effects as well as adjusting single pictures. The tools used will overwrite the log already written in batch-processing.

In general, reanalysis of pictures or recapitulation of analysis is easy, as all variables are automatically saved in log files and reloaded when one reopens the "project".

Aside from movie stacks, the FilamentSensor software can also be used for large batches of single images to illustrate "orientation fields" (filaments grouped by localization and angle), and to correlate stress fibers with focal adhesions (or any other fiber with any other point-like structure of interest-see Section 3.4 for more details).

All results of the analysis are given in three different formats. First, pictures with labeled fibers are produced. These can also be used as learning data sets for neuronal networks. Second, XML files are written, which are used when projects are reimported. Third, CSV files are produced in a serialized fashion for every image analyzed. As every file produced uses the same structure, they are easily imported into MatLab or Python for further analysis or for plain visualization.

\subsection{Results}

In this section, I present data on the differentiation of hMSCs in the first 24 hours after seeding. This data was collected by monitoring the stress fiber patterns in live cells, as well as measuring large batches of fixed cells. However, before showing results (see Section 5.6), I want to show some of the problems one faces while working with stem cells. These problems-when not taken into consideration-can obscure significant results. In order to illustrate these problems I mostly show plots of the area and aspect ratio, as those are two very clear and easy-to-obtain descriptors.

\subsubsection{Different lots of hMSCs}

As already described in 5.1, the behavior and morphology of stem cells vary based on the donor and, respectively, the lot number. In Figure 5.5 it can be seen how, for cells from four different lots, cell area and aspect ratio of the cells vary for two different substrates while following the same protocol.

Especially in the comparison of the area, one can see that for Lot 1 the area is similar for the cells under both conditions, with those grown on glass being slightly larger starting hour 9 . In Lot 2, cells on glass are bigger from the start with the discrepancy growing continuously. In the end, Lot 2 cells are 1,000 $\mu \mathrm{m}^{2}$ bigger on glass than on muscle-like $(10 / 11 \mathrm{kPa})$ substrate. In contrast, in Lot 3 the cells on glass are 
around 2,000 $\mu \mathrm{m}^{2}$ bigger continuously. In conclusion, these lots show three very distinct behaviours that cannot be grouped.
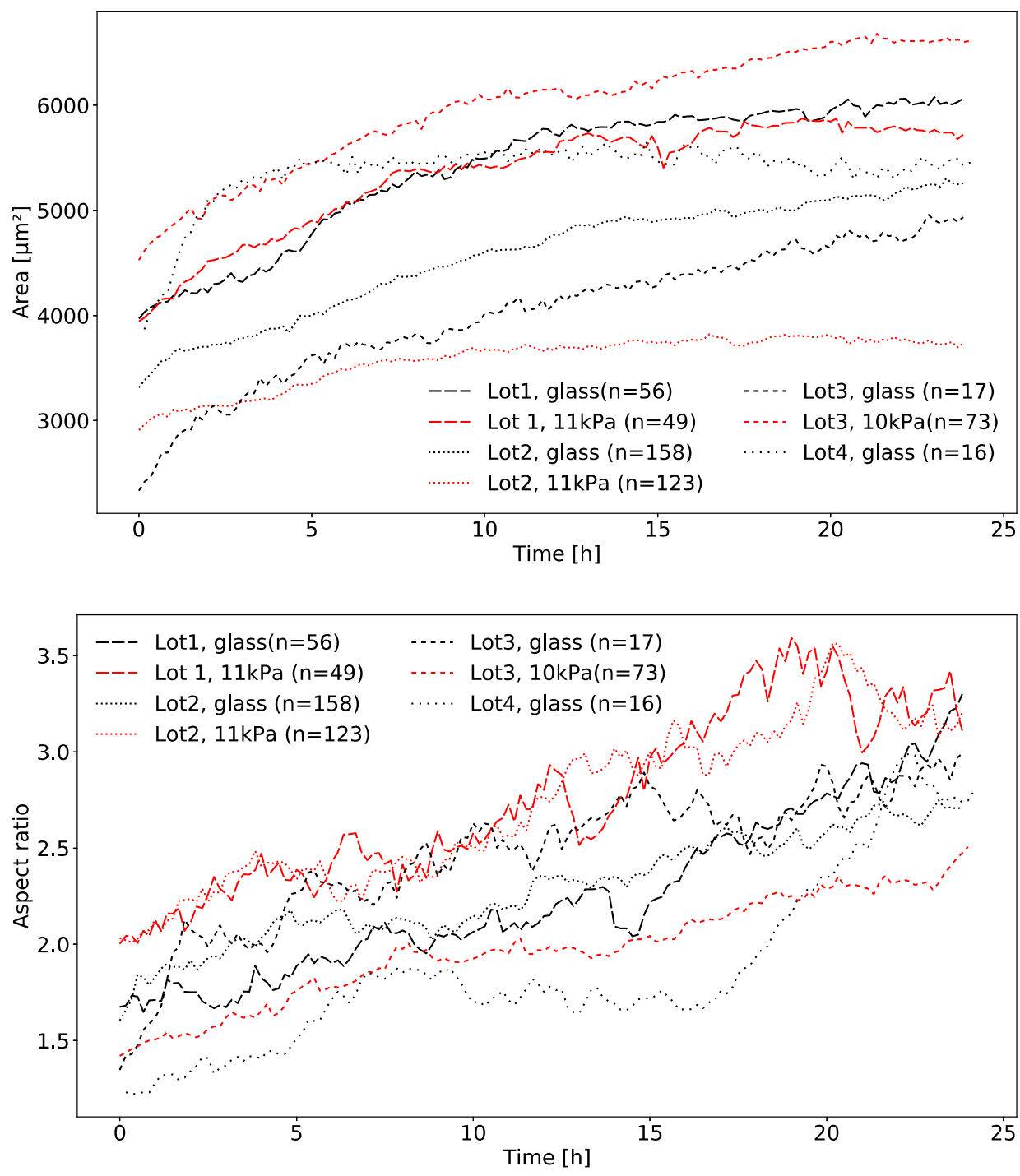

Figure 5.5.: Development of area and aspect ratio over the course of 24 hours of four different Lots of hMSC on $10 / 11 \mathrm{kPa}$ gels and glass. Top: Area. Bottom: Aspect ratio.

However, for the aspect ratio, which is a descriptor of relations in the cell, Lots 1 and 2 seem to follow the same pattern. Both lots have an aspect ratio 0.2-0.4 times higher on muscle-like stiffness than on glass, while for Lot 3 this is switched. For other indirect descriptors the differences between lots (i.e., orientation) is smaller as well (data not shown). From this, one can draw the conclusion that different lots of stem cells behave significantly differently. This might be more visible in direct cell measurements than in indirect descriptors. Thus, one has to think about the stem cells one is using and whether results are transferable. For experiments used in the MIET chapter (Chapter 6), abundance of focal adhesions was key. As all tested lots of hMSC (Lonza, PT-2501) were found to have a different mean number of focal adhesions per cell, the lot with the most focal adhesions was chosen. In that case, as testing the method is the crucial point, we did not worry about representativeness of the cells. For Subchapter 5.6, in which the evolution of stress fiber patterns in large sets of live cells is shown, availability of cells was key. So, instead of choosing a lot with a very distinct reaction to substrate stiffness, a lot with a less distinct reaction but 
plenty of vials in stock was used. Ironically, with the stiffness effect decreasing, necessary sample size for reliable results increases in turn.

\subsubsection{Sources of statistical variation in hMSC data}

Even when using just one lot of hMSCs, there is still some observable variation. There are multiple reasons for this. One is that there is a remaining fraction of fibroblasts in the vials purchased. Because the density and surface marker are mostly identical to the desired stem cells, there is no possibility to remove them. This leads to a subset in the data not undergoing differentiation but not distinguishable from the main set.
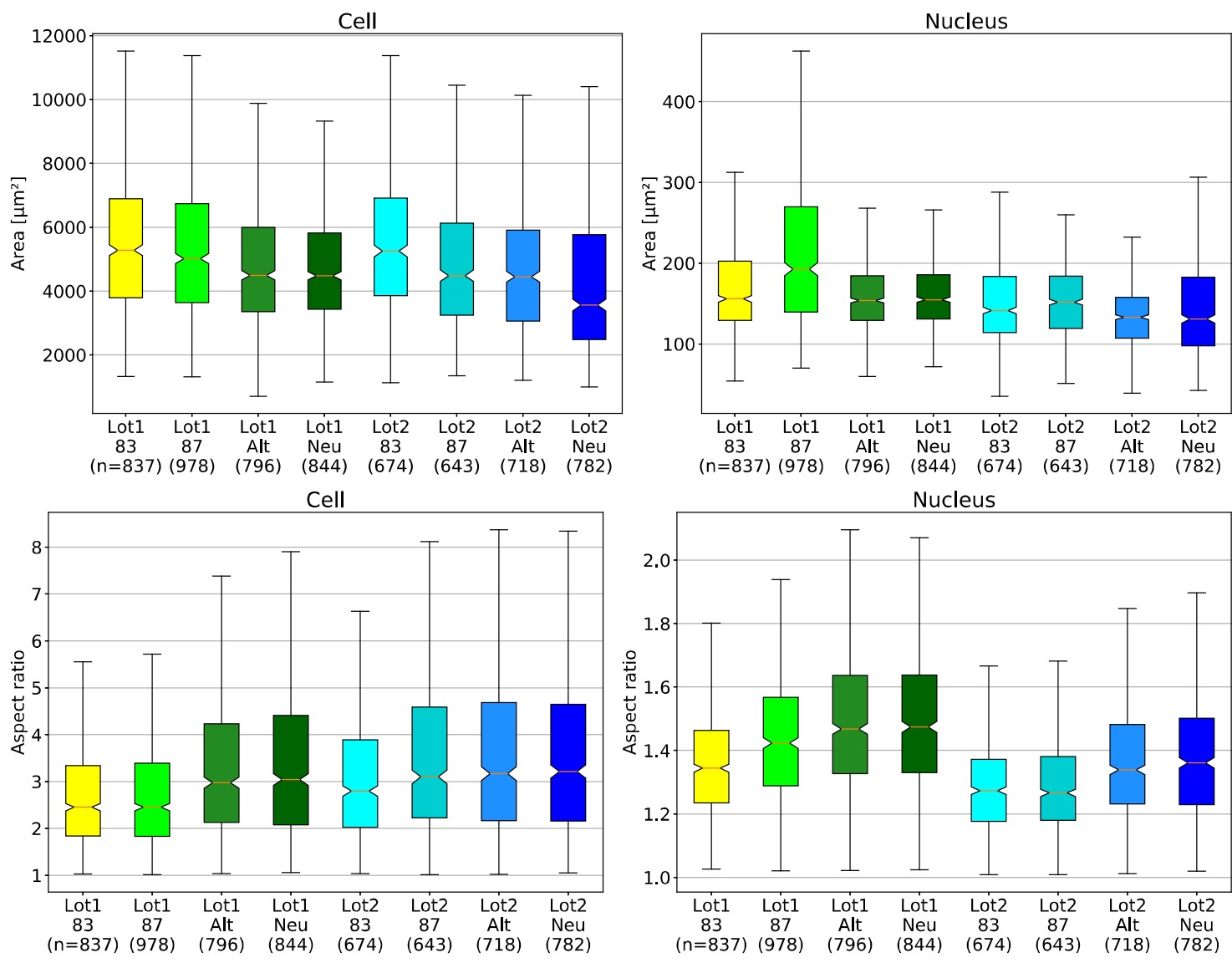

Figure 5.6.: Results for the effect of different batches of FBS on two Lots of hMSCs. Top: Measurements of area. Bottom: Measurements of the aspect ratio. Left: Cells stained with phalloidin. Right: Nuclei stained with Hoechst. All: Lot1=451491, Lonza PT-2501, Lot2=603525, Lonza PT-2501, 83=EU-FBS, Sigma, F0804, 87=EU-FBS, Sigma, F0804, Alt=USFBS, Sigma, F2442, Neu=US-FBS, Sigma, F2442.

Second is the effect of chemicals and media. The media composition used in this thesis for each cell type can be found in Table A.2. For hMSCs we used a combination of low glucose DMEM, Penicillin/Streptomycin $(\mathrm{P} / \mathrm{S})$, and fetal bovine serum (FBS). This solution was compared to Lonza's "MSCGM Mesenchymal Stem Cell Growth Medium BulletKit" (PT-3001) - recommended for hMSCs - but was found to have no downsides in the early passages (\#4-8) we used for differentiation experiments. Most labs will order media and supplements from the same supplier for years and have reserved batches of FBS, which has large batch-to-batch variation. If at some point the composition of the used media varies, this may have a visible effect in the data obtained. Because our FBS batch ran out, I had the opportunity to compare 
effects of different batches of Sigma FBS on my cells. Cells were grown in parallel following protocols mentioned in the Appendix (Section A) and treated the same, the only difference being the FBS used. For this I used two different batches (x-83 \& x-87) of \#F0408 European-grade FBS and two batches (Alt \& Neu) of \#F2442 US-grade FBS, each paired with two different lots of hMSC. In Figure 5.6 these variations can be seen both for the whole cell and for the nucleus alone. To measure the whole cell, a phalloidin staining was done; the nucleus was stained with Hoechst (see Appendix 5.3). Looking onto the area, European-grade FBS seems to lead to a slightly larger cell area, while there is no trend in the nuclear area. For the aspect ratio, European-grade FBS seems to result in more elongated cells as well as nuclei, the elongation of the nuclei being a side effect of the stretched cells' pull. These variations might not be statistically significant in themselves, but can add up with other factors and thus be considered.

\begin{tabular}{l|l|r|r|r|r} 
Lot & FBS & $\begin{array}{r}\text { Mean Area } \\
\text { Cell }\left[\mu \mathrm{m}^{2}\right]\end{array}$ & $\begin{array}{c}\text { Mean Area } \\
\text { Nucleus }\left[\mu \mathrm{m}^{2}\right]\end{array}$ & $\begin{array}{c}\text { Difference } \\
\text { Mean Area Cell }\end{array}$ & $\begin{array}{c}\text { Difference } \\
\text { Mean Area Nucleus }\end{array}$ \\
\hline Old & 83 & 5669.42 & 171.51 & $100.00 \%$ & $100.00 \%$ \\
Old & 87 & 5595.47 & 215.93 & $98.70 \%$ & $125.90 \%$ \\
Old & alt & 4961.44 & 160.36 & $87.51 \%$ & $93.50 \%$ \\
Old & neu & 4923.84 & 159.12 & $76.00 \%$ & $92.78 \%$ \\
New & 83 & 5811.62 & 149.12 & $102.50 \%$ & $86.95 \%$ \\
New & 87 & 5026.07 & 153.39 & $88.62 \%$ & $89.44 \%$ \\
New & alt & 4850.06 & 135.10 & $85.55 \%$ & $78.77 \%$ \\
New & neu & 4446.95 & 146.64 & $78.44 \%$ & $85.50 \%$ \\
\hline Lot & FBS & Mean AR & Mean AR & Difference & Difference \\
& & Cell & Nucleus & Mean AR Cell & Mean AR Nucleus \\
\hline Old & 83 & 2.78 & 1.36 & $100.00 \%$ & $100.00 \%$ \\
Old & 87 & 2.80 & 1.44 & $100.72 \%$ & $105.89 \%$ \\
Old & alt & 3.40 & 1.50 & $122.30 \%$ & $110.29 \%$ \\
Old & neu & 3.49 & 1.50 & $125.54 \%$ & $110.29 \%$ \\
New & 83 & 3.25 & 1.29 & $116.90 \%$ & $94.85 \%$ \\
New & 87 & 3.60 & 1.30 & $129.50 \%$ & $95.59 \%$ \\
New & alt & 3.63 & 1.37 & $130.58 \%$ & $100.74 \%$ \\
New & neu & 3.62 & 1.39 & $130.22 \%$ & $102.21 \%$
\end{tabular}

Table 5.3.: Differences of means of area and aspect ratio measurements of two lots of hMSCs grown in different FBS supplements and fixed and stained after 24 hours.

A well-documented effect of transfection with LifeAct is the change of cell morphology and behavior. This has already been discussed in Subsection 5.1.1.1. As this also has a significant effect on the differentiation of hMSCs-and thus on the data presented in Section 5.6-it will be discussed in a separate subsection (see Subsection 5.5.4).

In contrast, an almost unnoticeable effect on differentiation is caused by the cell's environment during experiments. Differences in $\mathrm{CO}_{2}$ levels, humidity, and temperature might affect proliferation and, to some extent, cell behavior. In Figure 5.7 cells are compared that have been either sitting in a cell culture incubator or imaged on an inverted microscope $\left(37^{\circ} \mathrm{C}, 5 \% \mathrm{CO}_{2}\right.$, humidifier) for 24 hours. 

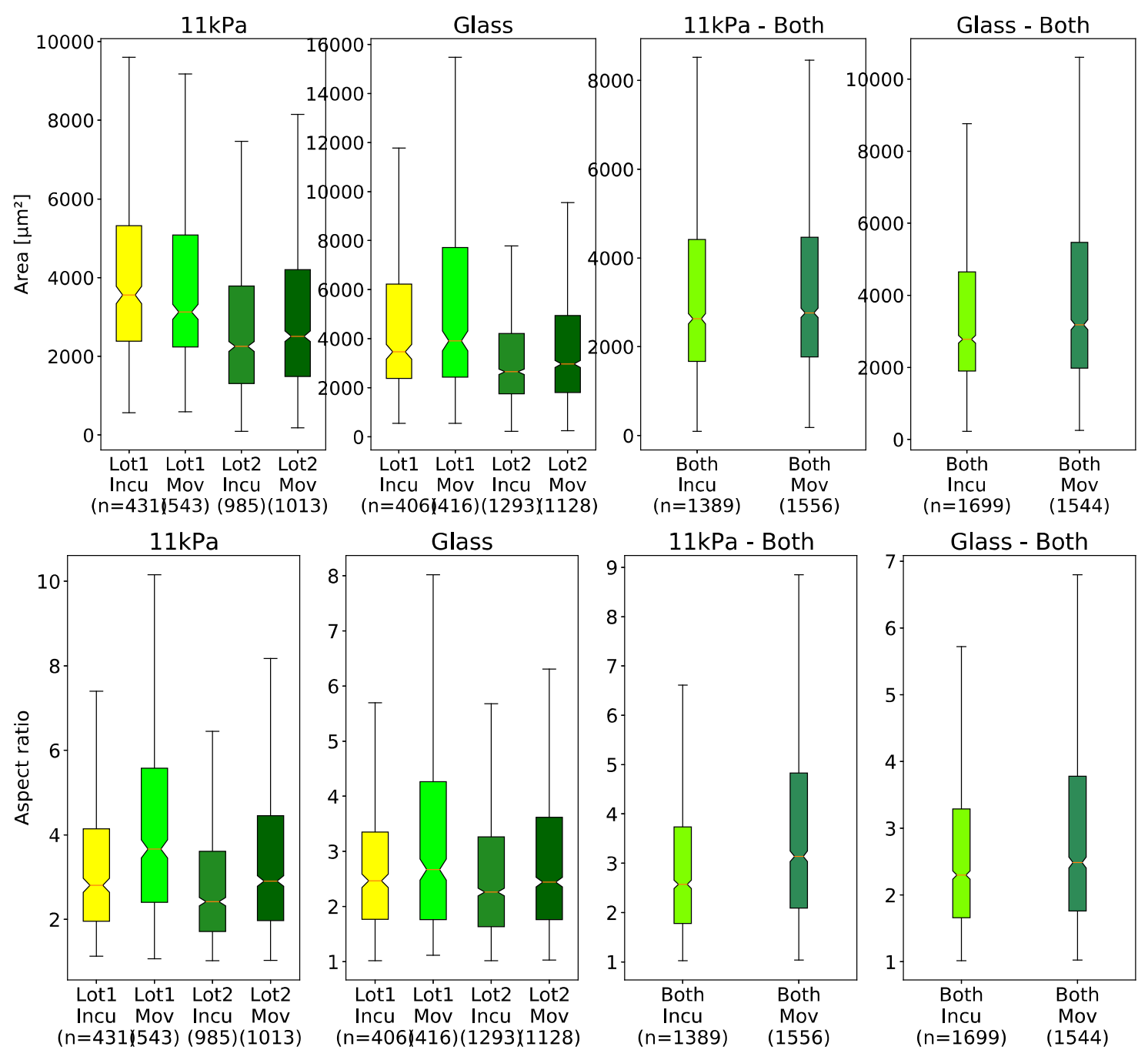

Figure 5.7.: Comparison of cell area and aspect ratio of hMSC cells treated exactly the same except for being either in the incubator or on a microscope $\left(37^{\circ} \mathrm{C}, 5 \% \mathrm{CO}_{2}\right.$, humidifier $)$ for 24 hours. Left: Area. Right: Aspect ratio. 


\begin{tabular}{l|l|l|r|r|r|r} 
Source & Substrate & Lot & $\begin{array}{r}\text { Mean Area } \\
\text { Cell }\left[\mu \mathrm{m}^{2}\right]\end{array}$ & Difference & $\begin{array}{r}\text { Mean AR } \\
\text { Cell }\end{array}$ & Difference \\
\hline Inc & 11 & 42 & 4577.93 & $100.00 \%$ & 3.43 & $100.00 \%$ \\
Mov & 11 & 42 & 4229.17 & $93.06 \%$ & 4.32 & $125.95 \%$ \\
Inc & 11 & 45 & 3062.20 & $66.89 \%$ & 3.02 & $88.05 \%$ \\
Mov & 11 & 45 & 3268.37 & $71.39 \%$ & 3.50 & $106.06 \%$ \\
Inc & Gl & 42 & 5487.95 & $119.88 \%$ & 2.89 & $84.26 \%$ \\
Mov & Gl & 42 & 5986.95 & $130.78 \%$ & 3.42 & $99.71 \%$ \\
Inc & Gl & 45 & 3501.24 & $76.48 \%$ & 2.68 & $78.14 \%$ \\
Mov & Gl & 45 & 3775.96 & $107.87 \%$ & 2.97 & $86.59 \%$ \\
\hline Inc & 11 & & 3532.52 & $100.00 \%$ & 3.15 & $100.00 \%$ \\
Mov & 11 & & 3603.66 & $102.01 \%$ & 3.79 & $94.01 \%$ \\
Inc & Gl & & 3976.00 & $112.55 \%$ & 2.73 & $75.04 \%$ \\
Mov & Gl & & 4371.67 & $123.75 \%$ & 3.09 & $98.10 \%$
\end{tabular}

Table 5.4.: Mean differences comparing transfected cells stained with LifeAct and immunostained cells stained with phalloidin. Results are given as difference of live cell movie (LifeAct) in relation to fixed cells (phalloidin).

Although both incubator and microscope samples have nominally the same physiological environment $\left(37^{\circ} \mathrm{C}, 5 \% \mathrm{CO}_{2}\right.$, humidifier $)$, there are visible differences in their large data sets ranging from a number of 406 to 1293 observed cells.

After getting to know the statistical effects arising during cell culture and experiments, there is also the analysis itself that can lead to errors. While in physics there is often a set of standard methods employed, for statistics of biological processes different methods have to be used in some areas. In Figure 5.8 one set of data from Figure 5.7 is shown as a fitted histogram. Here, a standard Gaussian fit is compared to a Weibull fit. The Weibull fit is often used for weather forecasts or in engineering; but in biology, it models best the minimal cell size given by nucleus size and sparse occurrence of large cells. Even at the first glance its mean is more reasonable than the Gaussian fit.

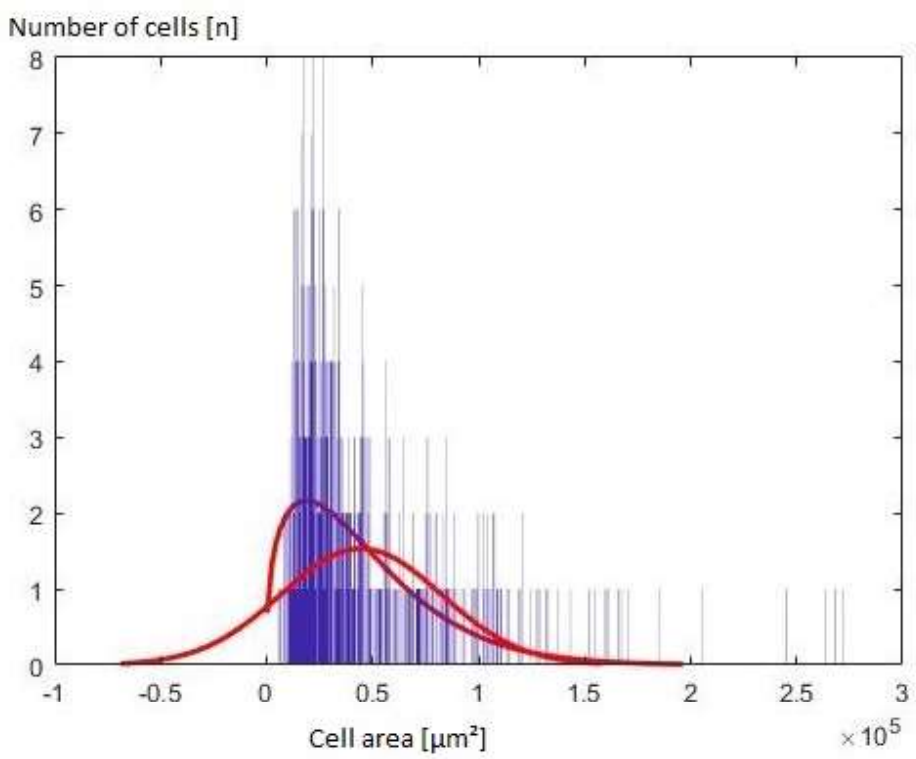

Figure 5.8.: One data set from Figure 5.6 fitted with both Gaussian and Weibull fit. 
When comparing these fits for different data sets the fitting issue gets more obvious. In Figure 5.9 fitted histograms of three data sets from Figure 5.7 are shown fitted with Gaussian and Weibull fits. While for the Weibull fit the means overlap, for the Gaussian fit they are shifted. Although this provides a clear example of how these fits diverge, errors made in statistical analysis can obscure experimental results or give false results when the cases are not so obvious.
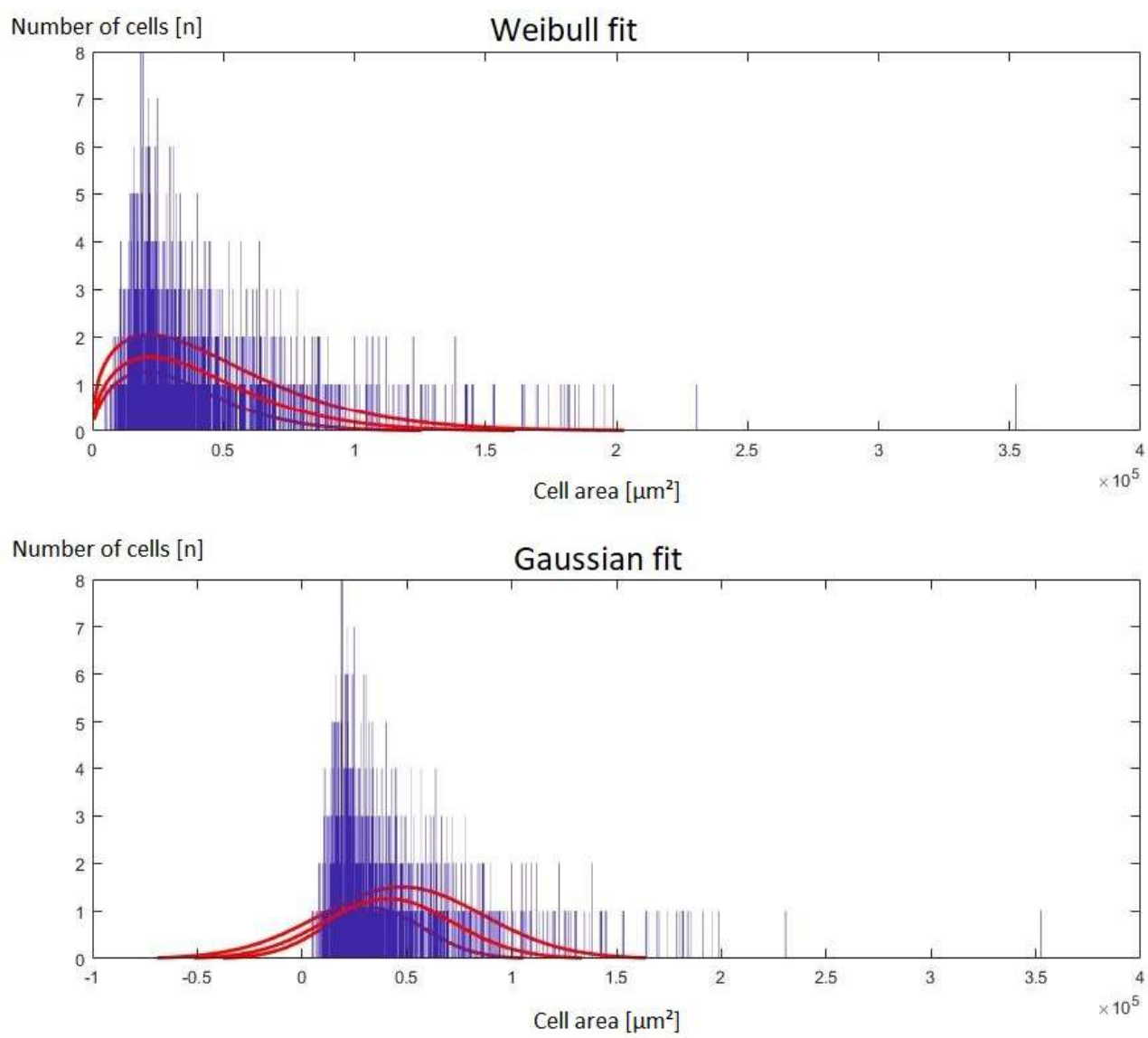

Figure 5.9.: Three different data sets from Figure 5.6 compared with different fits. Top: Weibull fit. Bottom: Gaussian fit. 


\subsubsection{Effect of stiffness on untransfected hMSC}

The effect of substrate stiffness on hMSCs has already been described in Subsection 2.1.3 and Section 4.5. Moreover, many papers have shown dependency of spreading area and spindle factor on substrate stiffness, includes some produced by our own lab (see Figure 5.10). However, in most cases the number of measured cells is either small or not mentioned.
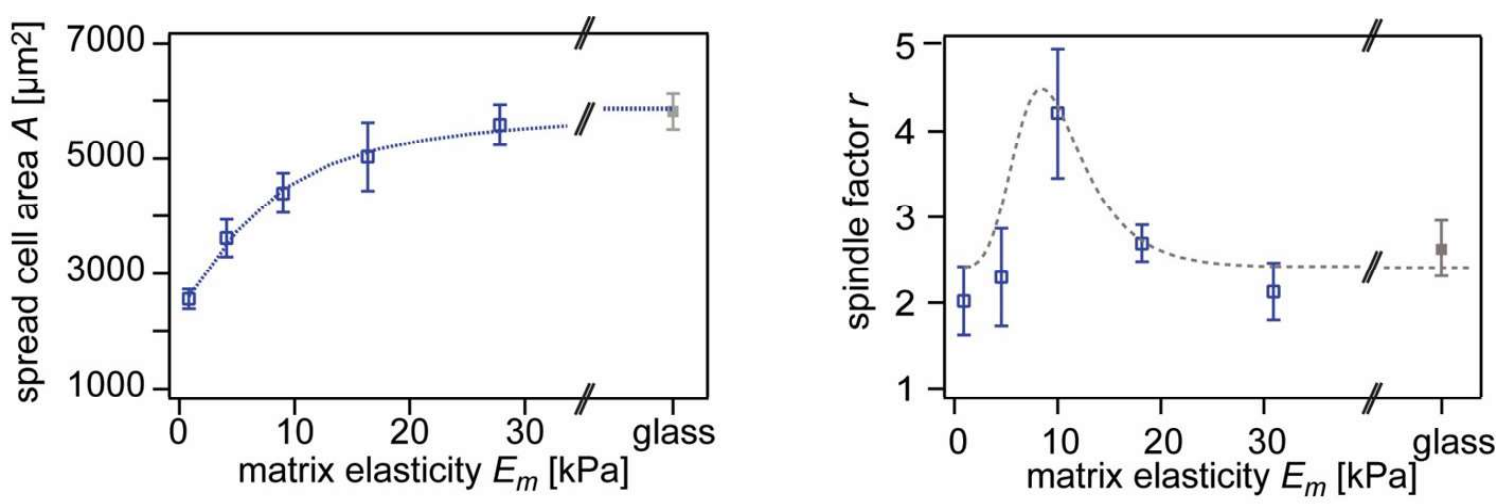

Figure 5.10.: Behavior of stem cells on PA-gels of different stiffness. Left: Spreading area increases with stiffness. Right: Spindle factor has a maximum at $11 \mathrm{kPa}$. Figure adapted from [169], number of cells not mentioned in paper.

This is why I decided to fabricate a unique, large, and thus statistically sound set of fixed, untransfected hMSCs on different stiffnesses. Furthermore, as we use LifeAct transfection (see Subsection A.4), I introduced a LifeAct protein with an RFP fluorophore into hMSC cells for live cell movies. These proteins bind reversibly to the fibres. While they do not stall the fibers movements per se, as intercalating phalloidin does, changes in morphology can be observed (see Subsection 5.1.1.1). Findings by Dupont et.al. [58] seem to hint at stalling effects exerted by the transient binding of the introduced LifeAct proteins accumulating to a strong enough distortion to alter stress fiber network and thus cell behavior. This large untransfected set might also serve to quantify the LifeAct effect in these movies (see Section 5.6).

Looking at the results in Figure 5.11 and comparing them to Figure 5.10 from [169], I want to first look at the area. While the range of area seems to be the same, basically ranging from 2,000 to $5,000 \mu \mathrm{m}^{2}$, the slope differs. While in [169] cell size doubles from $1 \mathrm{kPa}$ to $5 \mathrm{kPa}$ and again to $10 \mathrm{kPa}$, before slowly reaching a plateau from $20 \mathrm{kPa}$ onward to glass (the stiffest substrate), I found my cells' area to only slowly grow and double from $1 \mathrm{kPa}$ to $32 \mathrm{kPa}$. Further, cells grown on glass were found to be significantly larger than $32 \mathrm{kPa}$. The size of the nuclei, spreading of which is a result of the spreading and tension of the cell itself, increases in the same pattern. The spindle factor (or aspect ratio) in [169] shows a clear maximum of 4.1 around $10 \mathrm{kPa}$, with $20 \mathrm{kPa}$ also slightly higher compared to other stiffnesses. All other measurements, soft as well as stiff $(1 \mathrm{kPa}, 5 \mathrm{kPa}, 30 \mathrm{kPa}$, glass) revealed an aspect ratio lying around 2 . For my dat set, the very soft and stiff conditions $(1 \mathrm{kPa}, 2 \mathrm{kPa}, 5 \mathrm{kPa}$, glass) have an aspect ratio of around 1.5 , while $11 \mathrm{kPa}$ and $32 \mathrm{kPa}$ are higher-around 2.5 - and $20 \mathrm{kPa}$ produces the maximum at around 2.9 . For the nuclei, the aspect ratio is around 1.4 for all conditions, with $20 \mathrm{kPa}$ only slightly larger. In conclusion, the general findings are the same, with the main difference being where the cells' reaction on the substrate has its maximum. This aligns well with other studies on substrate-cell interaction on fixed hMSCs (e.g., in $[67,152,225])$. 

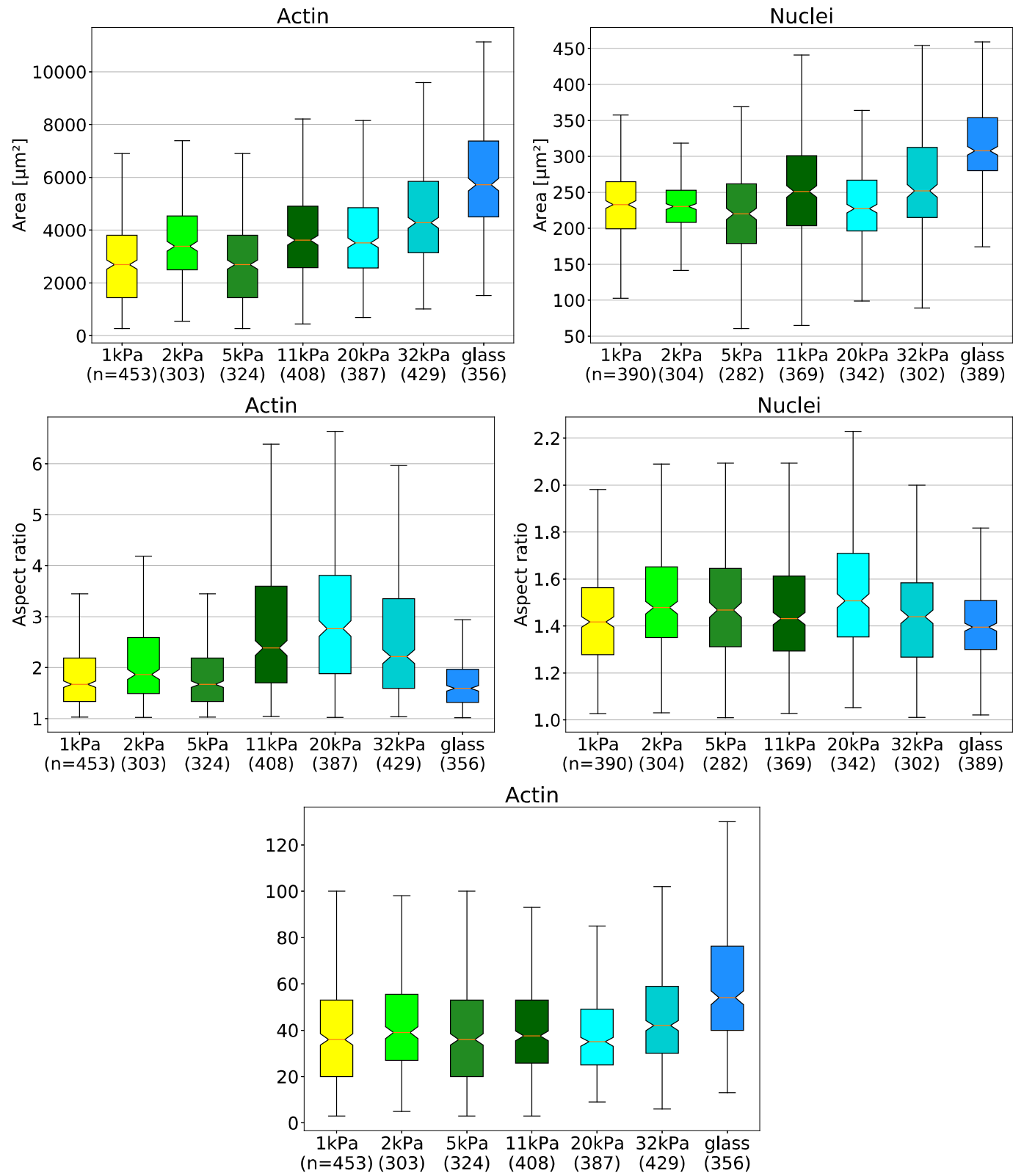

Figure 5.11.: Measurements of untransfected hMSCs seeded on substrates of different stiffness for 24 hours, fixed and stained with protocols from Appendix 5.3. Number of imaged cells indicated under the respective boxes. Top: Area. Middle: Aspect ratio. Bottom: Filament number. Left: Whole cells, stained with phalloidin. Right: Nuclei, stained with Hoechst. 


\begin{tabular}{|c|c|c|c|c|}
\hline Stiffness & $\begin{array}{c}\text { Mean Area } \\
\text { Cell }\left[\mu \mathrm{m}^{2}\right]\end{array}$ & $\begin{array}{c}\text { Mean Area } \\
\text { Nucleus }\left[\mu \mathrm{m}^{2}\right]\end{array}$ & $\begin{array}{c}\text { Difference } \\
\text { Mean Area Cell }\end{array}$ & $\begin{array}{c}\text { Difference } \\
\text { Mean Area Nucleus }\end{array}$ \\
\hline $1 \mathrm{kPa}$ & 2992.19 & 235.52 & $100.00 \%$ & $100.00 \%$ \\
\hline $2 \mathrm{kPa}$ & 3704.31 & 228.85 & $129.17 \%$ & $97.17 \%$ \\
\hline $5 \mathrm{kPa}$ & 2992.19 & 220.28 & $100.00 \%$ & $93.53 \%$ \\
\hline $11 \mathrm{kPa}$ & 4033.37 & 255.25 & $134.80 \%$ & $108.38 \%$ \\
\hline $20 \mathrm{kPa}$ & 3988.29 & 233.84 & $179.67 \%$ & $99.29 \%$ \\
\hline $32 \mathrm{kPa}$ & 4846.59 & 279.84 & $161.97 \%$ & $118.82 \%$ \\
\hline Glass & 6550.07 & 327.03 & $218.90 \%$ & $138.85 \%$ \\
\hline Stiffness & $\begin{array}{c}\text { Mean AR } \\
\text { Cell }\end{array}$ & $\begin{array}{c}\text { Mean AR } \\
\text { Nucleus }\end{array}$ & $\begin{array}{c}\text { Difference } \\
\text { Mean AR Cell }\end{array}$ & $\begin{array}{c}\text { Difference } \\
\text { Mean AR Nucleus }\end{array}$ \\
\hline $1 \mathrm{kPa}$ & 1.92 & 1.45 & $100.00 \%$ & $100.00 \%$ \\
\hline $2 \mathrm{kPa}$ & 2.20 & 1.51 & $114.58 \%$ & $104.14 \%$ \\
\hline $5 \mathrm{kPa}$ & 1.92 & 1.50 & $100.00 \%$ & $103.45 \%$ \\
\hline $11 \mathrm{kPa}$ & 2.88 & 1.48 & $150.00 \%$ & $102.07 \%$ \\
\hline $20 \mathrm{kPa}$ & 3.15 & 1.54 & $161.46 \%$ & $106.20 \%$ \\
\hline $32 \mathrm{kPa}$ & 2.79 & 1.45 & $145.31 \%$ & $100.00 \%$ \\
\hline Glass & 1.73 & 1.42 & $90.10 \%$ & $97.93 \%$ \\
\hline Stiffness & $\begin{array}{c}\text { Mean } \\
\text { Filament number }\end{array}$ & & $\begin{array}{c}\text { Difference } \\
\text { Filament number }\end{array}$ & \\
\hline $1 \mathrm{kPa}$ & 40.58 & & $100.00 \%$ & \\
\hline $2 \mathrm{kPa}$ & 43.41 & & $106.97 \%$ & \\
\hline $5 \mathrm{kPa}$ & 40.58 & & $100.00 \%$ & \\
\hline $11 \mathrm{kPa}$ & 43.25 & & $106.98 \%$ & \\
\hline $20 \mathrm{kPa}$ & 41.65 & & $102.64 \%$ & \\
\hline $32 \mathrm{kPa}$ & 49.97 & & $123.14 \%$ & \\
\hline Glass & 65.21 & & $160.69 \%$ & \\
\hline
\end{tabular}

Table 5.5.: Differences of means of area, aspect ratio, and filament number measurements of hMSC grown on PAA gels of different stiffnesses and fixed and stained after 24 hours.

Additionally, I counted the number of fibers in those fixed cells. In theory, on brain-like substrates hMSCs should develop a multitude of short, unordered fibers. On muscle-like substrates hMSCs should form elongated cells with few, long fibers. On bone-like substrates and glass there should be a multitude of fibers of all lengths, again unordered. In the bottom of Figure 5.11 it can be seen that the filament number for stiffnesses ranging 1 to $11 \mathrm{kPa}$ is fairly constant, then drops slightly at $20 \mathrm{kPa}$ consistent with aspect ratio maximum, before rising for $32 \mathrm{kPa}$ and even more for glass. So, the data measured seem to conform to theory with the exception of $20 \mathrm{kPa}$ showing the strongest myogenic differentiation, and measurements overall diverge less.

\subsubsection{Comparing live cell movies to existing results of different experimental origin}

One concern when doing analysis with live cell movies over long periods of time is how to compare these results with existing findings, which are most of the time done on fixed cells or living cells imaged only on specific time points. The reason this provides a difficulty is that the sets of cells imaged are not the same. While some requirements for the cells apply in all conditions (e.g., nondividing, single, not polynucleated), requirements are more restrictive for live cell movies, as the cells should not divide or interact with other cells over the whole span of a movie. This leads on the one hand to an effect in which most of the cells imaged (and not dividing during the 24-hour-long movie) seem to have divided shortly before the start of 
the movie, and so grow and prepare for the next division during it. On the other hand, these cells seem to behave uniformly, as they are all at about the same point in the cell cycle. When imaging on a single time point, each cell's history is unknown; they are at different stages of the cell cycle and results are more divergent.
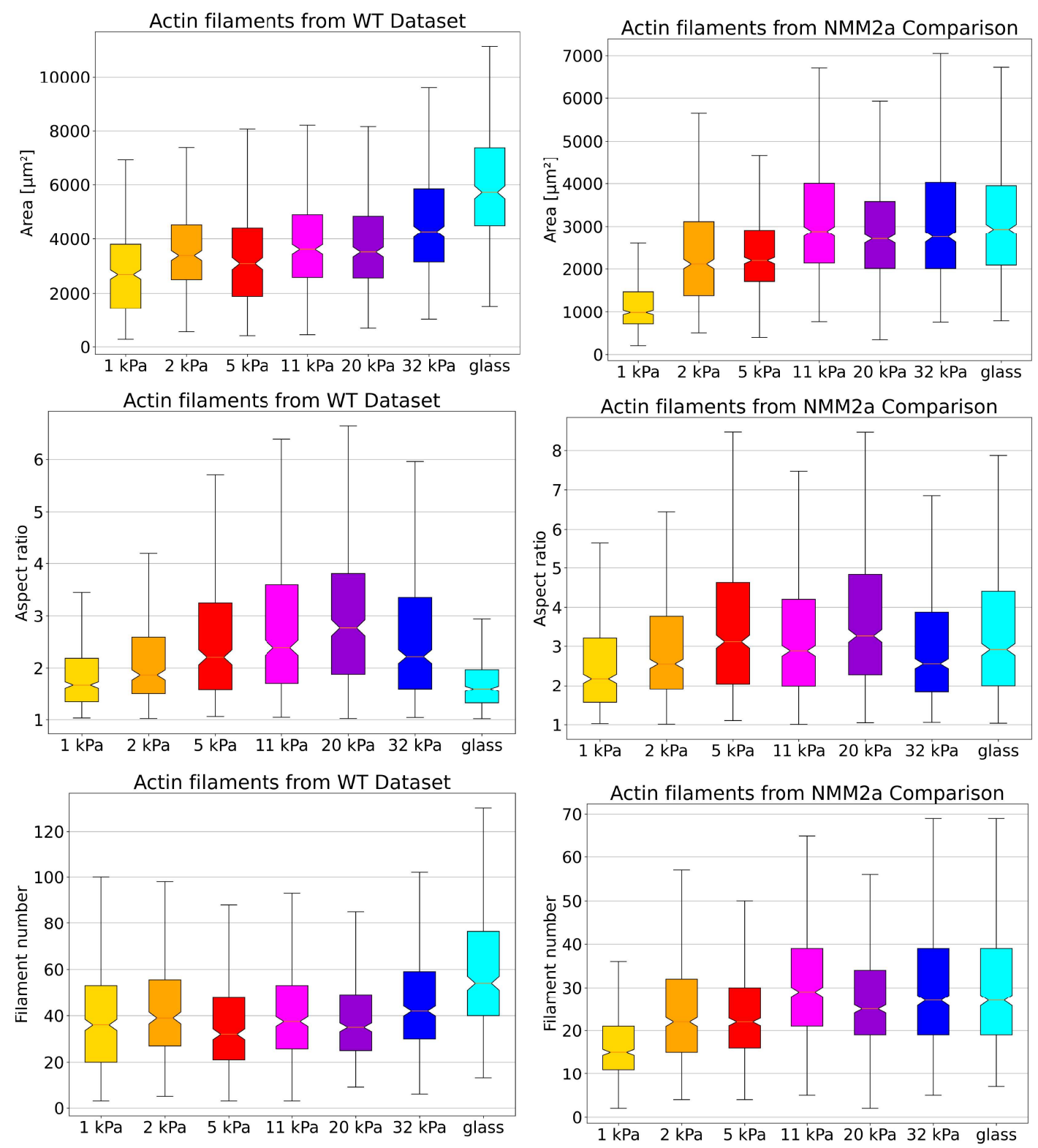

Figure 5.12.: All: Two different experiments with hMSCs seeded on substrates of different stiffness. All cells untransfected, fixed after 24 hours and stained. Cells imaged when single, nondividing, mono-nucleated. Top: Area. Middle: Aspect ratio. Bottom: Filament number.

So, the basic question is: how much influence has this subsetting in live cell movies? Can we directly compare data obtained from movies with fixed cell data or data obtained from a specific time point? 


\begin{tabular}{|c|c|c|c|}
\hline Stiffness & $\begin{array}{c}\text { Mean Area }\left[\mu \mathrm{m}^{2}\right] \\
\text { WT Data }\end{array}$ & $\begin{array}{c}\text { Mean Area }\left[\mu \mathrm{m}^{2}\right] \\
\text { NMM2a Data }\end{array}$ & $\begin{array}{c}\text { Difference } \\
\text { Between Sets }\end{array}$ \\
\hline $1 \mathrm{kPa}$ & 2992.19 & 1192.75 & $250.86 \%$ \\
\hline $2 \mathrm{kPa}$ & 3704.31 & 2569.32 & $144.17 \%$ \\
\hline $5 \mathrm{kPa}$ & 3332.41 & 2453.33 & $114.04 \%$ \\
\hline $11 \mathrm{kPa}$ & 4033.37 & 3299.83 & $135.83 \%$ \\
\hline $20 \mathrm{kPa}$ & 3988.29 & 3006.00 & $132.68 \%$ \\
\hline $32 \mathrm{kPa}$ & 4846.59 & 3467.73 & $139.76 \%$ \\
\hline Glass & 6550.07 & 3458.48 & $189.39 \%$ \\
\hline Stiffness & $\begin{array}{l}\text { Mean AR } \\
\text { WT Data }\end{array}$ & $\begin{array}{c}\text { Mean AR } \\
\text { NMM2a Data }\end{array}$ & $\begin{array}{c}\text { Difference } \\
\text { Between Sets }\end{array}$ \\
\hline $1 \mathrm{kPa}$ & 1.92 & 3.41 & $56.30 \%$ \\
\hline $2 \mathrm{kPa}$ & 2.70 & 3.15 & $85.71 \%$ \\
\hline $5 \mathrm{kPa}$ & 2.20 & 3.85 & $57.14 \%$ \\
\hline $11 \mathrm{kPa}$ & 3.14 & 2.79 & $112.54 \%$ \\
\hline $20 \mathrm{kPa}$ & 2.73 & 3.21 & $85.05 \%$ \\
\hline $32 \mathrm{kPa}$ & 3.69 & 1.73 & $213.29 \%$ \\
\hline Glass & 2.88 & 3.50 & $82.29 \%$ \\
\hline Stiffness & $\begin{array}{c}\text { Mean Filaments } \\
\text { WT Data }\end{array}$ & $\begin{array}{c}\text { Mean Filaments } \\
\text { NMM2a Data }\end{array}$ & $\begin{array}{c}\text { Difference } \\
\text { Between Sets }\end{array}$ \\
\hline $1 \mathrm{kPa}$ & 40.58 & 32.17 & $126.14 \%$ \\
\hline $2 \mathrm{kPa}$ & 16.68 & 41.65 & $40.04 \%$ \\
\hline $5 \mathrm{kPa}$ & 43.41 & 28.04 & $154.84 \%$ \\
\hline $11 \mathrm{kPa}$ & 27.01 & 49.97 & $54.05 \%$ \\
\hline $20 \mathrm{kPa}$ & 37.62 & 33.27 & $113.07 \%$ \\
\hline $32 \mathrm{kPa}$ & 24.37 & 65.21 & $37.37 \%$ \\
\hline Glass & 43.25 & 32.26 & $134.07 \%$ \\
\hline
\end{tabular}

Table 5.6.: Comparison of mean measurements of two data sets of hMSCs fixed and stained after 24 hours. Means given for area, aspect ratio, and filament number of cells on different stiffness and percentage of difference in relation to the WT Data set.

Figure 5.12 shows the two large transfected data sets (one already shown in Subchapter 5.5.3). While some of the measurements, especially for glass, differ, one would say on the first glance that the trends are similar.

When directly compared, however, the differences in both absolute measurement and standard deviation become obvious. In Figure 5.13 the same data is plotted for direct comparison, showing that for the "WT Data set" area is higher (SD higher), aspect ratio lower (SD lower), and filament number higher (SD higher). We can compare the data from the substrates of $11 \mathrm{kPa}$ PAA gel and glass to the live cell movies, as data from live cell experiments, as well as from fixed and stained cells, were gathered at these stiffnesses to check for the presence of other effects. Thus, we see that the measurements obtained from transfected cells are in the same range, and have similar standard deviations, as the fixed data sets. Additionally, variation between the large fixed data sets-WT data (orange) and NMM2a data (cyan) - is larger than the variation from the transfected cells. Hence, we should be able to directly compare results obtained from fixed cells with those of live cell movies. 

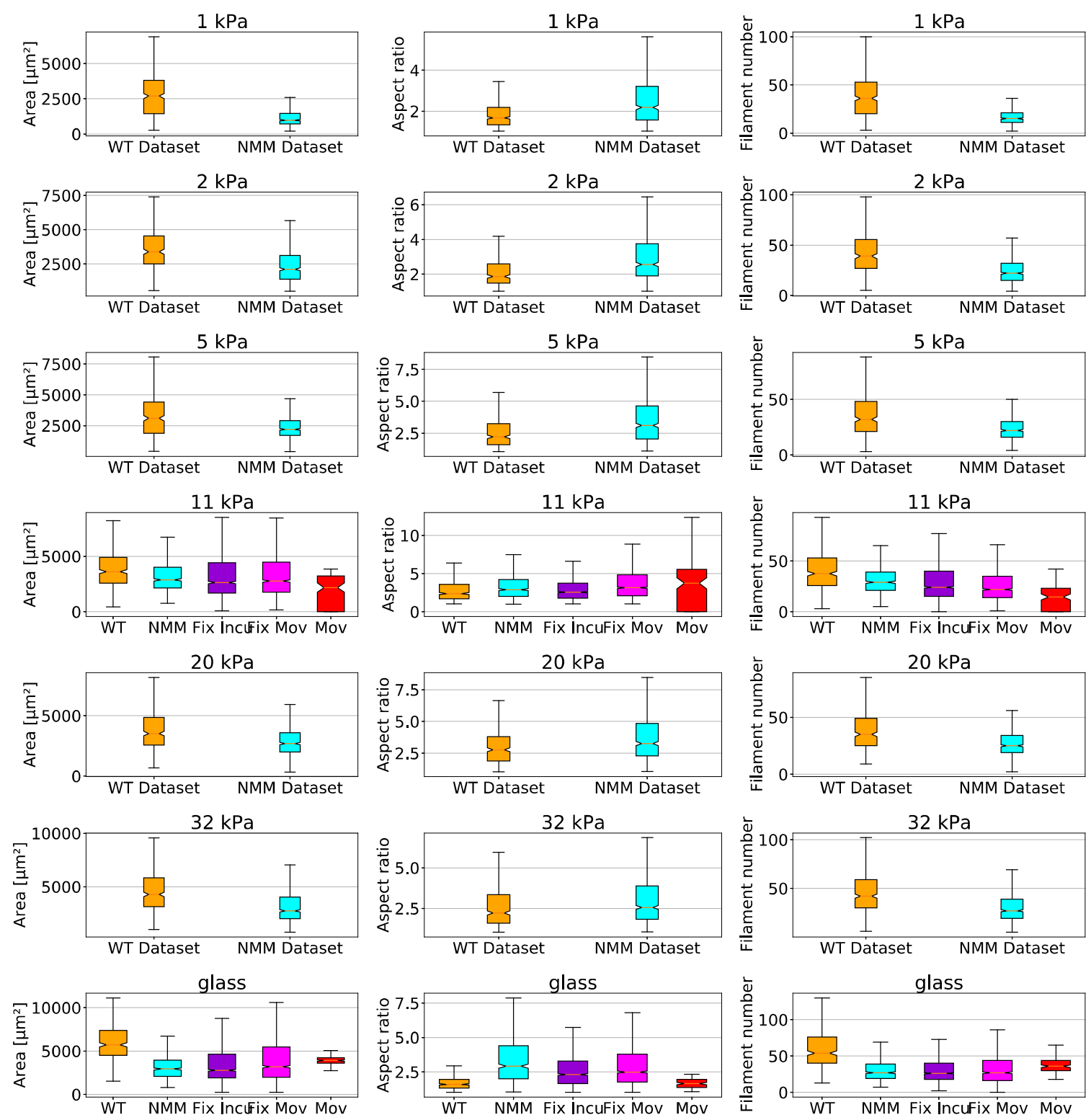

Figure 5.13.: All: Comparison of 5 different sets of data from different experiments for 7 stiffness. WT Data set (orange) and NMM2a Data set (cyan) single experiments from one passage, fixed and stained. Fixed cells done from samples prepared for live cell movies ('Fix Incu' (purple), 'Fix Mov' (pink)) from different passages, fixed and stained, over long period of time. Movie data ('Mov'(red)) obtained from transfected cells, last frame of usable live cell movies, from different passages over long period of time. Left: Area. Middle: Aspect ratio.

Right: Filament number. 


\begin{tabular}{l|r|r|r|r} 
Data set & $\begin{array}{r}\text { Mean Area } \\
\mathbf{1 1} \mathbf{~ k P a}\left[\mu \mathrm{m}^{2}\right]\end{array}$ & $\begin{array}{r}\text { Mean Area } \\
\text { glass }\left[\mu \mathrm{m}^{2}\right]\end{array}$ & $\begin{array}{c}\text { Difference } \\
\mathbf{1 1} \mathbf{~ k P a}\end{array}$ & $\begin{array}{c}\text { Difference } \\
\text { glass }\end{array}$ \\
\hline WT Data & 4033.37 & 6550.07 & $100.00 \%$ & $100.00 \%$ \\
NMM2a & 3299.83 & 3458.48 & $81.81 \%$ & $52.80 \%$ \\
Incubator & 3532.52 & 3976.00 & $87.58 \%$ & $60.70 \%$ \\
Microscope & 3603.66 & 4371.66 & $89.35 \%$ & $66,74 \%$ \\
Movie & 4072.24 & 4779.09 & $100.96 \%$ & $72.96 \%$ \\
\hline Data set & Mean AR & Mean AR & Difference & Difference \\
& $\mathbf{1 1 ~ k P a}$ & glass & $\mathbf{1 1} \mathbf{k P a}$ & glass \\
\hline WT Data & 2.88 & 1.73 & $100.00 \%$ & $100.00 \%$ \\
NMM2a & 3.41 & 3.50 & $118.40 \%$ & $202.31 \%$ \\
Incubator & 3.15 & 2.73 & $109.38 \%$ & $157.80 \%$ \\
Microscope & 3.79 & 3.09 & $131.60 \%$ & $178.61 \%$ \\
Movie & 2.73 & 2.27 & $94.79 \%$ & $131.21 \%$ \\
\hline Data set & Mean Filament & Mean Filament & Difference & Difference \\
& number $\mathbf{1 1} \mathbf{k P a}$ & number glass & $\mathbf{1 1} \mathbf{k P a}$ & glass \\
\hline WT Data & 43.25 & 65.21 & $100.00 \%$ & $100.00 \%$ \\
NMM2a & 32.17 & 32.26 & $74.38 \%$ & $49.47 \%$ \\
Incubator & 30.96 & 33.87 & $71.58 \%$ & $51.94 \%$ \\
Microscope & 28.60 & 34.74 & $66.13 \%$ & $53.27 \%$ \\
Movie & 41.72 & 44.68 & $96.46 \%$ & $68.52 \%$
\end{tabular}

Table 5.7.: Comparison of 5 different sets of data from different experiments on $11 \mathrm{kPa}$ PAA gel and glass. WT data set and NMM2a data set single experiments from one passage, fixed and stained. Fixed cells done from samples prepared for live cell movies ('Incubator', 'Microscope') from different passages, fixed and stained, over a long period of time. Movie data ('Movie') obtained from transfected cells, last frame of usable live cell movies, from different passages over a long period of time. 


\subsubsection{Comparison of cells transfected (LifeAct) and immunostained (phal- loidin)}

Another issue is the discrepancy in staining when comparing transfected and immunostained cells. While immunostaining labels all available matching structures in the cell, fluorescent proteins introduced into the cell via transfection might be distributed unevenly (see also Subsection 5.1.1.1). To evaluate the effect of the staining differences, cells were transfected with LifeAct, fixed, stained with phalloidin, and imaged for both.
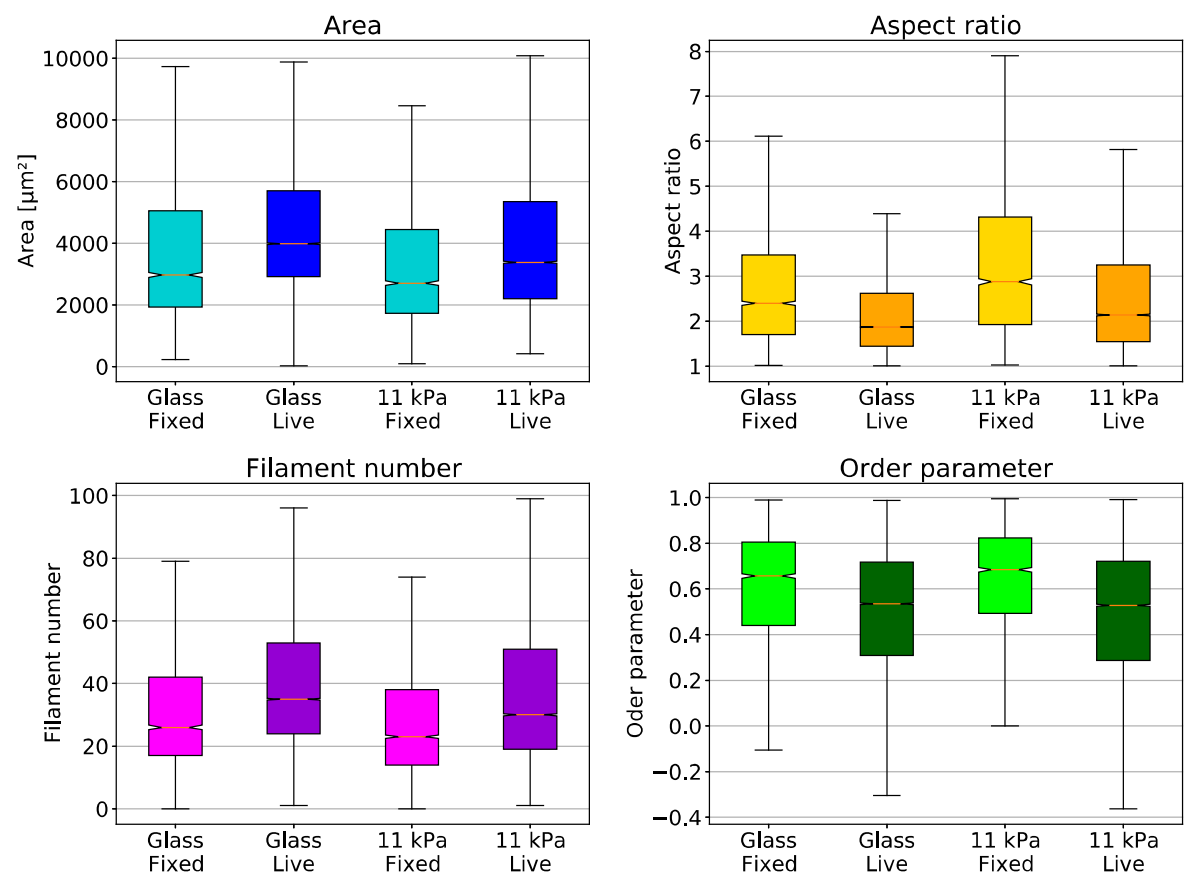

Figure 5.14.: All: LifeAct-transfected hMSC grown on $11 \mathrm{kPa}$ PAA substrates and glass, both coated with collagen, for 24 hours, fixed and stained with phalloidin, according to Appendix (Section D). Top left: Area. Top right: Aspect ratio. Bottom left: Filament number. Bottom right Order parameter.

The results show that in live cells more, but less ordered, filaments are detected while also registering a larger, but less elongated, area. Table 5.8 states difference of live cell movie mean results in comparison to fixed cells.

\begin{tabular}{l|r|r|r|r|r|r} 
& $\begin{array}{r}\text { glass } \\
\text { fixed }\end{array}$ & $\begin{array}{c}\text { glass } \\
\text { live }\end{array}$ & $\begin{array}{c}\text { Difference } \\
\text { glass }\end{array}$ & $\begin{array}{c}\text { 11kPa } \\
\text { fixed }\end{array}$ & $\begin{array}{c}\text { 11kPa } \\
\text { live }\end{array}$ & $\begin{array}{c}\text { Difference } \\
\mathbf{1 1} \mathbf{~ k P a}\end{array}$ \\
\hline Area $\left[\mu \mathrm{m}^{2}\right]$ & 4164 & 4779 & $114.77 \%$ & 3570 & 4072 & $114.06 \%$ \\
Aspect ratio & 2.90 & 2.27 & $78.28 \%$ & 3.48 & 2.73 & $78.45 \%$ \\
Filament number & 34.28 & 44.68 & $130.34 \%$ & 30.06 & 41.72 & $138.79 \%$ \\
Order parameter & 0.59 & 0.49 & $83.05 \%$ & 0.63 & 0.49 & $77.78 \%$
\end{tabular}

Table 5.8.: Mean differences comparing transfected cells stained with LifeAct and immunostained cells stained with phalloidin. Results are given as difference of LifeAct in relation to phalloidin. 


\subsubsection{Comparison of phalloidin and NMM2a staining for stress fibers}

In some papers, instead of using a fluorescent actin marker, NMM2a is labelled. As this would be a way to avoid LifeAct effects (see Subsection 5.1.1.1), I stained a large set of untransfected fixed hMSCs with phalloidin, anti-NMM2a, and Hoechst (see Appendix Section D) to compare for fiber recognition. Because fiber network dynamics are the prime interest, a switch to NMM2a labelling is only feasible with clear association of fluorophores to fibers, hence offering a good base for downstream analysis.
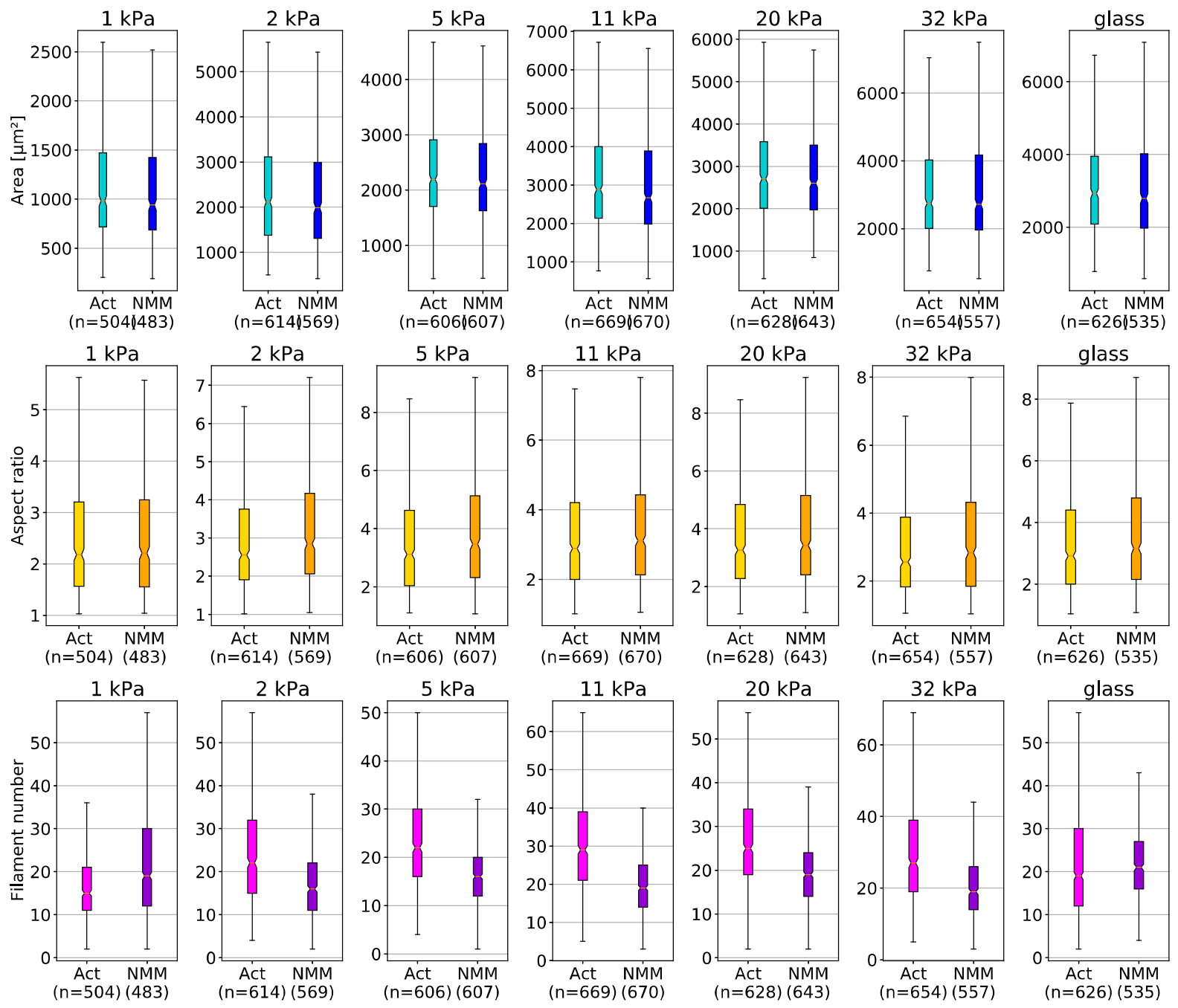

Figure 5.15.: All: Untransfected hMSC grown on substrates of different stiffness for 24 hours, fixed and stained with phalloidin, anti-NMM2a, and Hoechst according to Appendix (Section D). Phalloidin (Actin) and NMM2a (NMM) shown next to each other, seperated for substrate stiffness. Top: Area. Middle: Aspect ratio. Bottom: Filament number.

And while, as can be seen in Figure 5.15, the area detection seems to be similar using direct actin staining compared to NMM2a staining, in the aspect ratio comparison the NMM staining shows slightly higher means consistently. Looking to the filament number comparison, we see, except for cells on $1 \mathrm{kPa}$ and glass, significantly lower counts for NMM2a staining. In elongated cells, the stress fibers running along the long axis of the fiber are very persistent and thicker than stress fibers running in other directions. Thus, they also attract more fluorophores. In NMM2a-stained elongated cells, the thinner stress fibers seem not to attract enough fluorophores to pass thresholding, leading to fewer detected fibers and a higher aspect 
ratio.

\begin{tabular}{l|r|r|r} 
Stiffness & $\begin{array}{r}\text { Mean Area } \\
\text { Actin }\left[\mu \mathrm{m}^{2}\right]\end{array}$ & $\begin{array}{r}\text { Mean Area } \\
\text { NMM2a }\left[\mu \mathrm{m}^{2}\right]\end{array}$ & Difference \\
\hline $1 \mathrm{kPa}$ & 1192.75 & 1137.16 & $95.34 \%$ \\
$2 \mathrm{kPa}$ & 2569.32 & 2447.71 & $95.27 \%$ \\
$5 \mathrm{kPa}$ & 2453.33 & 2380.63 & $97.04 \%$ \\
$11 \mathrm{kPa}$ & 3299.83 & 3170.49 & $96.08 \%$ \\
$20 \mathrm{kPa}$ & 3006.00 & 2980.35 & $99.15 \%$ \\
$32 \mathrm{kPa}$ & 3467.73 & 3504.61 & $101.06 \%$ \\
$\mathrm{Glass}$ & 3458.48 & 3542.63 & $102.43 \%$ \\
\hline Stiffness & Mean AR & Mean AR & Difference \\
\hline $1 \mathrm{kPa}$ & Actin & 3.74 & $102.62 \%$ \\
$2 \mathrm{kPa}$ & 3.67 & 3.41 & $108.60 \%$ \\
$5 \mathrm{kPa}$ & 3.69 & 3.10 & $111.11 \%$ \\
$11 \mathrm{kPa}$ & 3.41 & 4.00 & $106.45 \%$ \\
$20 \mathrm{kPa}$ & 3.85 & 3.48 & $108.41 \%$ \\
$32 \mathrm{kPa}$ & 3.21 & 3.83 & $109.43 \%$ \\
Glass & 3.50 & 21.22 & $63.78 \%$ \\
\hline Stiffness & Mean Filament & Mean Filament & Difference \\
\hline $1 \mathrm{kPa}$ & Number Actin & Number NMM2a & \\
$2 \mathrm{kPa}$ & 16.68 & 23.60 & $141.49 \%$ \\
$5 \mathrm{kPa}$ & 27.01 & 17.57 & $65.05 \%$ \\
$11 \mathrm{kPa}$ & 24.37 & 16.31 & $66.93 \%$ \\
$20 \mathrm{kPa}$ & 32.17 & 20.18 & $62.73 \%$ \\
$32 \mathrm{kPa}$ & 23.04 & 19.59 & $69.86 \%$ \\
$\mathrm{Glass}$ & & 21.31 & $98.77 \%$
\end{tabular}

Table 5.9.: Mean measurements of untransfected hMSCs grown on substrates of different stiffnesses for 24 hours, fixed and stained with phalloidin and anti-NMM2a. Measurements for area, aspect ratio, and filament number are shown and percentage of NMM2a staining in comparison to actin data given as percentage.

While this data provides a reason to reject NMM2a staining for stress fiber analysis, we can also use this setup for other purposes. A follow-up experiment is currently being done by master's student Nicole Drewes, who will compare untransfected, empty-vector-transfected and LifeAct-RFP-transfected hMSCs parallel stained with phalloidin and anti-NMM2a, while also recording brightness of LifeAct-RFP-transfected cells. This will yield a data set that, on the one hand, effects of LifeAct transfection (mentioned in 5.1.1.1) can be quantified; on the other hand, this data set can also be tested to determine whether transfection per se or the LifeAct transfection specifically leads to the morphological changes. 


\subsection{Evolution of stem cells in the first 24 hours after seeding}

In this section, stress fiber network changes in the first 24 hours of mechanically differentiating stem cells on two stiffnesses will be shown.

As my whole thesis deals with the question of whether or not one can see definitive differences as soon as 24 hours after seeding with different methods, the two conditions with the biggest assumed difference were picked. An in-depth analysis of these differences would be beyond the scope of this thesis.

For all experiments shown in this section, hMSCs from Lot 451491 (Lonza, PT-2501) were used. Cells were transfected with LifeAct-RFP (see Subsection 5.1.1.1) and seeded on freshly prepared $11 \mathrm{kPa}$ PAA gels (for rheological measurements see Section 4.5) and glass covered with collagen I (for methods see Section C). Both conditions were measured in parallel to ensure the same treatment besides their different substrate stiffnesses. Movies were started 1 hour after seeding, when most of the hMSCs were adherent but not fully spread. To obtain the set of 321 movies on $11 \mathrm{kPa}$ gel and 369 movies on glass, 26 experiments were pooled. Number of movies obtained per experiment varies, as only nondividing cells with no cell-to-cell contact over the whole 24-hour period were used. In general, fewer cells on $11 \mathrm{kPa}$ gels were selected for imaging. This is mainly due to the necessary distance to the gel edge or to gel holes caused by air bubbles during polymerization. Furthermore, with this layer added, brightness is generally lower in the $11 \mathrm{kPa}$ cells compared to cells on glass. I first show differences in the whole cell behavior of hMSCs on different substrates, then behavior of the stress fiber network.

\subsubsection{Whole cell behavior}

As already described in Section 4.5 and Figure 4.12, there is an agreement that for different stiffnesses hMSCs converge against specific aspect ratios. Thus, we expect to see smaller, more elongated cells on 11 $\mathrm{kPA}$ gels and round, more spread-out cells on glass.

In Figure 5.16, measurements of cell area, aspect ratio, and length of the long half axis are shown. While the long half axis remains similar for both conditions over time, area is significantly larger for cells on glass, as expected. This larger area based on the roundness of the cell, and thus larger short axis, is reflected in the aspect ratio. The trend of these measurements is consistent with Rehfeldt's findings from 2012 [169], in which a different lot and fixed hMSCs were used. There, the area for cells was around 4,500 $\mu \mathrm{m}^{2}$ on $11 \mathrm{kPa}$ PAA gels and around $5,900 \mu \mathrm{m}^{2}$ on glass, yielding a $1,400 \mu \mathrm{m}^{2}$ difference, or cells on glass being one third larger. In my data, cells on $11 \mathrm{kPa}$ measure around $4,150 \mu \mathrm{m}^{2}$ and those on glass measure around $5,150 \mu \mathrm{m}^{2}$, yielding a $1,000 \mu \mathrm{m}^{2}$ difference, or cells on glass being one fourth larger. For the aspect ratio, Rehfeldt reported around 4.1 for cells on $11 \mathrm{kPa}$ and around 2.8 on glass, yielding a 1.3 difference in aspect ratio. For my experiments, aspect ratio for cells converged to 3.0 on $11 \mathrm{kPa}$ and to 2.5 on glass, yielding only a 0.5 difference. However, as the Rehfeldt paper uses fixed cells, a discrepancy is to be expected. Another source to compare would be the thesis of Carina Wollnik [225], who did live cell movies in the same fashion. However, she does not show cells seeded on glass. For $10 \mathrm{kPa}$, her cell area is around $6,000 \mu \mathrm{m}^{2}$ and aspect ratio around $2.4(\mathrm{n}=79)$. This is, in terms of area, significantly larger and, in terms of aspect ratio, lower than my results. 

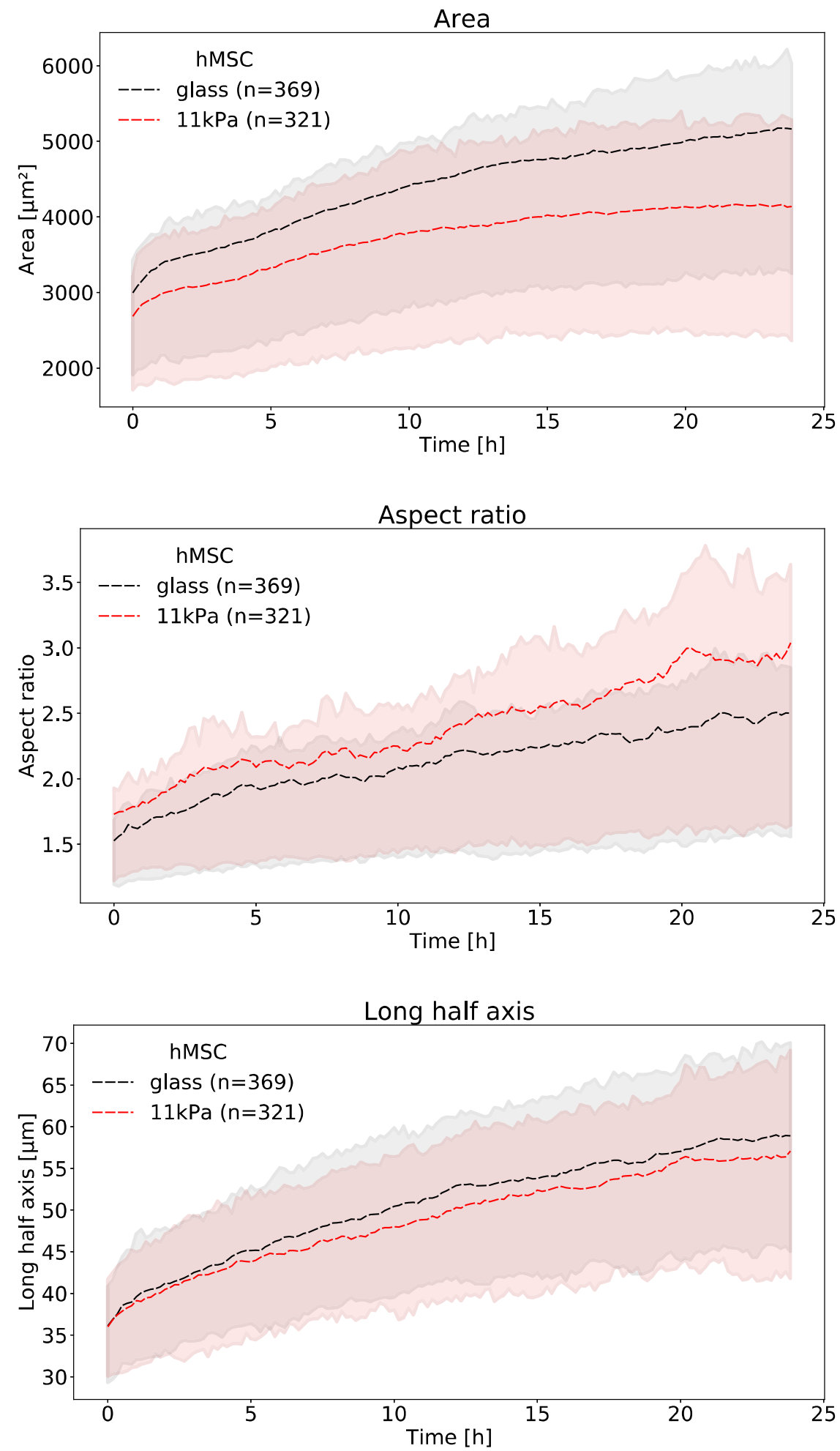

Figure 5.16.: All: LifeactRFP transfected hMSC (Lot 451491, Lonza PT-2501) seeded 48 hours after transfection onto $11 \mathrm{kPa}$ PAA gels or glass, both covered with collagen I. 24-hour imaging recorded in 10 min intervals. Data shown as mean over all cells $(11 \mathrm{kPa}: \mathrm{n}=321$, glass: $\mathrm{n}=369)$ with quantiles. Top: Area. Middle: Aspect ratio. Bottom: Long Half Axis. 


\subsubsection{Filament network behavior}

For the analysis of the stress fiber network, one can either use descriptors of the whole network inside the cells and average over the single cell before averaging over these again; or one can gather all fibers of all cells and average over them. Each of the two approaches will lead to different results, as number of filaments averaged per condition varies significantly.

One descriptor of the whole network is the order parameter (see Subsection 3.2.4). It describes how well-aligned all fibers are with the long axis of the cell. An order parameter of 1 means all filaments are parallel to the long axis of the cell, -1 means all are parallel to the short axis. As one can see in Figure 5.17 , most of the filaments in both conditions are aligned with the long axis and development of the order parameter in both conditions is similar.

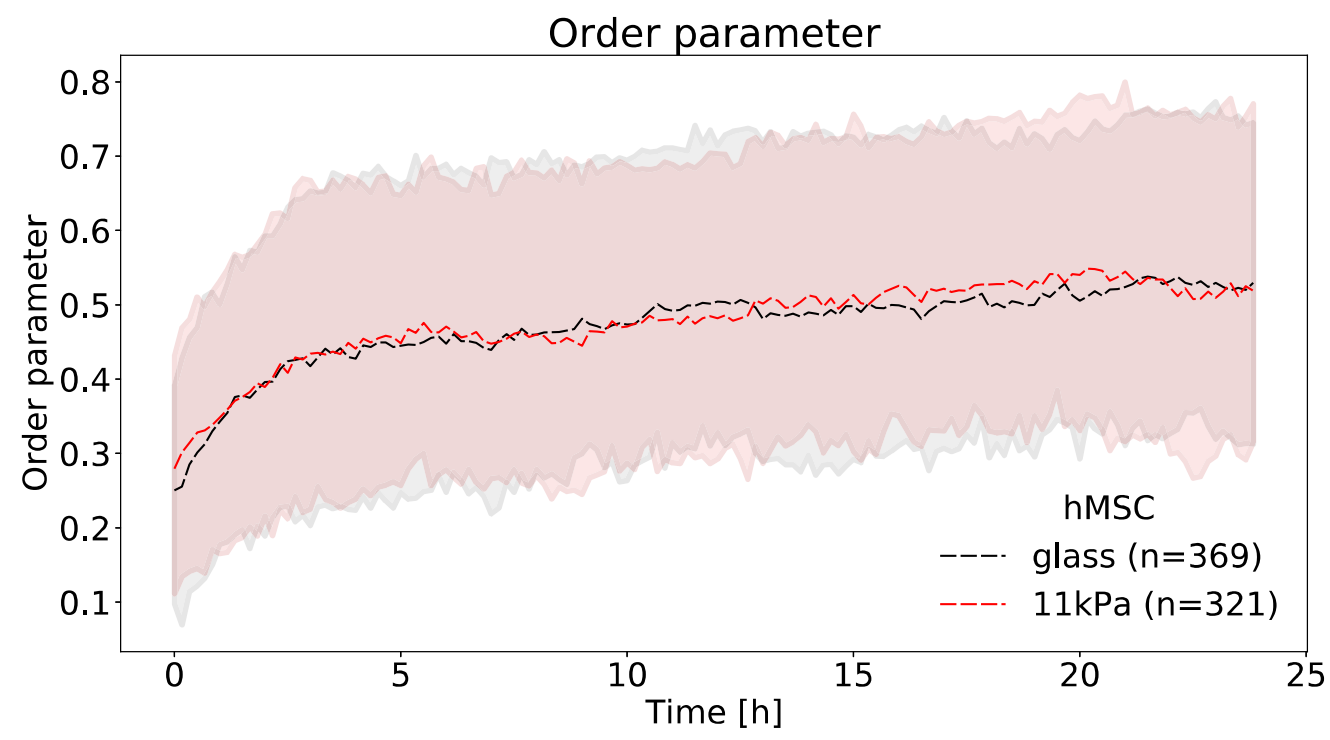

Figure 5.17.: Depiction of the order parameter in movies of lifeactRFP transfected hMSCs (Lot 451491, Lonza PT-2501) seeded 48 hours after transfection onto $11 \mathrm{kPa}$ PAA gels or glass, both covered with collagen I. 24-hour imaging recorded in 10 min intervals. Data shown as mean over all cells $(11 \mathrm{kPa}: \mathrm{n}=321$, glass: $\mathrm{n}=369)$ with quantiles.

In theory, one would expect the order parameter to be lower in cells on glass, where cells are wider and as a result more filaments should deviate from the long axis. In Figure 5.17 it can be seen that this is not the case. Quite the contrary, curves for both conditions overlap and converge against 0.5 , where most fibers are aligned with the long axis. This behavior can also be found in Wollnik's thesis [225], where live cell movies on three different PAA gel stiffnesses also all merge at an order parameter of 0.5 (see Figure 5.18). 

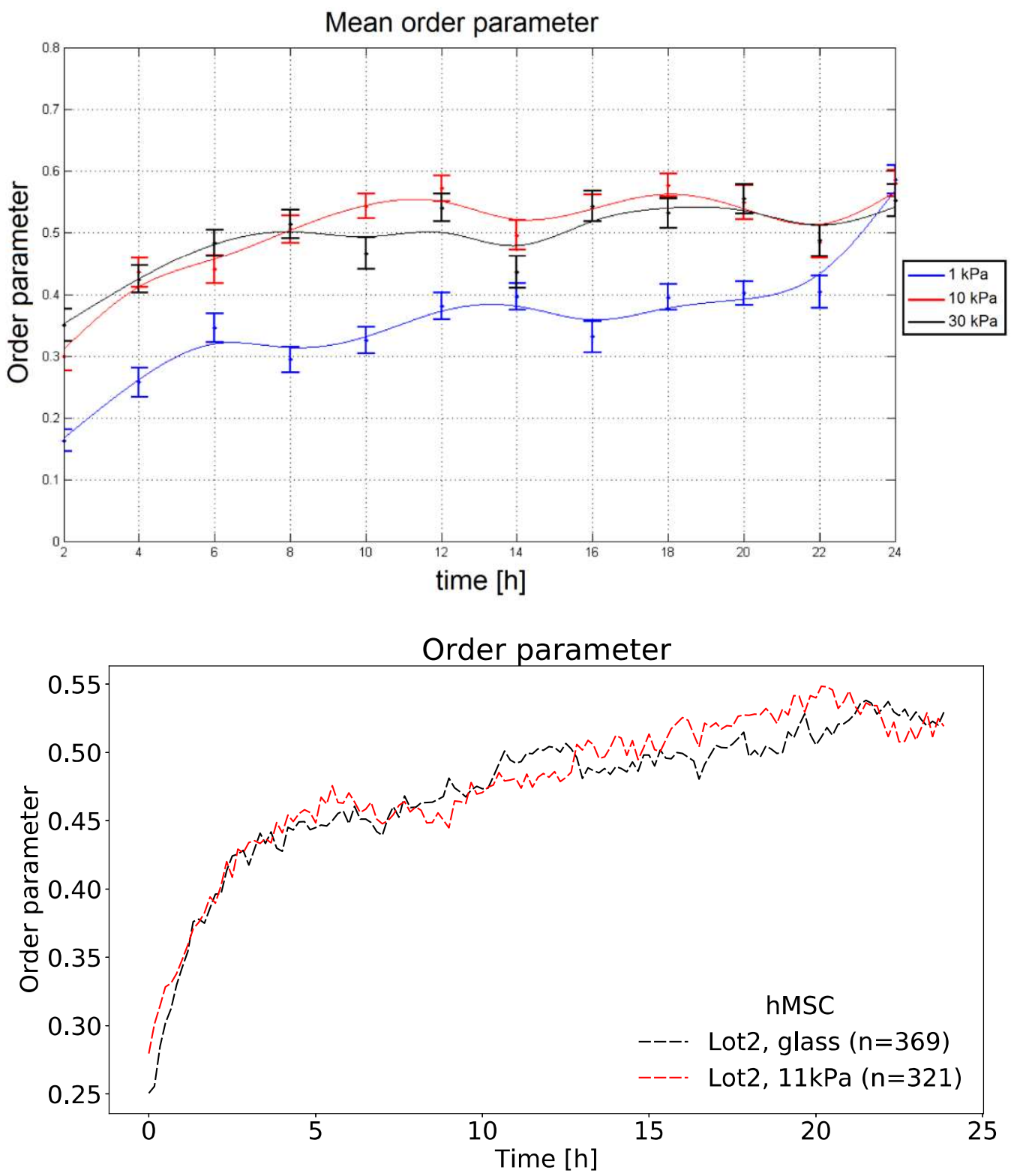

Figure 5.18.: Depiction of the order parameter in movies of lifeactRFP transfected hMSCs (Lonza PT-2501) seeded 48 hours after transfection onto 1, 10, and $30 \mathrm{kPa}$ PAA gels covered with collagen I. 24-hour imaging recorded in 2 hour intervals. Data shown as mean over all cells $(n \leq 120)$. Top: Figure from [225]. Bottom: Data already used in 5.6.

A distinct difference can be seen only when imaging fixed cells. In Figure 5.19, stained, untransfected hMSCs of the same lot are shown. Here, we see a stiffness-dependant behavior of the cells. This implies that the unordered component arises from recently divided cells. Those are excluded from the movies but cannot be excluded in the fixed data, as we have no information about the cells' fates in that setting. 


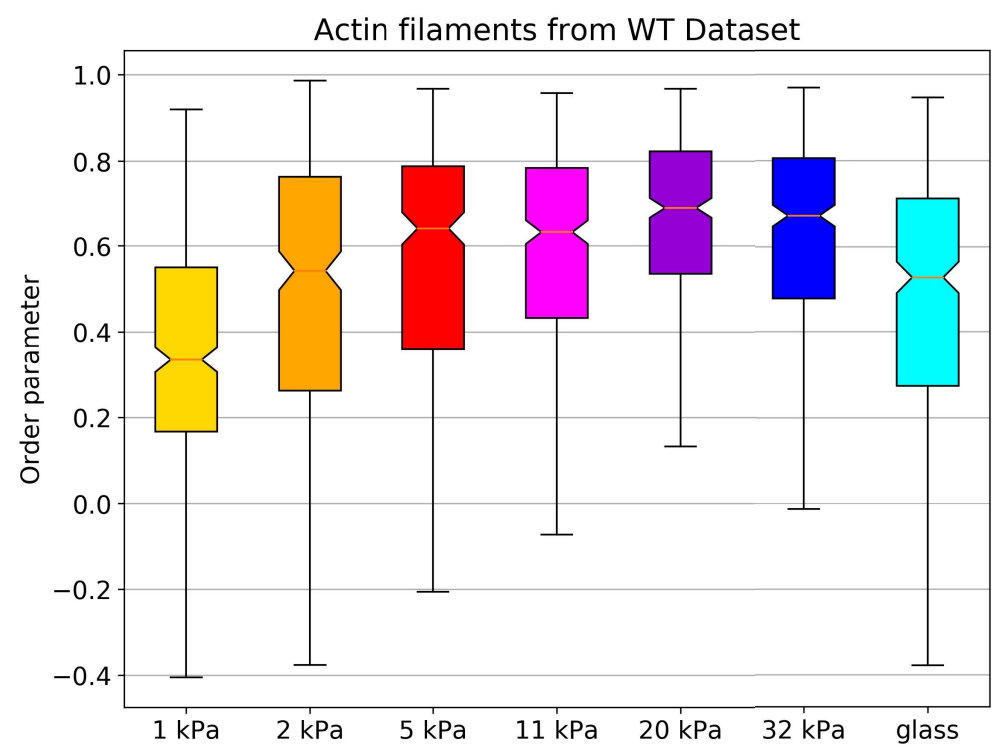

Figure 5.19.: Order parameter measurements of untransfected hMSCs seeded on substrates of different stiffness for 24 hours, fixed and stained with protocols from Appendix 5.3. Number of imaged cells indicated under the respective boxes.

When looking into how the filament network is composed, Figure 5.20 shows the filament number per cell, averaged over all cells. Here, cells exhibit more stress fibers on glass throughout the whole imaging time, with two exceptions towards the end of the movie. On average, cells on glass express around $16 \%$ more stress fibers than on $11 \mathrm{kPa}$ PAA gels. This means that, when averaging over cells instead of over fibers, fewer fibers are taken into account for cells on $11 \mathrm{kPa}$ gels. Unfortunately, because cells on gels are darker, filament number of the cells on $11 \mathrm{kPa}$ gels are also overcounted by introduction of stray fibers in strongly bleached cells.

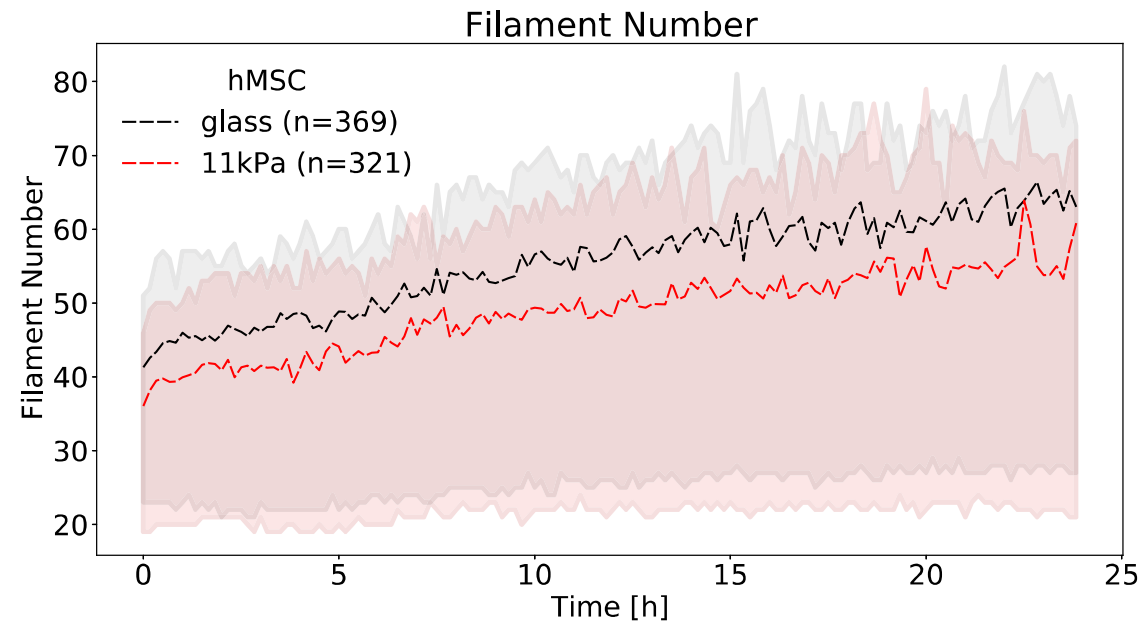

Figure 5.20.: Depiction of the filament number per cell in movies of lifeactRFP transfected hMSCs (Lot 451491, Lonza PT-2501) seeded 48 hours after transfection onto $11 \mathrm{kPa}$ PAA gels or glass, both covered with collagen I. 24-hour imaging recorded in 10 min intervals. Data shown as mean over all cells $(11 \mathrm{kPa}: \mathrm{n}=321$, glass: $\mathrm{n}=369)$ with quantiles. 
To answer biological questions, however, it is not the number of filaments that is interesting, as the number might be statistically significant for larger sample sets but is highly variable from cell to cell. As described in Subsection 2.2.2, stress fibers can be classified into subtypes. Those have different composition, binding partners, functionalities, and, supposedly, lifetimes. To distinguish differentiation by observation of the stress fiber network is a major goal. For this, the theory of different stress fiber subtypes being predominant in different differentiation pathways is most important. In Section 3.4 an approach to differentiate stress fiber subtypes via colocalization has been introduced. However, a technique using only stress fibers and that is as noninvasive as possible is still needed. A classification of bulk filaments in live cells is a start. However, the optimal solution would be to follow single filaments over the course of the movies. This will not be discussed here, but is referenced in Section 10 as it is currently not part of the officially available software.
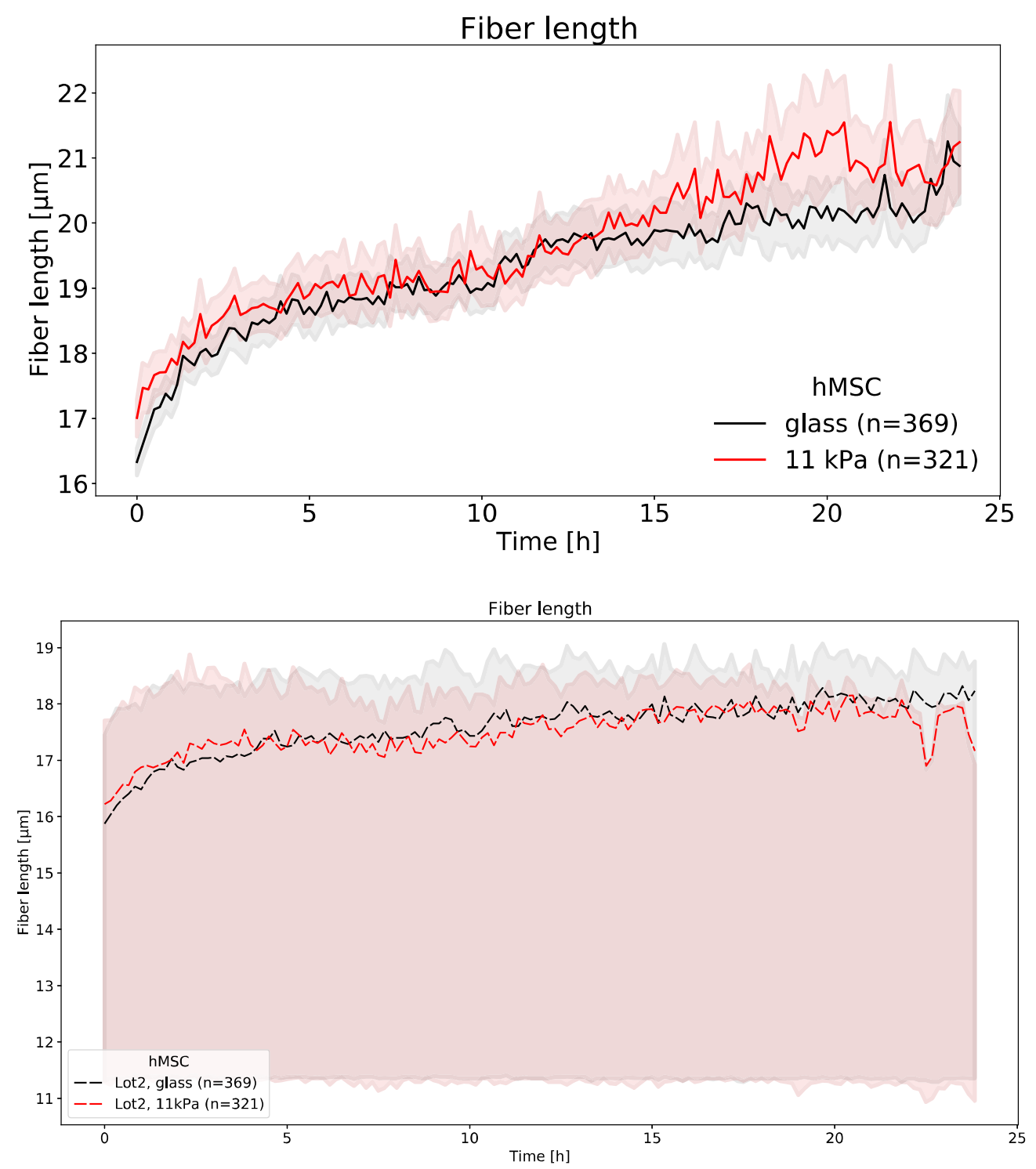

Figure 5.21.: Depiction of the fiber length per cell in movies of lifeactRFP transfected hMSCs (Lot 451491, Lonza PT-2501) seeded 48 hours after transfection onto $11 \mathrm{kPa}$ PAA gels or glass, both covered with collagen I. 24-hour imaging recorded in $10 \mathrm{~min}$ intervals. Top: Averaged per cell, then over all cells. Bottom: Averaged over all fibers of all cells. (11 kPa: $\mathrm{n}=321$, glass: $\mathrm{n}$ $=369$; top with SEM, bottom with quantiles). 
Without single filament tracking to derive individual lifetimes of fibers, length is a handy descriptor to be used. One could also resort to localization of fibers, as some subtypes are typical for the cell's leading or trailing edge. However, this is not a task that can be performed for large data sets, as for now a human is needed to determine which is the leading edge of the cell in each frame. When resorting to the length as a proxy for lifetime, there is a noticeable difference depending on whether it is first averaged over all fibers in a single cell and then over all cells, or just averaged over all fibers in all cells in the data set.

In Figure 5.21 effects of different methods of averaging can be seen. While averaging over all fibers in all cells to assess bulk filament dynamic, we end up with almost nonexisting standard error of the mean (SEM) but the same effects visible in Figure 5.20 (Filament number) show through. When averaging the filaments for the individual cells first, we remove the effect of filament number per cell and yield a smoother curve, although with larger SEM. As the filament number per cell seems like an important effect to remove given variations between cells and bleaching effects, all further figures will feature the mean of the cells' average fiber.

In conclusion, on $11 \mathrm{kPa}$ PAA gels the cells express longer filaments than on glass most of the time, although this difference is not statistically significant. This aligns with higher aspect ratios and smaller filament numbers in hMSCs on softer substrates. On these substrates the cells undergo myogenic differentiation, in which cells tend to be elongated with few but long fibers along the cell axis. Further, these fibers can be classified by width, curvature and angle (please see Section 3.2 for details on how these are derived). The filament angle can be seen in Figure 5.22. The angle develops similarly in both conditions as it rises in the first 3 to 5 hours, until the stress fiber network is fully developed after adhesion. Afterwards fluctuations occur in both conditions around the same values.

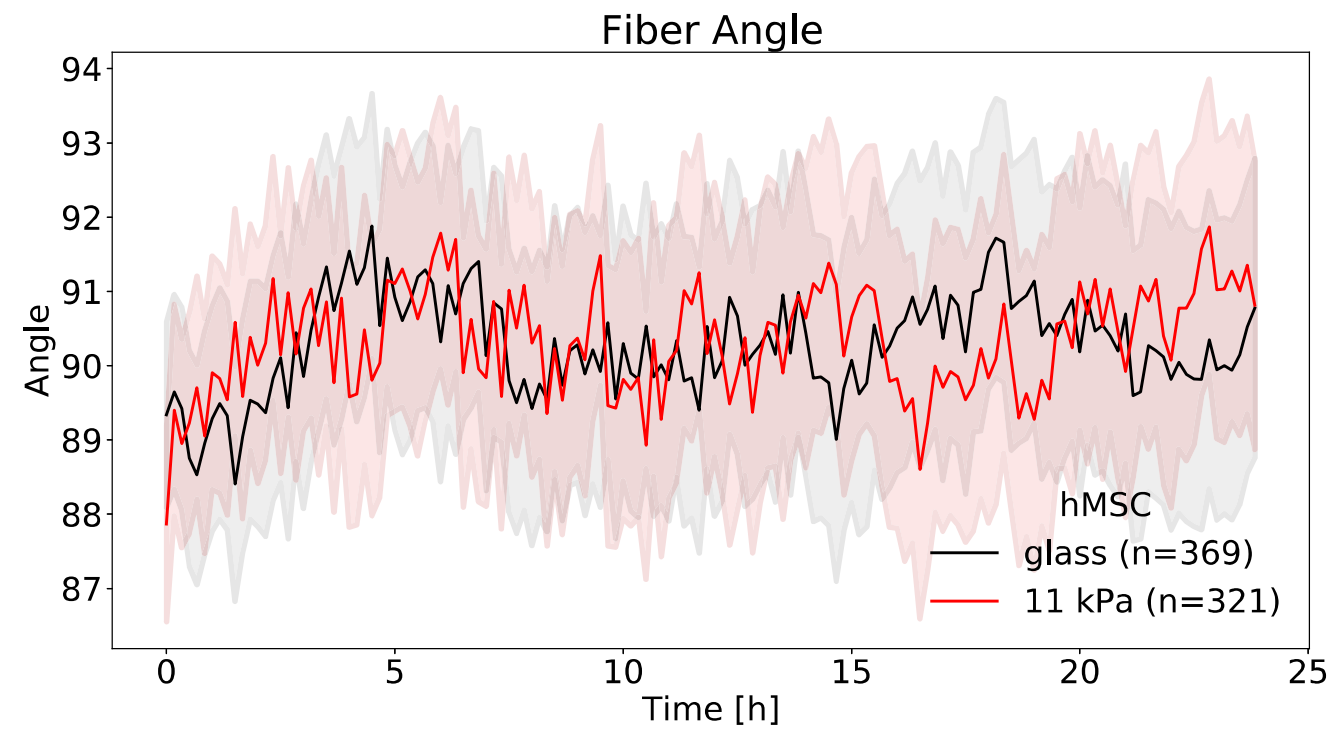

Figure 5.22.: Depiction of the fiber angle in movies of lifeactRFP transfected hMSCs (Lot 451491, Lonza PT-2501) seeded 48 hours after transfection onto $11 \mathrm{kPa}$ PAA gels or glass, both covered with collagen I. 24-hour imaging recorded in 10 min intervals. Averaged per cell, then over all cells. (11 $\mathrm{kPa}: \mathrm{n}=321$, glass: $\mathrm{n}=369$; with $\mathrm{SEM})$.

The fibers differ, however, in the width and curvature expressed. In Figure 5.23 it can be seen that cells on $11 \mathrm{kPa}$ express thicker stress fibers. This is consistent with them expressing few long fibers along the cell axis. In contrast, cells on glass tend to express multiple, interlacing, fine fibers. However, this difference is not statistically significant. The same has to be said for the fiber curvature, depicted in Figure 5.24. Here, 
cells on glass express, on average, more curved fibers.

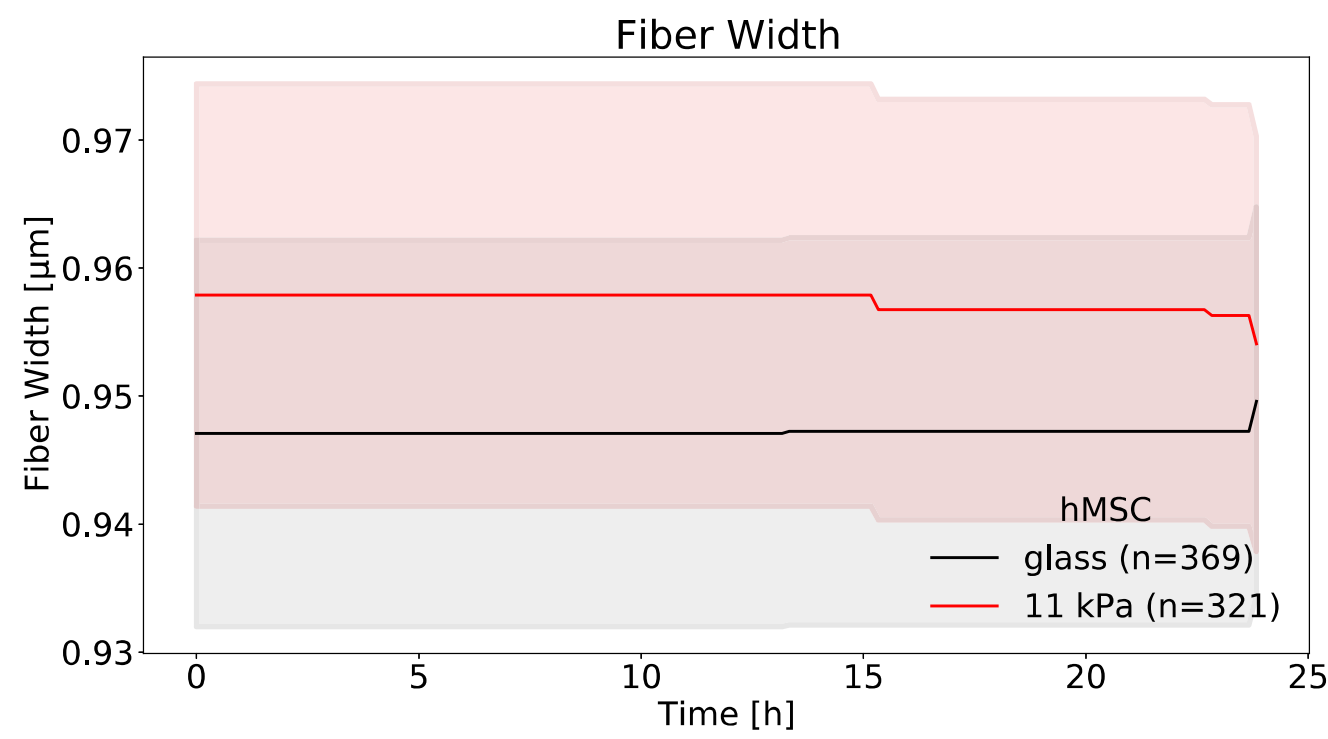

Figure 5.23.: Depiction of the fiber width in movies of lifeactRFP transfected hMSCs (Lot 451491, Lonza PT-2501) seeded 48 hours after transfection onto $11 \mathrm{kPa}$ PAA gels or glass, both covered with collagen I. 24-hour imaging recorded in $10 \mathrm{~min}$ intervals. Averaged per cell, then over all cells. (11 $\mathrm{kPa}: \mathrm{n}=321$, glass: $\mathrm{n}=369$; with $\mathrm{SEM})$.

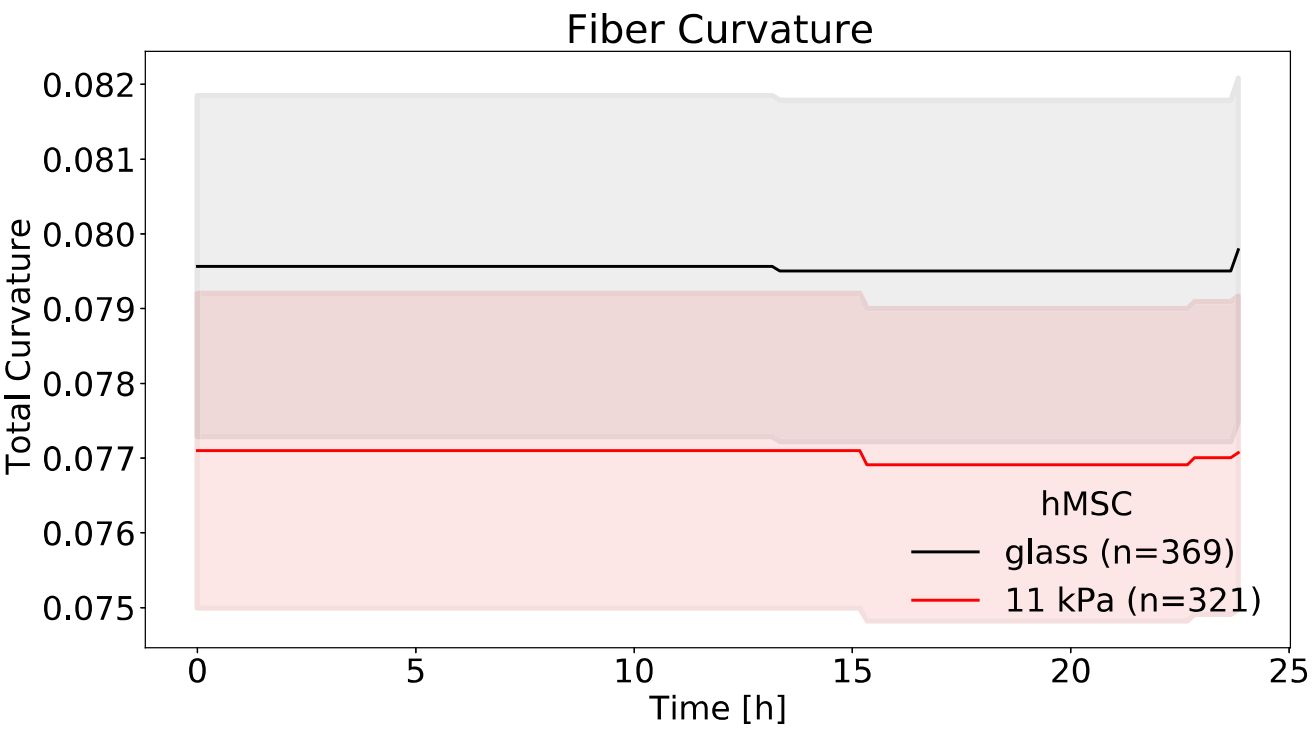

Figure 5.24.: Depiction of the fiber curvature in movies of lifeactRFP transfected hMSCs (Lot 451491, Lonza PT-2501) seeded 48 hours after transfection onto $11 \mathrm{kPa}$ PAA gels or glass, both covered with collagen I. 24-hour imaging recorded in $10 \mathrm{~min}$ intervals. Averaged per cell, then over all cells. (11 $\mathrm{kPa}: \mathrm{n}=321$, glass: $\mathrm{n}=369$; with $\mathrm{SEM})$.

This is, again, founded on the contrast between long cells on $11 \mathrm{kPa}$ and more round and spread-out cells on glass. Hereby, cells on glass show a more star-like morphology, with adhesive regions interconnected by 
stress fibers along the cell periphery. These fibers get pulled inward by the forces exerted from the rest of the fiber network.

In conclusion, the length of the long axis of hMSCs on $11 \mathrm{kPa}$ and glass does not differ. The same can be said for the order parameter of the network, the fiber length, and angle. In the fiber width and curvature differences can be seen, but those are not statistically significant. The only parameters with statistically significant differences are the cell area, the cells' aspect ratio, and the filament number per cell.

\subsubsection{Migration}

As described in Subsection 3.2.5, the FilamentSensor software also derives the center of the cell. This can be used to derive the mean square displacement (MSD) and the trajectory. For hMSCs, we expect different rates of movement for different differentiation pathways, also dependent on the substrate. Whereas very soft substrates do not offer enough resistance for cells to form a functional stress fiber network and move through pulling, very hard substrates offer too much resistance. Cells on very hard substrates are "locked" in place, generating huge forces but not moving.

Thus, we expect to see a higher MSD, in relation to to diffusion (see Figure 5.25), for cells on $11 \mathrm{kPa}$. This higher relative MSD puts the cells' movement in the realm of directed movement.

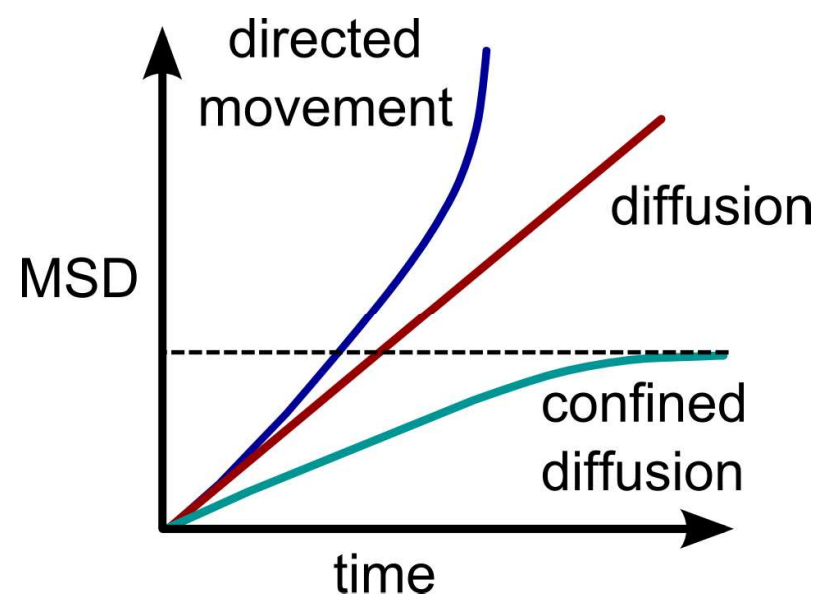

Figure 5.25.: Schematic of mean square displacement (MSD) curves. Diffusion is used as reference to distinguish between confined diffusion (slower) and directed movement (faster). Image from [225].

However, when looking at the MSD of 24-hour movies of live cells, cells seeded on $11 \mathrm{kPa}$ PAA gel exceed the diffusion limit only slightly, while cells seeded on glass stay below. But, as for many other variables described in this section, we have to take into account the subsampling. Because only cells that stay within the field of view for 24 hours are selected for analysis, we subsample for slowly moving cells. In Figure 5.26 it can be seen how the MSD changes for cells that leave the field of view.

In the top, both cells remaining and staying are depicted in the same graph (log transformed for better visibility), while in the bottom the data is split. In the bottom left, cells that leave in the last 4 hours of imaging are analyzed. One can assume that for cells leaving even earlier, the MSD would be even higher. Most interestingly, while the cells seeded on glass show no directed movement in the 24-hour movies, cells on glass leaving the field of view do exhibit directed movement. However, for cells on $11 \mathrm{kPa}$ PAA gels, the MSD of leaving cells compared to those on glass is 140-fold higher. 

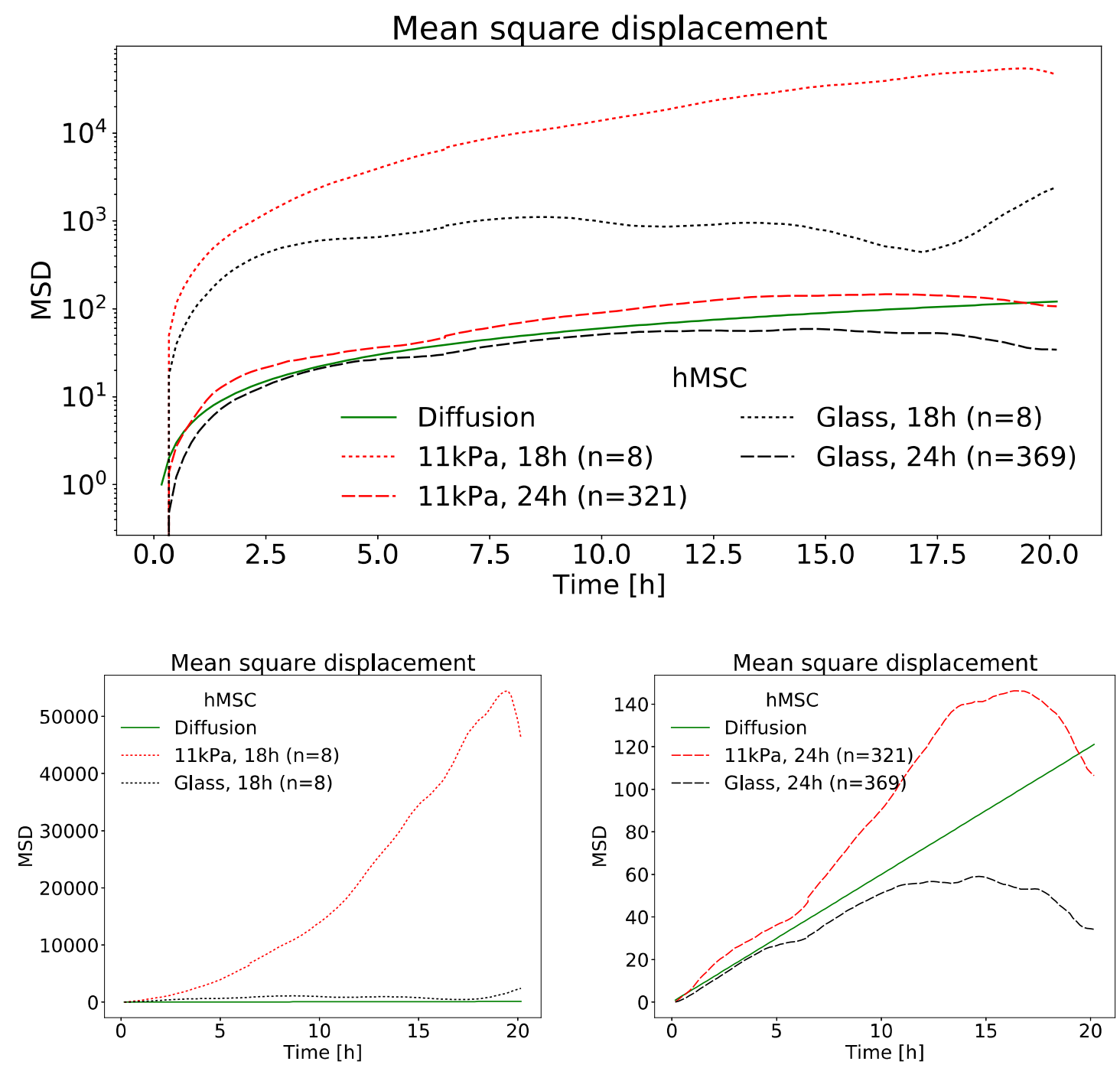

Figure 5.26.: Depiction of the mean square displacement of lifeactRFP transfected hMSCs (Lot 451491, Lonza PT-2501) seeded 48 hours after transfection onto $11 \mathrm{kPa}$ PAA gels or glass, both covered with collagen I. 24-hour imaging recorded in $10 \mathrm{~min}$ intervals, cut to 20 hours. Top: Data from cells used in 24-hour movies and cells leaving the field of view. Bottom left: Cells leaving the field of view after 20 hours. Bottom right: Cells not leaving the field of view within 24 hours.

Additionally, we can look into where the cells move. Figure 5.27 again shows data for cells within and leaving the field of view combined (top), and at the bottom the data are split. For all cells the current distance to the point of origin is measured, using the center of mass. For cells staying within the field of view for 24 hours, we see that cells move back towards their origin. Also, total trajectory is 13 -fold smaller for cells that stay within the field of view. Of the cells leaving the field of view in the last 24 hours of imaging, cells on glass seem to barely move for a long time and then suddenly start moving at the end of the time frame. For the cells on $11 \mathrm{kPa}$, cells leaving the field of view seem to start a constant movement away from their origin at about 5 to 6 hours after seeding.

To conclude, we see the expected differences in movement speed in live cell movies of hMSCs. To get a real measurement of the MSD, however, one would have to measure every cell independent of how long they stay within the field of view. This is beyond the aim of this thesis and thus was not done. 

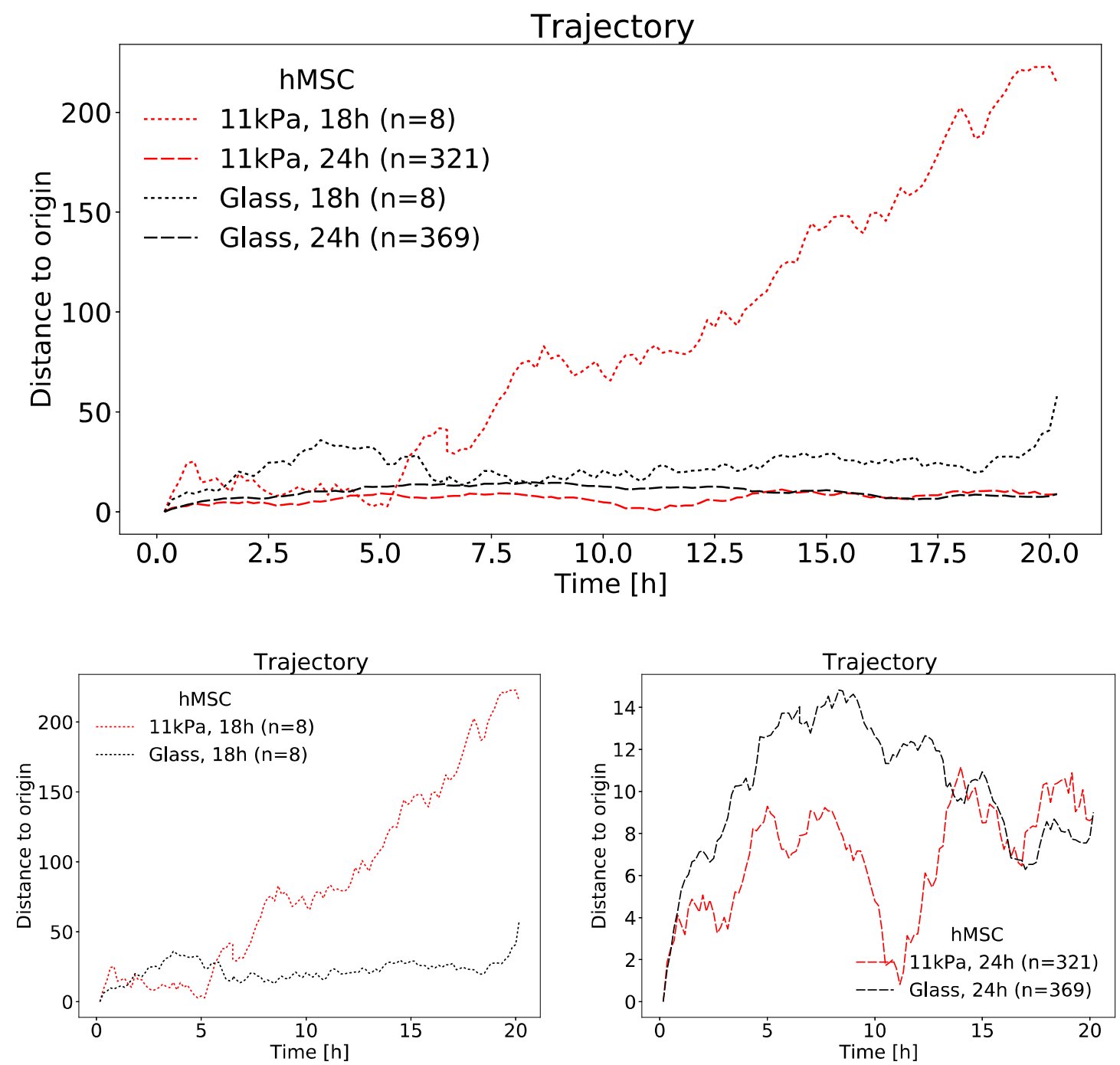

Figure 5.27.: Depiction of the trajectorie of lifeactRFP transfected hMSCs (Lot 451491, Lonza PT-2501) seeded 48 hours after transfection onto $11 \mathrm{kPa}$ PAA gels or glass, both covered with collagen I. 24-hour imaging recorded in 10 min intervals, cut to 20 hours. Top: Data from cells used in 24-hour movies and cells leaving the field of view. Bottom left: Cells leaving the field of view after 20 hours. Bottom right: Cells not leaving the field of view within 24 hours. 


\title{
6. MIET
}

\subsection{MIET}

It might seem otherwise, but some facts we seem to know about cells are assumptions. When in the past cells were drawn containing focal adhesions and stress fibers, it was assumed the fibers span from the adhesions on the edge of the cell to the top of the nucleus quite direct and with an angle, although small, still bigger than measured nowadays. With the Dual-Color Metal-Induced and Förster Resonance Energy Transfer for Cell Nanoscopy a precise measurement of the height of a labeled structure above the gold surface is possible.

This measurement seems trivial in itself, but as forces in the cell depend on the angle of the pull, it can be quite significant. I will paste here a paper on MIET measurements on fixed hMSC cells labeled for actin stress fibers and vinculin. This was published in Molecular Biology of the Cell in 2018, Volume 29, 1.4.18. To this paper, I contributed fixed and stained samples of BM-hMSCs I cultured.

\section{Dual-color metal-induced and Förster resonance energy transfer for cell nanoscopy}

Anna M. Chizhik ${ }^{a,+}$, Carina Wollnik ${ }^{a,+}$, Daja Ruhlandt ${ }^{\mathrm{a}}$, Narain Karedla ${ }^{\mathrm{a}, \mathrm{b}}$, Alexey I. Chizhik ${ }^{\mathrm{a}}$, Lara Hauke $^{\mathrm{a}}$, Dirk Hähnel ${ }^{\mathrm{a}}$, Ingo Gregor ${ }^{\mathrm{a}, \mathrm{b}}$, Jörg Enderlein ${ }^{\mathrm{a}, \mathrm{b}, *}$, and Florian Rehfeldt ${ }^{\mathrm{a}, *}$

${ }^{a}$ Third Institute of Physics-Biophysics, University of Göttingen, 37077 Göttingen, Germany; ${ }^{b}$ Center for Nanoscale Microscopy and Molecular Physiology of the Brain (CNMPB), 37077 Göttingen, Germany

\begin{abstract}
We report a novel method, dual-color axial nanometric localization by metal-induced energy transfer, and combine it with Förster resonance energy transfer (FRET) for resolving structural details in cells on the molecular level. We demonstrate the capability of this method on cytoskeletal elements and adhesions in human mesenchymal stem cells. Our approach is based on fluorescence-lifetime-imaging microscopy and allows for precise determination of the three-dimensional architecture of stress fibers anchoring at focal adhesions, thus yielding crucial information to understand cell-matrix mechanics. In addition to resolving nanometric structural details along the z-axis, we use FRET to gain precise information on the distance between actin and vinculin at focal adhesions.
\end{abstract}

\section{INTRODUCTION}

The mechano-induced differentiation of human mesenchymal stem cells (hMSCs) is an example where stress fibers and focal adhesions are key players of translating mechanical cues from the extracellular environment into biochemical signaling that leads to lineage decision (Engler et al., 2006). It was shown that stress fiber structure critically depends on the mechanical interactions with the environment and 
is an early morphological marker for differentiation (Zemel et al., 2010a,b; Paluch et al., 2015). The coupling of stress fibers to focal adhesions became a widely studied topic in mechano-biology and the exact geometry of this architecture is important to deduce the acting forces. However, current studies, albeit even using superresolution in $\mathrm{x}$ and $\mathrm{y}$, lack sufficient height information and therefore a detailed view on the three-dimensional (3D) architecture, essential to understand force transmission from and to the substrate, and to elucidate the mechano-sensing of cells. Particularly crucial for the mechanical coupling of the cell's cytoskeleton to the surrounding are focal adhesions, where vinculin plays an essential role (Geiger et al., 2009; Case et al., 2015; Burridge and Guilluy, 2016; Livne and Geiger, 2016).

There are several coherent optical microscopy methods such as reflection interference contrast microscopy (see, e.g., Limozin and Sengupta, 2009), or interferometric scattering microscopy (see, e.g., Kukura et al., 2009), which routinely achieve nanometer and subnanometer localization accuracy of scattering/reflecting entities along the optical axis. However, these methods lack the specificity and, to some extent, the sensitivity of fluorescence microscopy methods. Over recent years, various fluorescence microscopy techniques for also gaining superresolution along the optical axis have been proposed and implemented. Within the context of stochastic optical reconstruction microscopy (STORM; Rust et al., 2006) or photoactivated localization microsocopy (PALM; Betzig et al., 2006), the most widely used techniques are astigmatic imaging (Huang et al., 2008), biplane imaging (Juette et al., 2008), or helical wavefront shaping (Pavani et al., 2009). They all achieve localization accuracies of single molecules along the optical axis on the order of $\sim 50 \mathrm{~nm}$. Two distinct but powerful techniques, which are not based on single-molecule localization, are supercritical angle fluorescence imaging (Ruckstuhl and Verdes, 2004; Deschamps et al., 2014), and variable-angle total internal reflection fluorescence microscopy (see, e.g., Stock et al., 2003, and Dos Santos et al., 2016). They achieve, under optimal conditions, an axial resolution around $10 \mathrm{~nm}$. Along the same line, there is a class of methods based on fluorescence interference contrast (FLIC), initially developed by Braun and Fromherz (1997), and recently further improved as variable incidence angle FLIC (Ajo-Franklin et al., 2005) and scanning angle interference microscopy (Paszek et al., 2012). Although the last one also achieves subnanometer precision, all of these techniques require the use of optically opaque silicon wafers with a precisely controlled silicon oxide layer, and for the latter two an extremely accurate tuning of the angle of incidence.

One class of techniques which indeed achieves single nanometer localization accuracy along the optical axis is represented by interferometric PALM (iPALM) or 4pi-STORM (Shtengel et al., 2009; Aquino et al., 2011). Using iPALM with a plethora of constructed photoactivatable fluorescent proteins fused to focal adhesion proteins in U2OS cells, it was possible to localize different proteins in the focal adhesion cluster with a resolution of $10 \mathrm{~nm}$ to $15 \mathrm{~nm}$ along the z-axis (Kanchanawong et al., 2010). However, complex instrumentation, requirement of specialized fluorescent protein fusion constructs (photoactivatable fluorescent proteins), and double transfection of cells render this method rather tedious and complicated. Here, we present a novel method of height and colocalization measurements of two dyes in cells with nanometer accuracy. We used MIET imaging (Chizhik et al., 2014), which allows us to measure the axial localization of a fluorophore with an accuracy of $\sim 3 \mathrm{~nm}$ in both living and fixed cells. Additionally, we extended our setup with a second fluorescence emission channel that allows for dual-color MIET imaging (dcMIET) and Förster resonance energy transfer (FRET) measurements (see Figure 1).

The principle of MIET imaging is based on the energy transfer from a fluorescent molecule to surface plasmons within a thin metal film on a glass surface, which results in the acceleration of its deexcitation rate. This can be observed as a reduction of the molecule's fluorescence lifetime (Karedla et al., 2014). Because, within the first $200 \mathrm{~nm}$, the energy transfer rate is monotonically dependent on the distance of a molecule from the metal layer, the fluorescence lifetime can be directly converted into a distance between the emitter and the metal surface within this distance range (see Supplemental Figure S3; not included). Here, for the first time, we present a detailed description of dc-MIET and its combination with FRET imaging. This allows us to measure the axial localization of both actin filaments and vinculin, and additionally to monitor their distance within FRET range $(<10 \mathrm{~nm})$. By combining the capabilities 
of dcMIET and FRET microscopy, we achieve unprecedented axial resolution and demonstrate their applicability to elucidate the 3D structure of actin and vinculin at focal adhesions in hMSCs.

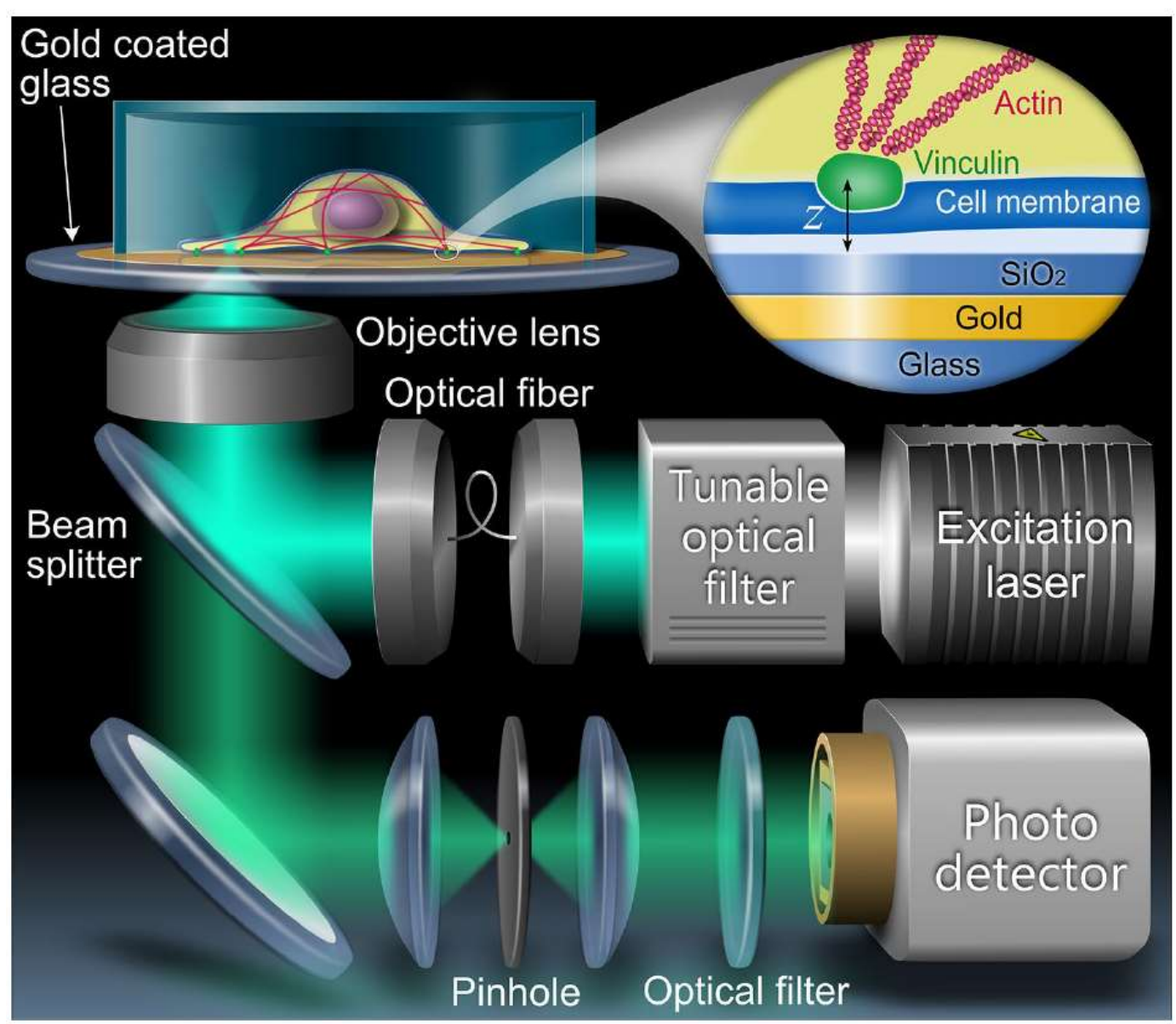

Schematic of the setup for dcMIET. The inset shows the anchoring of actin stress fibers to ensembles of the focal adhesion protein vinculin. The measured height $\mathrm{z}$ is always counted from the top of the $\mathrm{SiO} 2$-spacer.

\section{RESULTS}

To study the developing actomyosin stress fiber structure and the architecture of focal adhesions, we fixed hMSCs at distinct time points after seeding, and fluorescently stained actin (Atto 647N phalloidin) and vinculin (Atto 488/anti-mouse/anti-vinculin; see Materials and Methods for further details). Figure 2 shows a time series of developing actin stress fibers starting at heights of $>150 \mathrm{~nm}$ in the early regime (1-6 h). Later, from $12 \mathrm{~h}$ on, they form tight connections to focal adhesions at low distances to the substrate $(\sim 40 \mathrm{~nm}$ ), and finally span throughout the cell (increasing above $180 \mathrm{~nm}$ ). For better visualization, the height-profile images are weighted by fluorescence intensity to reflect the amount of actin within the stress fibers.

For a detailed view of the 3D architecture of stress fibers and focal adhesions, we imaged selected areas using dcMIET with reduced pixel size. Because we labeled the two structures with dyes that constitute a FRET pair, we can also measure the distance between actin and vinculin at the adhesion sites. However, one needs to be careful when analyzing the vinculin height using MIET, because FRET also affects the lifetime of this fluorophore (acting as FRET donor). 

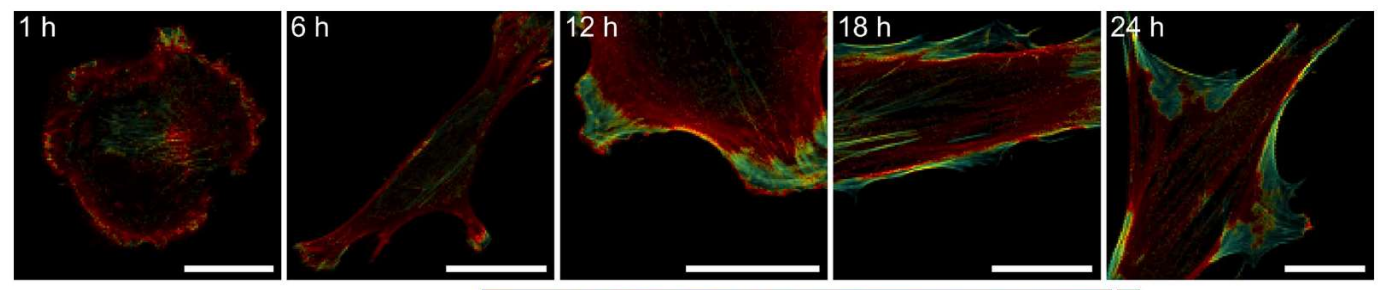

Height $\mathrm{z}(\mathrm{nm})$
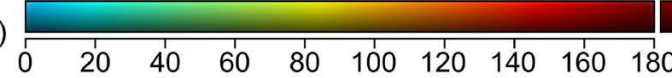

$>180$

Time series of actin height imaged by MIET. Intensity-weighted height images of actin filaments stained with Atto 647N-phalloidin in human mesenchymal stem cells (hMSCs) fixed on goldcoated coverslips at $1 \mathrm{~h}(\mathrm{~N}=8$ cells $), 6 \mathrm{~h}(\mathrm{~N}=11), 12 \mathrm{~h}(\mathrm{~N}=10), 18 \mathrm{~h}(\mathrm{~N}=6)$, and $24 \mathrm{~h}(\mathrm{~N}$ $=27$ ) after seeding. Scale bar is $30 \mu \mathrm{m}$.
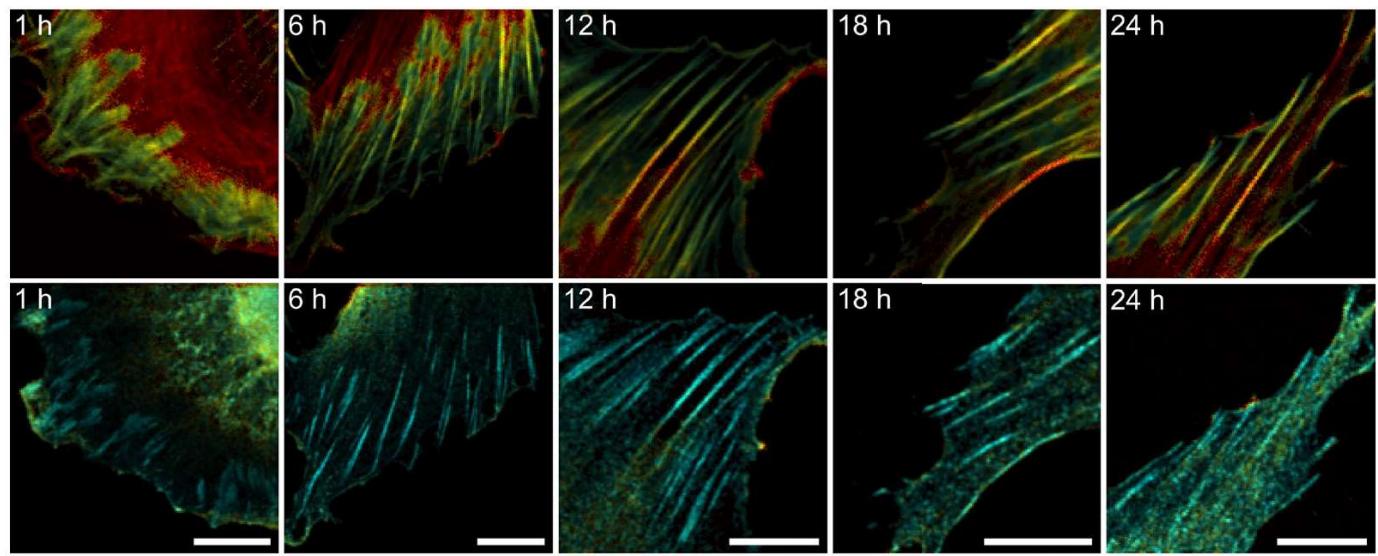

Height z $(\mathrm{nm})$

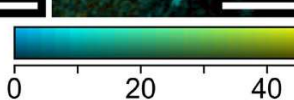

60

Time series of dual-color MIET imaging of actin and vinculin. Intensity-weighted height images of actin (top row) and vinculin (bottom row) focal adhesions for the same cell areas. Cells were fixed on gold-coated coverslips at $1 \mathrm{~h}(\mathrm{~N}=4), 6 \mathrm{~h}(\mathrm{~N}=3), 12 \mathrm{~h}(\mathrm{~N}=4), 18 \mathrm{~h}(\mathrm{~N}=4)$, and 24 $\mathrm{h}(\mathrm{N}=7)$ (from left to right) after seeding. Scale bar is $10 \mu \mathrm{m}$.

For an undisturbed measurement of the vinculin height, we completely bleached the actin fluorophore (the FRET acceptor) before performing MIET measurements and analysis. Figure 3 illustrates the temporal maturation of focal adhesions. In particular, it shows how actin filaments develop into stress fibers and move closer to the substrate over time.

The growing focal adhesions show that the height of the vinculin ensembles above the surface is smaller than the height of the actin ensembles, which is in agreement with the standard picture of stress fibers anchoring at focal adhesions, as was also shown with iPALM (Kanchanawong et al., 2010). During the early phase of adhesion $(1-6 \mathrm{~h})$ vinculin is close to the surface $(11 \pm 2 \mathrm{~nm})$, and during maturation and growth of the focal adhesions this value increases to $19 \pm 5 \mathrm{~nm}$ after $24 \mathrm{~h}$ (see Supplemental Figure S4; not included). When analyzing vinculin in seven different cells, we get an average height of $(20 \pm 8) \mathrm{nm}$ at $24 \mathrm{~h}$ (see Supplemental Figure S6; not included). This height coincides well with the height of paxillin where we observed $17 \pm 19 \mathrm{~nm}$ (see Supplemental Figure S7; not included).

With the height information of actin within stress fibers, and of vinculin as an integral part of the focal adhesion, we are now able to draw a detailed 3D picture of stress fiber anchoring as depicted in Figure 4. Here, we analyzed the obtained height information along selected actin filaments and the associated vinculin complex after 12 and $24 \mathrm{~h}$. Although the vinculin height remains more or less constant $(\sim 10-15 \mathrm{~nm}$ above 
the substrate), the actin filaments exhibit an inclination toward the surface. Over their first several micrometers, they increase by $\sim 20-30 \mathrm{~nm}$, which corresponds to a very shallow inclination angle of $0.15 \pm 0.01^{\circ}$ and $0.18 \pm 0.04^{\circ}$ for three fibers at 12 and $24 \mathrm{~h}$, respectively (see Figure 4 ). Remarkably, we observe a gap in height between the "centers of mass" of the ensembles of actin and vinculin that decreases from roughly 20 $\mathrm{nm}$ at $12 \mathrm{~h}$ to $10 \mathrm{~nm}$ at $24 \mathrm{~h}$, which is indicative of the maturation of stress fiber anchoring at focal adhesions.
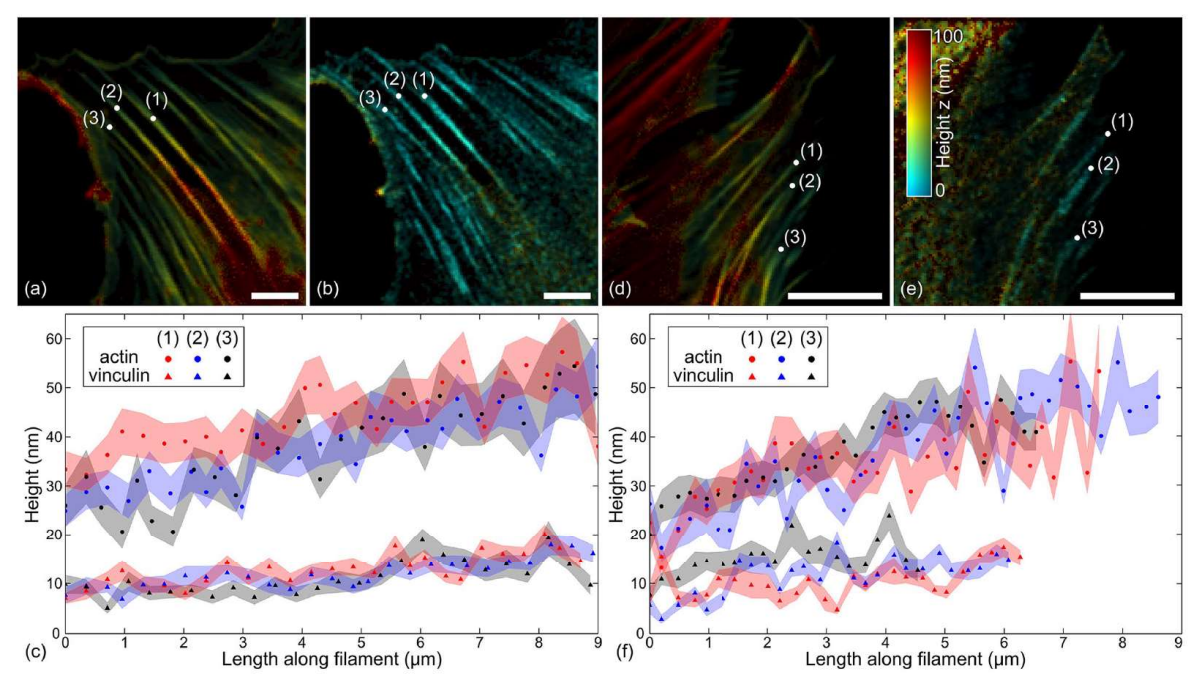

3D architecture of stress fibers at focal adhesions changes from 12 to $24 \mathrm{~h}$. Height profiles along actin filaments and vinculin complexes after 12 and $24 \mathrm{~h}$. Images a and b correspond to intensity-weighted ensemble heights of actin and vinculin, respectively, for a cell fixed $12 \mathrm{~h}$ after seeding. Images $\mathrm{d}$ and e correspond to intensity-weighted ensemble heights of actin and vinculin, respectively, for a cell fixed $24 \mathrm{~h}$ after seeding. White points (1), (2), and (3) on the intensity-weighted height images indicate the starting points of the height profiles shown in images $\mathrm{c}$ and $\mathrm{f}$. They show the height of actin filaments (circles) and vinculin clusters (triangles) at the same focal adhesion. The shaded areas mark the 1-regions of the height values. Scale bar is $10 \mu \mathrm{m}$.
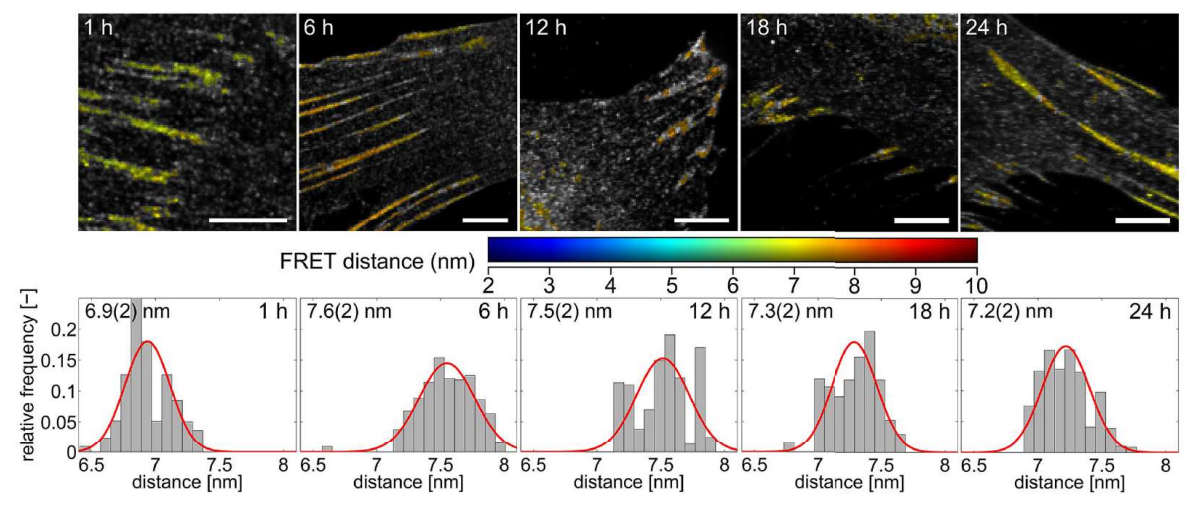

Constant FRET distance of actin and vinculin over time. Images show the color coded FRET distance between actin and vinculin in focal adhesions overlaid on the intensity image of vinculin (after bleaching of Atto 647N-phalloidin). As can be seen, this distance does not significantly change over time as imaged $1 \mathrm{~h}(\mathrm{~N}=4$ cells $), 6 \mathrm{~h}(\mathrm{~N}=4), 12 \mathrm{~h}(\mathrm{~N}=5), 18 \mathrm{~h}(\mathrm{~N}=4)$, and 24 $\mathrm{h}(\mathrm{N}=6)$ after seeding. Scale bar is $5 \mu \mathrm{m}$. 
After having measured and analyzed the distinct 3D architecture of actin and vinculin using MIET, we use the same experimental system with uncoated glass coverslips to measure FRET for determining the distance between the two proteins. For obtaining quantitative FRET values, we performed donor lifetime measurements before and after acceptor bleaching (see Supplemental Figures S1, S2, and S5; not included; Jares-Erijman and Jovin, 2003, 2006; Beutler et al., 2008). Figure 5 shows the temporal evolution of the distance between the two proteins. Although the focal adhesion areas were growing, there was no significant change in the mean distance during a time span from 6 to $24 \mathrm{~h}$. Here, FRET data reveal the proximity between $\mathrm{F}$-actin from stress fibers and vinculin in focal adhesions that is stable throughout the maturation process. Furthermore, we observed that FRET occurs not in all focal adhesions, and that some focal adhesions show FRET only within distinct small areas, which shows the complexity and heterogeneity of these supramolecular structures. For Figure 5, we have selected only cells where the mean values of the lifetime difference of the vinculin label before and after acceptor bleaching is larger than the SD of the lifetime values.

\section{DISCUSSION}

Our results show that dcMIET is a powerful method to spatially resolve the z-positions of two different dye-labeled proteins (actin and vinculin) in cells. By combining it with FRET, we obtained additional insight into the 3D structure of the anchoring of stress fibers at focal adhesions. The analysis of the temporal evolution of actin heights as shown in Figure 2 nicely illustrates how the actin filaments move closer to the surface while the cell is spreading and firmly adhering. Although the fibers are distributed over a broad height range during an early phase (1-6 h), their distance to the surface reduces around $12 \mathrm{~h}$ and later time points (to $\sim 40 \mathrm{~nm}$ ). We could also successfully demonstrate that it is possible to quantitatively disentangle the effects of MIET and FRET on the donor lifetime, so that we could measure distances of two different molecules from a surface, and their mutual distance from each other. Using dcMIET, we confirmed the standard picture of stress fibers attached to focal adhesions, and could also observe that vinculin is below the actin filaments. Moreover, during maturation of focal adhesion complexes, vinculin aggregates grow larger as indicated by an increase in height and SD, and the mean height of the actin bundles above the surface is decreasing. The measured values for vinculin height above the substrate $(19 \pm 5 \mathrm{~nm})$ are within the range of previously published values where vinculin was only slightly higher than the plasma membrane (90-106 nm vs. $100 \mathrm{~nm}$; Paszek et al., 2012, 2014), or roughly $22.7 \pm$ $5.5 \mathrm{~nm}$ higher than the membrane as iPALM measurements suggest (Kanchanawong et al., 2010). The nanometer-precise height information along the fibers and of the vinculin clusters shown in Figure 3 gives us a detailed picture of stress fibers anchoring at focal adhesions and spanning the cell at a slight inclination of below $1^{\circ}$. Although we observe a gap of roughly 10-20 nm between actin and vinculin at focal adhesions, the FRET data shows a close proximity of both proteins of $\sim 7 \mathrm{~nm}$. This latter distance is based on a Förster radius of $5.0 \mathrm{~nm}$ (see Materials and Methods). However, it is unlikely that vinculin and actin form stoichiometric complexes at focal adhesions. Instead, there are many more labels at the actin fibers than at the vinculin site. Therefore, the FRET donor can interact with multiple acceptor dyes. For such arrangements, the transfer rates add up, and a considerably larger effective Förster radius should be used (Maliwal et al., 2012). Thus, the FRET-determined distance has to be considered as a lower bound. In summary, the combination of dcMIET and FRET is a powerful method to dissect and elucidate the detailed 3D structure of molecular architecture within cells (e.g., nuclear envelope, synaptic vesicles, exocytosis, and endocytosis) that will be very helpful for many structural aspects of the basal part of the cell.

Our method of combined dcMIET and FRET imaging in biological cells is a straightforward and versatile tool for elucidating the 3D architecture of molecular structures. It can be easily implemented in standard fluorescence lifetime microscopes. In contrast to other existing axial superresolution techniques, it does not require specialized fluorescent labels, and it is compatible with commercially available dyes and antibodies. In particular, our method can also be used with fluorescent fusion proteins, thus allowing for live cell measurements. Concerning focal adhesions, our results confirm the standard picture of stress fibers 
anchoring at focal adhesions that maturate within the first $24 \mathrm{~h}$ and show that stress fibers are slightly inclined and above the vinculin complexes. Although we focused in this study on actin and vinculin, dcMIET with FRET can also be used to study other structures in cells within the first $150 \mathrm{~nm}$ from the basal membrane.

\section{MATERIALS AND METHODS}

Experimental setup

Photoluminescence (PL) measurements of cells on gold were performed with a homebuilt confocal microscope equipped with an objective lens of high numerical aperture (Apo N, 60 oil, 1.49 NA; Olympus Europe, Hamburg, Germany). A pulsed, linearly polarized white light laser (SC400-4-20; Fianium, UK; pulse width $\sim 50 \mathrm{ps}$, repetition rate $20 \mathrm{MHz}$ ) equipped with a tunable filter (AOTFnC- 400.650- TN; Pegasus Optik $\mathrm{GmbH}$, Wallenhorst, Germany) served as excitation source, delivering light with a tunable wavelength of 488 or $635 \mathrm{~nm}$. The light was reflected by a nonpolarizing beam-splitter toward the objective, and the backscattered excitation light was blocked with long-pass filters (EdgeBasic BLP01-488R; Semrock, New York, for the green channel, and BLP01-635R; Semrock, New York, for the red channel). An additional band-pass filter 550/88 (BrightLine FF01-550/88; Semrock, New York) was used for the vinculin (Atto 488) measurements. Emission light was focused onto the active area of an avalanche photodiode (PDM Series; MicroPhoton Devices), and data recording was performed with a multichannel picosecond event timer (HydraHarp 400; PicoQuant GmbH, Berlin, Germany).

Samples were scanned with a focused laser spot using a piezo nano-positioning stage (P-562.3CD; Physik Instrumente GmbH, Karlsruhe, Germany). PL spectra of Atto 488 and Atto $647 \mathrm{~N}$ molecules inside the cells were recorded using a spectrograph (SR 303i; Andor Technology, Belfast, UK) equipped with a charge-coupled device camera (iXon DU897 BV; Andor Technology, Belfast, UK). For measurement without FRET, the FRET acceptor (Atto 647N-phalloidin labeling actin) was bleached with a diode laser (MRL-FN-639, CNI laser; CNI Optoelectronics Tech., Changchun, People's Republic of China) at $640 \mathrm{~nm}$ excitation (laser power is $\sim 1 \mathrm{~mW}$ after the objective lens). Bleaching time was typically around $10 \mathrm{~min}$ per cell. For MIET measurements, glass cover slides were coated with the following multilayer structure: $3 \mathrm{~nm} \mathrm{Ti}, 15 \mathrm{~nm} \mathrm{Au}, 3 \mathrm{~nm} \mathrm{Ti}, 20 \mathrm{~nm} \mathrm{S_{0 }}{ }_{2}$. The metal and silica films were prepared by vapor deposition onto a cleaned glass cover slide (thickness $170 \mu \mathrm{m}$ ) using an electron beam source (Univex 350; Leybold) under high-vacuum conditions ( 106 mbar). During vapor deposition, film thickness was monitored using an oscillating quartz unit, and afterward verified by atomic force microscopy.

For FRET measurements, "donor-acceptor" images were first acquired by exciting the donor molecules present on the vinculin protein with the diode laser at $485 \mathrm{~nm}$. Subsequently, the fluorescent dyes used for labeling actin filaments were bleached using the diode laser at $640 \mathrm{~nm}$ excitation with a power of $\sim 140$ $\mu \mathrm{W}$ after the objective lens. The bleaching time usually was around 10 min per area. Thereafter, "donor only" images were acquired on the same area using the $485 \mathrm{~nm}$ excitation.

\section{Metal nanocavity for quantum yield measurements}

The nanocavity used for quantum yield measurements consists of two silver mirrors with subwavelength spacing. The bottom silver mirror was prepared by vapor deposition of a $30 \mathrm{~nm}$ silver film (see above for details) onto a commercially available cleaned microscope glass coverslip (thickness $170 \mu \mathrm{m}$ ). The top silver mirror was prepared by vapor deposition of a $60-\mathrm{nm}$-thick silver film onto the surface of a plano-convex lens (focal length of $150 \mathrm{~mm}$ ) under the same conditions.

The spherical shape of the upper mirror allowed for reversibly tuning the cavity length by moving the laser focus laterally away or toward the contact point between the lens and the cover slide. It should be noted that across the size of the diffraction-limited laser focus, the cavity can be considered to be a plane-parallel resonator. For a detailed presentation of the theoretical background, refer to Chizhik et al. (2013). The measured quantum yield of Atto 488/anti-mouse/anti-vinculin/ $3 \%$ bovine serum albumin (BSA) in 
phosphate-buffered saline (PBS) solution is $\phi_{0}=0.67$. The free space fluorescence lifetime of this sample is $\tau_{0}=2.9 \mathrm{~ns}$.

\section{Fluorescence lifetime data evaluation}

Both for dcMIET and FRET measurements, fluorescence photons were detected in time-tagged, timeresolved mode. Each photon carries two time tags, one with respect to the start of the experiment, which is counted as the number of preceding laser pulses, and a second with respect to the last laser pulse. Additionally, line change markers from the piezo driver are recorded as "virtual photons" which are used for recognizing a change of lines in the scan image, and for sorting the photons into individual pixels based on their arrival time in each line. By calculating histograms of the arrival times of the sorted photons with respect to the last laser pulses, one obtains time-correlated single-photon counting (TCSPC) curves for each individual pixel. In this way, one obtains intensity and lifetime information for all pixels of an image. One limitation of FLIM with TCSPC and single-photon detectors is that the photon count rate should not exceed some threshold (typically $1 \%$ of the laser excitation rate) to prevent data distortion due to pileup and detector/ electronics dead-times. Measuring at high count rates introduces severe distortions into the acquired TCSPC and intensity data. We determined the detector and electronics dead-times as 74 and $80 \mathrm{~ns}$, respectively, as described in Isbaner et al. (2016). These values were then used in the correction algorithm to obtain dead-time corrected TCSPC curves. We then calculated the decay with a least-square-error minimization algorithm using a multiexponential tail fitting model. All lifetime values presented in this paper are always the inverse of the average decay rate extracted from these fits. Furthermore, the dead-time correction algorithm also yielded corrected intensity values in each pixel. To estimate the lifetime uncertainties needed for determining the height uncertainties in Figure 3, we performed both simulations and bootstrapping of experimental data. We found the empirical formula $\sigma \tau \approx 4.8 \tau / \mathrm{N}$, where $\tau$ is the lifetime and $\mathrm{N}$ the number of photons. To avoid large fitting errors at low photon numbers, only pixels with at least 1000 photons were evaluated.

\section{Cell culture}

Adult hMSCs from bone marrow (Lonza Group, Basel, Switzerland; \#PT-2501), were cultured in T75 cell culture flasks (Corning; 43061) in DMEM (Life Technologies, Thermo Fisher Scientific, Waltham, MA; A18967-01) supplemented with $10 \%$ fetal bovine serum (Sigma-Aldrich, St. Louis, MO; F2442-500ML) and $1 \%$ antibiotics (penicillin/ streptomycin; Life Technologies, Thermo Fisher Scientific, Waltham, MA; 15140-122) at $37^{\circ} \mathrm{C}$ and $5 \% \mathrm{CO}_{2}$ and passaged every 2-3 d (passage \#4 was used in this study). Cells were seeded on $25 \mathrm{~mm}$ glass and gold-coated glass coverslips at a density of $10.000 \mathrm{hMSCs}$ per glass in six-well plates (Sarstedt AG \& Co., Nuembrecht, Germany; 83.3920) with $2 \mathrm{ml}$ growth medium per well and grown at $37^{\circ} \mathrm{C}$ and $5 \% \mathrm{CO}_{2}$. Cells were chemically fixed 1, 6, 12, 18, and $24 \mathrm{~h}$ after seeding in $10 \%$ formaldehyde (Sigma-Aldrich, St. Louis, MO; 47608-250ML-F) in PBS for 5 min. Subsequently, cells were permeabilized using 0.5 \% Triton X-100 (Carl Roth GmbH \& Co. KG, Karlsruhe, Germany; 6683.1) in PBS for 10 min and blocked with $3 \%$ BSA (Sigma-Aldrich, St. Louis, MO; A9418-100G) in PBS for 30 min, incubated again with Triton $\mathrm{X}$ for $5 \mathrm{~min}$ and thoroughly washed with PBS. All antibodies were kept in a $3 \%$ BSA PBS solution. Immunostaining was performed with anti-vinculin (Sigma-Aldrich, St. Louis, MO; V9131-.2ML) (1:1000) for $1 \mathrm{~h}$, then anti-mouse immunoglobulin G (IgG) Atto 488 (Sigma-Aldrich, St. Louis, MO; 62197) (1:500) for $1 \mathrm{~h}$, and then phalloidin Atto 647N (ATTO-TEC GmbH, Siegen, Germany; AD647N-82) (1:250) for $1.5 \mathrm{~h}$. Paxillin was stained using a primary anti-paxillin antibody (abcam; ab32084) (1:100) for $21 \mathrm{~h}$ followed by an anti-rabbit IgG Atto 488 (Sigma-Aldrich, St. Louis, MO; 40839) for $2.5 \mathrm{~h}$.

Samples were mounted on microscope slides (VWR; 631-1550) using Fluoroshield mounting medium (Sigma-Aldrich, St. Louis, MO; F6182-20ML).

Wide-field fluorescence images were obtained with an Axio Observer.Z1 microscope (Zeiss, Oberkochen, 
Germany; 431007- 0001-000), using a 20 objective (Zeiss, Oberkochen, Germany; 1006-591) and a sCMOS camera (Andor Technology, Belfast, Northern Ireland; Zyla 4.2).

\section{ACKNOWLEDGMENTS}

This work was supported in part by the Deutsche Forschungsgemeinschaft (DFG) through the Cluster of Excellence "Center for Nanoscale Microscopy and Molecular Physiology of the Brain (CNMPB)." A.M.C. is grateful to the Human Frontiers Science Program Organization for financial support under Grant no. RGP0061/2015. A.I.C. and D.R. are grateful to the DFG for financial support via SFB 937 (project A14). N.K. is grateful to the DFG for financial support of his position via SFB 860 (project A06). F.R. acknowledges funding by the DFG through SFB 755 (project B8), SFB 937 (project A13), and the Niedersachsen-Israel framework (MWK-VWZN2722).

\section{REFERENCES}

Ajo-Franklin CM, Ganesan PV, Boxer SG (2005). Variable incidence angle fluorescence interference contrast microscopy for Z-imaging single objects. Biophys J 89, 2759-2769.

Aquino D, Schönle A, Geisler C, v Middendorff C, Wurm CA, Okamura Y, Lang T, Hell SW, Egner A (2011). Two-color nanoscopy of three-dimensional volumes by 4Pi detection of stochastically switched fluorophores. Nat Methods 8, 353-359.

Betzig E, Patterson GH, Sougrat R, Lindwasser OW, Olenych S, Bonifacino JS, Davidson MW, LippincottSchwartz J, Hess HF (2006). Imaging intracellular fluorescent proteins at nanometer resolution. Science 313, 1642-1645.

Beutler M, Makrogianneli K, Vermeij RJ, Keppler M, Ng T, Jovin TM, Heintzmann R (2008). satFRET: estimation of Förster resonance energy transfer by acceptor saturation. Eur Biophys J 38, 69-82.

Braun D, Fromherz P (1997). Fluorescence interference-contrast microscopy of cell adhesion on oxidized silicon. Appl Phys A 65, 341-348.

Burridge K, Guilluy C (2016). Focal adhesions, stress fibers and mechanical tension. Exp Cell Res 343, $14-20$.

Case LB, Baird MA, Shtengel G, Campbell SL, Hess HF, Davidson MW, Waterman CM (2015). Molecular mechanism of vinculin activation and nanoscale spatial organization in focal adhesions. Nat Cell Biol 17, 880-892.

Chizhik AI, Gregor I, Ernst B, Enderlein J (2013). Nanocavity-based determination of absolute values of photoluminescence quantum yields. Chem Phys Chem 14, 505-513.

Chizhik AI, Rother J, Gregor I, Janshoff A, Enderlein J (2014). Metal-induced energy transfer for live cell nanoscopy. Nat Photon 8, 124-127.

Deschamps J, Mund M, Ries J (2014). 3D superresolution microscopy by supercritical angle detection. Opt Express 22, 29081-29091.

Dos Santos MC, Déturche R, Vézy C, Jaffiol R (2016). Topography of cells revealed by variable-angle total internal reflection fluorescence microscopy. Biophys J 111, 1316-1327.

Engler AJ, Sen S, Sweeney HL, Discher DE (2006). Matrix elasticity directs stem cell lineage specification. Cell 126, 677-689.

Geiger B, Spatz JP, Bershadsky AD (2009). Environmental sensing through focal adhesions. Nat Rev Mol Cell Biol 10, 21-33.

Huang B, Wang W, Bates M, Zhuang X (2008). Three-dimensional superresolution imaging by stochastic optical reconstruction microscopy. Science 319, 810-813.

Isbaner S, Karedla N, Ruhlandt D, Stein SC, Chizhik A, Gregor I, Enderlein J (2016). Dead-time correction of fluorescence lifetime measurements and fluorescence lifetime imaging. Opt Express 24, 9429-9445.

Jares-Erijman EA, Jovin TM (2003). FRET imaging. Nat Biotech 21, 1387-1395. 
Jares-Erijman EA, Jovin TM (2006). Imaging molecular interactions in living cells by FRET microscopy. Curr Opin Chem Biol 10, 409-416.

Juette MF, Gould TJ, Lessard MD, Mlodzianoski MJ, Nagpure BS, Bennett BT, Hess ST, Bewersdorf J (2008). Three-dimensional sub-100 $\mathrm{nm}$ resolution fluorescence microscopy of thick samples. Nat Methods $5,527-529$.

Kanchanawong P, Shtengel G, Pasapera AM, Ramko EB, Davidson MW, Hess HF, Waterman CM (2010). Nanoscale architecture of integrin-based cell adhesions. Nature 468, 580-584.

Karedla N, Chizhik AI, Gregor I, Chizhik AM, Schulz O, Enderlein J (2014). Single-molecule metal-induced energy transfer (smMIET): resolving nanometer distances at the single-molecule level. Chem Phys Chem $15,705-711$.

Kukura P, Ewers H, Muller C, Renn A, Helenius A, Sandoghdar V (2009). High-speed nanoscopic tracking of the position and orientation of a single virus. Nat Methods 6, 923-927.

Limozin L, Sengupta K (2009). Quantitative reflection interference contrast microscopy (RICM) in soft matter and cell adhesion. Chem Phys Chem 10, 2752-2768.

Livne A, Geiger B (2016). The inner workings of stress fibers-from contractile machinery to focal adhesions and back. J Cell Sci 129, 1293-1304.

Maliwal BP, Raut S, Fudala R, D'Auria S, Marzullo VM, Luini A, Gryczynski I, Gryczynski Z (2012). Extending Förster resonance energy transfer measurements beyond $100 \AA$ using common organic fluorophores: enhanced transfer in the presence of multiple acceptors. J Biomed Opt 17, 0110061-0110068.

Paluch EK, Nelson CM, Biais N, Fabry B, Moeller J, Pruitt BL, Wollnik C, Kudryasheva G, Rehfeldt F, Federle W (2015). Mechanotransduction: use the force(s). BMC Biol 13, 1-14.

Paszek MJ, DuFort CC, Rossier O, Bainer R, Mouw JK, Godula K, Hudak JE, Lakins JN, Wijekoon AC, Cassereau L, et al. (2014). The cancer glycocalyx mechanically primes integrin-mediated growth and survival. Nature 511, 319-325.

Paszek MJ, DuFort CC, Rubashkin MG, Davidson MW, Thorn KS, Liphardt JT, Weaver VM (2012). Scanning angle interference microscopy reveals cell dynamics at the nanoscale. Nat Methods 9, 825-827.

Pavani SRP, Thompson MA, Biteen JS, Lord SJ, Liu N, Twieg RJ, Piestun R, Moerner W (2009). Threedimensional, single-molecule fluorescence imaging beyond the diffraction limit by using a double-helix point spread function. Proc Natl Acad Sci USA 106, 2995-2999.

Ruckstuhl T, Verdes D (2004). Supercritical angle fluorescence (SAF) microscopy. Opt Express 12, 4246-4254.

Rust MJ, Bates M, Zhuang X (2006). Sub-diffraction-limit imaging by stochastic optical reconstruction microscopy (STORM). Nat Methods 3, 793-796.

Shtengel G, Galbraith JA, Galbraith CG, Lippincott-Schwartz J, Gillette JM, Manley S, Sougrat R, Waterman CM, Kanchanawong P, Davidson MW (2009). Interferometric fluorescent super-resolution microscopy resolves 3D cellular ultrastructure. Proc Natl Acad Sci USA 106, 3125-3130.

Stock K, Sailer R, Strauss W, Lyttek M, Steiner R, Schneckenburger H (2003). Variable-angle total internal reflection fluorescence microscopy (VATIRFM): realization and application of a compact illumination device. J Microsc 211, 19-29.

Zemel A, Rehfeldt F, Brown AEX, Discher DE (2010a). Cell shape, spreading symmetry, and the polarization of stress-fibers in cells. J Phys Condens Matter 22, 194110.

Zemel A, Rehfeldt F, Brown AEX, Discher DE, Safran SA (2010b). Optimal matrix rigidity for stress-fibre polarization in stem cells. Nat Phys 6, 468-473. 


\section{2. liveMIET}

All analysis, and figures in this Section were done by Sebastian Isbaner. All biological samples including cell culture, staining, and microinjection was done by me. Microscopy has been done by Sebastian Isbaner and me collaboratively. Protein purification has been done by Ulrike Schulz. The quoted parts of this Section are taken from the method part of the yet unpublished manuscript that Sebastian and I wrote for our respective parts.

As shown in the previous Section 6.1, MIET is a great tool to resolve intra-cellular structures in height with nanometer resolution. Cells fixed on multiple time-points showed the spreading behavior of the cells and continuously closer localization to the gold surface over time. An improvement of the MIET setup to enable live measurements was a logical step.

But while for the MIET measurement of fixed cells the main issues are on the technical side, for live cells there are some difficulties. While imaging, cells have to either be in $\mathrm{CO}_{2}$-independent medium or a surrounding containing $5 \% \mathrm{CO}_{2}$. The phenol red in common $\mathrm{CO}_{2}$-independent medium, however, interferes with the measurement and thus can not be used and phenol red-free $\mathrm{CO}_{2}$-independent medium is quite expensive in comparison. Thus, phenol-red free DMEM supplemented with $1 \% \mathrm{P} / \mathrm{S}$ and $12.5 \%$ FBS was used in combination with a climatic chamber for optimal temperature, atmosphere, and humidity. This leads to the introduction of $z$-drift, as the heated chamber causes thermal expansion in other parts of the microscope. As MIET measures in the nanometer resolution, this drift is quite substantial.

Another issue is the hunt for a usable fluorophore. As MIET relies on a mono-exponential decay for an optimal fit, an optimal fluorophore should be exactly that. However, after extensive screening by the authors named in Section 6, no fluorophore purely mono-exponential in living cells could be found. The additional bi-exponential component from the fluorophore (in this case LifeAct-mScarlet-i) leads to several nanometer loss in accuracy. However, as we image living, moving cells there is a certain uncertainty nevertheless. During experiments, it can be seen that cells move away from the area of illumination. As they are capable of movements in the micron range during single measurements, the uncertainty in the nanometer range should be neglected for the time being.

Cells were imaged in Phenol red free DMEM (Gibco, Thermo Fisher Scientific Inc., Waltham, MA, USA, 11880-028) supplemented with 12,5\% fetal bovine serum (Sigma-Aldrich Co., St. Louis, MO, USA, F2442-500ML) and 1\% antibiotics (penicillin/streptomycin, Life Technologies, Thermo Fisher Scientific Inc., Waltham, MA, USA, 15140- 122) at $37^{\circ} \mathrm{C}, 5 \%$ $\mathrm{CO}_{2}$ and $80 \%$ humidity using a climatic chamber (ibidi, Gräfeling, Ger, 10918) and gas mixer with humidifier column (ibidi, Gräfeling, Ger, 11922-DS).

$12.5 \%$ FBS as supplement in the imaging medium was used to accommodate both SAOS-2 and hMSC cells, which prefer $15 \%$, respectively $10 \%$ FBS in their growth medium.

hMSC (Lonza Ref. \#PT-2501, Lot 603525) were grown in T75 cell culture flasks (Corning Inc., New York, NJ, USA, 430641U) in DMEM (Gibco, Thermo Fisher Scientific Inc., Waltham, MA, USA, 31885-023) supplemented with 10\% fetal bovine serum (Sigma-Aldrich Co., St. Louis, MO, USA, F2442-500ML) and 1\% antibiotics (penicillin/streptomycin, Life Technologies, Thermo Fisher Scientific Inc., Waltham, MA, USA, 15140-122) at $37^{\circ} \mathrm{C}$ and $5 \% \mathrm{CO}_{2}$ and passaged every 2-3 days (passage \#6 was used in this experiments).

SAOS-2 (ACC 243, DSMZ) were grown in T75 cell culture flasks (Sarstedt, Nümbrecht, Ger, 833.911.002) in McCoy's 5A (Gibco, Thermo Fisher Scientific Inc., Waltham, MA, USA, 26600-23) supplemented with $15 \%$ fetal bovine serum (Sigma-Aldrich Co., St. Louis, MO, USA, F2442-500ML) and 1\% antibiotics (penicillin/streptomycin, Life Technologies, Thermo Fisher Scientific Inc., Waltham, MA, USA, 15140-122) at $37^{\circ} \mathrm{C}$ and $5 \% \mathrm{CO}_{2}$ and passaged every 4 days (passage \#13 was used in this experiments). 
In the original MIET paper (see Section 6), only hMSCs were used. We chose SAOS-2 as an additional imaging target as those cells usually spread nicely and their stress fibers are closer to the surface in comparison with the hMSCs. This is necessary as we use

Glass coverlips of $25 \mathrm{~mm}$ diameter (VWR, Darmstadt, Ger, ECN631-1584) were covered with $2 \mathrm{~nm} \mathrm{Ti}, 15 \mathrm{~nm} \mathrm{Au}, 1 \mathrm{~nm} \mathrm{~nm} \mathrm{Ti,} 20 \mathrm{~nm} \mathrm{~nm} \mathrm{SiO}{ }_{2}$. Both, gold coated and regular glass coverslips were cleaned and functionalized with collagen-I using a hetero-bifunctional crosslinking chemistry as described earlier [102]. In brief, all slides were plasma cleaned for $15 \mathrm{~min}$, sonicated first using 99\% EtOH, second using 2\% APTES (Sigma-Aldrich Co., St. Louis, MO, USA, 440140) in EtOH and treated with a $0.2 \%$ gluteraldehyde solution (Sigma-Aldrich Co., St. Louis, MO, USA, C7651). Glasses were rinsed twice with PBS and once with HEPES buffer. Sulfo-SANPAH was added to the surface and activated under UV for $10 \mathrm{~min}$. A solution of $0,02 \%$ Collagen I (in PBS, with $0,02 \%$ Acetic Acid; Rat tail Collagen I, Corning Inc., New York, NJ, USA, 354236) is added and incubated over night at $4^{\circ} \mathrm{C}$, resulting in a 25 $\mathrm{nm}$ thick collagen network. Glasses are rinsed twice with PBS and glued into bottomless ibidi dishes (ibidi, Gräfeling, Ger, DIO01110) using UV-curable glue (NOA68, Norland products inc., Cranbury, NJ, USA, 6801). Prepared dishes are UV sterilized for 2 hours and rinsed three times with PBS before seeding of cells.

The final $\mathrm{SiO}_{2}$ as well as the collagen coating act as spacer reducing the region from the bottom membrane up where quenching can be seen. Both SAOS-2 and hMSC

were transfected with the plasmid pLifeAct_mScarlet-i_N1, a gift from Dorus Gadella (Addgene plasmid \#85056, http://n2t.net/addgene:85056, RRID:Addgene_85056), using the Lonza 4D Nucleofector (Lonza, Cologne, Ger, AAF-1002B AAF-1002X). hMSCs were transfected using the P1 Kit (Lonza, Cologne, Ger, V4XP-1012) and pulse code FF-104. SAOS-2 cells were transfected using the SF Kit (Lonza, Cologne, Ger, V4XC-2012) and pulse code DS-150. Subsequent to transfection cells were seeded onto prepared dishes at 80.000 cells per well. Dead cells were removed by rinsing with medium $12 \mathrm{~h}$ after transfection.

As the quantum yield reported by the vendor differed significantly from the measured quantum yield in live cells, we expressed a LifeAct-mScarlet-6His in E.coli. The 6His-Tag facilitates purification with a NiNTA column.

The lifeact-mScarlet sequence was cut out using NdeI and XhoI sites and inserted into pET24b+ vector (Sigma-Aldrich Co., St. Louis, MO, USA, 39750), yielding a 6-His Tag on the mScarlet. Construct was electroporated into E.coli (BL21-Gold Competend Cells) (Agilent Technologies, Santa Clara, CA, USA, \#230130) and plated on LB plates (Sigma-Aldrich Co., St. Louis, MO, USA, 52062) with kanamycin (Sigma-Aldrich Co., St. Louis, MO, USA, 70560-51-9). Growing cultures were sequenced (Sanger Sequencing, Microsynth Seqlab, Göttingen, Ger) and expressed in $500 \mathrm{ml}$ LB Medium (Sigma-Aldrich Co., St. Louis, MO, USA, 51208) for 5 hours before harvesting. Cultures were spun down and pallets subjected to lysis buffer (50 mM Tris/Cl pH 8.0, $250 \mathrm{mM} \mathrm{NaCl}, 10 \mathrm{mM} \beta$-Mercaptoethanol, $1 \mathrm{mM} \mathrm{PMSF}$ ). Lifeact-mScarlet-6His was bound to Protino NiNTA Agarose (Macherey-Nagel, Düren, Ger, $745400.25)$ and washed (50 mM Tris/Cl pH 8.0, $250 \mathrm{mM} \mathrm{NaCl}, 10 \mathrm{mM} \beta$-Mercaptoethanol, $20 \mathrm{mM}$ Imidazol). NiNTA beads were given into a empty Protino column (Macherey-Nagel, Düren, Ger, 745400.10) and washed again. Final elution was done with 5 times $1 \mathrm{ml}$ elution buffer (50 mM Tris/Cl pH 8.0, $250 \mathrm{mM} \mathrm{NaCl}, 10 \mathrm{mM} \beta$-Mercaptoethanol, $250 \mathrm{mM}$ Imidazol) for $30 \mathrm{~min}$ each and samples frozen at $-80^{\circ} \mathrm{C}$. Samples were checked with a SDS-Page gel.

Additionally, we extracted LifeAct-mScarlet from a reasonably sized population of transfected SAOS-2 cells using a RFP-Trap. 
For quantum yield measurements, SAOS-2 (passage \#13) were grown and transfected as previously mentioned. Protein (Lifeact-mScarlet) was extracted using a RFP-Trap (Chromotek, Planegg-Martinsried, Ger, rta-20). Cells were harvested, lysed, and protein extracted as advised by vendor. For elution, we bound proteins by adding $50 \mu \mathrm{l} 0.2 \mathrm{M}$ glycine (pH 2.5) under $30 \mathrm{sec}$ of constant mixing. After 2 min centrifugation at $2.500 \mathrm{x}$ g supernatand was transfered to a new tube and $\mathrm{pH}$ neutralized by addition of $5 \mu \mathrm{l} 1 \mathrm{M}$ Tris base (pH 10.4).

To ensure that binding affinity and localization inside the cell were undisturbed, we microinjected SAOS cells with purified LifeAct-mScarlet-6His.

Microinjection of SAOS-2 (ACC 243, DSMZ) cells, grown as described (passage 16 was used), was done using a Transjector 5246 (Eppendorf, Hamburg, Ger, 5246 01084) and Femtotips (Eppendorf, Hamburg, Ger, 930000035). The lifeact-mScarlet-6His protein extracted from E.coli was used concentrated $0.78 \mathrm{mg} / \mathrm{ml}$ in elution buffer $(50 \mathrm{mM}$ Tris $/ \mathrm{Cl} \mathrm{pH} 8.0,250 \mathrm{mM}$ $\mathrm{NaCl}, 10 \mathrm{mM} \beta$-Mercaptoethanol, $250 \mathrm{mM}$ Imidazol) with $2 \%$ succrose added.

During liveMIET measurements, microinjected SAOS-2 showed the same distribution and intensity of fluorophores as transfected ones.

All lifeMIET measurements were done on a custom-built confocal setup.

Fluorescence lifetime images were acquired on a custom-built confocal setup. The excitation light was generated by an $80 \mathrm{MHz}$ pulsed white-light laser (SuperK Power, Koheras) and the wavelength was selected by an AOTF (SpectraK Dual, Koheras). The beam was coupled into a single-mode fibre (PMC-460Si-3,0-NA012-3APC-150-P and fibre coupler 60SMS-1-4-RGBV11-47, both Schäfter + Kirchhoff) and after the fibre recollimated by an objective (UPlanSApo $10 \times 0.40$ N.A., Olympus). After passing a clean-up filter (F37-563, AHF), a 90/10 beam splitter was used to reflect the excitation light into the microscope and separate it from the emission. The reflected beam was directed into a laser scanning system (FLIMbee, PicoQuant) and then into a custom sideport of the microscope (Olympus IX73). The three galvo mirrors in the scanning system are imaged onto the backfocal plane of the objective (UApo N 100× 1.49 N.A. oil, Olympus) with $180 \mathrm{~mm}$ and $90 \mathrm{~mm}$ achromatic lenses. The sample could be moved by a manual xy stage (Olympus) and a z-piezo stage (Nano-ZL100, MadCityLabs). Fluorescence in the sample was collected by the same objective and descanned in the scanning system. The fluorescence light that passed the 90/10 beam splitter was then focused onto a pinhole $(100 \mu \mathrm{m}$, Thorlabs) with a $180 \mathrm{~mm}$ achromatic lens. Backscattered laser light was blocked by a long-pass filter (568 LP Edge Basic, Semrock). The light was collimated by a $100 \mathrm{~mm}$ lens and passed through a bandpass filter (FF01-593/40-25, AHF) before a lens ( $f=30 \mathrm{~mm}$, Thorlabs) focused the light onto the detector ( $\tau$-SPAD, PicoQuant). The signal of the photon detector was recorded by a TCSPC system (HydraHarp 400, PicoQuant) together with the trigger signal by the laser. FLIM images were recorded with the SymPhoTime 64 software (Picoquant) which controlled the TCSPC system and the laser scanner. Typically, a pixel size of $100 \mathrm{~nm}$ was chosen with a pixel dwell time of $20 \mu$ s and a TCSPC resolution of $16 \mathrm{ps}$.

To monitor the axial focus position, back-reflected light was coupled out from the excitation beam with an additional $90 / 10$ beam splitter and focused $(f=200 \mathrm{~mm}$, Thorlabs) onto a camera (Guppy GF036B ASG, Allied Vision Technologies).

Data analysis was carried out in MATLAB by Sebastian Isbaner for all results shown here.

Data analysis was carried out using custom routines written in MATLAB (MathWorks). First, scan lines were aligned using a linear shift. Then, TCSPC histograms of individual pixels were generated and corrected for dead-time artefacts[88]. A mono-exponential fit including a 
measured instrument response function (IRF) was used to estimate the fluorescence lifetime. The TCSPC curve was fitted with the function

$$
f\left(t \mid \tau, a, b, s, a_{s}\right)=\int \mathrm{d} t^{\prime} \operatorname{IRF}\left(t-t^{\prime}-s\right) \frac{a}{\tau} e^{-\frac{t^{\prime}}{\tau}}+a_{s} \operatorname{IRF}(t-s)+b
$$

where $\tau$ denotes the fluorescence lifetime, $a$ denotes the fit amplitude, $b$ the background, $a_{s}$ the scattering amplitude, and $s$ the shift of the IRF. The scattering amplitude accounts for the luminescence of the gold, and the shift $s$ was introduced as the IRF depends on the count rate. We found for our detectors that a linear shifts was sufficient to account for this effect. The lifetime was converted into a height using the appropriate MIET curve for the sample. Images and videos were created with the lifetime or height information in color, using the intensity (rescaled to $0-1$ ) as the transparency of the image.

For MIET curve calculations, the

theoretical curve for converting measured lifetimes into heights were computed based on the model by Chance, Prock, and Silbey [33, 63, 64]. For the substrate, we assumed a layered system of (from bottom to top): glass, $2 \mathrm{~nm}$ titanium, $15 \mathrm{~nm}$ gold, $1 \mathrm{~nm}$ titanium, and $20 \mathrm{~nm}$ silica. The medium of the fluorophores and above was assumed to have the refractive index of water, refractive indices of the metals were taken from literature [168].

We were able to show that neither SAOS-2 nor hMSC shows any quenching on glass substrates covered with collagen I. These measurements also serve as a baseline to calculate MIET quenching. However, they will not be shown here. For both cell lines, we could observe quenching at the stress fiber ends. This was visible on the cell edges for both SAOS-2 and hMSC but for SAOS-2 it was in some cases also possible to see quenching in the cell center when the cell was thin enough and unquenched upper fibers did not cover quenched ones. In Figure 1, an exemplary hMSC cells is shown with selected regions color-coded for both measured lifetime and height. Additionally, Figure 2 shows height curves along selected stress fibers in a representative hMSC cell.

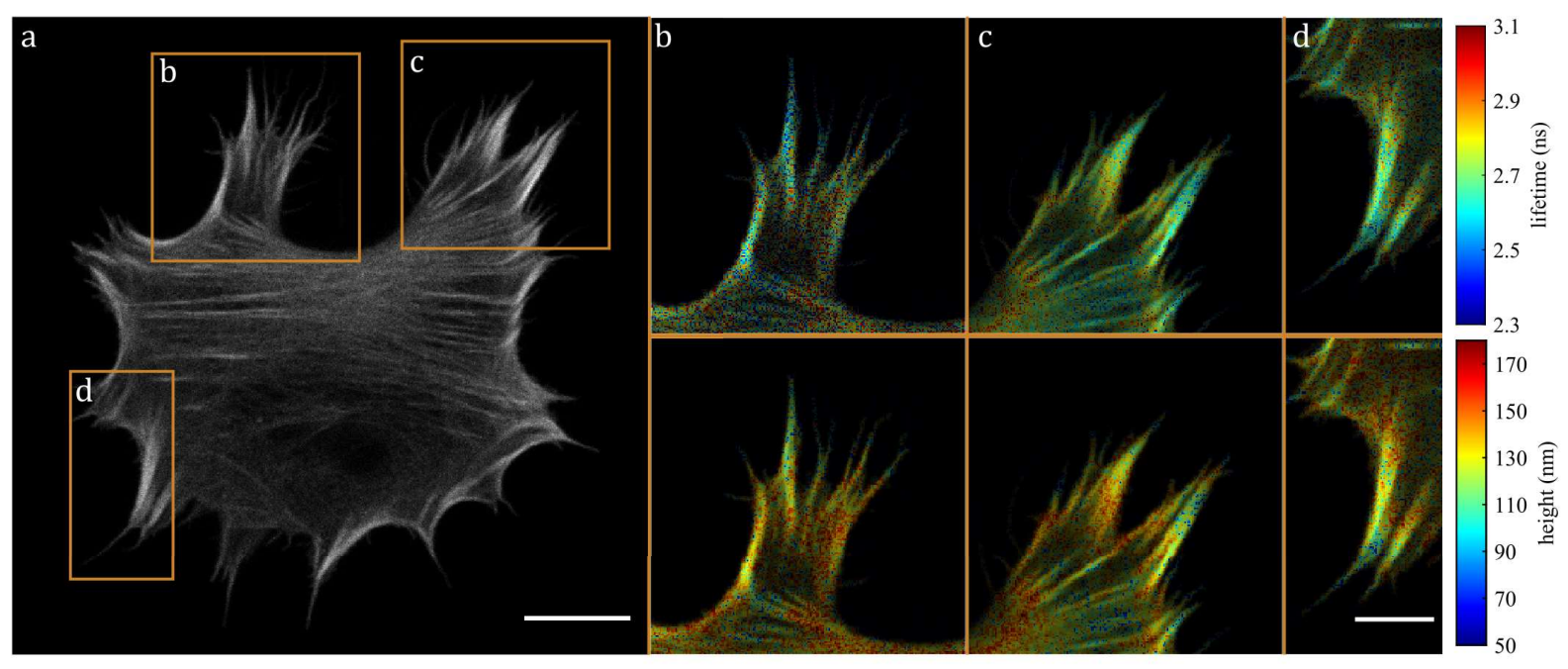

Fig.1: Height measurement along several fibers in a LifeAct-mScarlet transfected hMSC cell. Stress fibers used for measurements are marked white and labeled, omitted stress fibers are marked red.

Interestingly, while the original MIET paper (see Section 6) reports an inclination of stress fibers of below $1^{\circ}$, here we also see larger inclinations. This variety of inclinations might be coupled to the localization 
and mechanical load of the respective fibers.

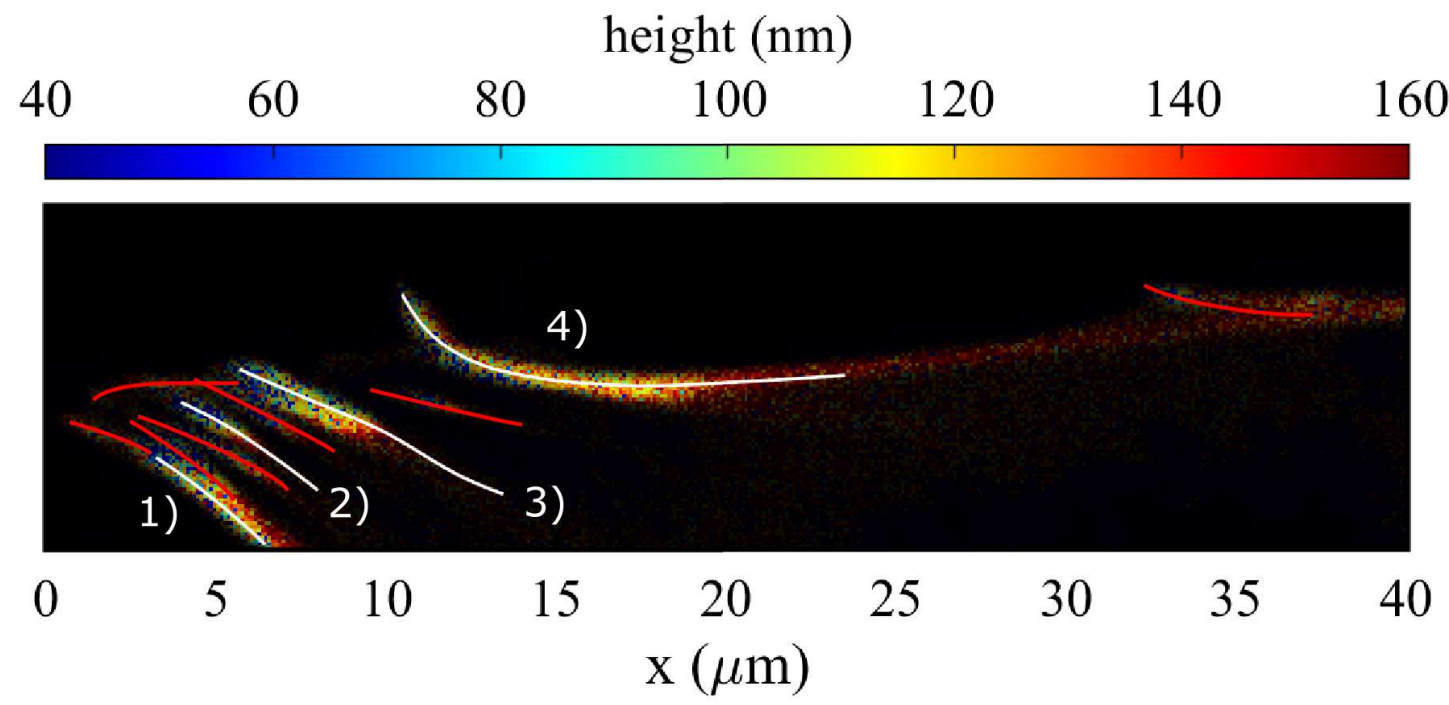

1)

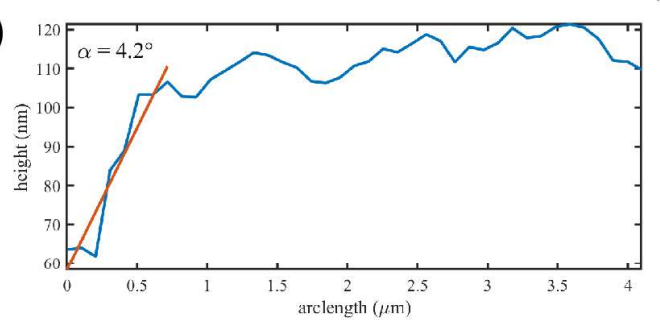

2)

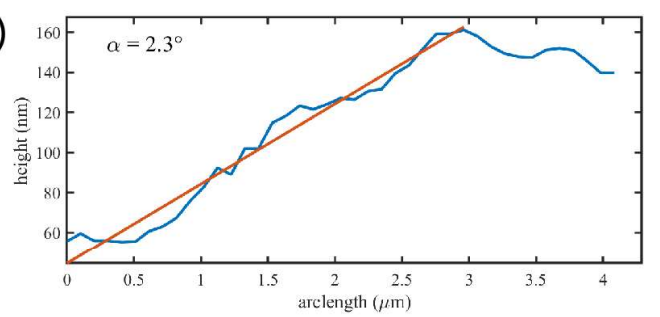

3)

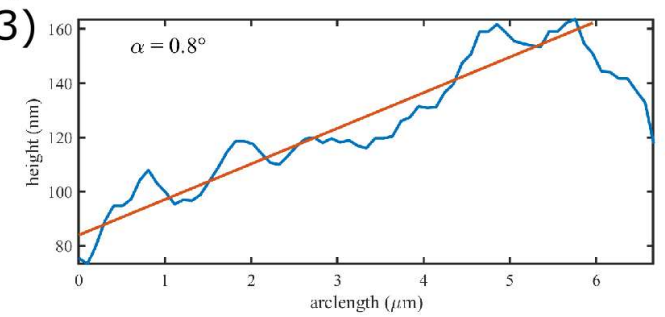

4)

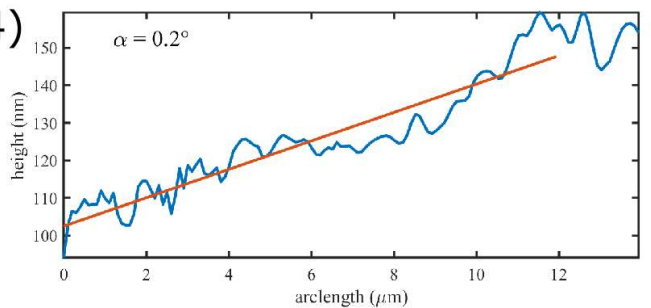

Fig.2: Constant FRET distance of actin and vinculin over time. Images show the color coded FRET distance between actin and vinculin in focal adhesions overlaid on the intensity image of vinculin (after bleaching of Atto 647N-phalloidin). As can be seen, this distance does not significantly change over time as imaged $1 \mathrm{~h}(\mathrm{~N}=4$ cells $), 6 \mathrm{~h}(\mathrm{~N}=4), 12 \mathrm{~h}(\mathrm{~N}=5), 18 \mathrm{~h}(\mathrm{~N}$ $=4)$, and $24 \mathrm{~h}(\mathrm{~N}=6)$ after seeding. Scale bar is $5 \mu \mathrm{m}$.

In summary, we can prove that the liveMIET technique works with living cells and yields results comparable to MIET measurements of fixed cells. Examples shown here were generated within minutes and with high intensity but in the published paper we will show that prolonged measurements are possible. We tested liveMIET with an 8 hour long continuous measurement of a SAOS-2 cell. In this video, lateral shifting of stress fibers during the migration can be seen as well as changes in height and quenching. 



\section{Enucleation}

\subsection{The contribution of the nucleus to force sensing}

Whenever we talk about force sensing in cells, we assume that there is a force recognition network in the cell consisting of focal adhesions, stress fibers, and the nucleus. It has been shown that the nucleus is necessary for 3D migration and strongly affects 2D migration [79]. This seems to be due to the nucleus acting as a counterweight for the forces pulling in different directions inside the cell [110]. However, thinking of it merely as a counterweight underplays the role of the nucleus and misconceives its properties. As the nuclear envelope is not completely rigid but is folded, it can be stretched and squished (see Figure 7.1).

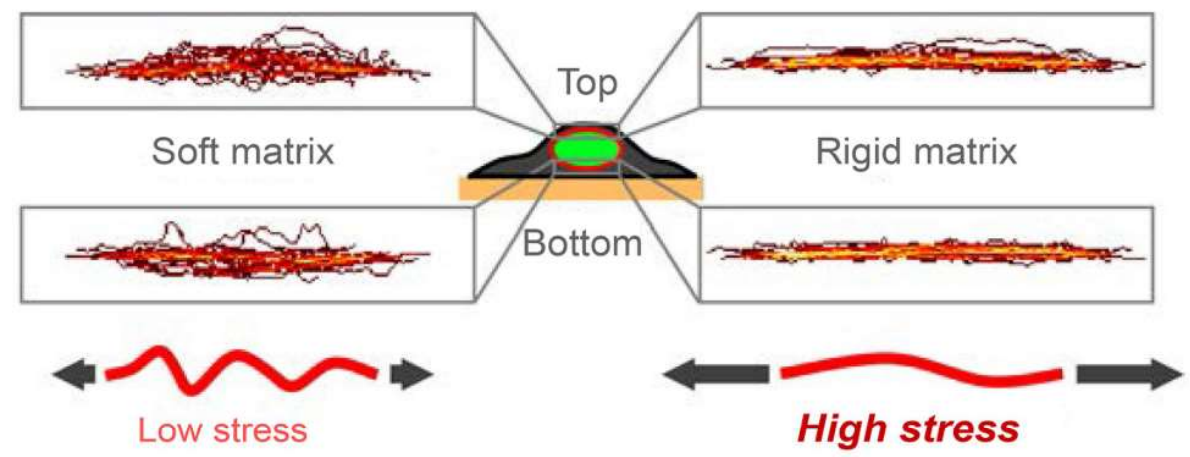

Figure 7.1.: Schematic drawing of the stretching of the nuclear envelope. From [28].

The deformation of the nucleus occurs in connection with stress on the whole cell. Area of the nucleus scales with the cell area [28]. Shape is influenced, too, as can be shown with micro-patterning [111]. A big portion of this deformation is mediated by the nuclear actin cap. The nuclear actin cap is linked both to the nuclear envelope-by the LINC (linker of nucleoskeleton and cytoskeleton) complex [111] —and to the substrate-by a special subset of focal adhesions (actin cap associated focal adhesions, or ACAFAs) that can sense forces over a broader range of forces compared to common focal adhesions [112]. This former connection, to the cytoskeleton, also facilitates the nuclear positioning necessary for the proper function of many cells. After LINC complex disruption, force propagation, organization of perinuclear actin and intermediate filaments, nuclear positioning, cell polarization, and migration speed are all significantly reduced [135]. For both Drosophila and cultured mammalian myotubes, mutants with disturbed linkage and aberrant nuclear positioning show decreased muscle contractility [142].

However, the proteins and composition of the nuclear envelope seem to be of greater interest for the force sensing, as they are linked to altered gene expression. Here, the proteins directly linked to the coupling are of greatest interest. These proteins are SUN1 and SUN2, lamins, and nesprins (the last are connected to SUN proteins and often linked to muscle dystrophies [135]). 


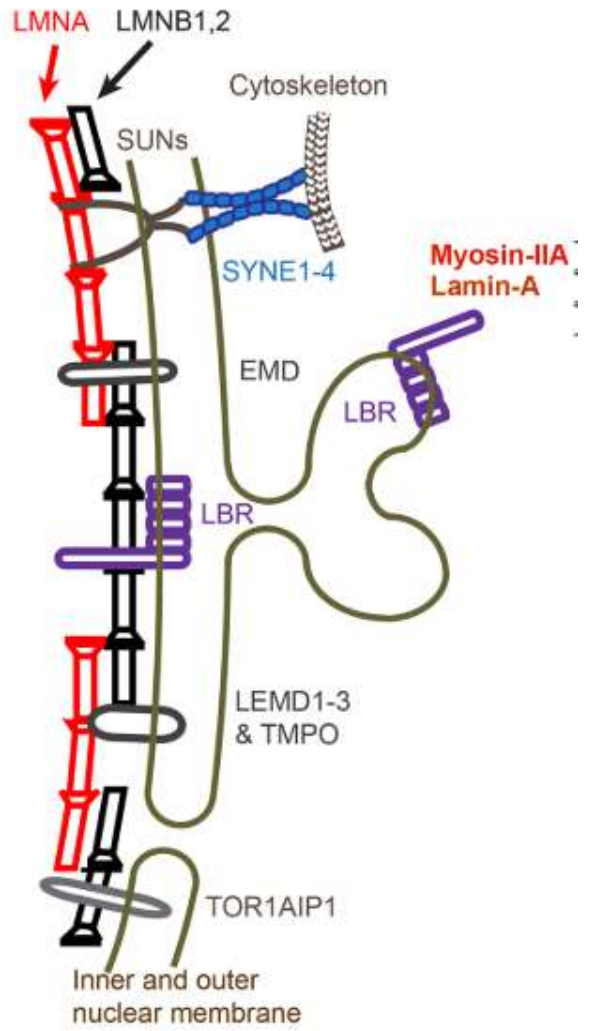

Figure 7.2.: Schematic drawing proteins connecting cytoskeleton with nuclear envelope. From $[28]$.

One of the most important effects of SUN2 is the promotion of focal adhesion assembly by activation of the small GTPase RhoA. In this process, the transcription factor/coactivator complex composed of SRF/Mkl1 is used. But as a constitutively active form of SRF/Mkl1 was not sufficient to induce focal adhesion assembly in cells lacking SUN2, it can be assumed that there is a transcription-independent mechanism of RhoA activation mediated by the LINC complex [205]. This concurs with the finding that SUN2-null cells express aberrant intercellular adhesions and changed morphology [196]. Additionally, SRF (nuclear transcription factor serum response factor) is directly induced by the liberation of myocardin-related transcription factor (MRTF) cofactors during globular actin polymerization [151]. However, SUN1 antagonizes SUN2 LINC complexes and inhibits RhoA activation and focal adhesion assembly. So, SUN1 and SUN2 LINC complexes have different roles in the transcription-independent control of the actin cytoskeleton through the small GTPase RhoA.[205].

As the nuclear lamins are part of the regulation of replication, transcription, and epigenetic modifications of chromatin, they are equally of interest. In the nuclear envelope, A- and B-type lamins form separate, but interacting, stable meshworks of different mobility [183].

Silencing of lamin B1 leads to increased meshwork size and higher mobility of lamin A. The change in mesh size seems to induce lamin B2-deficient nuclear-envelope blebs. The blebs, which are composed of lamin A and C, are associated with euchromatin and transcriptionally active. This suggests chromatin organization and regulation is influenced by interconnections of the lamin microdomains [183]. Lamin A levels also seem to scale with tissue elasticity, with cells on stiffer substrates expressing more lamin A. It could be shown that, during regulation of stem cell differentiation by the vitamin $\mathrm{A} /$ retinoic acid (RA) pathway, nuclear entry of RA receptors are modulated by lamin A, which stabilizes the nucleus and contributes to lineage determination [201].

Because the chromosomes are attached via nuclear pore complexes to certain and determinable regions 
of the nuclear envelope [71], it is likely that nuclear distension has an influence on the remodeling of chromosome territories and chromatin-envelope interactions [201]. This would also affect and alter gene activities.[218].

To determine the influence of the nucleus on the stress fiber network, one can either disconnect the nucleus from the actin network by disturbing the LINC complex $[28,21]$ or remove the nucleus entirely $[79,222]$.

\subsection{Enucleation}

Enucleation was done with the help of technical assistant Kerstin von Roden, who has contributed much to the improvement of the protocol.

To accomplish the enucleation of various cell types (see Table 7.1), we used an ultracentrifugation approach, yielding two million cytoplasts. Other possible methods would be burning the nucleus with a UV laser or extracting it with a micro-needle, but simply pushing it out seems to be the most straightforward way, leading to fewer artifacts.

\begin{tabular}{|c|c|c|c|}
\hline Cell line & Supplier & Product No. & Additional Information \\
\hline $\mathrm{hMSC}$ & Lonza & PT-2501 & human mesenchymal stem cells \\
\hline $3 \mathrm{~T} 3$ & DSMZ & ACC 173 & murine fibroblasts \\
\hline NIH-3T3 & ECACC & 88022401 & murine embryonal fibroblasts \\
\hline REF-52 (WT) & Berchadsky Lab & described in [79] & rat embryonal fibroblasts \\
\hline REF-52 (YFP-Pax) & Berchadsky Lab & described in [79] & rat embryonal fibroblasts \\
\hline SAOS-2 & DSMZ & ACC 243 & human osteogenic sarcoma \\
\hline C2-C12 & DSMZ & ACC 565 & murine myoblasts \\
\hline HeLa & ECACC & 93021013 & 17A001, cervix epithelial \\
\hline $\mathrm{MCF}-7$ & DSMZ & ACC 115 & $\begin{array}{c}\text { human mammary gland, } \\
\text { metastasis }\end{array}$ \\
\hline 293 & DSMZ & ACC 305 & human embryonal kidney \\
\hline Cos-7 & DSMZ & ACC 60 & African green monkey kidney \\
\hline Vero & prob. Sigma & prob. 84113001 & $\begin{array}{l}\text { African green monkey } \\
\text { kidney epithelial }\end{array}$ \\
\hline
\end{tabular}

Table 7.1.: Cell line details.

The cell lines used were mainly purchased from companies, but some were brought to the lab by postdocs or gifted from other labs. There is no reliable information on origin for three of these cell lines. The Vero cells from Eugenia Butkevic were likely originally purchased from Sigma, but there is no paperwork to prove this. In experiments, the cell lines perform as expected. For the REF-52 cells, we know that there is a mutation leading to a larger-than-normal amount of p53 [90], making it a poor metric for comparing "healthy" cells. This cell line was chosen explicitly for enucleation because of the prior usage in [79]. They were gifted from the Berchadsky Lab in wild type and YFP-paxillin (CRISPR/Cas9) form.

Media were used according to (Table A.2). Cells were kept at $37^{\circ} \mathrm{C}$ and $5 \% \mathrm{CO}_{2}$. Protocols for thawing (Subsection A.1), freezing (Subsection A.2), and splitting (Subsection A.3), as well as other cell culturerelated protocols can be found in the Appendix (Chapter A). General materials are used according to A.1 for each cell line and application. Where special reagents are used, as for enucleation (Subsection A.5) and transfection (Subsection A.4), additional reagents can be found in the respective special sections. The Ficoll approach for enucleation is not the only possible method, as shear stress applied with various methods can be utilized as well. Use of ultracentrifugation in a Ficoll 400 gradient while inhibiting actin 
polymerization by cytochalasin B [106] has already been done in 1975 by Wigler [222]; the same protocol was adjusted and refined in 2018 by Graham [79].

For my thesis, I used both of these papers for general instructions and adapted them to the machines available. Graham clarifies that the properties of Ficoll 400 vary significantly from company to company and recommends Product No. BP525 from Fisher, which is used throughout the thesis.

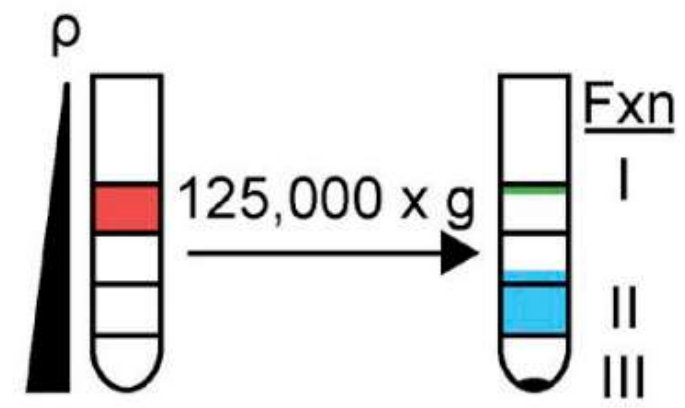

Figure 7.3.: Schematic drawing of the gradients for ultracentrifugation. Black triangle shows stiffness of gradient. Red layer shows cells in $15 \%$ solution, green layer (I) cell debris after ultracentrifugation, blue layer (II) cytoplasts, black dot at the bottom of the tube (III) nuclei. Figure from [79].

Gradient-based cell sorting is used for multiple applications-for example, extraction of hMSCs from donor bone marrow. Ficoll, as inert substance, is commonly used for this gradients. Ficoll solution for the gradient was prepared in PBS in a ratio of 50/50 overnight and under rotation. Gradients were prepared on the second day, mixing Ficoll solution with the respective cell medium to obtain 30, 20, 18 and 15\% Ficoll solutions. To each of these solutions cytochalasin B was added. The refractive index of the $30 \%$ Ficoll 400 solution was measured on a photometer at a $600 \mathrm{~nm}$ wavelength, setting PBS as blank to control the quality of the gradients. A refractive index of 1.373 was given by [79] as optimal for HeLa and other cell lines of similar size; we stick to that value. Gradients were layered starting with $30 \%$ solution at the bottom and finished with half the volume of the $15 \%$ solution.

The gradients were prewarmed overnight together with adapters and the SW32-Ti rotor used for ultracentrifugation at $37^{\circ} \mathrm{C}$. Cells used for enucleation were grown for at least one passage as described in protocols in Section A to minimize stress effects. Cell line-specific materials were used according to Table A.2. For transfection of cells to obtain cytoblasts with fluorescent-labeled stress fibers, materials described in Table A.3 and protocols described in Subsection A.4 were used. Cells were trypsinized and pallet-solved in the remaining volume of $15 \%$ solution. This was carefully layered onto the gradient and the tube filled to top with the respective cell medium used (see the red layer in Figure 7.3). Before ultracentrifugation, gradients with cells were incubated at $37^{\circ} \mathrm{C}$ for 1 hour. Ultracentrifugation was performed at $30^{\circ} \mathrm{C}$ and 27,000 rpm. Cells were retrieved from the respective layer in the gradient (see the blue layer in Figure 7.3) and washed three times with cell culture medium and twice with PBS before being plated on substrates (see 4.1). These cytosplasts were used for live-cell imaging (see 5.2) as well as for immunostaining (see Section 5.3).

\subsubsection{Difference in the enucleation of multiple cell lines}

Because I am interested in how the stress fiber network develops differently when the nucleus is removed, this research included comparing the enucleated cells to cells with intact nuclei. Originally, a comparison 
of wild-type (WT) cells, WT enucleated cells, LifeAct-RFP transfected cells, and LifeAct-RFP transfected enucleated cells was intended. However, as there is no adequate software to analyze phase contrast movies of cells at the required level of accuracy — and writing the softward would be beyond the scope of the thesis - the movies of WT cells (normal and enucleated) were not analyzed. Nevertheless, these experiments were necessary, and were done in the process of experiment evaluation and improvement.

The original Wigler paper [222] mentions successful enucleation for L (Mouse) cells, NRK (Normal Rat Kidney) cells, Vero (African green monkey) cells, and rat liver epithelial cells. The Graham paper [79] uses REF-52 and HUVEC cell, and concludes that REF-52 cells yield cytoplasts of longer viability.

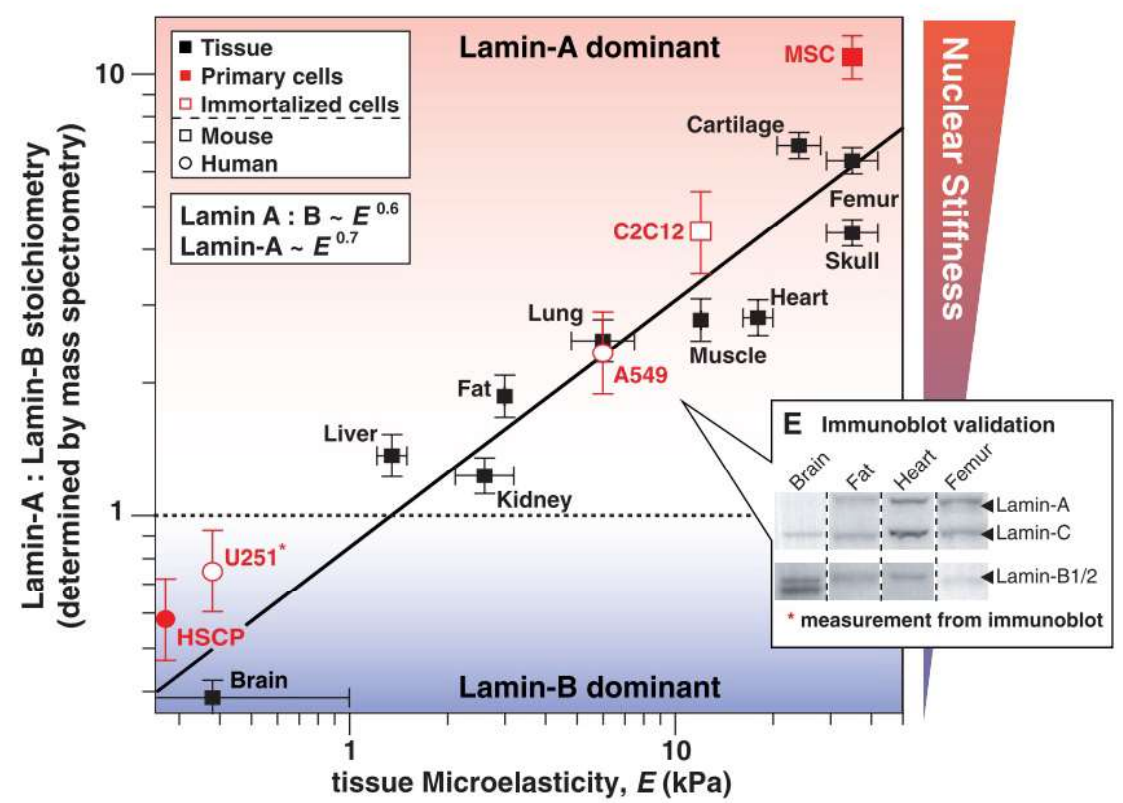

Figure 7.4.: Nuclear stiffness and lamin composition of different tissues and primary cell lines in comparison. Figure from [201].

All of the cells studied in the Wigler and Graham papers were one of three types: fibroblasts, endothelial, or kidney cells. Initial tests with hMSCs, which we wanted to enucleate to assess the contribution of the nucleus in differentiation, were not successful. This might be due to the comparatively higher nuclear stiffness and lamin composition (see Figure 7.4). Because a stiffness gradient is used to achieve enucleation, it is reasonable to assume that it needs to be adjusted according to both the cells' nuclear and overall stiffness. Furthermore, there might be cell lines that cannot cope with enucleation and this has to be distinguished from failed enucleation.

Thus, aside from fibroblasts, endothelial, and kidney cells, as used by Wigler and Graham, we also enucleated muscle cells and osteoclasts in addition to hMSCs. Each of these cell lines presents additional struggles for stress fiber imaging of non-enucleated cells. Some may have too few stress fibers; some form clusters after dividing; some may polynucleate. In enucleated cells, division and related problems do not occur. Instead, we have to deal with nuclear residues and reduced viability.

To exclude cells with nuclear residues from analysis, ibidi dishes were prepared for live cell movies to allow the addition of Hoechst into the medium without disturbance of the microscopy setup. Hoechst was added after 24 hours of imaging (1:10,000, $20 \mathrm{~min})$ and cells were imaged again. Afterwards, dishes were fixed and stained with phalloidin to yield a larger set of images of enucleated cells (for immunostaining see Section D). 


\subsubsection{Stress fiber networks of different cell lines}

When analysing non-enucleated, LifeAct-transfected cells exposed to the same conditions used in Chapter $5(11 \mathrm{kPa}$ PAA gels, glass, both coated with collagen I), three cells groups can be differentiated based on their behaviors. First, there are cells that spread more on glass, have mostly a higher aspect ratio on 11 $\mathrm{kPa}$, and have more filaments on glass. In this group we also find hMSCs, and it can be assumed that after enucleation these cells lines should also behave in a manner similar to how enucleated hMSCs would. Second, there are cell lines that behave similarly in terms of cell area, aspect ratio, and filament number whether plated on $11 \mathrm{kPa}$ PAA gels or glass. Third, there are cell lines for which the cell area and filament number are higher on $11 \mathrm{kPa}$, whereas the aspect ratio does not differ between the two media. Each of these subgroups is described in detail in the following subsections.

It should be noted that these behaviors cannot be clustered according to the suggested respective tissue stiffness for most of the cell lines, as would be expected. Cell stiffnesses mentioned in this subsection are measured via atomic force microscopy (AFM). AFM is used to prove surfaces by indentation of a lever, which locally probes resistance. Results obtained with this technique may differ from techniques measuring whole cell or even tissue properties.

\subsubsection{Spreading on glass}

For the first group of cell lines (Cos-7, hMSC, MCF-7, and SAOS-2), the group that spreads more and has a higher filament count on glass, but a larger aspect ratio on $11 \mathrm{kPa}$ PAA gel, this non-dependency on supposed stiffness can be seen quite well. However, even for single cell lines, different suggested values can be found. For MCF-7, one source mentions a stiffness of $4 \mathrm{kPa}$ [157], while another gives a span of 20-30 $\mathrm{kPa}$ [130]. Cos7 cells and hMSCs seem to be in the same order of stiffness, with Cos7 around 5-10 kPa [81] and hMSC with $8.4 \mathrm{kPa}$ [121]. In contrast, SAOS-2 cells inhabit a very high range, with a Young's modulus of $56.4 \mathrm{kPa}[121]$.
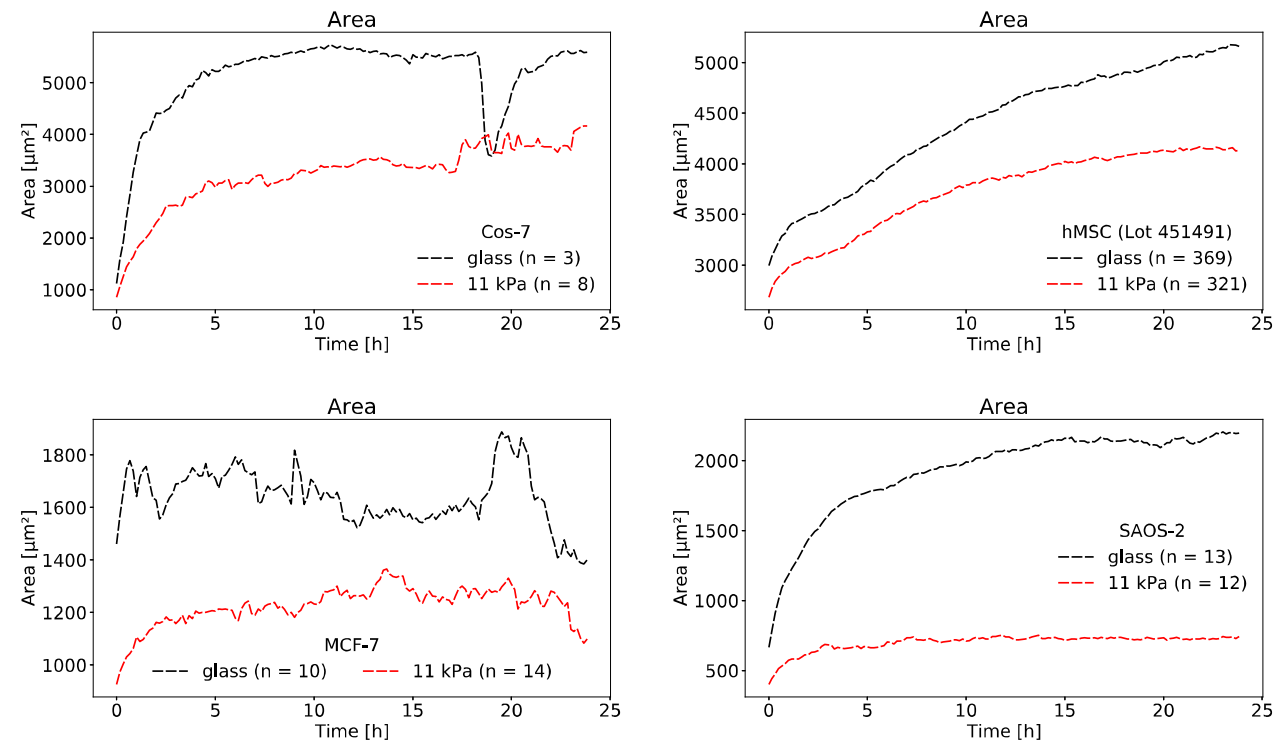

Figure 7.5.: Cell area development during 24-hour live cell imaging on collagen I coated glass and $11 \mathrm{kPa}$ gels with LifeAct-RFP transfected, single, nondividing cells. $10 \mathrm{~min}$ interval between images. Top left: Cos-7. Top right: hMSC (Lot 451491). Bottom left: MCF-7. Bottom right: SAOS-2. 

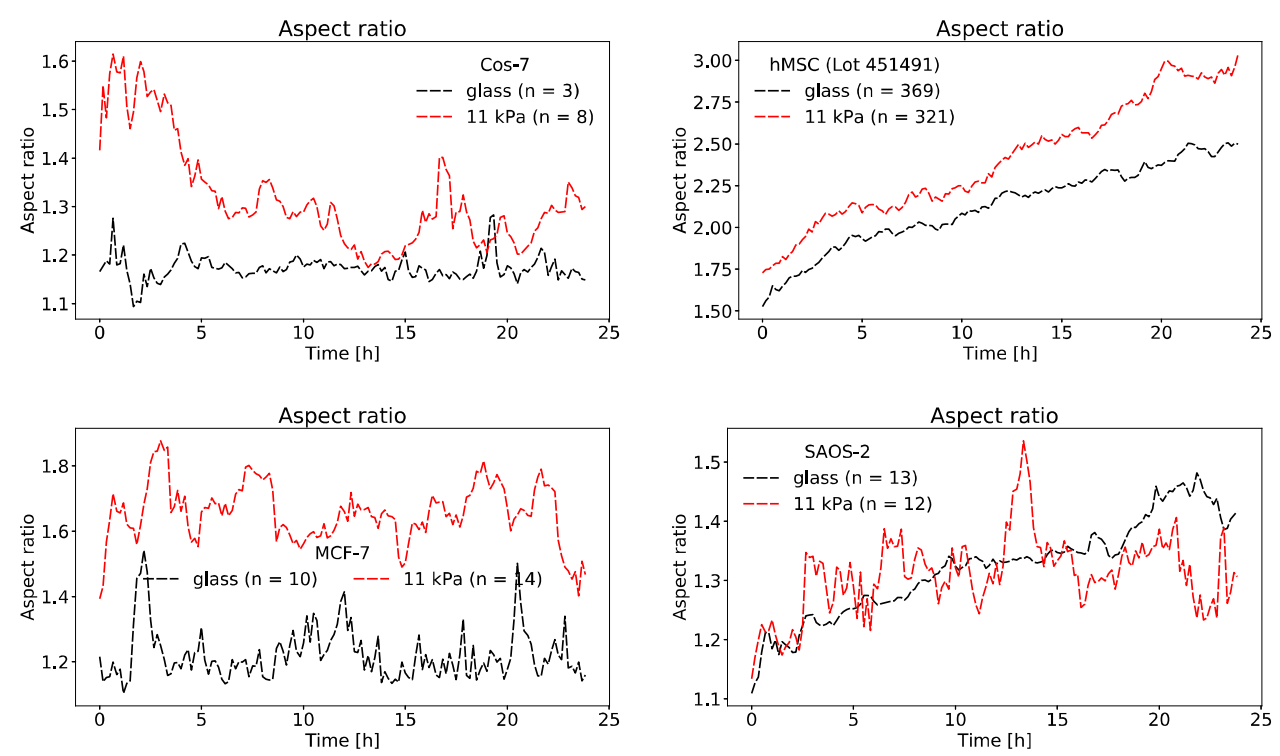

Figure 7.6.: Aspect ratio development during 24-hour live cell imaging on collagen I coated glass and $11 \mathrm{kPa}$ gels with LifeAct-RFP transfected, single, nondividing cells. $10 \mathrm{~min}$ interval between images. Top left: Cos-7. Top right: hMSC (Lot 451491). Bottom left: MCF-7. Bottom right: SAOS-2.
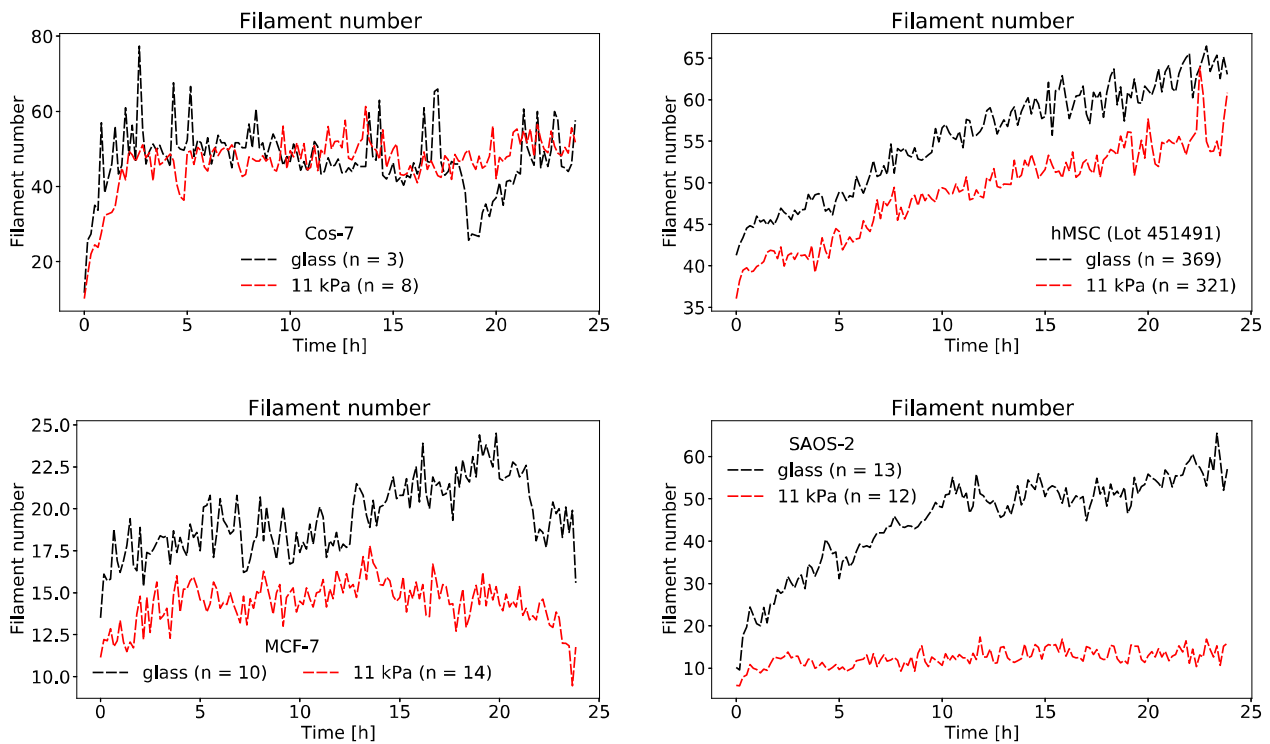

Figure 7.7.: Filament number development during 24-hour live cell imaging on collagen I coated glass and $11 \mathrm{kPa}$ gels with LifeAct-RFP transfected, single, nondividing cells. $10 \mathrm{~min}$ interval between images. Top left: Cos-7. Top right: hMSC (Lot 451491). Bottom left: MCF-7. Bottom right: SAOS-2. 
In Figure 7.5 one can see that, although the trend of the curves and the cell volume and - thus spreadable area - is quite different, for these four cell lines there is significantly higher spreading area on glass.

For the aspect ratio, shown in Figure 7.6, the cell lines show a higher aspect ratio on $11 \mathrm{kPa}$ with the exception of SAOS-2. Thus, they are collectively rounder than hMSCs, whose aspect ratio develops from 1.5 to 3.0 while the other cell lines show values between 1.1 and 1.8 .

Looking at the filament numbers for these cell lines, with the exception of Cos-7 all cell lines show more stress fibers on glass. Comparing these, Cos-7, hMSC, and SAOS-2 on glass all express around 60 stress fibers per cell. MCF-7 on glass express around 20 fibers per cell, whereas MCF-7 and SAOS-2 only express around 12 fibers per cell on $11 \mathrm{kPA}$.

\subsubsection{Ambivalent behavior}

For those cells not showing differences in behavior on the two tested substrates, cell stiffness is similarly ill-defined. For Vero cells, some report 5-10 kPa [81], others twice as much (with 15-20 kPa) [153]. For $3 \mathrm{~T} 3,32.4 \mathrm{kPa}$ was measured [121]. For NIH-3T3, no values could be found but it can be expected that they behave very similarly to 3T3s.

Thus, one could assume that those cells range around $30 \mathrm{kPa}$, and that neither $11 \mathrm{kPa}$ nor glass is the optimal substrate stiffness for these cells. All of these cell lines show very similar behavior on $11 \mathrm{kPa}$ PAA gels and glass with regard to area (see Figure 7.8), aspect ratio (see Figure 7.9), and filament number (see Figure 7.10).
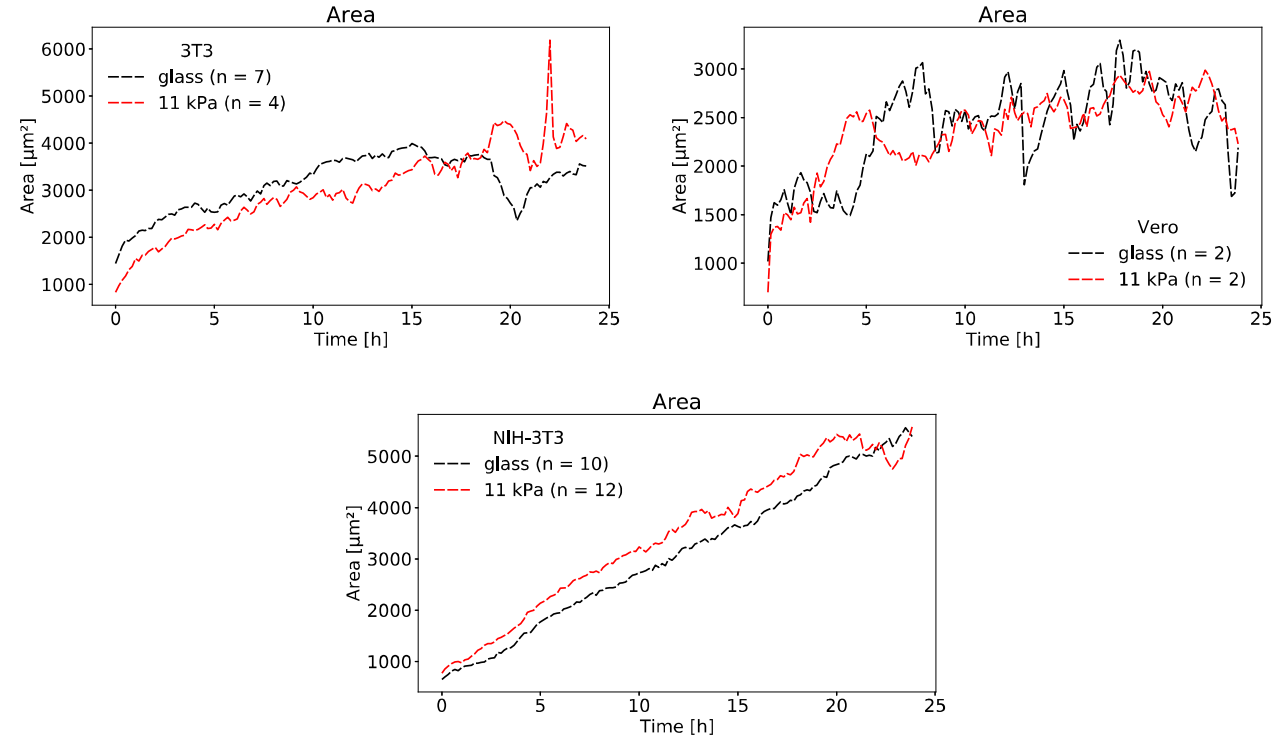

Figure 7.8.: Cell area development during 24-hour live cell imaging on collagen I coated glass and $11 \mathrm{kPa}$ gels with LifeAct-RFP transfected, single, nondividing cells. 10 min interval between images. Top left: 3T3. Top right: Vero. Bottom: NIH-3T3. 

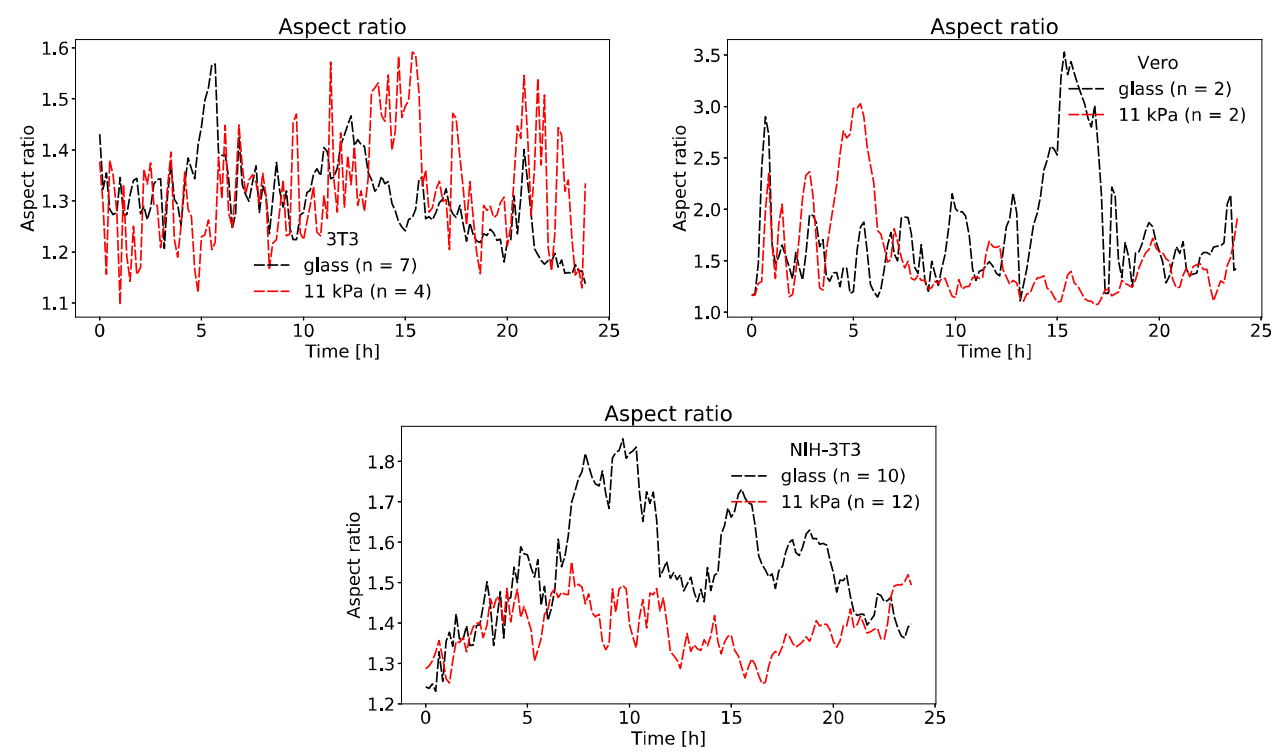

Figure 7.9.: Aspect ratio development during 24-hour live cell imaging on collagen I coated glass and $11 \mathrm{kPa}$ gels with LifeAct-RFP transfected, single, non-dividing cells. $10 \mathrm{~min}$ interval between images. Top left: 3T3. Top right: Vero. Bottom: NIH-3T3.
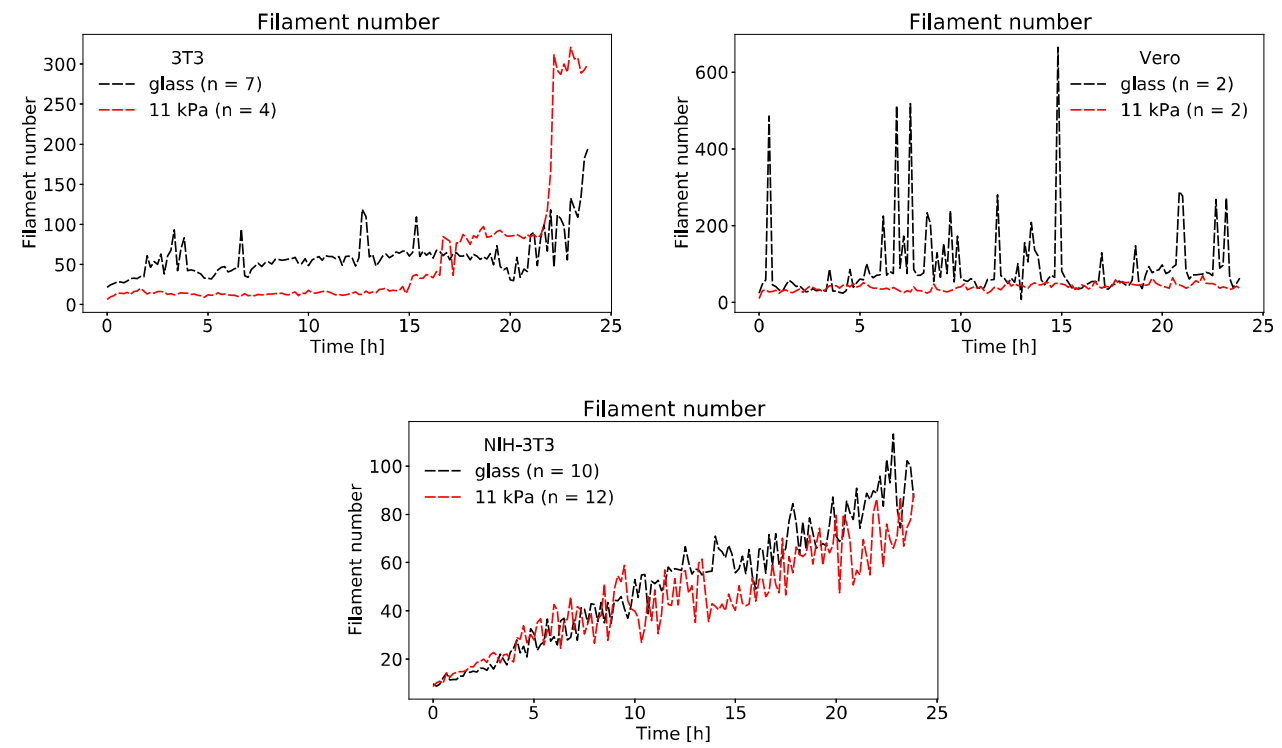

Figure 7.10.: Filament number development during 24-hour live cell imaging on collagen I coated glass and $11 \mathrm{kPa}$ gels with LifeAct-RFP transfected, single, nondividing cells. $10 \mathrm{~min}$ interval between images. Top left: 3T3. Top right: Vero. Bottom: NIH-3T3.

\subsubsection{Spreading on $11 \mathrm{kPa}$ PAA gel}

For the last subset, which spreads more on $11 \mathrm{kPa}$ PAA gel, reported optimal cell stiffness varies as well. For 293 (human embryonal kidney) cells, the optimal stiffness was $0.15 \mathrm{kPa}$ [184]; for REF-52, 18-59 kPa [75]; and finally, for HeLa cells, 100-200 kPa [130]. This span is extremely large and seems not to be explainable in any way. For all three cell lines, we see a credible difference between cell area on both substrates (see Figure 7.11). 

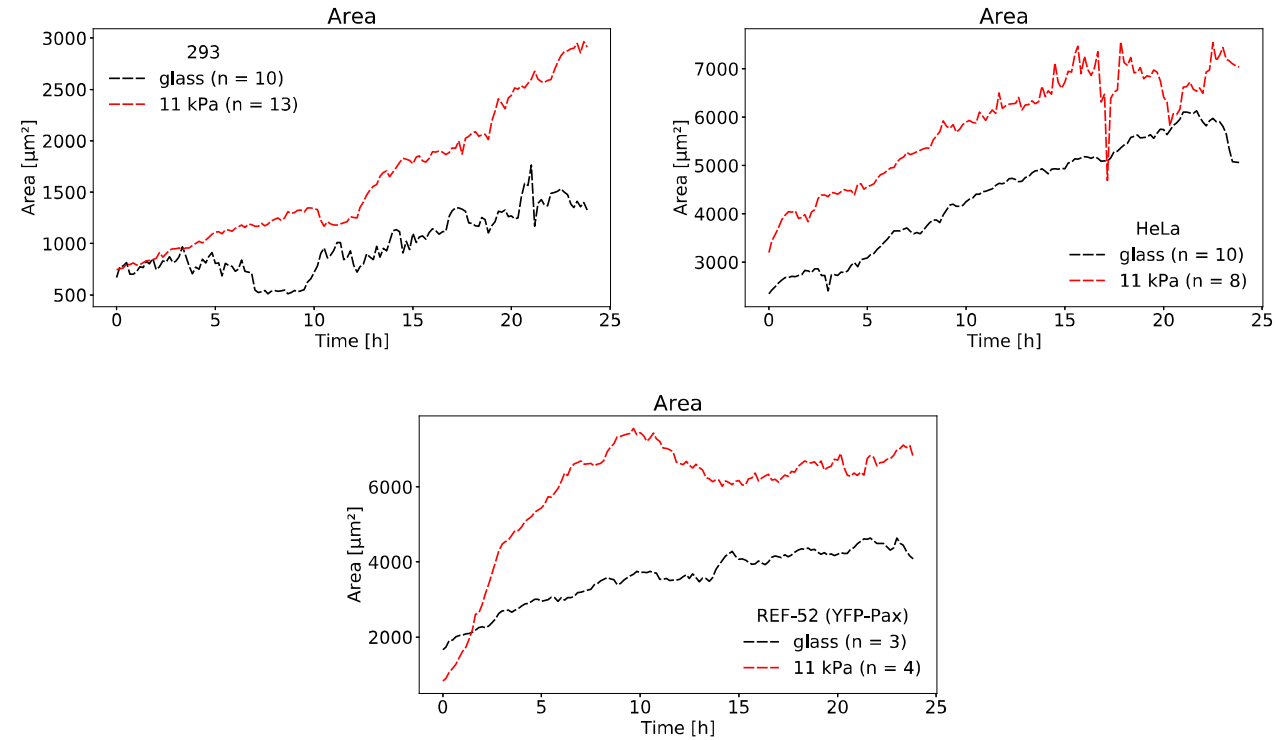

Figure 7.11.: Cell area development during 24-hour live cell imaging on collagen I coated glass and $11 \mathrm{kPa}$ gels with LifeAct-RFP transfected, single, nondividing cells. 10 min interval between images. Top left: 293. Top right: HeLa.Bottom: REF-52 (YFP-Pax).
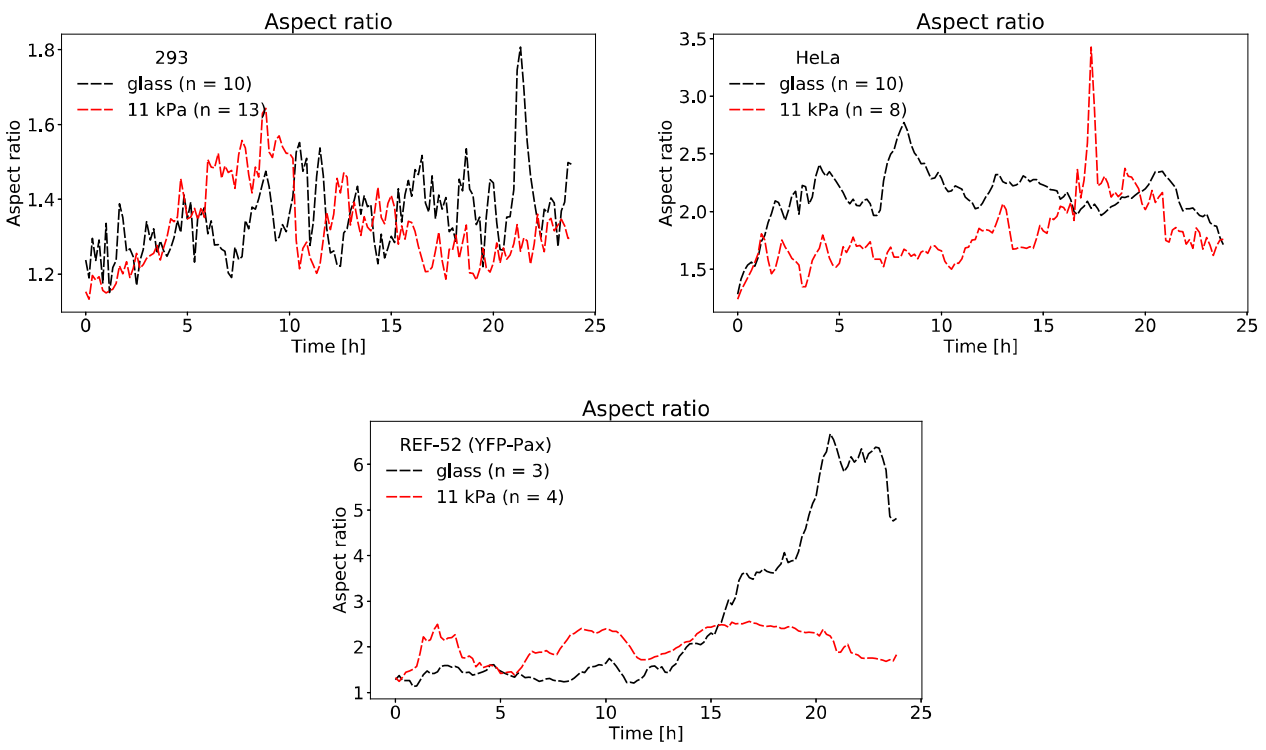

Figure 7.12.: Aspect ratio development during 24-hour live cell imaging on collagen I coated glass and $11 \mathrm{kPa}$ gels with LifeAct-RFP transfected, single, nondividing cells. 10 min interval between images. Top left: 293. Top right: HeLa.Bottom: REF-52 (YFP-Pax). 
In respect to aspect ratio only REF-52 cells show a clear difference between the two substrates, with aspect ratio being higher on glass. This mirrors cell lines that have higher area on glass but higher aspect ratio on $11 \mathrm{kPa}$. For 293 and HeLa cells, the aspect ratio fluctuates more and does not show a clear difference, although aspect ratio on glass is higher on the 24-hour mark in both cases (see Figure 7.12). Further mirroring the behavior of cells spreading more on glass, for which the filament number on glass is higher, here cells on $11 \mathrm{kPa}$ show by far more filaments (see Figure 7.13). This is especially surprising for the 293 cells, which are human embryonal kidney cells that showed very few stress fibers in earlier experiments.
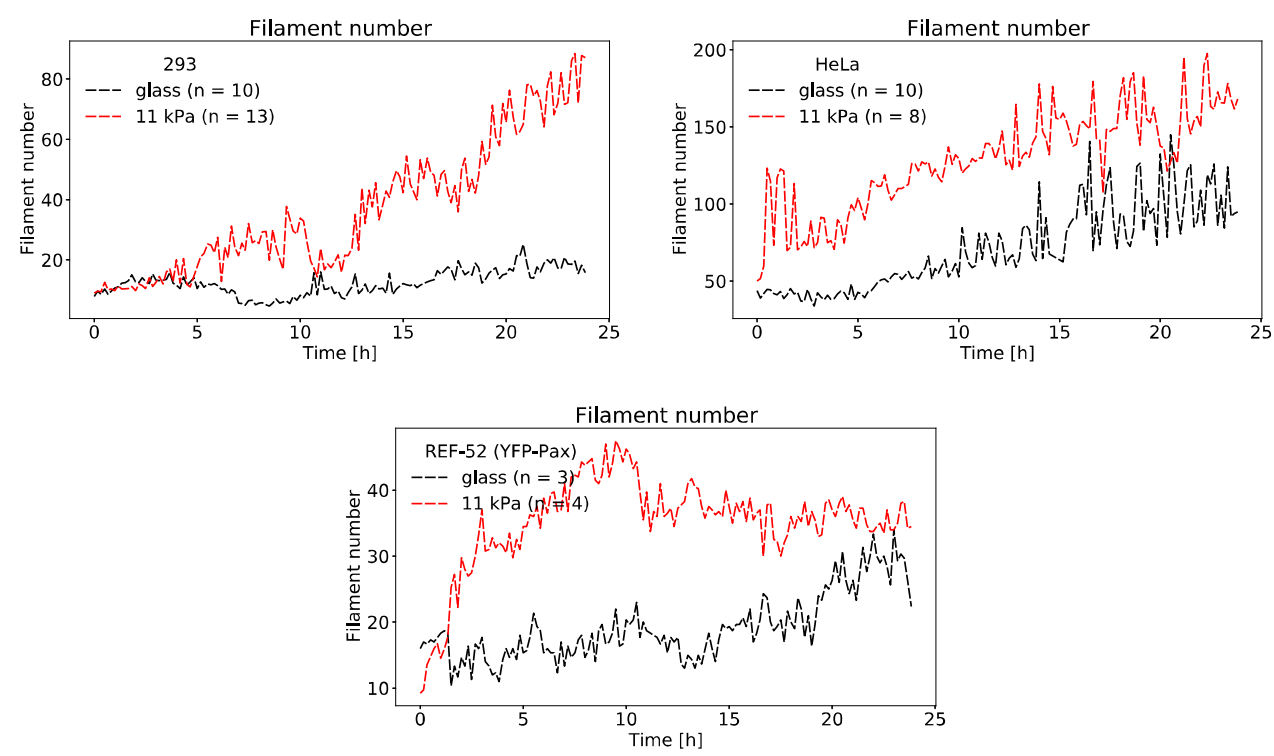

Figure 7.13.: Filament number development during 24-hour live cell imaging on collagen I coated glass and $11 \mathrm{kPa}$ gels with LifeAct-RFP transfected, single, nondividing cells. $10 \mathrm{~min}$ interval between images. Top left: 293. Top right: HeLa.Bottom: REF-52 (YFP-Pax).

In conclusion, all cell lines used show cell line-specific substrate-related behaviors that differed significantly from other cell lines that should have had the same mechanical responses based on cell and tissue stiffness. Thus, for a significant set of enucleation experiments, a large database of cells on respective substrates should be imaged to compare the cytoplasts to. As in this thesis all cell lines - with exception of hMSCs-below 15 cells were deemed usable for analysis, all enucleation results are just a proof of method and a guideline for further experiments with suitable cell lines.

\subsubsection{Results of enucleation}

In this first round of enucleation experiments, in which the main focus was to find cell lines surviving enucleation for at least 24 hours, only four cell lines yielded viable cells on both conditions (see Table 7.2). For the REF-52 cells this was no surprise, as these cells were used in the paper by Graham [79]. The NIH-3T3 cells are fibroblasts as well, so the results were similarly expected. Additionally, this cell line has proven to be very resilient in other experiments run in our lab, always yielding more surviving cells after stress than other cell lines. On the other hand, MCF-7 and Cos-7 cell lines surviving enucleation was unexpected. As the Graham protocol [79] is tailored to fibroblasts with higher cell and nuclear stiffness, it was expected that kidney and fat tissue cells would not survive without adapting the protocol. 


\begin{tabular}{l|c|l|c} 
Cell line & Supplier & Product No. & Additional Information \\
\hline NIH-3T3 & ECACC & 88022401 & murine embryonal fibroblasts \\
REF-52 (YFP-Pax) & Berchadsky Lab & described in [79] & $\begin{array}{c}\text { rat embryonal fibroblasts } \\
\text { human mammary gland, }\end{array}$ \\
MCF-7 & DSMZ & ACC 115 & metastasis \\
Cos-7 & DSMZ & ACC 60 & African green monkey kidney
\end{tabular}

Table 7.2.: Cell line details for cells surviving at least 24 hours after enucleation.

For all of these cell lines, spreading area and filament number is significantly lower after enucleation, as expected. However, the number of cells imaged in the 24-hour movie fashion (already explained in Chapter 5) is quite low, thus the statistics of fixed cytoplasts for all cell lines (see Subchapter 7.2.4) should give more insight.

All results in this subchapter show results from Subchapter 7.2.2 to compare cytoplasts to, as well as the respective results of fixed cells. Those results of fixed cells are then not repeated in Subchapter 7.2.4), where cell lines for which live cell movies of cytoplasts were not possible are shown and discussed.

\subsubsection{Cos-7 cells}

Cos-7 cells are green monkey kidney cells and were measured with a stiffness of around $5-10 \mathrm{kPa}$ [81]. As described in Subsection 7.2.2.1, these cells usually show larger cell area on glass, a larger aspect ratio on $11 \mathrm{kPa}$ PA gel, and more filaments on glass.

During control movies with transfected cells the main issue with Cos-7 cells was their tendency to form clusters, making it hard to find single cells to image. What catches the eye first is the huge discrepancy between cell area of non-enucleated and enucleated cells (see Figure 7.14). Although enucleated cells, both live and fixed, still spread more on glass, total area in enucleated cells is around one-tenth of non-enucleated ones. This is not explainable by the absence of the nucleus alone but has to be caused by insufficient spreading.

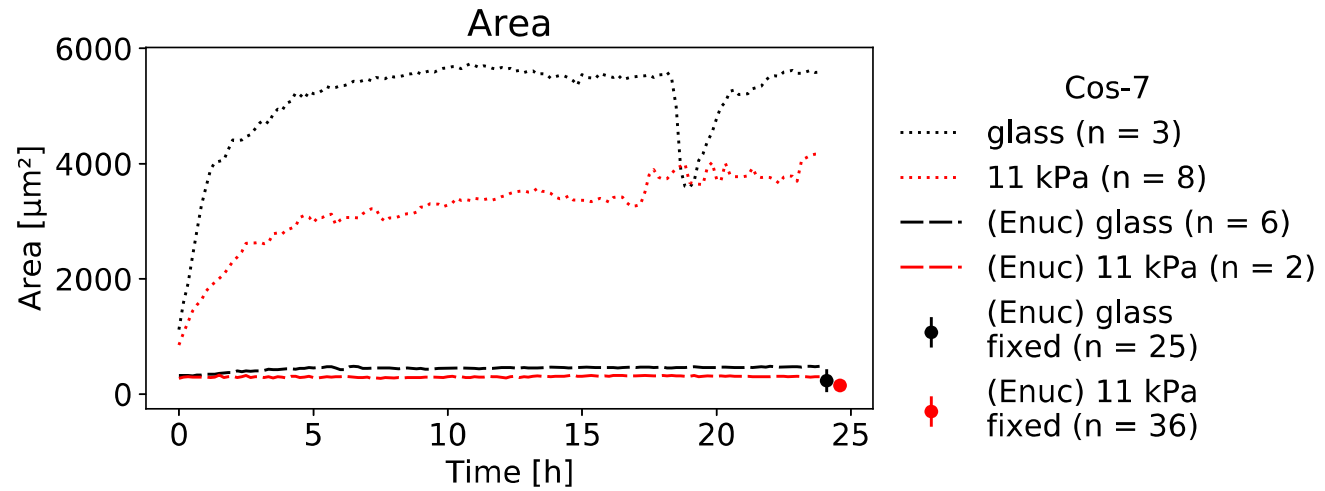

Figure 7.14.: Area of enucleated Cos-7 cells in 24-hour live movies and samples fixed after 24 hours compared to non-enucleated Cos-7 cells. All transfected with LifeAct-RFP. Errorbars of fixed cells shown as SD.

Looking at the aspect ratio for all conditions, it overlaps, showing that all cells are relatively round with an aspect ratio between 1 and 1.5. While non-enucleated Cos- 7 cells on $11 \mathrm{kPa}$ show a higher value, for enucleated cells this is only true between hours 14 and 20 (see Figure 7.15). 


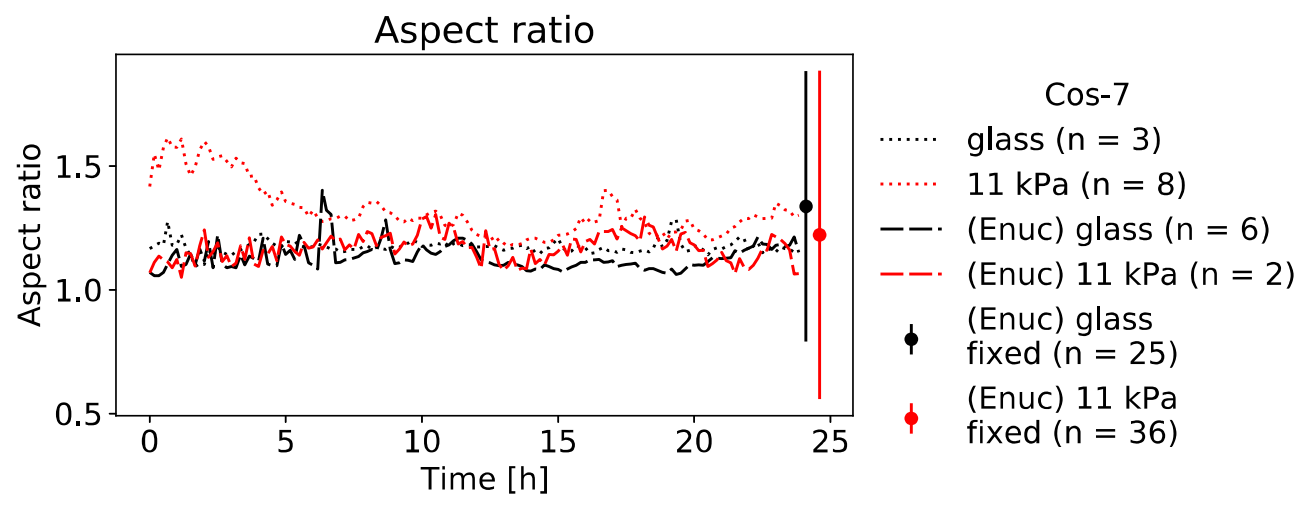

Figure 7.15.: Aspect ratio of enucleated Cos-7 cells in 24-hour live movies and samples fixed after 24 hours compared to non-enucleated Cos-7 cells. All transfected with LifeAct-RFP. Errorbars of fixed cells shown as SD.

For the filament number, non-enucleated Cos-7 cells did not behave like the rest of the group spreading more on glass, which expressed more filaments. Instead, for Cos-7 cells the curves overlap and show about 50 filaments on both conditions after spreading and stress fiber network development is completed at around 4 hours after seeding. For enucleated cells in movies the number is around one-third of this, with cells on $11 \mathrm{kPa}$ PAA gel showing more filaments throughout the whole imaging period. However, in fixed enucleated cells the filament number is even lower but shows a slightly higher value on glass (see Figure 7.15). The number of imaged cells is quite low for all of these conditions while the fluctuations in live movies and standard diviation (SD) in fixed cells is surprisingly small. Again, this might be attributed to insufficient spreading, which may lead to low turnover in the stress fiber network.

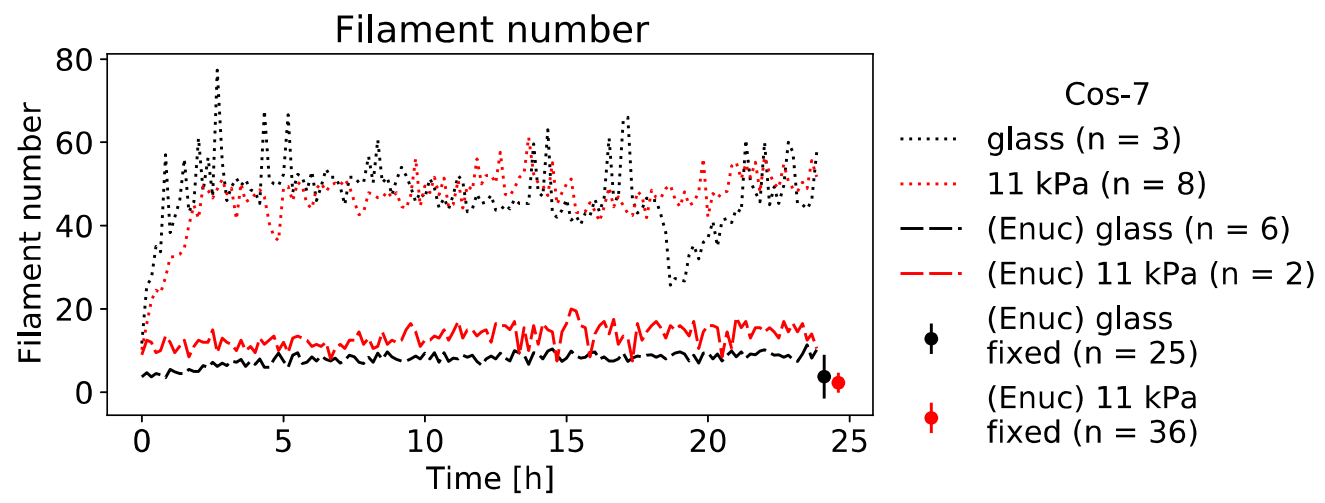

Figure 7.16.: Filament number of enucleated Cos-7 cells in 24-hour live movies and samples fixed after 24 hours compared to non-enucleated Cos-7 cells. All transfected with LifeAct-RFP. Errorbars of fixed cells shown as SD.

\subsubsection{MCF-7 cells}

As is the case with Cos-7 cells, MCF-7 cells tend to form clusters; also making it hard to image single cells for comparison. Non-enucleated, they belong in the category of spreading more on glass, having higher aspect ratio on $11 \mathrm{kPa} \mathrm{PAA}$ gel, and having more filaments on glass. For enucleated cells, this is also true 
in the fixed population. In the movie, there is only one imaged cell on $11 \mathrm{kPa}$ that was usable. Thus, this cell's area surpassing the combined cells on glass could be a coincidence. The area of enucleated cells in this case is one-third that of non-enucleated ones. Furthermore, SD on the fixed enucleated ones is higher. However, with an area of around $500 \mu \mathrm{m}^{2}$, the enucleated cell area matches that of Cos-7 enucleated cells.

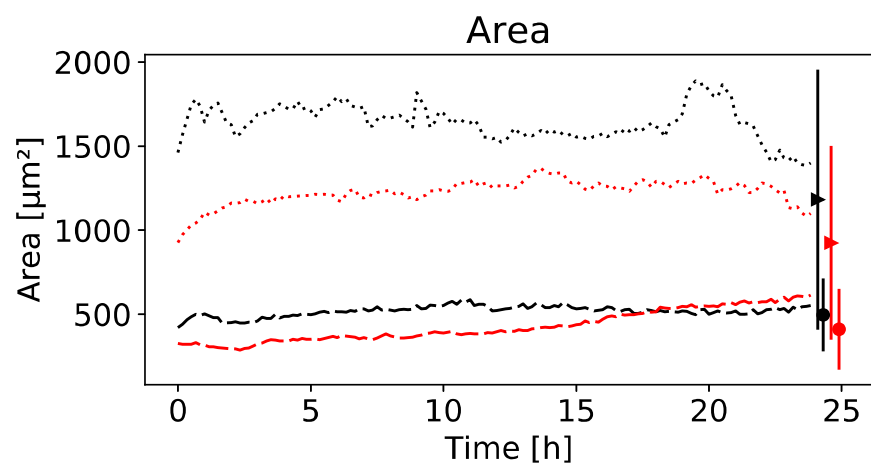

$$
\begin{array}{ll} 
& \multicolumn{1}{c}{\text { MCF-7 }} \\
\cdots & \text { glass }(n=14) \\
\cdots & 11 \mathrm{kPa}(\mathrm{n}=10) \\
-\cdot & \text { (Enuc) glass }(\mathrm{n}=6) \\
\ldots \cdot & \text { (Enuc) } 11 \mathrm{kPa}(\mathrm{n}=1) \\
& \text { glass } \\
\text { fixed }(\mathrm{n}=196) \\
11 \mathrm{kPa} \\
\text { fixed }(\mathrm{n}=118) \\
\text { (Enuc) glass } \\
\text { fixed }(\mathrm{n}=22) \\
\text { (Enuc) } 11 \mathrm{kPa}
\end{array}
$$

Figure 7.17.: Area of enucleated MCF-7 cells in 24-hour live movies and samples fixed after 24 hours compared to non-enucleated MCF-7 cells. All transfected with LifeAct-RFP. Errorbars of fixed cells shown as SD.

Regarding the aspect ratio (see Figure 7.18), for non-enucleated cells both live and fixed cells on $11 \mathrm{kPa}$ PAA gel show higher aspect ratios, while enucleated cells do not. For non-enucleated cells, both conditions show values just above 1, which means these cells are almost round. For the fixed enucleated cells, this value is higher, falling between the aspect ratios of live and fixed non-enucleated MCF-7. However, the cells on glass show a higher aspect ratio.

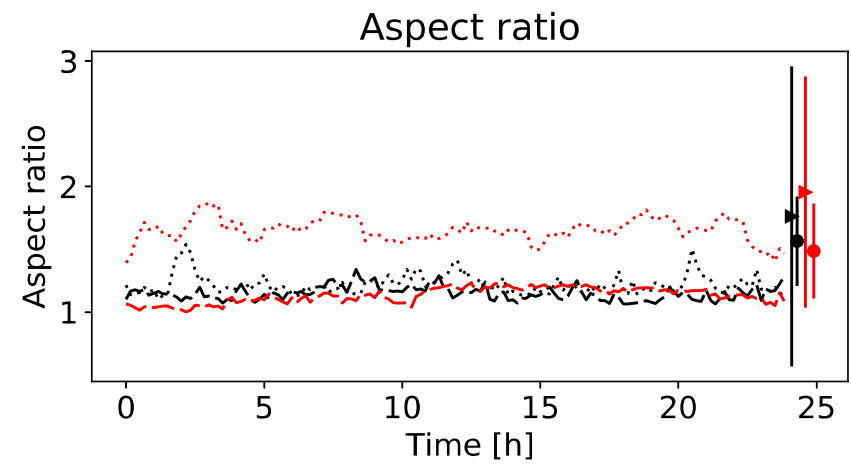

$$
\begin{aligned}
& \multicolumn{1}{c}{\text { MCF-7 }} \\
\ldots . . . & \text { glass }(n=14) \\
\ldots . . . & 11 \mathrm{kPa}(\mathrm{n}=10) \\
--. & \text { (Enuc) glass }(\mathrm{n}=6) \\
--. & \text { (Enuc) } 11 \mathrm{kPa}(\mathrm{n}=1) \\
+\quad & \text { glass } \\
& \text { fixed }(\mathrm{n}=196) \\
& 11 \mathrm{kPa} \\
& \text { fixed }(\mathrm{n}=118) \\
& \text { (Enuc) glass } \\
& \text { fixed }(\mathrm{n}=22) \\
& \text { (Enuc) } 11 \mathrm{kPa}
\end{aligned}
$$

Figure 7.18.: Aspect ratio of enucleated MCF-7 cells in 24-hour live movies and samples fixed after 24 hours compared to non-enucleated MCF-7 cells. All transfected with LifeAct-RFP. Errorbars of fixed cells shown as SD.

Looking at the filament numbers in all conditions (see Figure 7.19), all live cell movies show higher filament counts than the respective fixed cells. 

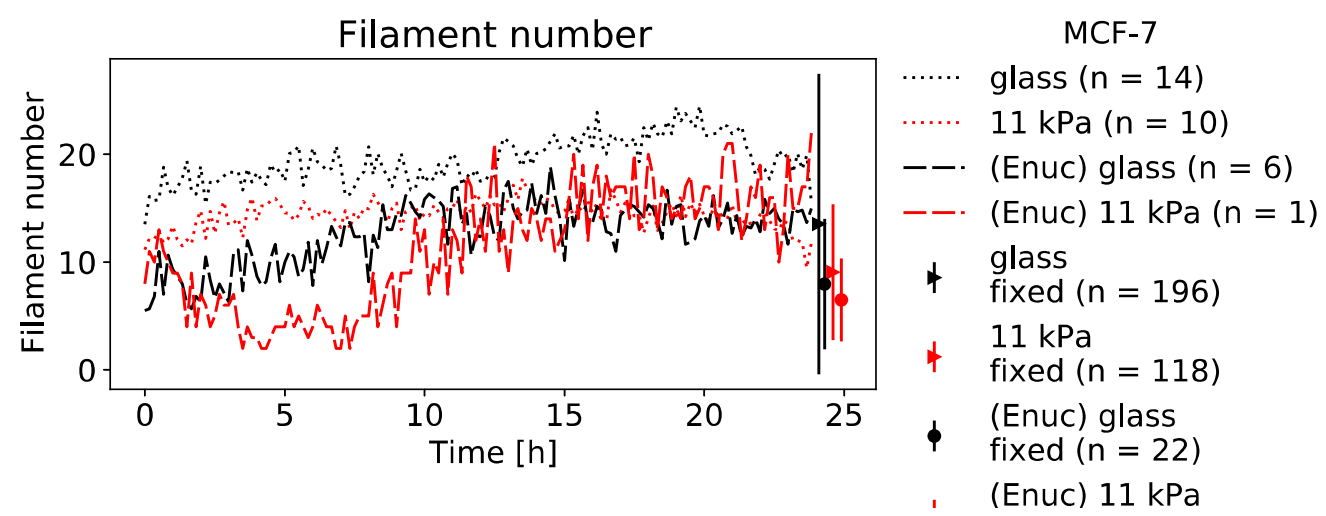

Figure 7.19.: Filament number of enucleated MCF-7 cells in 24-hour live movies and samples fixed after 24 hours compared to non-enucleated MCF-7 cells. All transfected with LifeAct-RFP. Errorbars of fixed cells shown as SD.

conditions show around 15 filaments per cell. This is lower than for other cell lines but matches experiments conducted by other lab members and should be attributed to the MCF-7 originating in breast tissue. While for live movies of non-enucleated cells the filament count is higher on glass, this is not the case for enucleated movies, where the curves of both conditions overlap. In fixed cells cells on glass show more filaments for both enucleated and non-enucleated cells, albeit with overall counts lower than in the live movies. The aberrant result of the enucleated cell on $11 \mathrm{kPa}$ PAA gel is again attributed to the fact that these are values from a single cell.

In conclusion, MCF-7 seems to tolerate the enucleation procedure better than Cos-7. While Cos-7 results seem to hint at impaired spreading, MCF-7 cytoplasts appear to exhibit a decent stress fiber network. However, this latter claim has to be proven with more experiments.

\subsubsection{NIH-3T3 cells}

Although NIH-3T3 cells survive enucleation in great numbers, they seem unsuitable for cytoplast studies of the stress fiber network.

Comparing the cell area measurements on all conditions, non-enucleated NIH-3T3 spread over the whole 24 hours of imaging without reaching a plateau. Enucleated, there is only little spread visible with cell area being around $100 \mu \mathrm{m}^{2}$, significantly lower than both Cos-7 and MCF-7 cells. Fixed non-enucleated cells show only around one-third of cell area compared to live cell movies at 24 hours while fixed enucleated cells show double the cell area. However, in both fixed and live enucleated cells, the cell area is higher on glass in contrast to non-enucleated live movies (see Figure 7.20).

For the aspect ratio, curves in live cell movies are similar. However, in enucleated live cells the aspect ratio is higher. This repeats in fixed enucleated cells, in which the discrepancy is more visible. Enucleated cells have an aspect ratio of around 2.4 in contrast to non-enucleated cells, which show one around 1.8. Also, while in both live imaging conditions aspect ratio for glass is slightly higher, in fixed cells those in $11 \mathrm{kPa}$ show a higher value and in enucleated cells both conditions show the same aspect ratio (see Figure 7.21). Repeating the pattern already seen in the area measurements, enucleated NIH-3T3 cells show barely any filaments (see Figure 7.22). Again, filament count in nonenucleated cells grows without reaching a plateau while fixed non-enucleated cells only show about half the filament number. Also, filament count in fixed enucleated cells is again lower than in the live imaging. While in non-enucleated cells, both live and fixed, there are slightly more filaments in cells on glass, in enucleated ones the values are the same. 


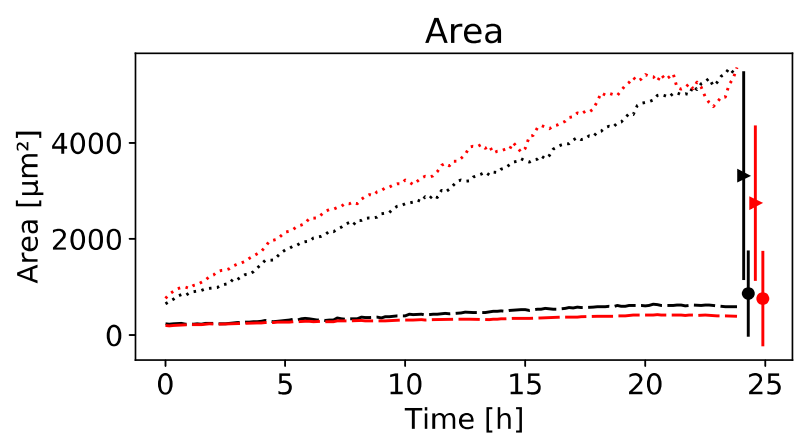

NIH-3T3

glass $(n=12)$

$11 \mathrm{kPa}(\mathrm{n}=10)$

(Enuc) glass $(n=5)$

-- (Enuc) $11 \mathrm{kPa}(\mathrm{n}=19)$

glass

fixed $(n=618)$

$11 \mathrm{kPa}$

fixed $(n=772)$

(Enuc) glass

fixed $(n=183)$

(Enuc) $11 \mathrm{kPa}$

Figure 7.20.: Area of enucleated NIH-3T3 cells in 24-hour live movies and samples fixed after 24 hours compared to non-enucleated NIH-3T3 cells. All transfected with LifeAct-RFP. Errorbars of fixed cells shown as SD.
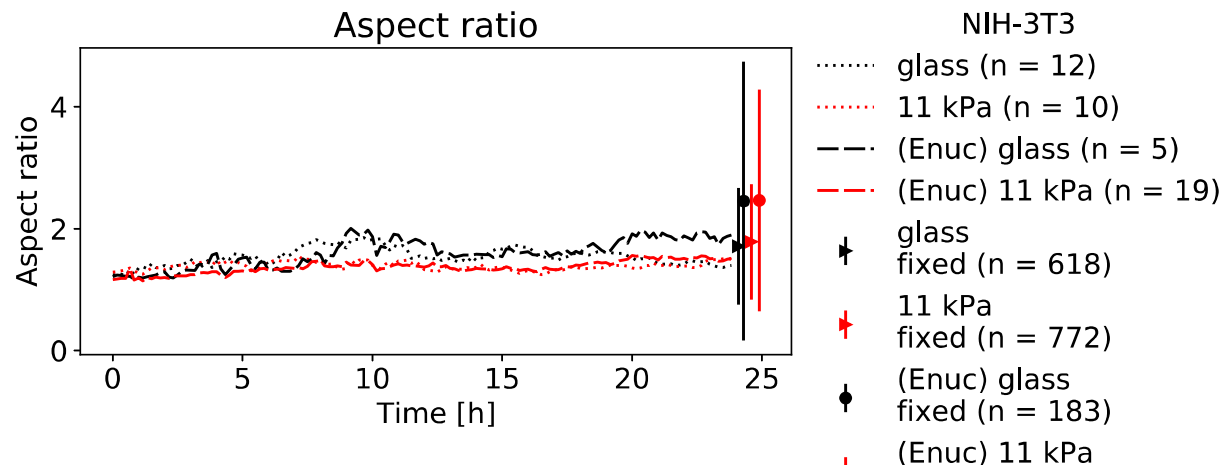

Figure 7.21.: Aspect ratio of enucleated NIH-3T3 cells in 24-hour live movies and samples fixed after 24 hours compared to non-enucleated NIH-3T3 cells. All transfected with LifeAct-RFP. Errorbars of fixed cells shown as SD.
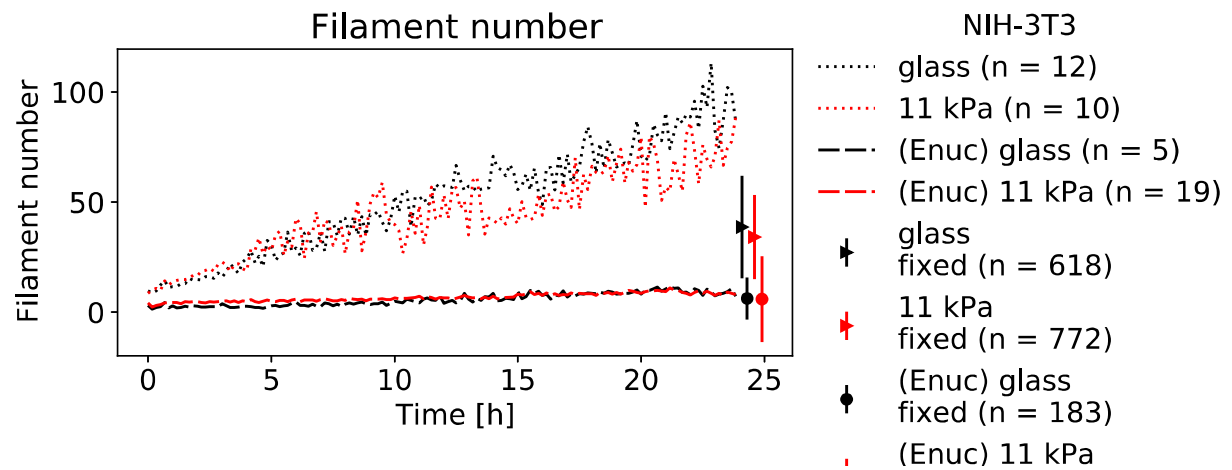

Figure 7.22.: Filament number of enucleated NIH-3T3 cells in 24-hour live movies and samples fixed after 24 hours compared to non-enucleated NIH-3T3 cells. All transfected with LifeAct-RFP. Errorbars of fixed cells shown as SD. 
Concluding for NIH-3T3 cells, both very small cell area and low filament count after enucleation seem to render them useless for live cell imaging of cytoplast stress fiber network despite their higher tolerance for enucleation procedure.

\subsubsection{REF-52 (YFP-Pax) cells}

REF-52 cells were kindly provided from the Berchadsky lab, which used the WT as well as the CRISPR/Cas9fabricated YFP-Paxillin ones in the Graham paper [79] and adapted and improved upon the original Wigler paper on enucleation via ultracentrifugation [222]. However, the process of obtaining these cells was lengthy. Ideally, experiments on REF-52 cells should have been done first to check whether we executed the protocol correctly and obtained similar results as Graham. But due to delays, most other cell lines were imaged before the REF-52 cells arrived at our lab.

Unfortunately, the movies with WT REF-52 did not turn out with a signal-to-noise ratio sufficient for analysis. Whether this was due to temporary issues with the electroporation protocol, or with the plasmid or fluorophore itself, was not determined. However, the YFP-Pax cells usually showed to be less stress tolerant then the wild-type variant. Thus, for future experiments, the issues with electroporation of WT REF-52 should be solved.

For REF-52 (YFP-Pax), results match those of NIH-3T3 to some degree. Non-enucleated REF-52 (YFPPax) do, like non-enucleated NIH-3T3, spread more on $11 \mathrm{kPa}$ gel and show less area when fixed. Similarly, when enucleated there seems to be no spreading (see Figure 7.23). In contrast to NIH-3T3, though, enucleated fixed cells show larger area than in live movies. Interestingly, in all conditions the cell area is larger on $11 \mathrm{kPa}$ PAA gel.
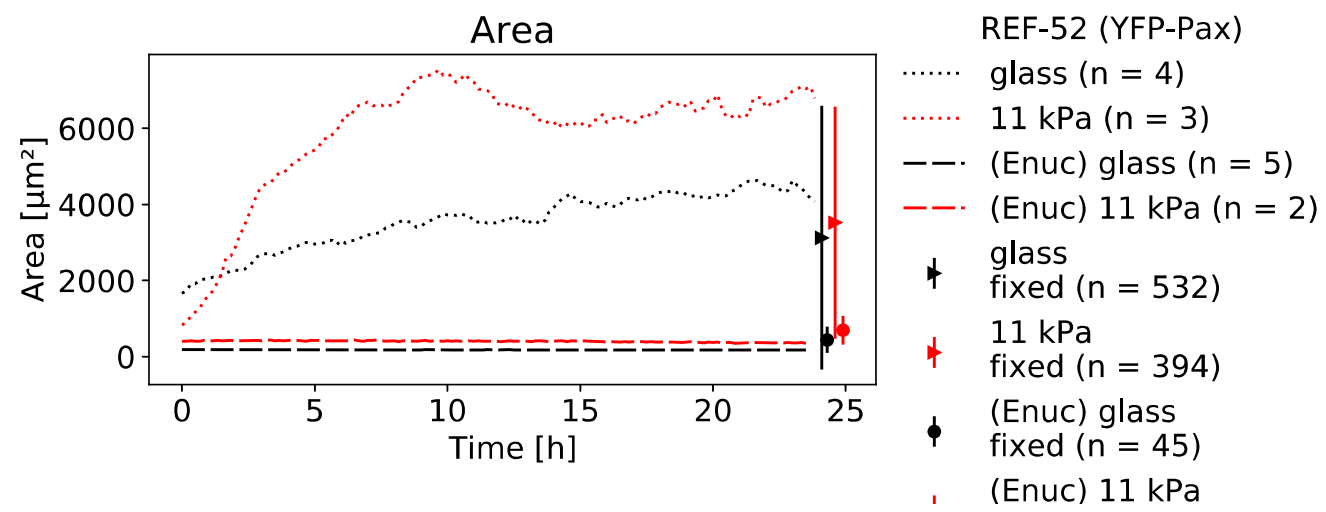

Figure 7.23.: Area of enucleated REF-52 (YFP-Pax) cells in 24-hour live movies and samples fixed after 24 hours compared to non-enucleated REF-52 (YFP-Pax) cells. All transfected with LifeAct-RFP. Errorbars of fixed cells shown as SD.

The same consistency is visible in the aspect ratio, where for all conditions cells on glass display a larger aspect ratio. However, in live imaging of enucleated cells the value is barely over 1 , which describes round cells; in this, case non-spreading ones. While for the non-enucleated cells the fixed cell values are again below the live movie ones, the fixed enucleated cells show higher values than the corresponding movies (see Figure 7.24). 

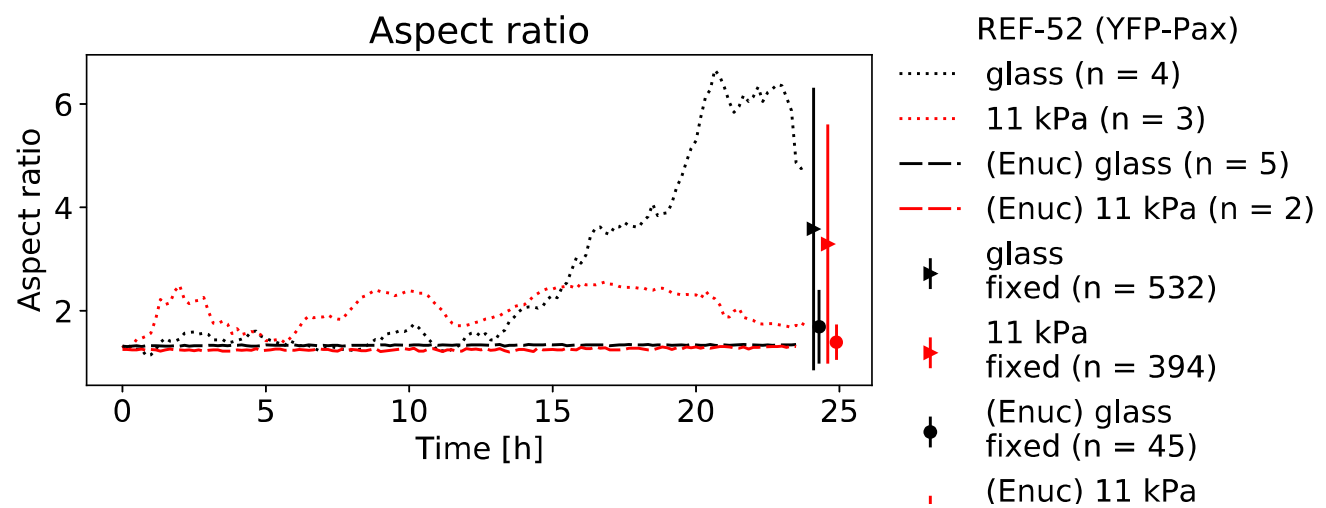

Figure 7.24.: Aspect ratio of enucleated REF-52 (YFP-Pax) cells in 24-hour live movies and samples fixed after 24 hours compared to non-enucleated REF-52 (YFP-Pax) cells. All transfected with LifeAct-RFP. Errorbars of fixed cells shown as SD.

The results of the filaments per cell data are in this case the only proof that the imaged cells are in fact not dead, but rather are simply not spreading. Here again the results are consistent, with cells on $11 \mathrm{kPa}$ expressing more filaments. Also, the live movies of enucleated cells show around 15 filaments per cell and some fluctuations in the numbers per time point. On glass, only around five filaments per cell and fewer fluctuations are found. Non-enucleated cells express approximately three times more filaments. Interestingly, for the filament number the measurements for live and fixed cells are in the same range.
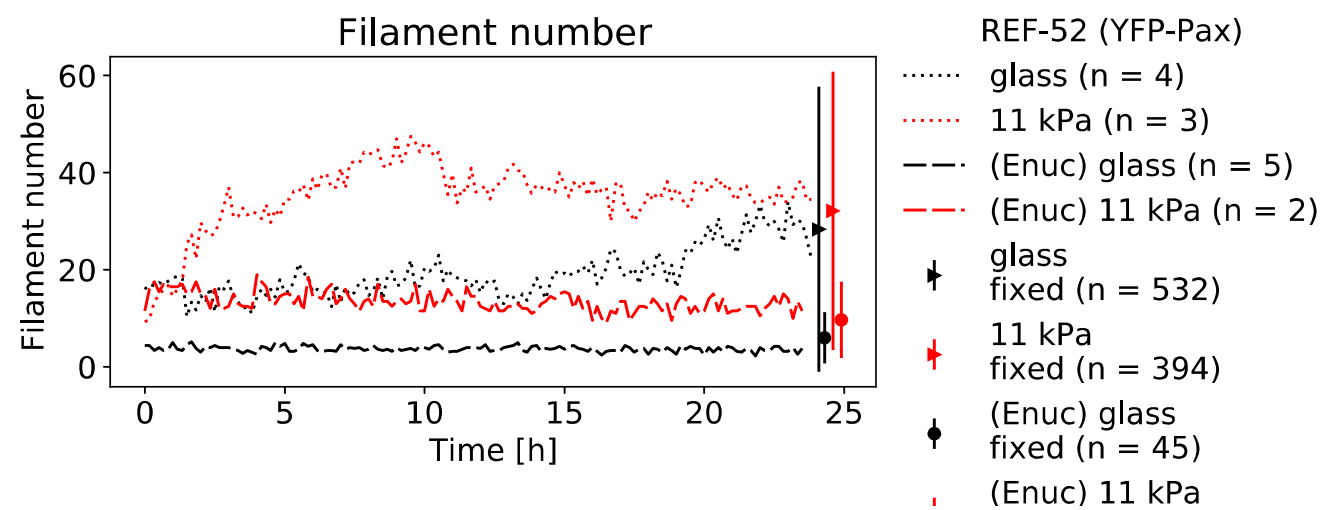

Figure 7.25.: Filament number of enucleated REF-52 (YFP-Pax) cells in 24-hour live movies and samples fixed after 24 hours compared to non-enucleated REF-52 (YFP-Pax) cells. All transfected with LifeAct-RFP. Errorbars of fixed cells shown as SD.

To conclude, although the area and aspect ratio measurements are not promising, we see a stress fiber network with enough filaments to analyse in a cell line that seems to tolerate enucleation. However, as the YFP-Pax in this setting is not bright enough to be imaged and the YFP-Pax cell line is less stress tolerant than the WT, these experiments should be repeated with the WT cell line for meaningful results.

In my experiments, only MCF-7 and REF-52 seem to be usable for live-cell imaging of cytoplasts gained by ultracentrifugation. However, many of the tested cell lines can be used to generate fixed cytoplasts. 


\subsubsection{Statistics of cytoplasts fixed after 24 hours}

For the fixation of cytoplasts, there are some details to be considered. First, the Graham protocol [79] calls for one million cells per ultracentrifugation tube, meaning one ultracentrifugation yields possibly two million cytoplasts. However, only a small percentage of cytoplasts seem to survive more than 10 hours. Thus, we resorted to high-density seeding but removing non-adherent cytoplasts and cell debris very carefully before imaging through medium change. Second, even after fixation with the standard $3.4 \%$ PFA solution, cytoplasts kept detaching. After testing multiple fixation protocols, 20 min of $3 \%$ glutaraldehyde at room temperature was found to be sufficient, yielding similar results to mixtures of PFA and glutaraldehyde and better results than alcohols.

Data shown here is from samples originally prepared for live cell movies, thus LifeAct-RFP transfected, fixed after 24 hours of imaging, and stained with phalloidin and Hoechst. Cells bearing leftover nuclear fragments were excluded. Additionally, it has to be mentioned that, dependent on cell line, a significant amount of bare nuclei was found in the samples. Those should, after enucleation, be located on the bottom of the ultracentrifugation tube. However, for some cell lines this is not the case and nuclei stay in less dense fractions of the gradient.

From all tested cell lines, there was only one where enucleation yielded not a single usable cell. C2-C12, a murine myoblast cell line, forms multinucleated myotubes under starvation conditions. In my experiments, despite going into the gradient layering and ultracentrifugation with mononucleated C2-C12 cells, I would often end up with fused, multinucleated cells instead of cytoblasts. However, of the fixed, non-enucleated cells, C2-C12 cells alone fall into the category of cells with higher area on glass, higher aspect ratio on 11 $\mathrm{kPa}$, and higher filament count on glass, although in Figure 7.26 the glass filament count is only slightly higher.

\section{$\mathrm{C} 2 \mathrm{C} 12$}
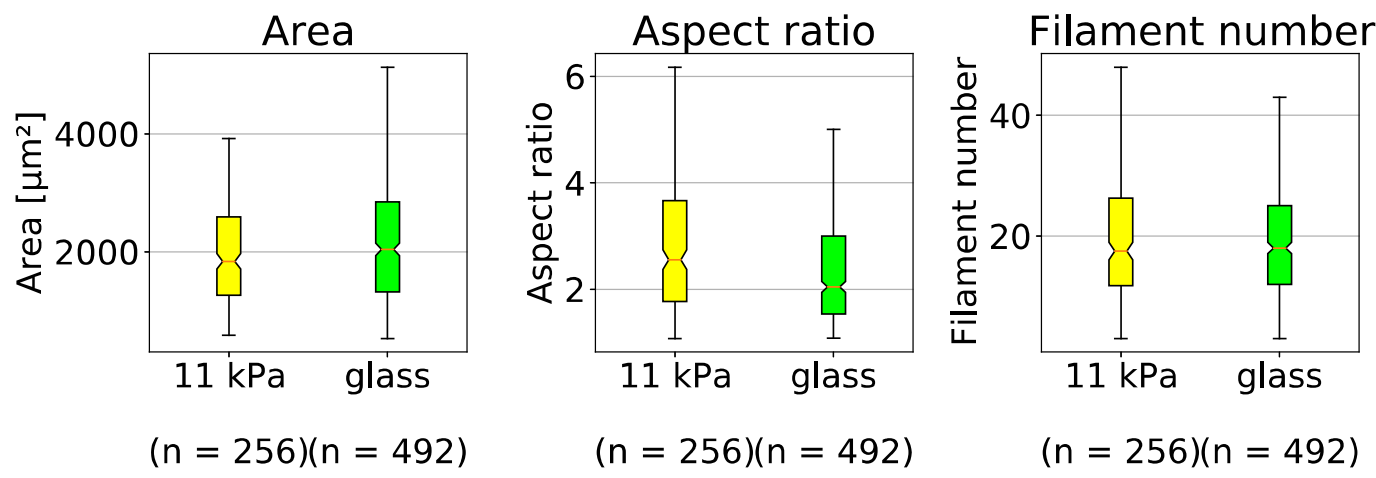

Figure 7.26.: Area, aspect ratio and filament number of enucleated and non-enucleated C2-C12 cells originally transfected with LifeAct-RFP for live cell movies. Fixed after 24 hours and stained with phalloidin and Hoechst.

For kidney cells, 293 (human embryonal kidney) and Vero (African green monkey kidney) should behave similarly. In comparison to the Cos-7 (green monkey kidney) cells, 293 and Vero cells did not yield favorable results in enucleation, with only single cells on $11 \mathrm{kPa}$ PAA gels surviving. When looking at 293 cells (see Figure 7.27), fixed non-enucleated cells show higher values on glass for area, aspect ratio, and filament number. For non-enucleated Vero cells only aspect ratio is higher on glass, mean area is the same in both conditions, and mean filament number is smaller on glass. Both of these cell lines, however, tend to form clusters; thus, only a small number of single cells could be imaged. For cluster-forming cell lines all single cells can be assumed to have not divided. As already mentioned in the discussion of live cell 
movies of differentiating hMSCs (see Section 5.6), cells that do not divide for a long time might behave aberrantly. However, as spreading area and cell outline in clusters behave differently from single cells, and cell borders of clustered cells are often not segmentable, as they overlap, I stuck to single cells.
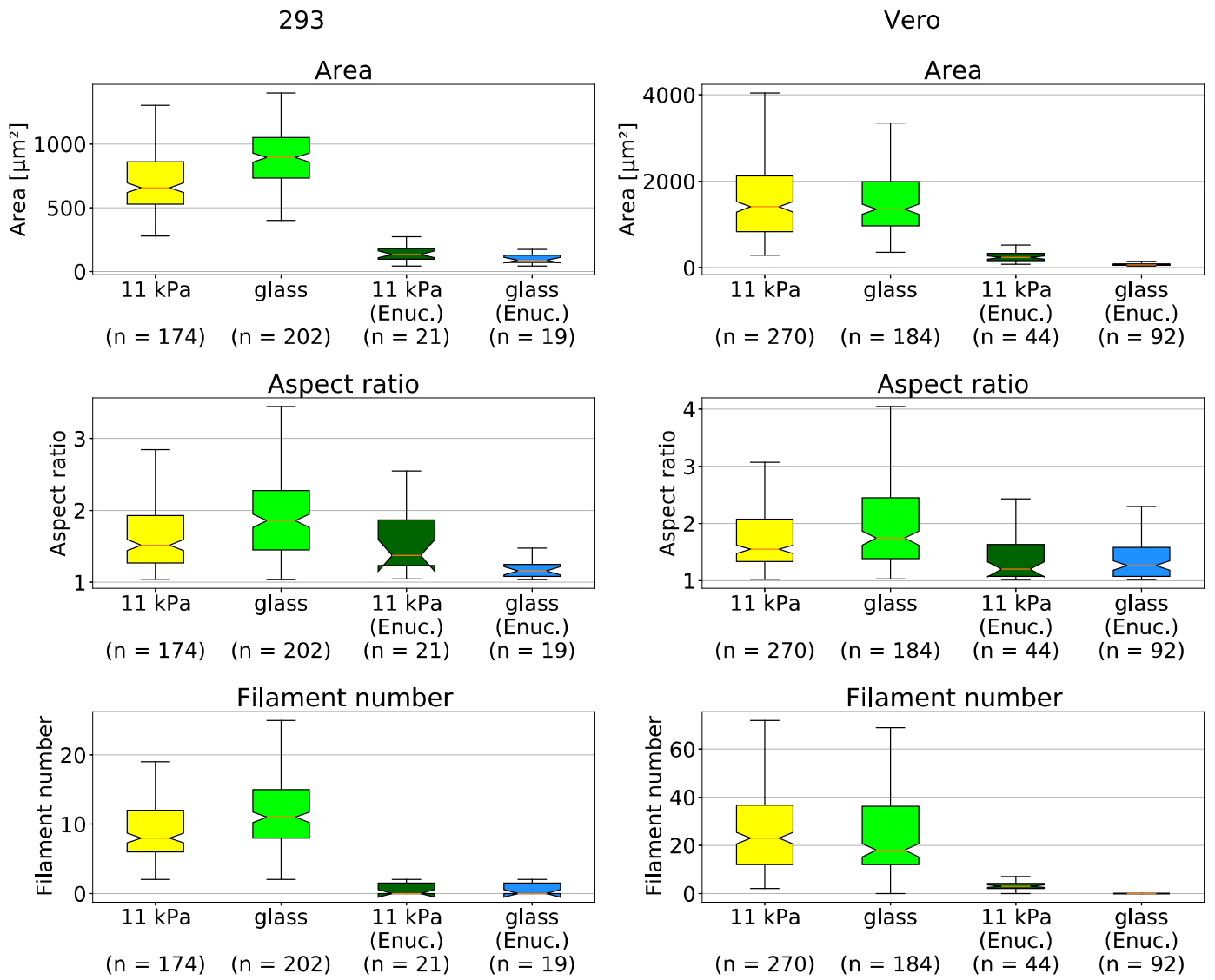

Figure 7.27.: Area, aspect ratio and filament number of enucleated and non-enucleated 293and Vero cells originally transfected with LifeAct-RFP for live cell movies. Fixed after 24 hours and stained with phalloidin and Hoechst.

Comparing the enucleated fixed cells, 293 cells seem to perform better than Vero cells, although there are more enucleated cells imaged for Vero. This assumption is based on the number of filaments, which is consistent for 293 cells but nonexistent for Vero on glass. Furthermore, cytoplasts on glass have a significantly lower area for Vero. In contrast, the aspect ratio is very similar on both conditions, whereas for the 293 cells there is a clear difference. In conclusion, one would assume that Vero cells do not spread on glass when enucleated, although they remain viable.

For HeLa cells, live cell movies of enucleated cells were also not possible. Most of the cytoplasts died around 6-8 hours into imaging. As HeLa cells are easy to cultivate, maintain, and electroporate, have a large body of information about the cell line, and in non-enucleated state exhibit a decent stress fiber network, their inability to withstand enucleation is unfortunate. Cytoplasts remaining viable and adherent after 24 hours, however, do in most cases not exhibit any stress fiber network. Therfore, although the spreading area on glass is higher and there are cytoplasts with higher aspect ratios (see Figure 7.28), HeLa cells do not seem to be a worthwhile target for further enucleation experiments. 

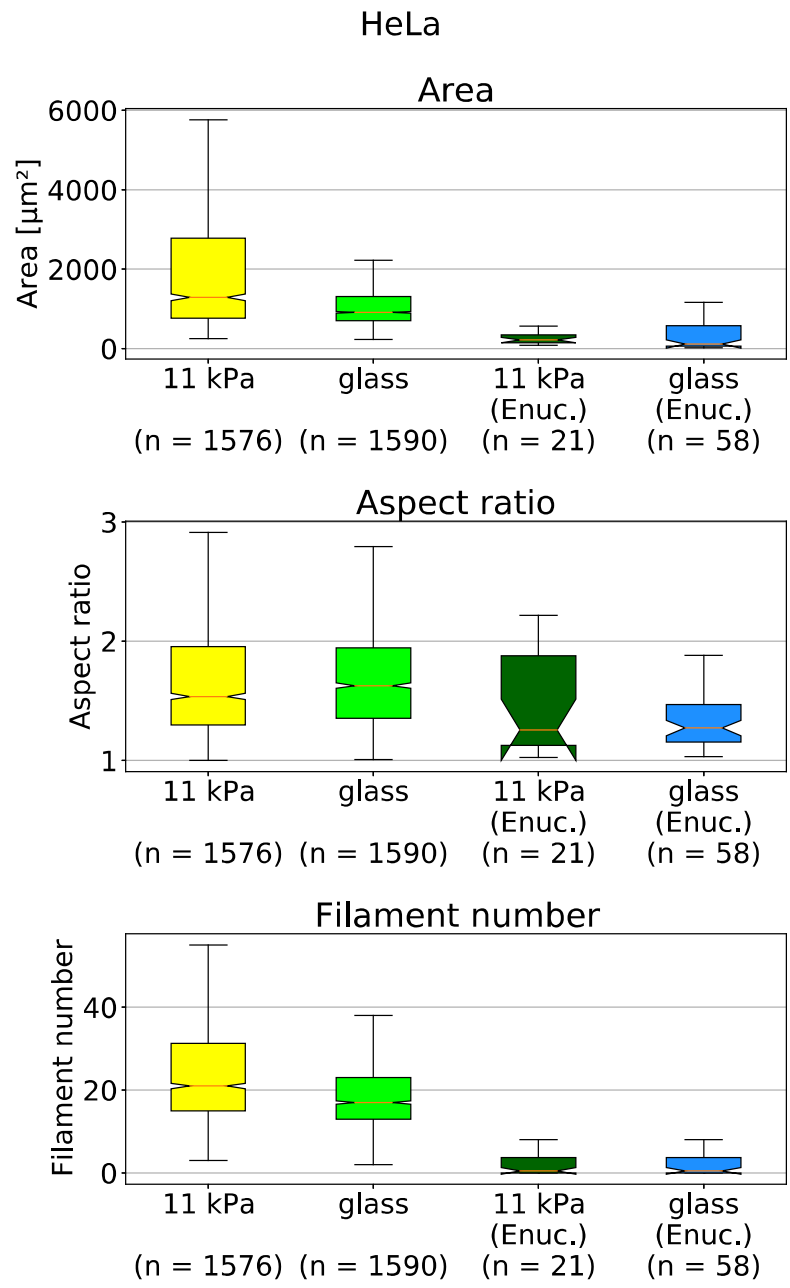

Figure 7.28.: Area, aspect ratio and filament number of enucleated and non-enucleated HeLa cells originally transfected with LifeAct-RFP for live cell movies. Fixed after 24 hours and stained with phalloidin and Hoechst.

Another cell line often used for stress fiber studies is SAOS-2, human osteogenic sarcoma cells. These cells seem to tolerate enucleation quite well. However, it was not possible to gather live cell imaging data for them as our experiments yielded just one surviving cell each on $11 \mathrm{kPa}$ PAA gel and glass, and viable cytoplasts after 24 hours were sparse, too. With SAOS-2 enucleated cells being about $500 \mu \mathrm{m}^{2}$ in area, one-third the size of non-enucleated cells, SAOS-2 seems to behave similarly to MCF-7 and NIH-3T3; enucleated cells for these latter two cell lines also measured around this number, too. It should perhaps be investigated whether all cytoplasts, when viable with stress fiber network and seeded on a suitable substrate, measure a similar area. Additionally, enucleated SAOS-2 cells express roughly one-third the number of filaments compared to non-enucleated SAOS-2 cells (see Figure 7.29). But, while cytoplasts on $11 \mathrm{kPa}$ show only a slightly higher aspect ratio than their non.enucleated counterparts, the aspect ratio for cytoplasts on glass is significantly higher. However, as there are only few cytoplasts measured, these experiments need to be repeated with larger cell numbers and possibly an altered enucleation protocol. 


\section{SAOS-2}
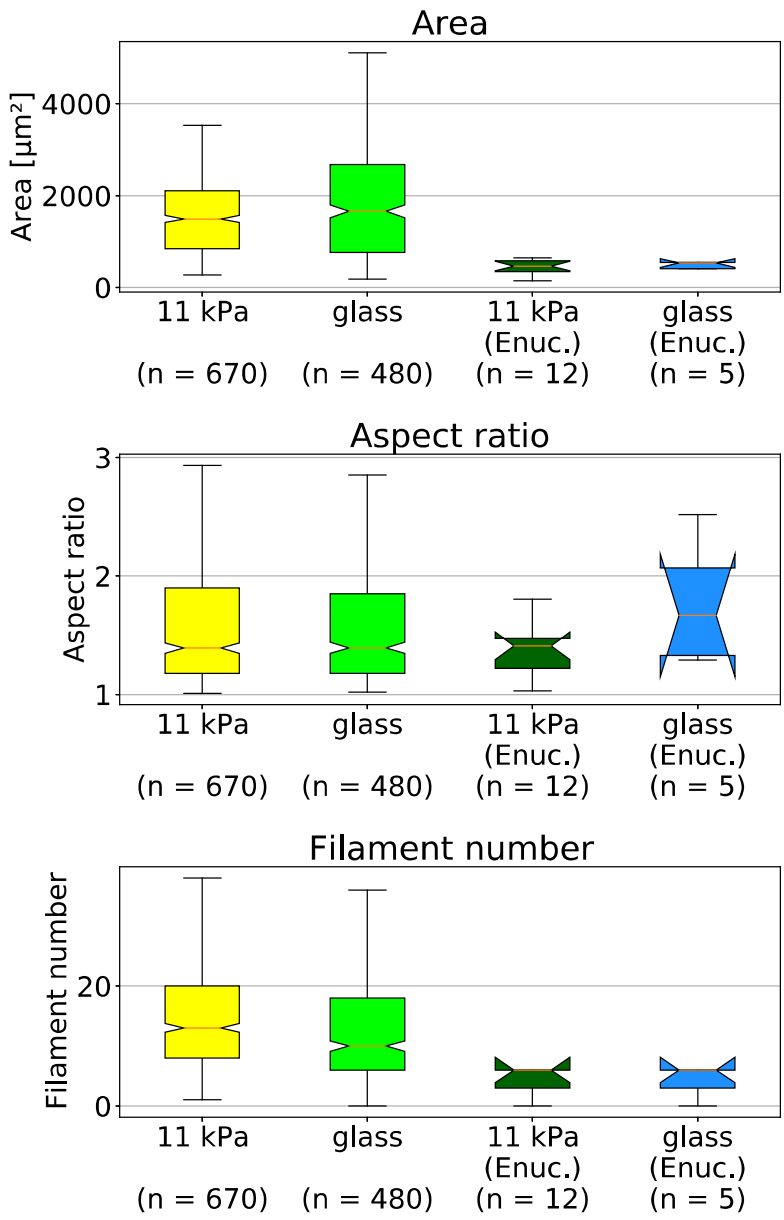

Figure 7.29.: Area, aspect ratio and filament number of enucleated and non-enucleated SAOS-2 cells originally transfected with LifeAct-RFP for live cell movies. Fixed after 24 hours and stained with phalloidin and Hoechst.

NIH-3T3 cells were already discussed in Subsection 7.2.3.3 but are in general very similar to 3T3 cells, as can be seen in Figure 7.30. There, for fixed cells and cytoplasts mean area, aspect ratios, and filament numbers match between the cell lines, with NIH-3T3 having only slightly larger cell area on glass and overall around one-quarter more stress fibers. During enucleation experiments it was not possible to image viable 3T3 cytoplasts on $11 \mathrm{kPa}$ PAA gel. However, the cell line yielded the most cytoplasts after 24 hours and fixation, with overall percentage of cytoplasts with nuclear residues being very low. Troubles obtaining cytoplasts for live movies might be due to overall problems electroporating this cell line. Although growing, maintaining, and enucleating NIH-3T3 does not pose problems in our lab, 3T3 cells usually proliferate slower and only yield around $20 \%$ of transfected cells after electroporation.

Thus, 3T3 cells seem to be favorable for generation of untransfected cytoplasts or imaging of fixed cytoplasts. However, for live cytoplast imaging, NIH-3T3 should be preferred when looking for cytoplasts of fibroblasts. 
$3 T 3$

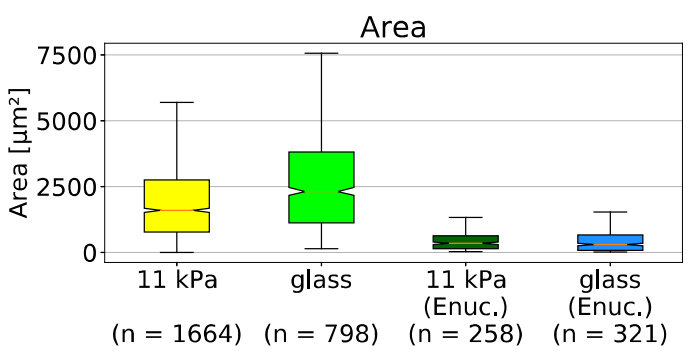

Aspect ratio

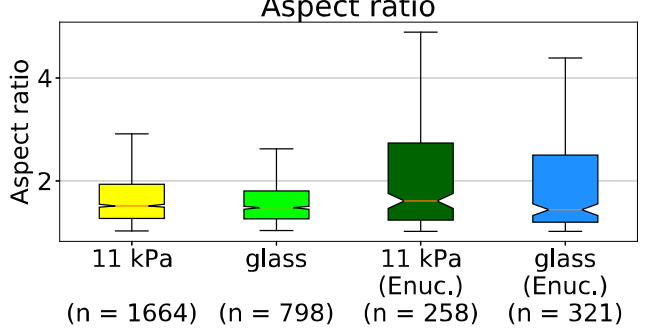

Filament number

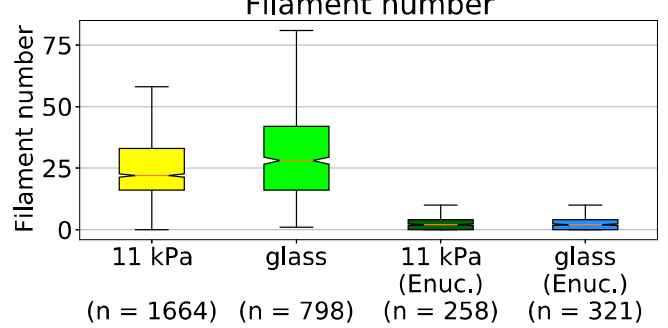

$\mathrm{NIH}-3 \mathrm{T3}$

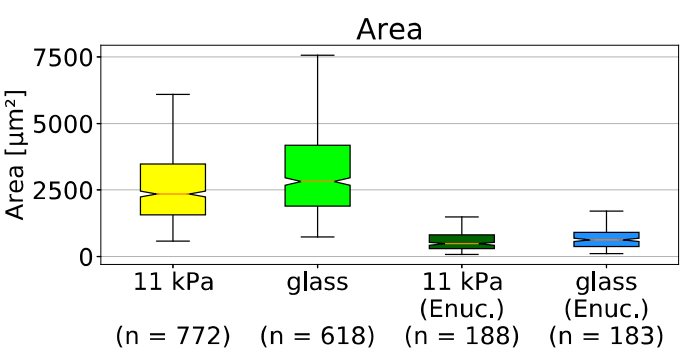

Aspect ratio

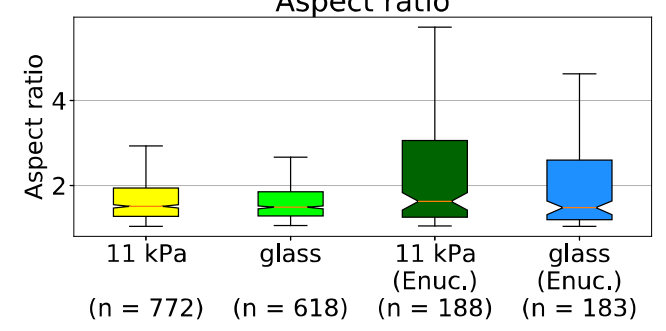

Filament number

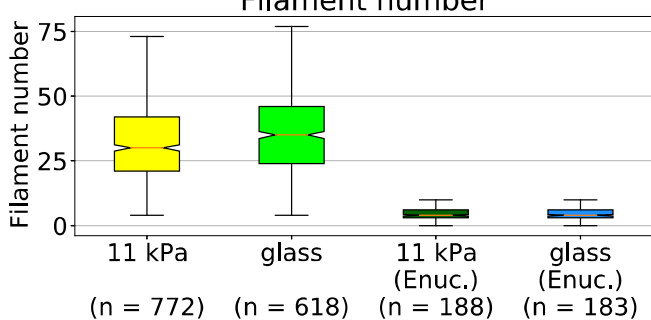

Figure 7.30.: Area, aspect ratio and filament number of enucleated and non-enucleated 3T3 cells originally transfected with LifeAct-RFP for live cell movies. Fixed after 24 hours and stained with phalloidin and Hoechst.

The original idea behind enucleation was to investigate the contribution of the nucleus to the mechanosensitive differentiation of hMSCs, seeding hMSC cytoplasts on substrates of different stiffness and analyzing the stress fiber network as done in Chapter 5. This original research design itself have posed a series of problems as only hMSCs up to passage \#8 can be used and two million cells would have had to be grown. But from the start it became clear that hMSCs did not tolerate the enuclation protocol, with cytoblasts dying 4-8 hours into experiments, which triggered the search for other cell lines to either use to conduct experiments or to find a better starting point to improve the protocol.

As can be seen in Figure 7.31, few viable cytoplasts can be found after 24 hours with cell area and filament number close to zero and aspect ratio indicating a very round shape. Those cytoplasts, although viable, seem not to exhibit a stress fiber network, nor to act as functioning cells, in contrast to other cell lines like NIH-3T3, MCF-7, REF-52, SAOS-2, and 3T3. 
hMSC
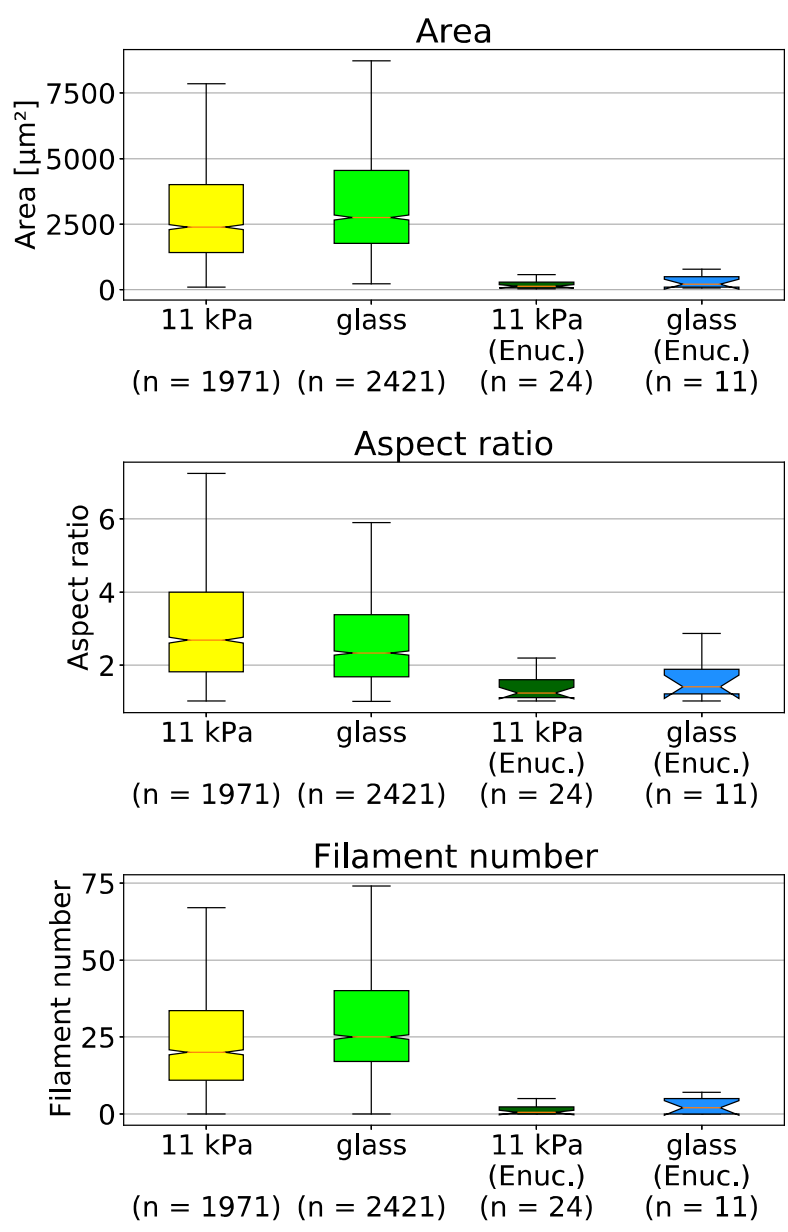

Figure 7.31.: Area, aspect ratio and filament number of enucleated and non-enucleated hMSC cells originally transfected with LifeAct-RFP for live cell movies. Fixed after 24 hours and stained with phalloidin and Hoechst.

In conclusion, the formerly mentioned cell lines might be used for future enucleation experiments. Also, there are existing phase-contrast movies of non-transfected, thus less stressed and possibly more viable, cytoplasts. The analysis of these is beyond the scope of this thesis as it does not show the stress fiber network I am interested in, but might be done as a follow-up while finalizing development of a software for high-throughput phase contrast analysis. 


\section{Investigating the transcriptome of differentiating hMSCs}

Investigating the transcriptome of differentiating hMSCs is nothing new. However, this is usually done after several days or weeks, when tissue-specific transcription factors are present in large amounts [67]. However, since differences in stress fiber network - and thus connected structures and progresses - are visible after the first 24 hours, there should be changes in transcriptome by that time as well. In this chapter, I present data on a full transcriptome analysis via RNA Sequencing (RNA-Seq) of hMSCs grown for 24 hours on PAA gels of different stiffnesses, as well as qRT-PCR (quantitative reverse transcription polymerase chain reaction) of different time points measured from 0 to 24 hours after plating on PAA gels of different stiffnesses, for targets selected based on the full transcriptome.

\subsection{RNA extraction of hMSCs on substrates of different stiffnesses}

RNA extraction of hMSCs was done using the same methods for both types of experiments, RNA-Seq and qRT-PCR. RNA-Seq was done by an external laboratory and should provide insight into the complete metabolism of our cells on different stiffnesses $(1,2,5,11,20$, and $30 \mathrm{kPa}$ PAA gel, glass, all coated with collagen I) and two time points ( 0 and 24 hours). Cells for qRT-PCR were grown in the same environment, but more time points have been analyzed $(0,1,4,12,18,24$ hours) for some chosen target proteins.

The same lot of hMSCs were used for all RNA-Seq and qRT-PCR experiments, in order to facilitate comparing these experiments. After thawing (see Subsection A.1) the hMSCs were grown for one passage (see Subsection A.3) to minimize stress effects. Afterward, they were seeded on glass coverslips and polyacrylamide gels (see Section C), both collagen-coated (see Subsection C.4), as well as T-75 Corning cell culture flasks. PAA gel mixtures were measured with a rheometer to ensure correct stiffness. The results of these measurements can be found in Section 4.4. They have been removed by trypsinization (also described in Subsection A.3) from the respective substrates. RNA extraction was done according to protocol in Subsection B.1 with commercially available Qiagen kits, and samples were frozen at $-20^{\circ} \mathrm{C}$. Samples for RNA Sequencing in the TAL laboratory were brought there on ice. For qRT-PCR, RNA samples were written into cDNA (see Subsection B.2). cDNA was stored at $-20^{\circ} \mathrm{C}$ and RNA, as backup, at $-80^{\circ} \mathrm{C}$.

\subsection{Full RNA Sequencing after 24 hours}

RNA-Seq gives insight into the full transcriptome of a certain time point and can be employed on multiple-cells or single-cell levels. It can be used with RNA selection or depletion to build libraries of total RNA, mRNA, tRNA or miRNA. Besides giving the presence and quantity of a certain RNA, it can also identify alternative splicing, mutations, SNPs, gene fusions, or post-transcriptional modifications. The resulting library can be analyzed de novo or genome guided with a reference genome as close to the sample 
properties as possible. In these experiments, we generate a full mRNA library. After annotation one can choose between multiple approaches for analysis. One of the most common is differential analysis, which compares two or more conditions and selects up- and downregulated genes. The tool chosen in this thesis is count-based DESeq2, which uses negative binomial-generalized linear models and FDR (false discovery rate) adjusted p-value adjustment to account for multiple hypotheses. However, poor experiment design and misplaced assumptions during analysis can lead to statistical errors or wrong interpretations, and so must be kept in mind. After differential analysis, a candidate gene approach or gene set enrichment analysis can be employed to gather an understanding of the larger biological implementation. Here, I use KEGG (Kyoto Encyclopedia of Genes and Genomes) analysis to facilitate this.

Up to the analysis of up- and downregulated genes, this experiment had been done before in some parts by Carina Wollnik in 2015. Getting the results quite shortly before her submission deadline, only preliminary but very promising data was shown in her thesis [225]. She kindly allowed me to reanalyze and use her data in my thesis. To allow for comparison with Wollnik's data, I used the same gel stiffness and, as was done in her research, selected a control of trypzinized cells (zero-hour control). However, as this control is unsuitable for analyzing cells plated for 24 hours, additional controls grown on collagen coated glass and T-75 Corning cell culture flasks are used. Another difference in experiment design can be seen in the cell seeding and lysing. Wollnik

cultured 50,000 cells per gel stiffness each on 4 large polyacrylamide gels with a diameter of $5 \mathrm{~cm}$. As a control, [we] used cells thawed on the same day of the same passage from the same person, frozen the same day. The control cells and the cells used for the experiment were allowed to incubate 3 days after thawing in a T75 Corning cell culture flask. Then, the control cells were lysed by trizol treatment and stored at $-80^{\circ} \mathrm{C}$. At the same time, the other cells were seeded onto the different gels. 24 hours later, the cells were lysed as well. [225]

, I seeded around 150,000 cells on $5 \times 8 \mathrm{~cm}$ glasses with or without polyacrylamide gels, manufactured according to protocol in Section C, in 1-well dishes (gels and glass both coated with collagen I) and T-75 Corning cell culture flasks. Cells for zero hour samples were lysed right away, 24-hour samples were removed from the gels and lysed with the Qiagen RNeasy and DNase Kits as described in protocol in Subsection B.1. Samples were stored at $-20^{\circ}$ C. In both cases, all produced samples were given for sequencing, independent of quality. Samples of minor quality were excluded by Dr. Shomroni, who was responsible for both experiments at the Transcriptome and Genome Analysis Laboratory in Göttingen (TAL), from analysis after sequencing.

An important detail with this repeated experiment, besides the additional control samples, is the difference in the replicates. While the Wollnik experiment uses four technical replicates, I collected samples from four different passages, thus generating biological replicates.

In both cases, RNA samples were handed personally to the TAL, sequenced, and resulting sequencing data analyzed by Dr. Orr Shomroni according to the materials and methods paper provided by him and given in the Appendix in Section F. Sample data of Carina Wollnik's from 2015 were reanalyzed with the new $\mathrm{R}$ pipeline (updated software and library versions).

Additional analyses like ToppGene and KEGG pathways were done by me starting from raw data after redoing the DESeq analysis, for comparison with Dr. Shomroni's results. Samples (cells after 24 hours on polyacrylamide gels of different stiffnesses) were compared to non-plated cells of the same passage (which had been the control in Wollnik's experiment), to collagen-coated glass (what is usually used in fixed cell experiments, in contrast to polyacrylamide gels, as "infinite stiffness") and regular cell culture flasks. Furthermore, the resulting significant genes of the sets of samples versus zero hours have been compared for overlapping genes in the two data sets, as well as for the results of collagen-coated glass versus cell culture flask in the new data set. 


\subsubsection{KEGG pathway analysis}

While analysis of specific genes can be of use for many approaches, for this experimental question a KEGG pathway analysis will most likely yield the best results. As the cells are seeded for a fairly short time, distinct transcriptomal changes or tissue differentiation markers will most likely not have emerged. In contrast, probing general pathways consisting of regulatory changes of a multitude of genes might already show distinct changes.

KEGG data links gene catalogs to systemic functions in the cell, organism, and ecosystem. It integrates genomics, chemical, and systemic functional information. Hereby, the information on systemic functions was gathered from experiments and transferred to KEGG pathway maps. There is also a cross-species annotation to link genomes of molecular networks through the KEGG Orthology (KO) system. KEGG is widely used as a reference knowledge base for data sets generated by genome sequencing. In recent years it has also been expanded with applications for human diseases, drugs, and health-related substances.

The transcriptome data will be discussed in three subsections: first the overlap (Subsection 8.3.1), second the Wollnik data (Subsection 8.4.1), and lastly my data (Subsection 8.4.2). The KEGG pathway analysis will be found at the end of each of these subsections.

\subsection{Comparison of my results and Wollnik (2015) results regarding sample quality}
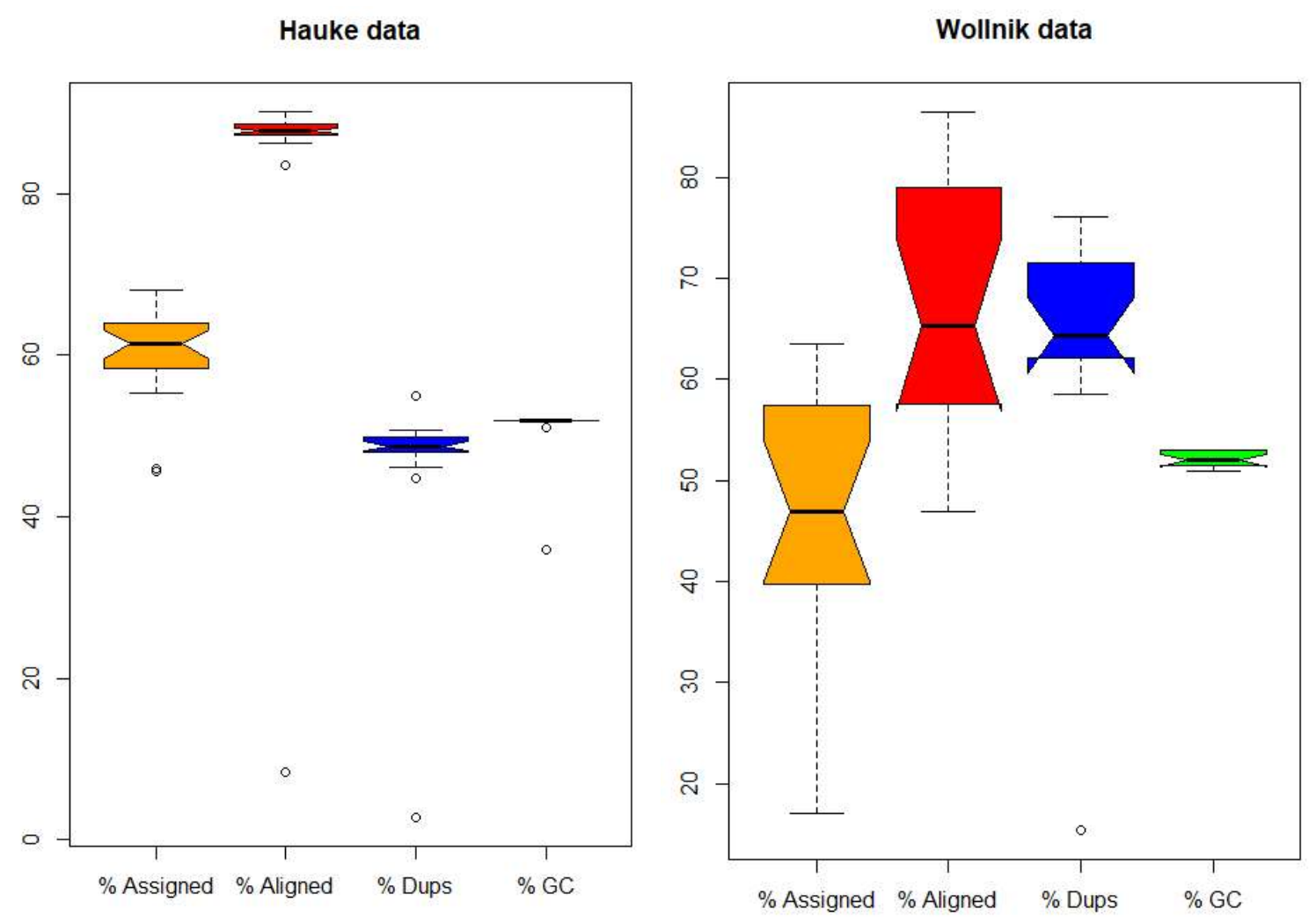

Figure 8.1.: Comparison of percentages for aligned and assigned reads, duplicates and GC content. Wollnik data shows broader distributions in all variables and in general lower values for assigned and aligned reads and higher values for duplicates.

Before comparison of both data sets, there is an issue that must be considered. This is the dependence on alignment in the DESeq2 analysis of the sample quality. As the FASTQ files are aligned to a selected 
database and their reads counted, better sample quality will lead to longer strands and more detailed identification. This will result, finally, in a lower count number of more individual genes.

Hauke data

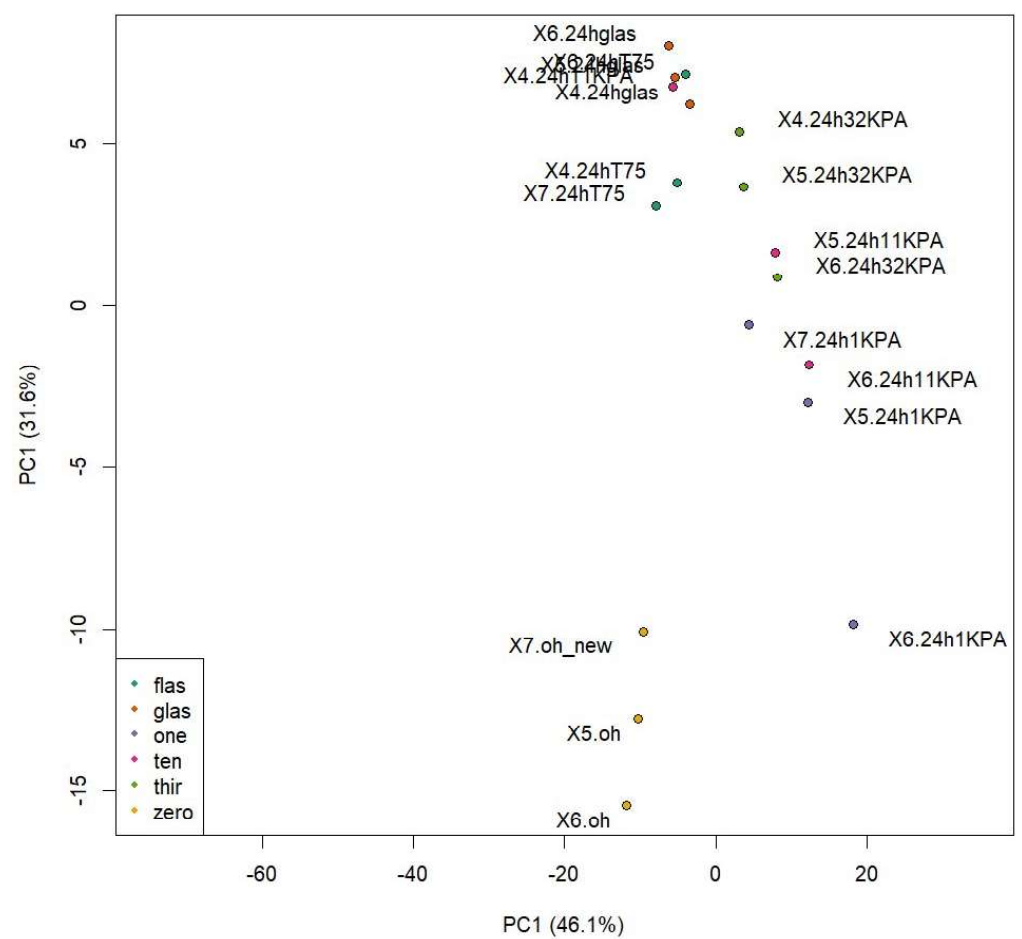

Wollnik data

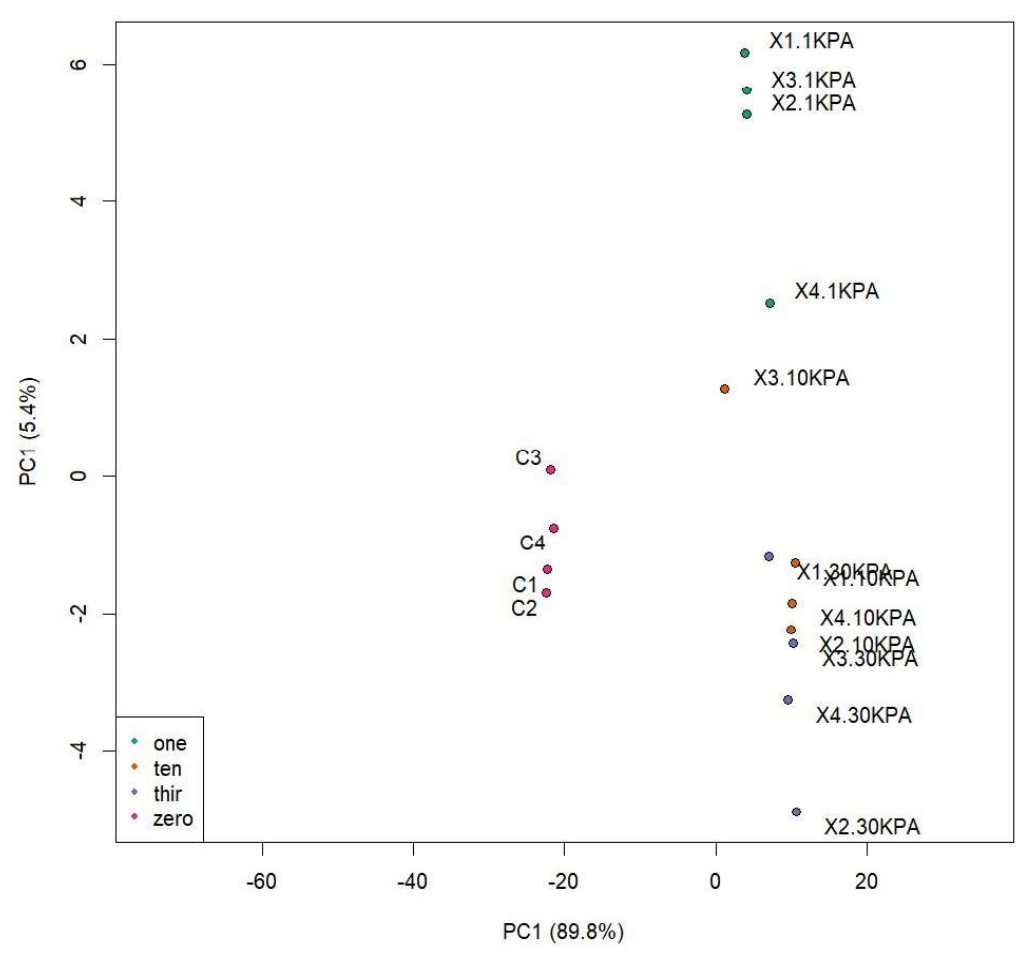

Figure 8.2.: PCA plots for Hauke (top) and Wollnik (bottom) data. Samples shown with three replicates each, outmost replicate already excluded according to Dr. Shamroni. 

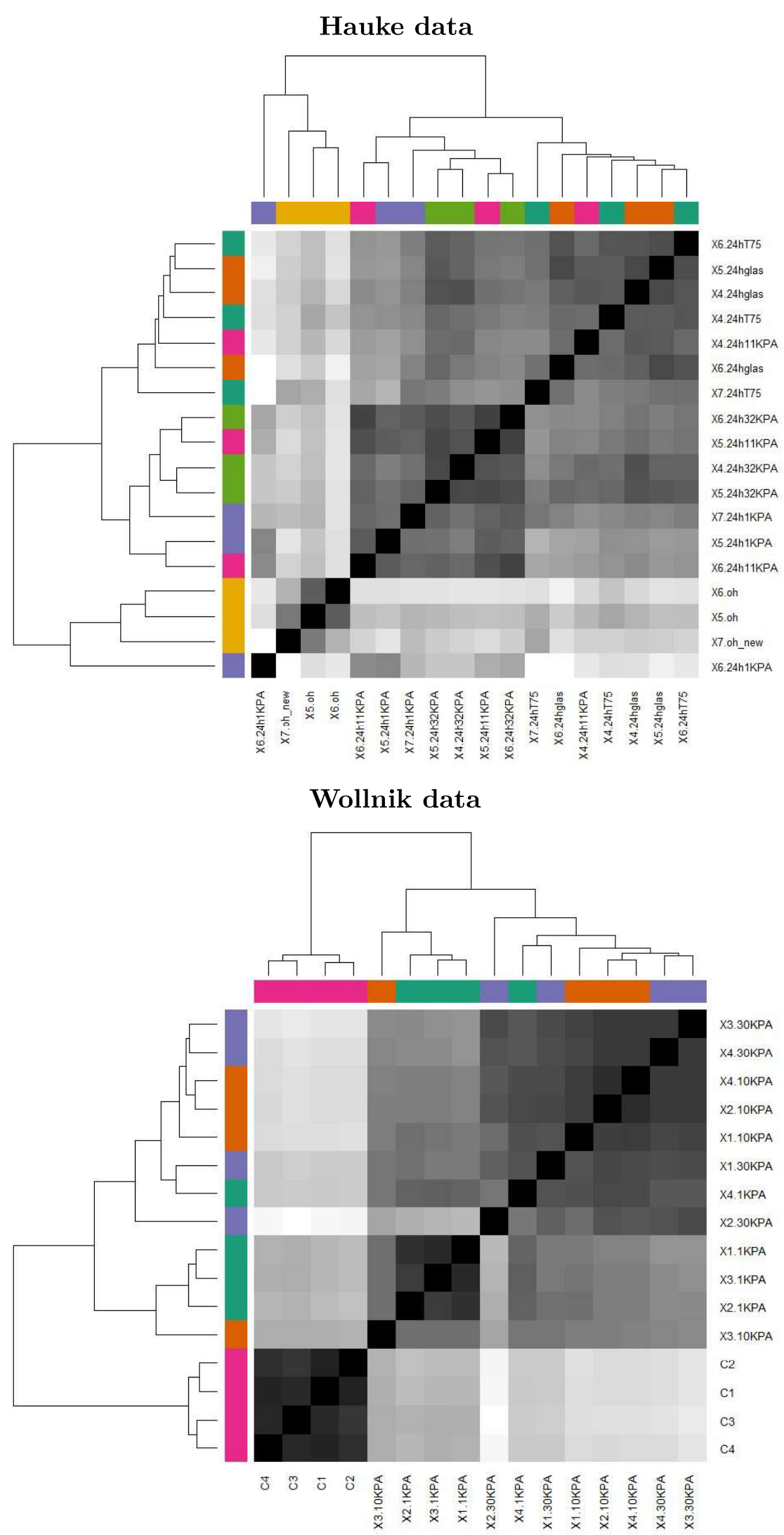

Figure 8.3.: Comparison of sample distance heatmaps. Left: Wollnik data set, right my data set. Three replicates of each condition shown, of three separate experiments each. 
Thus, sample quality has to be considered when comparing different RNA-Seq experiments. As shown in Figure 8.1, the Wollnik data has fewer assigned reads, fewer aligned reads, and more duplicates, while guanine/cytosine (GC) content is about the same. Also, distribution between samples is larger, showing more variable sample quality. Given those initial differences, I expect the analysis of my data to show less significant up- and downregulations then the Wollnik data.

Following the steps of the DESeq2 protocol, dispersion plots and mean gene expression do not differ significantly between both data sets. In the primary component analysis (PCA), however, differences in the sample sets can clearly be seen.

These PCA plots are a means of checking for quality control as well as accessing general experimental issues such as contamination or mislabeled data. The different samples should be spread along the axes per condition but replicates clustered. For the Wollnik data, this works as expected with controls and 1 $\mathrm{kPa}$ samples well-clustered and separate, while 10 and $30 \mathrm{kPa}$ samples overlap. Also, zero-hour controls are well separated from 24-hour samples seeded on PAA gels. For my own data, zero-hour controls cluster as well, but 24-hour samples show a larger overlap. However, clustering of T-75 cell culture flasks and glass seems to occur in my data and should be investigated. Samples of $1 \mathrm{kPa}$ PAA gel show a large variance in themselves, but could be seen as somewhat clustered. As seen also in the Wollnik data, 11 and $32 \mathrm{kPa}$ gel samples seem to be very similar. This is also shown in the sample distance heatmaps (see Figure 8.3). For the sample distance heatmap of the Wollnik data set, the controls are clustered perfectly, as well as most of the 1 and $10 \mathrm{kPa}$ samples where one outlier each can be seen. The $32 \mathrm{kPa}$ samples mix with the other PAA gel samples. For my data, one $1 \mathrm{kPa}$ sample clusters with the zero-hour control group. While the PAA gel samples are quite mixed, again the glass and T-75 Corning flask samples seem to be quite similar, with one $11 \mathrm{kPa}$ PAA gel sample mixing in.

From this PCA plot's heatmaps we can get a general idea of the relative distances of the samples and the clustering. The next step is to look into the genes showing the largest expression differences, which will be done in the next section. To conclude, we see differences even before detailed analysis. While the zero-hour control group is distinguishable in both sets, 10 and 30 (Wollnik data set), respective 11 and 32 (my data set) $\mathrm{kPa}$ PAA gels seem to overlap in both. $1 \mathrm{kPa}$ is distinguishable in Wollnik's data, but not mine. The two additional 24-hour controls on glass and T-75 Corning flasks in my data seem to be nearly identical.

\subsubsection{Gene and pathway overlap}

In both the Wollnik and Hauke data sets, around 16,000 single genes were identified. To get a insight into how big the overlap is, I searched for genes expressed in the same fashion-meaning up- or downregulated in regards to either zero-hour control or the respective other 24-hour PAA gel sample. I did this for the zero-hour controls and the 1, 10 (11), and 30 (32) $\mathrm{kPa}$ PAA gel samples in each set.

However, genes expressed similarly between the same substrates in both sets are surprisingly sparse. Comparing $30 \mathrm{kPa}$ samples of both data sets, only HOXA3 is expressed in the same fashion. For $10 \mathrm{kPa}$ samples, ALOX12, C5orf56, and MYH3 are expressed similarly. Comparing the results of cells seeded on 1 $\mathrm{kPa}$ gels there are 538 genes of similar expression. Of these, ten seem to be relevant for actin remodeling, adhesion, and differentiation (ADGRA2, ACTA2, ADGRG6, MAPK8IP3, PPFIA4, MRNIP, VCAM1, CADM1, BCAM, and NPIPB2). Relevant genes were selected from various papers and reviews for relation to mechanosensing and ECM remodeling linkage $[20,36,91,125,140,185,193,200]$. Between the samples used at zero hours without plating, there were 1,010 similarly expressed genes, of which 27 were deemed relevant (ADGRA2, CDON, PIK3IP1, TSNAXIP1, ACTA2, RIPOR2, ADGRG6, AUNIP, PSMC3IP, CKAP2, BRIP1, MAPK8IP3, PPFIA2, PPFIA4, TWF1, MRNIP, VCAM1, PBXIP1, NRIP3, ARL6IP6, CADM1, OPCML, FSCN2, BCAM, JAKMIP3, KAZN, and NPIPB2). 


\section{Upregulated pathways}

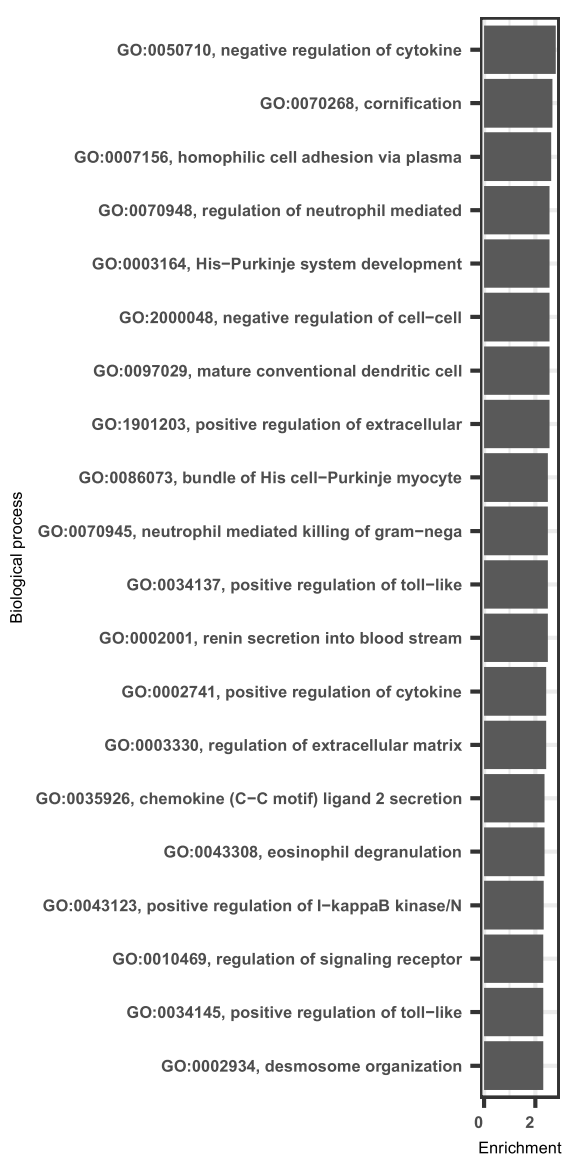

\section{Downregulated pathways}

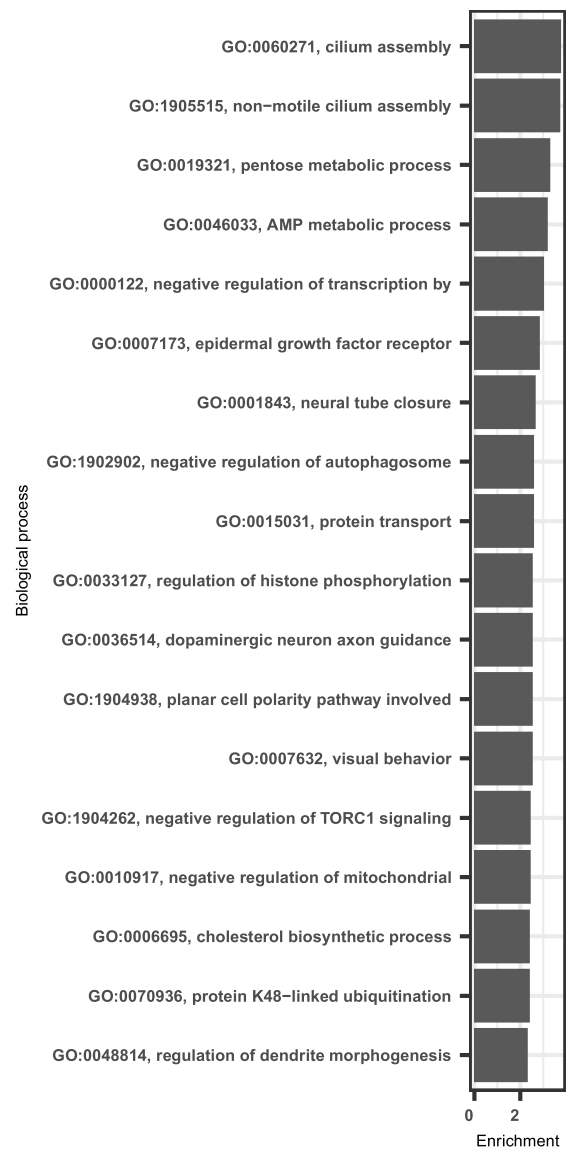

Figure 8.4.: Top 20 up- and downregulated pathways in both data sets according to KEGG pathway analysis with topGo package using built-in weight01 algorithm and Fisher test.

When looking for similarly expressed genes between 24-hour samples, the situation is alike. Comparing both sets of up- and downregulated genes between 10 (11) and 30 (32) kPa samples for Hauke and Wollnik data, there is no overlap found. For 1 and 10 (11) $\mathrm{kPa}$ samples of both sets, 5 genes overlap (PTHLH, NFATC2, ANO1, LHFPL2, and ATF3) of which none seems to be relevant for cell adhesion pathways and differentiation. Similar overlap can be found when comparing 1 and 30 (32) $\mathrm{kPa}$ samples (PTHLH, NFATC2, SLC16A6, LHFPL2, ATF3, and MTSS1). Four genes from this set overlap with the former comparison. In conclusion, while PCA and heatmaps appear at least somewhat similar, single gene expression differences differ vastly between the two data sets.

To scan for overlapping pathways, I combined Wollnik's data and my data, reduced to zero-hour control and PAA gel samples for 1, $10(11), 30(32) \mathrm{kPa}$. In this case the reference is the zero-hour control. Reiterating the generation of these samples, cells were thawed and grown for 3 days, then trypsinized. From the trypsinized cells, zero-hour controls are taken without replating. Remaining cells were seeded on PAA gels coated with collagen and incubated for 24 hours before harvesting. Harvesting method diverges between the two sets, trizol being used for the Wollnik cells and trypsinization for Hauke. All following steps were done alike. KEGG pathway analysis is done separately for up- and downregulated pathways and shown as such. In Figure 8.4 top pathways, both up- and downregulated, are shown, while Tables 8.1 and 8.2 show results in detail. 


\begin{tabular}{|c|c|c|c|c|c|}
\hline GO.ID & Term & Annotated & Significant & Expected & Result \\
\hline GO:0050710 & negative regulation of cytokine secretion & 109 & 2 & 0.06 & 0.0016 \\
\hline GO:0070268 & cornification & 128 & 2 & 0.07 & 0.0022 \\
\hline GO:0007156 & $\begin{array}{l}\text { homophilic cell adhesion via } \\
\text { plasma membrane adhesion molecules }\end{array}$ & 133 & 2 & 0.07 & 0.0024 \\
\hline GO:0070948 & regulation of neutrophil mediated cytotoxicity & 5 & 1 & 0 & 0.0028 \\
\hline GO:0003164 & His-Purkinje system development & 5 & 1 & 0 & 0.0028 \\
\hline GO: 2000048 & $\begin{array}{l}\text { negative regulation of cell-cell } \\
\text { adhesion mediated by cadherin }\end{array}$ & 5 & 1 & 0 & 0.0028 \\
\hline GO:0097029 & mature conventional dendritic cell differentiation & 5 & 1 & 0 & 0.0028 \\
\hline GO: 1901203 & positive regulation of extracellular matrix assembly & 5 & 1 & 0 & 0.0028 \\
\hline GO:0086073 & $\begin{array}{l}\text { bundle of His cell-Purkinje myocyte } \\
\text { adhesion involved in cell communication }\end{array}$ & 6 & 1 & 0 & 0.0033 \\
\hline GO:0070945 & neutrophil mediated killing of gram-negative bacterium & 6 & 1 & 0 & 0.0033 \\
\hline
\end{tabular}

Table 8.1.: Top 10 upregulated KEGG Pathways in both data sets when combined

The combination of both data sets was done by adding the total counts for each identified gene. Thus, we should get an exaggerated count for ubiquitous and overlapping genes, but also a broad range of medium expressed and discrete (not overlapping) ones. Pathway analysis for these overlapping genes with high count numbers should lead to some interesting results.

In the comparison of data sets in Subsection 8.3 we already saw more common downregulated genes then upregulated ones. Here, we see a similar distribution. We annotate fewer genes and of these almost none are significant. We see some effects on cell-to-cell adhesion pathways, but most prominent are ones repressing certain cytokines (see Table 8.2).

In contrast,in the downregulated top pathways of the combined data sets we see a large number of both annotated genes and significant genes for certain pathways. The top downregulated pathway is especially interesting. As RNA Polymerase II is responsible for the transfer of DNA into RNA, it is a typical candidate for getting downregulated in the stress response. In second place we see a pathway-facilitating hydrolysis of protein and peptides. Below that there are pathways for cilium assembly. According to GO annotation, cilium and microtubule-based flagellum are deemed equivalent as the related genes overlap significantly. And, taking a closer look into the 310 genes combined in this pathway, there are actin binding proteins; polarity related, centrosomal proteins; and dynein encoding genes also found in these pathways. Additionally, in the nonmotile cilium assembly pathway, fibroblast-exclusive genes for tubulin kinase 2 and Scl/Tal1 interrupting locus, as well as microtubule-associated proteins, can be found.

\begin{tabular}{|c|c|c|c|c|c|}
\hline GO.ID & Term & Annotated & Significant & Expected & Result \\
\hline GO:0006357 & $\begin{array}{l}\text { regulation of transcription by } \\
\text { RNA polymerase II }\end{array}$ & 2617 & 289 & 179,75 & $8.9 \mathrm{e}-28$ \\
\hline GO:0043161 & $\begin{array}{l}\text { proteasome-mediated ubiquitin-dependent } \\
\text { protein catabolic process }\end{array}$ & 373 & 37 & 25,62 & $6.6 \mathrm{e}-05$ \\
\hline GO:0060271 & cilium assembly & 358 & 42 & 24,59 & 0.00017 \\
\hline GO: 1905515 & non-motile cilium assembly & 51 & 11 & 3,5 & 0.00018 \\
\hline GO:0019321 & pentose metabolic process & 11 & 5 & 0,76 & 0.00049 \\
\hline GO:0046033 & AMP metabolic process & 12 & 5 & 0,82 & 0.00065 \\
\hline GO:0000122 & $\begin{array}{l}\text { negative regulation of transcription } \\
\text { by RNA polymerase II }\end{array}$ & 797 & 78 & 54,74 & 0.00092 \\
\hline GO:0007173 & epidermal growth factor receptor signaling pathway & 117 & 17 & 8,04 & 0.00141 \\
\hline GO:0001843 & neural tube closure & 89 & 14 & 6,11 & 0.00212 \\
\hline GO:1902902 & negative regulation of autophagosome assembly & 15 & 5 & 1,03 & 0.00255 \\
\hline
\end{tabular}

Table 8.2.: Top 10 downregulated KEGG Pathways in both data sets when combined

\subsection{Influence of stiffness on early hMSC transcriptome}

Given the differences in hMSC source (different donors), replicates (biological vs. technical), method (different recovery of cells for RNA-Seq), and samples (additional controls in my data), I decided to do in-depth gene and pathway analysis separately for both data sets. 


\subsubsection{Wollnik data (2015)}

As already described, the Wollnik data set consists of technical replicates of four different conditions: zero-hour controls, and cells seeded for 24 hours on PAA gels of three different stiffnesses (1, 10, $30 \mathrm{kPa})$. The zero-hour controls are taken from the trypsinized cells before seeding on gels.

What has already been made visible in the PCA plots and sample distance heatmaps continues with the top differently expressed genes (see Figure 8.5).

\section{Top 50 differently expressed genes in Wollnik data}

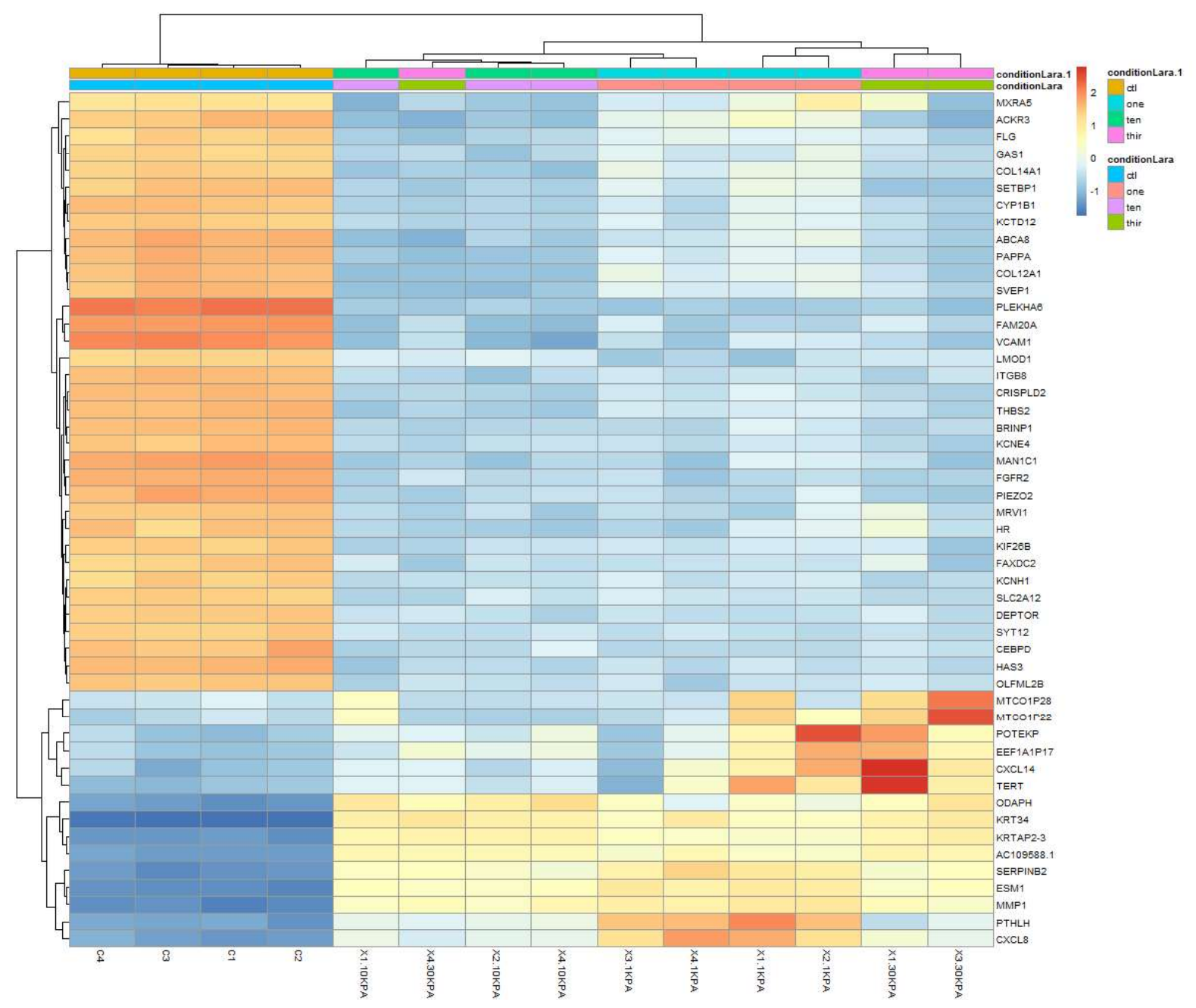

Figure 8.5.: Wollnik data set, heatmap, and clustering of the top 50 differently expressed genes.

The top 50 differently expressed genes for the Wollnik data show obvious differences between zero-hour controls and 24-hour PAA gel samples. Additionally, even when just clustering the samples according to the top regulated genes, not only the zero-hour controls but also the 1 and $10 \mathrm{kPa}$ PAA gel samples seem to behave very similarly. Filtering these genes for those linkable to stress fiber remodeling, adhesion, and differentiation, we see none except VCAM1 and two collagen subtypes (VCAM1, COL12A1, COL14A1). Additionally, FGFR2, BRINP1, ITGB8, PIEZO2, DEPTOR, and MMP1 can be seen as related to matrix remodeling and mechanosensing.

Looking into KEGG pathway analysis for the Wollnik data set (TopGo weight01 algorithm and Fischer test), extracellular matrix remodeling is the top upregulated pathway (see Table 8.3). However, other 
upregulated pathways seem rather unrelated, with an upregulation in ethanol response even hinting at a possible reaction to the RNA extraction protocol used.

\begin{tabular}{|c|c|c|c|c|c|}
\hline GO.ID & Term & Annotated & Significant & Expected & Result \\
\hline GO:0003330 & $\begin{array}{l}\text { regulation of extracellular matrix } \\
\text { constituent secretion }\end{array}$ & 7 & 2 & 0,04 & 0.00058 \\
\hline GO:0035774 & $\begin{array}{l}\text { positive regulation of insulin secretion } \\
\text { involved in cellular response } \\
\text { to glucose stimulus }\end{array}$ & 31 & 3 & 0,16 & 0.00059 \\
\hline GO:0045471 & response to ethanol & 131 & 5 & 0,7 & 0.00067 \\
\hline GO:0042758 & long-chain fatty acid catabolic process & 9 & 2 & 0,05 & 0.00098 \\
\hline GO:0006617 & $\begin{array}{l}\text { SRP-dependent cotranslational protein targeting } \\
\text { to membrane, signal sequence recognition }\end{array}$ & 9 & 2 & 0,05 & 0.00098 \\
\hline GO:0014894 & $\begin{array}{l}\text { response to denervation involved } \\
\text { in regulation of muscle adaptation }\end{array}$ & 10 & 2 & 0,05 & 0.00122 \\
\hline GO:0010918 & $\begin{array}{l}\text { positive regulation of mitochondrial } \\
\text { membrane potential }\end{array}$ & 10 & 2 & 0,05 & 0.00122 \\
\hline GO:0000353 & formation of quadruple SL/U4/U5/U6 snRNP & 12 & 2 & 0,06 & 0.00178 \\
\hline GO:0051712 & $\begin{array}{l}\text { positive regulation of killing of } \\
\text { cells of other organism }\end{array}$ & 12 & 2 & 0,06 & 0.00178 \\
\hline GO:0032570 & response to progesterone & 46 & 3 & 0,24 & 0.00187 \\
\hline
\end{tabular}

Table 8.3.: Top 10 upregulated KEGG pathways in the Wollnik data set analysed with TopGo package, weight01 algorithm and Fischer test.

Consistent with possible reactions to the experiment and handling, RNA polymerase II regulation appears again as the top downregulated pathway (see Table 8.4). Other downregulated pathways consist of many essential processes like protein transport, protein transport, MAPKK activity, and GTPase activity.

\begin{tabular}{l|c|l|l|l|l} 
GO.ID & Term & Annotated & Significant & Expected & Result \\
\hline GO:0006357 & $\begin{array}{c}\text { regulation of transcription } \\
\text { by RNA polymerase II }\end{array}$ & 2617 & 620 & 412,54 & $1.3 \mathrm{e}-30$ \\
GO:0007030 & Golgi organization & 96 & 38 & 15,13 & $8.8 \mathrm{e}-09$ \\
GO:0000186 & activation of MAPKK activity & 51 & 25 & 8,04 & $8.1 \mathrm{e}-08$ \\
GO:0043547 & positive regulation of GTPase activity & 363 & 98 & 57,22 & $2.6 \mathrm{e}-07$ \\
GO:0015031 & $\begin{array}{l}\text { protein transport } \\
\text { negative regulation of transcription }\end{array}$ & 1895 & 379 & 298,72 & $2.7 \mathrm{e}-07$ \\
GO:0000122 & $\begin{array}{l}\text { by RNA polymerase II } \\
\text { embryonic digit morphogenesis }\end{array}$ & 61 & 180 & 125,64 & $5.2 \mathrm{e}-07$ \\
GO:0042733 & intraciliary transport involved in & 40 & 25 & 9,62 & $2.1 \mathrm{e}-06$ \\
GO:0035735 & cilium assembly & 19 & 6,31 & $2.5 \mathrm{e}-06$ \\
GO:0006886 & intracellular protein transport & 905 & 191 & 142,66 & $9.5 \mathrm{e}-06$ \\
GO:0001701 & in utero embryonic development & 366 & 82 & 57,7 & $1.5 \mathrm{e}-05$
\end{tabular}

Table 8.4.: Top 10 downregulated KEGG pathways in the Wollnik data set analysed with TopGo package, weight01 algorithm and Fischer test.

\subsubsection{Transcriptomal differences between gel stiffnesses with controls omitted}

Because the differences between controls and PAA gel samples are more pronounced than the differences between gel samples themselves, I omitted controls to find the most diverse genes between gel sample sets. As before, the DESeq2 pipeline was used to filter outliers and nonsignificant genes. Finally, mean expression difference of all technical replicates is compared and shown, simplified, in Table 8.5 for easy comparison. 


\begin{tabular}{|c|c|c|c|c|}
\hline Gene tag & Long term & $\begin{array}{l}\text { Mean } \\
1 \mathrm{kPa}\end{array}$ & $\begin{array}{c}\text { Mean } \\
10 \mathrm{kPa}\end{array}$ & $\begin{array}{c}\text { Mean } \\
30 \mathrm{kPa}\end{array}$ \\
\hline NTN1 & netrin 1 & + & - & - \\
\hline WNT11 & Wnt family member 11 & + & - & - \\
\hline PTHLH & parathyroid hormone like hormone & + & - & - \\
\hline NFATC2 & nuclear factor of activated $\mathrm{T}$ cells 2 & + & - & - \\
\hline MXRA5 & matrix remodeling associated 5 & + & - & - \\
\hline COL12A 1 & collagen type XII alpha 1 chain & + & - & - \\
\hline PRG4 & proteoglycan 4 & + & - & - \\
\hline PTGIS & prostaglandin I2 synthase & + & - & + \\
\hline C3 & complement $\mathrm{C} 3$ & + & - & + \\
\hline FOSB & FosB proto-oncogene, AP-1 transcription factor subunit & + & - & - \\
\hline LRP4 & LDL receptor related protein 4 & + & - & + \\
\hline HMCN1 & hemicentin 1 & + & - & - \\
\hline CXCL14 & $\mathrm{C}-\mathrm{X}-\mathrm{C}$ motif chemokine ligand 14 & + & - & + \\
\hline MMP3 & matrix metallopeptidase 3 & + & - & - \\
\hline DST & dystonin & + & - & - \\
\hline SMG1 & SMG1, nonsense mediated mRNA decay associated PI3K related kinase & + & - & - \\
\hline COLEC12 & collectin subfamily member 12 & + & - & + \\
\hline ACTC1 & actin, alpha, cardiac muscle 1 & - & + & + \\
\hline TPPP3 & tubulin polymerization promoting protein family member 3 & + & - & - \\
\hline ACTG2 & actin, gamma 2 , smooth muscle, enteric & - & - & + \\
\hline ITGA2 & integrin subunit alpha 2 & + & - & - \\
\hline TERT & telomerase reverse transcriptase & + & - & + \\
\hline $\mathrm{ABCA} 1$ & ATP binding cassette subfamily A member 1 & + & - & - \\
\hline CXCL8 & $\mathrm{C}-\mathrm{X}-\mathrm{C}$ motif chemokine ligand 8 & + & - & - \\
\hline $\mathrm{PCDH} 7$ & protocadherin 7 & + & - & + \\
\hline HSPB7 & heat shock protein family B (small) member 7 & - & + & + \\
\hline ACTG1P1 & actin gamma 1 pseudogene 1 & + & - & + \\
\hline АСТВP11 & actin, beta pseudogene 11 & + & - & + \\
\hline SNORA73B & small nucleolar RNA, H/ACA box $73 \mathrm{~B}$ & - & + & - \\
\hline RNU4-2 & RNA, U4 small nuclear 2 & - & + & - \\
\hline POTEKP & POTE ankyrin domain family member $\mathrm{K}$, pseudogene & + & - & + \\
\hline EEF $1 \mathrm{~A} 1 \mathrm{P} 16$ & eukaryotic translation elongation factor 1 alpha 1 pseudogene 16 & - & - & + \\
\hline EEF $1 \mathrm{~A} 1 \mathrm{P} 15$ & eukaryotic translation elongation factor 1 alpha 1 pseudogene 15 & + & - & + \\
\hline MEG3 & maternally expressed 3 & + & - & - \\
\hline RPS2P55 & ribosomal protein $\mathrm{S} 2$ pseudogene 55 & + & - & + \\
\hline AC016739.1 & ribosomal protein, large, P1 (RPLP1) pseudogene & - & - & + \\
\hline AC010970.1 & RNA, $18 \mathrm{~S}$ ribosomal pseudogene & - & - & + \\
\hline ACTG1P12 & actin gamma 1 pseudogene 12 & + & _- & + \\
\hline ACTG1P21 & actin gamma 1 pseudogene 21 & + & _- & + \\
\hline ACTG1P19 & actin gamma 1 pseudogene 19 & + & - & + \\
\hline PEG10 & paternally expressed 10 & + & - & - \\
\hline NME2 & NME/NM23 nucleoside diphosphate kinase 2 & + & + & - \\
\hline NEAT1 & nuclear paraspeckle assembly transcript 1 & - & - & + \\
\hline MALAT1 & metastasis associated lung adenocarcinoma transcript 1 & - & + & + \\
\hline EEF1A1P17 & eukaryotic translation elongation factor 1 alpha 1 pseudogene 17 & + & - & + \\
\hline MTCO1P28 & MT-CO1 pseudogene 28 & - & - & + \\
\hline MTCO1P22 & MT-CO1 pseudogene 22 & - & - & + \\
\hline RN7SL2 & RNA, 7SL, cytoplasmic 2 & - & + & - \\
\hline FP671120.4 & novel transcript, similar to YY1 associated myogenesis RNA 1 YAM1 & - & + & + \\
\hline AD000090.1 & novel transcript, antisense to ATP4A & - & + & + \\
\hline
\end{tabular}

Table 8.5.: Top 50 genes from Wollnik data set with control group omitted, expression differences calculated as rowwise means for the respective gene and sample set and transcribed according to $\mathrm{x}$-fold expression. ( $+++=<2$-fold expression, $++=1$-2-fold expression, $+=0$-1-fold expression, - = 0-(-1)-fold expression, - - = (-1)-(-2)-fold expression, - - - = <(-2)-fold expression).

Comparing the top 50 differently expressed genes in Table 8.5, we see that for 1 and $30 \mathrm{kPa}$ samples genes are usually expressed more highly than on $10 \mathrm{kPa}$ PAA gels. Furthermore, while in the set comparing with control samples almost no genes linkable with mechanosensitivity are found, comparing only the stiffness samples shows multiple actin genes and pseudogenes as well as genes for integrins, MMPs, and RNA transcription. Also, for almost all found actin-encoding genes, $10 \mathrm{kPa}$ samples are significantly downregulated in comparison to 1 and $30 \mathrm{kPa}$ samples. This could be because cells on $10 \mathrm{kPa}$ gels have significantly fewer actin fibers than on either very soft or stiff substrates. 


\subsubsection{Hauke data}

As already stated, the differences between my data and the 2015 Wollnik data set do partly result from better alignment as well as from using biological instead of technical replicates.

While the Wollnik data set consists of zero hour nonplated controls and 24-hour samples plated on 1, 10, and $30 \mathrm{kPa}$ PAA gels coated with collagen I, in my data set 24-hour samples of cells seeded on glass coated with collagen and T-75 Corning cell culture flasks are introduced as additional controls. Less pronounced clustering was already visible in the PCA plots (see Figure 8.2) and sample distance heatmaps (see Figure 8.3) for my data. Looking into the expression differences on gene level, the Wollnik data showed differences for single genes of above 1/-1 consistently when comparing zero-hour controls against 24-hour samples of cells seeded on PAA gels, while also showing clear clustering and around one-third of top 50 genes downand two-thirds upregulated in controls (compare Figure 8.5).

\section{Top 50 differently expressed genes in Hauke data}

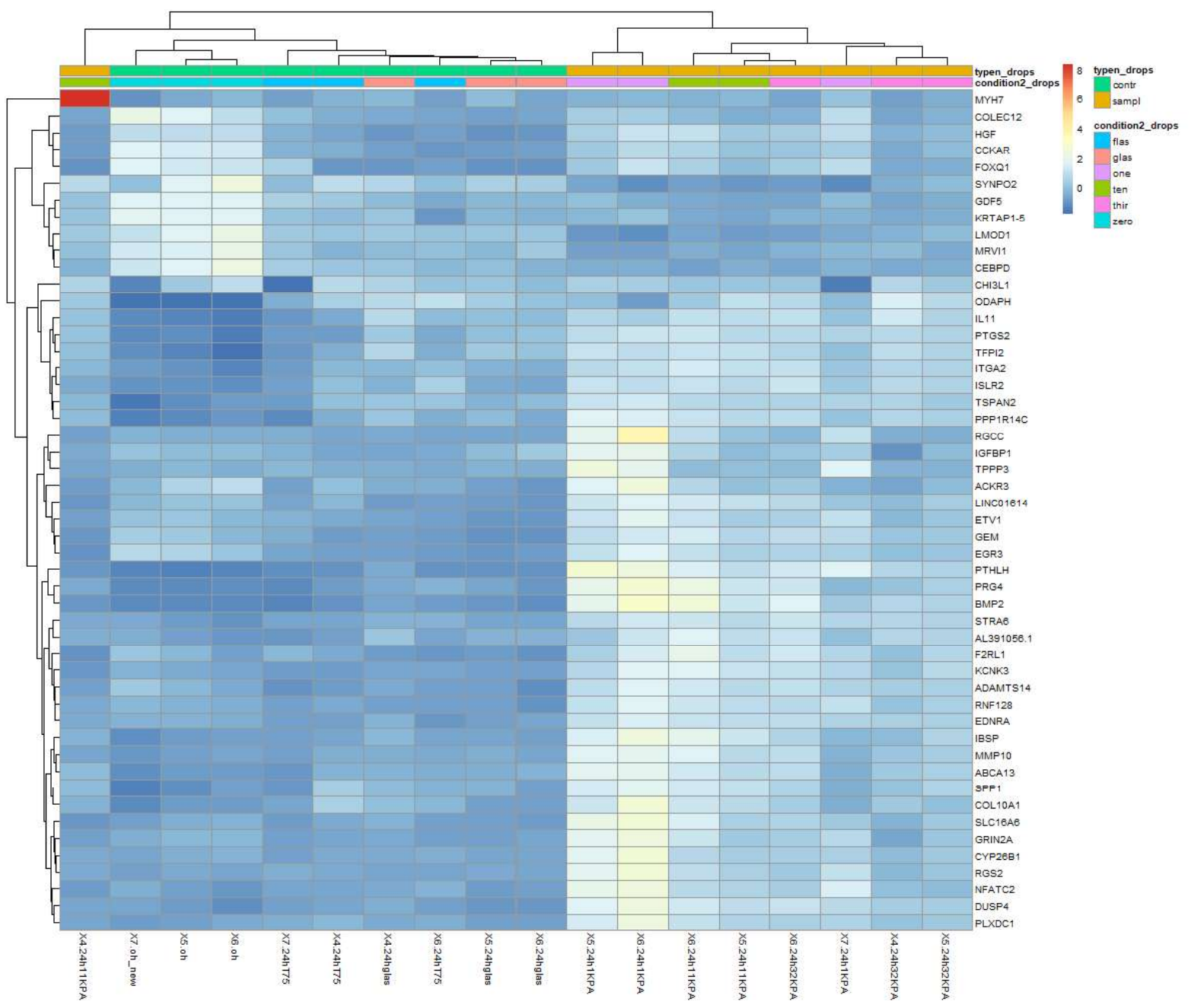

Figure 8.6.: My data set, heatmap, and clustering of the top 50 differently expressed genes.

In comparison, my data - as shown in Figure 8.6-seems less distinct. However, one has to keep in mind from the start that the color code is shifted because of one outlier in one $11 \mathrm{kPa}$ sample, seen at the top left corner, showing eightfold expression. So, instead of from -2 to 2 , the range here is from -2 to 8 , with a distorted color code. So, most of the gene expression difference here is in the range of -1 to 3 without looking quite as impressive. Additionally, when leaving the $11 \mathrm{kPa}$ outlier out of the picture, it can be 
seen that for the top 50 genes clustering occurs for the control groups (zero hours, glass, cell culture flask) versus 24-hour PAA gel samples; and, within the control groups, zero-hour controls are also clustered. Glancing into possibly relevant genes for cell mechanics, we see MYH7, ITGA2, IBSP, and several proteins involved in the MAPK-/JAK-signalling pathways. In total, IBSP, MYH7, NFATC2, RGCC, COL10A1, BMP2, GDF5, ITGA2, GEM, and MMP10 are the relevant proteins for adhesion, mechanosensing, and matrix remodeling. Also, there seem to be some gene clusters up- and downregulated significantly (please see Figure 8.7); these could be interesting to investigate.

\section{Top 50 differently expressed genes in Hauke data}

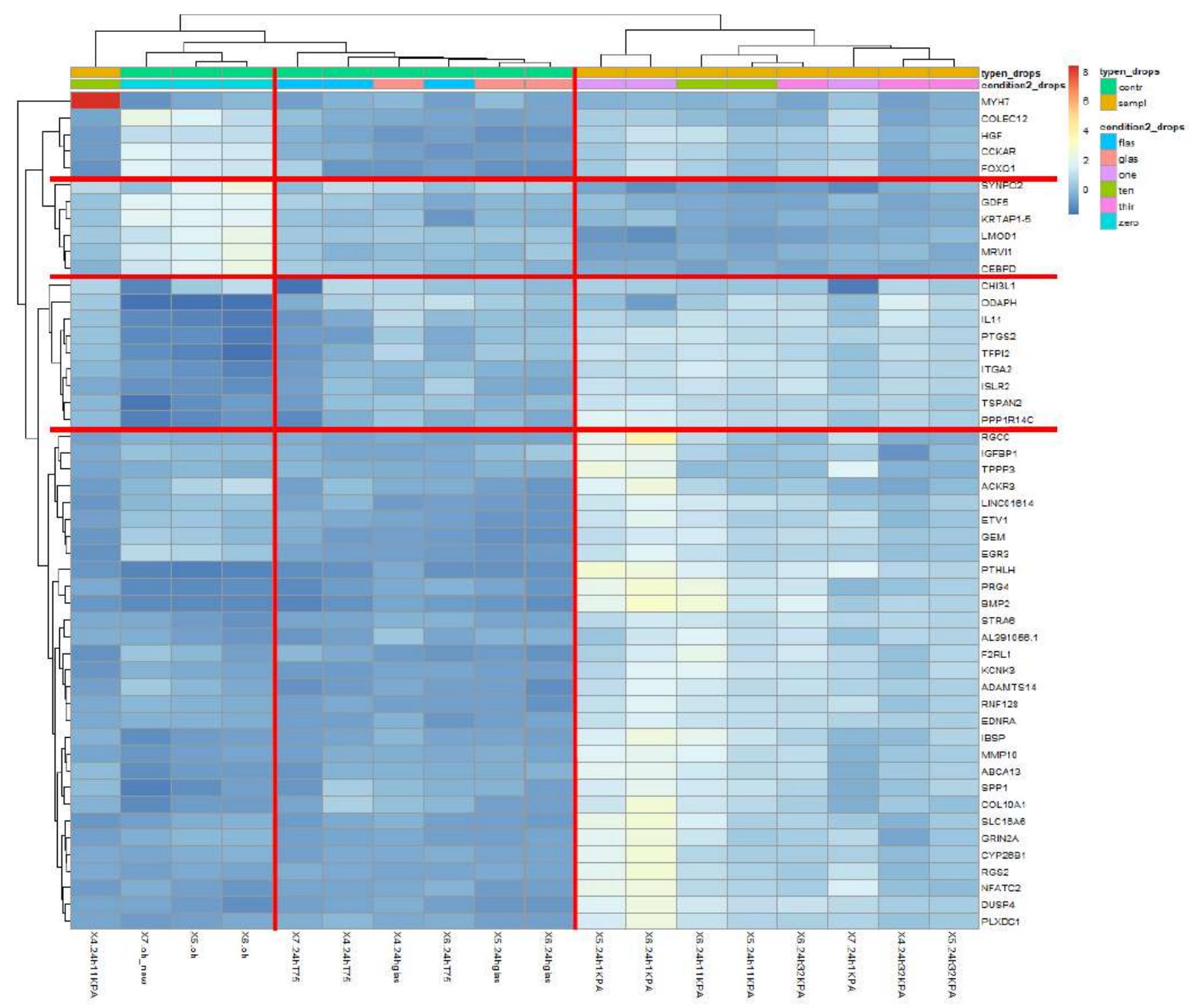

Figure 8.7.: My data set, heatmap, and clustering of the top 50 differently expressed genes.

In Table 8.6 rowmeans for the genes according to the groups hinted at in Figure 8.7 were calculated and coded for expression difference. And while those genes, with exceptions already named, seem rather unrelated - and thus need to be subjected to pathways analysis - there are a few interesting results. First, there is a group of genes strongly overexpressed in zero-hour controls to which 24-hour gels react differently compared to glass and plastic samples. As the cells should react similarly to all surface coatings, given cell culture plastic is the standard and collagen coating is applied in the same way to PAA gels and glass, the disparate reactions may be due to the PAA gel substrate. The other two subset of genes hint in a similar direction, with one set that is strongly underexpressed in zero-hour controls overexpressed in glass and plastic, but underexpressed in PAA gel samples. Lastly, there is a group that is underexpressed in both zero-hour controls and PAA gel samples but overexpressed in glass and plastic samples. All of this 
suggests a strong effect from the presence of PAA gels, probably making it impossible to find real stiffness effects.

\begin{tabular}{|c|c|c|c|c|}
\hline Gene tag & Long term & $\begin{array}{c}\text { Mean } \\
\text { o hours }\end{array}$ & $\begin{array}{c}\text { Mean } \\
\text { glass/plastic }\end{array}$ & $\begin{array}{c}\text { Mean } \\
\text { 24-hour gels }\end{array}$ \\
\hline MYH7 & myosin heavy chain 7 & - & + & - \\
\hline COLEC12 & collectin subfamily member 12 & ++ & - & - \\
\hline HGF & hepatocyte growth factor & + & + & - \\
\hline CCKAR & cholecystokinin A receptor & ++ & + & - \\
\hline FOXQ1 & forkhead box Q1 & ++ & + & - \\
\hline SYNPO2 & synaptopodin 2 & ++ & - & + \\
\hline GDF 5 & growth differentiation factor 5 & ++ & - & - \\
\hline KRTAP1-5 & keratin associated protein $1-5$ & ++ & - & - \\
\hline LMOD1 & leiomodin 1 & ++ & - & + \\
\hline MRVI1 & murine retrovirus integration site 1 homolog & ++ & - & - \\
\hline CEBPD & CCAAT enhancer binding protein delta & ++ & - & - \\
\hline CHI3L1 & chitinase 3 like 1 & - & + & - \\
\hline ODAPH & odontogenesis associated phosphoprotein & - & + & + \\
\hline IL11 & interleukin 11 & - & + & - \\
\hline PTGS2 & prostaglandin-endoperoxide synthase 2 & -- & + & - \\
\hline TFPI2 & tissue factor pathway inhibitor 2 & - & + & - \\
\hline ITGA2 & integrin subunit alpha 2 & -- & + & - \\
\hline ISLR2 & immunoglobulin superfamily containing leucine rich repeat 2 & -- & + & - \\
\hline TSPAN2 & tetraspanin 2 & -- & + & - \\
\hline PPP1R14C & protein phosphatase 1 regulatory inhibitor subunit $14 \mathrm{C}$ & -- & + & - \\
\hline RGCC & regulator of cell cycle & - & + & - \\
\hline IGFBP1 & insulin like growth factor binding protein 1 & - & + & - \\
\hline TPPP3 & tubulin polymerization promoting protein family member 3 & - & + & - \\
\hline ACKR3 & atypical chemokine receptor 3 & + & + & - \\
\hline LINC01614 & long intergenic nonprotein coding RNA 1614 & - & + & - \\
\hline ETV1 & ETS variant 1 & - & + & - \\
\hline GEM & GTP binding protein overexpressed in skeletal muscle & + & + & - \\
\hline EGR3 & early growth response 3 & + & + & - \\
\hline PTHLH & parathyroid hormone like hormone & - & ++ & ++ \\
\hline PRG4 & proteoglycan 4 & - & + & - \\
\hline BMP2 & bone morphogenetic protein 2 & - & ++ & - \\
\hline STRA6 & stimulated by retinoic acid 6 & - & + & - \\
\hline AL391056.1 & novel transcript & - & + & - \\
\hline F2RL1 & F2R like trypsin receptor 1 & - & + & - \\
\hline KCNK3 & potassium two pore domain channel subfamily $\mathrm{K}$ member 3 & - & + & - \\
\hline ADAMTS14 & ADAM metallopeptidase with thrombospondin type 1 motif 14 & - & + & - \\
\hline RNF128 & ring finger protein $128, \mathrm{E} 3$ ubiquitin protein ligase & - & + & - \\
\hline EDNRA & endothelin receptor type $\mathrm{A}$ & - & + & - \\
\hline IBSP & integrin binding sialoprotein & - & + & - \\
\hline MMP10 & matrix metallopeptidase 10 & - & + & - \\
\hline $\mathrm{ABCA} 13$ & ATP binding cassette subfamily A member 13 & - & + & - \\
\hline SPP1 & secreted phosphoprotein 1 & - & + & - \\
\hline COL10A1 & collagen type $\mathrm{X}$ alpha 1 chain & - & + & - \\
\hline SLC16A6 & solute carrier family 16 member 6 & - & + & - \\
\hline GRIN2A & glutamate ionotropic receptor NMDA type subunit $2 \mathrm{~A}$ & - & + & - \\
\hline CYP26B1 & cytochrome P450 family 26 subfamily B member 1 & - & + & - \\
\hline RGS2 & regulator of $\mathrm{G}$ protein signaling 2 & - & + & - \\
\hline NFATC2 & nuclear factor of activated $\mathrm{T}$ cells 2 & - & + & - \\
\hline DUSP4 & dual specificity phosphatase 4 & - & + & - \\
\hline PLXDC1 & plexin domain containing 1 & - & + & - \\
\hline
\end{tabular}

Table 8.6.: Top 50 genes from Figure 8.7, expression differences calculated as rowwise means for the respective gene and sample set and transcribed according to $\mathrm{x}$-fold expression. $(+++=$ $<2$-fold expression, $++=1$-2-fold expression, $+=0$-1-fold expression, $-=0$-(-1)-fold expression, $--=(-1)-(-2)$-fold expression, $---=<(-2)$-fold expression) .

Looking into the pathways resulting from these, I did three separate sets of KEGG pathways analyses that will each be shown in Subsections. First, I tailored my data to match the Wollnik data-of zero-hour controls and 24 hours samples on gels of 1, 10, and $30 \mathrm{kPa}$ stiffness - and compare them (see also Subsection 8.3.1). These use the zero-hour controls as reference points. Second, I did pathway analysis on a set of all 24-hour samples, excluding the zero-hour controls, which I deemed to be insufficient controls. For this the reference consists of the samples on collagen-coated glass and cell culture plastic combined. This Subsection also features a comparison of these two control groups. Third, in a last attempt, I compare the different passages used in the biological replicates looking for potential genes associated with loss of differentiation during passage progression. 


\subsubsection{Reduced data set according to Wollnik samples compared to zero-hour con- trols}

For my reduced data set, overall gene expression and top genes do not hint in any particular direction, especially when looking for stress fiber-related transcriptome changes. There are 189 genes upregulated in the 24-hour gel samples in comparison to the zero-hour controls, and 472 downregulated. Of those, 29 upregulated genes can be loosely tied to mechanosensing - mainly ECM remodeling. Of the downregulated genes, 41 can be loosely tied to mechanosensing, mostly consisting of transcription factors and interacting proteins. Genes encoding actin, actin cross-linkers, and myosin motors cannot be found.

After the pathway clustering, however, results hint in a certain direction. In Table 8.7, top 10 upregulated pathways are shown. Of these, seven are directly linked to proliferation and division. Keeping in mind both that this analysis is done of cells seeded 24 hours compared to a zero-hour control, as well as results from Section 5.6, this finding makes sense. The doubling rate of hMSCs used in Chapter 5 varied, with cells generally doubling in around 2-3 days in optimal environments. However, in many experiments a large percentage of cells underwent division approximately 16-18 hours after seeding. Thus, having a large amount of unstressed hMSCs plated for 24 hours expressing more proliferation-related genes than ones trypsinized after a regular passaging period of three to four days could be normal.

\begin{tabular}{l|c|l|l|l|l} 
GO.ID & Term & Annotated & Significant & Expected & Result \\
\hline GO:0051301 & cell division & 611 & 44 & 7,23 & $7.8 \mathrm{e}-16$ \\
GO:0000281 & mitotic cytokinesis & 70 & 11 & 0,83 & $9.2 \mathrm{e}-10$ \\
GO:0051256 & mitotic spindle midzone assembly & 7 & 4 & 0,08 & $6.5 \mathrm{e}-07$ \\
GO:0007094 & mitotic spindle assembly checkpoint & 30 & 7 & 0,35 & $8.4 \mathrm{e}-07$ \\
GO:0000070 & mitotic sister chromatid segregation & 139 & 26 & 1,64 & $2.6 \mathrm{e}-06$ \\
GO:0008283 & cell proliferation & 2174 & 59 & 25,71 & $4.9 \mathrm{e}-06$ \\
GO:0010469 & regulation of signaling receptor activity & 660 & 26 & 7,81 & $7.4 \mathrm{e}-06$ \\
GO:0045840 & positive regulation of mitotic nuclear division & 49 & 9 & 0,58 & $2.7 \mathrm{e}-05$ \\
GO:0015816 & glycine transport & 6 & 3 & $3.2 \mathrm{e}-05$ \\
GO:0051988 & regulation of attachment of spindle & 14 & 4 & 0,17 & $5.5 \mathrm{e}-05$
\end{tabular}

Table 8.7.: Top 10 upregulated KEGG pathways in the new data set without collagen coated glass and cell culture plastic samples

\begin{tabular}{|c|c|c|c|c|c|}
\hline GO.ID & Term & Annotated & Significant & Expected & Result \\
\hline GO:0006357 & $\begin{array}{l}\text { regulation of transcription by } \\
\text { RNA polymerase II }\end{array}$ & 2617 & 719 & 539,38 & $1.8 \mathrm{e}-26$ \\
\hline GO:0006413 & translational initiation & 211 & 70 & 43,49 & $1.4 \mathrm{e}-10$ \\
\hline GO:0000184 & $\begin{array}{l}\text { nuclear-transcribed mRNA catabolic } \\
\text { process, nonsense-mediated decay }\end{array}$ & 138 & 60 & 28,44 & $1.0 \mathrm{e}-09$ \\
\hline GO:0032981 & $\begin{array}{l}\text { mitochondrial respiratory chain } \\
\text { complex I assembly }\end{array}$ & 80 & 41 & 16,49 & $1.2 \mathrm{e}-09$ \\
\hline GO:0006614 & $\begin{array}{l}\text { SRP-dependent cotranslational protein } \\
\text { targeting to membrane }\end{array}$ & 118 & 51 & 24,32 & $3.6 \mathrm{e}-08$ \\
\hline GO:0015031 & protein transport & 1895 & 489 & 390,57 & $4.4 \mathrm{e}-07$ \\
\hline GO:0019083 & viral transcription & 221 & 69 & 45,55 & $4.5 \mathrm{e}-07$ \\
\hline GO:0000122 & $\begin{array}{c}\text { negative regulation of transcription } \\
\text { by RNA polymerase II }\end{array}$ & 797 & 217 & 164,27 & $1.4 \mathrm{e}-06$ \\
\hline GO:0006120 & $\begin{array}{l}\text { mitochondrial electron transport, } \\
\text { NADH to ubiquinone }\end{array}$ & 71 & 33 & 14,63 & $3.3 \mathrm{e}-06$ \\
\hline GO:0006895 & Golgi to endosome transport & 16 & 12 & 3,3 & $4.6 \mathrm{e}-06$ \\
\hline
\end{tabular}

Table 8.8.: Top 10 downregulated KEGG pathways in the new data set without collagen coated glass and cell culture plastic samples

In the downregulated pathways, again RNA polymerase II regulation is the top differently expressed pathway. Here it is accompanied with translational initiation, Golgi to endosome transport, protein transcription, and mitochondiral transport. This seems to exceed the stress-related downregulation of 
RNA polymerase II regulation and could be an effect of mitosis rather than stress. However, as seen in Table 8.6, cells seem to react to the presence of PAA gel. So, each difference seen here could also be attributed to comparing cells that have never come into contact with PAA gels to those that have.

\subsubsection{Full data set compared to glass and cell culture flasks as controls}

For the analysis of the full transcriptomal changes, there are multiple controls one could use. What Wollnik did in 2015 was compare cells seeded on gels of different stiffnesses to nonplated cells as zero-hour controls. This was done by lysing the cells with trizol, preserving the mRNA of interest. However, the difference between doing this on trypsinated versus still-adherent cells could lead to side effects. This said, I planned on comparing my data set to 24 -hour plated samples from the start. Glass coated with collagen in the same fashion as the PAA gels should act as a sufficient control with infinitive stiffness in comparison to the PAA gels of significant lower stiffness. However, as seen in Table 8.6, translational differences seem to originate rather from the presence of PAA gel than from stiffness difference.

\begin{tabular}{l|c|l|l|l|l} 
GO.ID & Term & Annotated & Significant & Expected & Result \\
\hline GO:0006954 & inflammatory response & 926 & 21 & 7,68 & $1.7 \mathrm{e}-07$ \\
GO:0044597 & daunorubicin metabolic process & 9 & 4 & 0,07 & $5.6 \mathrm{e}-07$ \\
GO:0044598 & doxorubicin metabolic process & 9 & 4 & 0,07 & $5.6 \mathrm{e}-07$ \\
GO:0070098 & chemokine-mediated signaling pathway & 102 & 8 & 0,85 & $2.2 \mathrm{e}-06$ \\
GO:0071799 & cellular response to prostaglandin D stimulus & 5 & 3 & 0,04 & $5.5 \mathrm{e}-06$ \\
GO:0016115 & terpenoid catabolic process & 6 & 3 & 0,05 & $1.1 \mathrm{e}-05$ \\
GO:0007267 & cell-cell signaling & 1812 & 34 & 15,03 & 0.00010 \\
GO:0071222 & cellular response to lipopolysaccharide & 254 & 10 & 2,11 & 0.00011 \\
GO:0034310 & primary alcohol catabolic process & 15 & 3 & 0,12 & 0.00020 \\
GO:0042448 & progesterone metabolic process & 16 & 3 & 0,13 & 0.00029
\end{tabular}

Table 8.9.: Top 10 upregulated KEGG pathways in the new data set vs. glass and cell culture plastic

The top 50 differently expressed genes are aptly named. Referencing Figure 8.6, outliers can occur and impact the expressional differences of genes in relation to other sample groups. When comparing 24-hour gel samples to a combined set of glass and plastic samples, 3 genes distinctly upregulated in the gel samples and 20 genes distinctly downregulated in the gel samples can be found, none of which seem to be relevant. With pathway analysis, which takes into consideration the whole data instead of just significantly differently expressed genes, I hoped to find pathway effects, as a multitude of slightly over- or underexpressed genes can accumulate.

\begin{tabular}{|c|c|c|c|c|c|}
\hline GO.ID & Term & Annotated & Significant & Expected & Result \\
\hline GO:0051301 & cell division & 636 & 337 & 173,89 & $<1 \mathrm{e}-30$ \\
\hline GO:0019083 & viral transcription & 221 & 124 & 60,42 & $9.0 \mathrm{e}-24$ \\
\hline GO:0006413 & translational initiation & 222 & 120 & 60,7 & $5.4 \mathrm{e}-16$ \\
\hline GO:0006614 & $\begin{array}{l}\text { SRP-dependent cotranslational protein } \\
\text { targeting to membrane }\end{array}$ & 120 & 75 & 32,81 & $1.5 \mathrm{e}-15$ \\
\hline GO:0000184 & $\begin{array}{c}\text { nuclear-transcribed mRNA catabolic process, } \\
\text { nonsense-mediated decay }\end{array}$ & 145 & 84 & 39,64 & $8.7 \mathrm{e}-15$ \\
\hline GO:0006260 & DNA replication & 290 & 185 & 79,29 & $2.5 \mathrm{e}-14$ \\
\hline GO:0000398 & mRNA splicing, via spliceosome & 421 & 196 & 115,11 & $8.8 \mathrm{e}-14$ \\
\hline GO:0007049 & cell cycle & 2065 & 911 & 564,59 & $4.0 \mathrm{e}-13$ \\
\hline GO:0070125 & mitochondrial translational elongation & 98 & 60 & 26,79 & $2.1 \mathrm{e}-12$ \\
\hline GO: 1901796 & $\begin{array}{l}\text { regulation of signal transduction } \\
\text { by p } 53 \text { class mediator }\end{array}$ & 201 & 98 & 54,96 & $5.3 \mathrm{e}-12$ \\
\hline
\end{tabular}

Table 8.10.: Top 10 downregulated KEGG pathways in the new data set vs. glass and cell culture plastic

Unfortunately, results match those hypotheses. In the top upregulated pathways (see Table 8.9) we see, 
besides inflammatory response and cell-to-cell signalling, some very specific pathways unregulated that deal mostly with disrupting chemicals. The aforementioned pathway of cellular response to lipopolysaccharide includes different miRNAs, toll like receptors, zinc finger proteins, interleukins, defensins, and CD antigens. The downregulated pathways shown in Table 8.10 consist almost exclusively of those related to proliferation, mitosis, and intra-cellular transport. This again is consistent with stress response mediated by the presence of PAA gels, where cells will stop proliferation until circumstances become acceptable again.

\subsection{Comparison of control groups}

Comparing both glass and cell culture plastic to unplated zero-hour controls, there are a number of differences. First, between zero-hour controls and glass there are 91 genes upregulated in zero-hour controls, of which 17 can be connected to adhesion and mechanosensing-predominantly anillin, an actin binding protein. Searching for genes downregulated in zero-hour controls produces 73 hits, of which 26 can be related to mechanosensing, and also including a myosin interacting protein, JAK interacting protein, and fascin.

Checking genes upregulated in zero-hour controls, in comparison to cells seeded for 24-hour cell in culture flasks, return 67 hits. Ten of these can be linked to mechanosensing, again including anillin. In contrast, there are 749 genes significantly downregulated in zero-hour controls looked at in comparison to cells seeded in flasks. However, of those only 18 are tied to mechanosensing, with the exception of the JAK interacting protein also featuring Alpha-actin 2.

To compare both of the 24-hour control groups, collagen coated glass and T-75 Corning cell culture flasks, I look into the differences in up- and downregulated genes directly. As both groups constantly overlap in PCA analysis, sample distance, and top 50 genes, a significant difference in pathways is not to be expected. To compare these groups, significant differences in the filtered and adjusted sample sets during DESeq 2 pipeline are taken into account. Subsequently, an expression difference of $5 \%$ is set as a threshold for different expression when comparing mean expression of all biological replicates. According to this, there is one gene significantly upregulated in the cell culture flask samples-VWA5A (von Willebrand factor A domain containing 5A) - and three downregulated-BIRC3 (baculoviral IAP repeat containing 3), PTGS2 (prostaglandin-endoperoxide synthase 2), and SERPINB2 (serpin family B member 2). None of these genes' expressions can be connected to mechanosensing, stress response, or each other. As those seem to be either random effects or genes very close to the DESeq2 pipeline's threshold for significance, both control groups seem to show corresponding transcriptome.

While in the context of this thesis this seems to be only a footnote, it could hint at a way to simplify future experiments. For experiments with collagen-coated PAA gels, similarly coated glass is commonly used as a control. This is based on possible effects of surface interactions on cell behavior. But, if cells show neither visible nor translational differences on these two substrates, future researchers could use standard cell culture plastic and invest saved time in additional experiments.

\subsubsection{Full data set analyzed for passage effects}

In all the already discussed analysis, comparison of samples to control groups was done. With sample groups consisting of four biological replicates created from different passages, another possibility is to group samples accordingly and search for genes up- or downregulated with higher passage number. Admittedly, this would be more meaningful with bigger passage differences. I did this analysis twice: once with the reduced sample set containing only zero-hour controls and 24-hour PAA gel samples, and once with the glass and cell culture plastic samples included. As shown and discussed in Subsection 8.4.2, there are large differences between the three groups of zero-hour control, PAA gel samples, and glass and plastic flask samples, with glass and plastic showing aberration. Thus, excluding this third group from the set might lead to different results. 


\subsection{Reduced sample set}

With the exclusion of glass and cell culture plastic samples, a sample group with large numbers of induced proliferation pathways is eliminated from the data. Because differences between PAA gels, glass, and cell culture plastic seem to be quite strong, this reduced set might show more accurate results than the full set.

\begin{tabular}{l|c|l|l|l|l} 
GO.ID & Term & Annotated & Significant & Expected & Result \\
\hline GO:0010804 & $\begin{array}{r}\text { negative regulation of tumor necrosis } \\
\text { factor-mediated signaling pathway }\end{array}$ & 24 & 2 & 0,05 & 0.0011 \\
GO:0071353 & $\begin{array}{r}\text { cellular response to interleukin-4 } \\
\text { positive regulation of blood vessel diameter }\end{array}$ & 34 & 2 & 0,07 & 0.0021 \\
GO:0097755 & immune response & 2756 & 9 & 0,11 & 0.0055 \\
GO:0006955 & positive regulation of fat cell differentiation & 65 & 2 & 5,57 & 0.0061 \\
GO:0045600 & mesenchyme migration & 5 & 0,13 & 0.0076 \\
GO:0090131 & positive regulation of protein & 5 & 1 & 0,01 & 0.0101 \\
GO: 1904352 & catabolic process in the vacuole & 1 & 0,01 & 0.0101 \\
GO:0033153 & T cell receptor V(D)J recombination & 5 & 1 & 0,01 & 0.0101 \\
GO:1990349 & gap junction-mediated intercellular transport & 5 & 1 & 0,01 & 0.0101 \\
GO:0003418 & growth plate cartilage chondrocyte differentiation & 5 & & 0,01 & 0.0101
\end{tabular}

Table 8.11.: Top 10 upregulated KEGG pathways in the new data set without collagen coated glass and cell culture plastic samples. Analyzed with respect to differences in passages

Looking into genes upregulated with growing passage number (see Table 8.11), there are multiple pathways mentioned for signalling (TNF, IL-4) and differentiation, as well as one for intercellular transport. With the exception of the immune response pathway these are all very small and specific pathways consisting of only a handful of genes with one or two genes altered.

\begin{tabular}{l|r|l|l|l|l} 
GO.ID & Term & Annotated & Significant & Expected & Result \\
\hline GO:0006357 & $\begin{array}{r}\text { regulation of transcription by } \\
\text { RNA polymerase II }\end{array}$ & 2617 & 926 & 700,48 & $9.0 \mathrm{e}-28$ \\
GO:0035264 & $\begin{array}{r}\text { multicellular organism growth } \\
\text { negative regulation of transcription }\end{array}$ & 156 & 75 & 41,76 & $3.8 \mathrm{e}-08$ \\
GO:0000122 & by RNA polymerase II & 363 & 279 & 213,33 & $4.7 \mathrm{e}-08$ \\
GO:0043547 & positive regulation of GTPase activity & 89 & 150 & 97,16 & $3.1 \mathrm{e}-07$ \\
GO:0009791 & post-embryonic development & 49 & 23,82 & $3.6 \mathrm{e}-07$ \\
GO:0006897 & endocytosis & 366 & 126 & 2.67 \\
GO:0001701 & in utero embryonic development & 1223 & 97,97 & $7.2 \mathrm{e}-06$ \\
GO:0006355 & regulation of transcription, DNA-templated & 3598 & 243,06 & $1.3 \mathrm{e}-05$ \\
GO:0018105 & peptidyl-serine phosphorylation & 243 & 65,04 & $1.7 \mathrm{e}-05$ \\
GO:0051893 & regulation of focal adhesion assembly & 67 & 35 & 17,93 & $2.4 \mathrm{e}-05$
\end{tabular}

Table 8.12.: Top 10 downregulated KEGG pathways in the new data set without collagen coated glass and cell culture plastic samples. Analyzed with respect to differences in passages

In comparison, in the downregulated set (see Table 8.14) larger pathways are found. Of these, RNA polymerase II regulation can also be found as the top pathway in all analysis of downregulation up to this point. The rest of the top pathways also includes pathways related to proliferation and development like transcription, GTPase activity, multicellular organism growth, and development.

In conclusion, for the reduced data set the later passages seem to express more genes related to signalling, differentiation, and transport, and fewer genes for transcription and development. As later passages are generally known to proliferate more slowly and to loose stem characteristics, these results seem reasonable.

\subsection{Full sample set}

For the full set-including 24-hour controls of collagen-coated glass and cell-culture plastic, which show opposite gene expression in not only the top 50 but also the top 300 genes-I expect those trends to also extend on the passage effects. Again, I compared later passages to earlier ones, grouping all samples first 
according to passage number. Among the pathways upregulated in later passages for this set (see Table 8.13) are eight of the pathways also found in Table 8.9, in which PAA gel samples were compared to glass and cell culture plastic as a control group.

\begin{tabular}{l|r|l|l|l|l} 
GO.ID & Term & Annotated & Significant & Expected & Result \\
\hline GO:0010469 & regulation of signaling receptor activity & 660 & 22 & 5,5 & $2.0 \mathrm{e}-07$ \\
GO:0044597 & daunorubicin metabolic process & 9 & 4 & 0,08 & $5.7 \mathrm{e}-07$ \\
GO:0044598 & doxorubicin metabolic process & 9 & 4 & 0,08 & $5.7 \mathrm{e}-07$ \\
GO:0071799 & cellular response to prostaglandin D stimulus & 5 & 3 & 0,04 & $5.6 \mathrm{e}-06$ \\
GO:0016115 & terpenoid catabolic process & 6 & 3 & 0,05 & $1.1 \mathrm{e}-05$ \\
GO:0007267 & cell-cell signaling & 1659 & 32 & 13,83 & $9.6 \mathrm{e}-05$ \\
GO:0006954 & inflammatory response & 846 & 16 & 7,05 & 0.00012 \\
GO:0034310 & primary alcohol catabolic process & 15 & 3 & 0,13 & 0.00020 \\
GO:0042448 & progesterone metabolic process & 16 & 3 & 0,13 & 0.00029 \\
GO:0007155 & cell adhesion & 1515 & 22 & 12,63 & 0.00044
\end{tabular}

Table 8.13.: Top 10 upregulated KEGG pathways in the new data set. Analyzed with respect to differences in passages

Additionally, of the pathways downregulated in later pathways (see Table 8.14), nine pathways can also be found in Table 8.10. For the upregulated genes, similar pathways dealing with metabolic processes come up that I had attributed to a stress response and presence of PAA gels in the previous Subsection 8.4.2.2. Additionally, two divergent pathways can be seen. These are the chemokine-mediated signaling pathway and the cellular response to lipopolysaccharides. In the downregulated genes most pathways relate to cell cycle and transcription, with the one divergent pathway being that of the regulation of signal transduction by p 53 class mediator.

\begin{tabular}{l|r|l|l|l|l} 
GO.ID & Term & Annotated & Significant & Expected & Result \\
\hline GO:0051301 & cell division & 327 & 169,72 & $<1 \mathrm{e}-30$ \\
GO:0019083 & viral transcription & 221 & 61,39 & $3.0 \mathrm{e}-23$ \\
GO:0006260 & DNA replication & 274 & 76,11 & $8.9 \mathrm{e}-18$ \\
GO:0006413 & translational initiation & 211 & 175 & 58,61 & $4.5 \mathrm{e}-17$ \\
GO:0000184 & nuclear-transcribed mRNA catabolic process, & 138 & 84 & 38,33 & $4.0 \mathrm{e}-16$ \\
& nonsense-mediated decay & & & \\
GO:0006614 & SRP-dependent cotranslational protein & 118 & 74 & 32,78 & $1.6 \mathrm{e}-15$ \\
GO:0000398 & targeting to membrane & 379 & 181 & 105,28 & $1.3 \mathrm{e}-14$ \\
GO:0007049 & mRNA splicing, via spliceosome & cell cycle & 556 & 528,88 & $1.7 \mathrm{e}-13$ \\
GO:0070126 & mitochondrial translational termination & 97 & 59 & 26,94 & $9.9 \mathrm{e}-12$ \\
GO:0070125 & mitochondrial translational elongation & 95 & 58 & 26,39 & $1.2 \mathrm{e}-11$
\end{tabular}

Table 8.14.: Top 10 downregulated KEGG pathways in the new data set. Analyzed with respect to differences in passages

This overlap between the KEGG pathway differences in both comparisons of PAA gels with those on glass or plastic and earlier with later passages could mean that there is in fact a difference between earlier passages on gel and later passages on glass or plastic that appears in both analyses as samples are grouped, thus measuring an combined effect in two different ways.

\subsubsection{Transcriptomal differences between gel stiffnesses with control groups omit- ted}

As already mentioned when describing the Wollnik data set, the expression differences between control groups and stiffness samples are larger then those within these stiffness samples. Thus, I omitted all control groups from the data set and used the DESeq2 pipeline again on this smaller set. As before, outliers and genes with too few transcripts are filtered out and, for the top 50 differently expressed genes, 
means of all biological replicates are generated. These are shown simplified for easy comparison in Table 8.15 .

In the same analysis for the Wollnik data, multiple actin genes and pseudogenes emerged and 1 and 30 $\mathrm{kPa}$ gel samples showed upregulated expression while $10 \mathrm{kPa}$ samples showed downregulation. Conversely, my data shows mostly genes in $1 \mathrm{kPa}$ samples being upregulated and 11 and $32 \mathrm{kPa}$ gel samples being downregulated. However, one has to keep in mind that here the genes wtih the biggest differences among the sample groups are depicted. Among these genes we see some tied to mechanosensing, such as actin, myosin, MMP, integrin, tubulin, and ADAM. Most interesting is the strong upregulation in $11 \mathrm{kPa}$ samples of MYH7, as ACTA2 is upregulated only in 11 and $32 \mathrm{kPa}$ samples and ACTC1 only in $11 \mathrm{kPa}$ samples.

\begin{tabular}{|c|c|c|c|c|}
\hline Gene tag & Long term & $\begin{array}{l}\text { Mean } \\
1 \mathrm{kPa}\end{array}$ & $\begin{array}{c}\text { Mean } \\
11 \mathrm{kPa}\end{array}$ & $\begin{array}{c}\text { Mean } \\
32 \mathrm{kPa}\end{array}$ \\
\hline CYP26B1 & cytochrome P450 family 26 subfamily B member 1 & + & - & - \\
\hline ETV1 & ETS variant 1 & + & - & - \\
\hline IBSP & integrin binding sialoprotein & + & + & - \\
\hline PTHLH & parathyroid hormone like hormone & + & - & - \\
\hline MYH7 & myosin heavy chain 7 & - & ++ & - \\
\hline NFATC2 & nuclear factor of activated $\mathrm{T}$ cells 2 & ++ & - & - \\
\hline $\mathrm{RGCC}$ & regulator of cell cycle & + & - & - \\
\hline ACTA2 & actin, alpha 2 , smooth muscle, aorta & - & + & + \\
\hline SLC16A6 & solute carrier family 16 member 6 & + & - & - \\
\hline IGFBP5 & insulin like growth factor binding protein 5 & + & + & - \\
\hline PRG4 & proteoglycan 4 & + & - & - \\
\hline RGS2 & regulator of $\mathrm{G}$ protein signaling 2 & + & - & - \\
\hline TNNT2 & troponin $\mathrm{T} 2$, cardiac type & - & + & - \\
\hline SPP1 & secreted phosphoprotein 1 & + & + & - \\
\hline PGF & placental growth factor & - & - & + \\
\hline DUSP4 & dual specificity phosphatase 4 & + & - & - \\
\hline COL10A1 & collagen type $\mathrm{X}$ alpha 1 chain & + & - & - \\
\hline BMP4 & bone morphogenetic protein 4 & + & - & - \\
\hline FOSB & FosB proto-oncogene, AP-1 transcription factor subunit & + & - & - \\
\hline BMP2 & bone morphogenetic protein 2 & + & - & - \\
\hline $\mathrm{AOC} 2$ & amine oxidase, copper containing 2 & + & - & - \\
\hline ANO1 & anoctamin 1 & + & - & - \\
\hline CHI3L1 & chitinase 3 like 1 & - & + & + \\
\hline ZNF365 & zinc finger protein $365+$ & - & - & \\
\hline ADAMTS14 & ADAM metallopeptidase with thrombospondin type 1 motif 14 & + & - & - \\
\hline PHLDA1 & pleckstrin homology like domain family A member 1 & + & - & - \\
\hline ACKR3 & atypical chemokine receptor 3 & + & - & - \\
\hline IGFBP 1 & insulin like growth factor binding protein 1 & + & - & - \\
\hline ANKRD1 & ankyrin repeat domain 1 & - & + & + \\
\hline ANKH & ANKH inorganic pyrophosphate transport regulator & + & - & - \\
\hline TTN & titin & - & + & - \\
\hline ACTC1 & actin, alpha, cardiac muscle 1 & - & + & - \\
\hline TPPP3 & tubulin polymerization promoting protein family member 3 & ++ & - & - \\
\hline PLXDC1 & plexin domain containing 1 & + & - & - \\
\hline $\mathrm{PTX} 3$ & pentraxin 3 & - & + & + \\
\hline F2RL1 & F2R like trypsin receptor 1 & + & - & - \\
\hline FOXQ1 & forkhead box Q1 & + & - & - \\
\hline GEM & GTP binding protein overexpressed in skeletal muscle & + & - & - \\
\hline RRAD & RRAD, Ras-related glycolysis inhibitor and calcium channel regulator & + & - & - \\
\hline MMP10 & matrix metallopeptidase 10 & + & - & - \\
\hline SYNPO2 & synaptopodin 2 & - & + & + \\
\hline SYT12 & synaptotagmin 12 & + & - & - \\
\hline CMKLR1 & chemerin chemokine-like receptor 1 & + & - & - \\
\hline ODAPH & odontogenesis-associated phosphoprotein & - & + & + \\
\hline $\mathrm{ABCA} 13$ & ATP binding cassette subfamily A member 13 & + & - & - \\
\hline GRIN2A & glutamate ionotropic receptor NMDA type subunit $2 \mathrm{~A}$ & + & - & - \\
\hline $\mathrm{AKR} 1 \mathrm{C} 1$ & aldo-keto reductase family 1 member $\mathrm{C} 1$ & + & - & - \\
\hline LAMB3 & laminin subunit beta 3 & + & - & - \\
\hline LINC01614 & long intergenic nonprotein coding RNA 1614 & + & - & - \\
\hline NEAT1 & nuclear paraspeckle assembly transcript 1 & + & - & - \\
\hline
\end{tabular}

Table 8.15.: Top 50 genes from my data set, expression differences calculated as rowwise means for the respective gene and sample set and transcribed according to $\mathrm{x}$-fold expression. $(+++=$ $<2$-fold expression, $++=1$-2-fold expression, $+=0$-1-fold expression, $-=0$-(-1)-fold expression, $--=(-1)-(-2)$-fold expression, $---=<(-2)$-fold expression) . 


\section{5. qRT-PCR of selected genes for multiple time points}

Quantitative real time PCR is a technique based on fluorescence measurements during PCR, where fluorescence rises with increasing amounts of PCR products. Here, we start with full RNA and a specific primer pair to amplify out target for a robust quantification. It outperforms detection of mRNA on Northern blots. Quantification can be done either as relative or absolute. Absolute quantification relies on a standard curve with the same amplification efficiency to yield the number of target molecules. Relative quantification uses a reference gene to identify expression difference of the target gene. Using a housekeeping gene as a reference also corrects for difference of quantity and quality of RNA as housekeeping genes are unregulated and expressed at relatively constant rates. Expression of housekeeping genes varies according to experimental conditions, but to correct for RNA differences the selection of the most stable housekeeping gene is crucial.

A standard qRT-PCR program usually consists of multiple cycles of splitting of double strands, binding of primers to single strands, and polymerization through DNA polymerase. After each cycle, the amount of PCR product is measured under conditions that prevent primer dimers. For measurement, a fluorescent probe is added to the reaction. This can be done by unspecific intercalation through SYBR green, or by using specific fluorescent probes. Duration and temperature of each step depend on the polymerase used, primer binding temperature, target length, and dNTP concentration.

After amplification, melting of the product is used to identify the specific DNA fragments amplified. Primer dimers or side products due to unspecific binding can be identified in this step.

For analysis, the quantification cycle and exponential amplification phase is important. The quantification cycle is defined as number of cycles where the fluorescence exceeds a threshold level, usually set to three to five times the standard deviation of signal noise above background. Because the amplification efficiency is dependent on template-primer interaction, real amplification efficiency for every reaction is determined with an experiment of serial dilutions. The efficiency is $100 \%$ in case of a 1:2 dilution, resulting in a quantification cycle difference of 1 . In the exponential amplification phase the amount of target doubles every cycle. Therefore, difference in amplification is $2^{x}$ with $x$ being the difference of cycles. To quantify in reference to a housekeeping gene, the $\Delta \Delta$ Ct-method is used, in which the quantification cycle of the reference gene is subtracted from the target gene.

To gather data for multiple time points within the first 24 hours, we gathered samples on different stiffnesses of polyacrylamide gels, collagen coated glass, and cell culture plastic at $0,1,4,12,18$, and 24 hours after plating. This was done with passage five hMSCs in three separate experiments. For each condition, 150,000 cells were seeded on gels on large glass slides (see C. Cells had been removed using trypsinazation. As cDNA is more stable than RNA, we froze the RNA samples after RNA extraction (protocol given in Subsection B.1) at $-20^{\circ} \mathrm{C}$, but transcribed them into cDNA within a week (protocol given in Subsection B.2). Afterwards, RNA was stored at $-80^{\circ} \mathrm{C}$ and $\mathrm{cDNA}$ at $-20^{\circ} \mathrm{C}$. qRT-PCR was done according to the protocol in Subsection B.3, after all samples were collected, within 1 month and at a rate of one to two (96-well) plates per day. $q R T-P C R$ was done by technical assistant Ulrike Schulz who also provided the protocols in Subsection B.2 and B.3.

Targets for qRT-PCR were chosen according to RNA-Seq analysis. GAPDH was used as housekeeping gene throughout all measurements. Temperature for each run was chosen according to lowest melting temperature in the run. Primers were grouped to guarantee as uniform melting temperatures as possible. All primers were manufactured by Sigma-Aldrich and obtained via custom order.

Standard curves were manufactured from the zero hours samples of the three separate experiments. Each cDNA sample was run with two replicates, giving two technical replicates of three biological replicates, six measurements per condition in total.

Controls have been done with primer solutions without sample, samples without primers, and SYBR green master mix with water. The location of samples has been kept constant over all runs with different primers and for same conditions on different time points due to effects of possible heating inhomogeneities in the machine. 


\subsubsection{Why these targets?}

As the RNA sequencing did not yield a clear set of actin- or mechanosensitivity-linked target genes, I selected genes of interest found in some data sets with high count numbers. Vimentin showed up frequently in the top 100 differently regulated genes in all analyses. Myosin heavy chain 9 (MYH9) showed up in the top 50 in my data with up to an eightfold expression difference between sample sets. Actin Beta, Aniline, and Actin Alpha 2 were the only actin and actin cross-linker genes consistently present in all top 100 analyses. Also, all of these genes count numbers well in the thousands, which should yield reliable results and be well above the detection minimum.

\begin{tabular}{l|c|l|l} 
Alias & Long name & Tm & Sequence \\
\hline GAPDH for & $\begin{array}{c}\text { Glycerinaldehyd-3-phosphat- } \\
\text { Dehydrogenase for } \\
\text { Glycerinaldehyd-3-phosphat- } \\
\text { Dehydrogenase rev }\end{array}$ & $67.0^{\circ} \mathrm{C}$ & CTGCACCACCAACTGCTTAGC \\
GAPDG rev & $68.0^{\circ} \mathrm{C}$ & GGCATGGACTGTGGTCATGAG \\
VIM for & Vimentin for & $64.1^{\circ} \mathrm{C}$ & CCCTGAACCTGAGGGAAACTA \\
VIM rev & Vimentin rev & $64.3^{\circ} \mathrm{C}$ & CGTGATGCTGAGAAGTTTCGT \\
MYH9 for & Myosin heavy chain 9 for & $65.6^{\circ} \mathrm{C}$ & CATCTACACCTATTCAGGCCTGTTC \\
MYH9 rev & Myosin heavy chain 9 rev & $67.2^{\circ} \mathrm{C}$ & GCGGTGTCTGTGATGGCATA \\
ACTB for & Actin Beta for & $59.7^{\circ} \mathrm{C}$ & GACGACATGGAGAAAATCTG \\
ACTB rev & Actin Beta rev & $58.0^{\circ} \mathrm{C}$ & ATGATCTGGGTCATCTTCTC \\
ANLN for & Aniline for & $62.7^{\circ} \mathrm{C}$ & CATGCTAAGCGAGCTAGA \\
ANLN rev & Aniline rev & $61.9^{\circ} \mathrm{C}$ & ACAGGACTTGGAGAACAA \\
ACTA2 for & Actin Alpha 2 for & $59.8^{\circ} \mathrm{C}$ & CTCACTGACTACCTCATG \\
ACTA2 rev & Actin Alpha 2 rev & $59.4^{\circ} \mathrm{C}$ & GTCCAGAGCTACATAACA
\end{tabular}

Table 8.16.: qRT-PCR primers used for qRT-PCR experiments

\subsubsection{Results}

Measurements were done for three experiments of passage five hMSCs on PAA gels of different stiffnesses and at different time points. These yield three technical replicates. Furthermore, each sample is measured twice in the qRT-PCR, yielding a total of six measurements to average and compare to the housekeeping gene. Averaging was first done for the two measurements in the qRT-PCR, then again for all three experiments. When outliers or measurements below detection minimum occurred all samples of a specific time point, including the housekeeping gene measurements, were redone to verify. All data shown here is averaged for at least four and at most seven single measurements of a condition.

Vimentin is a type of intermediate filament (see Subsection 2.2) present in hMSCs. As part of the intermediate filaments, it connects to the nuclei and other cell organelles and plays an important role within the cytoskeleton and in movement. In hMSCs vimentin seems to play a role in the nuclear shape and overall cell flexibility [108]. As it is a protein characteristically expressed in mesenchymal cells, assumed to play a role in differentiation, and part of the cytoskeleton, it was picked for a quantitative analysis over time. As can be seen in Figure 8.8, there is a tendency for all stiffnesses to reach an expression maximum around 12 hours. 


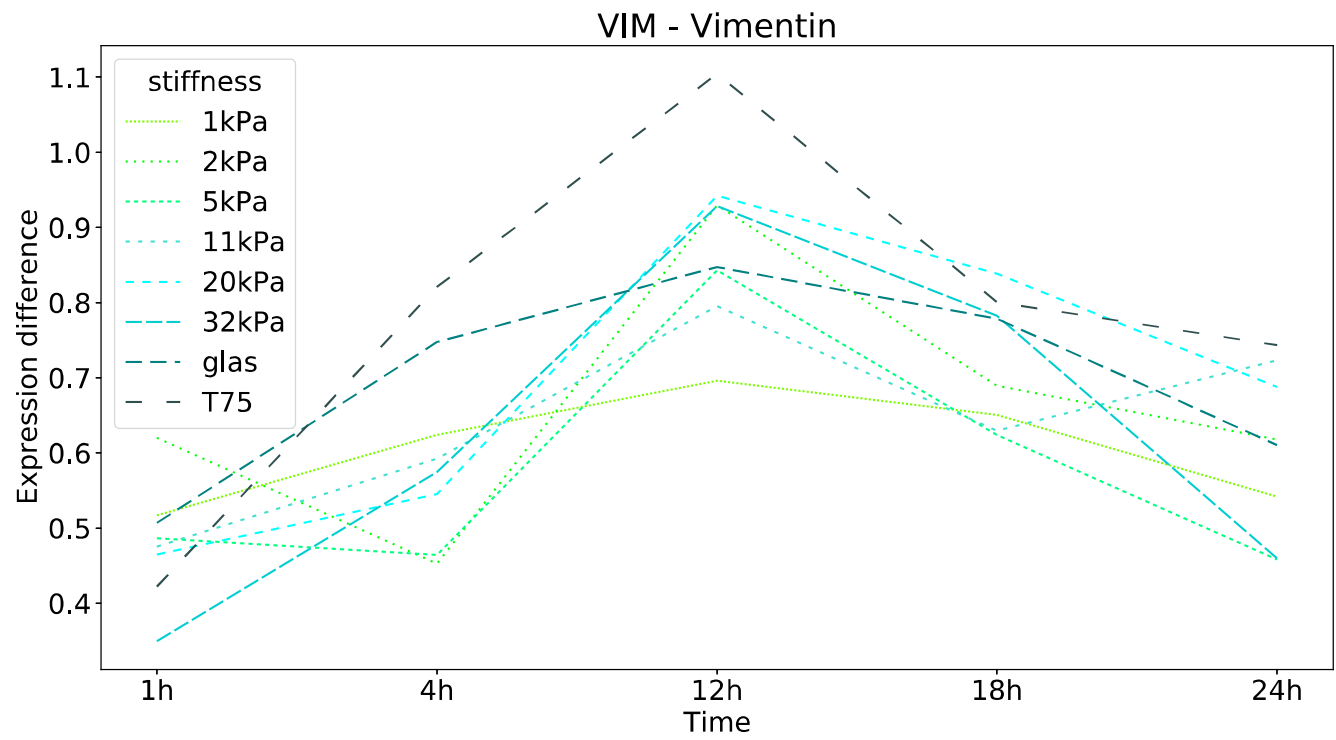

Figure 8.8.: Expression difference of vimentin, averaged over 2 technical replicates for each of 3 experiments and normalised against GAPDH as housekeeping gene.

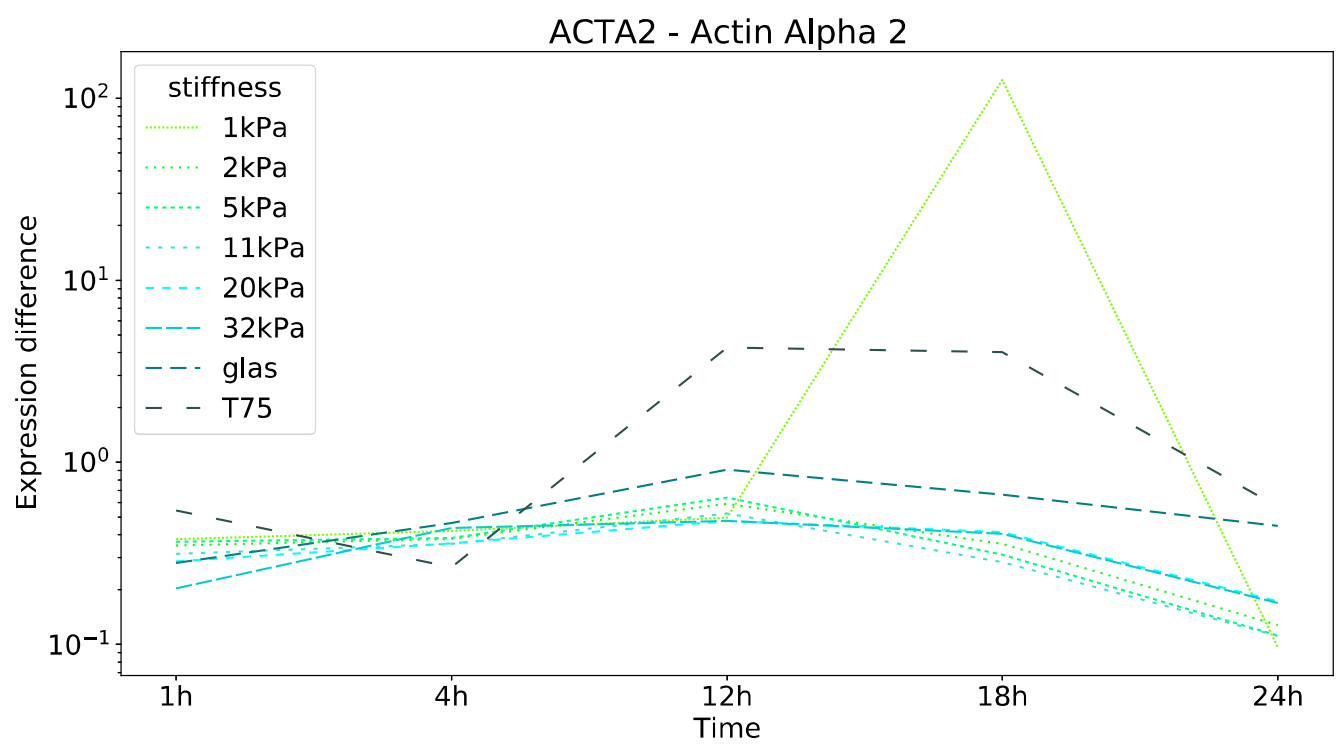

Figure 8.9.: Expression difference of Actin Alpha 2, averaged over 2 technical replicates for each of 3 experiments and normalised against GAPDH as housekeeping gene.

Actin Alpha 2 (ACTA2) is one of several genes encoding smooth muscle actin. In the TAL measurements, different smooth muscle actins were given as potential targets but ACTA2 emerged most frequently. It encodes for six different actin proteins and is usually overexpressed in contractile tissues lined with smooth muscles such as the esophagus, bladder, or blood vessels.

In Figure 8.9 we see an overexpression on $1 \mathrm{kPa}$ stiffness at around 18 hours. This is generated by a measurement of one out of three experiments. Usually this would be excluded as an outlier but in consecutive measurements results were similar. For the other stiffnesses and time points, expression seems to slightly rise leading up to 12 hours, then drop. This pattern with a maximum around 12 hours is similar to vinculin. 


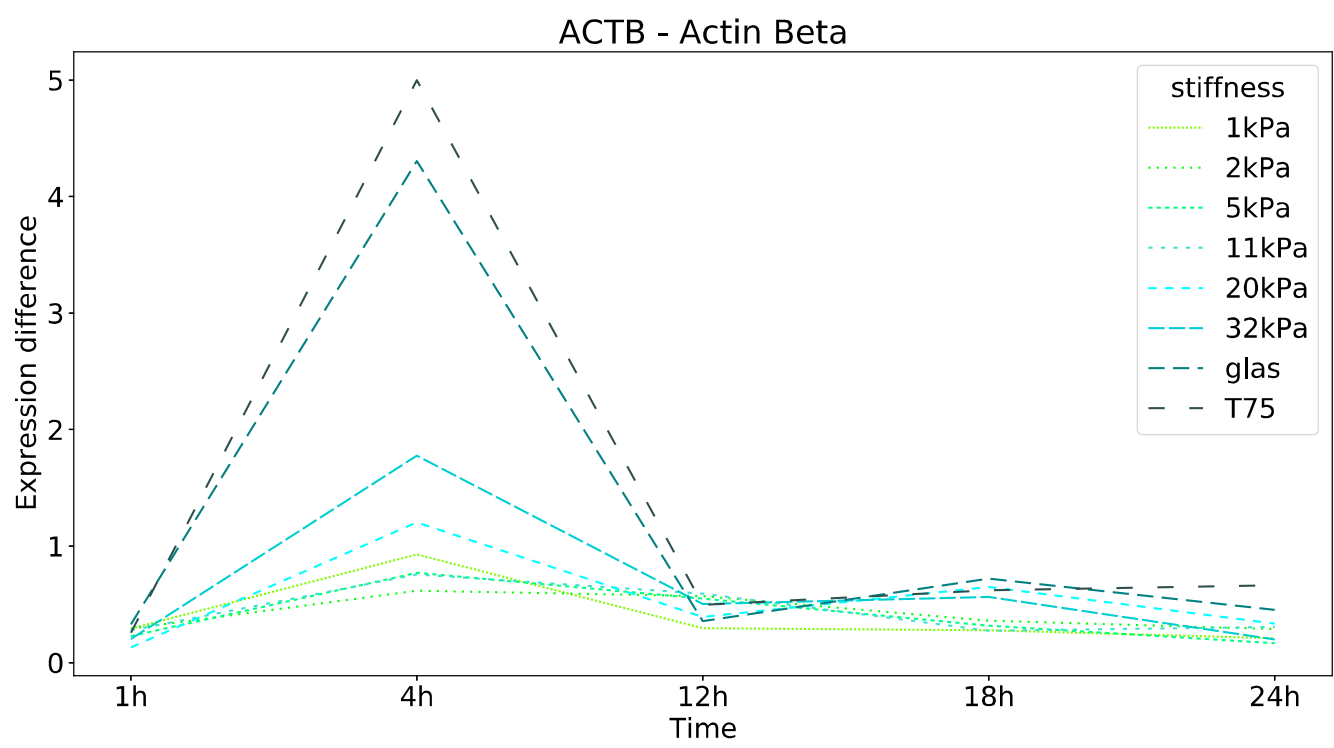

Figure 8.10.: Expression difference of Actin Beta, averaged over 2 technical replicates for each of 3 experiments and normalised against GAPDH as housekeeping gene.

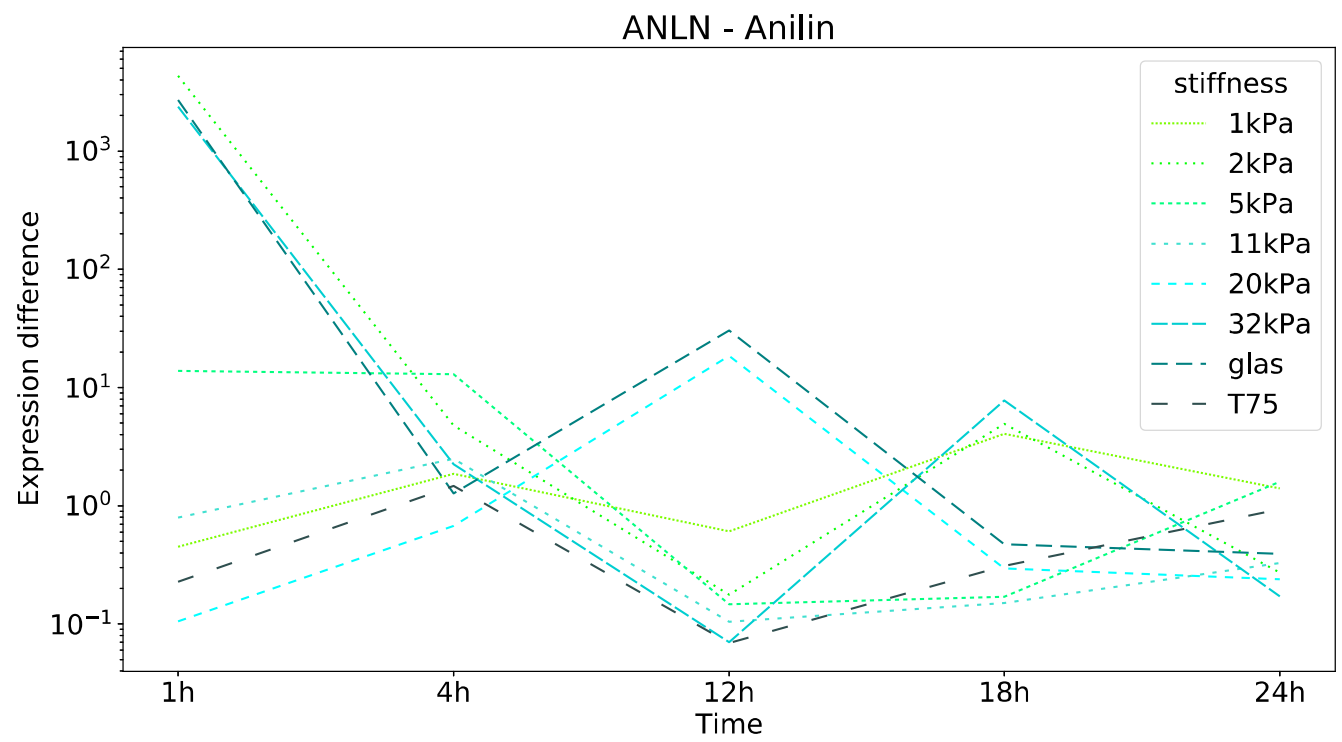

Figure 8.11.: Expression difference of Aniline, averaged over 2 technical replicates for each of 3 experiments and normalised against GAPDH as housekeeping gene.

For Actin Beta (ACTB), the pattern differs. Here, expression stays on a low level but shows maxima around 4 hours with the maxima scaling with the substrate stiffness, as can bee seen in Figure 8.10. This result seems valid but is bothersome, as ACTB is itself often used as housekeeping gene. Its expression should be steady at all times, merely being influenced by differentiation. Expression differences this pronounced could imply problems for other qRT-PCR experiments. Regarding this set of experiments, it seems safe to assume that the overexpression is linked with the cell spreading. The hMSCs used have shown in previous experiments and preparations thereof to need around 1 hour to firmly adhere to substrates 
of any kind. Within the first 6 hours spreading and formation of a cytoskeleton characteristic for the respective substrate and stiffness was finished (please see Chapter 5).

The actin cross-linker measured, aniline, shows less pronounced effects. There is a clear overexpression at the 1-hour time points for the stiffnesses of 2 and $32 \mathrm{kPa}$ gels as well as glass (see Figure 8.11). These measurements are consistent with their reruns but are not explainable as every effect seen should be influenced by either stiffness or time.

For myosin heavy chain 9 , which is associated with stress fibers, there seems to be slightly higher expression for $11 \mathrm{kPa}$ stiffness around 4 hours as well as for 2 and $5 \mathrm{kPa}$ stiffness around 12 hours. As myosins are connected to movement and contraction and should be overexpressed in cells differentiating in the myogenic lineage, this would make sense. However, the range of these expression differences is quite low and thus likely an outcome of variation rather than a real effect.

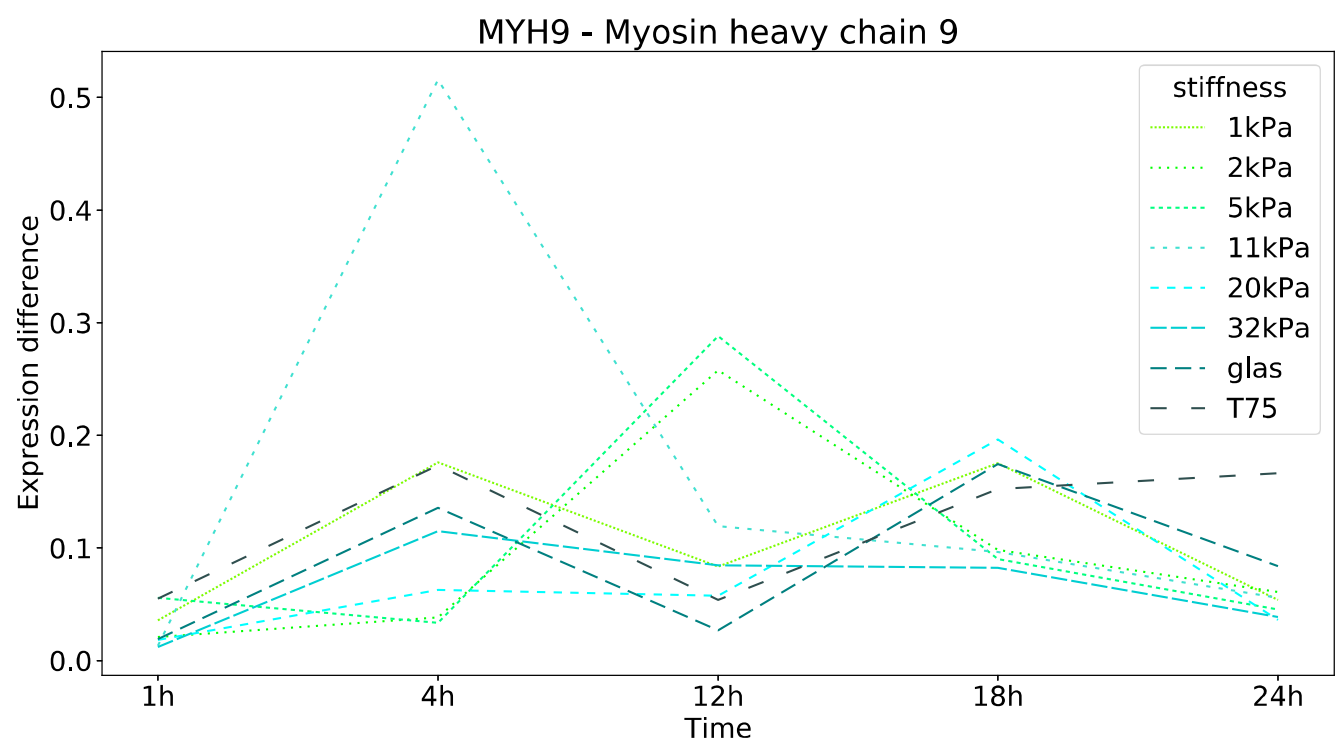

Figure 8.12.: Expression difference of myosin heavy chain 9, averaged over 2 technical replicates for each of 3 experiments and normalised against GAPDH as housekeeping gene. 



\section{Summary}

Apart from the experiments on differentiating hMSCs, the further improvement of the FilamentSensor has been a big part of my thesis. As the basic program was already developed [62] but too slow for high-throughput analysis in the scale needed for statistically reliable claims about cell behavior of a highly variable cell line, refactoring and runtime improvement were each a priority. On the associated website www.filament-sensor.de, the refactored source code is now available next to a variety of ready-to-use versions with full in-code documentation, to be reused under the GNU General Public License. After refactoring, various classes and functions were streamlined and runtime was reduced to one-sixth of the original version. Additionally, further functionalities have been added:

- area detection algorithm selectable

- expanding usable image types from .png to all image types compatible with ImageJ

- calculation of mechanically active area

- stack view

- split screen of all processing stages

- brightness and contrast adjustment for single pictures or stack

- variable filter list with possibility to import self-written filters, savable and reimportable

- saving and reimporting of settings

- bounding box for multi-cell analysis

- error $\log$

- clickable filament overlay for easy removal of fibers from data set

With these improvements, the FilamentSensor software is a valuable tool for high-throughput analysis of any filament—given curvature does not exceed a certain threshold, which is not only dependent on the filaments in question but also the image quality.

We further improved on FilamentSensor by developing a version extended by a cross-correlation subroutine. This software offers identification of point-like structures and the FilamentSensor workflow with the option to either use the routines on their own or correlate structures. Additionally, a ground truth database is included as well as a verification toolbox to compare pixel- or object-wise against a ground truth or the user's own annotations. This, in connection with advanced techniques like MS-TIRF or liveMIET, will help to analyze extended information about the interaction between stress fibers and focal adhesions can be gathered.

The improvements on FilamentSensor allowed for the analysis of a set of 690 hMSC cells of Lot 451491 on two conditions (glass and $11 \mathrm{kPa}$ PAA gel, both coated with collagen I). Additionally, several other sets of fixed and live cells were imaged and analyzed, leading to some important findings surrounding the main data set: 
- different lots of hMSCs show different, sometimes contrary, behavior

- cell culture chemicals like FBS measurably influence cell behavior

- culture conditions influence cell behavior, in this case cells on the microscope showing a larger area and aspect ratio than in the incubator (both $37^{\circ} \mathrm{C}, 5 \% \mathrm{CO}_{2}$ )

- cell and nuclei area correlate in fixed cells on different stiffnesses of PAA gels but nuclei aspect ratio stays constant while cell aspect ratio is highest around $20 \mathrm{kPa}$ in this data set

- with large numbers of fixed hMSCs, results are reproducible

- there are large differences between LifeAct-transfected live and phalloidin-stained fixed cells, with live cells having:

$-15 \%$ larger cell area

- $22 \%$ smaller aspect ratio

- $34 \%$ more stress fibers

- $20 \%$ smaller order parameter

When evaluating the large set of live movies, it can be said with high statistical certainty that:

- cells on glass show an approximately $25 \%$ larger cell area throughout the full 24 hours of imaging while long half axis in both conditions is the same length

- aspect ratio fluctuates but is consistently higher in cells on $11 \mathrm{kPa}$ PAA gel, at 24 hours being one-fourth larger

- order parameter develops in the same pattern and shows the same values on both conditions

- while in fixed cells order parameter varies between stiffnesses, in both Wollnik's [225] and my movies all live cell movies converge to an order parameter of 0.5

- cells on glass express consistently more filaments and $16 \%$ more after 24 hours

- mean fiber length does not vary between hMSC on $11 \mathrm{kPa}$ PAA gel and glass

- fibers are wider in cells on $11 \mathrm{kPa}$ PAA gels, but not statistically significant

- total curvature of the fibers in cells on glass is higher but not statistically significant

Furthermore, as FilamentSensor also saves the coordinates of the center of mass of the cells in all images, some migration statistics were done. In this analysis, cells on $11 \mathrm{kPa}$ PAA gels appear slightly superdiffusive, while cells on glass are subdiffuisve (please see Subsection 3.2.5). The trajectories of cells on glass also show that they often return to their original position. When looking on cells leaving the field of view after 18 hours, cells on both substrates exceed the diffusion threshold with cells on $11 \mathrm{kPa}$ clearly being in the realm of directed movement.

Considering this, we might only image a certain subsample during the live cell imaging, which might also affect the stress fiber network data as more movement requires higher stress fiber turnover. Thus, analysis of all cells, independent of how long they stay in the field of view, is called for. However, as the reason for the current protocol is to know the fate of the cell, this issue has to be solved first.

Before investigation of the transcriptome of hMSCs, I wanted to investigate transcriptional changes and de novo protein production in the nucleus to the early phase of mechanical differentiation by means of an enucleation protocol using ultracentrifugation. This did not yield viable cytoplasts for hMSCs. While testing other cell lines, however, NIH-3T3, MCF-7, SAOS-2, and 3T3 were identified as possible cell lines for future enucleation experiments. Also, live cell movies of various cell lines transfected with 
LifeAct-RFP on both $11 \mathrm{kPa}$ PAA gel and glass were done to provide reference measurements for the cytoplasts. During this, cell lines could be split into three groups according to area, aspect ratio, and filament number development. In the first group, there is no difference between behavior on the two substrates. The second group spreads more on glass, and has a higher aspect ratio on $11 \mathrm{kPa}$ PAA gel and higher filament number on glass. The third group behaves contrarily, with higher spreading area and filament count on $11 \mathrm{kPa}$ PAA gel and a higher aspect ratio on glass. Although the sample size is small for all of these movies, it seems to hint at an interesting follow-up project.

RNA-Seq was done with the same lot of hMSCs as was used for the large live cell movie data set. Here, unpublished data from former PhD student Carina Wollnik [225] was available. The Wollnik set compared PAA gels of 1, 10, and $30 \mathrm{kPa}$ stiffnesses against unplated cells as controls. For my set, I appended this sample set with cell culture plastic and collagen I coated glass as controls of plated cells. Comparison of sample qualities resulted in:

- Better clustering in the Wollnik set

- Better alignment and assignment in my set

- Different gene expression patterns between Wollnik and Hauke sets

The sub-par alignment and assignment in the Wollnik set is most likely the reason for the better clustering, as shorter reads tend to be grouped together and create higher counts, whereas in my set the counts are divided between more genes.

Regarding the full analysis of the Wollnik set:

- ECM-constituent secretion is upregulated in cells on PAA gels

- In direct comparison of PAA gel samples, many stress fiber network-related genes are upregulated on 1 and $30 \mathrm{kPa}$ but downregulated on $10 \mathrm{kPa}$, aligning with lower filament count in $10 \mathrm{kPa}$ samples

- Expression differences between PAA gel samples are significantly lower than against the zero-hour unplated control

As expected, using zero-hour unplated cells as the control against the samples plated for 24 hours on collagen I coated PAA gels is suboptimal. This is due not only to different cell treatment (24 hours longer plating) but also differences in stress levels. While zero-hour controls were trypsinized once, 24-hour samples were lysed with Trizol 24 hours after being trypsinized. Use of Trizol might, in this case, also explain upregulation of pathways induced by alcohols and cell poisons.

For my data set, although overall gene expression differences were less pronounced, some results could be found. Interestingly, four different expression patterns emerged when samples were grouped in zero-hour control versus glass and plastic versus samples on PAA gels: These results mean that samples on PAA

\begin{tabular}{c|c|c|}
$\begin{array}{c}\text { Mean } \\
\text { 0 hours }\end{array}$ & $\begin{array}{c}\text { Mean } \\
\text { glass/plastic }\end{array}$ & $\begin{array}{c}\text { Mean } \\
\text { 24-hour PAA gels }\end{array}$ \\
\hline++ & + & - \\
++ & - & - \\
- & + & - \\
- & + & -
\end{tabular}

gels have overall lower expression rates but, while there is a subset of genes where both unplated cells and cells on glass and plastic are upregulated, for other gene sets they behave contrarily. It has to be said that in the top 50 genes taking all samples into account, not many cytoskeleton-related ones can be found. Other results taking just my data set into account include: 
- When reduced to mirror Wollnik data set, main gene expression differences relate to proliferation and stress response

- Comparing samples on PAA gels to plastic and glass, on PAA gels more genes related to inflammatory response are upregulated and proliferation related genes downregulated

- Throughout PCA, ToppGene analysis, and KEGG pathway analysis, control samples on glass and cell culture plastic are almost identical

- When splitting according to passage number, "older" cells show downregulation of genes related to proliferation

- When analyzing only the PAA gel samples and selecting the top 50 genes, they are usually upregulated for $1 \mathrm{kPA}$ and downregulated for both 11 and $32 \mathrm{kPa}$ (one of six genes where this is reversed is Actin Alpha 2)

The downregulation of genes related to proliferation is especially interesting, as it occurs both on PAA gels and for older passages of cells. However, for my set, most gene expression and pathway differences relate in some way to stress response. To discriminate between stress response and substrate effect is not feasible for this data in my opinion, as even when only comparing between PAA gel samples percentage of genes related to the expected cytoskeletal reaction is low. A useful insight is the similarity between samples on collagen I coated glass and cell culture plastic. These substrates are usually both denoted as "infinite stiffness" and assumed to be similar but the matching gene expression profile proofs this.

As the RNA-Seq could only be done for a limited amount of samples, qRT-PCT was performed for selected targets with multiple time points up to 24 hours. This should show changes in expression during adhesion and differentiation. These targets were chosen for their relation to cytoskeletal development and turnover as well as read count during RNA-Seq, as the qRT-PCR has a detection threshold. In the end, hMSCs of the same lot as was used in live cell movies and RNA-Seq were seeded on cell culture plastic, glass, and PAA gels $(1,2,5,11,20,32 \mathrm{kPa})$. RNA was extracted from the cells after 1, 4, 12, 18 and 24 hours. This resulted in:

- Vinculin reaching a peek expression 12 hours after seeding and showing a tendency for higher values at higher stiffness

- Actin Alpha 2 expression staying low overall, with two outliers but less expression at 24 hours than at 1 hour

- Actin Beta having expression maximum at 4 hours with larger values on stiffer substrates. For time points $1,12,18$, and 24 hours however, expression is equally low

- Aniline expression being random with no relation to time or stiffness

- Myosin heavy chain 9 expression fluctuating as well, with some samples showing slightly higher expression at 4 and 18 hours

Concluding the qRT-PCR, only Vinculin and Actin Beta show interesting results with a distinct relation to time and stiffness.

To summarize these experiments: for hMSCs and other cell lines in the first 24 hours of plating, reactions of cells towards the substrates and the respective stress fiber network behavior is highly variable between cell lines and seems not to be connected to the cells or respective tissue stiffness. More experiments with cell lines not commonly used for stress fiber analysis could provide interesting results. Additionally, with some of these cell lines, enucleation with the ultracentrifugation method [79] is possible, yielding large amounts of viable cytoplasts. More experiments in this field have to be done to quantify the changes in the stress fiber network caused by absence of the nucleus.

For hMSCs, enucleation does not seem possible with this protocol. Transcriptome analysis after 24 hours 
of plating was not fruitful, either. The samples for RNA-Seq were generated after 24 hours as, in this thesis, I wanted to constrain myself to that timeframe. However, because other studies found significant changes after 1-2 weeks [67], it might be beneficial to repeat the experiment with samples plated for 3-4 days. For qRT-PCR, more samples should be generated to support the findings in this thesis. However, with the sample size of around 350 cells per condition, statements about stress fiber network behavior in the cell as well as general cell behavior can be made. This is restricted by the conclusion that cells have to be of the same lot and treated with the same materials to be comparable. Whenever this is the case, different experiments yield comparable and reproducible results. Also, for hMSCs, passage number has to be taken into consideration. A combination of combined stress fiber and focal adhesion imaging with high-throughput analysis of both structures and their correlation should provide more understanding of the stress fiber network development. 



\section{Outlook}

After implementing various new features in the FilamentSensor software, transferring them to 3D is a logical step.

For this, not only do changes need to made in the program algorithms and structure, but the experimental techniques have to match the objective as well.

Moving stem cells to the 3D environment is the first challenge to solve when trying to understand mechano-differentiation in tissue-like environments. For other cell lines and topics, 3D cell cultures are already commonly used. In-lab fabricated 3D gels or ready-made chambers or substrates can be used to tackle various topics. Cancer invasion and progression, as well as tissue interaction and cell migration in healthy tissues, are predominately of interest.

From this, we much is already known about the general behavior of cells in a 3D environment. We know that cell shape, position of focal adhesions [48], and stress fiber network morphology vary between 2D and 3D gels. Also, we know that movement in 3D is fundamentally different [235], as anchor points are distributed all around the membrane. Stress fiber patterns differ, as well as levels of related proteins like Arp2/3 and ROCK [46]. Also, it is known that during 3D migration of cancer cells the movement is dependent on a balance of tissue constraints and cell deformability. While cells can degrade ECM components to make way for migration, nondegradable substances will lead to substantial deformation of the nucleus [224]. This deformation, leading to rupture and repair, can in turn lead to DNA damage, altered transcriptome, and modified behavior [51]. All of this has to be taken into account and transferred to experiments with stem cells.

Also, while interaction with the environment is more general for immortalized cell lines, for stem cells matrices have to be well-defined in morphology, rheology, and composition [23]. An understanding of composition and stress transduction throughout the matrix and on interfaces will be crucial.

For basic material, PAA with a collagen coating, as used for $2 \mathrm{D}$ experiments, cannot be used, as it is cytotoxic. One common solution is a pure collagen gel [57] but manufacturing gels with stiffnesses as high as that of PAA gels is not possible. In our group, hyaluronic acid (HA) gels have proven to be a good substitute [53]. While cells are viable in the matrix for up to weeks, they lack ECM mimickers to aid movement. With addition of collagen into the HA mix, cells seeded into this mixture during polymerization remained viable and motile for several days afterwards [177]. To monitor gelation process and resulting gel properties, different techniques can be used. These techniques, some can also be employed to monitor gel behavior under the influence of seeded cells [177, 232, 233].

For analysis of 3D stress fiber patterns, the FilamentSensor software had to be altered. For these alterations, the necessary adjustments depend on the image files provided. In our lab, MicroManager is generally used to collect 3D samples. The integrated ImageJ software saves them as z-stacks (or hyperstacks, when stacks are imaged over time). Thus, we can analyse a 3D stack picture-by-picture and stitch filaments together when they are found in multiple pictures.

We already have in use a Wasserstein transport method for over-time tracking of single filaments. This can be adjusted to work in $3 \mathrm{D}$ stacks assuming that fibers overlap from picture to picture. 
A description of the idea and realization of the Wasserstein transport in the FilamentSensor was provided by Benjamin Eltzner:

\section{The Wasserstein Distance}

Assume two sets of objects $R=\left\{r_{j}\right\}_{j}$ and $S=\left\{s_{k}\right\}_{k}$, a base distance or cost function $d: R \times S \rightarrow \mathbb{R}_{\geq 0}$ and weights for every element of $R$ and $S$, which we denote by $w\left(r_{j}\right)$ and $w\left(s_{k}\right)$ respectively. Define an optimal transport as a matrix $T=\left(t_{j k}\right)_{j k}$ satisfying the following minimization with constraints

$$
\begin{gathered}
T=\sum_{j, k} d\left(r_{j}, s_{k}\right) t_{j k} \\
\forall j: \sum_{k} t_{j k}=w\left(r_{j}\right) \\
\forall k: \sum_{j} t_{j k}=w\left(s_{k}\right)
\end{gathered}
$$

where "argmin" simply denotes the minimum position, not the minimum value. As a side remark, the transport plan need not be unique in every instance, but for many choices of $d$ it is unique in practical situations.

For the optimal transport $T$, the Wasserstein distance is $\mathcal{W}(R, S)=\sum_{j, k} d\left(r_{j}, s_{k}\right) t_{j k}$. However, we will only use the transport plan.

\section{$\underline{\text { Filament Transport }}$}

In our case, the sets $R$ and $S$ contain line segments of roughly 10 pixels length from two subsequent images in a movie. The weights $w\left(r_{j}\right)$ denote the length of each line segment in pixels. Both $R$ and $S$ contain a "dummy" element, $r_{0}$ and $s_{0}$ respectively, which have distances $\forall j: d\left(r_{j}, s_{0}\right)=d_{\max }^{2}$ and $\forall k: d\left(r_{0}, s_{k}\right)=d_{\max }^{2}$ and "infinite weights" $w\left(r_{0}\right)=\sum_{k} w\left(s_{k}\right)$ and $w\left(s_{0}\right)=\sum_{j} w\left(r_{j}\right)$. Thus, a distance of more than $d_{\max }^{2}$ between two segments means that they will not be connected by the Wasserstein transport. In this sense the transport is "local". For all true line segments, the distance is composed of $d_{l}\left(r_{j}, s_{k}\right)$, which is the distance in pixels between the closest point of the two segments, and $d_{\phi}\left(r_{j}, s_{k}\right)$, the orientation difference between the two segments in degrees. Then the base distance is

$$
d\left(r_{j}, s_{k}\right):=4 d_{l}\left(r_{j}, s_{k}\right)^{2}+d_{\phi}\left(r_{j}, s_{k}\right)^{2},
$$

which, together with the dummy elements, means that only line segments that are at most $d_{\max } / 2$ pixels apart and whose orientation differs by at most $d_{\max }$ degrees can be joined. (We typically use $d_{\max } \approx 20$.) The transport plan $t_{j k}$ is then interpreted as something similar to a correlation matrix.

\section{Preliminary Visualization}

In the preliminary visualization all segments for which more than $50 \%$ of the weight is transported to the dummy element-i. e., $t_{j 0}>0.5 w\left(r_{j}\right)$-when compared with both the previous and the next image are marked in red. All others segments are marked in blue (see Figure 10.1). In other words, all blue segments have a "partner" segment on the previous or the next image, while the red lines are "lone" lines, which may be due to flawed line detection. 

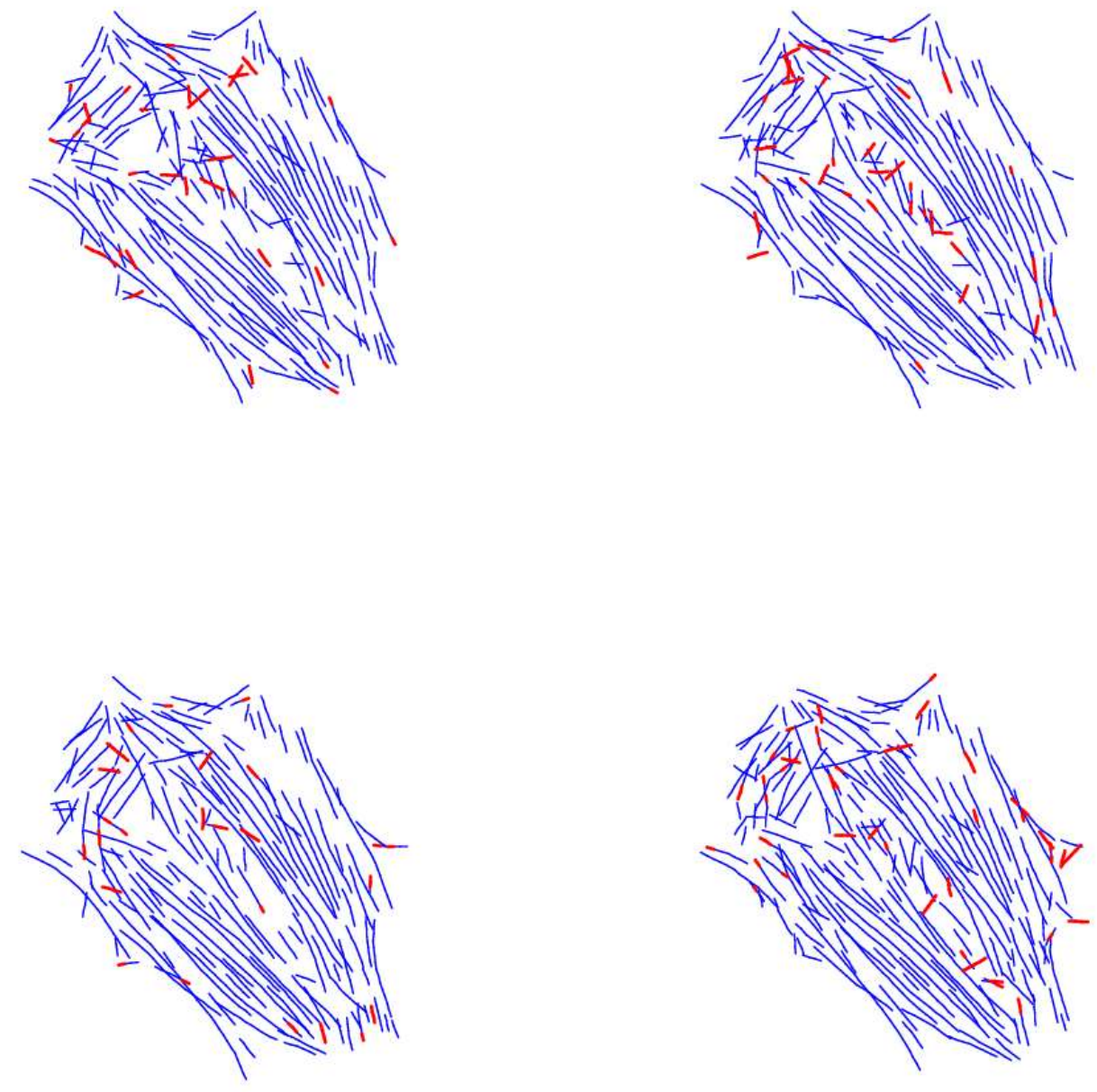

Figure 10.1.: Preliminary visualization of single filament tracking. Filaments stable over time marked in blue, 'noise' in red. Noise means this filaments only appear in one frame. 



\section{Acknowledgements}

This thesis would not exist without the help of several people. First, my supervisor Dr. Florian Rehfeldt selected me for this project and was always supportive of new ideas and side projects. He and Prof. Dr. Sarah Köster and Prof. Dr. Stefan Klumpp formed a great thesis committee, that supported this project not only with great suggestions and advice but with criticism that helped to regain focus whenever necessary. During the experiments, the technical assistants Kerstin von Rhoden, Ulrike Schulz, and Tanja Gall were incredibly helpful. They shared their experiences, suggested solutions, tested protocols, and protocol improvements as well as doing a lot of the routine work. Tanja established new qRT-PCR protocols and tested runs even shortly before she was leaving the lab. Ulrike took over and spared my all the dreaded 96-well pipetting. During the years she provided all of my plasmids, protein purification, and great antibody staining. Kerstin is the queen of gels. Many of my experiments would have taken twice or triple the time they did if I had to make gels myself instead of finding flawless gels in the fridge. Also, enucleation experiments would have been incredibly more complicated if I would not have been able to work in parallel with Kerstin. Also, work would have been much harder without Thomas Geiling, who dealt with all computer issues, Dieter Hille and the guys from the metal workshop, who provided $\mathrm{CO}_{2}$, customized parts of all sorts, and some emergency quick fixes, the guys from the electronics workshop, who repaired a lot of cables and power supplies, Nicole Rehbein for all the administrative work, and Dr. Dieter Klopfenstein, who set up the Sharepoint with the well-used machine sign-up calendars, the liquid nitrogen Raspberry Pi, and provided coffee supplies.

During the thesis, I had the opportunity to work with many people. Andreas Primeßnig was my working student for almost the whole time. He finished the immense task of refactoring the FilamentSensor and helped with improvements and new applications. Without him and his Java experience, it would have taken at least a year of my thesis to do it myself. The development of this software was done in the SFB 755 , which ended 1,5 years after the start of the project. In this project, I continued collaboration from my predecessor Dr. Carina Wollnik with Dr. Benjamin Eltzner and Prof. Dr. Stefan Huckemann. I have to thank them for their previous work, suggestions, and many fruitful discussions. Another collaboration I inherited from Dr. Carina Wollnik was with the group of Prof. Enderlein on the metal-induced energy transfer (MIET). This led to the follow-up collaboration with Dr. Sebastian Isbaner on the liveMIET, which had unbelievable few experimental issues and setbacks - it was a pleasure to work on it. With Shwetha Narasimhan and Prof. Irina Kaverina, we developed the FAFCK, the cross-correlation addition to the original FilamentSensor, which is now used in large parts of their department. Shwetha was very helpful by providing data, requests, and extensive testing of the provided software versions. Lastly, I want to thank Peleg Sapir and Victor Turrion for the live cell movie database and user interface they provided. Also, I want to thank my peers: Daniel for the Python introduction, data discussions, patterning project, and coffee breaks; Christina for a lot (still not enough) of Indian food, fun cooking lessons, group activity initiations, and all the long talks; Galina for being the "lab mom" and keeping the group together; Heidi for lots of coffee breaks, fun, corgi butts, and singing (not sure about the singing, though); all other members of the Schmidt/Rehfeld lab - Samaneh, Behzad, Amna, Eugenia, Constantin, Chonglin, Jiango, Sufi, Octavio, Renata, Nora, Martin, Matthias, and Stefanie - it was a great work environment, although I do not miss the three-hour group meetings on Friday afternoons. 
For wine, games, and distractions I want to thank Fie, Marina, Harald, Tibbs, and Charlotte. A late-night orc-killing session or discussion about doors and Nubian pharaohs sometimes helped more to solve some experimental issues than a scientific discussion. Also, you kept my work-life-balance in an acceptable range - as my family did as well. My family was always supportive of this thesis and I am glad I can finally tell them that I am done (yes, Dad, stop asking). Unfortunately, the two people that looked forward to my degree the most, will not be able to celebrate with me. Many things I needed to complete my thesis, I learned from them. My mother was a great role model for persistence, integrity, and efficiency. Also, she was curious and always eager to learn. She taught me more about the scientific mindset than most of my professors. My grandfather, with whom I seem to have never agreed on anything, taught me how to discuss while respecting the opponent and his view. Both of them loved and supported me. 




\section{A. Appendix}

This appendix section contains detailed protocols used for

- A - Cell culture

- A.5 - Enucleation

- B.1 - Preparation of RNA for qRT-PCR and Genome Analysis Laboratory (TAL)

- B.3 - qRT-PCR

- C - Preparation and coating of polyacrylamide gels

- D - Staining of fixed cells

- E - Live-cell imaging

- F - RNA-Sequencing (done by the Transcriptome and Genome Analysis Laboratory, Göttingen) 


\section{A. Cell culture}

All protocols are written in a generalized fashion. Changes due to the use of different cell lines only apply to the medium and cell culture flasks used (see Table A.2), as well as pulse code and kit for the transfection protocol in Subsection A.4. For REF-52 cells, which we received from the Berchadsky laboratory in Singapur, we use the protocol given from members of this laboratory and mentioned in [79].

Materials in Table A.1 were used in all cell culture related protocols.

\begin{tabular}{|c|c|c|c|}
\hline Abbr. & Product & Supplier & Product No. \\
\hline T25 flasks (red) & Cell culture flask & Sarstedt & 833.910 .002 \\
\hline T25 flasks (orange) & Cell culture flask & Corning & 430639 \\
\hline T75 flasks (red) & Cell culture flask & Sarstedt & 833.911.002 \\
\hline T75 flasks (orange) & Cell culture flask & Corning & $430641 \mathrm{U}$ \\
\hline T175 Flasks (red) & Cell culture flask & Sarstedt & 833.912 .002 \\
\hline T225 flasks (orange) & Cell culture flask & Corning & 431082 \\
\hline 1-well plate & 1-well cell culture dish & Greiner & GREI670190_32 \\
\hline 6-well plate & 6-well cell culture dish & Sarstedt & 833.920 \\
\hline $\mathrm{P} / \mathrm{S}$ & $\begin{array}{c}\text { Penicillin/Streptomycin } \\
10.000 \text { Units } / \mathrm{ml}\end{array}$ & Gibco & $151440-122$ \\
\hline 0,05\% Trypsine & $0,05 \%$ Trypsine-EDTA $(1 \mathrm{x})$ & Gibco & $25300-054$ \\
\hline L-glutamin & L-Glutamin $200 \mathrm{mM}$ & Gibco & $25030-024$ \\
\hline FBS & Fetal bovine serum & Sigma & F2442 \\
\hline Sterile PBS & $\begin{array}{c}\text { Dulbecco's Phosphate Buffered } \\
\text { Saline, tablets }\end{array}$ & Sigma & $\mathrm{P} 4417$ \\
\hline DMEM (low glucose) & DMEM (1 g/l Glucose) & Gibco & $31885-023$ \\
\hline DMEM (high glucose) & DMEM (4.5 g/l Glucose) & Sigma & D6429 \\
\hline McCoy's 5A & McCoy's 5A Medium & Gibco & $26600-23$ \\
\hline Gloves & gloves nitrile nextgen & Meditrade GmbH & $1283 \mathrm{M}$ \\
\hline Lint free wipes & precision wipes & Kimberly clark & 05511 \\
\hline Incubator & $\begin{array}{l}\text { BBD6220 Heraeus } \\
\mathrm{CO}_{2} \text { Incubator }\end{array}$ & Thermo Scientific & 51027763 \\
\hline Bench 1 & HeraSafe HS 18 & Kendro & 51013503 \\
\hline Bench 2 & HeraSafe KS 12 & Thermo Scientific & 51022515 \\
\hline Pipettboy & accu-jet pro & VWR & $612-2625 \mathrm{P}$ \\
\hline Single use pipettes & $10 \mathrm{ml}$ single use pipettes & Sarstedt & 86.1685 .001 \\
\hline Aspiration pipettes & $2 \mathrm{ml}$ Aspiration pipettes & Sarstedt & 86.1252 .011 \\
\hline Falcon tubes $(50 \mathrm{ml})$ & Falcon Tube size $50 \mathrm{ml}$ & Sigma & Z618985-2EA \\
\hline
\end{tabular}

Table A.1.: Materials in cell culture. 


\section{A.1. Thawing}

\section{Materials:}

- $70 \%$ ethanol (spray flasks, mixed from $99 \%$ ethanol and MilliQ water)

- Incubator $\left(37^{\circ} \mathrm{C}, 5 \% \mathrm{CO}_{2}\right)$

- Cells in cryo-vial (stored in liquid nitrogen before thawing)

- Ice

- Water bath, heated to $37^{\circ} \mathrm{C}$

- Medium suitable for cell line (+FBS, $+\mathrm{P} / \mathrm{S})$, heated to $37^{\circ} \mathrm{C}$

- $50 \mathrm{ml}$ Falcon tubes

- T75 or T225 cell culture flasks (suitable for cell line)

- Incubate the cells for two to three days at $37^{\circ} \mathrm{C}$ and $5 \% \mathrm{CO}_{2}$ )

- For some cell lines it is advised to change the medium on the following day to remove dead cells

\begin{tabular}{l|l|l} 
Cell line & Medium & Flask \\
\hline hMSC & DMEM low Glucose $+10 \% \mathrm{FBS}+1 \% \mathrm{P} / \mathrm{S}$ & Corning \\
HeLa & DMEM low Glucose $+10 \% \mathrm{FBS}+1 \% \mathrm{P} / \mathrm{S}$ & Sarstedt \\
MCF-7 & DMEM low/high Glucose $+10 \% \mathrm{FBS}+1 \% \mathrm{P} / \mathrm{S}$ & Sarstedt \\
Vero & DMEM high Glucose $+10 \% \mathrm{FBS}+1 \% \mathrm{P} / \mathrm{S}+1 \%$ L-Glutamin & Sarstedt \\
SAOS-2 & McCoy's 5A $+15 \% \mathrm{FBS}+1 \% \mathrm{P} / \mathrm{S}$ & Sarstedt \\
C2-C12 & DMEM high Glucose $+10 \% \mathrm{FBS}+1 \% \mathrm{P} / \mathrm{S}$ & Sarstedt \\
$3 \mathrm{~T} 3$ & DMEM low Glucose $+10 \% \mathrm{FBS}+1 \% \mathrm{P} / \mathrm{S}$ & Sarstedt \\
Cos-7 & DMEM low Glucose $+10 \% \mathrm{FBS}+1 \% \mathrm{P} / \mathrm{S}$ & Sarstedt \\
293 & DMEM low Glucose $+10 \% \mathrm{FBS}+1 \% \mathrm{P} / \mathrm{S}$ & Sarstedt \\
NIH-3T3 & DMEM high Glucose $+10 \% \mathrm{FBS}+1 \% \mathrm{P} / \mathrm{S}$ & Sarstedt \\
REF-52 (WT) & DMEM high Glucose $+10 \% \mathrm{FBS}+1 \% \mathrm{P} / \mathrm{S}$ & Sarstedt \\
REF-52 (YFP-Pax) & DMEM high Glucose $+10 \% \mathrm{FBS}+1 \% \mathrm{P} / \mathrm{S}$ & Sarstedt
\end{tabular}

Table A.2.: Medium and Flasks used for cell lines.

\section{Protocol:}

- Spray the bench with Ethanol

- Prepare bench and keep available $50 \mathrm{ml}$ Falcon tube and heated medium

- Take the cells out of the liquid nitrogen tank and store on ice until thawing

- Heat the cells in the water bath for $45 \mathrm{~s}$

- Add warm medium to the cell suspension

- Try to not pipette too many times while transferring the cells into the Falcon tube

- Fill the tube with $15 \mathrm{ml}$ warm medium

- Spin down for 5 min at $1000 \mathrm{rpm}$

- Remove medium, refill with $10 \mathrm{ml}$ PBS 
- Spin down for 5 min at $1000 \mathrm{rpm}$

- Remove PBS, solve the cell pellet in $13 \mathrm{ml}$ warm Medium (26 ml for $>5.000 .000$ cells)

- Transfer to T75 flask (or T175/T225 for $>5.000 .000$ cells)

- Gently move the cell culture flask to distribute cells evenly across the bottom 


\section{A.2. Freezing}

The same protocol is used for all different cell lines.

\section{Materials:}

- $70 \%$ Ethanol (spray flask, mixed from $99 \%$ ethanol and MilliQ water)

- Cells in culture flasks (T75 or larger)

- PBS, room temperature

- Trypsin, heated to $37^{\circ} \mathrm{C}$

- Medium suitable for cell line (Table A.2)

- $50 \mathrm{ml}$ Falcon tube

- FBS, heated to $37^{\circ} \mathrm{C}$

- Suitable Medium, see Table A.2, heated to $37^{\circ} \mathrm{C}$

- Cryo-vials

- Freezing box, inner box swimming in Isopropanol

- Freezer, $-80^{\circ} \mathrm{C}$

- Liquid nitrogen in storage container

\section{Protocol:}

- Spray cell culture bench with ethanol

- Discard medium in the cell culture flask

- Add $10 \mathrm{ml}$ PBS to the flask, gently move to dilute remaining medium

- Discard PBS

- Add $3 \mathrm{ml}$ warm trypsin to the flask, incubate for $3 \mathrm{~min}$ at $37^{\circ} \mathrm{C}$

- Check under the microscope if trypsin treatment was successful. When cells stick to the flask detach them by gently tapping the flask

- Under the bench add $10 \mathrm{ml}$ of warm Medium to stop the reaction

- Transfer to $50 \mathrm{ml}$ Falcon tube, take $1 \mathrm{ml}$ aliquot to count cells

- Spin for 5 min at $1000 \mathrm{rpm}$

- During centrifuging, prepare freezing medium (70 \% medium, $20 \%$ FBS, $10 \%$ DMSO) in an amount that you freeze 100.000 to 2.000 .000 cells (depending on cell line) per cryo-vial and $1 \mathrm{ml}$ freezing medium

- Discard the medium of the Falcon tube

- Resuspend the cells in the appropriate amount of freezing medium, aliquot into cryo-vials (should be labeled beforehand)

- Put the cryo-vials into the freezing box and store overnight in $-80^{\circ} \mathrm{C}$

- Transfer them into liquid nitrogen storage 


\section{A.3. Splitting}

\section{Materials:}

- $70 \%$ Ethanol (spray flask, mixed from $99 \%$ Ethanol and MilliQ water)

- Cells

- PBS, room temperature

- Trypsin, prewarmed at $37^{\circ} \mathrm{C}$

- Medium, prewarmed at $37^{\circ} \mathrm{C}$ (suitable for cell line)

- $50 \mathrm{ml}$ Falcon tube

- Culture flasks (T75 or larger, suitable for cell line)

\section{Protocol:}

- Spray cell culture bench with Ethanol

- Discard Medium in the cell culture flask

- Add $10 \mathrm{ml}$ PBS to the flask, gently move to dilute remaining medium

- Discard PBS

- Add $3 \mathrm{ml}$ warm trypsin to the flask, incubate for $3 \mathrm{~min}$ at $37^{\circ} \mathrm{C}$

- Check under the microscope if trypsin treatment was successful. When cells stick to the flask detach them by gently tapping the flask

- Under the bench add $10 \mathrm{ml}$ of warm Medium to stop the reaction

- Transfer to $50 \mathrm{ml}$ Falcon tube, take $1 \mathrm{ml}$ aliquot to count cells

- Spin for $5 \mathrm{~min}$ at $1000 \mathrm{rpm}$

- Discard medium

- Add $10 \mathrm{ml}$ PBS

- Spin 5 min at $1000 \mathrm{rpm}$

- Discard PBS

- Resuspend pallet in medium, split to 3-4 new flasks of the same size

- Add $13 \mathrm{ml}$ medium for T75, $26 \mathrm{ml}$ medium for T225

- Incubate for two to three days at $37^{\circ} \mathrm{C}$ and $\mathrm{CO}_{2}$ ) 


\section{A.4. Transfection}

\begin{tabular}{l|l|l|l} 
Abbr. & Product & Product No. & Supplier \\
\hline Lifeact-TagRFP & pCMV LifeAct-TagRFP & 60102 & ibidi \\
Nucleofector core & 4D Nucleofector Core unit & AAF-1002B & Lonza \\
Nucleofector unit & 4D Nucleofector X unit & AAF-1002X & Lonza \\
P1 Kit & P1 Primary cell D-Nucleofector X Kit L & V4XP-1012 & Lonza \\
SE Kit & SE Cell Line 4D-Nucleofector X Kit L & V4XC-1024 & Lonza \\
SF Kit & SF Cell Line 4D-Nucleofector X Kit L & V4XC-2012 & Lonza
\end{tabular}

Table A.3.: Transfection supplies, additional to Table A.1.

\section{Materials:}

- Cells to be transfected

- PBS, room temperature

- Medium (suitable for the cell line)

- Trypsin, preheated to $37^{\circ} \mathrm{C}$

- $50 \mathrm{ml}$ Falcon tube

- Nucleofector buffer solution suitable for cell line

- Nucleocuvette

- DNA (i.e. pCMV with Lifeact-TagRFP (see Figure 5.2))

- Flask (T75, suitable for cell line, see Table A.2), dishes or substrates to seed cells

\section{Protocol:}

- Spray cell culture bench $70 \%$ with ethanol

- Discard medium in the cell culture flask

- Add $10 \mathrm{ml}$ PBS to the flask, gently move to dilute remaining medium

- Discard PBS

- Add $3 \mathrm{ml}$ warm trypsin to the flask, incubate for $3 \mathrm{~min}$ at $37^{\circ} \mathrm{C}$

- Check under the microscope if trypsin treatment was successful. When cells stick to the flask detach them by gently tapping the flask

- Under the bench add $10 \mathrm{ml}$ of warm Medium to stop the reaction

- Transfer to $50 \mathrm{ml}$ Falcon tube, take $1 \mathrm{ml}$ aliquot to count cells

- Spin for 5 min at $1000 \mathrm{rpm}$

- Use waiting time to switch on the Nucleofector machine and select the desired slot, used nucleofection buffer and desired pulse code

- Discard medium

- Add $10 \mathrm{ml}$ PBS 
- Spin 5 min at $1000 \mathrm{rpm}$

- Meanwhile prepare the nucleofection solution

- $82 \mu \mathrm{l}$ Nucleofector Solution (according to requirements of cell line)

- $18 \mu \mathrm{l}$ Supplement 1 (according to requirements of cell line)

- DNA (according to requirements of cell line)

- DNA should be added to the mix 1 min before using it

- Prepare T75 bottle with $13 \mathrm{ml}$ of medium

- Discard PBS

- Resuspend pellet in nucleofector solution and transfer to nucleocuvette

- Put into nucleofector machine, start the program (According to Table A.4)

- Gently remove cells in nucleofector solution from the nucleocuvette and transfer to the prepared bottle

- Gently move the bottle to distribute cells evenly

- Incubate at $37^{\circ} \mathrm{C}$ and $5 \% \mathrm{CO}_{2}$ )

- Change the medium on the following day to remove dead cells

- Use 48 hours after transfection

\begin{tabular}{l|l|l|l|l} 
Cell line & Transfection Kit & Pulse & n of cells & amount Plasmid \\
\hline hMSC & P1 & FF-104 & min 50.00 & $2 \mu \mathrm{g}$ \\
HeLa & SE & DS-150 & 1 Mio & $2 \mu \mathrm{g}$ \\
MCF-7 & SE & EN-130 & 1 Mio & $2 \mu \mathrm{g}$ \\
Vero & SF & DS-100 & 1 Mio & $2 \mu \mathrm{g}$ \\
SAOS-2 & SF & CA-137 & 1 Mio & $2 \mu \mathrm{g}$ \\
C2-C12 & SE & CD-133 & 1 Mio & $2 \mu \mathrm{g}$ \\
3 T3 & SE & CA-137 & 2 Mio & $2 \mu \mathrm{g}$ \\
Cos-7 & SE & FF-113 & 1 Mio & $2 \mu \mathrm{g}$ \\
293 & SF & CM-103 or CA-137 & 1 Mio & $2 \mu \mathrm{g}$ \\
NIH-3T3 & SE & DS-150 & 1 Mio & $2 \mu \mathrm{g}$ \\
REF-52 (WT) & SE & EN-158 & 1 Mio & $2 \mu \mathrm{g}$ \\
REF-52 (YFP-Pax) & SE & EN-158 & 1 Mio & $2 \mu \mathrm{g}$
\end{tabular}

Table A.4.: Transfection solutions, pulse codes and number of cells used for transfection of different cell lines. 


\section{A.5. Enucleation}

While using Ficoll 400 of different companies and Lots, a variation of enucleation efficacy is reported by [222]. Therefore, we purchased our Ficoll 400 from the distributor suggested in the paper, which is OrderNr. BP525 from Fischer.

\begin{tabular}{l|l|l} 
Product & Supplier & Product No. \\
\hline Ficoll 400 & Fisher & BP525 \\
Cytochalasin B & Sigma & C2743-200UL \\
Optima LE-90K Ultracentrifuge & Beckman Coulter & DS-9339A \\
Polypropylene centrifuge tubes (10.4 ml volume) & Beckman Coulter & 4415 \\
Adapter tubes 10.4 ml to SW32 Ti & Beckman Coulter & 11035 \\
SW32 Ti - Swing bucket rotor & Beckman Coulter & 369650
\end{tabular}

Table A.5.: Materials for enucleation, additional to Table A.1.

\section{Materials:}

- Ficoll 400 (BP525, Fischer)

- Cytochalasin B (Enzo Life Sciences)

- DMSO

- Medium suitable for cell line (see Table A.2)

- PBS

- Beckman Coulter Optima LE-90K Ultracentrifuge

- Polypropylene centrifuge tubes (10.4 ml volume)

- $\mathrm{SW} 32 \mathrm{Ti}$ - Swing bucket rotor

- Adapter tubes $10.4 \mathrm{ml}$ to $\mathrm{SW} 32 \mathrm{Ti}$

- Cells trypsinated and washed according to Protocol in Subsection A.3

\section{Protocol:}

- Dissolve Ficoll $40050 \%$ (wt/vol) in PBS $\left(\mathrm{Ca}_{2+} / \mathrm{Mg}_{2+}\right.$ free) over night at room temperature on shaker

- Sterile filter the solution using a $0,4 \mu \mathrm{m}$ pore size

- Dilute to $30 \%$ (vol/vol) with suitable medium and all required additions

- Measure refractive index of $30 \%$ solution on photometer at $600 \mathrm{~nm}$ wavelength using PBS as blank (for REF-52, HeLa and other cell lines of similar size optimal refractive index is 1.373)

- Dilute $30 \%$ solution to $20 \%, 18 \%$ and $15 \%$ (vol/vol) using the suitable medium

- Add $10 \mu \mathrm{g} / \mathrm{ml}$ Cytochalasin B (in $100 \%$ Ethanol) and $0.2 \%$ DMSO to the prepared solutions

- Layer into $10.4 \mathrm{ml}$ polypropylene centrifuge tubes as following:

$-2 \mathrm{ml} 30 \%$ solution at bottom 
$-2 \mathrm{ml} 20 \%$ solution

$-2 \mathrm{ml} 18 \%$ solution

$-1 \mathrm{ml} 15 \%$ solution

- Store the remaining $15 \%$ solution at $4^{\circ} \mathrm{C}$

- Seal the gradients with parafilm, store overnight at $37^{\circ} \mathrm{C}$

- Incubate SW32 Ti rotor bucket and adapter tubes at $37^{\circ} \mathrm{C}$ overnight

- Next day trypsinate the cells according to protocol in Subsection A.3

- Resuspend the pellet containing up to $2 \times 10 \hat{7}$ cells in $1 \mathrm{ml}$ prewarmed $15 \%$ Ficoll solution

- Layer on top of gradient

- Fill tube with medium to top

- Incubate gradient at $37^{\circ} \mathrm{C}$ and $5 \% \mathrm{CO} 2$ for $60 \mathrm{~min}$

- Prewarm Centrifuge at $30^{\circ} \mathrm{C}$

- Spin gradients at $125.000 \mathrm{~g}(27.000 \mathrm{rpm})$ for 1 hour at $30^{\circ} \mathrm{C}$

- Stop at minimal breaking

- Collect cell fraction from gradient (or nucleus fraction from tube bottom)

- Wash twice with PBS

- Wash twice with DMEM

- Resuspend pellet in DMEM

- Plate on substrates (for example gels manufactured according to protocol in Subsection C.3) 


\section{B. RNA}

\section{B.1. RNA extraction}

\begin{tabular}{l|l|l} 
Product name & Supplier & Product No. \\
\hline RNeasy Mini Kit (250) & Qiagen & 74106 \\
$\beta$-Mercaptoethanol & Roth & 4227.1 \\
RNase-Free DNase (250) & Qiagen & 79256
\end{tabular}

Table B.6.: RNA extraction reagents, additional to Table A.1.

\section{Materials:}

- Cells

- $70 \%$ Ethanol (spray flask, mixed from $99 \%$ Ethanol and MilliQ water)

- Trypsin, heated to $37^{\circ} \mathrm{C}$

- PBS, room temperature

- Medium, heated to $37^{\circ} \mathrm{C}$

- $50 \mathrm{ml}$ Falcon tube

- $99 \%$ Ethanol, RNA grade

- $\beta$-Mercaptoethanol

- Filter pipet tips

- Qiagen RNeasy Kit

- Qiagen DNAse

\section{Protocol:}

- Prepare buffers of the Qiagen RNeasy Kit

- Add Ethanol to RPE buffer

- Add $\beta$-Mercaptoethanol to RLT buffer

- Prepare DNAse

- Solve DNAse in RDD buffer (1:8 DNase in RDD buffer)

- DNAse can be stored aliquoted at $-20^{\circ} \mathrm{C}$ to prevent multiple thawing events

- Spray cell culture bench with ethanol

- Get cells (in bottles or on substrates)

- Discard medium in the cell culture flask

- Add a suitable amount of PBS to dilute the remaining medium

- Discard PBS

- Add a suitable amount of prewarmed trypsin, incubate for 3 min at $37^{\circ} \mathrm{C}$ 
- Check under the microscope if trypsin treatment was successful. When cells stick, gently tap

- Add a suitable amount of medium to stop the reaction

- Transfer to $50 \mathrm{ml}$ Falcon tube

- Spin for 5 min at $1000 \mathrm{rpm}$

- Add $10 \mathrm{ml}$ PBS

- Spin 5 min at $1000 \mathrm{rpm}$

- Discard the PBS carefully

- Resolve the pellet in $350 \mu \mathrm{l} \beta$-Mercaptoethanol in RLT-buffer

- Lysate the cells at least 5 times through a $0.9 \mathrm{~mm}$ diameter needle

- Add $350 \mu \mathrm{l} 70 \%$ Ethanol

- Mix well

- Transfer to RNeasy spin column

- Spin $15 \mathrm{~s}$ at $\geq 10000 \mathrm{rpm}$

- Discard the flow-through

- Add $350 \mu$ l Buffer RW1 to the RNeasy spin column

- Spin $15 \mathrm{~s}$ at $\geq 10000 \mathrm{rpm}$

- Discard the flow-through

- Add $80 \mu \mathrm{l}$ DNAse Stock solution

- incubate at RT for $20 \mathrm{~min}$

- Add $350 \mu$ l Buffer RW1 to the RNeasy spin column

- Spin $15 \mathrm{~s}$ at $\geq 10000 \mathrm{rpm}$

- Discard the flow-through

- Add $500 \mu$ l Buffer RPE to the RNeasy spin column

- Spin $15 \mathrm{~s}$ at $\geq 10000 \mathrm{rpm}$

- Discard the flow-through

- Add $500 \mu$ l Buffer RPE to the RNeasy spin column

- Spin 2 min at $\geq 10000 \mathrm{rpm}$

- Discard the collection tube with the flow-through

- place the spin column in a new collection tube

- Spin 2 min at $\geq 10000 \mathrm{rpm}$

- Place the spin column in a new 1.5 . ml collection tube

- Add $30 \mu$ l RNase-free water directly on the spin column

- Spin 1 min at $\geq 10000 \mathrm{rpm}$

- Store RNA at $-20^{\circ} \mathrm{C}$ for short times or $-80^{\circ} \mathrm{C}$ for prolonged storage 


\section{B.2. Writing RNA in cDNA}

Writing RNA into cDNA was done entirely by technical assistant Ulrike Schulz, who kindly provided the protocol.

\begin{tabular}{l|l|l} 
Product name & Supplier & Product No. \\
\hline SuperScript IV Reverse Transcriptase & Thermo Fisher & 18090010 \\
$5 x$ SuperScript IV buffer & Thermo Fisher & $18090050 \mathrm{~B}$ \\
$10 \mathrm{mM}$ dNTP Mmix & Thermo Fisher & 18427013 \\
$100 \mathrm{mM}$ DTT & Thermo Fisher & R0861 \\
$50 \mu \mathrm{M}$ Oligo d(T) primer & Thermo Fisher & 18418020 \\
T3000 Thermocycler & Biometra & 704384 \\
RT-PCR Grade Water & Thermo Fisher & AM9935 \\
PowerUp SYBR Green Master Mix & Thermo Fisher & A25779 \\
StepOne Real-Time PCR Machine & Thermo Fisher & 4376357 \\
MicroAmp Fast 96-Well Reaction Plate $(0.1 \mathrm{ml})$ & Fisher Scientific & 4346907
\end{tabular}

Table B.7.: qRT-PCR reagents, additional to Table A.1.

Materials:

- SuperScript IV Reverse Tranascriptase

- Nuclease free water

- $\mathrm{dNTPs}_{\mathrm{S}}$

- Oligo d(T) primer

- 5x SSIV buffer

- DTT

- Biometra T3000 thermocycler

- NanoPhotometer

\section{Protocol:}

- Premix per probe

$-1 \mu$ l Oligo $\mathrm{d}(\mathrm{T})$ primer

$-1 \mu \mathrm{ldNTP}$

$-5 \mu \mathrm{l}$ RNA

$-7 \mu \mathrm{l}$ Nuclease free water

- Heat the RNA-primer mix at $65^{\circ} \mathrm{C}$ for $5 \mathrm{~min}$

- Vortex and briefly centrifuge the 5x SSIV buffer

- Combine in a reaction tube

$-4 \mu \mathrm{l}$ SSIV buffer

- $1 \mu \mathrm{l} 100$ mM DTT 
- $1 \mu \mathrm{l}$ Superscript IV Reverse Transcriptase $(200 \mathrm{U} / \mu \mathrm{l})$

- (1 $\mu \mathrm{l}$ RNase Inhibitor was not added, hence smaller Volume)

- Mix and briefly centrifuge

- Incubate at $50-55^{\circ} \mathrm{C}$ for $10 \mathrm{~min}$

- Inactivate the reaction at $80^{\circ} \mathrm{C}$ for $10 \mathrm{~min}$

- Meassure the ssDNA content at NanoPhotometer

- Freeze at $-20^{\circ} \mathrm{C}$ 


\section{B.3. qRT-PCR}

Sample preparation and machine preparation was done entirely by technical assistant Ulrike Schulz, who kindly provided the protocol.

For materials used please see Table B.7 for specific reagents and Table A.1 for general materials.

\section{B.3.1. Sample and Plate Preparation}

Materials:

- PowerUp SYBR Green Master Mix

- Primer sets

- Samples

- Nuclease-free water

- 96-well optical reaction plates

- Adhesive covers

\section{Protocol:}

- Prepare for each reaction:

- $10 \mu \mathrm{l}$ PowerUp SYBR Green Master Mix

$-2 \mu \mathrm{l}$ forward primer

$-2 \mu \mathrm{l}$ reverse primer

- $2 \mu \mathrm{l}$ DNA template (diluted to the concentration set up in the run)

- $4 \mu \mathrm{l}$ Nuclease-free water

- Mix well

- centrifuge briefly

- Transfer sufficient amount to wells of optical plate

- Seal with adhesive cover

- Centrifuge briefly 


\section{B.3.2. Software settings and Run}

Materials:

- StepOne Real-Time PCR Machine

- StepOne Software v2.3

\section{$\underline{\text { Software settings: }}$}

- Experiment properties:

- StopOnePlus Instrument (96 Wells)

- Quantitation - Relative Standard Curve

- SYBR Green Reagents

- Standard ( 2 hours to complete a run)

- Plate setup:

- Add targets, choose SYBR as reporter

- Define Samples

- Design plate

* Assign standards, unknowns, and negative controls

- For standards set concentrations, replicates and locations

* Assign sample locations

* Choose reference sample

* Choose endogenous control

- Run method:

- Holding stage:

* $100 \%$ ramping

* $95^{\circ} \mathrm{C}, 10 \mathrm{~min}$

- Cycling stage:

* $95^{\circ} \mathrm{C}, 15 \mathrm{sec}$

* $100 \%$ ramping

* Temperature according to primer requirements, see Table 8.16, 1 min, data collection on

* 40 cycles

- Melt curve stage:

* $100 \%$ ramping

* $95^{\circ} \mathrm{C}, 15 \mathrm{sec}$

* $100 \%$ ramping

* Temperature according to primer requirements, see Table 8.16, 1 min

* Ramping $+0.3^{\circ} \mathrm{C}$, data collection on

* $95^{\circ} \mathrm{C}, 15$ sec, data collection on 


\section{Polyacrylamide gels}

\begin{tabular}{|c|c|c|c|}
\hline Abbr. & Product & Supplier & Product No. \\
\hline Large microscopy slides & $\begin{array}{l}\text { microscopy slides } \\
76-52-1 \mathrm{~mm}\end{array}$ & Marienfeld & MARI1100420 \\
\hline Large cover glasses & Cover slips $48 \times 64 \mathrm{~mm}$ & Agar scientific & AGL464864-15 \\
\hline Round glasses & $\begin{array}{c}\varnothing 25 \mathrm{~mm} \text { cover glasses, } 1, \\
0.13-0.16 \mathrm{~mm}\end{array}$ & VWR & ECN631-1584 \\
\hline Square cover glasses & $\begin{array}{c}24 \times 24 \mathrm{~mm} \text { square cover glasses, } 1 \\
0.13-0.16 \mathrm{~mm}\end{array}$ & VWR & ECN631-1571 \\
\hline PBS (unsterile) & 1x PBS tablets, $\mathrm{pH} 7.4$ & ChemSolute & 8435-100TABLETS \\
\hline HEPES & $\begin{array}{l}\text { 2-(4-(2-Hydroxyethyl)-1- } \\
\text {-piperazinyl) ethansulfon acid }\end{array}$ & Roth & 9105.3 \\
\hline $\mathrm{AA}$ & Acetic Acid & Riedel de Haën & 27221 \\
\hline APTES & (3-Aminopropyl) triethoxysilane & Sigma-Aldrich & 440140-100ML \\
\hline Sulfo-SANPAH & $\begin{array}{l}\text { Sulfosuccinimidyl 6-(4'-azido-2'- } \\
\text {-nitrophenylamino)hexanoate }\end{array}$ & Thermo Scientific & 22589 \\
\hline Glutaraldehyde & $\begin{array}{l}\text { Glutaraldehyde solution, Grade I, } \\
\qquad 50 \% \text { in } \mathrm{H}_{2} \mathrm{O}\end{array}$ & Sigma-Aldrich & G7651-10ML \\
\hline Ethanol, pure & $\begin{array}{c}\text { Ethanol absolute, } \\
\text { AnalaR NORMAPUR }\end{array}$ & VWR & 20821.321 \\
\hline Silanazation solution & $\begin{array}{l}\text { Silanization solution } 1, \\
5 \% \text { dimethyldichlorosilane in heptane }\end{array}$ & Sigma-Aldrich & 85126-1L \\
\hline Plasma cleaner & Expanded Plasma cleaner & Harrick Plasma & PDC-002 \\
\hline Ultrasonic bath & Ultrasonic unit & Elmasonic & S-100 \\
\hline Tweezers & Titanium Tweezers 7 & Aven Inc & $18072 \mathrm{TT}$ \\
\hline APS & Ammonium persulfate & Sigma & A3678 \\
\hline TEMED & $\begin{array}{c}\text { N,N,N',N'-Tetramethylethylendiamin, } \\
\text { 1,2-Bis(dimethylamino)-ethan }\end{array}$ & BioRad & $161-0800$ \\
\hline Acrylamide & Acrylamide $40 \%$ & BioRad & $161-0140$ \\
\hline Bis-acrylamide & Bis-acrylamide $2 \%$ & BioRad & $161-0142$ \\
\hline Collagen I & Rat tail Collagen I & Corning & 354236 \\
\hline
\end{tabular}

Table C.8.: Materials for polyacrylamide gel manufacturing. 


\section{C.1. Cover glass preparation}

\section{Materials:}

- Silanization solution

- $2.5 \times 2.5 \mathrm{~cm}$ glasses (or $6.8 \times 4.8 \mathrm{~cm}$ large microscopy slides)

- MilliQ water

\section{Protocol:}

- Place glasses in a petri dish without overlap

- Smear silanization solution onto every glass

- Incubate 10 min

- Rinse with MilliQ water

- Before use rinse with MilliQ water again, make sure glasses are still hydrophobic

- Glasses can be reused 


\section{C.2. Bottom glass preparation}

\section{Materials:}

- Plasma cleaner

- Diameter $2.5 \mathrm{~cm}$ glasses (or 7.6x5.2 cm large microscopy slides)

- Holder and glass bowl for washing

- $99 \%$ Ethanol

- APTES

- Gluteraldehyde

- MilliQ water

- Ultrasound bath

\section{Protocol:}

- Put round glasses into a holder

- Use plasma cleaner for 15 min at

- 1-1.2 mbar

- Power set to "high"

- Put into a glass bowl, add $250 \mathrm{ml} 99 \%$ Ethanol

- Sonicate for $5 \mathrm{~min}$

- Discard Ethanol

- Add $245 \mathrm{ml} 99 \%$ Ethanol + $5 \mathrm{ml}$ APTES (2\%)

- Sonicate for $5 \mathrm{~min}$

- Wash 2 times with $99 \%$ Ethanol

- Wash with MilliQ

- Put 8 glasses each into $9 \mathrm{~cm}$ diameter petri dish without overlap

- Add $10 \mathrm{ml}$ Glutaraldehyde solution per dish

- $200 \mu \mathrm{l}$ Glutaraldehyde in $40 \mathrm{ml}$ MilliQ

- Incubate $30 \mathrm{~min}$ at room temperature

- Put back into glass holder, fill with MilliQ

- Sonicate $15 \mathrm{~min}$

- Glasses can be stored in MilliQ at $4^{\circ} \mathrm{C}$ 


\section{C.3. Gels}

\begin{tabular}{l|c|l|l} 
Abbr. & Product & Supplier & Product No. \\
\hline Rheometer & $\begin{array}{c}\text { Physica MCR 501 rheometer } \\
\text { Type H-PTD200 }\end{array}$ & Anton Paar & 80696537 \\
25-2 probe & Messkegel CP25-2 & Anton Paar & 79039
\end{tabular}

Table C.9.: Rheometer equipment, additional to Table C.8.

\section{Materials:}

- APS

- TEMED

- $40 \%$ Acrylamide

- $2 \%$ Bisacrylamide

- Rheometer

- Rheometer steel bottom plate

- $2.5 \mathrm{~cm}$ steel probe, $2^{\circ}$ angle

- PBS

- Paper sheets

\begin{tabular}{|c|c|c|c|c|c|c|}
\hline $\mathrm{kPa}$ & $\begin{array}{c}\text { Acrylamide } \\
(40 \%)\end{array}$ & $\begin{array}{c}\text { Bis-acrylamide } \\
(2 \%)\end{array}$ & $\begin{array}{l}\text { Acrylamide } \\
(\mathrm{ml})\end{array}$ & $\begin{array}{l}\text { Bis-acrylamide } \\
(\mathrm{ml})\end{array}$ & $\begin{array}{l}\text { PBS } \\
(\mathrm{ml})\end{array}$ & $\begin{array}{l}\text { total } \\
(\mathrm{ml})\end{array}$ \\
\hline 0,75 & 3 & 0,14 & 0,75 & 0,7 & 8,55 & 10 \\
\hline 1 & 3 & 0,2 & 0,75 & 1 & 8,125 & 10 \\
\hline 2 & 4 & 0,25 & 1 & 1,25 & 7,75 & 10 \\
\hline 4 & 3,8 & 0,2 & 0,95 & 1 & 8,05 & 10 \\
\hline 5 & 5 & 0,14 & 1,25 & 0,7 & 8,05 & 10 \\
\hline 8 & 5 & 0,25 & 1,25 & 1,25 & 7,5 & 10 \\
\hline 10 & 6 & 0,14 & 1,5 & 0,7 & 7,8 & 10 \\
\hline 11 & 6 & 0,18 & 1,5 & 0,9 & 7,6 & 10 \\
\hline 20 & 8 & 0,14 & 2 & 0,7 & 7,3 & 10 \\
\hline 21 & 8 & 0,14 & 2 & 0,7 & 7,3 & 10 \\
\hline 30 & 8 & 0,3 & 2 & 1,5 & 6,5 & 10 \\
\hline 32 & 8,6 & 0,3 & 2,15 & 1,5 & 6,35 & 10 \\
\hline 64 & 13,2 & 0,3 & 3,3 & 1,5 & 5,2 & 10 \\
\hline 128 & 23,6 & 0,3 & 5,9 & 1,5 & 2,6 & 10 \\
\hline
\end{tabular}

Table C.10.: Polyacrylamide gel mixtures.

Protocol:

- Prepare a stock of Polyacrylamide mix according to Table C.10, the solutions can be stored for 6-8 weeks 
- Measure gel solutions on a rheometer to confirm stiffness using $140 \mu \mathrm{l}$ on a steel plate with $2.5 \mathrm{~cm}$ diameter steel probe, $2^{\circ}$ angle

- Time sweep (1 hour, spacing $30 \mathrm{~s}, 1 \%$ strain, $1 \mathrm{~Hz}$ frequency)

- Frequency sweep (3 measurements per decade, $1 \%$ strain, 0.01-100 Hz)

- Amplitude Sweep (0.01 \% - $100 \%$ strain, $1 \mathrm{~Hz}$ frequency)

- Mix desired amount of solution with 1/1000 volume TEMED and vortex thoroughly

- Prepare wet paper sheets as flat as possible on the benchtop

- Dry round bottom glasses from MilliQ, put on sheets (prepared according to Subsection C.1)

- Rinse square cover glasses with MilliQ

- Add 1/100 volume APS to mix and vortex thoroughly

- Add $35 \mu \mathrm{l}$ mix per bottom glass (or $300 \mu \mathrm{l}$ for the large microscopy glasses; glasses prepared according to Subsection C.2)

- Cover with square glass

- Cover with a lid to prevent drying

- Let polymerize 1-2 hours

- Remove square cover glasses

- Transfer into 6-Well plates

- Rinse 3 times with PBS

- Uncoated gels can be stored in PBS for up to 2 weeks 


\section{C.4. Coating}

\section{Materials:}

- PBS

- HEPES

- Rat tail Collagen I

- Sulfo-SANPAH

- Acetic Acid

\section{$\underline{\text { Protocol: }}$}

- Incubate twice 5 min with PBS on shaker

- Incubate once 5 min with HEPES on shaker

- Dry bottom of glasses, transfer to new a 6-Well plate

- Add Sulfo-SANPAH to the surface, incubate 10 min under $365 \mathrm{~nm}$ UV lamp with max. $10 \mathrm{~cm}$ spacing between lamp and gel

- Wash 3 times with HEPES

- Mix per well on ice by pipetting

- $100 \mu$ l Collagen I $(0.02 \%)$

- $100 \mu$ l Acetic acid $(0.02 \%)$

- Add $1800 \mu \mathrm{l}$ chilled HEPES and mix well

- Fill $2 \mathrm{ml}$ Collagen solution into wells

- Incubate overnight at $4^{\circ} \mathrm{C}$

- Wash thoroughly 3 times with PBS

- Sterilize 2 hours under UV light in cell culture bench 


\section{C.5. Preparing dishes for live cell imaging}

\begin{tabular}{l|c|l} 
Product & Supplier & Product No. \\
\hline Raw $\mu$-Dish 35mm, high & Ibidi & DIO01110 \\
Raw $\mu$-Dish 35mm, lid & Ibidi & DIO01110 \\
Isopropanol & VWR & 20880.320 \\
Lint free wipes & Kimberly clark & 05511 \\
UV curable glue NOA 68 & Norland products inc. & 6801 \\
UV-Lamp Typ NU-8 KL & Benda & 7721008
\end{tabular}

Table C.11.: Materials for dish preparation, additional to Table C.8.

\section{Materials:}

- Substrates manufactured according to protocol in Subsection C.3

- Bottomless ibidi dishes

- Norland Optical Adhesive 68

- PBS

- Isopropanol

\section{Protocol:}

- Wipe bottomless ibidi dishes with isopropanol

- Line edge of the bottom with optical glue

- Dry bottoms of gel glasses

- Place glasses into the bottom

- Gently tap edges, the glass should be flat and the glue without a hole

- Incubate $90 \mathrm{~s}$ under $365 \mathrm{~nm}$ UV lamp for each site

- Fill with $2 \mathrm{ml}$ PBS

- Sterilize 2 hours under UV in cell culture bench

- Rinse 2 times with sterile PBS

- Fill with cell culture medium

- Seed cells (trypsinated according to protocol in Subsection A.3) in desired density 


\section{Imunostaining}

\begin{tabular}{|c|c|c|c|}
\hline Abbr. & Product & Supplier & Product No. \\
\hline PBS & 1xPBS Tablets, $\mathrm{pH} 7,4$ & ChemSolute & 8418.0100 \\
\hline $\begin{array}{l}\text { Formaldehyde } \\
(3.6 \% \text { in PBS })\end{array}$ & $\begin{array}{c}\text { Formaldehyde solution } \\
36 \% \text { in } \mathrm{H} 2 \mathrm{O}\end{array}$ & Sigma-Aldrich & 47608-250ML-F \\
\hline Glutaraldehyde & $\begin{array}{l}\text { Glutaraldehyde } \\
\text { solution }\end{array}$ & Sigma & G7651 \\
\hline Triton X & Triton X 100 & $\begin{array}{c}\text { Carl Roth } \\
\text { GmbH }\end{array}$ & 6683.1 \\
\hline BSA & $\begin{array}{l}\text { Bovine Serum Albumin } \\
\text { lyophilised powder }\end{array}$ & Sigma-Aldrich & A9418-100G \\
\hline Microscope & Axio Observer.Z1 & Zeiss & 431007-0001-000 \\
\hline $20 \mathrm{x}$ objective & $\begin{array}{c}\text { Objective N-Achroplan } \\
\text { 20x/0.45 Ph2 M27 }\end{array}$ & Zeiss & 420951-9911-000 \\
\hline DAPI filter & $\begin{array}{c}\text { Filtersatz } 49 \\
\text { DAPI shift free }\end{array}$ & Zeiss & 488049-9901-000 \\
\hline GFP filter & Filtersatz eGFP & Zeiss (AHF) & F36-527 \\
\hline RFP filter & Filtersatz RFP & Zeiss (AHF) & F46-008 \\
\hline Colibiri & $\begin{array}{c}\text { illumination system } \\
\text { Colibri.2 }\end{array}$ & Zeiss & 423052-9501-000 \\
\hline 365 LED-Modul & $\begin{array}{l}365 \text { nm excitation } \\
\text { for Colibri }\end{array}$ & Zeiss & 423052-9011-000 \\
\hline 470 LED-Modul & $\begin{array}{l}470 \mathrm{~nm} \text { excitation } \\
\text { for Colibri }\end{array}$ & Zeiss & $423052-9052-000$ \\
\hline 540 LED-Modul & $\begin{array}{l}540-580 \mathrm{~nm} \text { excitation } \\
\text { for Colibri }\end{array}$ & Zeiss & 423052-9121-000 \\
\hline Mounting medium & $\begin{array}{l}\text { Fluoroshield histology } \\
\text { mounting medium }\end{array}$ & Sigma-Aldrich & F6182-20ML \\
\hline Camera & Zyla sCMOS 4.2 & Andor & via acal-bfi \\
\hline
\end{tabular}

Table D.12.: Imunostaining materials.

Materials:

- PBS

- $3,4 \%$ formaldehyde in PBS

- $0.5 \%$ Triton X-100 in PBS

- $3 \%$ BSA in PBS

- Antibodies in $3 \%$ BSA in PBS

- For mounting

- Microscope slides

- Fluoroshield mounting medium

- Nail polish 


\begin{tabular}{|c|c|c|c|c|c|}
\hline Abbr. & Product & Host & Usage & Supplier & Product No. \\
\hline Phalloidin 488 & $\begin{array}{l}\text { Phalloidin } \\
\text { ATTO } 488\end{array}$ & $\begin{array}{l}\text { Amanita } \\
\text { palloides }\end{array}$ & $\begin{array}{c}{[1: 150]} \\
2 \mathrm{~h}\end{array}$ & Atto Tec & $\mathrm{AD} 488-81$ \\
\hline Phalloidin 550 & $\begin{array}{l}\text { Phalloidin } \\
\text { ATTO } 550\end{array}$ & $\begin{array}{l}\text { Amanita } \\
\text { palloides }\end{array}$ & $\begin{array}{l}{[1: 150]} \\
2,5 \mathrm{~h}\end{array}$ & Atto Tec & AD 550-81 \\
\hline Phalloidin $647 \mathrm{~N}$ & $\begin{array}{c}\text { Phalloidin } \\
\text { ATTO 647N }\end{array}$ & $\begin{array}{l}\text { Amanita } \\
\text { palloides }\end{array}$ & $\begin{array}{c}{[1: 150]} \\
2 \mathrm{~h}\end{array}$ & Atto Tec & AD $647 \mathrm{~N}-81$ \\
\hline Hoechst-DAPI & $\begin{array}{c}\text { Hoechst } 33342, \\
\text { trihydrochloride, } \\
\text { trihydrate }\end{array}$ & none & $\begin{array}{l}{[1: 10.000]} \\
20 \mathrm{~min}\end{array}$ & Thermo Fisher & H3570 \\
\hline Anti-Pax & Anti-Paxillin IgG & Mouse & $\begin{array}{c}{[1: 400]} \\
\text { over night }\end{array}$ & Abcam & ab32084 \\
\hline Anti-NMM2a & $\begin{array}{l}\text { Anti-Myosin IIA, } \\
\text { non muscle }\end{array}$ & Rabbit & $\begin{array}{c}{[1: 250]} \\
\text { over night }\end{array}$ & Sigma & M8064 \\
\hline Anti-rabbit $647 \mathrm{~N}$ & $\begin{array}{l}\text { Anti-rabbit-IgG } \\
\text {-Atto } 647 \mathrm{~N}\end{array}$ & Goat & $\begin{array}{c}{[1: 400]} \\
2 \mathrm{~h}\end{array}$ & Sigma & 40839 \\
\hline
\end{tabular}

Table D.13.: Antibodies used in immunostaining.

\section{Protocol:}

- Remove cell medium

- Wash with PBS

- Incubate 10 min with $2 \mathrm{ml} 10 \%$ formaldehyde per well

- Rinse with PBS

- Incubate for 10 min with $2 \mathrm{ml}$ Triton X-100 (0,5\% (vol/vol) in PBS per well on rocker

- Wash with PBS

- Add $2 \mathrm{ml} 3 \%$ BSA in PBS to each well, incubate $30 \mathrm{~min}$ at room temperature on the rocker

- Discard BSA solution

- Add $2 \mathrm{ml} 0.5 \%$ Triton X-100 in PBS to each well, incubate 5 min on the

- Rinse with PBS

- Add $1 \mathrm{ml}$ of the premixed solution of primary antibody in $3 \%$ BSA in PBS in the desired concentration to each well

- Incubate on the rocker for at least one hour to one day at room temperature

- Remove primary antibody solution (can be stored at $4{ }^{\circ} \mathrm{C}$ and reused

- Add $2 \mathrm{ml} 0.5 \%$ Triton X-100 solution to each well and incubate for 5 min on the rocker at room temperature

- Rinse with PBS

- Now keep the samples covered in aluminum foil to prevent bleaching

- Ad $1 \mathrm{ml}$ premixed solution of secondary antibody in $3 \%$ BSA in PBS in the desired concentration to each well 
- Incubate on the rocker for one or two hours at room temperature

- Remove secondary antibody solution (can be stored at $4^{\circ} \mathrm{C}$ and reused)

- Add $2 \mathrm{ml} 0.5 \%$ Triton X-100 to each well and incubate for $5 \mathrm{~min}$ on the rocker at room temperature

- Rinse with PBS

- Add other antibodies if desired with the respective washing steps

- Samples can now be filled with PBS and sealed with parafilm to store at $4^{\circ} \mathrm{C}$ or be mounted

\section{Mounting:}

- Rinse your samples 3 times with MilliQ water

- Clean microscope slides with $70 \%$ ethanol, let dry

- Place a drop of fluoroshield mounting solution on the microscope slide

- Add the glass slide with the cell side towards the mounting medium

- Incubate in the dark for 2-3 hours

- Seal the edges with nail polish and let dry entirely overnight at $4{ }^{\circ} \mathrm{C}$ 


\section{E. Live-cell movies}

\begin{tabular}{|c|c|c|c|}
\hline Abbr. & Product & Supplier & Product No. \\
\hline Microscope & Axio Observer.Z1 & Zeiss & $431007-0001-000$ \\
\hline Microscope stage & 120x100 STEP scanning stage & Zeiss & 342029-0000-000 \\
\hline Lamp & HXP 120 illumination equipment & Zeiss & 423013-0000-000 \\
\hline Gas mixer & $\begin{array}{c}\text { ibidi gas incubation system for } \mathrm{CO} 2 \text { and } \mathrm{O} 2 \text {, } \\
\text { humidifying column }\end{array}$ & ibidi & 11920 \\
\hline Heating & $\begin{array}{c}\text { ibidi heating system system Universal fit: } \\
\text { ibidi Temperature Controller, } \\
\text { Heated Plate in Multi-Well Format, } \\
1 \text { Heating insert, } \\
\text { with heated lid }\end{array}$ & ibidi & 10918 \\
\hline Petri dish insert & heating insert $\mu$-Dish $35 \mathrm{~mm}$, high & ibidi & 10934 \\
\hline 20x objective & LD A-Plan 20x/0.30 Ph1, $1 / 1.0$ & Zeiss & $1006-591$ \\
\hline Micro manager & Micro-Manager Software & $\begin{array}{l}\text { Ron Vale Lab } \\
\text { Uni California }\end{array}$ & version 1.4 .21 \\
\hline Camera & Zyla sCMOS 4.2 & Andor & via acal-bfi \\
\hline
\end{tabular}

Table E.14.: Live cell imaging microscope parts.

\section{Materials:}

- Inverse microscope

- Appropriate filter cubes and light source for chosen fluorophore

- Transfected cells, rested 48 hours and replated into $35 \mathrm{~mm}$ wells

- Ibidi gas mixer with humidifier column

- Ibidi heating cube

- Appropriate heating chamber for the sample holder

- Camera

- Software able to perform multi-channel, -timepoint and -position measurements

\section{Protocol:}

- Preheat microscope at least 2 hours before the start of imaging in desired settings

- Set $\mathrm{CO}_{2}$-System to $5 \% \mathrm{CO}_{2}, 3$ liters per hour, $75 \%$ humidity

- Preheat chamber bottom to $37^{\circ} \mathrm{C}$ and chamber lid to $40^{\circ} \mathrm{C}$

- Empty dishes should be added into the chambers while heating

- Set up Köhler illumination

- Switch to desired objective and camera and turn illumination on

- Put dishes into the imaging chamber

- Check Köhler illumination

- Use "Multi-dimensional Acquisition" in $\mu$ Manager 
- Do 24 hour imaging with 144 time points, 10 min interval (shorter interval leads to too much bleaching)

- No z-Stacks

- $300 \mathrm{~ms}$ exposure time in RFP-channel (pCMV Lifeact-TagRFP) seems to be sufficient in most cases for lifeact-RFP transfected cells

- Take phase contrast (30 ms exposure time) to check for non-transfected cells and dirt interfering with transfected cells 


\section{F. RNA-Sequencing (TAL)}

This section was provided by Dr. Orr Shomroni as "Materials and Methods" paper alongside the results.

Library preparation:

RNA sequencing was performed at the Transcriptome and Genome Analysis Laboratory, University Medical Center, Göttingen, in biological triplicates for murine hearts and in technical triplicates for cells. RNA was extracted using Protocol descriped in Subsection B.1. Quality and integrity of RNA was assessed with the Fragment Analyzer from Advanced Analytical by using the standard sensitivity RNA Analysis Kit (DNF-471). All samples selected for sequencing exhibited an RNA integrity number over 8.

RNA-seq libraries were performed using a modified stranded-specific, massively-parallel cDNA sequencing (mRNA-Seq) protocol from Illumina, the TruSeq stranded mRNA prep Kit (Cat. No. RS-122-2101). Specifically we first optimized the protocol during the ligation step diluting the adapters concentration to increase ligation efficiency ( $>94 \%$ ), and finally we reduce the number of PCR cycles to avoid PCR duplication artifacts as well as primer dimers in the final library product.

Using strand-specificity protocols we observed differences in coverage, agreement with known annotations, and accuracy for expression profiling. The incorporation of dUTP during second strand synthesis and the RNA ligation method is the leading protocol to perform total stranded RNA Seq.

For accurate quantitation of cDNA libraries a fluorometric based system, the QuantiFluor ${ }^{\mathrm{TM}} \mathrm{dsDNA}$ System from Promega were used. The size of final cDNA libraries was determined by using the dsDNA 905 Reagent Kit (Fragment Analyzer from Advanced Bioanalytical) exhibiting a sizing of $300 \mathrm{bp}$ in average. Libraries were pooled and and sequenced on an Illumina HiSeq 4000 (Illumina) generating 50 bp single-end reads (30-40 Mio reads/sample).

Raw read \& Quality check:

Generation of raw data files and their quality check was performed by Orr Shomroni from the TAL. Sequence images were transformed with Illumina software BaseCaller to BCL files, which was demultiplexed to fastq files with bcl2fastq v2.17.1.14. The sequencing quality was asserted using FastQC (Andrews, S. (2014). FastQC A Quality Control tool for High Throughput Sequence Data.

(http://www.bioinformatics.babraham.ac.uk/projects/fastqc/)(version 0.11.5).

Mapping \& Normalization:

Mapping of sequenced reads and read counting for all samples was performed by Orr. Sequences were aligned to the reference genome Homo sapiens (hg38 version 89,

https://www.ensembl.org/Homo $a_{i}$ sapiens/Info/Index) using the STAR aligner (Dobin, Alexander, et al. "STAR: ultrafast universal RNA-seq aligner." Bioinformatics 29.1 (2013): 15-21.)(version 2.5.2a) allowing for 2 mismatches within 50 bases. Subsequently, read counting was performed using featureCounts (Liao, Yang, Gordon K. Smyth, and Wei Shi. "featureCounts: an efficient general purpose program for assigning sequence reads to genomic features." Bioinformatics 30.7 (2013): 923-930.) (version 1.5.0-p1).

Differential expression analysis:

Differential expression analysis was performed by Orr. Read counts were analyzed in the R/Bioconductor environment (version 3.4.2, www.bioconductor.org) using the DESeq2 (Love, Michael I., Simon Anders, and Wolfgang Huber. "Moderated estimation of fold change and dispersion for RNA-seq data with DESeq2." Genome biology 15.12 (2014): 550.) package version 1.16.1. Candidate genes were filtered using an absolute $\log 2$ fold-change $>1$ and FDR-corrected p-value $<0.05$. Gene annotation was performed using Homo sapiens entries via biomaRt R package version 2.32.1 (Durinck S, Spellman P, Birney E and Huber $\mathrm{W}$ (2009). "Mapping identifiers for the integration of genomic datasets with the R/Bioconductor package biomaRt." Nature Protocols, 4, pp. 1184-1191.). 


\section{Bibliography}

[1] B. Akpinar, B. Arda, T. Bagci-Onder, M. Bencze, G. Butler-Browne, P. C. Demircan, C. Deng, M. Erdem, F. M. Frame, C. H. Güvercin, M. Hansson, C. Honore, A. Z. Ilter, E. Karaoz, S. Klein, G. T. Kose, G. Liu, N. J. Maitland, V. Mouly, T. T. Onder, J. Pruszak, M. Ramazanoglou, T. Redmer, C. R. A. Regenbrecht, I. Riederer, A. E. Sariboyaci, W. Savino, R. Schäfer, K. A. Schlegel, D. Schumacher, G. Sobaci, B. Stratmann, D. Telci, R. Thomas, D. Tschoepe, D. Uckan, W. Wruck, and Y. Zhang. Stem Cells: Current Challenges and New Directions. Springer Science+Business Media, New York, 2013.

[2] B. Alberts, A. Johnson, J. Lewis, D. Morgan, M. Raff, K. Roberts, and P. Walter. Molecular Biology of the Cell. Garland Science, New York, 6th edition, 2015.

[3] A. Y. Alexandrova, K. Arnold, S. Schaub, J. M. Vasiliev, J. J. Meister, A. D. Bershadsky, and A. B. Verkhovsky. Comparative dynamics of retrograde actin flow and focal adhesions: Formation of nascent adhesions triggers transition from fast to slow flow. PLoS ONE, 3(9):1-9, 2008.

[4] J.-H. Alixt. Molecular Aspects of the In Vivo and In Vitro Effects of Ethionine, an Analog of Methionine Adenosine-Trapping Actiity of Ethionine in Rat Liver Cells. MICROBiOLOOICAL REVIEWS, 46(3):281-295, 1982.

[5] R. D. A. M. Alves, J. A. A. Demmers, K. Bezstarosti, B. C. J. Van Der Eerden, J. A. N. Verhaar, M. Eijken, and J. P. T. M. Van Leeuwen. Unraveling the Human Bone Microenvironment beyond the Classical Extracellular Matrix Proteins: A Human Bone Protein Library. Journal of Proteome Research, 10(10):4725-4733, oct 2011.

[6] N. Amariglio, A. Hirshberg, B. W. Scheithauer, Y. Cohen, R. Loewenthal, L. Trakhtenbrot, N. Paz, M. Koren-Michowitz, D. Waldman, L. Leider-Trejo, A. Toren, S. Constantini, G. Rechavi, and A. Fischer. Donor-Derived Brain Tumor Following Neural Stem Cell Transplantation in an Ataxia Telangiectasia Patient. PLoS Medcine, 6(2), 2009.

[7] Y. Aratyn-Schaus, P. W. Oakes, J. Stricker, S. P. Winter, and M. L. Gardel. Preparation of Complaint Matrices for Quantifying Cellular Contraction. Journal of Visualized Experiments, (46):1-6, 2010.

[8] M. Atakhorrami, J. I. Sulkowska, K. M. Addas, G. H. Koenderink, J. X. Tang, A. J. Levine, F. C. MacKintosh, and C. F. Schmidt. Correlated fluctuations of microparticles in viscoelastic solutions: Quantitative measurement of material properties by microrheology in the presence of optical traps. Physical Review E, 73(6):061501, jun 2006.

[9] P. Au, J. Tam, D. Fukumura, and R. K. Jain. Bone marrow-derived mesenchymal stem cells facilitate engineering of long-lasting functional vasculature. 2008.

[10] M. Bahat-Stroomza, Y. Barhum, Y. S. Levy, O. Karpov, S. Bulvik, E. Melamed, and D. Offen. Induction of Adult Human Bone Marrow Mesenchymal Stromal Cells into Functional Astrocyte-Like Cells: Potential for Restorative Treatment in Parkinson's Disease. J Mol Neurosci, 39:199-210, 2009. 
[11] B. Balana, C. Nicoletti, I. Zahanich, E. M. Graf, T. Christ, S. Boxberger, and U. Ravens. 5Azacytidine induces changes in electrophysiological properties of human mesenchymal stem cells. Cell Research, 16(12):949-960, dec 2006.

[12] B. L. Bangasser, G. A. Shamsan, C. E. Chan, K. N. Opoku, E. Tüzel, B. W. Schlichtmann, J. A. Kasim, B. J. Fuller, B. R. McCullough, S. S. Rosenfeld, and D. J. Odde. Shifting the optimal stiffness for cell migration. Nature communications, 8:15313, may 2017.

[13] J. R. Beach, K. S. Bruun, L. Shao, D. Li, Z. Swider, K. Remmert, Y. Zhang, M. A. Conti, R. S. Adelstein, N. M. Rusan, E. Betzig, and J. A. Hammer. Actin dynamics and competition for myosin monomer govern the sequential amplification of myosin filaments. Nature Cell Biology, 19(2):85-93, 2017.

[14] V. Belaud, T. Petithory, A. Ponche, C. Mauclair, C. Donnet, L. Pieuchot, S. Benayoun, K. Anselme, V. Belaud, T. Petithory, A. Ponche, C. Mauclair, C. Donnet, L. Pieuchot, S. Benayoun, and K. Anselme. Influence of multiscale and curved structures on the migration of stem cells. Biointerphases, 13(6):14, 2018.

[15] B. J. Belin, L. M. Goins, and R. D. Mullins. Comparative analysis of tools for live cell imaging of actin network architecture. BioArchitecture, 4(6):189-202, 2014.

[16] M. F. Berry, A. J. Engler, Y. J. Woo, T. J. Pirolli, T. Lawrence, V. Jayasankar, K. J. Morine, T. J. Gardner, D. E. Discher, H. L. Sweeney, W. Hiesinger, M. J. Brukman, R. C. Mccormick, J. R. Fitzpatrick, J. R. Frederick, E. C. Yang, J. R. Muenzer, N. A. Marotta, M. F. Berry, P. Atluri, Y. J. Woo, C. Suire, N. Brouard, K. Hirschi, P. J. Simmons, E. T. H. Yeh, M. F. Berry, A. J. Engler, Y. J. Woo, T. J. Pirolli, L. T. Bish, V. Jayasankar, K. J. Morine, T. J. Gardner, D. E. Discher, H. L. Sweeney, F. Mark, A. J. Engler, Y. J. Woo, T. J. Pirolli, L. T. Bish, V. Jayasankar, K. J. Morine, J. Timothy, A. J. Physiol, H. Circ, F. February, M. F. Berry, A. J. Engler, Y. J. Woo, T. J. Pirolli, T. Lawrence, V. Jayasankar, K. J. Morine, T. J. Gardner, D. E. Discher, H. L. Sweeney, W. Hiesinger, M. J. Brukman, R. C. Mccormick, J. R. Fitzpatrick, J. R. Frederick, E. C. Yang, J. R. Muenzer, N. A. Marotta, M. F. Berry, P. Atluri, Y. J. Woo, C. Suire, N. Brouard, K. Hirschi, P. J. Simmons, E. T. H. Yeh, M. F. Berry, A. J. Engler, Y. J. Woo, T. J. Pirolli, L. T. Bish, V. Jayasankar, K. J. Morine, T. J. Gardner, D. E. Discher, H. L. Sweeney, F. Mark, A. J. Engler, Y. J. Woo, T. J. Pirolli, L. T. Bish, V. Jayasankar, K. J. Morine, and J. Timothy. Mesenchymal stem cell injection after myocardial infarction improves myocardial compliance Regulation and Function of Stem Cells in the Cardiovascular System Mesenchymal stem cell injection after myocardial infarction improves myocardial compliance. Am J Physiol Heart Circ Physiol 290, 6085(February 2006):2196-2203, 2006.

[17] A. D. Bershadsky, N. Q. Balaban, and B. Geiger. Adhesion-dependent cell mechanosensitivity. Annu. Rev. Cell Dev. Biol, 19(1):677-95, nov 2003.

[18] A. Blais, M. Tsikitis, D. Acosta-Alvear, R. Sharan, Y. Kluger, and B. D. Dynlacht. An initial blueprint for myogenic differentiation. Genes \& Development, 19:553-569, 2005.

[19] L. Blanchoin, R. Boujemaa-Paterski, C. Sykes, and J. Plastino. Actin dynamics, architecture, and mechanics in cell motility. Physiol Rev, 94:235-263, 2014.

[20] N. R. Blondheim, Y. S. Levy, T. Ben-Zur, A. Burshtein, T. Cherlow, I. Kan, R. Barzilai, M. BahatStromza, Y. Barhum, S. Bulvik, E. Melamed, and D. Offen. Human Mesenchymal Stem Cells Express Neural Genes, Suggesting a Neural Predisposition. Stem Cells and Development, 15(2):141-164, apr 2006 .

[21] J. Borrego-Pinto, T. Jegou, D. S. Osorio, F. Auradé, M. Gorjánácz, B. Koch, I. W. Mattaj, and E. R. Gomes. Samp1 is a component of TAN lines and is required for nuclear movement. Journal of Cell Science, 125:1099-1105, 2012. 
[22] P. Bossolasco, L. Cova, C. Calzarossa, S. G. Rimoldi, C. Borsotti, G. L. Deliliers, V. Silani, D. Soligo, and E. Polli. Neuro-glial differentiation of human bone marrow stem cells in vitro. Experimental Neurology, 193:312-325, 2005.

[23] J. Brábek, C. T. Mierke, D. Rösel, P. Veselý, and B. Fabry. The role of the tissue microenvironment in the regulation of cancer cell motility and invasion. Cell communication and signaling: CCS, 8:22, 2010 .

[24] D. T. Burnette, S. Manley, P. Sengupta, R. Sougrat, M. W. Davidson, B. Kachar, and J. LippincottSchwartz. A role for actin arcs in the leading-edge advance of migrating cells. Nature Cell Biology, 2011.

[25] D. T. Burnette, L. Shao, C. Ott, A. M. Pasapera, R. S. Fischer, M. A. Baird, C. Der Loughian, H. Delanoe-Ayari, M. J. Paszek, M. W. Davidson, E. Betzig, and J. Lippincott-Schwartz. A contractile and counterbalancing adhesion system controls the 3D shape of crawling cells. Journal of Cell Biology, 205(1):83-96, 2014.

[26] K. Burridge and C. Guilluy. Focal adhesions, stress fibers and mechanical tension. Experimental Cell Research, 343(1):14-20, 2016.

[27] K. Burridge and E. S. Wittchen. The tension mounts: Stress fibers as force-generating mechanotransducers. Journal of Cell Biology, 200(1):9-19, 2013.

[28] A. Buxboim, J. Irianto, J. Swift, A. Athirasala, J.-W. Shin, F. Rehfeldt, and D. E. Discher. Coordinated increase of nuclear tension and lamin-A with matrix stiffness outcompetes lamin-B receptor that favors soft tissue phenotypes. Molecular Biology of the Cell, 28(23):mbc.E17-06-0393, nov 2017.

[29] A. Buxboim, K. Rajagopal, E. Brown, and D. E. Discher. How deeply cells feel: methods for thin gels. J Phys Condens Matter, 22(19), 2010.

[30] A. B. Cadwallader, H. J. Yost, A. Czirok, B. J. Rongish, C. D. Little, B. J. Dzamba, D. W. DeSimone, W. P. Daley, K. M. Yamada, S. Astrof, Y. S. Choi, A. W. Holle, A. J. Engler, X. Zhang, M. P. Sarras Jr, N. A. Mundell, J. R. Jessen, P. J. Roughley, and P. Moffat. Extracellular Matrix in Development. Springer Verlag, Berlin Heidelberg, 2013.

[31] Y. Cai, N. Biais, G. Giannone, M. Tanase, G. Jiang, J. M. Hofman, C. H. Wiggins, P. Silberzan, A. Buguin, B. Ladoux, and M. P. Sheetz. Nonmuscle Myosin IIA-Dependent Force Inhibits Cell Spreading and Drives F-Actin Flow. Biophysical Journal, pages 3907-3920, 2006.

[32] D. Chan, S. R. Lamande, W. G. Cole, and J. F. Bateman. Regulation of procollagen synthesis and processing during ascorbate-induced extracellular matrix accumulation in vitro. Biochem. $J$, 269:175-181, 1990.

[33] R. Chance, A. Prock, and R. Silbey. Molecular fluorescence and energy transfer near interfaces. Advances of Chemical Physics, 37(1):65, 1978.

[34] C.-W. Chang and S. Kumar. Vinculin tension distributions of individual stress fibers within cell-matrix adhesions. Journal of Cell Science, 126(14):3021-3030, 2013.

[35] C. W. Chang and S. Kumar. Differential Contributions of Nonmuscle Myosin II Isoforms and Functional Domains to Stress Fiber Mechanics. Scientific Reports, 5(March):1-13, 2015.

[36] B. P. Chen, Y.-S. S. Li, Y. Zhao, K.-D. D. Chen, S. Li, J. Lao, S. Yuan, J. Y. Shyy, S. Chien, B. P. C Chen, Y. Zhao, K.-D. D. Chen, J. Lao, S. Yuan, J. Y-J Shyy, S. Chien, B. P. C, Y.-S. S. Li, K.-D. D. Chen, S. Li, J. Y-J Shyy, B. P. Chen, Y.-S. S. Li, Y. Zhao, K.-D. D. Chen, S. Li, J. Lao, S. Yuan, J. Y. Shyy, and S. Chien. DNA microarray analysis of gene expression in endothelial cells in response to 24-h shear stress. Physiological genomics, 7(1):55-63, oct 2001. 
[37] G. Chen, A. Yue, Z. Ruan, Y. Yin, R. Wang, Y. Ren, and L. Zhu. Monitoring the biology stability of human umbilical cord-derived mesenchymal stem cells during long-term culture in serum-free medium. Cell and Tissue Banking, 15(4):513-521, 2014.

[38] S. Chetty, F. W. Pagliuca, C. Honore, A. Kweudjeu, A. Rezania, and D. A. Melton. A simple tool to improve pluripotent stem cell differentiation. Nat Methods, 10(60):553-556, 2013.

[39] A. Chizhik, C. Wollnik, D. Ruhlandt, N. Karedla, A. Chizhik, L. Hauke, D. Hähnel, I. Gregor, J. Enderlein, and F. Rehfeldt. Dual-color metal-induced and Förster resonance energy transfer for cell nanoscopy. Molecular Biology of the Cell, 29(7), 2018.

[40] H. Cho, Y.-K. Seo, H.-H. Yoon, S.-M. S.-C. Kim, S.-M. S.-C. Kim, K.-Y. Song, and J.-K. Park. Neural stimulation on human bone marrow-derived mesenchymal stem cells by extremely low frequency electromagnetic fields. Biotechnology Progress, 28(5):1329-1335, sep 2012.

[41] J. Choi, M. L. Costatt, C. S. Mermelsteintt, C. Chagast, S. Holtzert, and H. Holtzert. MyoD converts primary dermal fibroblasts, chondroblasts, smooth muscle, and retinal pigmented epithelial cells into striated mononucleated myoblasts and multinucleated myotubes (myogenesis/myoflbrils/desmin/master switch genes). Developmental Biology, 87:7988-7992, 1990.

[42] Y.-K. Choi, D. Heon Lee, Y.-K. Seo, H. Jung, J.-K. Park, H. Cho, K. Choi, K. Seo, K. Park, D. H. Lee, H. Jung, and H. Cho. Stimulation of Neural Differentiation in Human Bone Marrow Mesenchymal Stem Cells by Extremely Low-Frequency Electromagnetic Fields Incorporated with MNPs. Appl Biochem Biotechnol, 174:1233-1245, 2014.

[43] M. Chojkiers, K. Houglum, J. Solis-Herruzog, and D. A. Brennerl. Stimulation of Collagen Gene Expression by Ascorbic Acid in Cultured Human Fibrcoblasts - A role for liquid peroxidation? THE JOURNAL OF BIOLOGICAL CHEMISTRY, 266(28):16957-16962, 1989.

[44] D. L. Christiansen, E. K. Huang, and F. H. Silver. Assembly of type I collagen: Fusion of fibril subunits and the influence of fibril diameter on mechanical properties. Matrix Biology, 19(5):409-420, sep 2000.

[45] E. Colucci-Guyon, M. Giménez Y Ribotta, T. Maurice, C. Babinet, and A. Privat. Cerebellar defect and impaired motor coordination in mice lacking vimentin. Glia, 25(1):33-43, jan 1999.

[46] T. Company, F. Barros-Becker, P.-Y. Lam, R. Fisher, and A. Huttenlocher. Live imaging reveals distinct modes of neutrophil and macrophage migration within interstitial tissues. Journal of Cell Science, 130(September):3801-3808, 2017.

[47] C. M. Creecy, C. F. O'Neill, B. P. Arulanandam, V. L. Sylvia, C. S. Navara, and R. Bizios. Mesenchymal Stem Cell Osteodifferentiation in Response to Alternating Electric Current. Tissue Engeneering, 19(3/4):467-474, 2013.

[48] E. Cukierman, R. Pankov, D. R. Stevens, and K. M. Yamada. Taking Cell-Matrix Adhesions to the Third Dimension. Science, 294(5547):1708-1712, nov 2001.

[49] M. W. Curtis and B. Russel. Cardiac Tissue Engineering Matthew. J Cardiovasc Nurs., 24(2):1-7, 2009.

[50] R. L. Davis, H. Weintraub, and A. B. Lassar. Expression of a Single Transfected cDNA Converts Fibmblasts to Myoblasts. Cell, 51:987-1000, 1987.

[51] C. M. Denais, R. M. Gilbert, P. Isermann, A. L. McGregor, M. te Lindert, B. Weigelin, P. M. Davidson, P. Friedl, K. Wolf, and J. Lammerding. Nuclear envelope rupture and repair during cancer cell migration. Science (New York, N.Y.), 352(6283):353-8, apr 2016. 
[52] F. Derksen. Master' s Thesis Mechanically Tunable Biomimetic Hyaluronic Acid Based Hydrogels Mechanisch abstimmbare biomimetische Hydrogele auf Basis von Hyaluronsäure. (August), 2015.

[53] F. Derksen. Mechanically Tunable Biomimetic Hyaluronic Acid Based Hydrogels. PhD thesis, Georg-August-Universität Göttingen, 2015.

[54] D. E. Discher, P. Janmey, and Y.-L. Wang. Tissue Cells Feel and Respond to the Stiffness of Their Substrate. Science, 310(5751):1139-1143, nov 2005.

[55] M. Dogterom and G. H. Koenderink. Actin-microtubule crosstalk in cell biology. Nature Reviews Molecular Cell Biology, 2018.

[56] M. Dominici, K. Le Blanc, I. Mueller, I. Slaper-Cortenbach, F. Marini, D. Krause, R. Deans, A. Keating, D. Prockop, and E. Horwitz. Minimal criteria for defining multipotent mesenchymal stromal cells. The International Society for Cellular Therapy position statement. Cytotherapy, $8(4): 315-7,2006$.

[57] A. D. Doyle, N. Carvajal, A. Jin, K. Matsumoto, and K. M. Yamada. Local 3D matrix microenvironment regulates cell migration through spatiotemporal dynamics of contractility-dependent adhesions. Nature communications, 6:8720, nov 2015.

[58] S. Dupont, L. Morsut, M. Aragona, E. Enzo, S. Giulitti, M. Cordenonsi, F. Zanconato, J. Le Digabel, M. Forcato, S. Bicciato, N. Elvassore, and S. Piccolo. Role of YAP/TAZ in mechanotransduction. Nature, 474(7350):179-183, jun 2011.

[59] J. Dyachok, J. A. Sparks, F. Liao, Y. S. Wang, and E. B. Blancaflor. Fluorescent protein-based reporters of the actin cytoskeleton in living plant cells: Fluorophore variant, actin binding domain, and promoter considerations. Cytoskeleton, 71(5):311-327, 2014.

[60] A. Edelstein, N. Amodaj, K. Hoover, R. Vale, and N. Stuurman. Computer Control of Microscopes Using $\mu$ Manager. (October):1-17, 2010.

[61] A. D. Edelstein, M. A. Tsuchida, N. Amodaj, H. Pinkard, R. D. Vale, and N. Stuurman. Advanced methods of microscope control using $\mu$ Manager software. J Biol Methods., 1(2):18, 2015.

[62] B. Eltzner, C. Wollnik, C. Gottschlich, S. Huckemann, and F. Rehfeldt. The Filament Sensor for Near Real-Time Detection of Cytoskeletal Fiber Structures. PLoS ONE, 10(5):1-28, 2015.

[63] J. Enderlein. Single-molecule fluorescence near a metal layer. Chemical Physics, 247(1):1-9, 1999.

[64] J. Enderlein. A theoretical investigation of single-molecule fluorescence detection on thin metallic layers. Biophysical Journal, 78(4):2151-2158, 2000.

[65] A. Engler, L. Bacakova, C. Newman, A. Hategan, M. Griffin, and D. Discher. Substrate Compliance versus Ligand Density in Cell on Gel Responses. Biophysical Journal, 86:617-628, 2004.

[66] A. J. Engler, M. A. Griffin, S. Sen, C. G. Bönnemann, H. L. Sweeney, and D. E. Discher. Myotubes differentiate optimally on substrates with tissue-like stiffness: pathological implications for soft or stiff microenvironments. The Journal of Cell Biology, 166(6):877-887, 2004.

[67] A. J. Engler, S. Sen, H. L. Sweeney, and D. E. Discher. Matrix Elasticity Directs Stem Cell Lineage Specification. Cell, 126(4):677-689, aug 2006.

[68] J. E. Eriksson, T. Dechat, B. Grin, B. Helfand, M. Mendez, H.-M. Pallari, and R. D. Goldman. Introducing intermediate filaments: from discovery to disease. The Journal of Clinical Investigation, 119(7):1763-1771, 2009. 
[69] L. R. Flores, M. C. Keeling, X. Zhang, K. Sliogeryte, and N. Gavara. Lifeact-GFP alters F-actin organization, cellular morphology and biophysical behaviour. Scientific Reports 2019 9:1, 9(1):3241, 2019 .

[70] L. E. Fox, J. Shen, K. Ma, Q. Liu, G. Shi, G. D. Pappas, T. Qu, and J. Cheng. Membrane Properties of Neuron-Like Cells Generated from Adult Human Bone-Marrow-Derived Mesenchymal Stem Cells. Stem Cells and Development, 19(12), 2010.

[71] C. Franz, R. Walczak, S. Yavuz, R. Santarella, M. Gentzel, P. Askjaer, V. Galy, M. Hetzer, I. W. Mattaj, and W. Antonin. MEL-28/ELYS is required for the recruitment of nucleoporins to chromatin and postmitotic nuclear pore complex assembly. EMBO reports, 8(2):165-172, 2007.

[72] E. J. J. Gang, J. A. Jeong, S. H. Hong, S. H. Hwang, S. W. Kim, I. H. Yang, C. Ahn, H. Han, and H. Kim. Skeletal Myogenic Differentiation of Mesenchymal Stem Cells Isolated from Human Umbilical Cord Blood. Stem Cells, 22(4):617-624, jul 2004.

[73] M. L. Gardel, I. C. Schneider, Y. Aratyn-Schaus, and C. M. Waterman. Mechanical Integration of Actin and Adhesion Dynamics in Cell Migration. Annual Review of Cell and Developmental Biology, 2010 .

[74] P. J. Gianakopoulos, V. Mehta, A. Voronova, Y. Cao, Z. Yao, J. E. Coutu, X. Wang, M. S. Waddington, S. J. Tapscott, and I. S. Skerjanc. MyoD Directly Up-regulates Premyogenic Mesoderm Factors during Induction of Skeletal Myogenesis in Stem Cells. The Journal of Biological Chemistry, 286(4):2517-2525, 2010.

[75] J. M. Goffin, P. Pittet, G. Csucs, J. W. Lussi, J. J. Meister, and B. Hinz. Focal adhesion size controls tension-dependent recruitment of $\alpha$-smooth muscle actin to stress fibers. Journal of Cell Biology, 172(2):259-268, 2006.

[76] M. Gordon and M. Prisco. Stem Cells Handbook, volume 21. 2004.

[77] A. Gore, Z. Li, H.-L. Fung, J. Young, S. Agarwal, J. Antosiewicz-Bourget, I. Canto, A. Giorgetti, M. Israel, E. Kiskinis, J.-H. Lee, Y.-H. Loh, P. D. Manos, N. Montserrat, A. D. Panopoulos, S. Ruiz, M. Wilbert, J. Yu, E. F. Kirkness, J. Carlos, I. Belmonte, D. J. Rossi, J. Thomson, K. Eggan, G. Q. Daley, L. S. B. Goldstein, and K. Zhang. Somatic coding mutations in human induced pluripotent stem cells. March, 3(7336):471-63, 2011.

[78] A. N. Gortari, S. Bouchoule, E. Cambril, A. Cattoni, L. Hauke, J. Enderlein, F. Rehfeldt, and A. Yacomotti. Metasurface-based total internal reflection microscopy. Biomedical Optics Express, 11:1967, 42020 .

[79] D. M. Graham, T. Andersen, L. Sharek, G. Uzer, K. Rothenberg, B. D. Hoffman, J. Rubin, M. Balland, J. E. Bear, and K. Burridge. Enucleated cells reveal differential roles of the nucleus in cell migration, polarity, and mechanotransduction. The Journal of cell biology, page jcb.201706097, 2018.

[80] M. A. Griffin, A. J. Engler, T. A. Barber, K. E. Healy, H. L. Sweeney, and D. E. Discher. Patterning, Prestress, and Peeling Dynamics of Myocytes. Biophysical Journal, 86:1209-1222, 2004.

[81] A. M. Handorf, Y. Zhou, M. A. Halanski, and W. J. Li. Tissue stiffness dictates development, homeostasis, and disease progression. Organogenesis, 11(1):1-15, 2015.

[82] A. K. Harris, P. Wild, and D. Stopak. Silicone rubber substrata: a new wrinkle in the study of cell locomotion. Science (New York, N.Y.), 208(4440):177-9, apr 1980.

[83] P. Hasty, A. Bradley, J. H. Morris, D. G. Edmondson, J. M. Venuti, E. N. Olson, and W. H. Klein. Muscle deficiency and neonatal death in mice with a targeted mutation in the myogenin gene. Nature, 364(6437):501-506, aug 1993. 
[84] B. Hinz, G. Celetta, J. J. Tomasek, G. Gabbiani, and C. Chaponnier. Alpha-Smooth Muscle Actin Expression Upregulates Fibroblast Contractile Activity. Molecular Biology of the Cell, 12:2730-2741, 2001.

[85] P. Hotulainen and P. Lappalainen. Stress fibers are generated by two distinct actin assembly mechanisms in motile cells. Journal of Cell Biology, 173(3):383-394, 2006.

[86] N. Huebsch, P. R. Arany, A. S. Mao, D. Shvartsman, O. A. Ali, S. A. Bencherif, J. Rivera-Feliciano, and D. J. Mooney. Harnessing Traction-Mediated Manipulation of the Cell-Matrix Interface to Control Stem Cell Fate. Nat Mater, 9(6):518-526, 2010.

[87] L. Inverardi and C. Ricordi. Tolerance and pancreatic islet transplantation. Philosophical Transactions of the Royal Society B: Biological Sciences, 356(1409):759-765, 2001.

[88] S. Isbaner, N. Karedla, D. Ruhlandt, S. C. Stein, A. Chizhik, I. Gregor, and J. Enderlein. Dead-time correction of fluorescence lifetime measurements and fluorescence lifetime imaging. 24(9):9429-9445.

[89] B. C. Isenberg, P. A. DiMilla, M. Walker, S. Kim, and J. Y. Wong. Vascular smooth muscle cell durotaxis depends on substrate stiffness gradient strength. Biophysical Journal, 97(5):1313-1322, 2009.

[90] A. V. Ivanov, P. B. Kopnin, R. V. Kondratov, V. S. Osovskaya, B. P. Kopnin, and P. M. Chumakov. A p53 mutation is required for stable transformation of REF52 cells by the myc and ras oncogenes. Molecular Biology, 34(2):277-285, 2000.

[91] N. Jain, K. V. Iyer, A. Kumar, and G. V. Shivashankar. Cell geometric constraints induce modular gene-expression patterns via redistribution of HDAC3 regulated by actomyosin contractility. Proceedings of the National Academy of Sciences, 110(28):11349-11354, 2013.

[92] N. Jaiswal, S. E. Haynesworth, A. I. Caplan, and S. P. Bruder. Osteogenic differentiation of purified, culture-expanded human mesenchymal stem cells in vitro. Journal of cellular biochemistry, 64(2):295-312, feb 1997.

[93] P. A. Janmey, P. C. Georges, and S. Hvidt. Basic Rheology for Biologists. In Methods in Cell Biology, pages 1-27. 2007.

[94] R. Janoštiak, J. Brábek, V. Auernheimer, Z. Tatárová, L. A. Lautscham, T. Dey, J. Gemperle, R. Merkel, W. H. Goldmann, B. Fabry, and D. Rösel. Cellular and Molecular Life Sciences CAS directly interacts with vinculin to control mechanosensing and focal adhesion dynamics strongly implicate a crucial role of CaS-vinculin interaction in mechanosensing and focal adhesion dynamics. Cell. Mol. Life Sci, 71:727-744, 2014.

[95] K. A. Jansen, D. M. Donato, H. E. Balcioglu, T. Schmidt, E. H. Danen, and G. H. Koenderink. A guide to mechanobiology: Where biology and physics meet. Biochimica et Biophysica Acta Molecular Cell Research, 1853(11):3043-3052, 2015.

[96] M. Jasnin and A. H. Crevenna. Quantitative Analysis of Filament Branch Orientation in Listeria Actin Comet Tails. Biophysical Journal, 110(4):817-826, 2016.

[97] L. M. Jawerth, S. Münster, D. A. Vader, B. Fabry, and D. A. Weitz. A blind spot in confocal reflection microscopy: The dependence of fiber brightness on fiber orientation in imaging biopolymer networks. Biophysical Journal, 98(3):3-5, 2010.

[98] C. Jayachandran. Molecular dna sensors to measure distribution of cytoskeletal forces, 2019.

[99] N. R. Jørgensen, Z. Henriksen, O. H. Sørensen, and R. Civitelli. Dexamethasone, BMP-2, and 1,25-dihydroxyvitamin $\mathrm{D}$ enhance a more differentiated osteoblast phenotype: validation of an in vitro model for human bone marrow-derived primary osteoblasts. Steroids, 69:219-226, 2004. 
[100] F. P. Jori, M. A. Napolitano, M. A. Melone, M. Cipollaro, A. Cascino, L. Altucci, G. Peluso, A. Giordano, and U. Galderisi. Molecular pathways involved in neural in vitro differentiation of marrow stromal stem cells. Journal of Cellular Biochemistry, 94(4):645-655, mar 2005.

[101] L. Kaestner, A. Scholz, and P. Lipp. Conceptual and technical aspects of transfection and gene delivery. Bioorganic \& Medicinal Chemistry Letters, 25:1171-1176, 2015.

[102] S. Kaliman, C. Jayachandran, F. Rehfeldt, and A.-S. Smith. Novel growth regime of mdck ii model tissues on soft substrates. Biophysical Journal, 106(7):L25-L28, 2014.

[103] P. Kanachanawong, G. Shtengel, A. M. Pasapera, E. B. Ramko, M. W. Davidson, H. H. F. Hess, C. M. Waterman, P. Kanchanawong, G. Shtengel, A. M. Pasapera, E. B. Ramko, M. W. Davidson, H. H. F. Hess, and C. M. Waterman. Nanoscale architecture of integrin-based cell adhesions W. Nature, 468(7323):580-584, 2010.

[104] L. Kassar-Duchossoy, B. Gayraud-Morel, D. Gomès, D. Rocancourt, M. Buckingham, V. Shinin, and S. Tajbakhsh. Mrf4 determines skeletal muscle identity in Myf5:Myod double-mutant mice. Nature, 431(7007):466-471, sep 2004.

[105] E. Kassianidou, C. A. Brand, U. S. Schwarz, and S. Kumar. Geometry and network connectivity govern the mechanics of stress fibers. Proceedings of the National Academy of Sciences, 114(10):2622$2627,2017$.

[106] E. Kassianidou, S. Kumar, and U. States. A biomechanical perspective on stress fiber structure and function. Biochim Biophys Acta, 1853(11 0 0):3065-3074, 2016.

[107] E. Kassianidou, D. Probst, S. Lee, A.-l. Roguet, U. S. Schwarz, S. Kumar, S. Lee, A.-l. Roguet, and U. S. Schwarz. Extracellular Matrix Geometry and Initial Adhesive Position Determine Stress Fiber Network Organization during Cell Spreading Article Extracellular Matrix Geometry and Initial Adhesive Position Determine Stress Fiber Network Organization during Cell Sprea. pages 1897-1909, 2019.

[108] M. C. Keeling, L. R. Flores, A. H. Dodhy, E. R. Murray, and N. Gavara. Actomyosin and vimentin cytoskeletal networks regulate nuclear shape, mechanics and chromatin organization. Scientific Reports, 7(1):1-14, 2017.

[109] K. Kendall, M. Kendall, and F. Rehfeldt. Adhesion of Cells. In Adhesion of Cells, Viruses and Nanoparticles, pages 221-240. Springer, Dordrecht, 2010.

[110] S. B. Khatau, R. J. Bloom, S. Bajpai, D. Razafsky, S. Zang, A. Giri, P. H. Wu, J. Marchand, A. Celedon, C. M. Hale, S. X. Sun, D. Hodzic, and D. Wirtz. The distinct roles of the nucleus and nucleus-cytoskeleton connections in three-dimensional cell migration. Scientific Reports, 2, 2012.

[111] S. B. Khatau, C. M. Hale, P. J. Stewart-Hutchinson, M. S. Patel, C. L. Stewart, P. C. Searson, D. Hodzic, and D. Wirtz. A perinuclear actin cap regulates nuclear shape. PNAS, 106, 2009.

[112] D.-H. Kim, S. B. Khatau, Y. Feng, S. Walcott, S. X. Sun, G. D. Longmore, and D. Wirtz. Actin cap associated focal adhesions and their distinct role in cellular mechanosensing. Scientific Reports, $2(555), 2012$.

[113] S. Kim, O. Honmou, K. Kato, T. Nonaka, K. Houkin, H. Hamada, and J. D. Kocsis. Neural differentiation potential of peripheral blood-and bone- marrow-derived precursor cells. Brain Res., 1123(3):27-33, 2006.

[114] S.-M. Kim, Y.-G. Kim, J.-W. Park, J.-M. Lee, and J.-Y. Suh. The effects of dexamethasone on the apoptosis and osteogenic differentiation of human periodontal ligament cells. J Periodontal Implant Sci, 4343(4), 2013. 
[115] S. W. Kim, Y. H. Bae, and T. Okano. Hydrogels: Swelling, Drug Loading, and Release. Pharmaceutical Research, 9(3):283-290, 1992.

[116] E. Kingham and R. O. C. Oreffo. Embryonic and Induced Pluripotent Stem Cells: Understanding, Creating, and Exploiting the Nano-Niche for Regenerative Medicine. 7(3):1867-1881, 2013.

[117] G. H. Koenderink and E. K. Paluch. Architecture shapes contractility in actomyosin networks. Current Opinion in Cell Biology, 50:79-85, 2018.

[118] H. J. Kong, T. R. Polte, E. Alsberg, and D. J. Mooney. FRET measurements of cell-traction forces and nano-scale clustering of adhesion ligands varied by substrate stiffness. PNAS, 102(12):4300-4305, 2005.

[119] S. F. Konieczny and C. P. Emerson. 5-Azacytidine Induction of Stable Mesodermal Stem Cell Lineages from 1OT1/2 Cells: Evidence for Regulatory Genes Controlling Determination. Cell, 36:791-800, 1984.

[120] G. C. Kopen, D. J. Prockop, and D. G. Phinney. Marrow stromal cells migrate throughout forebrain and cerebellum, and they differentiate into astrocytes after injection into neonatal mouse brains. Cell Biology, 96:10711-10716, 1999.

[121] G. Kudryasheva. Acto-myosin based mechano-sensitivity of cells - comparing human mesenchymal stem cells and differentiated cells. Doktorarbeit, Georg-August-Universität Göttingen, 2017.

[122] S. Kumar, I. Z. Maxwell, A. Heisterkamp, T. R. Polte, T. P. Lele, M. Salanga, E. Mazur, and D. E. Ingber. Viscoelastic Retraction of Single Living Stress Fibers and Its Impact on Cell Shape, Cytoskeletal Organization, and Extracellular Matrix Mechanics. Biophysical Journal, 90:3762-3773, 2006.

[123] M. Kuragano, T. Q. Uyeda, K. Kamijo, Y. Murakami, and M. Takahashi. Different contributions of nonmuscle myosin IIA and IIB to the organization of stress fiber subtypes in fibroblasts. Molecular biology of the cell, 29:mbc.E17-04-0215, 2018.

[124] K. Kurima, M. L. Warman, S. Krishnan, M. Domowicz, R. C. Krueger, A. Deyrup, N. B. Schwartz, R. J. Pelham, and Y.-L. Wang. Cell locomotion and focal adhesions are regulated by substrate flexibility. Cell Biology, 15(94):8681-13661, 1998.

[125] C. M. Kurylo, M. M. Parks, M. F. Juette, B. Zinshteyn, R. B. Altman, J. K. Thibado, C. T. Vincent, and S. C. Blanchard. Endogenous rRNA Sequence Variation Can Regulate Stress Response Gene Expression and Phenotype. Cell Reports, (October), 2018.

[126] T. Kyung Kim and J. H. Eberwine. Mammalian cell transfection: the present and the future. 2010.

[127] F. Lecanda, L. V. Avioli, and S. L. Cheng. Regulation of bone matrix protein expression and induction of differentiation of human osteoblasts and human bone marrow stromal cells by bone morphogenetic protein-2. Journal of cellular biochemistry, 67(3):386-96, dec 1997.

[128] S. Lee, E. Kassianidou, and S. Kumar. Actomyosin stress fiber subtypes have unique viscoelastic properties and roles in tension generation. Molecular biology of the cell, pages 1-42, 2018.

[129] W. C. Lee, H. Shi, Z. Poon, L. M. Nyan, T. Kaushik, G. V. Shivashankar, J. K. Y. Chan, C. T. Lim, J. Han, and K. J. Van Vliet. Multivariate biophysical markers predictive of mesenchymal stromal cell multipotency. Proceedings of the National Academy of Sciences, 111(42):E4409-E4418, 2014.

[130] S. Leporatti, D. Vergara, A. Zacheo, V. Vergaro, G. Maruccio, R. Cingolani, and R. Rinaldi. Cytomechanical and topological investigation of MCF-7 cells by scanningforce microscopy. Nanotechnology, $20(5), 2009$. 
[131] I. Levental, P. C. Georges, and P. A. Janmey. Soft biological materials and their impact on cell function. Soft Matter, 3(3):299-306, feb 2007.

[132] Ż. Lewandowska, P. Piszczek, A. Radtke, T. Jȩdrzejewski, W. Kozak, and B. Sadowska. The evaluation of the impact of titania nanotube covers morphology and crystal phase on their biological properties. In Journal of Materials Science: Materials in Medicine, volume 26, page 163. Springer US, apr 2015.

[133] L. S. Liu, J. Kost, F. Yan, and R. C. Spiro. Hydrogels from biopolymer hybrid for biomedical, food, and functional food applications. Polymers, 4(2):997-1011, 2012.

[134] C.-M. Lo, H.-B. Wang, M. Dembo, and Y.-L. Wang. Cell Movement Is Guided by the Rigidity of the Substrate, 2000.

[135] M. L. Lombardi, D. E. Jaalouk, C. M. Shanahan, B. Burke, K. J. Roux, and J. Lammerding. The interaction between nesprins and sun proteins at the nuclear envelope is critical for force transmission between the nucleus and cytoskeleton. The Journal of biological chemistry, 286(30):26743-53, jul 2011.

[136] F. C. Mackintosh, . J. Kas, and P. A. Janmey'. NUMBER 24 PHYSICAL REVIEW LETTERS 11. Technical report, 1995.

[137] E. Makhija, A. Jagielska, L. Zhu, A. C. Bost, W. Ong, S. Y. Chew, G. V. Shivashankar, and K. J. Van Vliet. Mechanical Strain Alters Cellular and Nuclear Dynamics at Early Stages of Oligodendrocyte Differentiation. Frontiers in Cellular Neuroscience, 12(March):1-12, 2018.

[138] K. Malec, J. Góralska, M. Hubalewska-Mazgaj, P. Głowacz, M. Jarosz, P. Brzewski, G. D. Sulka, M. Jaskuła, I. Wybrańska, M. Hubalewska, P. Głowacz, and M. Jarosz. Effects of nanoporous anodic titanium oxide on human adipose derived stem cells. International Journal of Nanomedicine, 11:5349-5360, 2016.

[139] N. P. Mastroyiannopoulos, P. Nicolaou, M. Anayasa, J. B. Uney, and L. A. Phylactou. DownRegulation of Myogenin Can Reverse Terminal Muscle Cell Differentiation. PLoS ONE, 7(1), 2012 .

[140] S. H. McBride and M. L. Knothe Tate. Modulation of Stem Cell Shape and Fate A: The Role of Density and Seeding Protocol on Nucleus Shape and Gene Expression. Tissue Engineering Part A, 14(9):1561-1572, 2008.

[141] R. Melzi, B. Antonioli, A. Mercalli, M. Battaglia, A. Valle, S. Pluchino, R. Galli, V. Sordi, E. Bosi, G. Martino, E. Bonifacio, C. Doglioni, and L. Piemonti. Co-Graft of Allogeneic Immune Regulatory Neural Stem Cells (NPC) and Pancreatic Islets Mediates Tolerance, while Inducing NPC-Derived Tumors in Mice. PLosONE, 5(4), 2010.

[142] T. Metzger, V. Gache, M. Xu, B. Cadot, E. S. Folker, B. E. Richardson, E. R. Gomes, and M. K. Baylies. MAP and Kinesin dependent nuclear positioning is required for skeletal muscle function. Nature, 487(7392):120-124, 2012.

[143] S. Munevar, Y.-L. Wang, and M. Dembo. Traction Force Microscopy of Migrating Normal and H-ras Transformed 3T3 Fibroblasts. Biophysical Journal, 80:1744-1757, 2001.

[144] L. N. Munsie, N. Caron, C. R. Desmond, and R. Truant. Lifeact cannot visualize some forms of stress-induced twisted f-actin. Nature Methods, 6(5):317-317, may 2009.

[145] P. Nghe, S. Boulineau, S. Gude, P. Recouvreux, J. S. van Zon, and S. J. Tans. Microfabricated Polyacrylamide Devices for the Controlled Culture of Growing Cells and Developing Organisms. PLoS ONE, 8(9):1-11, 2013. 
[146] T. A. Nieminen, G. Kn??ner, N. R. Heckenberg, H. Rubinsztein-Dunlop, G. Knöner, N. R. Heckenberg, H. Rubinsztein-Dunlop, G. Kn??ner, N. R. Heckenberg, H. Rubinsztein-Dunlop, G. Knöner, N. R. Heckenberg, and H. Rubinsztein-Dunlop. Physics of Optical Tweezers. Methods in Cell Biology, 82(06):207-236, 2007.

[147] P. W. Oakes, Y. Beckham, J. Stricker, and M. L. Gardel. Tension is required but not sufficient for focal adhesion maturation without a stress fiber template. Journal of Cell Biology, 196(3):363-374, 2012.

[148] Y. Oda, K. Tani, T. Kanei, T. Haraguchi, K. Itamoto, H. Nakazawa, and Y. Taura. Characterization of neuron-like cells derived from canine bone marrow stromal cells. Vet Res Commun, 37:133-138, 2013.

[149] S. Oh and S. Jin. Titanium oxide nanotubes with controlled morphology for enhanced bone growth. Materials Science and Engineering: C, 26(8):1301-1306, sep 2006.

[150] R. K. Okolicsanyi, E. T. Camilleri, L. E. Oikari, C. Yu, S. M. Cool, A. J. Van Wijnen, L. R. Griffiths, and L. M. Haupt. Human mesenchymal stem cells retain multilineage differentiation capacity including neural marker expression after extended in vitro expansion. PLoS ONE, 10(9):1-29, 2015.

[151] E. N. Olson and A. Nordheim. Linking actin dynamics and gene transcription to drive cellular motile functions. 11(5):353-365, 2011.

[152] N. A. Olszok. The influence of dna damage on the mechanics of the nucleus, 2019.

[153] R. Opik, A. Hunt, A. Ristolainen, P. M. Aubin, and M. Kruusmaa. Development of high fidelity liver and kidney phantom organs for use with robotic surgical systems. Proceedings of the IEEE RAS and EMBS International Conference on Biomedical Robotics and Biomechatronics, (June):425-430, 2012.

[154] L. M. Owen, A. S. Adhikari, M. Patel, P. Grimmer, N. Leijnse, M. C. Kim, J. Notbohm, C. Franck, and A. R. Dunn. A cytoskeletal clutch mediates cellular force transmission in a soft, three-dimensional extracellular matrix. Molecular Biology of the Cell, 28(14):1959-1974, 2017.

[155] E. K. Paluch, C. M. Nelson, N. Biais, B. Fabry, J. Moeller, B. L. Pruitt, C. Wollnik, G. Kudryasheva, F. Rehfeldt, and W. Federle. Mechanotransduction: use the force(s) Four centuries of cells and mechanical forces. BMC Biology, 13(47), 2015.

[156] S. Park, D. Koch, R. Cardenas, J. Käs, and C. K. Shih. Cell Motility and Local Viscoelasticity of Fibroblasts. Biophysical Journal, 89:4330-4342, 2005.

[157] M. J. Paszek, N. Zahir, K. R. Johnson, J. N. Lakins, G. I. Rozenberg, A. Gefen, C. A. ReinhartKing, S. S. Margulies, M. Dembo, D. Boettiger, D. A. Hammer, and V. M. Weaver. Tensional homeostasis and the malignant phenotype. Cancer Cell, 8(3):241-254, 2005.

[158] S. Patel, S. Y. Fok, H. Stefen, T. Tomanić, E. Parić, R. Herold, M. Brettle, A. Djordjevic, and T. Fath. Functional characterisation of filamentous actin probe expression in neuronal cells. PLoS ONE, 12(11):1-18, 2017.

[159] R. J. Pelham, Y.-L. Wang, R. J. Wang, Yu-Li, Pelham, R. J. Pelham, and Y.-L. Wang. Cell locomotion and focal adhesions are regulated by substrate flexibility. Proceedings of the American Thoracic Society, 94:13661-13665, 1997.

[160] S. Pellegrin and H. Mellor. Actin stress fibres. Journal of Cell Science, 120(20):3491-3499, 2007.

[161] K. Phadwal, S. W. Alexander, and K. S. Anna. Tightrope act: autophagy in stem cell renewal, differentiation, proliferation, and aging. Cell. Mol. Life Sci., 70:89-103, 2013. 
[162] L. Pieuchot, J. Marteau, A. Guignandon, T. Dos Santos, I. Brigaud, P.-F. Chauvy, T. Cloatre, A. Ponche, T. Petithory, P. Rougerie, M. Vassaux, J.-L. Milan, N. T. Wakhloo, A. Spangenberg, M. Bigerelle, and K. Anselme. Curvotaxis directs cell migration through cell-scale curvature landscapes. Nature Communications, 9(3995):14, 2018.

[163] M. Prabhune. DNA-based molecular force sensors in cytoskeletal networks and cells DNA-based molecular force sensors in cytoskeletal networks and cells. $\mathrm{PhD}$ thesis.

[164] M. Prabhune, F. Rehfeldt, and C. F. Schmidt. Molecular force sensors to measure stress in cells. Journal of Physics D: Applied Physics, 50(23):233001, jun 2017.

[165] Q. Qian, H. Qian, X. Zhang, W. Zhu, Y. Yan, S. Ye, X. Peng, W. Li, Z. Xu, L. Sun, and W. Xu. 5-Azacytidine induces cardiac differentiation of human umbilical cord-derived mesenchymal stem cells by activating extracellular regulated Kinase. Stem Cells and Development, 21(1):67-75, 2012.

[166] X.-C. Qiu, H. Jin, R.-Y. Zhang, Y. Ding, X. Zeng, B.-Q. Lai, E.-A. Ling, J.-L. Wu, and Y.-S. Zeng. Donor mesenchymal stem cell-derived neural-like cells transdifferentiate into myelin-forming cells and promote axon regeneration in rat spinal cord transection. Stem Cell Research 83 Therapy, $6,2012$.

[167] N. B. M. Rafiq, Y. Nishimura, S. V. Plotnikov, V. Thiagarajan, Z. Zhang, S. Shi, M. Natarajan, V. Viasnoff, P. Kanchanawong, G. E. Jones, and A. D. Bershadsky. A mechano-signalling network linking microtubules, myosin IIA filaments and integrin-based adhesions. Nature Materials, 18(6):638-649, 2019.

[168] A. D. Rakić, A. B. Djurišić, J. M. Elazar, and M. L. Majewski. Optical properties of metallic films for vertical-cavity optoelectronic devices. Applied Optics, 37(22), Aug. 1998.

[169] F. Rehfeldt, A. E. X. A. E. X. A. E. Brown, M. Raab, S. Cai, A. L. Zajac, D. E. Discher, A. Zemel, D. E. Discher, S. Thrun, M. P. Lutolf, H. M. Blau, D. A. Hammer, V. M. Weaver, A. Zemel, D. E. Discher, S. Thrun, M. P. Lutolf, H. M. Blau, D. A. Hammer, and V. M. Weaver. Integrative Biology Hyaluronic acid matrices show matrix stiffness in 2D and 3D dictates cytoskeletal order and myosin-II phosphorylation within stem cells w z. Integrative Biology, 4(4):422-430, apr 2012.

[170] B. A. B. A. B. A. B. A. Reynolds, S. Weiss, W. S. Reynolds BA, W. S. Reynolds BA, B. A. B. A. B. A. B. A. Reynolds, and S. Weiss. Generation of neurons and astrocytes from isolated cells of the adult mammalian central nervous system. Science (New York, N.Y.), 255(5052):1707-10, mar 1992.

[171] D. J. Rickard, T. A. Sullivan, B. J. Shenker, P. S. Leboy, and I. Kazdan. Induction of Rapid Osteoblast Differentation in Rat Bone Marrow Stromal Cell Cultures by Dexamethasone and BMP-2. Developmental Biology, 161:218-228, 1994.

[172] J. Riegler, M. Tiburcy, A. Ebert, E. Tzatzalos, U. Raaz, O. J. Abilez, Q. Shen, N. G. Kooremann, E. Neofytou, V. C. Chen, M. Wang, T. Meyer, P. S. Tsao, A. J. Conolly, L. A. Couture, J. D. Gold, W. H. Zimmermann, J. C. Wu, M. P. Snyder, R. A. Kellogg, and J. Dunn. Linking Genetic Variants With Dynamic Phenotype Viewpoints Personal Omics for Precision Health. Circ Res, 122:720-730, 2015.

[173] J. P. Rodríguez, M. González, S. Ríos, and V. Cambiazo. Cytoskeletal organization of human mesenchymal stem cells (MSC) changes during their osteogenic differentiation. Journal of Cellular Biochemistry, 93(4):721-731, 2004.

[174] H. Rogge, N. Artelt, N. Endlich, and K. Endlich. Automated segmentation and quantification of actin stress fibres undergoing experimentally induced changes. Journal of Microscopy, 00(0):1-12, 2017. 
[175] P. Roy, S. Berger, and P. Schmuki. TiO 2 nanotubes: Synthesis and applications. Angewandte Chemie - International Edition, 50(13):2904-2939, 2011.

[176] J. Sanchez-Ramos, S. Song, F. Cardozo-Pelaez, C. Hazzi, T. Stedeford, A. Willing, T. B. Freeman, S. Saporta, W. Janssen, N. Patel, D. R. Cooper, and P. R. Sanberg. Adult Bone Marrow Stromal Cells Differentiate into Neural Cells in Vitro. Experimental Neurology, 164:247-256, 2000.

[177] M. Schilling. Rheology of Hydrogels Based on Chemically Modified Hyaluronic Acid. 2019.

[178] F. Schlosser, F. Rehfeldt, and C. F. Schmidt. Force fluctuations in three-dimensional suspended fibroblasts. Philosophical Transactions of the Royal Society B: Biological Sciences, page 8, 2017.

[179] N. Schulze, M. Graessl, A. Blancke Soares, M. Geyer, L. Dehmelt, and P. Nalbant. FHOD1 regulates stress fiber organization by controlling the dynamics of transverse arcs and dorsal fibers. Journal of Cell Science, 127(7):1379-1393, 2014.

[180] N. J. Sebire and M. Malone. Myogenin and MyoD1 expression in paediatric rhabdomyosarcomas. $J$ Clin Pathol, 56:412-416, 2003.

[181] L. Shahriyari and N. L. Komarova. Symmetric vs. Asymmetric Stem Cell Divisions: An Adaptation against Cancer? PLoS ONE, 8(10), 2013.

[182] A. M. J. Shapiro and J. R. T. Lakey. Future Trends in Islet Cell Transplantation. Diabetes Technology \& Therapeutics, 2(3):449-452, oct 2000.

[183] T. Shimi, K. Pfleghaar, S.-I. Kojima, C.-G. Pack, I. Solovei, A. E. Goldman, S. A. Adam, D. K. Shumaker, M. Kinjo, T. Cremer, and R. D. Goldman. The A-and B-type nuclear lamin networks: microdomains involved in chromatin organization and transcription. Genes $\& 3$ Development, 22:3409$3421,2008$.

[184] Y. Shimizu, T. Kihara, S. M. A. Haghparast, S. Yuba, and J. Miyake. Simple display system of mechanical properties of cells and their dispersion. PLoS ONE, 7(3), 2012.

[185] G. Shivashankar. Mechanosignaling to the Cell Nucleus and Gene Regulation. Annual Review of Biophysics, 40(1):361-378, jun 2011.

[186] V. I. Sikavitsas, J. S. Temenoff, and A. G. Mikos. Biomaterials and bone mechanotransduction. Biomaterials, 22(19):2581-2593, 2001.

[187] D. N. Simon and K. L. Wilson. The nucleoskeleton as a genome-associated dynamic 'network of networks'. Nature Reviews Molecular Cell Biology, 12(11):695-708, oct 2011.

[188] A. Singh, P. K. Sharma, V. K. Garg, and G. Garg. Hydrogels : A review. International Journal of Pharmaceutical Sciences Review and Research, 4(2):97-105, 2010.

[189] P. P. Singh, J. L. Hawthorne, C. A. Davis, and O. A. Quintero. Permeabilization activated reduction in fluorescence: A novel method to measure kinetics of protein interactions with intracellular structures. Cytoskeleton, 73(6):271-285, 2016.

[190] B. Sjöblom, A. Salmazo, and K. Djinović-Carugo. $\alpha$-Actinin structure and regulation. Cellular and Molecular Life Sciences, 65(17):2688-2701, 2008.

[191] K. Sliogeryte, S. D. Thorpe, Z. Wang, C. L. Thompson, N. Gavara, and M. M. Knight. Differential effects of LifeAct-GFP and actin-GFP on cell mechanics assessed using micropipette aspiration. Journal of Biomechanics, 49:310-317, 2016.

[192] M. A. Smith, E. Blankman, M. L. Gardel, L. Luettjohann, C. M. Waterman, and M. C. Beckerle. A Zyxin-Mediated Mechanism for Actin Stress Fiber Maintenance and Repair. Dev Cell, 19(3):365-376, 2010. 
[193] C. J. Sobers, S. E. Wood, and M. Mrksich. A gene expression-based comparison of cell adhesion to extracellular matrix and rgd-terminated monolayers. Biomaterials, 52:385-394, 2015.

[194] V. Sordi, R. Melzi, A. Mercalli, R. Formicola, C. Doglioni, F. Tiboni, G. Ferrari, R. Nano, K. Chwalek, E. Lammert, E. Bonifacio, and L. Piemonti. Mesenchymal cells appearing in pancreatic tissue culture are bone marrow-derived stem cells with the capacity to improve transplanted islet function. Stem Cells, 28(1):140-151, 2010.

[195] J. Steinwachs, C. Metzner, K. Skodzek, N. Lang, I. Thievessen, C. Mark, S. Münster, K. E. Aifantis, and B. Fabry. Three-dimensional force microscopy of cells in biopolymer networks. Nature Methods, 13(2):171-176, 2016.

[196] R. M. Stewart, A. E. Zubek, K. A. Rosowski, S. M. Schreiner, V. Horsley, and M. C. King. Nuclear-cytoskeletal linkages facilitate cross talk between the nucleus and intercellular adhesions. The Journal of cell biology, 209(3):403-18, may 2015.

[197] C. Storm, J. J. Pastore, F. C. MacKintosh, T. C. Lubensky, and P. A. Janmey. Nonlinear elasticity in biological gels. Nature, 435(7039):191-194, may 2005.

[198] N. Stuurman, A. D. Edelstein, N. Amodaj, K. H. Hoover, and R. D. Vale. Computer Control of Microscopes using $\mu$ Manager. Curr Protoc Mol Biol, 2010.

[199] R. Sunyer, A. J. Jin, R. Nossal, and D. L. Sackett. Fabrication of Hydrogels with Steep Stiffness Gradients for Studying Cell Mechanical Response. PLoS ONE, 7(10), 2012.

[200] A. Supokawej, P. Kheolamai, K. Nartprayut, Y. U-pratya, S. Manochantr, M. Chayosumrit, and S. Issaragrisil. 5-Azasitidin Tedavisi Sonrasi\{Dotless\}Nda Mezenkimal Kök Hücrelerinde Kardiyojenik Ve Miyojenik Gen Sunumu. Turkish Journal of Hematology, 30(2):115-121, 2013.

[201] J. Swift, I. L. Ivanovska, A. Buxboim, T. Harada, P. C. D. P. Dingal, J. Pinter, J. D. Pajerowski, K. R. Spinler, J. W. Shin, M. Tewari, F. Rehfeldt, D. W. Speicher, and D. E. Discher. Nuclear Lamin-A Scales with Tissue Stiffness and Enhances Matrix-Directed Differentiation. Science, 341:17, 2013.

[202] K. Tanner, A. Boudreau, M. J. Bissell, and S. Kumar. Dissecting regional variations in stress fiber mechanics in living cells with laser nanosurgery. Biophysical Journal, 99(9):2775-2783, 2010.

[203] S. J. Tapscott, R. L. Davis, M. J. Thayer, P. F. Cheng, H. Weintraub, and A. B. Lassar. MyoD1: a nuclear phosphoprotein requiring a Myc homology region to convert fibroblasts to myoblasts. Science (New York, N.Y.), 242(4877):405-11, oct 1988.

[204] Y. H. Tee, T. Shemesh, V. Thiagarajan, R. F. Hariadi, K. L. Anderson, C. Page, N. Volkmann, D. Hanein, S. Sivaramakrishnan, M. M. Kozlov, and A. D. Bershadsky. Cellular chirality arising from the self-organization of the actin cytoskeleton. Nature Cell Biology, 17(4):445-457, 2015.

[205] K. Thakar, C. K. May, A. Rogers, and C. W. Carroll. Opposing roles for distinct LINC complexes in regulation of the small GTPase RhoA. Molecular biology of the cell, 28(1):182-191, jan 2017.

[206] D. Thirabanjasak, K. Tantiwongse, and P. S. Thorner. Angiomyeloproliferative lesions following autologous stem cell therapy. Journal of the American Society of Nephrology : JASN, 21(7):1218-22, jul 2010.

[207] S. Tojkander, G. Gateva, A. Husain, R. Krishnan, and P. Lappalainen. Generation of contractile actomyosin bundles depends on mechanosensitive actin filament assembly and disassembly. eLife, 4(DECEMBER2015):1-28, 2015.

[208] S. Tojkander, G. Gateva, and P. Lappalainen. Actin stress fibers - assembly, dynamics and biological roles. Journal of Cell Science, 125:1855-1864, 2012. 
[209] P. Tropel, N. Platet, J.-C. Platel, D. Noël, M. Albrieux, A.-L. Benabid, and F. Berger. Functional Neuronal Differentiation of Bone Marrow-Derived Mesenchymal Stem Cells. Stem Cells, 24(12):28682876 , dec 2006.

[210] J. R. Tse and A. J. Engler. Stiffness gradients mimicking in vivo tissue variation regulate mesenchymal stem cell fate. PLoS ONE, 6(1), 2011.

[211] Q. Tseng, I. Wang, E. Duchemin-Pelletier, A. Azioune, N. Carpi, J. Gao, O. Filhol, M. Piel, M. Théry, and M. Balland. A new micropatterning method of soft substrates reveals that different tumorigenic signals can promote or reduce cell contraction levels. Lab on a Chip, 11(13):2231-2240, 2011.

[212] S. Tudies, C. Fotino, C. Ricordi, V. Lauriola, R. Alejandro, A. Pileggi, S. Tudies, C. Fotino, C. Ricordi, V. Lauriola, R. Alejandro, and A. Pileggi. Bone marrow-derived stem cell transplantation for the treatment of insulin-dependent diabetes. The review of diabetic studies : RDS, 7(2):144-157, 2010.

[213] I. Ullah, R. B. Subbarao, and G. J. Rho. Human mesenchymal stem cells - current trends and future prospective. Bioscience Reports, 35(2):1-18, 2015.

[214] T. Vallenius. Actin stress fibre subtypes in mesenchymal-migrating cells. Open Biology, 2013.

[215] G. Villari, A. Jayo, J. Zanet, B. Fitch, B. Serrels, M. Frame, B. M. Stramer, B. T. Goult, and M. Parsons. A direct interaction between fascin and microtubules contributes to adhesion dynamics and cell migration. Journal of cell science, 128(24):4601-4614, 2015.

[216] V. Vogel and M. Sheetz. Local force and geometry sensing regulate cell functions. Nature Reviews Molecular Cell Biology, 7(4):265-275, apr 2006.

[217] J. H. C. Wang and J. S. Lin. Cell traction force and measurement methods. Biomechanics and Modeling in Mechanobiology, 6(6):361-371, 2007.

[218] N. Wang, J. D. Tytell, and D. E. Ingber. Mechanotransduction at a distance: mechanically coupling the extracellular matrix with the nucleus. Nature Reviews Molecular Cell Biology, 10(1):75-82, jan 2009.

[219] E. Warmt, Y. Nematbakhsh, K. T. Pang, C. T. Lim, H. Kubitschke, J. Schnauss, K. D. Nnetu, E. Warmt, R. Stange, J. Kaes, Y. Nematbakhsh, K. T. Pang, and C. T. Lim. Actin and microtubule networks contribute differently to cell response for small and large strains Actin and microtubule networks contribute differently to cell response for small and large strains. pages 0-13, 2017.

[220] H. Weintraub, S. J. Tapscott, R. L. Davis, M. J. Thayer, M. A. Adam, A. B. Lassar, and A. D. Miller. Activation of muscle-specific genes in pigment, nerve, fat, liver, and fibroblast cell lines by forced expression of MyoD (muscle regulatory gene/MyoD retrovirus). Developmental Biology, 86:5434-5438, 1989.

[221] J. H. Wen, L. G. Vincent, A. Fuhrmann, Y. S. Choi, K. Hribar, H. Taylor-Weiner, S. Chen, and A. J. Engler. Interplay of Matrix Stiffness and Protein Tethering in Stem Cell Differentiation. Nat Mater., 13(10):979-987, 2014.

[222] M. H. Wigler and I. Bernard Weinstein. A preparative method for obtaining enucleated mammalian cells. Biochemical and Biophysical Research Communications, 63(3):669-674, 1975.

[223] S. Wislet-Gendebien, G. Hans, P. Leprince, J.-M. Rigo, G. Moonen, and B. Rogister. Plasticity of Cultured Mesenchymal Stem Cells: Switch from Nestin-Positive to Excitable Neuron-Like Phenotype. Stem Cells, 23(3):392-402, mar 2005.

[224] K. Wolf, M. Te Lindert, M. Krause, S. Alexander, J. Te Riet, A. L. Willis, R. M. Hoffman, C. G. Figdor, S. J. Weiss, and P. Friedl. Physical limits of cell migration: control by ECM space and nuclear deformation and tuning by proteolysis and traction force. The Journal of cell biology, 201(7):1069-84, jun 2013. 
[225] C. Wollnik. Structure and dynamics of stress fibers in adult stem cells. 2016.

[226] C. Wong and T. Stearns. Centrosome number is controlled by a centrosome- intrinsic block to reduplication. NATURE CELL BIOLOGY, 5(6), 2003.

[227] J. Y. Wong, A. Velasco, P. Rajagopalan, Q. Pham, G.-c. Hydrogels, J. Y. Wong, A. Velasco, P. Rajagopalan, and Q. Pham. Directed movement of vascular smooth muscle cells on gradientcompliant hydrogels. Langmuir, 19(5):1908-1913, mar 2003.

[228] C. Wu, S. B. Asokan, M. E. Berginski, E. M. Haynes, N. E. Sharpless, J. D. Griffith, S. M. Gomez, and J. E. Bear. Arp2/3 is critical for lamellipodia and response to extracellular matrix cues but is dispensable for chemotaxis. Cell, 148(5):973-87, mar 2012.

[229] L. Xu, S. R. Gutbrod, A. P. Bonifas, Y. Su, M. S. Sulkin, N. Lu, H. J. Chung, K. I. Jang, Z. Liu, M. Ying, C. Lu, R. C. Webb, J. S. Kim, J. I. Laughner, H. Cheng, Y. Liu, A. Ameen, J. W. Jeong, G. T. Kim, Y. Huang, I. R. Efimov, and J. A. Rogers. 3D multifunctional integumentary membranes for spatiotemporal cardiac measurements and stimulation across the entire epicardium. Nature Communications, 5, 2014.

[230] T. Xu, C. Langouras, M. A. Koudehi, B. E. Vos, N. Wang, G. H. Koenderink, X. Huang, and D. Vavylonis. Automated Tracking of Biopolymer Growth and Network Deformation with TSOAX. bioRxiv preprint, pages 1-21, 2018.

[231] T. Xu, D. Vavylonis, F. C. Tsai, G. H. Koenderink, W. Nie, E. Yusuf, I. J. Lee, J. Q. Wu, and X. Huang. SOAX: A software for quantification of 3D biopolymer networks. Scientific Reports, 5, 2015.

[232] Y. L. Yang and L. J. Kaufman. Rheology and confocal reflectance microscopy as probes of mechanical properties and structure during collagen and collagen/hyaluronan self-assembly. Biophysical Journal, 96(4):1566-1585, 2009.

[233] Y.-l. L. Yang, S. Motte, and L. J. Kaufman. Pore size variable type I collagen gels and their interaction with glioma cells. Biomaterials, 31(21):5678-5688, jul 2010.

[234] N. Zaari, P. Rajagopalan, S. K. Kim, A. J. Engler, J. Y. Wong, N. Zaari, P. Rajagopalan, S. K. Kim, A. J. Engler, J. Y. Wong, N. Zaari, P. Rajagopalan, S. K. Kim, A. J. Engler, and J. Y. Wong. Photopolymerization in Microfluidic Gradient Generators: Microscale Control of Substrate Compliance to Manipulate Cell Response. Advanced Materials, 16(23-24):2133-2137, dec 2004.

[235] M. H. Zaman, L. M. Trapani, A. L. Sieminski, D. MacKellar, H. Gong, R. D. Kamm, A. Wells, D. A. Lauffenburger, and P. Matsudaira. Migration of tumor cells in 3D matrices is governed by matrix stiffness along with cell-matrix adhesion and proteolysis. Proceedings of the National Academy of Sciences, 103(29):10889-10894, 2006.

[236] E. Zamir and B. Geiger. Molecular complexity and dynamics of cell-matrix adhesions. Journal of Cell Science, 114:3583-3590, 2001.

[237] R. Zeng, L.-W. Wang, Z.-B. Hu, W.-T. Guo, J.-S. Wei, H. Lin, X. Sun, L.-X. Chen, and L.-J. Yang. Differentiation of Human Bone Marrow Mesenchymal Stem Cells into Neuron-Like Cells In Vitro. Spine, 36(13):997-1005, jun 2011.

[238] X. Zeng, X.-C. Qiu, Y.-H. Ma, J.-J. Duan, Y.-F. Chen, H.-Y. Gu, J.-M. Wang, E.-A. Ling, J.-L. $\mathrm{Wu}, \mathrm{W}$. Wu, and Y.-S. Zeng. Integration of donor mesenchymal stem cell-derived neuron-like cells into host neural network after rat spinal cord transection. Biomaterials, 53:184-201, 2015.

[239] X. Zhang, K. Lei, X. Yuan, X. Wu, Y. Zhuang, T. Xu, R. Xu, and M. Han. SUN1/2 and Syne/Nesprin-1/2 complexes connect centrosome to the nucleus during neurogenesis and neuronal migration in mice. oct 2009. 
[240] Y. Zhang, Y. Chu, W. Shen, and Z. Dou. Effect of 5-azacytidine induction duration on differentiation of human first-trimester fetal mesenchymal stem cells towards cardiomyocyte-like cells. Interactive Cardio Vascular and Thoracic Surgery, 9:943-946, 2009.

[241] Z. Zhang, Y. Nishimura, P. Kanchanawong, and J. Lippincott-schwartz. Extracting microtubule networks from superresolution single-molecule localization microscopy data. Molecular Biology of the Cell, 28:333-345, 2017.

[242] X. Zheng, C. Bi, M. Brooks, and D. Hage. Mechanical Integration of Actin and Adhesion Dynamics in Cell Migration. Annu Rev Cell Dev Biol. 2010, 26:315-333, 2010.

[243] P. Zorlutuna, N. Annabi, G. Camci-Unal, M. Nikkah, J. M. Cha, J. Nichol, A. Manbachi, H. Bae, S. Chen, and A. Khademhosseini. Microfabricated Biomaterials for Engineering 3D Tissues Dr. Adv Mater., 24(14):1782-1804, 2012. 